\title{
Nuclear Effects in Neutrino Detection
}

\author{
By \\ Steven James Gardiner \\ DisSERTATION
}

Submitted in partial satisfaction of the requirements for the degree of

Doctor of Philosophy

in

Physics

in the

Office of Graduate Studies

of the

UNIVERSITY OF CALIFORNIA

DAVIS

Approved:

Robert Svoboda, Chair

Michael Mulhearn

Ramona Vogt

Committee in Charge

2018 
Dedicated to Lindsey, Elizabeth, and Jared 


\section{Contents}

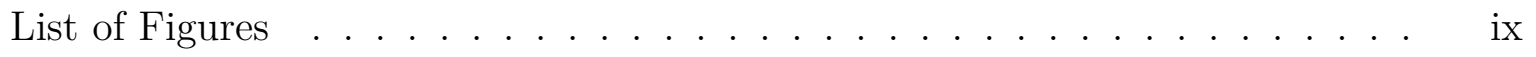

List of Tables . . . . . . . . . . . . . . . . . . . xxiv

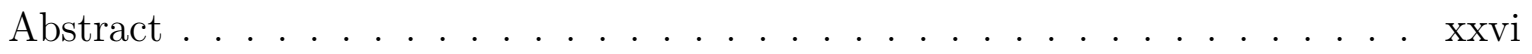

Acknowledgments . . . . . . . . . . . . . . . . . . xxviii

1 Introduction $\quad 1$

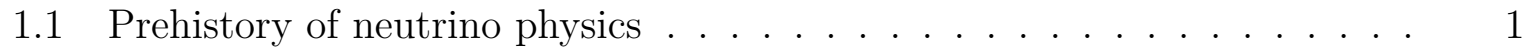

1.2 The trouble with beta decay . . . . . . . . . . . . . . 3

1.3 Discovery of the three neutrino flavors $\ldots \ldots \ldots \ldots \ldots$

1.4 Neutrino interactions . . . . . . . . . . . . . . . . . . . . . . 6

1.5 Neutrino oscillations . . . . . . . . . . . . . . . . 9

1.6 Neutrino masses . . . . . . . . . . . . . . . . . . . . . . . . . 13

1.6.1 Dirac masses . . . . . . . . . . . . . . . . . . 14

1.6 .2 Majorana masses . . . . . . . . . . . . . . . . 15

1.7 Neutrino detection techniques $\ldots \ldots \ldots \ldots$

1.7.1 Water Cherenkov detectors . . . . . . . . . . . . 18

1.7.2 Liquid scintillator detectors . . . . . . . . . . . . . 23

1.7.3 Neutron tagging in water and liquid scintillator . . . . . . . 24

1.7.4 Liquid argon time projection chambers . . . . . . . . . 25

2 Motivation for studying neutrino-nucleus reactions 28

2.1 Supernova neutrinos . . . . . . . . . . . . . . . . . . . . . . . . . . 29

2.2 Supernova neutrinos in DUNE . . . . . . . . . . . . . . 33 
2.3 Supernova neutrino energy reconstruction in argon $\ldots \ldots \ldots \ldots$

2.4 Monte Carlo methods for neutrino energy reconstruction . . . . . . . . 40

2.5 MARLEY: A new event generator for supernova neutrinos . . . . . . . 41

2.6 Neutron production in medium-energy neutrino scattering . . . . . . . 43

2.6.1 Oscillation measurements . . . . . . . . . . . . . . . 43

2.6.2 Rare event searches: proton decay . . . . . . . . . . . . . . 48

2.6.3 Rare event searches: the Diffuse Supernova Neutrino Background 49

2.7 The ANNIE experiment $\ldots \ldots \ldots \ldots \ldots$

I MARLEY $\quad 55$

3 Cross section model $\quad 56$

3.1 Formalisms for low-energy neutrino-nucleus cross sections . . . . . . . . 57

3.1 .1 Microscopic models . . . . . . . . . . . . . . . . . . . . . . 57

3.1.2 Macroscopic models . . . . . . . . . . . . . . . . . . . . . 59

3.1.3 A cross section formalism for MARLEY . . . . . . . . . . . 60

3.2 From neutrino-quark to neutrino-nucleon scattering . . . . . . . . . 62

3.2 .1 Isospin in nuclear physics . . . . . . . . . . . . . . . . 64

3.2.2 Four-vector expression for the free nucleon matrix element . . . 67

3.3 Calculation of $2 \rightarrow 2$ scattering cross sections $\ldots \ldots \ldots \ldots$

3.4 Free nucleon matrix element calculation $\ldots \ldots \ldots \ldots$

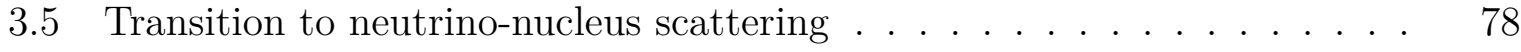

3.6 Charged current lepton tensor . . . . . . . . . . . . . . . . . . . . . 82

3.7 Charged current nucleon form factors . . . . . . . . . . . . . . 85

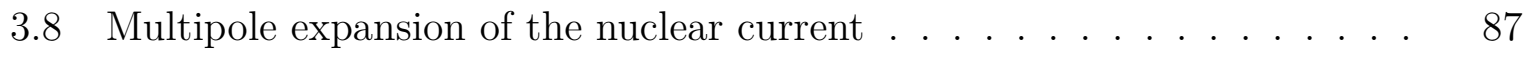

3.8 .1 Plane wave expansion $\ldots \ldots \ldots \ldots \ldots$

3.8.2 The Wigner-Eckhart theorem . . . . . . . . . . . . . . 88

3.8.3 The reduced nuclear matrix element tensor . . . . . . . . . . . 89

3.8.4 Simplified form of the lepton tensor elements . . . . . . . . . 90 
3.9 First-order approximation in $1 / m_{N} \ldots \ldots \ldots \ldots \ldots$

3.10 Correction for the final-state Coulomb interaction . . . . . . . . . . . 95

3.10 .1 The Fermi function . . . . . . . . . . . . . . . . . . . 95

3.10 .2 Effective momentum approximation . . . . . . . . . . . 98

3.10 .3 Coulomb-corrected differential cross section . . . . . . . . . 100

3.11 Allowed approximation cross section _. . . . . . . . . . . . . . . 101

3.12 Extension to charged current antineutrino scattering . . . . . . . . 106

3.13 Extension to neutral current scattering . . . . . . . . . . . . . 108

4 Nuclear de-excitation model $\quad 118$

4.1 Compound nuclear reactions . . . . . . . . . . . . . . . . . . . . . 118

4.2 The Hauser-Feshbach statistical model . . . . . . . . . . . . . . . . . . . 122

4.3 Level density model . . . . . . . . . . . . . . . . . . . . . . . . . . . . . . 129

4.4 Transmission coefficient model . . . . . . . . . . . . . . . . . . . . 134

4.4.1 Optical model parameterization for nucleons . . . . . . . . . . 134

4.4.2 Optical model parameterization for composite nuclear fragments . 136

4.4 .3 Optical model calculations . . . . . . . . . . . . . . . . . . . . . . 139

4.4.4 Gamma-ray transitions in nuclei . . . . . . . . . . . . . 141

4.4.5 Gamma-ray strength functions . . . . . . . . . . . . . . 143

5 Allowed nuclear matrix elements for ${ }^{40} \operatorname{Ar}\left(\nu_{\mathrm{e}}, \mathrm{e}^{-}\right)^{40} \mathrm{~K}^{*} \quad 147$

5.1 Model-independent predictions . . . . . . . . . . . . . . . . . 147

5.1.1 The Fermi matrix element . . . . . . . . . . . . . . . . . 148

5.1.2 The Gamow-Teller matrix element . . . . . . . . . . . . . 150

5.2 Measurement via mirror beta decay . . . . . . . . . . . . . . . . . . . 152

5.2.1 Equality of the allowed matrix elements for mirror nuclei . . . . 153

$5.2 .2{ }^{40} \mathrm{Ti} \boldsymbol{\beta}^{+}$decay measurement . . . . . . . . . . . . . . . 155

5.3 Measurement via $(p, n)$ scattering . . . . . . . . . . . . 158

5.3.1 Technique for extraction of Gamow-Teller strength $\ldots \ldots \ldots . . \quad 159$

$5.3 .2 \quad$ Experimental procedure . . . . . . . . . . . . . . . . 161 
5.4 Evaluated nuclear matrix elements for MARLEY . . . . . . . . . . . 161

5.4.1 Disagreements between the ${ }^{40} \mathrm{Ti} \beta^{+}$decay and ${ }^{40} \operatorname{Ar}(p, n)$ experiments 162

5.4.2 High-lying Gamow-Teller strength . . . . . . . . . . . . 166

6 Monte Carlo implementation 172

6.1 Generator initialization . . . . . . . . . . . . . . . . 172

$6.22 \rightarrow 2$ scattering reaction . . . . . . . . . . . . . . . . 174

6.3 Nuclear de-excitations . . . . . . . . . . . . . . . 176

6.4 Completing the event . . . . . . . . . . . . . . . . . . 177

7 Example simulation results $\quad 179$

7.1 Previous studies of low-energy neutrino- ${ }^{40} \mathrm{Ar}$ cross sections . . . . . . . . 179

7.2 Total cross section comparison . . . . . . . . . . . . . . . . . 181

7.3 Exclusive cross sections . . . . . . . . . . . . . . . . . . . . 182

7.4 Neutrino energy reconstruction . . . . . . . . . . . . . . . . . . . . . . 184

7.5 Truth-level studies in LArSoft . . . . . . . . . . . . . . . . . . . 187

7.6 Future prospects for MARLEY . . . . . . . . . . . . . . . . . 192

II ANNIE $\quad 196$

8 Neutron background characterization in ANNIE Phase I 197

8.1 The Fermilab Booster Neutrino Beam . . . . . . . . . . . . . . . . 198

8.2 Beam monitoring . . . . . . . . . . . . . . . . 203

8.2.1 Resistive wall current monitor . . . . . . . . . . . . . . . . 203

8.2.2 Beam current toroids . . . . . . . . . . . . . . . . . 204

8.3 Beam-associated neutron backgrounds . . . . . . . . . . . . 206

8.3.1 Dirt neutrons ...................... 208

8.3.2 Skyshine neutrons ..................... 209

8.4 Phase I detector description . . . . . . . . . . . . . . . . . . . . . . . 212

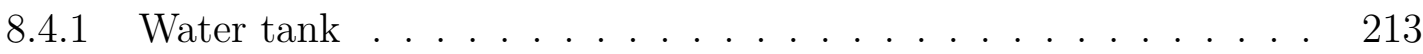


8.4.2 Neutron Capture Volume (NCV) _. . . . . . . . . . . . . . 214

8.4 .3 Front veto . . . . . . . . . . . . . . . . . . . . . . 215

8.4.4 Muon Range Detector . . . . . . . . . . . . . . . . . . . . 216

8.4 .5 Cosmic ray trigger $\ldots \ldots \ldots \ldots \ldots \ldots$

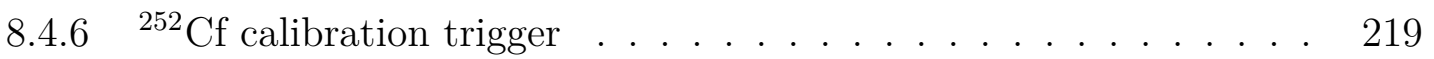

8.5 Phase I Data Acquisition . . . . . . . . . . . . . . . . . . . . . . . 219

8.6 Measurement strategy . . . . . . . . . . . . . . . . . . . . . . . 222

9 Neutron candidate event selection $\quad 228$

$9.1 \quad$ PMT waveform processing . . . . . . . . . . . . . . . . . . . 228

9.1.1 Waveform calibration . . . . . . . . . . . . . . . . 229

$9.1 .2 \quad$ PMT pulse finding . . . . . . . . . . . . . . . . . . . . 232

9.2 Determination of beam conditions . . . . . . . . . . . . . 234

9.3 Event selection . . . . . . . . . . . . . . . . . . . . . . 235

9.3.1 NCV coincidence requirement . . . . . . . . . . . . . . 236

9.3 .2 Afterpulsing cut . . . . . . . . . . . . . . . . . 238

9.3 .3 Total charge cut . . . . . . . . . . . . . . . . . . . . 239

9.3.4 Outer PMT veto cut . . . . . . . . . . . . . . . . . . . . . 239

9.4 Cut corrections . . . . . . . . . . . . . . . . . . . . . . . . . . . . 242

9.4 .1 Afterpulsing cut . . . . . . . . . . . . . . . . . . 243

9.4 .2 Outer PMT veto cut . . . . . . . . . . . . . . . . . . 245

9.4 .3 Total charge cut . . . . . . . . . . . . . . . . . . . 247

9.5 Results . . . . . . . . . . . . . . . . . . . . . . . . . . . 247

10 Efficiency calibration of the Neutron Capture Volume 249

$10.1{ }^{252} \mathrm{Cf}$ source calibration method . . . . . . . . . . . . . . . . . . . 249

10.1.1 Triggering apparatus . . . . . . . . . . . . . . . 251

10.1.2 Timing data . . . . . . . . . . . . . . . . . . 252

10.1.3 Simulations . . . . . . . . . . . . . . . . . . . 254

10.2 Cosmic muon calibration method . . . . . . . . . . . . . . . . . 264 
10.2.1 Estimate of the charge-to-energy conversion factor . . . . . . . 264

10.2.2 Charge and energy thresholds . . . . . . . . . . . . . . . 266

10.2.3 Efficiency determination . . . . . . . . . . . . . . . 268

10.3 Adopted value of the NCV efficiency . . . . . . . . . . . . . . . 270

11 Background neutron event rates $\quad 272$

$\begin{array}{ll}\text { Appendices } & 277\end{array}$

A Derivation of the compound nucleus formation cross section $\quad 278$

B Tensor product lemma $\quad 283$

C Allowed beta decay 286 


\section{List of Figures}

1.1 Feynman diagrams showing examples of the two general categories of neutrino interactions. LEFT: A muon neutrino undergoes a charged current interaction with a down quark, producing a muon and an up quark in the final state. RIGHT: A tau antineutrino elastically scatters off an electron in a neutral current interaction. . . . . . . . . . . . . . . . .

1.2 Neutrinoless double beta decay generated via the exchange of a virtual Majorana neutrino between two decaying nucleons. Figure from reference

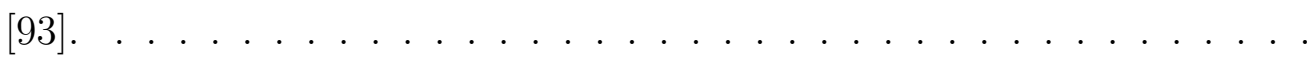

1.3 Induced dipoles created by an electrically charged particle traveling through a dielectric material. LEFT: For a particle traveling with a speed less than the phase velocity of light in the material, the distribution of the induced dipoles is symmetric. RIGHT: If the particle exceeds the local phase velocity of light, then an asymmetric pattern of induced dipoles is formed, resulting in the emission of Cherenkov radiation. Figure from reference

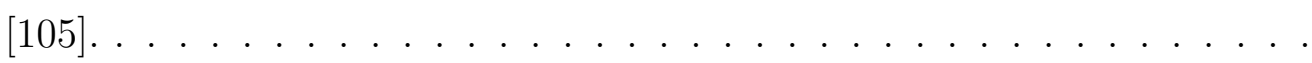

1.4 Cherenkov radiation induced by a charged particle is emitted (blue arrows) in a cone around the particle's direction of motion (red arrow). The cone has half opening angle $\theta=\arccos \left(n^{-1} \beta^{-1}\right)$, where $n$ is the index of refraction of the medium and $\beta$ is the (dimensionless) speed of the particle. Cherenkov radiation is emitted only for $\beta \geq 1 / n$. Figure from reference

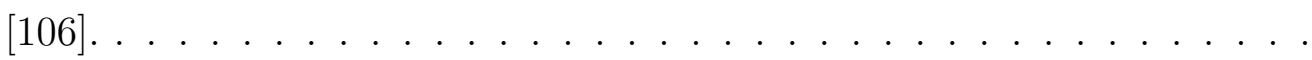


1.5 Cherenkov radiation is seen as a bright blue glow around the fuel plates of the Advanced Test Reactor at Idaho National Laboratory. Figure from

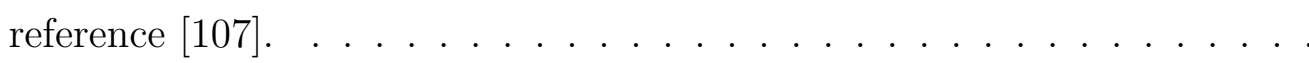

1.6 The basic components of a photomultiplier tube. The dynodes are held at different voltages by connecting the stem pins to a suitable base containing a voltage divider. Figure from reference [108] . . . . . . . . . . . . 22

1.7 Conceptual design of a liquid argon time projection chamber. Figure from reference $[133] . \ldots \ldots \ldots \ldots \ldots$

2.1 Onion-like structure of a massive star (not drawn to scale) just before a core-collapse supernova. Figure from reference $[138] . \ldots$. . . . . . . .

2.2 Conceptual design of the DUNE experiment. Measurements of a neutrino beam will be performed using liquid argon time projection chambers at two locations to study neutrino oscillations. DUNE will also pursue a rich science program beyond the oscillation measurements, including the detection of $\nu_{e}$ from a possible nearby core-collapse supernova. . . . . . .

2.3 Predicted event time distribution for the detection of supernova neutrinos in a DUNE-like $40 \mathrm{kt}$ LArTPC. Only events from the earliest stages of the supernova, assumed to have occurred at a distance of $10 \mathrm{kpc}$ from Earth, are shown. All error bars in the figure are statistical. Under the specific flux model (described in reference [157]) used in this calculation, the presence or absence of a clear excess of events during the neutronization burst provides a means of determining whether the neutrino mass hierachy is normal or inverted. Figure from reference $[158] . \quad \ldots \ldots$. . . . . . . 
2.4 Charged current $\nu_{e}$ scattering on ${ }^{40} \mathrm{Ar}$ will sometimes induce a transition to a highly-excited ${ }^{40} \mathrm{~K}$ nuclear energy level above the proton and neutron emission thresholds $S_{p}$ and $S_{n}$. When this occurs, de-excitation of the level may proceed via many competing channels, including those shown in the diagram: $\gamma$-ray emission, neutron emission, and proton emission. If the daughter nucleus is itself left in an excited state, one or more additional particles will be emitted in a cascade of de-excitation steps. . . . . . . .

2.5 Demonstration of the kinematic method for neutrino energy reconstruction using simulated $1 \mathrm{GeV} \nu_{\mu}$ scattering events. In each of the three plots, which are described in detail in the text, the black histogram represents the reconstructed neutrino energy distribution for all events, while the red and blue histograms show the contributions of "stuck pion" and $2 \mathrm{p}-2 \mathrm{~h}$ events, respectively. Selecting a subset of events with zero final-state neutrons (as shown in the lower left plot) noticeably improves the quality of the energy reconstruction. Figure from reference $[126]$. . . . . . . . . . . . .

2.6 TOP: A concept drawing of the completed Phase II ANNIE detector. The neutrino beam travels from left to right in the perspective shown here. BOTTOM: The stages of a typical ANNIE signal event, shown from left to right and described in the text. Figure from reference $[126]$. . . . . .

2.7 Schematic of a Large Area Picosecond Photodetector (LAPPD). Figure from reference $[192] . \ldots \ldots \ldots$

2.8 Results of a simulation study of neutrino vertex reconstruction in ANNIE. Raw (left) and cumulative (right) distributions of $\Delta r$, the difference between the reconstructed and true values of the vertex radial coordinate, are shown for simulated events detected by 128 conventional PMTs (blue) and the same number of PMTs plus 5 LAPPDs (red). The gray lines in the right-hand plot mark the location of the 68th percentile value for the

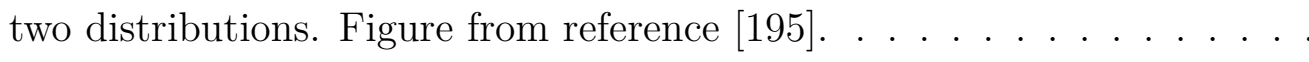


4.1 Measurement by Flowers et al. [302] of the energy spectra of alpha particles emitted in ${ }^{60} \mathrm{Ni}(e, \alpha) e^{\prime} X$ reactions at $\theta_{\alpha}=90^{\circ}$. Data were obtained for electron energies of $120 \mathrm{MeV}$ (curve A, upper left-hand scale), $60 \mathrm{MeV}$ (curve B, right-hand scale), and $33 \mathrm{MeV}$ (curve C, lower left-hand scale). The solid lines show predicted spectra obtained using a compound nucleus model. Angular distribution measurements (not shown here) were made at the mean energies marked by the vertical dashed lines. . . . . . . . .

5.1 Early observations of the Gamow-Teller giant resonance (GTGR) in ${ }^{90} \mathrm{Zr}(p, n){ }^{90} \mathrm{Nb}$ scattering experiments. LEFT: Measurement of the differential cross section at $0^{\circ}$ performed by Doering et al. [367]. An incident proton kinetic energy of $45 \mathrm{MeV}$ was used. The broad peak to the left of that corresponding to the isobaric analog state (labeled IAS in the figure) is identified as the GTGR. RIGHT: Neutron time-of-flight spectra obtained by Bainum et al. [368] for a proton kinetic energy of $120 \mathrm{MeV}$ and laboratory scattering angles of $0.2^{\circ}, 5.0^{\circ}$, and $9.9^{\circ}$. The peaks labeled $d$ and $e$ correspond to the isobaric analog state and the GTGR, respectively. . . . . . . . . .

5.2 Decay scheme for ${ }^{40} \mathrm{Ti} \beta^{+}$decay. Bhattacharya et al. [382] observed beta-delayed proton transitions to both the ground state (" $p_{0}$ ") and the first excited state (" $p_{1}$ ") of ${ }^{39} \mathrm{Ca}$. Without a determination of whether a particular proton line corresponds to a $p_{0}$ or a $p_{1}$ decay, the energy level of the excited ${ }^{40} \mathrm{Sc}^{*}$ nucleus originally accessed by the $\beta^{+}$decay is ambiguous. 157

5.3 Gamow-Teller strengths $B(\mathrm{GT})$ from two independent measurements of ${ }^{40} \mathrm{Ti} \beta^{+}$decay. The second dataset is shown with an inverted vertical axis to facilitate comparisons. . . . . . . . . . . . . . . . 163 
5.4 Comparison of the Gamow-Teller strengths $B(\mathrm{GT})$ measured using ${ }^{40} \mathrm{Ti}$ $\beta^{+}$decay (see fig. 5.3) with those obtained using a $0^{\circ}(p, n)$ scattering experiment. The sensitivity of the $\beta$ decay experiments above $6.5 \mathrm{MeV}$ (marked by the dashed line) was very limited. Liu et al. note that, for a ${ }^{40} \mathrm{Sc}$ level at this excitation energy, a $B(\mathrm{GT})$ value of 0.1 corresponds to a branching fraction of only $0.1 \%$ due to the phase space factor for allowed $\beta$ decay. Detector effects further complicate extraction of $B(\mathrm{GT})$ values from $\beta$ decays to higher excitation energies [379]. . . . . . . . . . 165

5.5 Theoretical Gamow-Teller strengths $B\left(\mathrm{GT}_{-}\right)$for ${ }^{40} \operatorname{Ar}\left(\nu_{e}, e^{-}\right){ }^{40} \mathrm{~K}$ calculated using the Quasiparticle Random Phase Approximation (QRPA) . .

5.6 Running sums of $B(\mathrm{GT})$ from three experimental measurements and a QRPA calculation as a function of ${ }^{40} \mathrm{~K}$ excitation energy. Although the theoretical prediction fails to reproduce the observed fragmentation of the Gamow-Teller strength at low energies, the predicted integrated strength at $7.5 \mathrm{MeV}$ nearly matches that of the ${ }^{40} \mathrm{Ti} \beta^{+}$decay measurements at around the same excitation energy. . . . . . . . . . . . . . . . 168

5.7 The three allowed matrix element datasets for charged current $\nu_{e}$ scattering on ${ }^{40} \mathrm{Ar}$ distributed with the current version of MARLEY . . . . . . 170

5.8 Comparison of the integrated $B(\mathrm{GT})$ curves for the three allowed matrix element datasets for ${ }^{40} \operatorname{Ar}\left(\nu_{e}, e^{-}\right)^{40} \mathrm{~K}$ distributed with the current version of MARLEY. A corresponding curve for the QRPA calculation that provided $B(\mathrm{GT})$ values for high-lying Gamow-Teller transitions in all three datasets is also shown. . . . . . . . . . . . . . . . 171

6.1 Flowchart showing the procedure followed by MARLEY for creating simulated neutrino-nucleus scattering events. . . . . . . . . . . . 173 
7.1 MARLEY ${ }^{40} \operatorname{Ar}\left(\nu_{e}, e^{-}\right)^{40} \mathrm{~K}$ total cross section compared to other calculations. Datasets A and B give similar results for the total cross section. The compilation of non-MARLEY cross sections shown here was originally presented in reference [415] and obtained via private communication with the author. GTBD: Unpublished calculation by A. Samana et al. that used a macroscopic neutrino cross section model based on the Gross Theory of Beta Decay. Reference [255] describes the model and presents some energy-averaged cross sections. RQRPA: Relativistic Quasiparticle Random Phase Approximation calculation by N. Paar et al. Energy-averaged cross sections obtained using this approach were published in reference [281]. SM+RPA: A "hybrid model" calculation by T. Suzuki and M. Honma [282]. Partial cross sections for $0^{+}$and $1^{+}$multipole transitions (which correspond to Fermi and Gamow-Teller transitions, respectively, in the low momentum transfer limit) were obtained using the nuclear shell model, while those for other multipoles were computed using the Random Phase Approximation. RPA: Random Phase Approximation calculation by Martinez-Pinedo et al. [409]. PQRPA: Unpublished Projected Quasiparticle Random Phase Approximation calculation performed by A. Samana et al. using the QRAP computer code [245]. QRPA: Quasiparticle Random Phase Approximation calculation by M. Cheoun et al. [412]. The dashed portion of the curve shows an extrapolation (based on a sixth degree polynomial fit) of this cross section beyond the maximum energy of $80 \mathrm{MeV}$ tabulated by A. Samana. . . . . . . . . . . . . . . 183

7.2 MARLEY prediction for the total cross section for $\nu_{e} \mathrm{CC}$ absorption on ${ }^{40} \mathrm{Ar}$, together with several partial cross sections to exclusive final states. For all of the exclusive channels considered here, the final state contains $X \gamma$-rays, where $X \geq 0$. . . . . . . . . . . . . 185 
7.3 TOP: Truth-level energy reconstruction study performed using MARLEY for simulated events generated according to the time-integrated $\nu_{e}$ spectrum predicted by the Livermore supernova model [419]. The definitions of the three histograms shown in the plot are described at length in the text. BOTTOM: A similar MARLEY study performed using the $\nu_{e}$ spectrum for muon decays at rest. . . . . . . . . . . . . . . . . . . . . . . . 188

7.4 Displays of the true trajectories of the outgoing particles for representative MARLEY events simulated using LArSoft. The particles were tracked through the simulated geometry of one of the proposed $10 \mathrm{kt}$ single-phase LArTPC modules for the DUNE far detector. Event vertices were sampled uniformly throughout the active TPC volumes within the simulated detector. TOP LEFT: A ${ }^{40} \operatorname{Ar}\left(\nu_{e}, e^{-} 5 \gamma\right){ }^{40} \mathrm{~K}$ event induced by a $16.1 \mathrm{MeV}$ neutrino. TOP RIGHT: A ${ }^{40} \operatorname{Ar}\left(\nu_{e}, e^{-} n\right)^{39} \mathrm{~K}$ event induced by a $16.3 \mathrm{MeV}$ neutrino. The secondary $\gamma$-rays emitted close to the vertex were produced via inelastic scatters of the primary neutron. Those emitted far away were produced when the primary neutron was captured on an ${ }^{40} \mathrm{Ar}$ nucleus. BOTTOM: A ${ }^{40} \operatorname{Ar}\left(\nu_{e}, e^{-} p \gamma\right)^{39} \mathrm{Ar}$ event induced by a $17.8 \mathrm{MeV}$ neutrino. 191

7.5 Stacked plot showing the separate contributions of MARLEY events involving at least one primary neutron (red) and zero primary neutrons (blue) to the total distribution of "visible energy sphere" radii observed in the LArSoft simulations discussed in this section. . . . . . . . . . . . 192

7.6 Distribution of neutron capture distances (defined as the distance between the neutrino vertex and the neutron capture vertex) observed in the LArSoft simulations described in this section. . . . . . . . . . . .

8.1 Diagram of the currently-operating proton accelerator beamlines at Fermilab. The line labeled "Low-Energy Neutrino Experiments"' is used to produce neutrinos for the Booster Neutrino Beam. Figure taken from http: // www.fnal.gov/pub/science/particle-accelerators/accelerator-complex. html. . . . . . . . . . . . . . . . . . 200 
8.2 Exploded view of the Booster Neutrino Beam target components. Figure

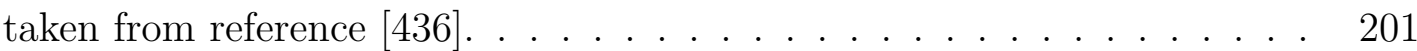

8.3 A magnetic horn used to produce the Booster Neutrino Beam. Figure taken from https://web.fnal.gov/project/TargetSystems/BNB_ target_hall/SitePages/BNB\%20Horn. aspx. . . . . . . . . . . .

8.4 Predicted neutrino flux (total and for individual neutrino species) from the Booster Neutrino Beam (running in neutrino mode) at SciBooNE hall, the location of the ANNIE detector. The spectra shown here represent averages within a $2.12 \mathrm{~m}$ radius of the beam center. They are normalized per unit area, per unit energy, and per proton on target (POT). Figure taken from reference [437]. . . . . . . . . . . . . . . . . 202

8.5 Map showing the detectors along the path of the Booster Neutrino Beam, including the planned sites of the far detector (ICARUS-T600) and near detector (SBND) for the Short-Baseline Neutrino Program at Fermilab. Although the near detectors for the MINOS and NOvA experiments are also relatively close to the production site of the BNB, their primary source of neutrinos is the Fermilab NuMI (Neutrinos at the Main Injector) beam. Figure taken from reference [84] and updated to include the ANNIE detector (shown near the bottom right of the drawing), which occupies an experimental hall previously used by the SciBooNE experiment [196]. . .

8.6 Summary of the procedure used to produce neutrinos for the Fermilab Booster Neutrino Beam. Figure taken from http://targets.fnal.gov/ BNB_neutrino_beam.html. . . . . . . . . . . . . . . . . . 204

8.7 Photograph of the resistive wall current monitor used to measure the proton beam arriving at the BNB target. The outer cover has been removed to expose the gap and the RWM electronics. Figure taken from reference

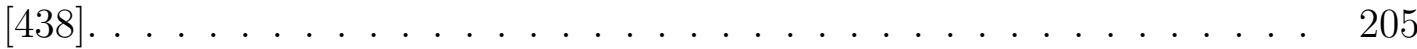


8.8 Basic design of a resistive wall current monitor (RWM). Resistors are connected across a ceramic gap in the beam pipe wall, and the current flowing through them is measured. Figure taken from reference [430]. . . 206

8.9 As protons from the beam pass through the beam pipe, they induce a negatively-charged image current on the wall of the pipe. Figure taken

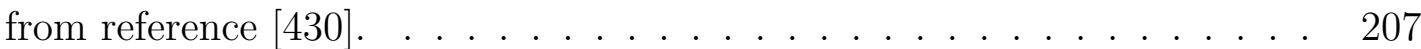

8.10 Conceptual design of a beam current toroid. The proton beam is directed perpendicularly into the page. Figure taken from [430]. . . . . . . . . . . 207

8.11 Beam-induced neutron backgrounds in the ANNIE detector. "Skyshine neutrons" originate from hadron interactions in the beam target and absorber, while "dirt neutrons" originate from neutrino interactions in the dirt and rock upstream of the ANNIE detector. Figure by Vincent Fischer. 208

8.12 Simulated spatial distributions of neutrino vertices outside the tank that produce neutrons that reach the tank (black points), neutrino vertices inside the tank (cyan points), and points where neutrons enter the tank (magenta). Figure from reference [126]. . . . . . . . . . . . . . . . . 210

8.13 Simulated arrival times for neutrino-induced neutrons entering the water volume. LEFT: All of the signal neutrons produced by neutrino interactions in the tank are emitted within a few ns. Most of the background dirt neutrons enter the water around the same time as the signal neutrons. RIGHT: The dirt neutron arrival time distribution includes a long tail that continues until tens of ms after the beam arrives in the detector. . . . .

8.14 Simulated distribution of the kinetic energies of neutrino-induced neutrons at the moment that they enter the ANNIE water volume. The signal neutrons (blue) produced by neutrino interactions in the tank have a similar energy distribution to the background dirt neutrons (red) produced outside of the tank. . . . . . . . . . . . . . . . . . . . 211 
8.15 Single-strip hit time distribution at the top of the SciBar plastic scintillator detector for a measurement at KEK. The beam bunch peaks sit on top of an increasing pedestal attributable to skyshine neutrons. Figure from

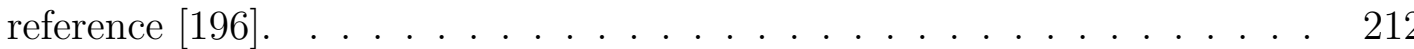

8.16 Reconstructed $y$ coordinates of event vertices in the SciBooNE detector while running at the BNB. Events that occur in time with the beam have a nearly uniform distribution of $y$ coordinates, while those that occur before and after have distributions that are biased toward the top of the detector. This bias is attributable to cosmic rays in the pre-beam timing region and to a combination of cosmic rays and skyshine in the post-beam timing region. Figure adapted from reference [447], which obtained the plots

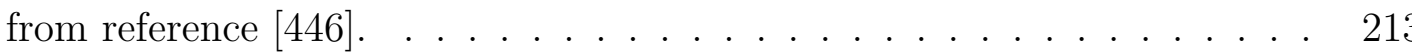

8.17 Concept drawings of the detector configuration used for each phase of the ANNIE experiment. In both drawings, the beam travels from left to right. LEFT: The Phase I configuration of the ANNIE detector. For Phase I, the tank contains pure water, and the bottom of the tank is instrumented with sixty PMTs. The Neutron Capture Volume (NCV), a movable neutronsensitive subvolume of the detector, is used to measure position-dependent neutron backgrounds within the tank. RIGHT: The Phase II configuration of the ANNIE detector. For Phase II, the tank is filled with gadoliniumloaded water, all inner surfaces of the tank are instrumented with PMTs, and LAPPDs (represented by thin gray rectangles in the drawing) are installed on the far side of the tank from the beam. Figure from reference [126].

8.18 LEFT: The Phase I stainless steel inner structure being lowered into the ANNIE water tank. UPPER RIGHT: The ANNIE water tank being installed in the experimental hall. LOWER RIGHT: The 8-inch PMTs used to instrument the bottom of the tank for Phase I. Figure from reference

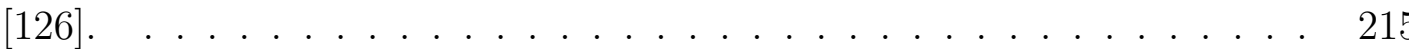


8.19 CAD (Computer Assisted Design) drawing of the Neutron Capture Volume from ANNIE Phase I. Figure by David Hemer. . . . . . . . . . . . . . . . 216

8.20 LEFT: The Neutron Capture Volume (NCV) from ANNIE Phase I before its installation in the water tank. Two PMTs, encased in waterproof housings and wrapped with black plastic, observe scintillation light induced by neutron captures in the acrylic vessel, which is filled with Gd-loaded liquid-scintillator. The NCV frame is attached to a winch system (partially shown near the top of the photograph), allowing the NCV to be moved to different locations inside the tank. RIGHT: The NCV after installation in the water tank. To optically isolate the NCV from the rest of the tank, the acrylic vessel and two PMTs were wrapped with a black plastic bag.

8.21 LEFT: Concept drawing of the ANNIE front veto. RIGHT: A photograph of the front veto after its installation in the experimental hall for ANNIE Phase I. . . . . . . . . . . . . . . . . . . . . . . . . . . . . . . 218

8.22 LEFT: Concept drawing of the SciBooNE Muon Range Detector, now repurposed for ANNIE. RIGHT: A side view of the MRD as it appeared during Phase I data taking. . . . . . . . . . . . . . . . . . . . . . . . . . 218

8.23 ANNIE Phase I cosmic ray trigger. LEFT: Concept drawing showing the paddle numbering scheme. As described in the text and in table 8.1, coincidences of multiple paddles are used to select cosmic muons traveling in three different directions. The yellow cylinder in the drawing represents the NCV. Drawing by Jingbo Wang. RIGHT: Photograph of the installed cosmic ray trigger during Phase I data taking. . . . . . . . . . . . . . 220 
8.24 NCV position scan performed during beam data taking in ANNIE Phase I. Each of the NCV positions, which are not drawn to scale, is numbered in chronological order. The beam had been shut down for maintenance before any data were taken at position \#8. Despite the lack of beam, additional test runs were taken with the NCV at position \#8 before the Phase I detector was decommissioned. Position \#8 is shown in red in the figure to indicate that data taken there are not included in the analysis described in this thesis. . . . . . . . . . . . . . . . .

9.1 Successive applications of each of the neutron candidate event criteria for zero-suppressed beam data taken at position \#2 (center of the tank). BLACK: Time distribution of all pulses recorded in zero suppression mode on NCV PMT \#1 at position \#2. No analysis cuts have been applied to these data. Starting from the left hand side of the plot, the first peak coincides with the neutrino beam crossing the ANNIE detector, while the second is attributable to afterpulsing. BLUE: Time distribution of all NCV coincidences from the same dataset. RED: Events from the blue histogram that passed the afterpulsing cut. GREEN: Events from the red histogram that passed the total charge cut. ORANGE: Events from the green histogram that passed the outer PMT veto cut. These are considered neutron candidates in the background event rate analysis. .

9.2 Distribution of the elapsed time since the previous event for NCV coincidences from the blue histogram in fig. 9.1. The plot includes only the small minority (about 4\%) of the NCV coincidence events that occurred within the first $100 \mu \mathrm{s}$. The remaining events, not shown in the plot, occurred far later (at least $10 \mathrm{~ms}$ since the previous event). . . . . . . . . . 
9.3 Results of an NCV containment simulation study performed using RATPAC. Thermal neutron tracks were started one at a time from locations sampled uniformly within a $50 \mathrm{~cm}$ radius sphere centered on the $\mathrm{NCV}$, which was placed at position \#2 (center of the tank). BLUE: Monte Carlo estimate of the probability distribution for the number of unique tank PMTs that will collect at least one photoelectron as a result of a neutron capture occurring within the NCV liquid. RED: The same distribution for neutrons that capture outside the NCV liquid. Only photoelectrons whose origin can be traced back to the neutron capture are considered in the simulation. . . . . . . . . . . . . . . .

9.4 Distributions of the number of unique water tank PMTs that recorded at least one pulse within 40 ns of activity in the NCV. For the blue dataset, "activity" is defined as an NCV coincidence, while for the red dataset it is defined as a pulse on NCV PMT \#2 without a coincident pulse on NCV PMT \#1. Both datasets were created using all runs analyzed for this thesis. No analysis cuts have been applied. . . . . . . . . . . . . . . . 241

9.5 BLUE: Distribution of the total charge collected on both NCV PMTs for NCV coincidence events that passed the afterpulsing cut. All events shown here were recorded within $2 \mu$ s of a cosmic trigger consistent with a downward muon passing through the NCV. RED: The corresponding charge distribution when all neutron candidate event cuts have been applied. 242

10.1 Calculated neutron emission rate of a californium source with an initial isotopic composition of $4.32 \%{ }^{249} \mathrm{Cf}, 10.82 \%{ }^{250} \mathrm{Cf}, 3.31 \%{ }^{251} \mathrm{Cf}, 81.50 \%$ ${ }^{252} \mathrm{Cf}, 0.04 \%{ }^{253} \mathrm{Cf}$, and $0.01 \%{ }^{254} \mathrm{Cf}$. Figure taken from [461]. . . . . . . 251

10.2 Distribution of neutron candidate event times from the ${ }^{252} \mathrm{Cf}$ source calibration runs taken with the NCV at position \#1. The large event rate in the third bin may be attributed to prompt fission $\gamma$-rays entering the NCV. 253

10.3 Exponential fit of the time distribution from fig. 10.2 after $15 \mu \mathrm{s} . \quad$. . . 254 
10.4 Experimental distribution measured by Hambsch and Oberstedt [477] of the mass number $A$ of fission fragments produced by spontaneous fission of ${ }^{252}$ Cf. . . . . . . . . . . . . . . . . . . . . 257

10.5 Predicted time distribution for neutron capture events in the NCV liquid. Time $t=0$ corresponds to a fission event simulated using the FREYA event generator. . . . . . . . . . . . . . . . . . . . 258

10.6 Comparison of the ${ }^{252} \mathrm{Cf}$ calibration source data with the result of a maximum likelihood fit of the model defined in eq. (10.12) . . . . . . . . . . . 261

10.7 TOP: The distribution of the pull $g_{\mathrm{NCV}}$ (see eq. (10.22)) for 10000 fictitious ${ }^{252} \mathrm{Cf}$ calibration source datasets generated using a toy Monte Carlo simulation. A Gaussian fit to the simulation results is shown in blue. BOTTOM: The pull distribution with the estimated 1-sigma error rescaled by a factor of 0.165 . Correcting the error in this way improves the agreement with the expected standard normal distribution. . . . . . . . . . 263

10.8 Distribution of deposited energies for simulated cosmic muons passing through the cosmic trigger paddles and through the NCV at position \#2 (center of the tank). . . . . . . . . . . . . . . . 266

10.9 Distribution of the total charge collected on the two NCV PMTs at position \#2 (center of the tank) for NCV coincidence events recorded in response to cosmic muon triggers. Only triggers consistent with a muon traveling nearly directly downward through the NCV are included in the plot. . . 267

10.10Distribution of the total charge collected on the two NCV PMTs for NCV coincidence events recorded at all positions. Only events with low charges (less than $100 \mathrm{pC}$ ) are shown. The afterpulsing cut has been applied to these data to avoid biasing the distribution toward an erroneously low

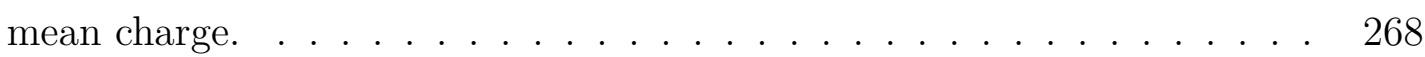


10.11Histogram of the total energy deposited in the scintillator for simulated neutron captures that occurred within the NCV liquid volume. Two events above $8.6 \mathrm{MeV}$, attributable to rare neutron captures on ${ }^{14} \mathrm{~N}$, are not shown. . . . . . . . . . . . . . . . . . . . . . . . 269

11.1 Neutron candidate event rates measured during ANNIE Phase I. The inset diagram shows the NCV positions included in the red and blue datasets. Position \#2 (the center of the tank) is shown in purple to indicate that it is included in both the red and blue data. For the blue dataset, the "water thickness" is the depth of the water above the top of the NCV. For the red dataset, it is the smallest distance between the side of the tube forming the NCV vessel and the beam side of the tank. Error bars shown in the plot include both statistical and systematic contributions. . . . . 


\section{List of Tables}

1.1 Results of a 2018 global fit of the 3-flavor model neutrino oscillation parameters [79]. Where applicable, best-fit values are given assuming a normal neutrino mass hierarchy $\left(m_{3}>m_{2}\right)$ or an inverted hierarchy $\left(m_{3}<m_{2}\right)$.

3.1 Quantities needed to compute the vector $g_{V}^{F}$ and $g_{A}^{F}$ axial-vector coupling constants for neutral current scattering amplitudes. . . . . . . . . . .

4.1 Lowest-order multipoles for $\gamma$-ray transitions between an initial nuclear level with spin (parity) $J_{i}\left(\Pi_{i}\right)$ and a final nuclear level with spin (parity) $J_{f}\left(\Pi_{f}\right)$. This table was taken from reference $[260] . \ldots$. . . . . . .

4.2 Giant resonance parameters used by MARLEY for $\gamma$-ray strength function calculations. Energies $E_{X \ell}$ and widths $\Gamma_{X \ell}$ are given in $\mathrm{MeV}$, and peak cross sections $\sigma_{X \ell}$ are given in mb. . . . . . . . . . . . . . 146

5.1 Level assignments and measured $B(\mathrm{~F})$ and $B(\mathrm{GT})$ values for ${ }^{40} \operatorname{Ar}\left(\nu_{e}, e^{-}\right)^{40} \mathrm{~K}$

7.1 Branching ratios to final states from the MARLEY simulations shown in fig. 7.3. The "single neutron emission" and "single proton emission" channels include cases where a single nucleon emission is followed by a gamma cascade. The channel labeled "other" includes both multi-step nucleon emissions (e.g., the emission of a neutron followed by the emission of a proton) and the emission of deuterons, alphas, etc. . . . . . . 
8.1 The three distinct combinations of coincident paddle hits that gave rise to cosmic muon triggers in ANNIE Phase I. The three combinations were distinguished during data taking by the width of the NIM logic pulse issued by the cosmic ray trigger. Each combination is indicative of a different physical situation, as described in the "Interpretation" column of the table. 219

8.2 Summary of beam and cosmic data taken during Phase I. See fig. 8.24 for a diagram of the NCV positions. An uncertainty of $2 \mathrm{~cm}$ was estimated for all NCV position measurements. . . . . . . . . . . . . . . 224

9.1 Neutron candidate events observed at each NCV position. Starting with the third column, the quantities $N_{\text {pass }}, N_{10 \mu \mathrm{s}}, N_{\text {tank }}$, and $N_{n}$ listed in the table represent event counts observed in the region of interest. They are, respectively, the number of events that pass all cuts, the number of events that fail only the afterpulsing cut and occur within the first $10 \mu \mathrm{s}$, the number of events that fail only the outer PMT veto cut, and the accepted number of neutron candidate events. . . . . . . . . . . . . . . . 248

10.1 Results of the maximum likelihood fit (described in section 10.1.3.3) to the ${ }^{252} \mathrm{Cf}$ source calibration data. Only statistical errors are given here. 260

10.2 Results of the fits used to estimate an energy threshold for the NCV . . . 265

11.1 Measured rates of neutron candidate events from ANNIE Phase I. A nominal value of $5 \times 10^{12}$ POT per spill is assumed in the second column. . . 273 


\section{Abstract \\ Nuclear Effects in Neutrino Detection}

Although the interactions of neutrinos with elementary particles are described with impressive precision by the Standard Model, neutrino-nucleus reactions remain less well understood. Improvements in our knowledge of neutrino-nucleus scattering will be an important requirement for the success of future experimental efforts involving neutrinos at both low $(\sim 10 \mathrm{MeV})$ and medium $(\sim 1 \mathrm{GeV})$ energies.

In the low energy regime, the planned DUNE experiment will attempt to observe neutrinos from a nearby core-collapse supernova using liquid argon time projection chambers (LArTPCs). Unlike other large neutrino detectors, which are primarily sensitive to electron antineutrinos, DUNE will observe mostly charged current absorptions of electron neutrinos on ${ }^{40} \mathrm{Ar}$ in response to a supernova, providing a unique window into the physics of stellar collapse. Despite the importance of low-energy neutrino-nucleus reactions to DUNE's supernova physics goals, prior to the work presented in this thesis, no thorough consideration of the many possible final states generated by neutrino-argon scattering, including those involving the emission of nucleons or heavier nuclear fragments, had yet been attempted in the literature for the energy range of interest for supernova neutrinos. To aid DUNE's supernova physics program, this thesis presents a detailed theoretical model of low-energy neutrino-argon scattering. This model has been implemented within a new event generator called MARLEY (Model of Argon Reaction Low Energy Yields) which may be used to simulate realistic ${ }^{40} \operatorname{Ar}\left(\nu_{e}, e^{-}\right){ }^{40} \mathrm{~K}^{*}$ events for LArTPC supernova neutrino sensitivity studies.

At medium energies, large theoretical uncertainties in predictions of neutrino-induced neutron production present a problem for precision neutrino oscillation experiments, attempts to discover the Diffuse Supernova Neutrino Background, and searches for proton decay. To constrain the widely-varying predictions of current nuclear models, the Accelerator Neutrino Neutron Interaction Experiment (ANNIE) collaboration plans to make a systematic measurement of neutron production by neutrino interactions in water. Be- 
cause the ANNIE experimental hall is located only $100 \mathrm{~m}$ away from the Fermilab Booster Neutrino Beam target, background neutrons correlated in time with the beam could potentially interfere with this proposed measurement. As a first step toward ANNIE's ultimate physics goals, this thesis presents an analysis of background neutron rates as a function of position within the ANNIE detector. These rates are found to be small enough for the neutron yield measurements to proceed as planned. 


\section{ACKNOWLEDGMENTS}

My efforts to produce this thesis have benefited enormously from the support of many people, only a fraction of whom can be enumerated here. I consider myself remarkably fortunate to have chosen Bob Svoboda to be my PhD advisor. Since the early days of graduate school, I have been the constant beneficiary of Bob's scientific acumen, witty sense of humor, consistently good advice, and seemingly inexhaustible supply of new ideas, all of which I will sorely miss as I move on to the next stage of my physics career. To the extent that I have already made useful contributions to physics, I owe Bob a debt of gratitude for pointing me in the right direction. Special thanks are also due to the other two members of my thesis committee: Ramona Vogt and Mike Mulhearn. At the risk of dooming her to a sudden increase in the number of requests that she receives to serve on thesis committees, I must note that Ramona's service on mine can only be described as "ideal." Her detailed and unfailingly helpful comments on the drafts of each chapter were always returned far more quickly than I could produce new material, and she frequently reached out to see how my writing was progressing. Mike has likewise been very kind to me, even taking time out of his busy schedule to serve as my faculty presenter at commencement. Despite his assurance (delivered with a smile) that this small sacrifice was simply an "occupational hazard" borne by all academics, his presence at the ceremony meant a lot to me and my family.

Beyond the members of my official thesis committee, I am deeply indebted to a number of unofficial advisors whose guidance and encouragement were instrumental in seeing this work to fruition. Chris Grant, whose time as a postdoctoral researcher at UC Davis happily coincided with much of my tenure as a graduate student, provided invaluable feedback throughout the development of MARLEY. I have learned much from his mentorship and example. Kate Scholberg's extensive expertise in supernova neutrinos has been a boon to the entire MARLEY project. I have greatly benefited from many discussions with her, both during regular meetings of the DUNE Supernova and Low-Energy Neutrinos Working Group and in a variety of other settings. Additionally, several "DUNE

Supernova Hack Days" events that she organized provided a wonderful opportunity to 
focus on developing a MARLEY/LArSoft interface, receive comments in real time from early users, and get to know other DUNE collaborators in an informal but productive atmosphere. Emilija Pantic has been very generous with her time and knowledge, both while serving as chair of my $\mathrm{PhD}$ qualifying exam committee and while enduring many a long-winded presentation before offering helpful comments. Matt Wetstein's willingness to explore the finer details of the ANNIE Phase I analysis with me was crucial to the success of the entire undertaking, and his patience, kindness, and contagious enthusiasm did much to make my work on the ANNIE experiment enjoyable.

While performing the work described in this thesis, I have had the privilege of working alongside many talented people as part of the ANNIE, CAPTAIN, and DUNE collaborations. To all of my colleagues who provided feedback on this work, taught me something new, helped me troubleshoot problems, and supported me in countless other ways, I offer my heartfelt thanks.

Before concluding these acknowledgements, I would be remiss not to mention at least some of those whose contributions predate the start of my graduate studies. Although I wish to thank all of the many teachers who have helped me get to this point, the names Kathy Archer, Jim Costello, David Ho, Mary Plecas, and Anne Turner come to mind especially quickly. My undergraduate advisors, Larry Rees and Bart Czirr, deserve special recognition for introducing me to the exciting world of experimental nuclear physics. Between finishing my bachelor's degree and starting graduate school, I spent a year as a postbaccalaureate intern at Los Alamos National Laboratory. I am greatly indebted to Jeremy Conlin, Kent Parsons, Morgan White, Kevin Honnell, and Brian Kiedrowski for their efforts to make my internship rewarding and productive. The nuclear data experience that I gained from my time in Los Alamos has been a valuable asset ever since.

During the travails of graduate school, my beloved wife Lindsey has been a constant support who has helped me keep my sanity. Although my studies have robbed her and our two young children of much-needed quality time with me, she has been incredibly patient and understanding. I owe a tremendous debt to my parents, whose love and sacrifices on my behalf have enabled me to get to this point. My extended family (on both sides) has 
also done much to help me succeed. I can only offer my love and gratitude in response.

Finally, I would like to gratefully acknowledge that the work presented in this thesis was supported in part by the Nancy and John Jungerman Graduate Award in Physics and by a Nuclear Science and Security Consortium Fellowship, the latter of which was funded by the U. S. Department of Energy National Nuclear Security Administration under Award Number DENA0000979. 


\section{Chapter 1}

\section{Introduction}

\subsection{Prehistory of neutrino physics}

The ideas which led to the birth of neutrino physics first took shape with the discovery of radioactivity near the turn of the twentieth century. In 1896, prompted by Wilhelm Röntgen's discovery of X-rays during the previous year [1, 2], the French physicist Henri Becquerel performed a series of experiments using photographic film to determine if Xrays were the result of photoluminescence. ${ }^{1}$ His choice to use uranium salts as the first fluorescent materials to be tested would prove fortuitous. In a series of papers presented to the French Academy of Sciences that year, ${ }^{2}$ Becquerel reported that

The salts of uranium emit radiations the existence of which had not as yet been recognised.... The radiations of the uranium salts are emitted not only when the substances are exposed to light, but even when they have been kept in darkness, and for more than two months the same fragments of various salts kept secluded from any known exciting radiation have continued to emit new radiations almost without any appreciable decrease....

All the uranium salts which I have studied, whether phosphorescent to light or not, and whether crystalline, melted, or dissolved, have given me comparable results. I have therefore been led to think that the effect was due to the presence

\footnotetext{
${ }^{1}$ The absorption and reemission of light, typically with a change of frequency

${ }^{2}$ See [3] and references therein. Reference [4] provides English translations of several of the original papers.
} 
of the element uranium in these salts, and that the metal would give effects more intense than its compounds.

The experiment made some weeks ago with commercial powder of uranium which had been kept for a long time in my laboratory confirmed this prevision; the photographic effect is notably stronger than that produced by one of the salts of uranium, and by uranium-potassium sulphate. [5, 6]

Soon after Becquerel's discovery of radioactivity in uranium, Marie Curie and Gerhard Carl Schmidt independently noticed similar emissions from thorium. The previously unknown elements polonium and radium were soon thereafter discovered by Marie Curie and her husband Pierre, the latter element also in collaboration with Gustave Bémont, by means of the radiation that they produce. These achievements resulted in Becquerel and the Curies sharing the 1903 Nobel Prize in Physics.

While the Curies were finding new radioactive materials, a graduate student named Ernest Rutherford was among the first to try to understand the properties of the radiation emitted by uranium and thorium. From his early experiments in which he observed the absorption of this radiation by varying thicknesses of aluminum foil, Rutherford concluded that

There are present at least two distinct types of radiation-one that is very readily absorbed, which will be termed for convenience the $\alpha$ radiation, and the other of a more penetrative character, which will be termed the $\beta$ radiation. $[7]$

In 1900, Paul Villard reported on his experiments with radium that showed that the more penetrating radiation could be separated into a component whose path could be altered by a magnetic field (which retained the name $\beta$ radiation) and a component which showed no such response. The "undeviable" component was given the name $\gamma$ radiation within a few years [8], probably by Rutherford, although the context in which the name was first used is somewhat unclear [9]. The labels $\alpha, \beta$, and $\gamma$ radiation are still in common use today, and refer respectively to the emission of helium nuclei, (anti)electrons, and photons from an atomic nucleus. 


\subsection{The trouble with beta decay}

While the $\alpha$ and $\gamma$ radiation were both found to be emitted with discrete energies (indicating that the production mechanism was a simple two-body decay), James Chadwick reported measurements of $\beta$ radiation in 1914 [10] that revealed a continuous spectrum. Although currently understood to proceed via the processes

$$
\begin{aligned}
& n \rightarrow p+e^{-}+\bar{\nu}_{e} \\
& p \rightarrow n+e^{+}+\nu_{e}
\end{aligned}
$$

beta decays appeared to Chadwick and his contemporaries to involve the emission of only a single particle (an electron or positron) from a nucleus. This result had troubling implications that were debated over the next two decades. ${ }^{3}$ One possible explanation, championed by Lise Meitner and others [13], was that beta particles are indeed emitted with discrete energies, but they lose varying amounts of energy as they undergo finalstate interactions while leaving the nucleus. In 1927, however, calorimetric measurements by Ellis and Wooster [14] of the average energy released per $\beta$ decay of "radium E" (known today as ${ }^{210} \mathrm{Bi}$ ) yielded a value consistent with the mean of the continuous $\beta$ particle spectrum. This contradicted the final-state energy loss model, under which one would expect the average energy release to be greater than or equal to the endpoint of the $\beta$ spectrum. Meitner and Orthmann [15] confirmed these results with an improved experiment in 1930, leading the former to write in a letter to Ellis,

We have verified your results completely. It seems to be now that there can be absolutely no doubt that you were completely correct in assuming that beta radiations are primarily inhomogeneous. But I do not understand the result at all. [12]

With the possibility of secondary interactions eliminated, only more outlandish explanations of the continuous $\beta$ spectrum remained. Neils Bohr was willing to suggest that conservation of energy must be reinterpreted as a statistical law, so that $\beta$ decays need

\footnotetext{
${ }^{3}$ For detailed historical reviews of the early struggles to understand $\beta$ decay, see references $[11,12]$.
} 
only conserve energy on average. At a lecture delivered to the Royal Society of Chemistry on May 8, 1930, Bohr claimed that

At the present stage of atomic theory we have no argument, either empirical or theoretical, for upholding the energy principle in the case of $\beta$-ray disintegrations, and are even led to complications and difficulties in trying to do so. ... In atomic theory, notwithstanding all the recent progress, we must still be prepared for new surprises. [16]

One of Bohr's predicted "new surprises" came on December 4, 1930, when Wolfgang Pauli wrote an open letter ${ }^{4}$ to a conference of physicists in Tübingen, Germany. In the letter, he proposed a "desperate remedy" to the $\beta$ spectrum problem: the existence of a new electrically neutral particle called the "neutron":

The continuous $\beta$-spectrum would then become understandable by the assumption that in $\beta$ decay a neutron is emitted together with the electron, in such a way that the sum of the energies of the neutron and electron is constant. ... I admit that my remedy may appear to have a small a priori probability ... However, only those who wager can win, and [given] the seriousness of the situation of the continuous $\beta$-spectrum ... one should earnestly discuss every way of salvation. - So, dear radioactives, put it to the test and set it right. [13, 17]

With the discovery of the modern neutron in early 1932 by James Chadwick [18, 19], Pauli's proposed neutral particle, which would need to be far lighter than Chadwick's in order for it to have avoided detection up to that point, was in need of a new name. After hearing of Pauli's proposed "light neutron" during a conversation with Enrico Fermi, Edoardo Amaldi jokingly dubbed it the neutrino, a name which he would later note ${ }^{5}$ was, "a funny and grammatically incorrect contraction of 'little neutron' in Italian: neu-

\footnotetext{
${ }^{4}$ The full text of the letter is reprinted in the original German in reference [17]. An English translation is given in reference [13].

${ }^{5}$ See footnote 277 of reference [20].
} 
tronino.' The name stuck, and it was quickly adopted by both Fermi and Pauli himself $[20]$.

\subsection{Discovery of the three neutrino flavors}

While Pauli's proposed solution to the $\beta$ decay problem was little more than a guess at the time that he wrote his famous letter, the idea was soon made more scientifically rigorous by Enrico Fermi, who published a highly successful theory of $\beta$ decay [21, 22] in 1934. Fermi's theory, which featured the neutrino as an indispensable component, was soon used by Gian Carlo Wick [23] and Hideki Yukawa and Shoichi Sakata [24] to predict the existence of electron capture, a nuclear process related to $\beta$ decay in which an atomic electron is absorbed by a proton and an electron neutrino is emitted. Nuclear recoil energy spectra observed in electron capture experiments by James Allen [25] and Raymond Davis [26] were found to be consistent with the hypothesis of single neutrino emission and provided the earliest experimental evidence (other than the continuous $\beta$ decay spectrum itself) for the existence of neutrinos.

Despite an earlier detection attempt by Horace Richard Crane ${ }^{6}$ [27] in 1939, the first direct evidence for the existence of neutrinos would emerge in 1953 when Frederick Reines and Clyde Cowan published [28] preliminary results of an antineutrino detection experiment performed at the Hanford Site, a plutonium production facility established during the Manhattan project. They confirmed their discovery using a second experiment at the Atomic Energy Commission's Savannah River Site in 1956 [29, 30]. In both of the experiments, a nuclear reactor was used as a source of antineutrinos, which were detected via the inverse $\beta$ decay reaction

$$
\bar{\nu}_{e}+p \rightarrow n+e^{+}
$$

using a cadmium-loaded liquid scintillator target. Reines was awarded the 1995 Nobel Prize in Physics for this work.

The discovery of the muon [31] in 1937 paved the way toward the realization that there

\footnotetext{
${ }^{6}$ Crane studied the reaction $\bar{\nu}_{e}+{ }^{37} \mathrm{Cl} \rightarrow{ }^{35} \mathrm{~S}+e^{+}$but did not come close to achieving the needed sensitivity.
} 
are multiple kinds of neutrinos, now referred to as flavors. Early experiments [32] with muons showed that they decay into an electron, a neutrino, and an antineutrino. However, since muon decay to an electron and a photon was not observed [33], it was thought that the two neutrinos were distinct, with one corresponding to the type produced in nuclear beta decay (now known as the electron neutrino) and the second being referred to at the time as the "neutretto" (now known as the muon neutrino) [34]. These expectations were confirmed years later during the first neutrino beam experiment performed by Danby et al. [35] in 1962, which was an implementation of an idea originally devised by Bruno Pontecorvo [36] and Melvin Schwartz [37] independently of each other. High-energy muon neutrinos created by pion decays were observed to produce only muons (as opposed to the electrons produced by $\nu_{e}$ ) via charged current interactions in a spark chamber detector, demonstrating the difference between the neutrino flavors. For this achievement, the leaders of the experiment, Leon Lederman, Melvin Schwartz, and Jack Steinberger, were awarded the 1988 Nobel Prize in Physics.

In light of the discovery of the muon neutrino during the previous decade, when the tau lepton was discovered by Perl et al. [38] in 1975, a corresponding neutrino was expected to exist. However, due to the experimental challenges involved, a direct observation of this particle would not be achieved until the year 2000, when the DONUT (Direct Observation of the $\mathrm{Nu} \mathrm{Tau}$ ) collaboration reported the observation of 4 tau neutrino interaction events in a nuclear emulsion detector [39]. This detection confirmed the results of four earlier experiments (ALEPH [40], DELPHI [41], L3 [42], and OPAL [43]) at the Large ElectronPositron Collider (LEP) at CERN, ${ }^{7}$ which all indirectly pointed to the existence of three active $^{8}$ neutrino flavors with masses lighter than half the $Z$ boson mass.

\subsection{Neutrino interactions}

Our modern understanding of neutrino interactions (and weak processes in general) originated in theoretical work performed in the late 1950s and 1960s by Sheldon Glashow

\footnotetext{
${ }^{7}$ European Organization for Nuclear Research

${ }^{8}$ Active neutrinos are those that undergo weak interactions. In addition to the three active neutrino flavors that are currently known, there may exist one or more sterile neutrino species which do not participate in the weak interaction.
} 
[44, 45], Abdus Salam (together with John Clive Ward) [46, 47], and Steven Weinberg [48]. The Glashow-Weinberg-Salam (GWS) model, for which the authors were awarded the 1979 Nobel Prize in Physics, provides a unified treatment of the electromagnetic and weak interactions and resolves a number of problems with previous refinements to Fermi's 1934 theory (such as a lack of renormalizability).

Under the GWS theory, the Lagrangian governing the electromagnetic and weak interactions is invariant under symmetry transformations of the local gauge group $S U(2) \otimes$ $U(1)$. Three massless gauge bosons $\left(W_{1}, W_{2}\right.$, and $\left.W_{3}\right)$ are associated with the generators of $S U(2)$ (Pauli matrices whose eigenvalues are the three components of weak isospin), while a single massless gauge boson, $B$, is associated with weak hypercharge, the generator of $U(1)$. Via the Higgs mechanism, ${ }^{9}$ the $S U(2) \otimes U(1)$ symmetry is spontaneously broken, leaving only the $U(1)$ phase symmetry of electromagnetism. The four gauge bosons of the theory couple to the Higgs field, which acquires a nonzero vacuum expectation value. As a result, one of the linear combinations of $W_{3}$ and $B$, the $Z$ boson field

$$
Z^{0}=-B \sin \theta_{W}+W_{3} \cos \theta_{W}
$$

gains a nonzero mass, while the other, the photon,

$$
\gamma=B \cos \theta_{W}+W_{3} \sin \theta_{W}
$$

remains massless. The other two gauge bosons likewise combine to form the massive $W^{ \pm}$ bosons ${ }^{10}$ via

$$
W^{ \pm}=\frac{1}{\sqrt{2}}\left(W_{1} \mp i W_{2}\right) .
$$

The electromagnetic and weak processes observed in experiments are mediated by the exchange of the photon and the three massive bosons $\left(Z^{0}, W^{ \pm}\right)$, respectively [49]. The weak mixing angle $\theta_{W}$ is given in terms of the $W$ and $Z$ boson masses by

$$
\cos \theta_{W}=\frac{m_{W}}{m_{Z}}
$$

\footnotetext{
${ }^{9}$ For a review of the Higgs mechanism, see any recent textbook on the Standard Model, e.g., reference [49].

${ }^{10}$ Note that $W^{+}$is the antiparticle of $W^{-}$.
} 

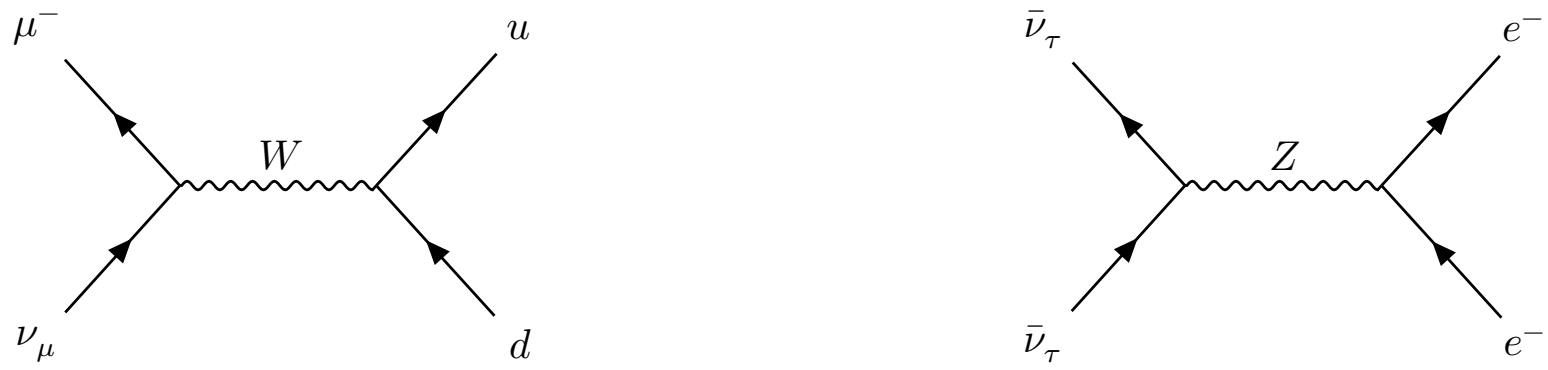

Figure 1.1: Feynman diagrams showing examples of the two general categories of neutrino interactions. LEFT: A muon neutrino undergoes a charged current interaction with a down quark, producing a muon and an up quark in the final state. RIGHT: A tau antineutrino elastically scatters off an electron in a neutral current interaction.

Because neutrinos interact only via the weak force and gravity, the latter of which is negligible in typical experimental situations, one may quite generally classify reactions involving them into two categories. In the first of these, a neutral current (NC) interaction, a neutrino exchanges a $Z^{0}$ boson with another particle, recoils, and emerges with its flavor unchanged. In the second, a charged current (CC) interaction, a neutrino exchanges a $W$ boson (either $W^{+}$or $W^{-}$) with another particle and is transformed into the charged lepton of the same flavor (e.g., $\nu_{e}$ becomes $e^{-}$). Section 1.4 illustrates examples of each of these two processes using Feynman diagrams.

The GWS theory forms a key part of the Standard Model of particle physics and has been validated by numerous experimental results, including the discovery of neutral current neutrino scattering on hadrons [50] and electrons [51] by the Gargamelle experiment in the 1970s, the direct detection of the $W[52,53]$ and $Z[54,55]$ bosons by the UA1 and UA2 experiments in 1983, and the discovery of the Higgs boson (and thus the Higgs field) in 2012 by the ATLAS [56] and CMS [57] experiments at the Large Hadron Collider (LHC). Additional tests of the model, with impressive experimental precision, continue to be made by LHC experiments (see reference [58] for a recent review).

Further discussion of the theory of neutrino interactions, including the Lagrangians describing $\mathrm{CC}$ and $\mathrm{NC}$ processes and a derivation of low-energy neutrino scattering cross sections, will be given in chapter 3 . 


\subsection{Neutrino oscillations}

In the Standard Model, neutrinos are assumed to be massless, and lepton flavor is conserved. ${ }^{11}$ Around the turn of the last century, the discovery of neutrino oscillations, a phenomenon in which a neutrino produced with a definite flavor can later be measured to have a different flavor, demonstrated both of these assumptions to be false. This discovery provided some of the first evidence of inadequacies in the Standard Model and has opened up an entirely new area of particle physics research.

To see why neutrino oscillations occur, it is sufficient to note that a neutrino in a state of definite flavor $\left|\nu_{\ell}\right\rangle$ (for $\ell \in\{e, \mu, \tau\}$ ) corresponds to a superposition of states $\left|\nu_{j}\right\rangle$ with definite mass, and the two eigenbases are connected via the relation

$$
\left|\nu_{\ell}\right\rangle=\sum_{j} U_{\ell j}\left|\nu_{j}\right\rangle
$$

where the $U_{\ell j}$ are elements of a unitary matrix called the Pontecorvo-Maki-NakagawaSakata (PMNS) matrix. For freely-propagating neutrinos in vacuum, the states $\left|\nu_{j}\right\rangle$ are eigenstates of the Hamiltonian with total energy ${ }^{12}$

$$
E_{j}=\sqrt{|\mathbf{p}|^{2}+m_{j}^{2}}
$$

where $\mathbf{p}$ is the neutrino 3 -momentum and $m_{j}$ is the mass of the $j$ th mass eigenstate. According to the Schrödinger equation, the time evolution of the mass states may be described using the plane wave expression

$$
\left|\nu_{j}(t)\right\rangle=e^{-i E_{j} t}\left|\nu_{j}\right\rangle
$$

where $\left|\nu_{j}\right\rangle$ is the $j$ th mass state at time $t=0$. Using the unitarity of the PMNS matrix, it can be shown [59] that the flavor state $\left|\nu_{\alpha}(t)\right\rangle$ at time $t$ can be expressed in terms of the flavor states $\left|\nu_{\beta}\right\rangle$ as

$$
\left|\nu_{\alpha}(t)\right\rangle=\sum_{\beta}\left(\sum_{j} U_{\alpha j} e^{-i E_{j} t} U_{\beta j}^{*}\right)\left|\nu_{\beta}\right\rangle .
$$

\footnotetext{
${ }^{11}$ That is, the number of leptons minus the number of antileptons of each flavor (electron, muon, and tau) must be the same before and after a reaction.

${ }^{12}$ Unless otherwise noted, natural units (with $\hbar=c=1$ ) are used throughout this thesis.
} 
The probability $P_{\nu_{\alpha} \rightarrow \nu_{\beta}}$ that a neutrino in a pure flavor state $\left|\nu_{\alpha}\right\rangle$ at time $t=0$ would be observed to have flavor $\beta$ at time $t$ is therefore given by

$$
P_{\nu_{\alpha} \rightarrow \nu_{\beta}}=\left|\left\langle\nu_{\beta} \mid \nu_{\alpha}(t)\right\rangle\right|^{2}=\sum_{j, k} U_{\alpha k} U_{\beta k}^{*} U_{\alpha j}^{*} U_{\beta j} e^{-i\left(E_{k}-E_{j}\right) t}
$$

Since neutrinos have tiny masses and travel at speeds close to the speed of light, the factors in the exponential are usually approximated by

$$
\begin{aligned}
& E_{k}-E_{j} \approx \frac{\Delta m_{k j}^{2}}{2 E} \\
& t \approx L
\end{aligned}
$$

where (in natural units) $E \approx|\mathbf{p}|$ is the neutrino energy, ${ }^{13} L$ is the distance traveled by the neutrino in time $t$, and

$$
\Delta m_{k j}^{2} \equiv m_{k}^{2}-m_{j}^{2}
$$

is the difference of the squared masses of the mass eigenstates $\left|\nu_{k}\right\rangle$ and $\left|\nu_{j}\right\rangle$. These approximations lead to the standard expression for the oscillation probability

$$
P_{\nu_{\alpha} \rightarrow \nu_{\beta}}(L, E)=\sum_{j, k} U_{\alpha k} U_{\beta k}^{*} U_{\alpha j}^{*} U_{\beta j} \exp \left(-i \frac{\Delta m_{k j}^{2} L}{2 E}\right) .
$$

Neutrino oscillations were first anticipated theoretically in the late 1950s and 1960s in a series of papers by Bruno Pontecorvo [60, 61]; Ziro Maki, Masami Nakagawa, and Shoichi Sakata [62]; and Vladimir Gribov and Bruno Pontecorvo [63]. Although it was not correctly understood by the physics community for many years, the first experimental evidence for neutrino oscillations was obtained by the Homestake solar neutrino experiment [64, 65] led by Raymond Davis. The Homestake neutrino detector consisted of a 100000 gallon tank of perchloroethylene, a liquid often used to dry clean clothing, which was installed underground in a gold mine near Lead, South Dakota. Electron neutrinos produced by fusion processes in the Sun were detected via the charged current reaction

$$
\nu_{e}+{ }^{37} \mathrm{Cl} \rightarrow{ }^{37} \mathrm{Ar}+e^{-}
$$

\footnotetext{
${ }^{13}$ The momentum $\mathbf{p}$ is the same for all of the flavor states.
} 
which produced radioactive ${ }^{37} \mathrm{Ar}$ argon atoms in the tank. These atoms were chemically extracted every few months and counted to determine the reaction rate and the corresponding solar neutrino flux. Over several decades, the Homestake experiment consistently reported a $\nu_{e}$ flux from the Sun that was about one third of the value predicted by standard solar models, including calculations by Davis's collaborator John Bahcall [66]. This solar neutrino problem was confirmed when similar deficits were seen by other solar neutrino experiments, including Kamiokande [67] SAGE [68], and GALLEX [69, 70].

A second set of anomalies that hinted at the existence of neutrino oscillations were measurements of atmospheric neutrinos by IMB [71] and Kamiokande [72, 73], both of which found an unexpectedly low value of the ratio of atmospheric $\nu_{\mu}$ to $\nu_{e}$ events. Reviews of this atmospheric neutrino problem written by participants in the related analyses may be found in references [74, 75].

Compelling evidence that both of these problems could be explained by neutrino oscillations came from the Super-Kamiokande (Super-K) and SNO experiments. In 1998, the Super-Kamiokande collaboration published an analysis in which they found a directional asymmetry in the number of detected atmospheric muon neutrino events, with more events being observed for neutrinos traveling downward when compared with those traveling upward through the detector [76]. The asymmetry could readily be explained by $\nu_{\mu} \rightarrow \nu_{\tau}$ oscillations: since the upward-going muon neutrinos had to travel through the earth to reach the detector, more time was available for them to oscillate into tau neutrinos, which were not detectable by Super-Kamiokande.

In 2002, the SNO collaboration reported a measurement of the relative rates of the charged current reaction

$$
\nu_{e}+{ }^{2} \mathrm{H} \rightarrow p+p+e^{-}
$$

and the neutral current reaction

$$
\nu_{x}+{ }^{2} \mathrm{H} \rightarrow p+n+\nu_{x} \quad x \in\{e, \mu, \tau\}
$$

induced by solar neutrinos within their heavy-water-based detector [77]. Because only the $\nu_{e}$ component of the flux could participate in the $\mathrm{CC}$ reaction, while all neutrino 
flavors participated in the NC reaction, a simultaneous measurement of the two reaction rates allowed a determination of the total solar neutrino flux and the $\nu_{e}$ fraction. Under the assumption that neutrino oscillations were taking place, SNO's results were shown to be consistent with standard solar models, providing an elegant solution to the solar neutrino problem. The achievements of the Super-Kamiokande and SNO collaborations in establishing the existence of neutrino oscillations were recognized when leaders from the two experiments, Takaaki Kajita and Arthur McDonald, were awarded the 2015 Nobel Prize in Physics.

In the years since these pioneering measurements, a wealth of new data on neutrino oscillations has been obtained in a worldwide experimental effort. To date, nearly all oscillation measurements can be described using a 3-flavor model of neutrino mixing, in which the PMNS matrix from eq. (1.8) is parameterized in terms of three mixing angles $\left(\theta_{12}, \theta_{13}\right.$, and $\left.\theta_{23}\right)$ and a charge-parity $(\mathrm{CP})$ symmetry violating phase $\delta_{C P}$, which generates differences in the oscillation probabilities for neutrinos and antineutrinos. Under the 3-flavor model, the PMNS matrix may be written in the form [78]

$$
U_{\ell j}=\left(\begin{array}{ccc}
j=1 & j=2 & j=3 \\
c_{12} c_{13} & s_{12} c_{13} & s_{13} e^{-i \delta_{C P}} \\
-s_{12} c_{23}-c_{12} s_{23} s_{13} e^{i \delta_{C P}} & c_{12} c_{23}-s_{12} s_{23} s_{13} e^{i \delta_{C P}} & s_{23} c_{13} \\
s_{12} s_{23}-c_{12} c_{23} s_{13} e^{i \delta_{C P}} & -c_{12} s_{23}-s_{12} c_{23} s_{13} e^{i \delta_{C P}} & c_{23} c_{13}
\end{array}\right)_{\ell=e}^{\ell=\mu}
$$

where $s_{i j} \equiv \sin \theta_{i j}$ and $c_{i j} \equiv \cos \theta_{i j}$. Table 1.1 gives recently obtained best-fit values for the three mixing angles $\theta_{j k}$, the CP violating phase $\delta_{C P}$, and two of the three squared mass differences ${ }^{14} \Delta m_{j k}^{2}$ needed to compute oscillation probabilites using the 3 -flavor model. ${ }^{15}$ If neutrinos are Majorana fermions (see section 1.6), then two additional CP-violating phases are needed to write down the PMNS matrix. However, factors containing the Majorana phases cancel out in the expressions for oscillation probabilities (see eq. (1.16)), so they are not observable in neutrino oscillation experiments.

\footnotetext{
${ }^{14}$ Note that $\Delta m_{23}^{2}$ may be obtained from the other two squared mass differences via $\Delta m_{23}^{2}=\Delta m_{21}^{2}-$ $\Delta m_{31}^{2}$.

${ }^{15}$ See eq. (1.16).
} 
Table 1.1: Results of a 2018 global fit of the 3-flavor model neutrino oscillation parameters [79]. Where applicable, best-fit values are given assuming a normal neutrino mass hierarchy $\left(m_{3}>m_{2}\right)$ or an inverted hierarchy $\left(m_{3}<m_{2}\right)$.

\begin{tabular}{|c|c|c|c|c|c|c|c|}
\hline & Parameter & $\begin{array}{c}\Delta m_{21}^{2} \\
\left(10^{-5} \mathrm{eV}^{2}\right)\end{array}$ & $\begin{array}{c}\left|\Delta m_{31}^{2}\right| \\
\left(10^{-3} \mathrm{eV}^{2}\right)\end{array}$ & $\begin{array}{l}\theta_{12} \\
\left({ }^{\circ}\right)\end{array}$ & $\begin{array}{l}\theta_{23} \\
\left(^{\circ}\right)\end{array}$ & $\begin{array}{l}\theta_{13} \\
\left(^{\circ}\right)\end{array}$ & $\begin{array}{c}\delta_{C P} \\
\left(^{\circ}\right)\end{array}$ \\
\hline \multirow{2}{*}{$\begin{array}{c}\text { Best fit } \\
\pm 1 \sigma\end{array}$} & Normal hierarchy & \multirow{2}{*}{$7.55_{-0.16}^{+0.20}$} & $2.50 \pm 0.03$ & \multirow{2}{*}{$34.5_{-1.0}^{+1.2}$} & $47.7_{-1.7}^{+1.2}$ & $8.45_{-0.14}^{+0.16}$ & $218_{-27}^{+38}$ \\
\hline & Inverted hierarchy & & $2.42_{-0.04}^{+0.03}$ & & $47.9_{-1.7}^{+1.0}$ & $8.53_{-0.15}^{+0.14}$ & $281_{-27}^{+23}$ \\
\hline
\end{tabular}

It is currently unknown whether the third neutrino mass, $m_{3}$, is larger or smaller than the second $m_{2}$, although the latest experimental results favor the former at the $3 \sigma$ level [79]. In the literature, the $m_{3}>m_{2}$ hypothesis is commonly referred to as the normal hierarchy (or normal ordering) of the neutrino masses, while $m_{3}<m_{2}$ is referred to as the inverted hierarchy. Determining the correct neutrino mass hierarchy and obtaining a precise measurement of $\delta_{C P}$ (which is poorly constrained at present) are primary goals of current and future neutrino oscillation experiments.

While the 3-flavor model has been highly successful in describing the majority of existing neutrino oscillation measurements, there exist several experimental anomalies whose disagreements with the 3-flavor model have not yet been satisfactorily explained. Examples of these anomalies ${ }^{16}$ include an excess of low-energy events seen in the LSND and MiniBooNE neutrino beam experiments [81] and a deficit in the $\bar{\nu}_{e}$ fluxes measured by reactor neutrino experiments $[82,83]$. Current experimental efforts to address these anomalies include the Short-Baseline Neutrino Program at Fermilab [84] and the PROSPECT experiment [85] at Oak Ridge National Laboratory. If confirmed, the anomalies may signal the existence of a fourth sterile neutrino flavor which does not participate in weak interactions.

\subsection{Neutrino masses}

While neutrino oscillation data provide compelling evidence that neutrinos have nonzero masses, the absolute scale of their masses remains unknown. Currently, the most stringent

\footnotetext{
${ }^{16}$ Reference [80] provides a detailed review of anomalies in the neutrino oscillation literature.
} 
model-independent constraints on the neutrino mass scale are provided by measurements of the effective electron antineutrino mass

$$
m_{\bar{\nu}_{e}}^{2} \equiv \sum_{j}\left|U_{e j}\right|^{2} m_{j}^{2}
$$

obtained using the electron energy spectrum near the endpoint of tritium beta decay

$$
{ }^{3} \mathrm{H} \rightarrow{ }^{3} \mathrm{He}+e^{-}+\bar{\nu}_{e}
$$

In 2011, data from the Troitsk experiment were reanalyzed to obtain the upper bound $[86]$

$$
m_{\bar{\nu}_{e}}<2.05 \mathrm{eV}
$$

Future measurements of tritium beta decay by the KATRIN [87] and Project 8 [88] experiments aim to improve this limit by one (KATRIN) or two (Project 8) orders of magnitude.

At present, strict constraints on the sum of the neutrino masses are available from cosmological observations, e.g., the Planck Collaboration's upper limit of [89]

$$
\sum_{k} m_{k}<0.170 \mathrm{eV}
$$

but certain model assumptions must be made to interpret the data in this way [78].

\subsubsection{Dirac masses}

Because the neutrino masses are nonzero and yet much smaller than those of all of the other fermions, there is considerable theoretical interest in identifying the mechanism by which neutrino masses are generated. The possible generation mechanisms depend on whether neutrinos are Dirac or Majorana fermions.

If neutrinos are Dirac fermions, i.e., if the chiral projections $\nu_{k, L}$ and $\nu_{k, R}$ of the $k$ th neutrino field $\nu_{k}$, defined by

$$
\nu_{k, L} \equiv \frac{1}{2}\left(1-\gamma_{5}\right) \nu_{k} \quad \nu_{k, R} \equiv \frac{1}{2}\left(1+\gamma_{5}\right) \nu_{k}
$$

are independent of each other, then it is possible that neutrinos obtain their masses via the Higgs mechanism in the same way as all other massive fermions in the Standard Model. 
For three neutrino species, this possiblity would be represented by adding the terms

$$
\mathcal{L}_{\nu, h}=-\sum_{k=1}^{3} \frac{y_{k}}{\sqrt{2}} \bar{\nu}_{k} \nu_{k}(v+H)=-\sum_{k=1}^{3} m_{k} \bar{\nu}_{k} \nu_{k}-\sum_{k=1}^{3} \bar{\nu}_{k} \nu_{k} H
$$

to the Standard Model Lagrangian [59]. Here $H$ is the physical Higgs field, $\nu_{k}=\nu_{k, L}+\nu_{k, R}$ is the $k$ th neutrino field (in the mass basis), the first sum on the right-hand side includes the neutrino mass terms, and the second sum represents the interactions between the Higgs field and each of the neutrino fields. The mass of the $k$ th neutrino $m_{k}$ may be written in terms of the Higgs vacuum expectation value $v$ and a dimensionless coupling constant $y_{k}$ :

$$
m_{k}=\frac{y_{k} v}{\sqrt{2}}
$$

Although the generation of the Dirac masses described above requires only a minimal extension of the Standard Model (the addition of right-handed components of the neutrino fields $\nu_{k, R}$ and a nonzero coupling between neutrinos and the Higgs field), it might be considered theoretically unsatisfying due to the large difference between the neutrino mass scale (order $1 \mathrm{eV}$ at most) and the Higgs vaccum expectation value $v \approx 246 \mathrm{GeV}$. Since the Dirac neutrino masses are proportional to $v$, this implies that the coupling constants $y_{k}$ must be extremely small, e.g., for a neutrino with a mass of $1 \mathrm{eV}, y_{k} \approx 5.7 \times 10^{-12}$, while the next lightest fermion, the electron, has $y_{k} \approx 2.9 \times 10^{-6}$. The lack of an explanation for the remarkable smallness of the neutrino masses might therefore be seen as a defect of the theory, even while recognizing that all of the couplings between the fundamental fermions and the Higgs field are free parameters in the Standard Model that must be determined empirically.

\subsubsection{Majorana masses}

For Majorana neutrinos, the chiral projections $\nu_{k, L}$ and $\nu_{k, R}$ of the field $\nu_{k}$ are not independent. Instead, the right-handed projection may be written in terms of the left-handed one via the relation

$$
\nu_{k, R}=\nu_{k, L}^{C} \equiv \mathcal{C}{\overline{\nu_{k, L}}}^{T}=\mathcal{C}\left(\nu_{k, L}^{\dagger} \gamma^{0}\right)^{T}
$$


where $\mathcal{C}$ is the charge conjugation matrix. Like all Majorana fermion fields, the Majorana neutrino field

$$
\nu_{k}=\nu_{k, L}+\nu_{k, R}=\nu_{k, L}+\nu_{k, L}^{C}
$$

has the important property

$$
\nu_{k}^{C}=\left(\nu_{k, L}+\nu_{k, L}^{C}\right)^{C}=\nu_{k, L}^{C}+\nu_{k, L}=\nu_{k},
$$

i.e., Majorana neutrinos are their own antiparticles. Although more exotic possibilities exist, the simplest Majorana mass term that can be constructed using the Standard Model fields and symmetries is [59] (restricting ourselves to a single neutrino species $\nu_{k}$ for simplicity)

$$
\mathcal{L}_{\text {Majorana mass }}=\frac{1}{2} m_{k}\left(\nu_{k, L}^{T} \mathcal{C}^{\dagger} \nu_{k, L}+\nu_{k, L}^{\dagger} \mathcal{C} \nu_{k, L}^{*}\right)
$$

The Majorana neutrino mass $m_{k}$ is given by

$$
m_{k}=\frac{g v^{2}}{M}
$$

where $g$ is a dimensionless coupling constant, and $M$ is a large mass characteristic of some high-energy unified theory.

Since it is assumed that $M \gg v$, the presence of $M$ in eq. (1.32) may be readily invoked to explain the smallness of the neutrino masses, thus eliminating one of the main theoretical objections to the Dirac mass mechanism considered above. However, the product of fields which generates the Majorana mass term given in eq. (1.31) is not renormalizable, necessitating an appeal to a currently unknown high-energy theory (which will solve the renormalizability problem) to justify the addition of such a term to the Standard Model.

An interesting feature of the Majorana mass term in eq. (1.32) is that it violates lepton number symmetry. While most lepton number violating processes that would enabled by the Majorana mass term would be far too rare to be detected in the foreseeable future, ${ }^{17}$ an important exception is neutrinoless double beta $(0 \nu \beta \beta)$ decay, a hypothesized

\footnotetext{
${ }^{17}$ See, e.g., reference [90] for a discussion of Majorana neutrinos undergoing neutrino-antineutrino oscillations.
} 


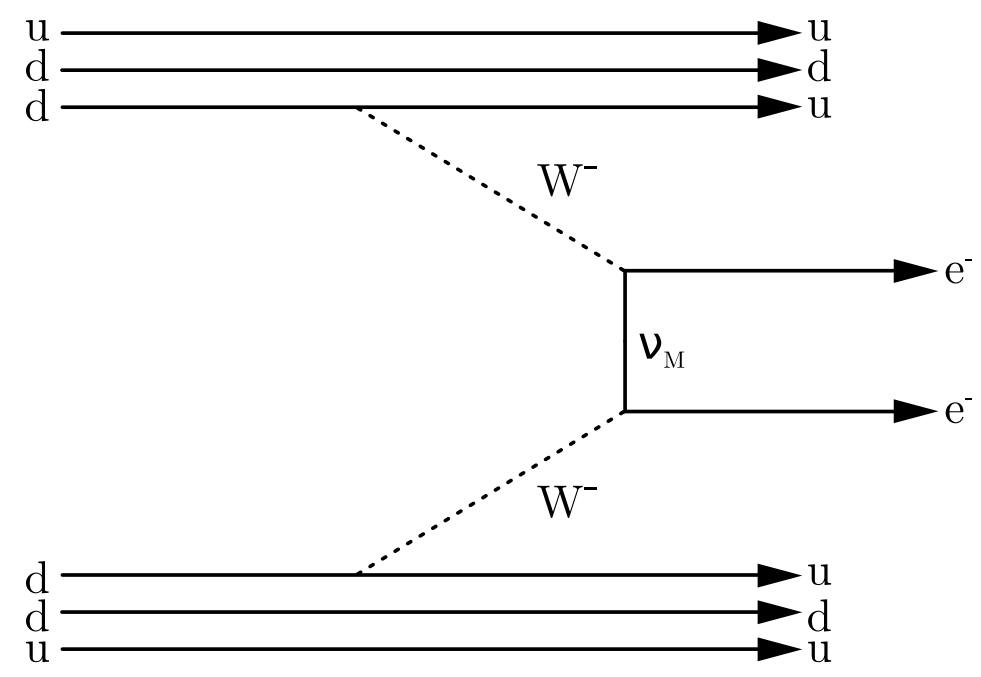

Figure 1.2: Neutrinoless double beta decay generated via the exchange of a virtual Majorana neutrino between two decaying nucleons. Figure from reference [93].

nuclear process in which two neutrons are transformed into two protons and two electrons without the emission of any neutrinos. While a number of different mechanisms could be responsible for $0 \nu \beta \beta$ decay, the most straightforward option is the exchange of a virtual Majorana neutrino between two decaying nucleons, as shown in fig. 1.2. Regardless of the underlying cause of the decay, in the absence of pathological fine-tuning, the observation of $0 \nu \beta \beta$ decay would imply that at least one neutrino species has a Majorana mass term [91]. Because this conclusion remains valid even in the presence of arbitrary new physics processes that contribute to $0 \nu \beta \beta$ decay, it is sometimes referred to in the literature as the "black box theorem" [92].

Many current and proposed experiments are attempting to discover neutrinoless double beta decay, including GERDA [94], Majorana [95], SNO+ [96], KamLAND-Zen [97], and EXO-200 [98], among others. While no credible observation of $0 \nu \beta \beta$ has yet been made, limits have been set on the half-life of $0 \nu \beta \beta$ decay, and significant effort is being invested in improving the sensitivity of the relevant detector technologies for nextgeneration searches. Recent reviews of the $0 \nu \beta \beta$ decay literature include references [93, 99, 100].

Although the discussion above has considered Dirac and Majorana mass terms for neutrinos as distinct possiblities, they are by no means mutually exclusive. In the most 
general case, each neutrino species $\nu_{k}$ could have an overall mass term $\mathcal{L}_{\text {mass }, k}$ of the form $[59]$

$$
\mathcal{L}_{\text {mass }, k}=\mathcal{L}_{\text {mass }, k}^{D}+\mathcal{L}_{\text {mass }, k}^{L}+\mathcal{L}_{\text {mass }, k}^{R}
$$

where $\mathcal{L}_{\text {mass }, k}^{D}$ is a Dirac mass term, and $\mathcal{L}_{\text {mass }, k}^{L}\left(\mathcal{L}_{\text {mass }, k}^{R}\right)$ are Majorana mass terms for the left-handed (right-handed) chiral projections of the $\nu_{k}$ field.

\subsection{Neutrino detection techniques}

Since the first experimental observation of reactor antineutrinos by Reines and Cowan [30], a variety of neutrino detectors have been developed using targets made of water, ice, iron, lead, argon, sodium iodide, and plastic and liquid scintillator, among other materials. Currently existing large (kiloton-scale) neutrino experiments typically use either water or liquid scintillator as the target material, while the planned Deep Underground Neutrino Experiment (DUNE) will use $40 \mathrm{kt}$ of liquid argon as a target. These three detector technologies are briefly reviewed in the following sections.

\subsubsection{Water Cherenkov detectors}

Among the largest neutrino detectors constructed to date are water Cherenkov detectors like Super-Kamiokande [101], which consist of a tank of purified water instrumented with photodetectors. Neutrino interactions within the water tank lead to the creation of charged particles. By means of a process called the Cherenkov effect, these charged particles produce light which may be used to reconstruct the neutrino interaction.

The Cherenkov effect was discovered experimentally by Pavel Cherenkov [102] in 1934 and explained theoretically by Igor Tamm and Ilya Frank [103] in 1937. These achievements led to the three scientists being awarded the Nobel Prize in Physics in 1958.

\subsubsection{Cherenkov radiation}

The Cherenkov effect is a phenomenon in which a fast-moving charged particle induces the emission of light by a surrounding dielectric medium. In response to the electric field of the particle, the material in its vicinity becomes temporarily polarized. After the charged particle passes, the molecules of the material reequilibrate by emitting electromagnetic 
radiation. When the speed of the charged particle is less than the phase velocity of light in the material, the distribution of the induced dipoles is symmetrical around the particle track, and there is no net radiation in the far field. However, when the particle speed $\beta$ exceeds the phase velocity of light in the material, i.e., when

$$
\beta>\frac{1}{n}
$$

where $n$ is the material's index of refraction, the polarized region of the material acquires a nonzero dipole moment and the electromagnetic radiation emitted from the induced dipoles interferes constructively at an angle $\theta$ with respect to the direction of the particle motion. The Cherenkov radiation emission angle $\theta$ obeys the relation

$$
\cos \theta=\frac{1}{n \beta}
$$

for $\beta \geq 1 / n$. The emitted light forms a cone around the direction of the charged particle with half opening angle $\theta$ (see fig. 1.4). The spectrum of Cherenkov radiation may be expressed in terms of the energy radiated $d E$ per unit length traveled by the particle $d x$ per unit of angular frequency $d \omega$ via the Frank-Tamm formula [104] (in Gaussian units)

$$
\frac{d^{2} E}{d x d \omega}=\frac{q^{2}}{c^{2}} \omega\left(1-\frac{1}{[n(\omega) \beta]^{2}}\right)
$$

where $q$ is the particle charge and $n(\omega)$ is the index of refraction of the medium, which is typically frequency-dependent. As the proportionality of the spectrum in eq. (1.36) to $\omega$ indicates, the emission of Cherenkov radiation is more intense for higher frequencies. As a result, Cherenkov radiation is perceived by the human eye as a blue glow (see fig. 1.5).

Equation (1.35) implies that there is a threshold kinetic energy

$$
E_{C}=m\left(\frac{n}{\sqrt{n^{2}-1}}-1\right)
$$

where $m$ is the particle mass, below which Cherenkov radiation will not be produced. For water (which has an index of refraction of about 1.33 for visible light), the Cherenkov threshold for electrons is $E_{C} \approx 0.26 \mathrm{MeV}$, while for muons it is about $55 \mathrm{MeV}$. In a water Cherenkov neutrino detector, an electron or muon created by a charged current neutrino interaction is detected using the Cherenkov radiation that it produces when 

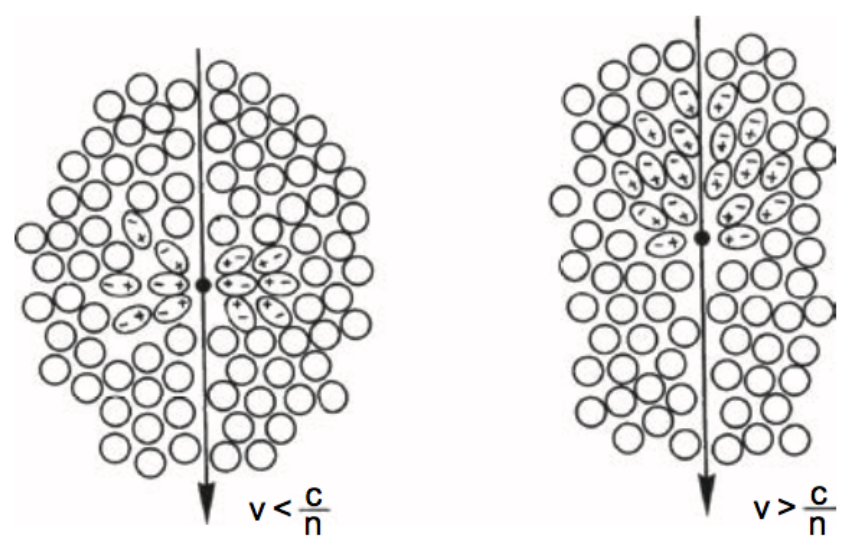

Figure 1.3: Induced dipoles created by an electrically charged particle traveling through a dielectric material. LEFT: For a particle traveling with a speed less than the phase velocity of light in the material, the distribution of the induced dipoles is symmetric. RIGHT: If the particle exceeds the local phase velocity of light, then an asymmetric pattern of induced dipoles is formed, resulting in the emission of Cherenkov radiation. Figure from reference [105].

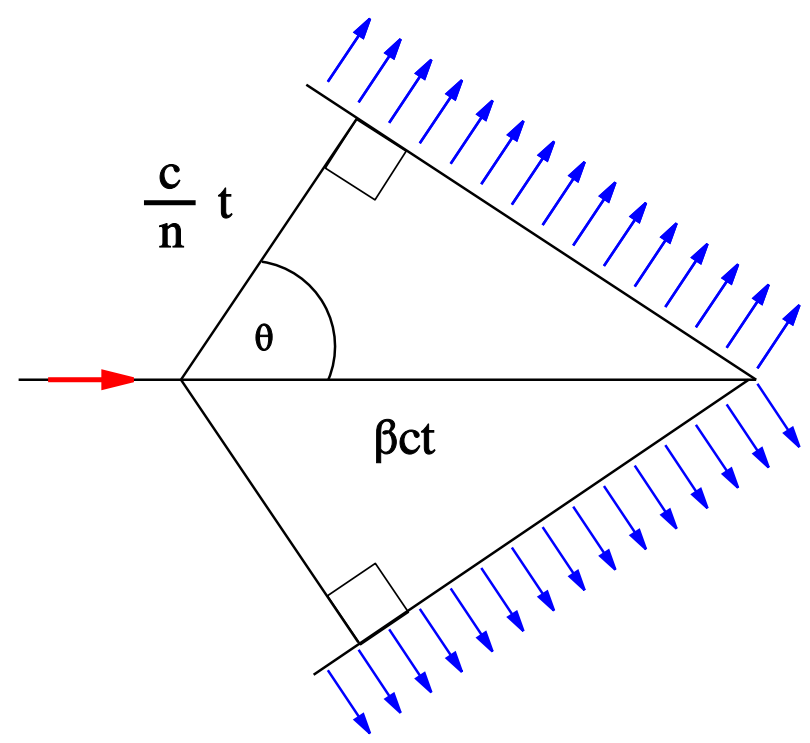

Figure 1.4: Cherenkov radiation induced by a charged particle is emitted (blue arrows) in a cone around the particle's direction of motion (red arrow). The cone has half opening angle $\theta=\arccos \left(n^{-1} \beta^{-1}\right)$, where $n$ is the index of refraction of the medium and $\beta$ is the (dimensionless) speed of the particle. Cherenkov radiation is emitted only for $\beta \geq 1 / n$. Figure from reference [106]. 


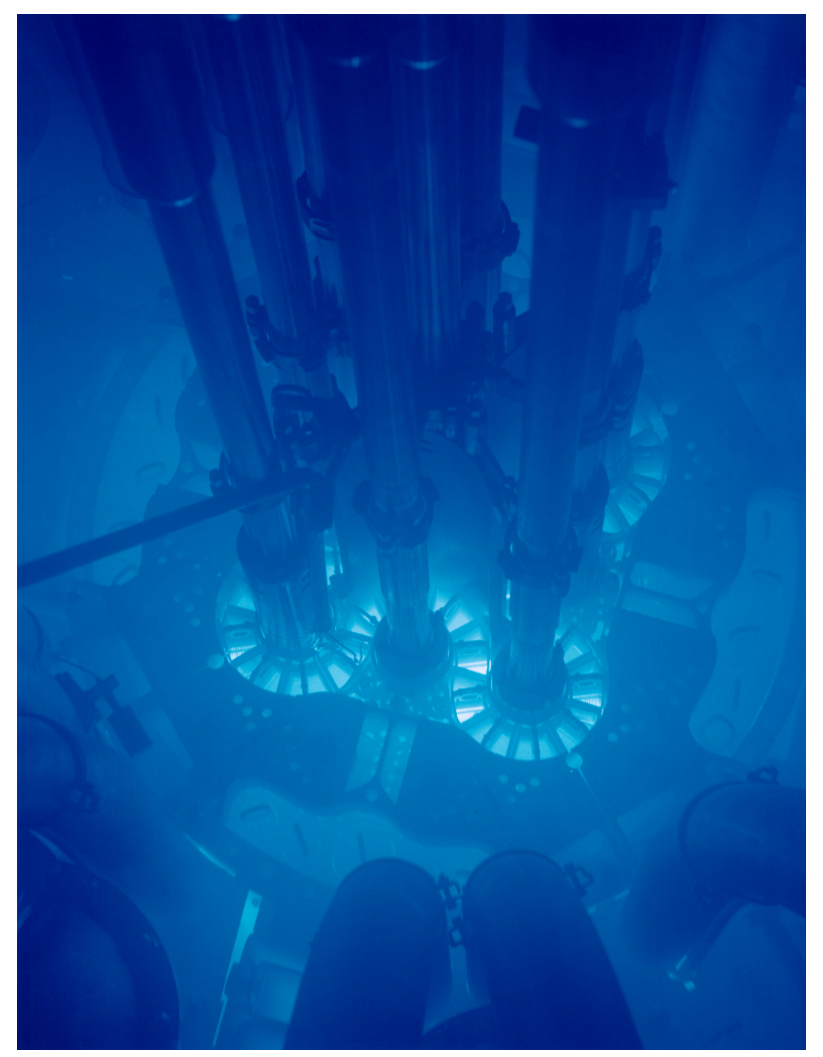

Figure 1.5: Cherenkov radiation is seen as a bright blue glow around the fuel plates of the Advanced Test Reactor at Idaho National Laboratory. Figure from reference [107].

above threshold. Imaging the cone of light created by the particle provides information about its energy and direction of propagation.

\subsubsection{Photomultiplier tubes}

The standard apparatus used to measure the flashes of light produced by charged particles in a water Cherenkov detector is the photomultiplier tube (PMT). Figure 1.6 is a schematic showing the basic components of a PMT. When photons, such as those emitted as Cherenkov radiation, pass through the PMT faceplate (typically a glass window) and strike the photocathode, they may eject an electron by means of the photoelectric effect. A vacuum is maintained within the PMT, allowing the electron to move freely in response to an electric field that accelerates it toward a series of dynodes. As the electron strikes each dynode, secondary electrons are emitted, amplifying the original single-electron signal created by the photon. After many such amplification steps, in which the secondary electrons are themselves multiplied, a total of around $10^{7}$ electrons per detected photon 


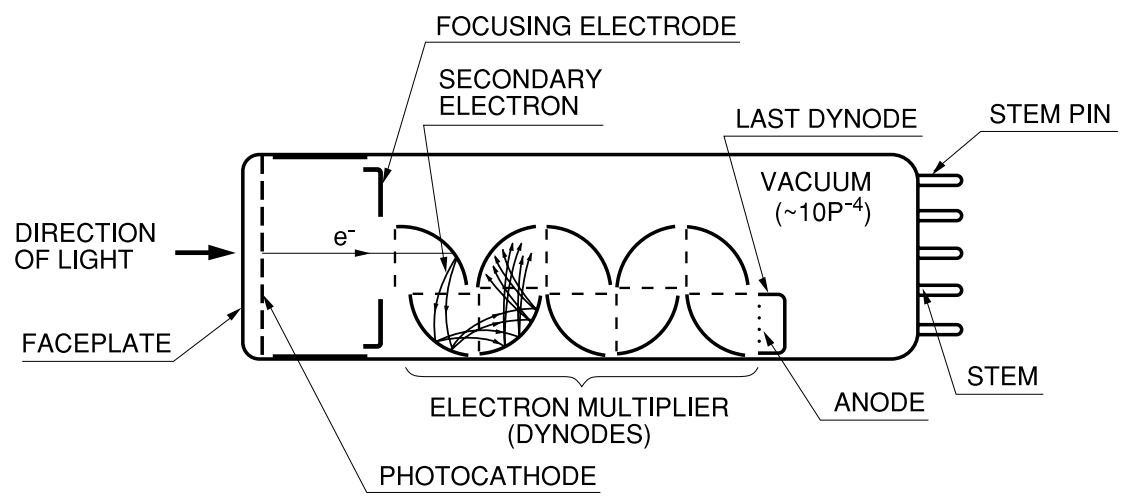

Figure 1.6: The basic components of a photomultiplier tube. The dynodes are held at different voltages by connecting the stem pins to a suitable base containing a voltage divider. Figure from reference [108].

is collected at the anode, resulting in a measurable pulse of current.

Although PMTs are exquisitely sensitive devices that are routinely used to detect individual photons, they are not without limitations. One significant issue that affects all PMTs is the presence of dark pulses, that is, current pulses registered by the PMT that do not correspond to a photon striking the photocathode. Dark pulses are primarily the result of random thermal excitations that cause an electron to be ejected from the photocathode. Since the electrons thermally produced at the dynodes will not pass through as many multiplication stages as electrons ejected from the photocathode, many dark pulses will have relatively small amplitudes compared with pulses produced by a true photoelectron.

Another problem that must be considered when interpreting PMT signals is afterpulsing: the tendency of a PMT to register one or more spurious small pulses (afterpulses) following a large-amplitude signal pulse. Afterpulses are largely produced by two distinct mechanisms [108]. Those that occur within nanoseconds to tens of nanoseconds after the signal pulse are created by electrons that elastically scatter off of the first dynode and are then reaccelerated toward it. Afterpulses that occur with a longer delay (tens of nanoseconds to several microseconds) occur when photoelectrons ionize trace amounts of gas within the PMT. The positively-charged gas ions are accelerated toward the photocathode and generate secondary electrons which may be mistaken for those produced by photon interactions. To distinguish between the two afterpulsing mechanisms, some authors (see, e.g., reference [109]) use the term late pulse to refer to an unwanted pulse 
that occurs at an early time while keeping the designation afterpulse for one that occurs later.

Chapter 9 presents an analysis of PMT event rates for the ANNIE experiment in which the importance of accounting for dark pulses and afterpulses will become apparent. References [108, 109] provide many more details about PMT operation, circuitry, and applications.

\subsubsection{Liquid scintillator detectors}

Like water Cherenkov neutrino detectors, liquid scintillator detectors, including Double Chooz [110], KamLAND [111], and the planned Jiangmen Underground Neutrino Observatory (JUNO) [112], consist of a tank filled with a liquid target and instrumented with photomultiplier tubes. In these detectors, however, the light that signals the presence of neutrinos is obtained via scintillation, the production of visible or near-visible light by certain materials in response to ionizing radiation.

Scintillating materials are typically chosen so that the light yield, i.e., the number of emitted scintillation photons per unit of deposited energy, is large and approximately linear in the deposited energy. Because the constant of proportionality will vary between different particle species, the absolute light yield of a scintillator is often expressed in terms of the amount of light produced by an electron with a given energy. Thus, an electron that deposits $1 \mathrm{MeV}$ in a scintillator is said to produce $1 \mathrm{MeV}$ electron equivalent (1 MeVee) of light (which will correspond to some particular number of scintillation photons), while a proton may need to deposit several $\mathrm{MeV}$ to achieve the same light yield. Beyond a high light yield, other desirable properties for scintillators include high transparency, fast light emission, an index of refraction close to that of glass (for optimal optical coupling to PMTs), and the ability to manufacture the material in large quantities [113]. Large scintillating neutrino detectors typically employ hydrocarbon liquid scintillators, such as linear alkylbenzene, as the target material.

While similar in many respects, water Cherenkov and liquid scintillator detectors differ in the information that they are able to provide about neutrino events. Because Cherenkov radiation is emitted in a cone around a particle's direction of travel, a water 
Cherenkov detector may be used to determine a charged particle's direction and kinetic energy, thus enabling kinematic reconstruction of neutrino interactions [114]. However, because of the energy threshold that must be exceeded before Cherenkov light may be produced, this reconstruction is only possible for sufficiently high-energy events. On the other hand, scintillator detectors are able to obtain signals from charged particles with much lower energies, but the detected light is typically emitted isotropically, precluding any detailed tracking of the neutrino reaction products.

Future large neutrino detectors, such as the proposed THEIA experiment [115], may combine the advantages of water Cherenkov and scintillator detectors by using a target made of water-based liquid scintillator (WbLS), a recently invented [116] chemically stable mixture of water and liquid scintillator. A WbLS-based detector could potentially achieve excellent energy resolution and a low energy threshold for particle detection while maintaining the directional sensitivity currently possible in water Cherenkov detectors $[117]$.

\subsubsection{Neutron tagging in water and liquid scintillator}

In both water Cherenkov and liquid scintillator detectors, the dominant reaction channel is inverse beta decay (see eq. (1.3)). While the positron may be detected using Cherenkov or scintillation light as appropriate, a detector containing pure water or pure hydrocarbon scintillator will be relatively insensitive to the final-state neutron. Assuming that the neutron remains in the detector, it is likely to capture on hydrogen to produce a $2.2 \mathrm{MeV}$ $\gamma$-ray. The low energy of this $\gamma$-ray and long capture time (about $200 \mu$ s on average for thermal neutrons in water), however, can limit the efficiency with which the neutron capture may be identified and associated with the prompt signal from the positron.

At least as early as 1958, it was recognized [118] that the sensitivity of organic scintillators to neutron captures might be increased by adding a small amount of a salt containing gadolinium. In the early 2000s, a similar technique was proposed [119, 120] for use in water Cherenkov detectors, with the first successful demonstration being reported by Dazeley et al. [121] in 2009. Natural Gd has a very large thermal neutron capture

cross section (about $4.97 \times 10^{4} \mathrm{~b}$ versus $0.3 \mathrm{~b}$ for ${ }^{1} \mathrm{H}$ ), with the isotope ${ }^{157} \mathrm{Gd}$ having the 
highest capture cross section (about $2.59 \times 10^{5} \mathrm{~b}$ ) of any stable nuclide [122]. As a result, the addition of even a modest amount of a gadolinium-containing material to pure water, say, $0.2 \%$ of $\mathrm{GdCl}_{3}$ by weight, ${ }^{18}$ reduces the mean capture time of thermal neutrons in the Gd-loaded water to $28 \mu \mathrm{s}$ [121]. Neutron capture on gadolinium produces a cascade of $\gamma$-rays with a total energy of about $8 \mathrm{MeV}$, making such captures considerably easier to detect than those on single protons [120].

The ability to tag neutrons using Gd-loaded scintillator or water provides considerable benefits to neutrino experiments, e.g., by allowing discrimination between neutrino elastic scattering on electrons (which will involve only a prompt Cherenkov or scintillation light signal from the struck electron) and inverse beta decay (which will include a delayed neutron capture signal). As a result, a number of liquid scintillator experiments, including Double Chooz and Daya Bay [123], have been constructed using a Gd-loaded target. Up to the present, two prototype Gd-loaded water Cherenkov neutrino detectors, EGADS [124] and WATCHBOY [125], have been successfully operated. Phase II of the ANNIE experiment is expected to be the first Gd-loaded water Cherenkov detector to observe beam neutrinos [126]. It will soon be followed by WATCHMAN [127], a kiloton-scale Gdloaded water Cherenkov detector designed to demonstrate remote monitoring of nuclear reactors via antineutrino detection, and Super-Kamiokande gadolinium (Super-K Gd) [128], an upgrade to the Super-Kamiokande detector in which it will be filled with Gdloaded water.

\subsubsection{Liquid argon time projection chambers}

The liquid argon time projection chamber (LArTPC) is a kind of neutrino detector first proposed in 1977 by Carlo Rubbia [129]. Examples of LArTPCs include MicroBooNE [130], ICARUS [131], and the four far detector modules for the proposed Deep Underground Neutrino Experiment (DUNE) [132]. Figure 1.7 illustrates the operational principle of a LArTPC. The detector consists of a volume of liquid argon placed within a uniform electric field generated between a cathode plane and a set of two or more anode planes. Each anode plane is composed of a set of closely-spaced parallel wires, gener-

\footnotetext{
${ }^{18}$ This corresponds to a Gd weight fraction of $0.1 \%$.
} 


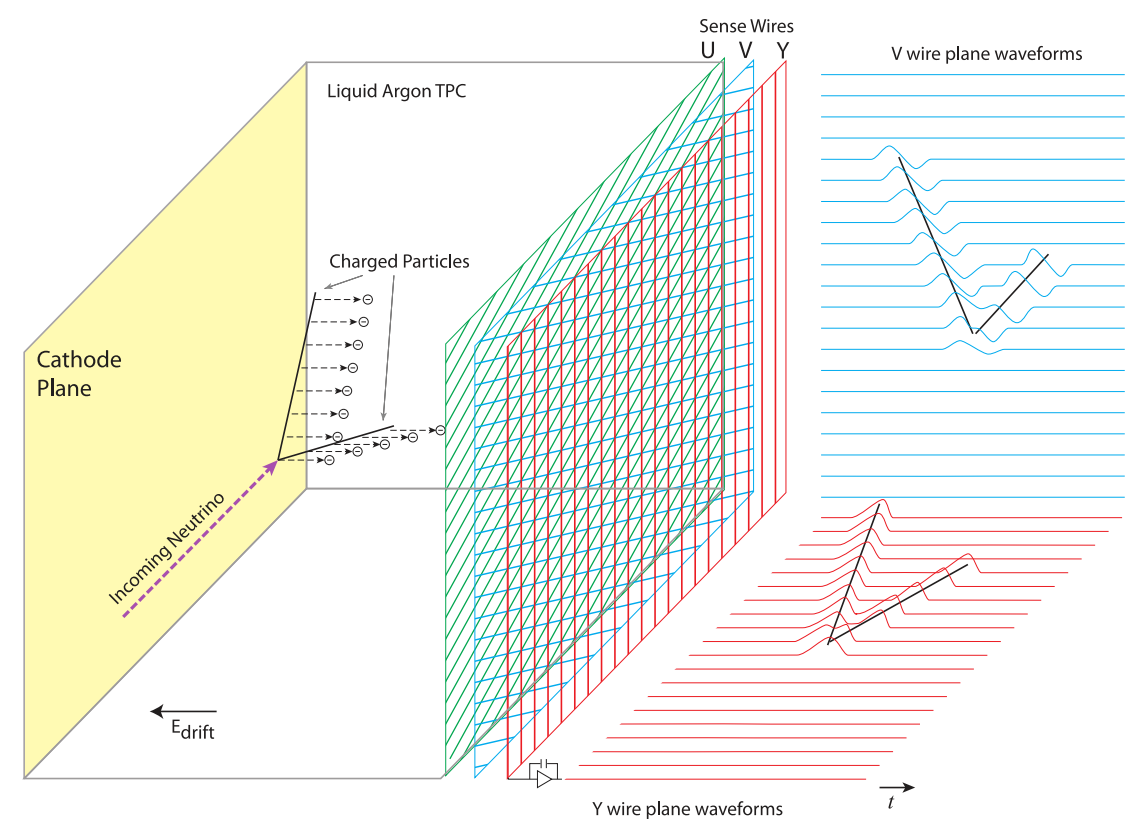

Figure 1.7: Conceptual design of a liquid argon time projection chamber. Figure from reference [133].

ally with a pitch of a few $\mathrm{mm}$. The anode planes are positioned a small distance apart and rotated with respect to each other so that no two anode planes share the same wire orientation.

When a neutrino interaction occurs within the chamber, the charged reaction products produce ionization tracks in the surrounding argon. The ionization electrons are pulled toward the anode wire planes by the electric field. The drift speed depends on the field strength, but the value of $1.1 \mathrm{~mm} / \mu$ s reported by MicroBooNE [134] for a $273 \mathrm{~V} / \mathrm{cm}$ field is typical. The choice of argon, a noble gas, as the target material enables the electrons to drift over long distances without being absorbed. However, the argon in the detector must be kept very pure since even small amounts of electronegative contaminants can greatly reduce the expected lifetime of the drifting electrons.

As the electrons reach the inner anode wire planes, called the induction planes, they induce electric currents in nearby wires. As shown in the portion of fig. 1.7 labeled "V wire plane waveforms," digitized signals coming from the induction planes exhibit a bipolar shape that reflects the change in direction of the induced currents as the electrons pass from one side of the plane to the other. The electrons continue to drift past the 
remaining induction planes until they reach the final anode plane, where they are collected on the wires. Digitized signals on the wires of the collection plane (labeled "Y wire plane waveforms" in the figure) are unipolar.

Thanks to the different wire orientations on each of the anode planes, digitized signals from independent planes represent different views of the same event. By comparing the pattern of wire hits registered on each plane, a two-dimensional image of the particle trackes (in a plane parallel to the wire planes) may be reconstructed. If the event start time (i.e. the time at which the neutrino interaction occurred) is known, then the times of wire hits may be combined with the known electron drift speed and the dimensions of the TPC to obtain full three-dimensional images of the charged particle tracks.

Because charged particles traveling through liquid argon create both ionization tracks and scintillation photons, LArTPCs are often instrumented with photomultiplier tubes in addition to the anode wire planes. The prompt flash of scintillation light created by the products of a neutrino interaction may be detected by the PMTs and used to assign an event start time. Since the intensity of the scintillation light also provides an independent measurement of the energy deposited in the liquid argon, the PMTs and the wire planes both record valuable information for detailed reconstruction of neutrino events. 


\section{Chapter 2}

\section{Motivation for studying neutrino-nucleus reactions}

Although neutrino interactions with elementary particles are described with high precision by the Standard Model, significant theoretical uncertainties arise in attempts to calculate cross sections for neutrino scattering on atomic nuclei. While much progress has been made in the past few decades on both theoretical and experimental fronts, the study of neutrino-nucleus reactions remains a challenging field with many open questions. Within the nuclear and particle physics communities, there is continuing interest in furthering our understanding of these reactions not only because they provide a unique means of probing nuclear structure, but also because of their importance for correctly interpreting the results of experiments, including measurements of neutrino oscillations, studies of astrophysical neutrinos, and searches for exotic new physics processes.

In this chapter, the relevance of neutrino-nucleus reactions to topics of current experimental interest at low $(\sim 10 \mathrm{MeV})$ and medium $(\sim 1 \mathrm{GeV})$ energies will be discussed. Inadequacies in our present understanding of these reactions will be used to motivate the two research projects that form the basis for this dissertation: (1) MARLEY (Model of Argon Reaction Low Energy Yields), a theoretical model and associated event generator describing low-energy neutrino scattering on ${ }^{40} \mathrm{Ar}$, and (2) a characterization of background neutron event rates in preparation for Phase II of the Accelerator Neutrino Neutron Interaction Experiment (ANNIE), which will perform measurements of neutron 


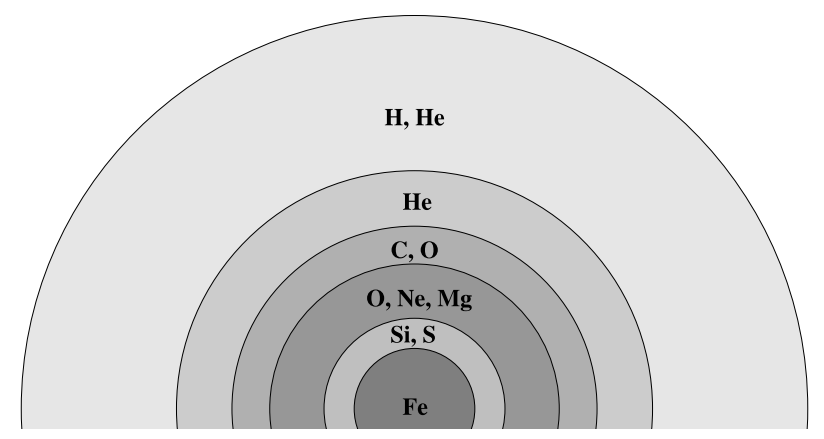

Figure 2.1: Onion-like structure of a massive star (not drawn to scale) just before a core-collapse supernova. Figure from reference [138].

production by neutrino interactions in water.

\subsection{Supernova neutrinos}

One area which will benefit from an improved understanding of neutrino-nucleus reactions is the study of neutrinos produced in supernovae, enormous explosions that end the lives of some stars. While these spectacular astronomical events have been observed for many centuries, ${ }^{1}$ the modern scientific study of supernovae was initiated in the 1930s by Walter Baade and Fritz Zwicky [136].

Supernovae may be sorted into two general categories based on the mechanism which generates the explosion. In a thermonuclear (or Type Ia) supernova, a carbon-oxygen white dwarf accretes matter from a neighboring star until it becomes sufficiently massive for carbon fusion reactions to take place. A thermonuclear explosion is generated by the sudden initiation of these reactions, ripping the white dwarf apart. Although this kind of supernova is thought to generate neutrinos, they are produced in relatively small numbers and with a low mean energy $(\sim 3 \mathrm{MeV})$ [137]. Such an event would therefore have to occur close to Earth (say, within a few kiloparsecs $(\mathrm{kpc})$ ) for its neutrinos to be detected, and even then, a large detector with a low threshold would be needed.

Roughly ten thousand times more neutrinos [139] are produced by core-collapse supernovae, the death throes of stars between about 8 and 40 solar masses. Near the end

\footnotetext{
${ }^{1} \mathrm{~A}$ description of a "guest star" by Chinese astronomers in $185 \mathrm{CE}$ is thought to be the earliest written record of a supernova [135].
} 
of its life, such a star has the onion-like structure shown in fig. 2.1, with a core made of nickel and iron ${ }^{2}$ enveloped by shells of progressively lighter elements. In the outer layers, the fusion of light elements into heavier ones generates heat that provides an outward pressure to counteract the inward pull of gravity. However, because nickel and iron are the most tightly bound of all nuclei, further fusion reactions involving these elements are endothermic. Therefore, the iron core is not supported against gravitational collapse by thermal pressure from fusion but rather by the pressure of degenerate electrons.

When the mass of the iron core exceeds the Chandrasekhar limit of 1.4 solar masses, the electron degeneracy pressure is insufficient to withstand gravity. Gravitational compression of the core causes its temperature and density to increase, and electron capture reactions on protons

$$
e^{-}+p \rightarrow n+\nu_{e}
$$

occur more readily. These reactions lower the electron pressure and allow the core collapse to accelerate. The collapse continues for about one second until the inner part of the core becomes as dense as nuclear matter (roughly $10^{14} \mathrm{~g} / \mathrm{cm}^{3}$ ). At this point, as long as the star is not too massive (less than about 40 solar masses), the degeneracy pressure of nucleons within the core prevents further collapse. The sudden rebound of the inner core, now referred to as a proto-neutron star, creates a shock wave that travels outwards and leads to the supernova explosion.

During late stages of the collapse and early stages of the shock, the inner part of the proto-neutron star is so dense that $\nu_{e}$ produced via electron captures remain trapped behind the shock wave. Within a few milliseconds of the core bounce, the shock wave reaches a region that has a low enough density to be transparent to neutrinos. When this occurs, an intense few-millisecond pulse of neutrinos, mostly $\nu_{e}$, is released. This pulse is often called the neutronization burst.

Although the collapse of the core stops abruptly, matter from outer parts of the star continues to fall inward. In this accretion phase, which lasts for up to a second or two

\footnotetext{
${ }^{2}$ Stars at the low end of the mass range for core-collapse supernovae may initially have a core composed of oxygen, neon, and magnesium rather than iron. However, these elements are converted to iron during the collapse [59], and the generation of the explosion proceeds in the manner outlined here.
} 
after the neutronization burst, the shock wave stalls and the proto-neutron star grows as it accretes the infalling material. The process that reenergizes the stalled shock wave and leads to the supernova explosion is not fully understood. One plausible explanation is that neutrino reactions generate heat in the region behind the stalled shock front. The buildup of thermal pressure behind the shock front drives it outward, allowing the explosion to proceed [140].

Following the revival of the shock, the supernova enters the cooling phase, in which the hot proto-neutron star radiates away energy in the form of neutrinos and antineutrinos of all flavors. These neutrinos are produced via a variety of weak processes within the star, including electron pair annihilation

$$
e^{-}+e^{+} \rightarrow \nu+\bar{\nu}
$$

electron-electron neutrino bremsstrahlung

$$
e^{ \pm}+e^{ \pm} \rightarrow e^{ \pm}+e^{ \pm}+\nu+\bar{\nu}
$$

electron-nucleon neutrino bremsstrahlung

$$
e^{ \pm}+N \rightarrow e^{ \pm}+N+\nu+\bar{\nu}
$$

photoannihilation

$$
\gamma+e^{ \pm} \rightarrow e^{ \pm}+\nu+\bar{\nu}
$$

and nuclear de-excitation by neutrino pair production

$$
{ }_{A}^{Z} \mathrm{X}^{*} \rightarrow{ }_{A}^{Z} \mathrm{X}+\nu+\bar{\nu}
$$

A typical core-collapse supernova emits on the order of $10^{58}$ neutrinos with tens of $\mathrm{MeV}$ energies in about ten seconds. For this brief period, the luminosity of the supernova in neutrinos is comparable to the optical luminosity of the entire observable universe [141]. Despite being well known for their exceptional brightness, the electromagnetic radiation produced by core-collapse supernovae represents only about $0.1 \%$ of the total energy release, compared to the $99 \%$ emitted in the form of neutrinos. 
To date, the only supernova to be observed via its neutrinos was SN1987A, which occurred in the Large Magellanic Cloud and was first detected on Earth on February 23, 1987. Several hours before the first optical signals arrived from SN1987A, three neutrino detectors, Kamiokande-II [142, 143], IMB [144], and Baksan [145] recorded 12, 8, and 5 electron antineutrino events, respectively, over a period of about ten seconds. Despite the limitations of such a small data sample, the neutrino signals from SN1987A have been the subject of published analyses on a wide range of topics. Some of these include the time profile of the expected neutrino signal [146], limits on a possible neutrino magnetic moment [147], and a test of the weak equivalence principle ${ }^{3}$ [149], among many others.

While the SN1987A data have helped to confirm our basic understanding of corecollapse supernovae, a high-statistics measurement of supernova neutrinos would be invaluable to both the astrophysics and neutrino physics communities. A few of the many things ${ }^{4}$ that could be learned from such a measurement include: the total energy of the explosion; whether collective effects (neutrino-neutrino scattering) significantly influence the development of the supernova; whether interesting dynamical features seen in some supernova models, such as the Standing Shock Accretion Instability (SASI) [152] or the Lepton-number Emission Self-sustained Asymmetry (LESA) [153], are present; neutrino properties, such as the mass hierarchy; and constraints on new physics beyond the Standard Model.

In recognition of the wide-ranging physics program that could be pursued using measurements of neutrinos from the next nearby supernova, a worldwide network of neutrino experiments called SNEWS (SuperNova Early Warning System) has been established to "optimize global sensitivity to a core-collapse supernova signal" and enable multimessenger supernova measurements by providing a prompt alert to astronomical observatories $[154,155]$. Currently, all large (kiloton scale) neutrino detectors that would be sensitive to a core-collapse supernova occurring in or near our galaxy are water Cherenkov or hydrocarbon scintillator detectors [156]. Both of these detector types are primarily sen-

\footnotetext{
${ }^{3}$ Also known as the "universality of free fall," the weak equivalence principle states that "all bodies fall with the same acceleration regardless of their mass and composition" [148].

${ }^{4}$ Reviews may be found in references $[150,151]$.
} 
sitive to electron antineutrinos via the inverse beta decay (IBD) reaction

$$
\bar{\nu}_{e}+p \rightarrow n+e^{+}
$$

While the usefulness of a high-statistics sample of supernova IBD events should not be understated, measurements of neutrinos of all flavors are desirable to maximally exploit the physics potential of a nearby core-collapse supernova. Although detectors based on water and hydrocarbon scintillator will observe some $\nu_{e}$ events, both from neutrino-electron elastic scattering and charged current absorption on composite nuclei (oxygen for water, carbon for scintillator), these events will likely be difficult to distinguish from those due to inverse beta decay, particularly in the absence of Gd loading. Additionally, since all neutrino flavors will participate in neutral current elastic scattering from electrons, only charged current reactions of $\nu_{e}$ with nuclei will allow a measurement of the supernova $\nu_{e}$ flux in isolation.

In contrast to neutrino detectors based on water and hydrocarbon scintillator, the dominant reaction channel for supernova neutrinos in a liquid argon time projection chamber (LArTPC) is charged current $\nu_{e}$ scattering on ${ }^{40} \mathrm{Ar}$ :

$$
\nu_{e}+{ }^{40} \mathrm{Ar} \rightarrow{ }^{40} \mathrm{~K}^{*}+e^{-} .
$$

Due to the large cross section for this process, a large LArTPC has the potential to achieve unique sensitivity to the $\nu_{e}$ component of the neutrino flux from a supernova, complementing and extending the physics reach of supernova neutrino measurements obtained using other detection technologies. Although existing LArTPCs, such as the 600-ton ICARUS and 170-ton MicroBooNE detectors, would record only modest numbers of neutrino events in response to a nearby core-collapse supernova (a combined total of about 80 events for a supernova at $10 \mathrm{kpc}$ from Earth), the planned Deep Underground Neutrino Experiment (DUNE) will increase the expected number of events by more than one and one-half orders of magnitude [156].

\subsection{Supernova neutrinos in DUNE}

Figure 2.2 depicts the basic components of the DUNE experiment. A neutrino beam produced at Fermilab will be directed toward two neutrino detectors. The near detector, 


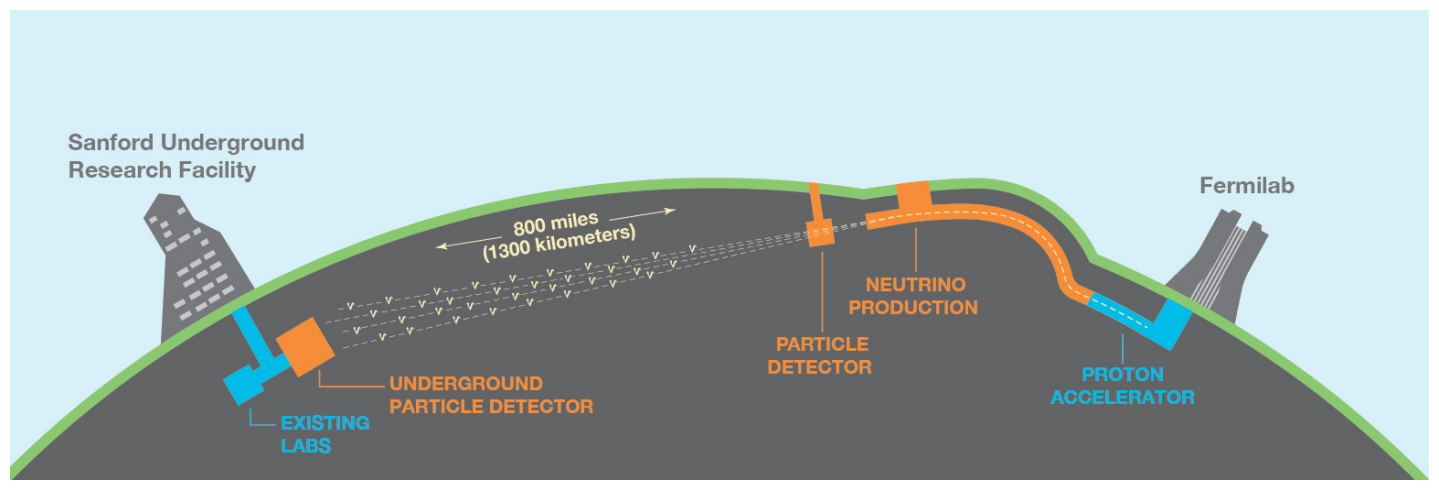

Figure 2.2: Conceptual design of the DUNE experiment. Measurements of a neutrino beam will be performed using liquid argon time projection chambers at two locations to study neutrino oscillations. DUNE will also pursue a rich science program beyond the oscillation measurements, including the detection of $\nu_{e}$ from a possible nearby core-collapse supernova.

located close to the beam production site, will measure the unoscillated neutrino flux using a detector technology that is yet to be finalized. The far detector, located $1300 \mathrm{~km}$ away at the Sanford Underground Research Facility near Lead, South Dakota, will include four large LArTPCs with a total active mass of $40 \mathrm{kt}$. DUNE will perform precision studies of neutrino oscillations by comparing measurements of the flavor content of the neutrino beam at the near and far detectors. Apart from the DUNE neutrino oscillation program, the two other primary science goals of the experiment are to search for proton decay ${ }^{5}$ and to "detect and measure the $\nu_{e}$ flux from a core-collapse supernova within our galaxy, should one occur" within the lifetime of the experiment [132].

Figure 2.3 shows an example of one of the unique contributions that could be made by DUNE to the worldwide supernova neutrino detection effort. Because the neutrinos released during the neutronization burst are almost entirely $\nu_{e}$, one might expect to see a large excess of events in DUNE at early times relative to the start of a supernova signal. This possibility is shown in the blue histogram in fig. 2.3, which displays the expected time distribution of supernova neutrino events (dominated by charged current $\nu_{e}$ scattering) in DUNE when neutrino oscillations are neglected. However, when the effects of oscillations are taken into account, the expected event time distribution in DUNE shows a strong

\footnotetext{
${ }^{5}$ See section 2.6.2 for a discussion of competing proton decay searches in water Cherenkov detectors.
} 


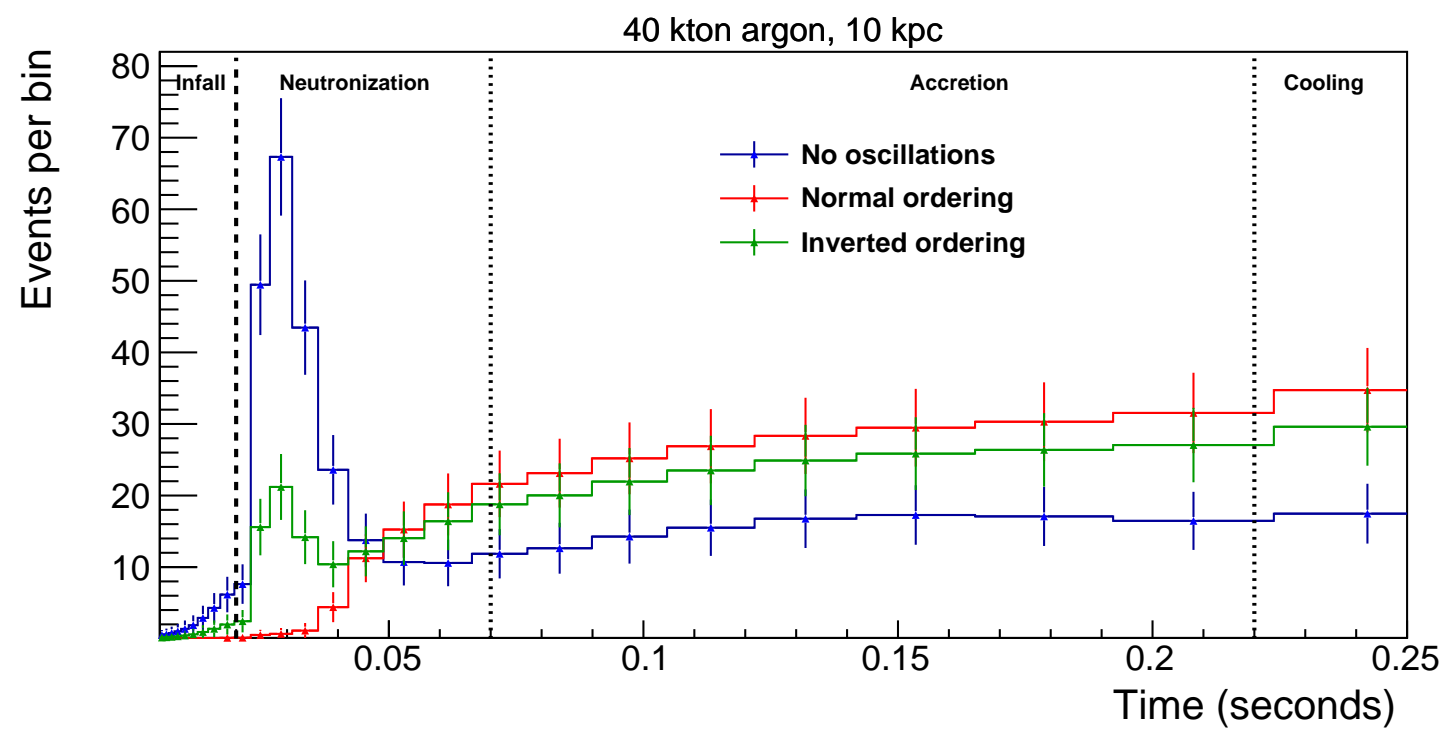

Figure 2.3: Predicted event time distribution for the detection of supernova neutrinos in a DUNE-like $40 \mathrm{kt}$ LArTPC. Only events from the earliest stages of the supernova, assumed to have occurred at a distance of $10 \mathrm{kpc}$ from Earth, are shown. All error bars in the figure are statistical. Under the specific flux model (described in reference [157]) used in this calculation, the presence or absence of a clear excess of events during the neutronization burst provides a means of determining whether the neutrino mass hierachy is normal or inverted. Figure from reference [158].

dependence on the neutrino mass hierarchy. For an inverted hierarchy, the excess due to the neutronization burst is suppressed but still detectable, while for the normal hierarchy it is altogether absent. Thus, if neutrinos from a core-collapse supernova were to be detected by DUNE, the presence or absence of the neutronization burst signal could provide a clear indication of which of the two neutrino mass orderings is realized in nature. While the results shown here were calculated for a specific supernova flux model [157], the general conclusion that a neutronization burst measurement could allow DUNE to determine the neutrino mass hierarchy is thought to be relatively model-independent [158].

Beyond the physics results that could be obtained by DUNE under fairly generic assumptions about supernova dynamics, there are also scenarios under which DUNE alone could provide a window into exotic supernova processes. A recent calculation by Friedland and Mukhopadhyay [159], for example, suggests that collective effects due to neutrino-neutrino interactions within a supernova may produce detectable signatures in 
the $\nu_{e}$ signal but not in the $\bar{\nu}_{e}$ signal. DUNE's high $\nu_{e}$ sensitivity and large active mass make it the only detector capable of robustly observing such signatures for the foreseeable future.

\subsection{Supernova neutrino energy reconstruction in argon}

While exciting as a means of performing a clean measurement of the $\nu_{e}$ flux from a supernova, detection of low energy neutrinos in liquid argon is not without significant challenges. In addition to the demanding technological requirements for DUNE's proposed supernova measurement, such as a low threshold for detecting the final-state electrons, there exist more fundamental difficulties due to the use of a composite nucleus, ${ }^{40} \mathrm{Ar}$, as the neutrino target. ${ }^{6}$ The complications for supernova neutrino detection in argon that are introduced by nuclear physics effects are perhaps best understood when one first considers neutrino energy reconstruction at low energies in a water Cherenkov or liquid scintillator detector. In such a detector, the dominant IBD reaction (see eq. (2.7)) leads to a positron and a neutron in the final state. The energy $E_{\nu}$ of the incident antineutrino for such an event can be expressed as

$$
E_{\nu}=E_{e}+\Delta_{n p}+T_{\text {recoil }}
$$

where $E_{e}$ is the total energy of the outgoing positron, $\Delta_{n p}$ is the neutron-proton mass difference, and $T_{\text {recoil }}$ is the kinetic energy of the recoiling neutron. At the low energies of interest for supernova neutrinos, $T_{\text {recoil }}$ is small enough to be neglected. Therefore, since $\Delta_{n p}$ is precisely known, ${ }^{7}$ the neutrino energy may be reliably determined using only a measurement of the positron energy.

Supernova neutrino energy reconstruction in a LArTPC is considerably more challenging than in water Cherenkov and scintillator detectors because of the excited ${ }^{40} \mathrm{~K}^{*}$ nucleus produced in the dominant charged current interaction (see eq. (2.8)). As in the IBD case, one may write the initial neutrino energy $E_{\nu}$ in terms of three other variables:

$$
E_{\nu}=E_{e}+\Delta_{f i}+T_{\text {recoil }}
$$

\footnotetext{
${ }^{6}$ The natural argon that will be used in DUNE is almost entirely (99.6\% [160]) ${ }^{40}$ Ar. As a first approximation, we will ignore the contributions of the other isotopes to the neutrino cross sections.

${ }^{7}$ Reference [161] gives the value $\Delta_{n p} \equiv m_{n}-m_{p}=1.29333205(48) \mathrm{MeV}$.
} 
The recoil kinetic energy $T_{\text {recoil }}$ remains negligible in this case, and the total energy of the outgoing electron $E_{e}$ may be measured if it produces a noticeable ionization track in the TPC. The difficulties introduced by nuclear effects arise when one considers the nuclear mass difference $\Delta_{f i}$, which is defined by

$$
\Delta_{f i} \equiv m_{f}-m_{i}
$$

where $m_{i}\left(m_{f}\right)$ is the mass of the initial ${ }^{40} \mathrm{Ar}\left(\right.$ final $\left.{ }^{40} \mathrm{~K}^{*}\right)$ nucleus. In contrast to $\Delta_{n p}$ from the IBD case, the value of $\Delta_{f i}$ varies from event to event because of the many excited states that exist within several tens of $\mathrm{MeV}$ above the ${ }^{40} \mathrm{~K}$ ground state. ${ }^{8}$ Each of these excited states corresponds to a different mass $m_{f}$ via the relation

$$
m_{f}=m_{f, \text {.s. }}+E_{x}
$$

where $m_{f, \text { g.s. }}$ is the ground-state mass of a ${ }^{40} \mathrm{~K}$ nucleus and $E_{x}$ is the excitation energy. Although it is possible for the charged current absorption of $\nu_{e}$ by ${ }^{40} \mathrm{Ar}$ to proceed via a transition directly to the ground state of ${ }^{40} \mathrm{~K}$, this channel will be strongly suppressed ("third-forbidden") due to the large spin-parity difference between the ${ }^{40} \mathrm{Ar}$ ground state $\left(0^{+}\right)$and the ${ }^{40} \mathrm{~K}$ ground state $\left(4^{-}\right)$. Nearly all $\nu_{e}$ absorption events in liquid argon will therefore involve transitions to excited ${ }^{40} \mathrm{~K}^{*}$ nuclear levels, and indirect measurements (discussed in detail in chapter 5) suggest that a substantial number of these levels will be readily accessible. Since the branching ratios to each of the final-state nuclear levels will depend on both the neutrino energy and on nuclear matrix elements representing the relevant ${ }^{40} \mathrm{Ar} \rightarrow{ }^{40} \mathrm{~K}^{*}$ transitions, the distribution of $\Delta_{f i}$ created by low energy neutrino scattering will be sensitive to nuclear structure details and to the incident neutrino spectrum in a complicated way.

To reconstruct $\Delta_{f i}$ (and thereby the incident neutrino energy $E_{\nu}$ ) in a LArTPC, one must observe the nuclear de-excitation products from the ${ }^{40} \mathrm{~K}^{*}$ nucleus. For neutrino reactions that populate low-lying nuclear levels, these will consist almost entirely of $\gamma$-rays ${ }^{9}$

\footnotetext{
${ }^{8}$ Although excited states of individual nucleons exist, the lightest of these are the Delta(1232) baryons, which have masses nearly $300 \mathrm{MeV}$ higher than the neutron and proton. Thus, at the tens-of-MeV energies of supernova neutrinos, IBD can only proceed via the creation of a final-state neutron, and therefore $\Delta_{n p}=m_{n}-m_{p}$ should be used for every event.

${ }^{9}$ Orbital electrons may occasionally be emitted from the ${ }^{40} \mathrm{~K}$ atom instead of a $\gamma$-ray in a process called internal conversion.
} 
with energies of up to several $\mathrm{MeV}$. While the $\gamma$-rays will be difficult to measure precisely with a LArTPC (which will only indirectly observe them as they scatter on atomic electrons), in principle one may obtain the value of $\Delta_{f i}$ for such events via the expression

$$
\Delta_{f i}=m_{\text {g.s. } \rightarrow \text { g.s. }}+\sum_{j} E_{\gamma, j}
$$

where the sum is over the measured $\gamma$-ray energies $E_{\gamma, j}$ and

$$
m_{\text {g.s. } \rightarrow \text { g.s. }} \equiv m_{\text {g.s. }}\left({ }^{40} \mathrm{~K}\right)-m_{\text {g.s. }}\left({ }^{40} \mathrm{Ar}\right)=1.5044 \mathrm{MeV}
$$

is the difference between the masses of the ${ }^{40} \mathrm{~K}$ and ${ }^{40} \mathrm{Ar}$ ground states.

The reconstruction of $\Delta_{f i}$ becomes even more difficult in cases where the excitation energy of the initial ${ }^{40} \mathrm{~K}$ level is sufficiently high for the nucleus to become unbound, i.e., it becomes energetically possible for de-excitation to proceed via the emission of a nucleon or a heavier nuclear fragment like a deuteron or an alpha particle. As shown in fig. 2.4, de-excitations from such a highly-excited nuclear level may proceed via many possible channels. Many of these channels will leave the daughter nucleus in an excited state, leading to the emission of additional $\gamma$-rays or nuclear fragments. The resulting cascade of successive de-excitations will continue until the ground state of the ultimate daughter nucleus is reached. In this more general case, the nuclear mass difference $\Delta_{f i}$ may be reconstructed via the relation

$$
\Delta_{f i}=m_{\text {g.s. } \rightarrow \text { g.s. }}+\sum_{j}\left(S_{j}+T_{j}\right)
$$

where $T_{j}$ is the kinetic energy of the $j$ th nuclear de-excitation product and $S_{j}$ is its separation energy, defined by

$$
S_{j} \equiv m_{j}+m_{X-j}-m_{X}
$$

Here $m_{j}$ is the mass of the $j$ th product, $m_{X}$ is the ground-state mass of the mother nucleus $X$ that emits the $j$ th product, and $m_{X-j}$ is the ground-state mass of the daughter nucleus. ${ }^{10}$ The sum over $j$ in eq. (2.15) includes all final-state particles emitted in the event except for the electron and the ultimate daughter nucleus.

\footnotetext{
${ }^{10}$ Note that the expression given in eq. (2.15) reduces to that of eq. (2.13) if only $\gamma$-rays are emitted. This follows immediately from the recognition that, for the definition of the separation energy given here, if the $j$ th de-excitation product is a $\gamma$-ray, then $S_{j}=0$.
} 


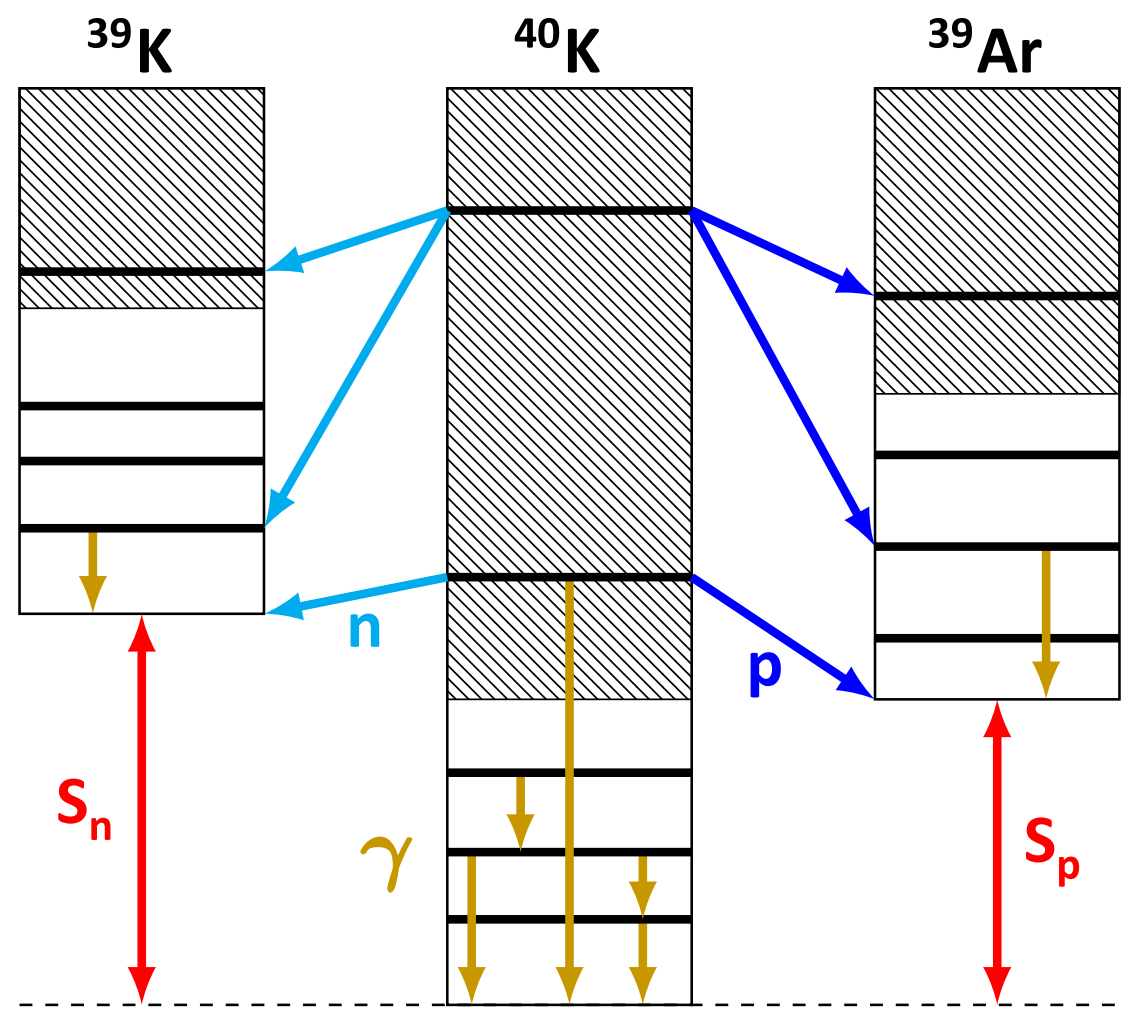

Figure 2.4: Charged current $\nu_{e}$ scattering on ${ }^{40} \mathrm{Ar}$ will sometimes induce a transition to a highly-excited ${ }^{40} \mathrm{~K}$ nuclear energy level above the proton and neutron emission thresholds $S_{p}$ and $S_{n}$. When this occurs, de-excitation of the level may proceed via many competing channels, including those shown in the diagram: $\gamma$-ray emission, neutron emission, and proton emission. If the daughter nucleus is itself left in an excited state, one or more additional particles will be emitted in a cascade of de-excitation steps.

Even for a LArTPC that achieves a low detection threshold and good energy resolution for charged particles, the sheer number of possible event topologies created by nuclear de-excitations introduces considerable complexity into the problem of determining the incident neutrino energy in a supernova $\nu_{e}$ event. A particularly worrisome prospect is that a significant fraction of such events will involve nucleon emission. The separation energy required to remove each emitted nucleon from the nucleus (typically about $8 \mathrm{MeV}$ ) represents a sizeable portion of the original tens-of-MeV supernova neutrino energy. This energy will be invisibly lost to the LArTPC unless a suitable means of tagging the emitted nucleons can be developed. Doing so is likely to be challenging: the millimeter-scale ionization tracks created by low-energy protons may be hard to distinguish from the start 
of the primary electron track, and, unless they are captured by an argon nucleus, lowenergy neutrons may escape without creating any discernible signals whatsoever in the detector.

\subsection{Monte Carlo methods for neutrino energy reconstruction}

A common technique for studying the potentially complicated response of a detector to incoming particles is the use of Monte Carlo simulations. Under this approach, a probabilistic model of the primary particle interactions, implemented within a computer code called an event generator, is used to randomly sample individual reactions occurring within a simulated detector geometry. These reactions are represented by a list of the final-state particles together with their 4-momenta at the moment that they emerge from the event vertex, the location where the primary particle first interacted. Although event generators produce results that are informative in their own right, they are most often used in experimental analyses as part of a larger simulation chain, a set of computer programs capable of producing simulated datasets that closely resemble the results of an actual measurement. In addition to the event generator, a typical simulation chain will include software tools to simulate secondary particle propagation through the detector, model electronic signal generation by the detector components, and reconstruct kinematic quantities of interest from these signals using the same methods employed for real data.

One common application of Monte Carlo simulations in the analysis of experimental data is the calculation of a smearing matrix $\mathbf{K}$ which connects the values of true kinematic variables from a detector event with their reconstructed counterparts. In the context of a measurement of the $\nu_{e}$ spectrum from a supernova, for instance, the elements $K_{i j}$ of such a matrix might be defined as

$$
K_{i j} \equiv P\left(E_{i} \leq E_{v}^{\text {reco }}<E_{i+1} \mid E_{j} \leq E_{v}^{\text {true }}<E_{j+1}\right)
$$

where $P$ is the probability that an event generated by a neutrino with true energy $E_{\nu}^{\text {true }}$ within the $j$ th true bin $\left[E_{j}, E_{j+1}\right)$ will be reconstructed so that the measured neutrino

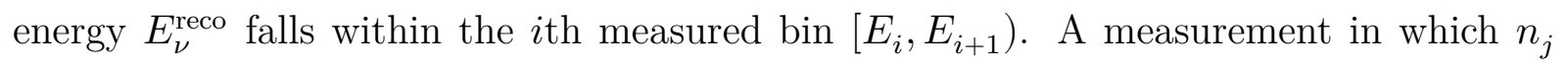
events were created by neutrinos with energies within the $j$ th true bin would then be 
expected to yield

$$
n_{i}^{\prime}=\sum_{j} K_{i j} n_{j}
$$

counts in the $i$ th measured bin, where the sum runs over all true bins. In matrix notation, one may rewrite eq. (2.18) as

$$
\mathbf{n}^{\prime}=\mathbf{K} \mathbf{n}
$$

Equation (2.19) may be solved for the true neutrino spectrum $\mathbf{n}$ by inverting the smearing matrix: ${ }^{11}$

$$
\mathbf{n}=\mathbf{K}^{-1} \mathbf{n}^{\prime}
$$

Because the exact smearing matrix needed for any particular physics measurement will be unknown, experiments must rely on approximate matrices. From the results of a Monte Carlo simulation, one may approximate the elements of the smearing matrix $\mathbf{K}$ via

$$
K_{i j} \approx \frac{n_{j \rightarrow i}}{n_{j}} .
$$

Here $n_{j}$ is the total number of simulated events in which the true neutrino energy $E_{\nu}^{\text {true }}$ falls within the $j$ th bin, and $n_{j \rightarrow i}$ is the number of these for which the simulated detector signals yield a reconstructed energy $E_{\nu}^{\text {reco }}$ in the $i$ th bin. For a sufficiently large sample of Monte Carlo events (i.e., sufficiently large $n_{j}$ ), the statistical error associated with making the approximation in eq. (2.21) will be negligible. The systematic errors that arise due to inadequacies in the simulation models, on the other hand, require careful consideration when a smearing matrix is used to interpret experimental data.

\subsection{MARLEY: A new event generator for supernova neutrinos}

In recent years, there has been extensive effort among LArTPC experiments to develop the software infrastructure needed for precise event reconstruction and detailed Monte Carlo simulations. The results of this effort are primarily embodied in LArSoft [165], a flexible LArTPC software framework used by the DUNE, MicroBooNE [130], LArIAT

\footnotetext{
${ }^{11}$ In practice, direct inversion of the smearing matrix often leads to statistical difficulties that can be avoided by using more sophisticated approaches. See references [162-164] for a detailed discussion of this point.
} 
[166], ICARUS [131], and SBND [167] experiments, among others. Although the LArSoft package is now sufficiently mature to have been used in a number of published physics analyses [168-170], most of the existing event reconstruction algorithms (either provided within LArSoft itself or via interfaces to external toolkits such as Pandora [171] and Wirecell [172]) are optimized for use by medium-energy neutrino beam experiments.

A promising first step toward detailed reconstruction of LArTPC events at supernova neutrino energies was made earlier this year by the ArgoNeuT collaboration, who reported a preliminary first observation of $\mathrm{MeV}$-scale energy depositions from neutrino-induced nuclear de-excitation $\gamma$-rays in a LArTPC [173]. In order for DUNE to meet its goal of providing a high-statistics $\nu_{e}$ flux measurement in the event of a nearby supernova, detailed simulation studies, informed by the ArgoNeuT results and any other relevant data that become available, will need to be performed to characterize DUNE's future sensitivity and guide the design of the far detector modules.

Prior to the work presented in this thesis, a major obstacle to such simulation studies was the lack of a suitable event generator. While several standard neutrino event generators (e.g., GENIE [174], NuWro [175], GiBUU [176], and NEUT [177]) currently enjoy widespread use in analyses of neutrino beam experiments, ${ }^{12}$ the nuclear models implemented in these generators universally adopt approximations that are inappropriate for supernova neutrino scattering on composite nuclei. For example, conventional neutrino event generators use a relativistic Fermi gas (noninteracting nucleons bound inside a constant potential well) as their baseline model of the target nucleus [178]. Even with more theoretically rigorous approaches, such as the GiBUU ${ }^{13}$ transport model [179], there remains no consideration of the nuclear level structure probed by low-energy neutrinos. As noted in section 2.3, because of the many possible final states that may be created by nuclear de-excitations, a realistic treatment of low-energy nuclear structure will likely be crucial to a correct understanding of supernova neutrino energy reconstruction in LArTPCs. Therefore, despite their successes at higher energies, the lack of a detailed low-energy

\footnotetext{
${ }^{12}$ The Particle Data Group gives a review of current neutrino event generators in section 42 of reference [78].

${ }^{13}$ Giessen-Boltzmann-Uehling-Uhlenbeck
} 
nuclear model in the standard neutrino event generators renders them inappropriate to use to study neutrino scattering at tens-of-MeV and below on composite nuclei, including ${ }^{40} \mathrm{Ar}$.

In the first half of the remainder of this thesis, a theoretical model of low-energy neutrino-nucleus scattering will be described, along with its implementation within a novel event generator for charged current scattering of $\nu_{e}$ on ${ }^{40} \mathrm{Ar}$ called MARLEY (Model of Argon Reaction Low Energy Yields). The description of MARLEY will culminate in chapter 7 with a presentation of example calculations performed using the generator. Prospects for extending MARLEY to include additional reaction channels and other target nuclei will also be discussed.

\subsection{Neutron production in medium-energy neutrino scattering}

At the intermediate energy scale (hundreds of $\mathrm{MeV}$ to few-GeV) of interest for atmospheric and accelerator neutrinos, nuclear effects are an important consideration for precision neutrino oscillation measurements and for background reduction in rare event searches.

\subsubsection{Oscillation measurements}

In a neutrino oscillation experiment, the quantity that is directly measured is the rate of detected events with a particular reconstructed topology. The selected event topology may be expressed in terms of a set of one or more reconstructed variables $\mathbf{p}_{\text {reco }}$, including kinematic quantities (e.g., momentum, scattering angle), final-state particle multiplicities, etc. Ignoring some potentially important complications, ${ }^{14}$ the expected event rate $N^{\alpha \rightarrow \beta}$ for an experiment measuring $\nu_{\alpha} \rightarrow \nu_{\beta}$ oscillations may be written in the form [178]

$N^{\alpha \rightarrow \beta}\left(\mathbf{p}_{\text {reco }}\right)=\sum_{j} \phi_{\alpha}\left(E_{\nu}^{\text {true }}\right) \times P_{\nu_{\alpha} \rightarrow \nu_{\beta}}\left(E_{\nu}^{\text {true }}\right) \times \sigma_{\beta}^{j}\left(\mathbf{p}_{\text {true }}\right) \times \epsilon_{\beta}\left(\mathbf{p}_{\text {true }}\right) \times R_{j}\left(\mathbf{p}_{\text {true }}, \mathbf{p}_{\text {reco }}\right)$

where $E_{\nu}^{\text {true }}$ is the true neutrino energy, $\phi_{\alpha}\left(E_{\nu}^{\text {true }}\right)$ is the energy-dependent flux of $\nu_{\alpha}$ (ignoring oscillations) at the detector location, and $P_{\nu_{\alpha} \rightarrow \nu_{\beta}}\left(E_{\nu}^{\text {true }}\right)$ is the oscillation prob-

\footnotetext{
${ }^{14}$ Examples of these include detector backgrounds and the production of (anti)neutrinos other than $\nu_{\alpha}$ by the source used in the experiment.
} 
ability (see section 1.5). The remaining factors represent the detector response:

- $\sigma_{\beta}^{j}\left(\mathbf{p}_{\text {true }}\right)$ is the cross section for the $j$ th reaction induced by $\nu_{\beta}$, which results in a final state described by the true event topology $\mathbf{p}_{\text {true }}$

- $\epsilon_{\beta}\left(\mathbf{p}_{\text {true }}\right)$ is the detection efficiency for an event with that topology

- $R_{j}\left(\mathbf{p}_{\text {true }}, \mathbf{p}_{\text {reco }}\right)$ is the probability that the true event toplogy $\mathbf{p}_{\text {true }}$ created by the $j$ th reaction will be reconstructed as $\mathbf{p}_{\text {reco }}$.

To obtain a measurement of neutrino oscillation parameters from such an experiment, the expected event rates are fit to data. By varying the model parameters used to compute the oscillation probability $P_{\nu_{\alpha} \rightarrow \nu_{\beta}}$ (see section 1.5), one may obtain their best-fit values. In order for the fit to produce accurate results, systematic errors, including those introduced by the various factors in eq. (2.22), must be carefully accounted for and controlled. A particularly difficult aspect of such analyses is that, in virtually all neutrino experiments, the true neutrino energy $E_{\nu}^{\text {true }}$ is not known on an event-by-event basis. ${ }^{15}$ It must therefore be inferred from the reconstructed event topology $\mathbf{p}_{\text {reco }}$ observed in the detector. Because the oscillation probability $P_{\nu_{\alpha} \rightarrow \nu_{\beta}}$ is a function of the true neutrino energy, reconstructing $E_{\nu}^{\text {true }}$ accurately is an unavoidable and critical part of any neutrino oscillation analysis.

Neutrino energy reconstruction in oscillation measurements is typically based on one of two general methods. The first of these, the kinematic method, relies on the selection of a pure sample of charged current quasi-elastic (CCQE) events. In a quasi-elastic event, an incident (anti)neutrino scatters on a single nucleon bound within the target nucleus, and the remaining nucleons participate in the interaction only as spectators. Assuming that the struck nucleon does not rescatter as it exits the nucleus, the final state of a CCQE event will consist of a charged lepton, a single ejected nucleon, and a recoiling nuclear remnant. A measurement of the total energy $E_{\ell}$ and scattering angle ${ }^{16} \theta_{\ell}$ of the outgoing charged lepton in a CCQE event may be used to determine the incident neutrino energy

\footnotetext{
${ }^{15}$ A notable exception is a recent measurement [180] by the MiniBooNE collaboration, in which a shapeonly differential cross section was measured for monoenergetic $236 \mathrm{MeV}$ muon neutrinos originating from charged kaon decays at rest $\left(K^{+} \rightarrow \mu^{+}+\nu_{\mu}\right)$.

${ }^{16}$ That is, the angle between the direction of the neutrino beam and the charged lepton 3-momentum
} 
$E_{\nu}^{\text {true }}$ via the relation

$$
E_{\nu}^{\text {true }} \approx E_{\nu}^{\text {reco,kin }} \equiv \frac{2\left(m_{N}-S_{N}\right) E_{\ell}+m_{N}^{2}-\left(m_{N}-S_{N}\right)^{2}-m_{\ell}^{2}}{2\left(m_{N}-S_{N}-E_{\ell}+\left|\mathbf{p}_{\ell}\right| \cos \theta_{\ell}\right)},
$$

where $m_{N}$ is the mass of the nucleon, $S_{N}$ is its separation energy, and $m_{\ell}\left(\mathbf{p}_{\ell}\right)$ is the mass (3-momentum) of the charged lepton. In the derivation of eq. (2.23), both the recoil kinetic energy of the remnant nucleus and the neutron-proton mass difference are neglected, and the separation energy of the struck nucleon is assumed to be constant [178].

While convenient to use because of its simplicity, the kinematic method is only valid when it is used to reconstruct neutrino energies for true CCQE events. Experimentally, these events are selected using topologies consistent with the CCQE hypothesis. For example, due to the high Cherenkov thresholds for the recoil nucleus and ejected proton produced in a $\nu_{\mu}$ CCQE event, only the outgoing muon is typically observed in a water Cherenkov detector. Therefore, a neutrino oscillation measurement might be performed in such a detector using a sample of events in which one muon and zero pions $(1 \mu+0 \pi)$ are detected. However, it should be noted that a number of other processes can lead to final states which satisfy this selection.

Figure 2.5 illustrates the bias that results when the kinematic method, with its underlying CCQE assumption, is used to reconstruct the neutrino energy for a sample of $1 \mu+0 \pi$ events. In the upper plot, the black histogram shows the distribution of reconstructed neutrino energies (predicted using the GENIE event generator) obtained using the kinematic method for $1 \mathrm{GeV}$ muon neutrinos scattering on a water target. Only CCQE candidates $(1 \mu+0 \pi$ events) are included in the plot. While true CCQE events make up the majority of those selected by the $1 \mu+0 \pi$ criterion, the black histogram includes other event types in significant numbers. In stuck pion events, whose contribution to the total is shown in red, a pion created by the initial neutrino interaction is reabsorbed before it leaves the nucleus. In two particle-two hole (" $2 \mathrm{p}-2 \mathrm{~h}$ ") events, shown in blue, the neutrino scatters off of a correlated pair of nucleons instead of a single nucleon. Although the black histogram would be expected to be symmetric about the true $1 \mathrm{GeV}$ neutrino energy for a pure CCQE sample, ${ }^{17}$ the asymmetric excess seen at low energies can largely

\footnotetext{
${ }^{17}$ Irreducible smearing around the true neutrino energy would still be present due to the nonzero initial
} 

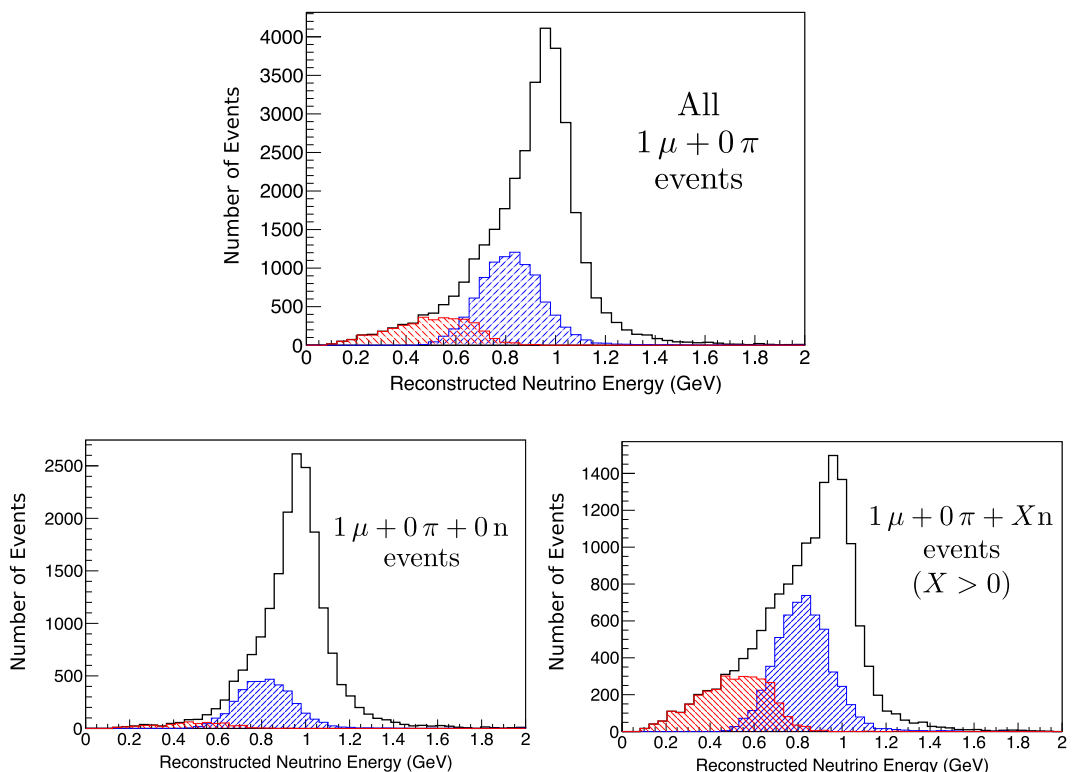

Figure 2.5: Demonstration of the kinematic method for neutrino energy reconstruction using simulated $1 \mathrm{GeV} \nu_{\mu}$ scattering events. In each of the three plots, which are described in detail in the text, the black histogram represents the reconstructed neutrino energy distribution for all events, while the red and blue histograms show the contributions of "stuck pion" and 2p-2h events, respectively. Selecting a subset of events with zero final-state neutrons (as shown in the lower left plot) noticeably improves the quality of the energy reconstruction. Figure from reference [126].

be attributed to the presence of these two types of nuisance events.

Efficient neutron tagging in a water Cherenkov detector, enabled using Gd-loaded water (see section 1.7.3), has the potential to significantly improve the selection of CCQE events and therefore the kinematic reconstruction of the neutrino energy. The lower plots in fig. 2.5 show the reconstructed neutrino energy distributions when events from the upper plot are selected based on whether they include zero final-state neutrons (lower left) or at least one neutron (lower right). According to the GENIE simulations shown here, selecting the zero neutron events leads to a noticeable reduction of the $2 \mathrm{p}-2 \mathrm{~h}$ and stuck pion contributions and therefore to a more symmetric distribution of the reconstructed neutrino energies.

A second method for neutrino energy reconstruction, the calorimetric method, may be momentum of the struck nucleon. 
used by detectors that are sensitive to final-state hadrons. At the intermediate energies of interest for beam-based neutrino oscillation measurements, the neutrino energy may be reconstructed for a charged current event via the approximate expression [178]

$$
E_{\nu}^{\mathrm{true}} \approx E_{\nu}^{\mathrm{reco,cal}} \equiv E_{\ell}+\epsilon_{n}+\sum_{i=1}^{n} T_{i}+\sum_{j=1}^{m} E_{j}
$$

Here $E_{\ell}$ is the total energy of the outgoing charged lepton, $\epsilon_{n}$ is the binding energy of the $n$ emitted nucleons, $T_{i}$ is the kinetic energy of the $i$ th nucleon, and $E_{j}$ is the total energy of the $j$ th of the $m$ emitted mesons. While the calorimetric method has the advantage that it does not make any assumptions about the true event topology, accurately identifying all of the emitted hadrons (since the detector response may vary with the particle type) and measuring their energies is far from trivial. Missing energy imparted to undetected final-state neutrons is a particularly troublesome problem for this method.

Regardless of the technique used to reconstruct the incident neutrino energy, oscillation analyses must rely on models, typically implemented within event generators, in order to assess systematic uncertainties that arise from nuclear effects. For kinematic reconstruction, a generator is needed to predict the contributions of non-CCQE reaction channels to a sample of events selected based on an observed topology, while one is needed in calorimetric reconstruction in order to account for energy loss to unreconstructed particles. Since nuclear model uncertainties are often among the largest sources of systematic error for neutrino oscillation measurements, ${ }^{18}$ a more accurate description of neutrinonucleus interactions in event generators will be needed if future precision oscillation experiments are to achieve their goals. Despite considerable recent progress in improving neutrino event generators and validating them against experimental measurements, neutron emission in medium-energy neutrino scattering remains poorly understood, with different generators giving markedly different predictions of neutron multiplicity and energy distributions [164]. A direct measurement of the kinematic dependence of the neutron yield from (anti)neutrino scattering events would therefore provide valuable constraints on the nuclear models to be used in future oscillation analyses.

\footnotetext{
${ }^{18}$ See, e.g., recent publications $[181,182]$ by the T2K collaboration.
} 


\subsubsection{Rare event searches: proton decay}

Ever since the development of the Glashow-Weinberg-Salam model, a unified theory of the electromagnetic and weak interactions (see section 1.4), there has been interest in creating a Grand Unified Theory (GUT) in which the strong and electroweak interactions are similarly explained in terms of a single fundamental interaction at high energies. A common prediction of all proposed GUTs [183] is the non-conservation of baryon number, an additive quantum number defined as the number of baryons in a system minus the number of antibaryons. Despite the current lack of experimental evidence for baryon number violation, GUTs remain theoretically well-motivated, and searches for baryon number violating processes are ongoing at ever-increasing sensitivity.

If baryon number is truly conserved, then, because the proton is the lightest baryon, it must be absolutely stable. Even a single unambiguous observation of proton decay would therefore provide conclusive evidence of the non-conservation of baryon number. Recently, the Super-Kamiokande experiment has set world-leading limits [184] on the partial lifetimes for two proton decay modes. If we define $\tau$ to be the mean lifetime of the proton and $B(p \rightarrow X)$ to be the branching ratio for the decay of the proton into final state $X$, then the limits on proton decay from Super-Kamiokande may be written as

$$
\begin{gathered}
\tau / B\left(p \rightarrow e^{+}+\pi^{0}\right)>1.6 \times 10^{34} \text { years, } \\
\tau / B\left(p \rightarrow \mu^{+}+\pi^{0}\right)>7.7 \times 10^{33} \text { years. }
\end{gathered}
$$

In the analysis used to obtain these limits, it was noted that proton decays could be distinguished from the dominant background, atmospheric neutrino interactions, using a neutron tagging technique. In contrast to proton decays in water, which will only rarely produce a neutron via the de-excitation of an oxygen nucleus [185], atmospheric neutrino interactions will often produce one or more neutrons in the final state via a variety of mechanisms [184]. In order to continue to improve these limits and, e.g., possibly observe the decay $p \rightarrow e^{+}+\pi^{0}$ with the partial lifetime of approximately $10^{35}$ years predicted by some GUTs [183], more precise estimates of the expected neutron yields from atmospheric neutrino reactions in water are desirable. Since these yields are not 
well-understood theoretically, a direct measurement of neutron production by neutrinos in the atmospheric energy range would greatly benefit future proton decay searches in water Cherenkov detectors.

\subsubsection{Rare event searches: the Diffuse Supernova Neutrino Background}

In addition to their relevance for proton decay, atmospheric neutrino interactions are also a hindrance to the effort to discover the Diffuse Supernova Neutrino Background (DSNB), the faint flux of neutrinos emitted from all past core-collapse supernovae [186]. The DSNB has not yet been directly observed, but some neutrino experiments, such as Super-Kamiokande [187] and KamLAND [188], have set upper limits on the DSNB $\bar{\nu}_{e}$ flux. Detection of the DSNB could lead to progress in a number of different areas, including constraints on the supernova rate within the observable universe, the fraction of "failed" supernovae that form black holes, and a variety of proposed exotic physics processes [189]. It could also enable a measurement of the average supernova neutrino spectrum, which could be compared to galactic core-collapse observations to determine how much the spectrum varies between stars [186].

Although almost certainly present, the DSNB remains unobserved because there are several backgrounds in the same energy range which are hard to eliminate [190]. Above about $20 \mathrm{MeV}$, for example, the dominant background comes from the production of sub-Cherenkov-threshold muons by low-energy atmospheric neutrinos [120]. Through the decays

$$
\mu^{+} \longrightarrow e^{+}+\nu_{e}+\bar{\nu}_{\mu}
$$

and

$$
\mu^{-} \longrightarrow e^{-}+\bar{\nu}_{e}+\nu_{\mu}
$$

these muons produce Michel (anti)electrons, which may be mistaken for antielectrons produced in a $\bar{\nu}_{e}$ inverse beta decay reaction.

In 2004, John Beacom and Mark Vagins proposed enabling high-effiency neutron tagging in water Cherenkov detectors by adding $0.2 \%$ gadolinium trichloride to the water [120]. Gadolinium has a remarkably high capture cross section for thermal neutrons, ${ }^{19}$

\footnotetext{
${ }^{19}$ The thermal neutron capture cross section for natural gadolinium is about 49000 barns versus 0.33
} 
and it emits a $\gamma$-ray cascade with a total energy of approximately $8 \mathrm{MeV}$ following each capture. An average of $4.3 \mathrm{MeV}$ of this energy is visible in a water Cherenkov detector, with most of the rest being lost to Compton-scattered electrons below the Cherenkov threshold [191]. Detecting the prompt $e^{+}$together with the delayed neutron capture gammas from inverse beta decay events is expected to reduce the atmospheric neutrino background for a DSNB measurement considerably, since muon decays do not produce neutrons [186]. However, some atmospheric neutrino reactions will produce both a lowenergy muon and a neutron in the final state. A measurement of the neutron yield from intermediate energy neutrino reactions in water would therefore be beneficial in the effort to discriminate between background and a DSNB signal.

\subsection{The ANNIE experiment}

Motivated by the usefulness of understanding neutron production by medium energy neutrinos, the upcoming second phase of the Accelerator Neutrino Neutron Interaction Experiment (ANNIE) will make a systematic measurement of neutrino-induced neutron production using the Fermilab Booster Neutrino Beam [126]. This measurement will be performed using the 26-ton water Cherenkov detector shown in the concept drawing in the top panel of fig. 2.6. From left to right, sketches depict the three major components of the detector: a wall of scintillator paddles used to detect and reject muons produced by neutrino interactions upstream of the detector (the front veto); a tank filled with Gdloaded water and lined with photodetectors; and a segmented steel-scintillator sandwich, called the Muon Range Detector (MRD), used to measure the direction and energy of muons produced in neutrino interactions within the tank.

The bottom panel of fig. 2.6 shows the stages of a typical ANNIE signal event (drawn from left to right) that will be selected for the neutron yield analysis. When a muon is produced inside the water volume by a charged current neutrino interaction, its trajectory and energy are reconstructed using the Muon Range Detector (MRD) and the tank photodetectors. Neutrons produced at the neutrino vertex scatter within the tank and reach barns for hydrogen. 

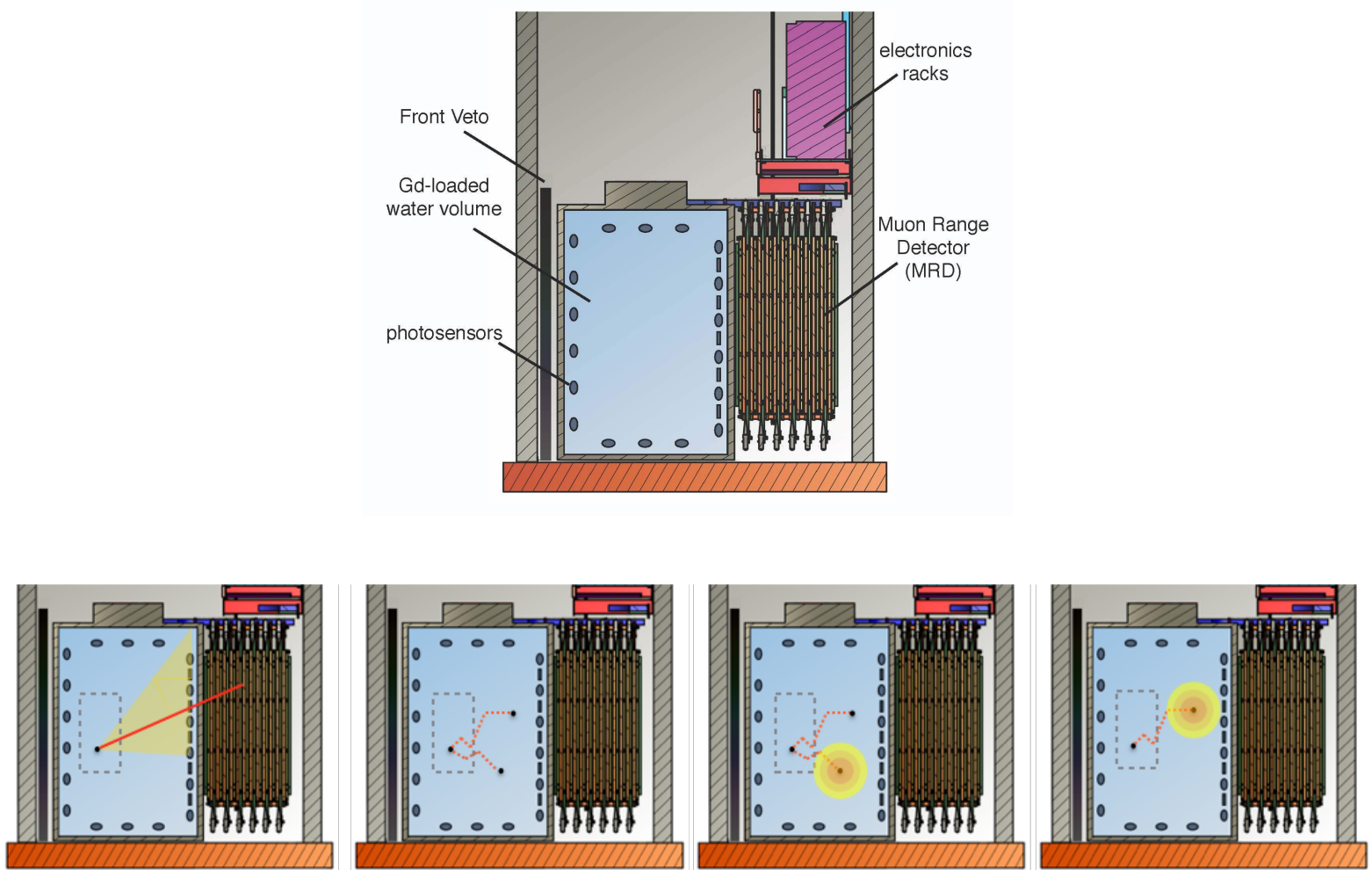

Figure 2.6: TOP: A concept drawing of the completed Phase II ANNIE detector. The neutrino beam travels from left to right in the perspective shown here. BOTTOM: The stages of a typical ANNIE signal event, shown from left to right and described in the text. Figure from reference [126].

thermal energies $(\sim 0.025 \mathrm{eV})$. Within tens of $\mu \mathrm{s}$, the neutrons capture on gadolinium nuclei. Each capture leads to a de-excitation $\gamma$-ray cascade with a total energy of about $8 \mathrm{MeV}$. The $\gamma$-rays from the neutron captures scatter within the water volume, leading to the production of Cherenkov light which is reconstructed by the photodetectors.

Because neutrino interactions near the edges of the tank may often produce neutrons that escape the water and thus go undetected, obtaining a sample of fully-contained events will be crucial to the success of the neutron yield analysis. In ANNIE, this will be accomplished via fiducialization, the designation of a portion of the active volume of a detector as the region of interest, or fiducial volume, within which all events used in an analysis must occur. For the neutron yield measurements, ANNIE will use a $2.5 \mathrm{~m}^{3}$ fiducial volume, represented in the lower panel of fig. 2.6 by the dashed box, which was 
chosen based on simulation studies of neutron containment, neutron detection efficiency, and the muon acceptance of the MRD. In one year of data taking, ANNIE is expected to observe about 14000 CCQE events occurring within the fiducial volume, about $30 \%$ of which will produce a muon that enters the MRD [126].

Unlike LArTPCs, which enable precise three-dimensional reconstruction of neutrino events, a water Cherenkov detector equipped solely with conventional photomultiplier tubes would be incapable of measuring the position of neutrino interaction vertices with sufficient resolution to achieve the fiducialization needed for the proposed ANNIE measurement. To address this problem, ANNIE will be the first experiment to make use of a recently-developed technology, the Large Area Picosecond Photodetector (LAPPD), in the context of an actual physics measurement. Figure 2.7 shows the operational principle of an LAPPD. A photon passes through a glass window and strikes the LAPPD's photocathode, liberating an electron via the photoelectric effect. The electric potential difference between the photocathode and the anode accelerates the electron toward a pair of microchannel plates. Each microchannel plate contains many pores with a diameter of around $10 \mu \mathrm{m}$ or less. Collisions of the primary electron with the walls of the pores, which are oriented at an angle with respect to the photocathode, lead to the generation of an exponentially-increasing number of secondary electrons. After passing through both of the plates, the electrons are collected at the anode, which is divided into micro-striplines that are instrumented and grounded at both ends. The position at which the photon hit the photocathode is inferred along the length of a stripline by measuring the time difference between the arrival of charge at each end. Calculation of a weighted centroid of the charge collected on neighboring striplines allows a determination of the position of the photon hit in the transverse direction [192]. The use of extremely fast electronics (e.g., PSEC4 integrated circuits [193]) to digitize LAPPD signals allows these devices to achieve a time resolution in the tens of ps and a photon hit position resolution better than $1 \mathrm{~mm}$ [194].

Figure 2.8 illustrates the dramatic improvement in vertex position reconstruction that the addition of even a few LAPPDs can provide for ANNIE. The left-hand plot shows the 


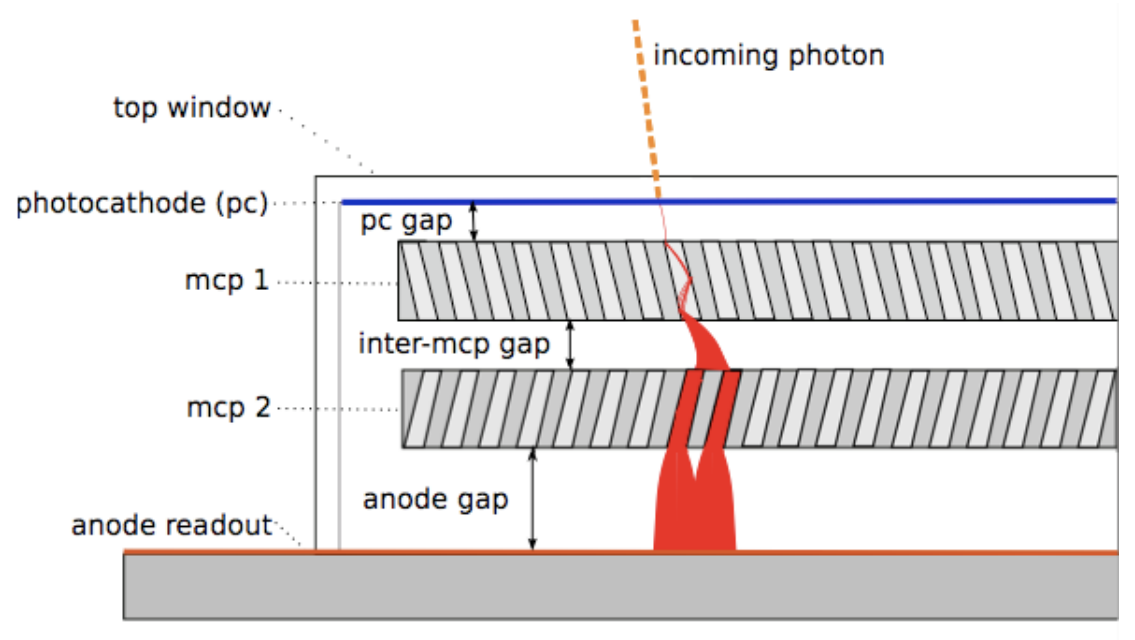

Figure 2.7: Schematic of a Large Area Picosecond Photodetector (LAPPD). Figure from reference [192].

distribution of $\Delta r$, the difference between the reconstructed and true vertex radial coordinates, for simulated charged current neutrino events in ANNIE. In the blue histogram, this reconstruction was carried out using 128 conventional PMTs (covering about 20\% of the inside of the tank) and zero LAPPDs. In the red histogram, five simulated LAPPDs were also used to reconstruct the vertex locations. Cumulative distributions for the two cases are shown in the right-hand plot. Using the conventional PMTs alone yields a radial position resolution (defined as the 68 th percentile value of $\Delta r$ ) of $38 \mathrm{~cm}$, while adding five LAPPDs improves the resolution to $12 \mathrm{~cm}$. Refining the existing reconstruction algorithms and including additional LAPPDs could both contribute to further improvements to the vertex resolution. However, early studies by members of the collaboration support the conclusion that five LAPPDs will be a sufficient number to achieve ANNIE's baseline physics goals, including the neutron yield measurements [126].

To obtain the data needed for the neutron yield analysis, the ANNIE detector will be placed in an experimental hall previously used by the SciBooNE experiment [196] and will be exposed to neutrinos from the Booster Neutrino Beam (BNB) at Fermilab. Because the hall is located only $100 \mathrm{~m}$ away from the beam target, the intense neutrino flux therein will enable ANNIE to make high-statistics measurements. However, this proximity 

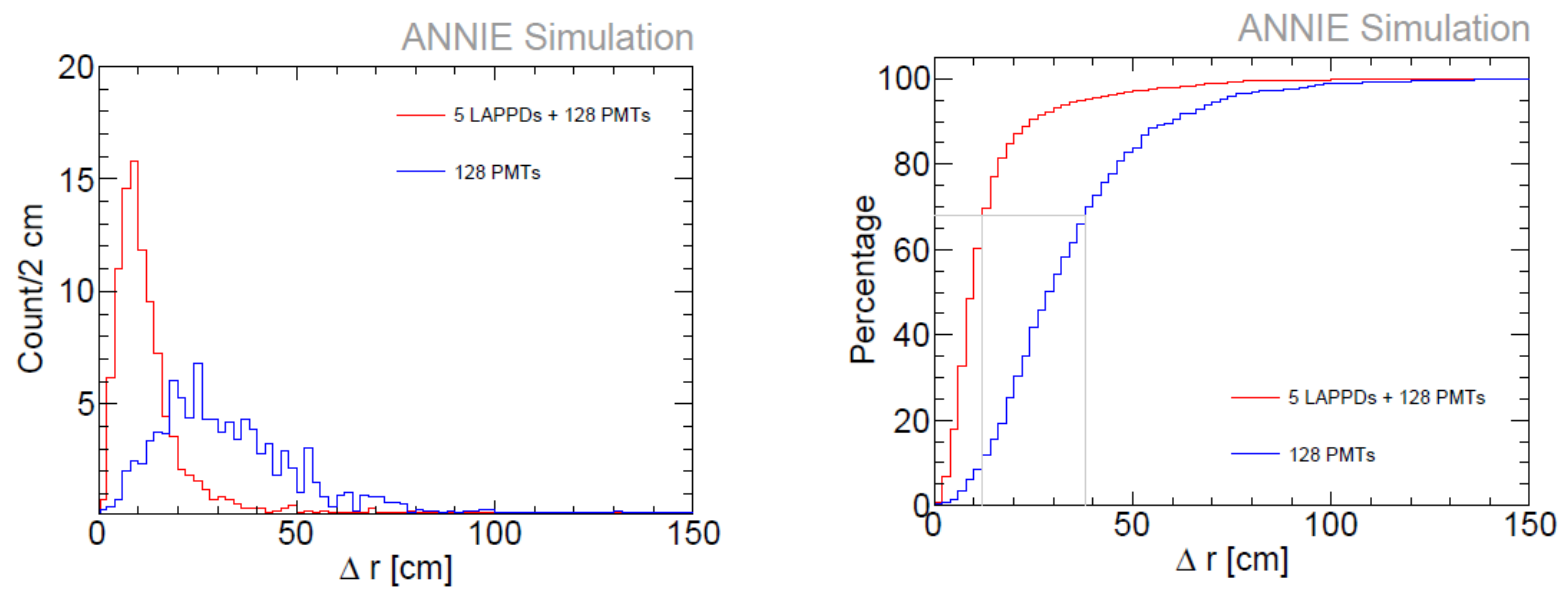

Figure 2.8: Results of a simulation study of neutrino vertex reconstruction in ANNIE. Raw (left) and cumulative (right) distributions of $\Delta r$, the difference between the reconstructed and true values of the vertex radial coordinate, are shown for simulated events detected by 128 conventional PMTs (blue) and the same number of PMTs plus 5

LAPPDs (red). The gray lines in the right-hand plot mark the location of the 68th percentile value for the two distributions. Figure from reference [195].

to the target comes with a cost: as will be discussed in detail in chapter 8, neutrons created by processes associated with the production of the beam could represent a large, potentially devastating source of background. To ensure that the beam-correlated neutron backgrounds will not preclude ANNIE's planned physics measurements, the collaboration has adopted a phased approach to the detector deployment. In preparation for ANNIE Phase II, in which the neutron yield measurement will be performed using the full detector, the ANNIE collaboration has successfully completed Phase I, an in situ characterization of neutron backgrounds using a partially instrumented version of the ANNIE detector. The second half of this thesis will describe this background measurement and present an analysis with the conclusion that, under very conservative assumptions, the neutron backgrounds at the planned site of ANNIE Phase II will be sufficiently low for the physics measurements to proceed. 


\section{Part I}

\section{MARLEY}




\section{Chapter 3}

\section{Cross section model}

Although accurate calculations of neutrino-nucleus cross sections are challenging in all energy regimes, the effects of nuclear structure present unique difficulties at the low energies of interest for supernova neutrinos $(\sim 10 \mathrm{MeV})$. Despite the vast theoretical literature that has emerged on this topic in the past half century, ${ }^{1}$ the only nuclear targets heavier than deuterium for which any cross section data are available in the supernova neutrino energy range are ${ }^{12} \mathrm{C},{ }^{56} \mathrm{Fe}$, and ${ }^{127} \mathrm{I}[200,202]$. Even for ${ }^{12} \mathrm{C}$, the best understood of these, the measured cross sections are known to a precision of no better than 10-20\% [156].

To maximize the physics impact of a future high-statistics measurement of neutrinos from a nearby supernova, our present limited understanding of tens-of-MeV neutrinonucleus scattering will need to be improved. This is particularly true for the proposed Deep Underground Neutrino Experiment (DUNE), since supernova neutrino events within its liquid-argon-based far detectors are expected to be dominated by charged current $\nu_{e}$ reactions on ${ }^{40} \mathrm{Ar}$, a medium-size nucleus [203]. By contrast, all other kiloton-scale neutrino detectors planned for the foreseeable future (which will be exclusively waterand oil-based) will detect much simpler $\bar{\nu}_{e}$ captures on protons as their primary supernova signal [156].

Because of the limitations of the currently available experimental data, theoretical

\footnotetext{
${ }^{1}$ See [197-199] for dedicated reviews and references [200, 201] for more recent review articles that include discussions of tens-of-MeV neutrino cross sections.
} 
models of low-energy neutrino-nucleus cross sections are needed as a means of estimating the sensitivity of future supernova neutrino detectors and informing their design. This chapter briefly reviews the current theoretical literature and then presents a derivation of approximate neutrino-nucleus cross section formulae that have been adopted for use in MARLEY.

\subsection{Formalisms for low-energy neutrino-nucleus cross sections}

Theoretical models of low-energy neutrino-nucleus scattering can be grouped into two major categories: (1) microscopic approaches, which consider the detailed structure of the nucleus using a model (such as the nuclear shell model or one of several variants of the random phase approximation) of the nuclear wavefunctions, and (2) macroscopic approaches, which parameterize cross sections in terms of overall nuclear properties without recourse to nuclear wavefunctions [204]. In the discussion of each of these approaches below, we will neglect for brevity a significant body of theoretical work that is specific to neutrino scattering on very light $(A \lesssim 4)$ nuclei, e.g., neutrino-deuterium cross section calculations via effective field theory (EFT) methods. The interested reader is encouraged to refer to recent reviews (examples include references [201, 205, 206]) which discuss that work.

\subsubsection{Microscopic models}

The most commonly used microscopic formalism for calculating neutrino-nucleus cross sections at low energies was introduced in the early 1970s by Walecka, Donnelly, and collaborators [207-213]. Based on previous theoretical descriptions of electron scattering [214] and muon capture (e.g., [215-217]), they put forward a unified treatment of semileptonic ${ }^{2}$ weak and electromagnetic interactions in nuclei, including beta decay, electron scattering, muon capture, and both charged and neutral current neutrino reactions. They then applied this formalism to a variety of specific calculations in a series of papers over the rest of the decade [218-224]. The Walecka-Donnelly approach was soon adopted by other researchers and continues to be used in many neutrino cross section calculations

\footnotetext{
${ }^{2}$ Involving a mixture of leptons and hadrons in the final state
} 
today (see, e.g., [225-230]).

The major steps in the derivation of the Walecka-Donnelly expressions for neutrino scattering cross sections include (1) neglecting the gauge boson field and writing the effective interaction Hamiltonian density at low energies as the product of a leptonic current and a nuclear current, (2) assuming that the matrix elements of the full nuclear current may be written as a sum of single-nucleon terms (the impulse approximation), (3) writing down the most general form of the single-nucleon current operator consistent with symmetry constraints, then approximating it to a given order (usually first, but see [231] for a detailed discussion of the second-order corrections) in inverse powers $1 / m_{N}$ of the nucleon mass, and (4) performing a multipole expansion of the nuclear current. The cross section that emerges from these steps is expressed in terms of the matrix elements of seven independent multipole operators, three from the vector current and four from the axial-vector current. These seven matrix elements may be conveniently evaluated for nucleon wavefunctions expressed in a harmonic oscillator basis using tables prepared by Donnelly and Haxton [224] or using a Mathematica [232] package created by Haxton and Lunardini [233].

Alongside the Walecka-Donnelly procedure, two related microscopic formalisms are present in the neutrino cross section literature. The first of these, developed by Kuramoto et al. [234-236] around 1990, is sometimes seen in publications by other authors (e.g., [237-239]), including a recent calculation by Civitarese and Tarutina [240]. Kuramoto's formalism makes the same basic assumptions as Walecka and Donnelly, but the single-nucleon current operator is approximated to third order in $|\mathbf{q}| / m_{N}$ (where $\mathbf{q}$ is the 3-momentum transfer) and zeroth order in $\left|\mathbf{p}_{N_{i}}\right| / m_{N}$, where $\mathbf{p}_{N_{i}}$ is the initial nucleon 3-momentum. This choice results in cross sections which depend on only three distinct nuclear matrix elements.

A second related microscopic formalism has been put forward in recent years by Krmpotić and Samana [241-244] and has been implemented in QRAP [245], a computer program for performing muon capture rate and charged current neutrino-nucleus cross section 
calculations using the $\mathrm{QRPA}^{3}$ and $\mathrm{PQRPA}^{4}$ models for the nuclear matrix elements. Krmpotić and Samana follow the same four steps described above for the Walecka-Donnelly formalism, including keeping terms up to first order in $1 / m_{N}$ when evaluating the singlenucleon current operator. However, they obtain simpler expressions for muon capture rates and charged current neutrino cross sections, both of which depend on only four nuclear matrix elements. ${ }^{5}$ As of this writing, the Krmpotić-Samana formalism has not appeared in published material outside of articles written by its creators (e.g., [204, 246, 247]). Since it is essentially equivalent to the popular Walecka-Donnelly approach, it may nevertheless see wider use in the future.

\subsubsection{Macroscopic models}

In contrast to the three formalisms described above, all of which rely on the impulse approximation and models for the nuclear wavefunctions, a macroscopic, model-independent alternative called the elementary particle treatment (EPT) was introduced in the 1960s for muon capture [248-250] and applied to neutrino scattering on ${ }^{12} \mathrm{C}$ by Fukugita in 1988 [251]. Under the EPT, the target nucleus is treated as a single "elementary particle," and the matrix elements describing neutrino interactions with it are parameterized in terms of nuclear (as opposed to single nucleon) form factors that must be determined using experimental measurements. In practice, obtaining the form factors needed to compute EPT neutrino cross sections is difficult, although successful calculations have been performed for ${ }^{2} \mathrm{H}[201]$ and ${ }^{12} \mathrm{C}[251]$. For the ${ }^{12} \mathrm{C}$ case, the EPT cross sections for neutrinos show good agreement with similar estimates performed using variations of the WaleckaDonnelly method, but the predictions differ markedly for antineutrinos [241, 252].

Models from a second class of macroscopic theories of neutrino-nucleus scattering, referred to as either "statistical" [253, 254] or "gross" [255] theories, are often used to estimate neutrino reaction rates in astrophysical nucleosynthesis calculations. These models are derived by starting with an approximate expression for a neutrino cross section

\footnotetext{
${ }^{3}$ Quasiparticle Random Phase Approximation

${ }^{4}$ Projected Quasiparticle Random Phase Approximation

${ }^{5}$ Equations relating the seven Walecka-Donnelly matrix elements and the three Kuramoto matrix elements to the four of Krmpotić and Samana are given in reference [242].
} 
obtained using a microscopic theory. This is typically $[253,255,256]$ the allowed approximation $^{6}$ cross section, i.e., the zero momentum transfer limit of the Walecka-Donnelly (or either of the other two microscopic formalisms mentioned above) cross section when the expansion of the nuclear current operator is carried out only to zeroth order in $1 / m_{N}$. However, rather than being evaluated directly, as they would be in a microscopic approach, the nuclear matrix elements are instead expressed in terms of strength functions, that is, semi-empirical parameterized functions of the excitation energy of the final-state nucleus that describe the average behavior of the matrix elements. Higher-order corrections beyond the allowed approximation are sometimes considered in this simplified treatment, either by using a more complicated starting expression for the cross section [254] or by adopting a value for the total cross section per nucleon based on the average of microscopic calculations of the cross sections for many different nuclei [256, see also reference 16 therein].

Statistical models of neutrino-nucleus scattering provide a simple means of obtaining total cross sections that agree reasonably well with microscopic approaches. However, because these models use strength functions that represent the energy-averaged behavior of the matrix elements, their ability to predict partial cross sections to individual nuclear levels will be more limited. While this problem can partially be remedied at high excitation energies $^{7}$ by averaging over groups of closely-spaced nuclear levels, such a solution fails at low excitation energies where the individual levels are well-separated and have small widths.

\subsubsection{A cross section formalism for MARLEY}

Due to the limitations of the macroscopic models reviewed above, a microscopic treatment of the cross sections for tens-of-MeV neutrino-nucleus scattering appears to be the best choice for implementation in a Monte Carlo event generator. Although any of the three microscopic formalisms described above would be a reasonable choice to use as a basis for

\footnotetext{
${ }^{6}$ This approximation originated in the beta-decay literature. See reference [257] for a review of the early years of theoretical research on beta decay, including a discussion of the allowed approximation in that context.

${ }^{7}$ Say, a little above the neutron emission threshold
} 
MARLEY, adopting them in their original form might limit the ability of MARLEY users to fully understand how the generator works. Because the Walecka-Donnelly approach to semileptonic nuclear reactions was developed decades ago when the Glashow-WeinbergSalam theory of the electroweak interaction had just emerged, the original publications describing the formalism use notation and conventions (e.g., a Euclidean Minkowski metric) which may be unfamiliar to readers who first learned about quantum field theory and neutrino interactions using recent textbooks. The same is true of the papers describing the Kuramoto and Krmpotić-Samana formalisms, which preserve many aspects of Walecka and Donnelly's original presentation.

In an effort to make the details of the calculation more accessible to current students, the remainder of this chapter presents a new derivation with more modern notation of cross section formulae for low-energy neutrino-nucleus scattering. This derivation begins with a discussion of neutrino-quark scattering at tree level, a relatively simple process for which a cross section calculation might be assigned as homework in a standard graduatelevel course on weak interactions. The discussion then follows Krmpotić and Samana's approach [242] the most closely as it proceeds through the four steps outlined above, culminating in expressions for neutrino cross sections, evaluated both to order $1 / m_{N}$ and under the allowed approximation, that are similar to theirs. Chapters 5 and 6 will revisit the allowed approximation cross sections and discuss how they have been used to create an initial version of the MARLEY generator. Prospects for improving the code to use the full $1 / m_{N}$ treatment will also be discussed in chapter 7

Beyond changes in notation, the derivations given in the remainder of this chapter differ from previous work in two major ways. First, the final expressions for the cross sections are evaluated in the center-of-momentum $(\mathrm{CM})$ frame instead of the usual laboratory frame. This choice of frame is far more convenient to use within the MARLEY event generator because the CM frame outgoing lepton energy is independent of the scattering angle, which facilitates straightforward Monte Carlo sampling of the latter. Second, the neutral current cross sections that are derived in this chapter are expressed in terms of matrix elements similar to those used by Krmpotić and Samana. Although Donnelly, 
Walecka, and collaborators [209, 211] provided a treatment of neutral current cross sections using their formalism, the same has not yet been done in published work for either of the other two microscopic formalisms mentioned above. The expressions for neutral current cross sections derived in this chapter, therefore, may be viewed as an extension of the Krmpotić-Samana formalism, albeit with somewhat modified notation.

For definiteness, the presentation below will initially be restricted to charged current neutrino scattering. The neutral current and antineutrino charged current cross sections will then be examined in light of the approach used previously.

\subsection{From neutrino-quark to neutrino-nucleon scattering}

In the Standard Model, the charged current interaction is described by the Lagrangian density

$$
\mathcal{L}_{W}=-\frac{g}{2 \sqrt{2}}\left(J_{W}^{\mu} W_{\mu}^{-}+J_{W}^{\mu \dagger} W_{\mu}^{+}\right)
$$

where $W_{\mu}^{ \pm}$is the $W$ boson field, $g$ is the $\mathrm{SU}(2)$ gauge coupling constant, and $J_{W}^{\mu \dagger}$ is the weak charge-raising current. This current may be written in the form ${ }^{8}$

$$
J_{W}^{\mu \dagger}=\left(\begin{array}{lll}
\bar{\nu}_{e} & \bar{\nu}_{\mu} & \bar{\nu}_{\tau}
\end{array}\right) \gamma^{\mu}\left(1-\gamma^{5}\right)\left(\begin{array}{l}
e^{-} \\
\mu^{-} \\
\tau^{-}
\end{array}\right)+\left(\begin{array}{lll}
\bar{u} & \bar{c} & \bar{t}
\end{array}\right) \gamma^{\mu}\left(1-\gamma^{5}\right) V_{\mathrm{CKM}}\left(\begin{array}{l}
d \\
s \\
b
\end{array}\right)
$$

where $\nu_{e}, \nu_{\mu}$, and $\nu_{\tau}$ are neutrino flavor eigenstate fields, the other field operators $\left(e^{-}\right.$, $u$, etc.) are expressed in the mass basis, and the $3 \times 3$ Cabibbo-Kobayashi-Maskawa matrix $V_{\mathrm{CKM}}$ connects the quark mass eigenstates to their weak eigenstates. The Lorentzinvariant amplitude $\mathcal{M}$ for charged current scattering of a neutrino on a free down quark may be computed to lowest order in the coupling constant $g$ using the tree-level diagram

\footnotetext{
${ }^{8}$ Following reference [49], we neglect neutrino masses in this expression and define the neutrino flavor eigenstate fields $\nu_{e}, \nu_{\mu}$, and $\nu_{\tau}$ as the weak interaction partners of the corresponding charged lepton mass eigenstate fields.
} 


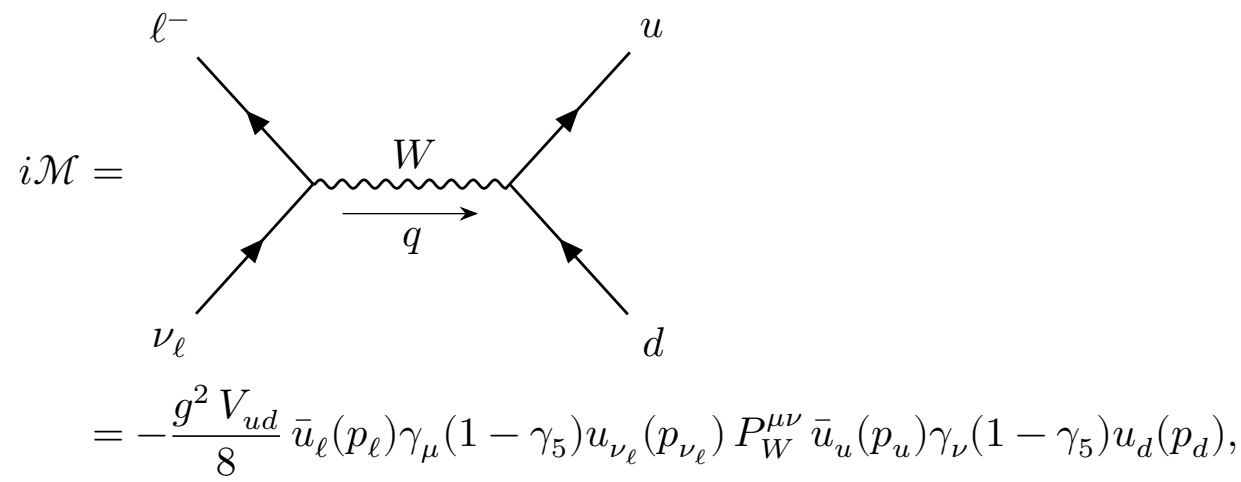

where $V_{u d}$ is the CKM matrix element connecting the up and down quarks, and

$$
P_{W}^{\mu \nu}=i \frac{-g^{\mu \nu}+\frac{q^{\mu} q^{\nu}}{m_{W}^{2}}}{q^{2}-m_{W}^{2}+i \epsilon}
$$

is the $W$ boson propagator. At supernova energies $(\sim 10 \mathrm{MeV})$, the square of the momentum transfer $q^{2}$ is small, i.e., $q^{2} \ll m_{W}^{2}$, and we may therefore approximate the propagator by its low-energy limit:

$$
P_{W}^{\mu \nu} \approx \frac{i g^{\mu \nu}}{m_{W}^{2}} .
$$

Under this approximation, the diagram shown in eq. (3.3) becomes

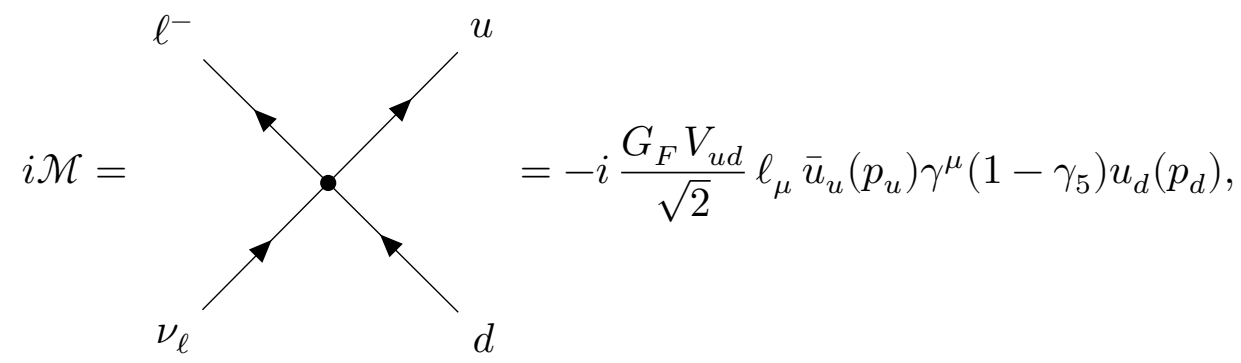

where the Fermi constant $G_{F}$ is given by

$$
\frac{G_{F}}{\sqrt{2}}=\frac{g^{2}}{8 m_{W}^{2}},
$$

and the leptonic matrix element $\ell_{\mu}$ is defined as

$$
\ell_{\mu}=\bar{u}_{\ell}\left(p_{\ell}\right) \gamma_{\mu}\left(1-\gamma_{5}\right) u_{\nu_{\ell}}\left(p_{\nu_{\ell}}\right)
$$

In reality, the struck quark is not free, but instead is bound in a nucleon. Therefore, the quark part of the amplitude shown above must be replaced by a suitable hadronic 
matrix element that accounts for the effects of strong interactions within the nucleon:

$$
\bar{u}_{u}\left(p_{u}\right) \gamma^{\mu}\left(1-\gamma_{5}\right) u_{d}\left(p_{d}\right) \rightarrow \mathrm{n}^{\mu}
$$

After making this substitution, the diagram in eq. (3.6) becomes

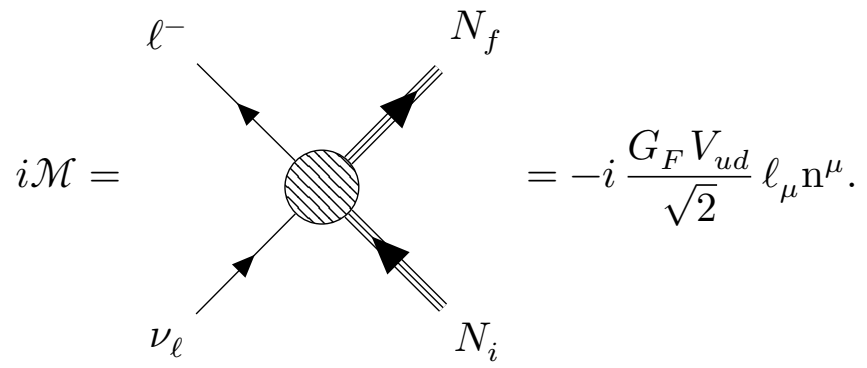

While writing down an explicit form for the hadronic matrix element $\mathrm{n}^{\mu}$, it will be helpful to work in the isospin formalism, which is reviewed in section 3.2.1.

\subsubsection{Isospin in nuclear physics}

In the isospin formalism, ${ }^{9}$ the proton and neutron are described as two distinct states of an abstract generic particle called the nucleon. By analogy with the quantum mechanical description of spin-half particles, the nucleon is taken to have total isospin quantum number $t=1 / 2$. The neutron is then defined by convention to be the nucleon's "isospin up" state (i.e., its third component of isospin $t_{3}=+1 / 2$ ), while the proton is defined to be the "isospin down" $\left(t_{3}=-1 / 2\right)$ state. $^{10}$ The components of the isospin operator $\mathbf{t}$ for isospin-half particles are defined by

$$
\mathbf{t} \equiv \frac{1}{2} \boldsymbol{\tau}
$$

where the components of $\boldsymbol{\tau}$ are given by the Pauli matrices

$$
\tau_{1}=\tau_{x}=\left(\begin{array}{cc}
0 & 1 \\
1 & 0
\end{array}\right) \quad \tau_{2}=\tau_{y}=\left(\begin{array}{cc}
0 & -i \\
i & 0
\end{array}\right) \quad \tau_{3}=\tau_{z}=\left(\begin{array}{cc}
1 & 0 \\
0 & -1
\end{array}\right)
$$

\footnotetext{
${ }^{9}$ The concept of isospin was first introduced by Heisenberg [258] in 1932, although it was Wigner [259] who coined the term "isotopic spin," which was later shortened to "isospin."

${ }^{10}$ This is the standard sign convention in nuclear physics. Note, however, that it is exactly the opposite of the particle physics convention in which up quarks have $+1 / 2$ as their third isospin component. The nuclear convention arises from the desire to have a positive third component of isospin for most naturallyoccurring nuclides, which tend to have an excess of neutrons [260].
} 
in isospace, the Hilbert space in which isospin states are defined. In this space, nucleon states are represented by isospinors. The two nucleon states with a definite third component of isospin, the neutron $|n\rangle$ and proton $|p\rangle$, are described by the isospinors

$$
|n\rangle \equiv\left|\frac{1}{2} \frac{1}{2}\right\rangle=\left(\begin{array}{l}
1 \\
0
\end{array}\right) \quad|p\rangle \equiv\left|\frac{1}{2}-\frac{1}{2}\right\rangle=\left(\begin{array}{l}
0 \\
1
\end{array}\right) .
$$

Because the isospin Pauli matrices $\tau_{1}, \tau_{2}$, and $\tau_{3}$ obey the same $\mathrm{SU}(2)$ Lie algebra in isospace that the spin Pauli matrices $\sigma_{1}, \sigma_{2}$, and $\sigma_{3}$ obey in spin space, ${ }^{11}$ one may immediately write down many of the properties of the isospin operators based on the well-known spin equivalents. For instance, the $\tau$ matrices obey the commutation and anticommutation rules

$$
\begin{gathered}
{\left[\tau_{a}, \tau_{b}\right]=2 i \epsilon_{a b c} \tau_{c}} \\
\left\{\tau_{a}, \tau_{b}\right\}=2 \delta_{a b} I
\end{gathered}
$$

where $\epsilon_{a b c}$ is the Levi-Civita tensor, $\delta_{a b}$ is the Kronecker delta, $I$ is the $2 \times 2$ identity matrix, and the indices $a, b, c \in\{1,2,3\}$. One may define an operator representing the square of the magnitude of the total isospin

$$
t^{2} \equiv \mathbf{t} \cdot \mathbf{t}=t_{x}^{2}+t_{y}^{2}+t_{z}^{2}
$$

as well as the isospin raising and lowering operators

$$
t_{ \pm} \equiv t_{x} \pm i t_{y}
$$

These operators satisfy the commutation relations

$$
\left[t^{2}, t_{a}\right]=0 \quad\left[t_{3}, t_{ \pm}\right]= \pm t_{ \pm} \quad\left[t_{+}, t_{-}\right]=2 t_{3}
$$

\footnotetext{
${ }^{11}$ The sole difference is that eigenvalues of the isospin operators do not contain factors of $\hbar$, even when one works in conventional units. Although its name might suggest the contrary, isospin is dimensionless. Apart from the isomorphism between $\tau$ and $\sigma$ matrices, it has nothing to do with angular momentum.
} 
The isospin operators described above act on the nucleon states of definite isospin projection in the following ways:

$$
\begin{aligned}
t^{2}|n\rangle & =\frac{3}{2}|n\rangle & t^{2}|p\rangle & =\frac{3}{2}|p\rangle \\
t_{+}|n\rangle & =0 & t_{+}|p\rangle & =|n\rangle \\
t_{-}|n\rangle & =|p\rangle & t_{-}|p\rangle & =0 \\
t_{3}|n\rangle & =\frac{1}{2}|n\rangle & t_{3}|p\rangle & =-\frac{1}{2}|p\rangle \\
\tau_{3}|n\rangle & =+|n\rangle & \tau_{3}|p\rangle & =-|p\rangle .
\end{aligned}
$$

Note that the isospin lowering operator $t_{-}$changes a neutron into a proton. This operator will be important in the description of charged current neutrino-nucleus scattering that follows. Its Hermitian conjugate $t_{-}^{\dagger}=t_{+}$, which changes a proton into a neutron, will appear in section 3.12 in the context of charged current antineutrino-nucleus scattering.

For a nucleus consisting of $A$ nucleons, one may define the total isospin operator $\mathbf{T}$, its third component $T_{3}$, and the total isospin ladder operators $T_{ \pm}$by

$$
\begin{gathered}
\mathbf{T} \equiv \sum_{k=1}^{A} \mathbf{t}(k)=\frac{1}{2} \sum_{k=1}^{A} \boldsymbol{\tau}(k), \\
T_{3} \equiv \sum_{k=1}^{A} t_{3}(k)=\frac{1}{2} \sum_{k=1}^{A} \tau_{3}(k),
\end{gathered}
$$

and

$$
T_{ \pm} \equiv T_{x} \pm i T_{y}=\sum_{k=1}^{A} t_{ \pm}(k)
$$

where an operator $\mathcal{O}(k)$ acts on the state vector for the $k$ th nucleon. The action of $T_{3}$ on a nuclear state $\left|\mathcal{T} \mathcal{T}_{3}\right\rangle$ with definite total isospin $\mathcal{T}$ and isospin projection $\mathcal{T}_{3}$ gives

$$
T_{3}\left|\mathcal{T} \mathcal{T}_{3}\right\rangle=\mathcal{T}_{3}\left|\mathcal{T} \mathcal{T}_{3}\right\rangle
$$

while the action of $T_{ \pm}$gives

$$
T_{ \pm}\left|\mathcal{T} \mathcal{T}_{3}\right\rangle=\sqrt{\left(\mathcal{T} \mp \mathcal{T}_{3}\right)\left(\mathcal{T} \pm \mathcal{T}_{3}+1\right)}\left|\mathcal{T} \mathcal{T}_{3} \pm 1\right\rangle
$$


The total isospin operators obey the commutation rules

$$
\left[T^{2}, T_{a}\right]=0 \quad\left[T_{3}, T_{ \pm}\right]= \pm T_{ \pm} \quad\left[T_{+}, T_{-}\right]=2 T_{3},
$$

where $T^{2} \equiv \mathbf{T} \cdot \mathbf{T}=T_{x}^{2}+T_{y}^{2}+T_{z}^{2}$ and $T_{z}=T_{3}$.

Just like operators in coordinate or spin space, the isospin operators may be classified by the way they transform under rotations in isospace. For example, the identity $I$ and the square of the total isospin $T^{2}$ are isoscalar operators, while $T_{3}$ is a component of an isovector operator.

\subsubsection{Four-vector expression for the free nucleon matrix element}

Using Lorentz invariance, translation invariance, and parity conservation in the strong interaction, it can be shown $[49,59]$ that the most general form ${ }^{12}$ of the hadronic matrix element $\mathrm{n}^{\mu}$ from eqs. (3.9) and (3.10) may be written for a free nucleon as:

$$
\begin{aligned}
\mathrm{n}^{\mu}= & \chi_{N_{f}}^{\dagger} \bar{u}_{N_{f}}\left(p_{N_{f}}\right)\left[\gamma^{\mu} F_{1}\left(Q^{2}\right)+\frac{i}{2 m_{N}} \sigma^{\mu \nu} q_{\nu} F_{2}\left(Q^{2}\right)+\frac{q^{\mu}}{m_{N}} F_{3}\left(Q^{2}\right)\right. \\
& \left.-\gamma^{\mu} \gamma^{5} G_{A}\left(Q^{2}\right)-\frac{q^{\mu}}{m_{N}} \gamma^{5} G_{P}\left(Q^{2}\right)+\frac{i}{m_{N}} \sigma^{\mu \nu} q_{\nu} \gamma^{5} G_{3}\left(Q^{2}\right)\right] t_{-} u_{N_{i}}\left(p_{N_{i}}\right) \chi_{N_{i}} .
\end{aligned}
$$

In the expression above, $m_{N}$ is the nucleon mass, ${ }^{13} q \equiv p_{N_{f}}-p_{N_{i}}$ is the 4-momentum transfer, ${ }^{14} Q^{2} \equiv-q^{2}, t_{-}$is the isospin lowering operator defined in eqs. (3.17) and (3.21), and $F_{1}\left(Q^{2}\right), F_{2}\left(Q^{2}\right), F_{3}\left(Q^{2}\right), G_{A}\left(Q^{2}\right), G_{P}\left(Q^{2}\right)$, and $G_{3}\left(Q^{2}\right)$ are the vector, weak magnetic (induced tensor), induced scalar, axial-vector, induced pseudoscalar, and weak electric (induced pseudotensor) nucleon form factors, respectively. The initial $\left(N_{i}\right)$ and final $\left(N_{f}\right)$ nucleon states are written in terms of the Dirac spinors $u_{N}\left(p_{N}\right)$ and the isospinors $\chi_{N}$ for $N \in\left\{N_{i}, N_{f}\right\}$

Before proceeding to a calculation of the amplitude for charged current neutrino scattering on a full nucleus, it will be helpful to briefly review the connection between the Lorentz invariant amplitude $\mathcal{M}$ and the differential cross section $d \sigma$ for a $2 \rightarrow 2$ scattering reaction.

\footnotetext{
${ }^{12}$ The expression in eq. (3.30) matches that of reference [59] (see their equations 5.73 and 5.74), except that the isospin representation has been used, and the Gordon identity $\bar{u}_{2}\left(p_{2}+p_{1}\right)^{\mu} \gamma^{5} u_{1}=$ $-i \bar{u}_{2} \sigma^{\mu \nu}\left(p_{2}-p_{1}\right)_{\nu} \gamma^{5} u_{1}$ has been used to rewrite the $G_{3}\left(Q^{2}\right)$ term.

${ }^{13}$ That is, the mean of the neutron and proton masses: $m_{N}=\left(m_{n}+m_{p}\right) / 2$

${ }^{14}$ Our definition for the momentum transfer $q$ matches that of references $[49,59]$. Note, however, that the opposite subtraction order $q=p_{N_{i}}-p_{N_{f}}$ is also used in the literature, e.g., references [210, 242].
} 


\subsection{Calculation of $2 \rightarrow 2$ scattering cross sections}

Let the Lorentz-invariant amplitude $\mathcal{M}$ describe an arbitrary reaction in which a group of initial particles becomes a group of final particles. Let $p_{i, j}$ represent the 4-momentum of the $j$ th initial particle and $p_{f, k}$ represent the 4 -momentum of the $k$ th final particle. Then, for a particular configuration of initial- and final-state spin orientations, ${ }^{15}$ the probability amplitude for the reaction is given by the scattering matrix (often referred to as simply the $S$-matrix) element $S_{f i}$ :

$$
S_{f i}=\delta_{f i}-\frac{i \mathcal{M}(2 \pi)^{4} \delta^{(4)}\left(\sum_{j} p_{i, j}-\sum_{k} p_{f, k}\right)}{\prod_{j} \sqrt{2 E_{i, j}} \prod_{k} \sqrt{2 E_{f, k}}}
$$

where the sum $\sum_{j}\left(\sum_{k}\right)$ and product $\prod_{j}\left(\prod_{k}\right)$ include all particles in the initial (final) state. The four-dimensional delta function in this expression enforces conservation of four-momentum. The Kronecker delta function $\delta_{f i}$ is unity if the initial and final states are identical and zero otherwise. Ignoring the trivial case of identical initial and final states, one may write the probability $P_{f i}$ for the reaction to occur in the form

$$
P_{f i}=\left|\overline{S_{f i}}\right|^{2}=\frac{|\overline{\mathcal{M}}|^{2}\left[(2 \pi)^{4} \delta^{(4)}\left(\sum_{j} p_{i, j}-\sum_{k} p_{f, k}\right)\right]^{2}}{\prod_{j} 2 E_{i, j} \prod_{k} 2 E_{f, k}},
$$

where a bar over a squared matrix element indicates that it should be averaged (summed) over all unobserved spin orientations in the initial (final) state.

The squared delta function in eq. (3.32) may be evaluated by imagining the system of reacting particles to be enclosed within a large but finite spacetime box [262]. The box includes the time interval $T$ and the spatial volume $V=L^{3}$, where $L$ is the length of one side of the box. The identity

$$
\delta(E)=\frac{1}{2 \pi} \int e^{i E t} d t
$$

\footnotetext{
${ }^{15}$ It is typically most convenient to specify the particle spin orientations using the helicity basis [261]. When this basis is used, the spin projection quantum number of interest for each particle is the helicity $h \equiv \boldsymbol{s} \cdot \mathbf{p} /|\mathbf{p}|$, where $\boldsymbol{s}$ is the particle's spin and $\mathbf{p}$ is its 3-momentum. In the rest frame of the particle, the spin direction may be measured along an arbitrary axis.
} 
implies that

$$
\left[(2 \pi) \delta\left(\sum_{j} E_{i, j}-\sum_{k} E_{f, k}\right)\right]^{2}=(2 \pi) \delta\left(\sum_{j} E_{i, j}-\sum_{k} E_{f, k}\right) \int e^{i\left(\sum_{j} E_{i, j}-\sum_{k} E_{f, k}\right) t} d t .
$$

Because of the remaining delta function, one may set the exponent of the integrand to zero in eq. (3.34), yielding

$$
\left[(2 \pi) \delta\left(\sum_{j} E_{i, j}-\sum_{k} E_{f, k}\right)\right]^{2}=T(2 \pi) \delta\left(\sum_{j} E_{i, j}-\sum_{k} E_{f, k}\right)
$$

since

$$
T=\int d t
$$

because the integral is evaluated only within the box. A similar argument gives us the relation

$$
\left[(2 \pi)^{3} \delta^{(3)}\left(\sum_{j} \mathbf{p}_{i, j}-\sum_{k} \mathbf{p}_{f, k}\right)\right]^{2}=V(2 \pi)^{3} \delta^{(3)}\left(\sum_{j} \mathbf{p}_{i, j}-\sum_{k} \mathbf{p}_{f, k}\right)
$$

since

$$
V=\int d^{3} \mathbf{x}
$$

if, once again, the integration takes place only within the box. To ensure that the reaction probability $P_{f i}$ remains properly normalized, the particle wavefunctions must be normalized to unity within the box. This can be accomplished by multiplying each energy factor in eq. (3.31) by $\sqrt{V}$. The probability for the reaction to occur within the box then may be written as

$$
P_{f i}=\left|\overline{S_{f i}}\right|^{2}=\frac{|\overline{\mathcal{M}}|^{2} V T(2 \pi)^{4} \delta^{(4)}\left(\sum_{j} p_{i, j}-\sum_{k} p_{f, k}\right)}{\prod_{j} 2 E_{i, j} V \prod_{k} 2 E_{f, k} V} .
$$

Because the box has a finite size, the three-momentum $\mathbf{p}$ of a particle within it is quantized so that

$$
\mathbf{p}=\frac{2 \pi}{L}\left(n_{1} \hat{\mathbf{e}}_{x}+n_{2} \hat{\mathbf{e}}_{y}+n_{3} \hat{\mathbf{e}}_{z}\right)
$$

where the $n_{a}$ are integers and the $\hat{\mathbf{e}}_{b}$ are Cartesian unit vectors. Therefore, the number $d n$ of single-particle states available within the box for a particle with 3-momentum between 
$\mathbf{p}$ and $\mathbf{p}+d^{3} \mathbf{p}$ is given by

$$
d n=\frac{V}{(2 \pi)^{3}} d^{3} \mathbf{p}
$$

The differential transition rate (probability per unit time) $d \Gamma_{f i}$ for the reaction described by $\mathcal{M}$ may be written as

$$
d \Gamma_{f i}=T^{-1}\left|\overline{S_{f i}}\right|^{2} \prod_{k} d n_{f, k}
$$

Specializing to a scattering reaction involving two initial particles with four momenta $p_{i, 1}$ and $p_{i, 2}$, allows one to write the differential cross section $d \sigma$ for the reaction in the form

$$
d \sigma=\frac{d \Gamma_{f i}}{\phi}
$$

Here $\phi$, the incident particle flux, may be written as [263]

$$
\phi=\frac{\left(p_{i, 1} \cdot p_{i, 2}\right) \beta_{i, \mathrm{rel}}}{V E_{i, 1} E_{i, 2}}
$$

where the relative speed of the two initial particles $\beta_{i \text {,rel }}$ is given in a manifestly Lorentzinvariant form by the expression

$$
\beta_{i, \mathrm{rel}}=\frac{\sqrt{\left(p_{i, 1} \cdot p_{i, 2}\right)^{2}-m_{i, 1}^{2} m_{i, 2}^{2}}}{p_{i, 1} \cdot p_{i, 2}} .
$$

If one further specializes to $2 \rightarrow 2$ scattering (a reaction with two initial particles and two final particles), then these results may be combined to obtain the following expression for the differential cross section:

$$
\begin{aligned}
d \sigma & =T^{-1} \frac{V E_{i, 1} E_{i, 2}}{\left(p_{i, 1} \cdot p_{i, 2}\right) \beta_{i, \mathrm{rel}}} \frac{|\overline{\mathcal{M}}|^{2} V T(2 \pi)^{4} \delta^{(4)}\left(\sum_{j} p_{i, j}-\sum_{k} p_{f, k}\right)}{16 V^{4} E_{i, 1} E_{i, 2} E_{f, 1} E_{f, 2}} \frac{V d^{3} \mathbf{p}_{f, 1}}{(2 \pi)^{3}} \frac{V d^{3} \mathbf{p}_{f, 2}}{(2 \pi)^{3}} \\
& =\frac{1}{4\left(p_{i, 1} \cdot p_{i, 2}\right) \beta_{i, \mathrm{rel}}}|\overline{\mathcal{M}}|^{2}(2 \pi)^{4} \delta^{(4)}\left(p_{i, 1}+p_{i, 2}-p_{f, 1}-p_{f, 2}\right) \frac{d^{3} \mathbf{p}_{f, 1}}{(2 \pi)^{3} 2 E_{f, 1}} \frac{d^{3} \mathbf{p}_{f, 2}}{(2 \pi)^{3} 2 E_{f, 2}} \\
& =F^{-1}|\overline{\mathcal{M}}|^{2}(2 \pi)^{4} \delta^{(4)}\left(p_{i, 1}+p_{i, 2}-p_{f, 1}-p_{f, 2}\right) d \Phi_{\text {LIPS }}
\end{aligned}
$$

where

$$
F \equiv 4\left(p_{i, 1} \cdot p_{i, 2}\right) \beta_{i, \mathrm{rel}}
$$


is called the Møller flux factor and

$$
d \Phi_{\mathrm{LIPS}} \equiv \frac{d^{3} \mathbf{p}_{f, 1}}{(2 \pi)^{3} 2 E_{f, 1}} \frac{d^{3} \mathbf{p}_{f, 2}}{(2 \pi)^{3} 2 E_{f, 2}}=\prod_{k} \frac{d^{3} \mathbf{p}_{f, k}}{(2 \pi)^{3} 2 E_{f, k}}
$$

is called the Lorentz invariant phase space.

In the case of $2 \rightarrow 2$ scattering where the helicities of all four of the involved particles are unobserved, note that the spin-summed squared amplitude $|\overline{\mathcal{M}}|^{2}$ introduced in eq. (3.32) may be written as

$$
|\overline{\mathcal{M}}|^{2} \equiv \frac{1}{\left(2 s_{i, 1}+1\right)\left(2 s_{i, 2}+1\right)} \sum_{h_{i, 1}} \sum_{h_{i, 2}} \sum_{h_{f, 1}} \sum_{h_{f, 2}}|\mathcal{M}|^{2} .
$$

where $s_{i, 1}$ is the spin of the first initial particle, $h_{i, 1}$ is its helicity, etc.

Because the factors of $T$ and $V$ in eq. (3.46) cancel, one may take the limits $T \rightarrow \infty$ and $V \rightarrow \infty$ at will, thus dispensing with the finite-size box enclosing the reaction. Integrating both sides of eq. (3.46) over $d^{3} \mathbf{p}_{f, 2}$ is trivial because of the delta function. Doing so yields

$$
d \sigma=F^{-1}|\overline{\mathcal{M}}|^{2}(2 \pi) \delta\left(E_{i, 1}+E_{i, 2}-E_{f, 1}-E_{f, 2}\right) \frac{1}{2 E_{f, 2}} \frac{d^{3} \mathbf{p}_{f, 1}}{(2 \pi)^{3} 2 E_{f, 1}}
$$

where it is understood that 3-momentum is conserved, i.e.,

$$
\mathbf{p}_{i, 1}+\mathbf{p}_{i, 2}-\mathbf{p}_{f, 1}-\mathbf{p}_{f, 2}=0
$$

Expressing $d^{3} \mathbf{p}_{f, 1}$ in spherical coordinates allows one to write

$$
\begin{aligned}
d^{3} \mathbf{p}_{f, 1} & =\left|\mathbf{p}_{f, 1}\right|^{2} d\left|\mathbf{p}_{f, 1}\right| d \cos \theta_{f, 1} d \phi_{f, 1} \\
& =\left|\mathbf{p}_{f, 1}\right|^{2} d\left|\mathbf{p}_{f, 1}\right| d \Omega_{f, 1}=\left|\mathbf{p}_{f, 1}\right| E_{f, 1} d E_{f, 1} d \Omega_{f, 1} .
\end{aligned}
$$

The relationship

$$
\frac{d E_{f, 1}}{d\left|\mathbf{p}_{f, 1}\right|}=\frac{d}{d\left|\mathbf{p}_{f, 1}\right|} \sqrt{\left|\mathbf{p}_{f, 1}\right|^{2}+m_{f, 1}^{2}}=\frac{\left|\mathbf{p}_{f, 1}\right|}{\sqrt{\left|\mathbf{p}_{f, 1}\right|^{2}+m_{f, 1}^{2}}}=\frac{\left|\mathbf{p}_{f, 1}\right|}{E_{f, 1}}
$$

was used in last step of eq. (3.52). The infinitesimal solid angle element $d \Omega_{f, 1}$ is defined by

$$
d \Omega_{f, 1} \equiv \sin \theta_{f, 1} d \theta_{f, 1} d \phi_{f, 1}=d \cos \theta_{f, 1} d \phi_{f, 1}
$$


In eq. (3.54), $\theta_{f, 1}$ denotes the polar angle and $\phi_{f, 1}$ denotes the azimuthal angle. These results may be used to rewrite eq. (3.50) as

$$
d \sigma=F^{-1}|\overline{\mathcal{M}}|^{2}(2 \pi) \delta\left(E_{i, 1}+E_{i, 2}-E_{f, 1}-E_{f, 2}\right) \frac{\left|\mathbf{p}_{f, 1}\right|}{4 E_{f, 2}} \frac{d E_{f, 1} d \Omega_{f, 1}}{(2 \pi)^{3}} .
$$

Thus far, this derivation of the differential cross section $d \sigma$ for general $2 \rightarrow 2$ scattering has not assumed any particular frame of reference. At this point, however, it will be convenient to specialize to $2 \rightarrow 2$ scattering in the center-of-momentum (CM) frame, i.e., the frame in which

$$
\mathbf{p}_{i, 1}=-\mathbf{p}_{i, 2} .
$$

In this frame, it is straightforward to show that

$$
E_{x, j}=\frac{s+m_{j}^{2}-m_{k}^{2}}{2 \sqrt{s}} \quad j, k \in\{1,2\}, j \neq k
$$

where the subscript $x \in\{i, f\}$ represents either the initial or final state and $j, k$ are the indices of either the first or second particle in the state of interest. The Mandelstam $s$, the square of the total energy in the CM frame, may be written in the manifestly Lorentz-invariant form

$$
s=\left(p_{i, 1}+p_{i, 2}\right)^{2}=\left(p_{f, 1}+p_{f, 2}\right)^{2} .
$$

Equation (3.57) implies that

$$
E_{f, 2}=\sqrt{E_{f, 1}^{2}-m_{f, 1}^{2}+m_{f, 2}^{2}}
$$

and therefore the delta function that appears in eq. (3.55) may be written in the CM frame as

$$
\begin{aligned}
\delta & \left(E_{i, 1}+E_{i, 2}-E_{f, 1}-E_{f, 2}\right) \\
& =\delta\left(\sqrt{s}-E_{f, 1}-\sqrt{E_{f, 1}^{2}-m_{f, 1}^{2}+m_{f, 2}^{2}}\right) \\
& =\left(1+\frac{E_{f, 1}}{\sqrt{E_{f, 1}^{2}-m_{f, 1}^{2}+m_{f, 2}^{2}}}\right)^{-1} \delta\left(E_{f, 1}-\frac{s+m_{f, 1}^{2}-m_{f, 2}^{2}}{2 \sqrt{s}}\right) \\
& =\frac{E_{f, 2}}{\sqrt{s}} \delta\left(E_{f, 1}-\frac{s+m_{f, 1}^{2}-m_{f, 2}^{2}}{2 \sqrt{s}}\right)
\end{aligned}
$$


The penultimate step above used the identity

$$
\delta(f(x))=\sum_{n} \frac{\delta\left(x-x_{n}\right)}{\left|f^{\prime}\left(x_{n}\right)\right|}
$$

where $x_{n}$ is the $n$th root of $f(x)$. Because the delta function will enforce energy conservation, the prefactor in the last step may be simplified using the result from eq. (3.57). Substituting the final expression from eq. (3.60) into eq. (3.55) and integrating over $E_{f, 1}$ yields

$$
d \sigma=F^{-1}|\overline{\mathcal{M}}|^{2} \frac{\left|\mathbf{p}_{f, 1}\right| d \Omega_{f, 1}}{16 \pi^{2} \sqrt{s}} .
$$

In the CM frame, the Møller flux factor may be written as

$$
F=4\left|\mathbf{p}_{i, 1}\right| \sqrt{s}
$$

which leads to the well-known result

$$
\frac{d \sigma}{d \Omega_{f, 1}}=|\overline{\mathcal{M}}|^{2} \frac{1}{64 \pi^{2} s} \frac{\left|\mathbf{p}_{f, 1}\right|}{\left|\mathbf{p}_{i, 1}\right|} .
$$

If the Lorentz-invariant amplitude has no dependence on the azimuthal angle ${ }^{16} \phi_{f, 1}$, then one may integrate both sides of eq. (3.64) over $\phi_{f, 1} \in[0,2 \pi)$ to obtain

$$
\frac{d \sigma}{d \cos \theta_{f, 1}}=\frac{|\overline{\mathcal{M}}|^{2}}{32 \pi s} \frac{\left|\mathbf{p}_{f, 1}\right|}{\left|\mathbf{p}_{i, 1}\right|} .
$$

This expression will be used to calculate a differential cross section for charged current neutrino-nucleus scattering in section 3.11.

Before examining the free nucleon matrix element $\mathrm{n}^{\mu}$ in the next section, we will pause to derive one additional result that will prove useful while discussing the transition from neutrino-nucleon to neutrino-nucleus scattering.

Consider the Fourier transform $\widetilde{M}$ of the Lorentz invariant amplitude, which is given by

$$
\widetilde{M} \equiv \int e^{i\left(\sum_{j} \mathbf{p}_{i, j}-\sum_{k} \mathbf{p}_{f, k}\right) \cdot \mathbf{x}} \mathcal{M} d^{3} \mathbf{x}
$$

\footnotetext{
${ }^{16}$ This will be the case for all cross sections that are examined in this thesis.
} 
Recall that, in the context of eq. (3.50) through the end of this section, 3-momentum conservation has been enforced by integrating over the spatial delta functions in the Smatrix element. Therefore

$$
\sum_{j} \mathbf{p}_{i, j}-\sum_{k} \mathbf{p}_{f, k}=\mathbf{0}
$$

With that understanding, the definition of $\widetilde{M}$ from eq. (3.66) may be used to write

$$
\widetilde{M}=\int e^{i(\mathbf{0}) \cdot \mathbf{x}} \mathcal{M} d^{3} \mathbf{x}=\mathcal{M} \int d^{3} \mathbf{x}=V \mathcal{M} . \quad \text { (3-momentum conserved, 3.68) }
$$

In the penultimate step above, we have noted that, since the Lorentz-invariant amplitude $\mathcal{M}$ is evaluated in momentum space, it depends on the particle momenta but not on their positions. It may therefore be moved outside of the integral over the spatial coordinates. Equation (3.68) will be used to relate $\mathcal{M}$ to the position-space nucleon wavefunctions in section 3.4 .

\subsection{Free nucleon matrix element calculation}

At the energy scale of interest for this calculation $(\sim 10 \mathrm{MeV})$, the wavefunctions describing individual nucleons within the target nucleus are typically expressed nonrelativistically using two-component Pauli spinors. This is inconvenient because the relativistic expression for the free-nucleon matrix element $\mathrm{n}^{\mu}$ (see eq. (3.30)) uses 4-component Lorentz spinors.

To resolve this mismatch, one may evaluate $\mathrm{n}^{\mu}$ using an explicit representation of the gamma matrices and the Lorentz spinors. Doing so will yield an expression involving Pauli spin matrices and two-component nucleon spinors.

In the Dirac representation, the gamma matrices may be written in $2 \times 2$ block form as

$$
\begin{aligned}
& \gamma^{0}=\left(\begin{array}{cc}
1 & 0 \\
0 & -1
\end{array}\right) \\
& \gamma^{j}=\left(\begin{array}{cc}
0 & \sigma_{j} \\
-\sigma_{j} & 0
\end{array}\right) \quad j \in\{1,2,3\}
\end{aligned}
$$




$$
\gamma^{5} \equiv i \gamma^{0} \gamma^{1} \gamma^{2} \gamma^{3}=\left(\begin{array}{ll}
0 & 1 \\
1 & 0
\end{array}\right)
$$

The Dirac spinor $u_{N}\left(p_{N}\right)$ for a free nucleon $N$ is written in this representation as

$$
u_{N}\left(p_{N}\right)=\sqrt{E_{N}+m_{N}}\left(\begin{array}{c}
\xi_{N} \\
\frac{\left(\mathbf{p}_{N} \cdot \boldsymbol{\sigma}\right)}{E_{N}+m_{N}} \xi_{N}
\end{array}\right)=\sqrt{2 E_{N}} \sqrt{\frac{E_{N}+m_{N}}{2 E_{N}}}\left(\begin{array}{c}
1 \\
\frac{\left(\mathbf{p}_{N} \cdot \boldsymbol{\sigma}\right)}{E_{N}+m_{N}}
\end{array}\right) \xi_{N}
$$

where $\xi_{N}$ is a two-component Pauli spinor. In the last step above, a factor of $\sqrt{2 E_{N}}$ has been extracted from the overall normalization constant for later convenience. Note that, with the normalization chosen in eq. (3.72) for the Dirac spinors,

$$
u\left(p_{N}\right)^{\dagger} u\left(p_{N}\right)=2 E_{N} \xi_{N}^{\dagger} \xi_{N}
$$

and

$$
\sum_{s=\downarrow, \uparrow} \bar{u}^{(s)}\left(p_{N}\right) u^{(s)}\left(p_{N}\right)=\not p_{N}+m_{N}=\gamma_{\mu} p_{N}^{\mu}+m_{N}
$$

where the sum is over the nucleon's two possible spin orientations. By plugging the explicit matrix representations above into eq. (3.30), one finds that the four components of the nucleon matrix element are given by

$$
\begin{array}{ll}
\mathrm{n}^{0}=\left(\sqrt{2 E_{N_{i}}} \sqrt{2 E_{N_{f}}}\right) \xi_{N_{f}}^{\dagger} \chi_{N_{f}}^{\dagger} \mathrm{j}^{0} \xi_{N_{i}} \chi_{N_{i}} & \\
\mathrm{n}^{b}=\left(\sqrt{2 E_{N_{i}}} \sqrt{2 E_{N_{f}}}\right) \xi_{N_{f}}^{\dagger} \chi_{N_{f}}^{\dagger} \mathrm{j}^{b} \xi_{N_{i}} \chi_{N_{i}} \quad b \in\{1,2,3\} .
\end{array}
$$

In the equations above, the free nucleon transition operator $\mathrm{j}^{\mu}$ has the time component

$$
\begin{gathered}
\mathrm{j}^{0}=\left(\sqrt{\frac{E_{N_{i}}+m_{N}}{2 E_{N_{i}}}} \sqrt{\left.\frac{E_{N_{i}}+m_{N}+q^{0}}{2\left(E_{N_{i}}+q^{0}\right)}\right)\left(F_{1}\left(Q^{2}\right)-\frac{(\mathbf{q} \cdot \boldsymbol{\sigma})}{m_{N}} G_{3}\left(Q^{2}\right)+\frac{q^{0}}{m_{N}} F_{3}\left(Q^{2}\right)\right.}\right. \\
+\frac{\left(\mathbf{p}_{N_{i}}+\mathbf{q}\right) \cdot \boldsymbol{\sigma}}{E_{N_{i}}+m_{N}+q^{0}}\left[\frac{\mathbf{q} \cdot \boldsymbol{\sigma}}{2 m_{N}} F_{2}\left(Q^{2}\right)-\frac{q^{0}}{m_{N}} F_{3}\left(Q^{2}\right)-G_{A}\left(Q^{2}\right)+\frac{q^{0}}{m_{N}} G_{P}\left(Q^{2}\right)\right] \\
+\left[\frac{q^{0}}{m_{N}} F_{3}\left(Q^{2}\right)-\frac{\mathbf{q} \cdot \boldsymbol{\sigma}}{2 m_{N}} F_{2}\left(Q^{2}\right)-G_{A}\left(Q^{2}\right)-\frac{q^{0}}{m_{N}} G_{P}\left(Q^{2}\right)\right] \frac{\mathbf{p}_{N_{i}} \cdot \boldsymbol{\sigma}}{E_{N_{i}}+m_{N}} \\
\left.+\frac{\left(\mathbf{p}_{N_{i}}+\mathbf{q}\right) \cdot \boldsymbol{\sigma}}{E_{N_{i}}+m_{N}+q^{0}}\left[F_{1}\left(Q^{2}\right)+\frac{\mathbf{q} \cdot \boldsymbol{\sigma}}{m_{N}} G_{3}\left(Q^{2}\right)-\frac{q^{0}}{m_{N}} F_{3}\left(Q^{2}\right)\right] \frac{\mathbf{p}_{N_{i}} \cdot \boldsymbol{\sigma}}{E_{N_{i}}+m_{N}}\right) t_{-}
\end{gathered}
$$


and the spatial components

$$
\begin{aligned}
\mathrm{j}^{b}= & \left(\sqrt{\frac{E_{N_{i}}+m_{N}}{2 E_{N_{i}}}} \sqrt{\frac{E_{N_{i}}+m_{N}+q^{0}}{2\left(E_{N_{i}}+q^{0}\right)}}\right)\left(-\sigma^{b} G_{A}\left(Q^{2}\right)+\frac{q^{b}}{m_{N}} F_{3}\left(Q^{2}\right)+\frac{q^{0} \sigma^{b}}{m_{N}} G_{3}\left(Q^{2}\right)\right. \\
& -\frac{i(\boldsymbol{\sigma} \times \mathbf{q})^{b}}{2 m_{N}} F_{2}\left(Q^{2}\right)+\frac{\left(\mathbf{p}_{N_{i}}+\mathbf{q}\right) \cdot \boldsymbol{\sigma}}{E_{N_{i}}+m_{N}+q^{0}}\left[\frac{q^{b}}{m_{N}}\left(G_{P}\left(Q^{2}\right)-F_{3}\left(Q^{2}\right)\right)+\sigma^{b} F_{1}\left(Q^{2}\right)\right. \\
& \left.-\frac{q^{0} \sigma^{b}}{2 m_{N}} F_{2}\left(Q^{2}\right)+\frac{i(\boldsymbol{\sigma} \times \mathbf{q})^{b}}{m_{N}} G_{3}\left(Q^{2}\right)\right]+\left[\sigma^{b} F_{1}\left(Q^{2}\right)+\frac{q^{0} \sigma^{b}}{2 m_{N}} F_{2}\left(Q^{2}\right)\right. \\
& \left.+\frac{q^{b}}{m_{N}}\left(F_{3}\left(Q^{2}\right)-G_{P}\left(Q^{2}\right)\right)-\frac{i(\boldsymbol{\sigma} \times \mathbf{q})^{b}}{m_{N}} G_{3}\left(Q^{2}\right)\right] \frac{\mathbf{p}_{N_{i}} \cdot \boldsymbol{\sigma}}{E_{N_{i}}+m_{N}} \\
& +\frac{\left(\mathbf{p}_{N_{i}}+\mathbf{q}\right) \cdot \boldsymbol{\sigma}}{E_{N_{i}}+m_{N}+q^{0}}\left[\frac{i(\boldsymbol{\sigma} \times \mathbf{q})^{b}}{2 m_{N}} F_{2}\left(Q^{2}\right)-\frac{q^{b}}{m_{N}} F_{3}\left(Q^{2}\right)\right. \\
& \left.\left.-\sigma^{b} G_{A}\left(Q^{2}\right)-\frac{q^{0} \sigma^{b}}{m_{N}} G_{3}\left(Q^{2}\right)\right] \frac{\mathbf{p}_{N_{i}} \cdot \boldsymbol{\sigma}}{E_{N_{i}}+m_{N}}\right) t_{-} .
\end{aligned}
$$

Here $(\boldsymbol{\sigma} \times \mathbf{q})^{b}$ is the $b$ th component of the cross product of $\boldsymbol{\sigma}$ and $\mathbf{q}$, e.g., in Cartesian coordinates

$$
(\boldsymbol{\sigma} \times \mathbf{q})^{1}=\sigma^{2} q^{3}-\sigma^{3} q^{2} .
$$

In the expressions above, the relations

$$
E_{N_{f}}=E_{N_{i}}+q^{0}
$$

and

$$
\mathbf{p}_{N_{f}}=\mathbf{p}_{N_{i}}+\mathbf{q}
$$

have been used to write the expressions in terms of the momentum transfer four-vector $q^{\mu} \equiv p_{N_{f}}^{\mu}-p_{N_{i}}^{\mu}$ and the initial nucleon energy $E_{N_{i}}$ and momentum $\mathbf{p}_{N_{i}}$.

Now consider the Fourier transform $\widetilde{M}$ of the Lorentz invariant matrix element $\mathcal{M}$ for charged current neutrino-nucleon scattering. Equations (3.10) and (3.66) show that it is 
given by

$$
\begin{aligned}
\widetilde{M} & =\int d^{3} \mathbf{x} e^{i\left(\mathbf{p}_{\nu_{\ell}}+\mathbf{p}_{N_{i}}-\mathbf{p}_{\ell}-\mathbf{p}_{N_{f}}\right) \cdot \mathbf{x}} \mathcal{M} \\
& =-\frac{G_{F} V_{u d}}{\sqrt{2}} \ell_{\mu} \int d^{3} \mathbf{x} e^{-i \mathbf{p}_{N_{f}} \cdot \mathbf{x}} e^{i \mathbf{q} \cdot \mathbf{x}} n^{\mu} e^{i \mathbf{p}_{N_{i}} \cdot \mathbf{x}} .
\end{aligned}
$$

In the second step, 3-momentum conservation has been invoked to determine that

$$
\mathbf{q} \equiv \mathbf{p}_{N_{f}}-\mathbf{p}_{N_{i}}=\mathbf{p}_{\nu_{\ell}}-\mathbf{p}_{\ell}
$$

Referring to eqs. (3.75) and (3.76) allows one to see that the integrand above may be written as

$$
\begin{aligned}
& e^{-i \mathbf{p}_{N_{f}} \cdot \mathbf{x}} e^{i \mathbf{q} \cdot \mathbf{x}} n^{\mu} e^{i \mathbf{p}_{N_{i}} \cdot \mathbf{x}} \\
& \quad=\sqrt{2 E_{N_{i}}} \sqrt{2 E_{N_{f}}} \xi_{N_{f}}^{\dagger} \chi_{N_{f}}^{\dagger} e^{-i \mathbf{p}_{N_{f}} \cdot \mathbf{x}} e^{i \mathbf{q} \cdot \mathbf{x}} \mathrm{j}^{\mu} \xi_{N_{i}} \chi_{N_{i}} e^{i \mathbf{p}_{N_{i}} \cdot \mathbf{x}} \\
& \quad=V \sqrt{2 E_{N_{i}}} \sqrt{2 E_{N_{f}}}\left(\frac{1}{\sqrt{V}} \xi_{N_{f}}^{\dagger} \chi_{N_{f}}^{\dagger} e^{-i \mathbf{p}_{N_{f}} \cdot \mathbf{x}}\right) e^{i \mathbf{q} \cdot \mathbf{x}} \mathrm{j}^{\mu}\left(\frac{1}{\sqrt{V}} \xi_{N_{i}} \chi_{N_{i}} e^{i \mathbf{p}_{N_{i}} \cdot \mathbf{x}}\right) .
\end{aligned}
$$

Recognizing the quantities in parentheses as the nonrelativistic position-space wavefunctions (including the spin and isospin dependence) for a free nucleon in the final and initial states allows one to cast eq. (3.82) in the form

$$
\widetilde{M}=-V \frac{G_{F} V_{u d}}{\sqrt{2}} \sqrt{2 E_{N_{i}}} \sqrt{2 E_{N_{f}}} \ell_{\mu}\left\langle N_{f}\left|\hat{\mathcal{O}}^{\mu}\right| N_{i}\right\rangle .
$$

Here the position-space free nucleon transition operator

$$
\hat{\mathcal{O}}^{\mu} \equiv e^{i \mathbf{q} \cdot \mathbf{x}} \hat{\mathrm{j}}^{\mu}
$$

is marked with a hat to indicate that the 4-momenta $p_{N_{i}}$ and $q=p_{N_{f}}-p_{N_{i}}$ appearing in eqs. (3.77) and (3.78) should now be interpreted as operators acting on the position-space wavefunctions ${ }^{17}$ for the initial and final nucleon states. Explicitly, we have

$$
\mathbf{p}_{N_{i}}=-i \nabla_{i}
$$

\footnotetext{
${ }^{17}$ The momentum operators should not, however, act on the factor of $e^{i \mathbf{q} \cdot \mathbf{x}}$ that appears in the definition of $\hat{\mathcal{O}}^{\mu}$.
} 
where the $i$ in the coefficient is the imaginary unit, while the $i$ in the subscript indicates that the operator acts on the initial nucleon wavefunction,

$$
\begin{aligned}
\mathbf{p}_{N_{f}} & =-i \nabla_{f}, \\
\mathbf{q} & =\mathbf{p}_{N_{f}}-\mathbf{p}_{N_{i}}=-i\left(\nabla_{f}-\nabla_{i}\right),
\end{aligned}
$$

and, for nonrelativistic free nucleons,

$$
q^{0}=\frac{\mathbf{p}_{N_{f}}^{2}-\mathbf{p}_{N_{i}}^{2}}{2 m_{N}}=\frac{\nabla_{i}^{2}-\nabla_{f}^{2}}{2 m_{N}} .
$$

Because the wavefunctions describing the free nucleon states are simple plane waves $e^{i \mathbf{p} \cdot \mathbf{x}}$ with definite momenta, replacing these operators with their eigenvalues will yield identical results at this point in the derivation. The distinction becomes important in section 3.5 during the transition from neutrino-nucleon to neutrino-nucleus scattering.

Note that the nonrelativistic nucleon wavefunctions are normalized to unity:

$$
\begin{aligned}
\langle N \mid N\rangle & =\int\left(\frac{1}{\sqrt{V}} \xi_{N}^{\dagger} \chi_{N}^{\dagger} e^{-i \mathbf{p}_{N} \cdot \mathbf{x}}\right)\left(\frac{1}{\sqrt{V}} \xi_{N} \chi_{N} e^{i \mathbf{p}_{N} \cdot \mathbf{x}}\right) d^{3} \mathbf{x} \\
& =\xi_{N}^{\dagger} \xi_{N} \chi_{N}^{\dagger} \chi_{N} V^{-1} \int d^{3} \mathbf{x}=1 .
\end{aligned}
$$

It follows from 3-momentum conservation (which will be explicitly enforced in any cross section calculation) and eqs. (3.68) and (3.85) that

$$
\mathcal{M}=-\frac{G_{F} V_{u d}}{\sqrt{2}} \sqrt{2 E_{N_{i}}} \sqrt{2 E_{N_{f}}} \ell_{\mu}\left\langle N_{f}\left|\hat{\mathcal{O}}^{\mu}\right| N_{i}\right\rangle
$$

From this result and eq. (3.10), it also follows that

$$
\mathrm{n}^{\mu}=\sqrt{2 E_{N_{i}}} \sqrt{2 E_{N_{f}}}\left\langle N_{f}\left|\hat{\mathcal{O}}^{\mu}\right| N_{i}\right\rangle
$$

\subsection{Transition to neutrino-nucleus scattering}

To further generalize to charged current neutrino scattering on a nucleus instead of a single nucleon, the free nucleon matrix element $\mathrm{n}^{\mu}$ must be replaced with a nuclear matrix element $\mathcal{N}^{\mu}$ representing the contributions of all nucleons to the amplitude. Equation (3.93) 
makes it easy to anticipate the needed expression for the nuclear matrix element:

$$
\begin{aligned}
\mathcal{N}^{\mu} \equiv \sqrt{2 E_{i}} \sqrt{2 E_{f}}\left\langle f\left|\hat{\mathcal{N}}^{\mu}\right| i\right\rangle & =\sqrt{2 E_{i}} \sqrt{2 E_{f}}\left\langle J_{f} M_{f}\left|\hat{\mathcal{N}}^{\mu}\right| J_{i} M_{i}\right\rangle \\
\mathrm{n}^{\mu} & \rightarrow \mathcal{N}^{\mu}
\end{aligned}
$$

In the second expression above for $\mathcal{N}^{\mu}$, the initial $(i)$ and final $(f)$ nuclear states are labeled with quantum numbers representing the total spin $J$ and the helicity $M$. The other quantum numbers needed to fully specify these states (e.g., the total isospin T) will not be referred to explicitly in the following discussion. Like the free nucleon state vectors in eq. (3.85), the nuclear state vectors represent nonrelativistic position-space wavefunctions normalized to unity:

$$
\langle i \mid i\rangle=\langle f \mid f\rangle=1
$$

Substituting the full nuclear matrix element $\mathcal{N}^{\mu}$ into eq. (3.10) yields the diagram

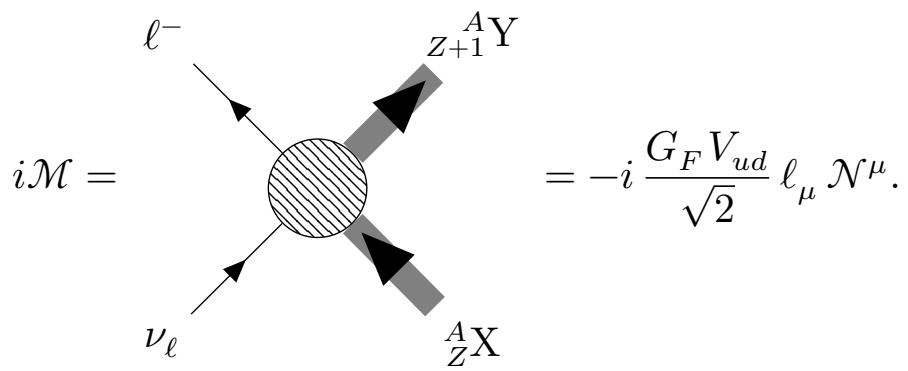

To calculate the nuclear matrix element $\mathcal{N}^{\mu}$, we rely on the impulse approximation, in which the target nucleus is seen as a collection of individual nucleons. Under this approximation, the nuclear current operator $\hat{\mathcal{N}}^{\mu}$ may be written as the sum of singlenucleon contributions, i.e.,

$$
\begin{aligned}
\hat{\mathcal{N}}^{\mu} & \approx \sum_{k=1}^{A} \hat{\mathcal{O}}^{\mu}(k)=\sum_{k=1}^{A} e^{i \mathbf{q} \cdot \mathbf{x}_{k}} \hat{\mathrm{j}}^{\mu}(k) \\
& =\sum_{N_{f}} \sum_{N_{i}}\left\langle N_{f}\left|\hat{\mathcal{O}}^{\mu}\right| N_{i}\right\rangle a_{N_{f}}^{\dagger} a_{N_{i}}
\end{aligned}
$$

where the first line gives the position-space representation, and the second gives the equivalent occupation number representation [260]. Here, the sums over $N_{i}$ and $N_{f}$ include 
complete sets of single-nucleon states, the single-nucleon operator $\hat{\mathcal{O}}^{\mu}(k)$ acts on the $k$ th nucleon's state vector, and $a_{N}^{\dagger}\left(a_{N}\right)$ creates (destroys) a nucleon in the state $N$. In diagrammatic form, this approximation may be stated as

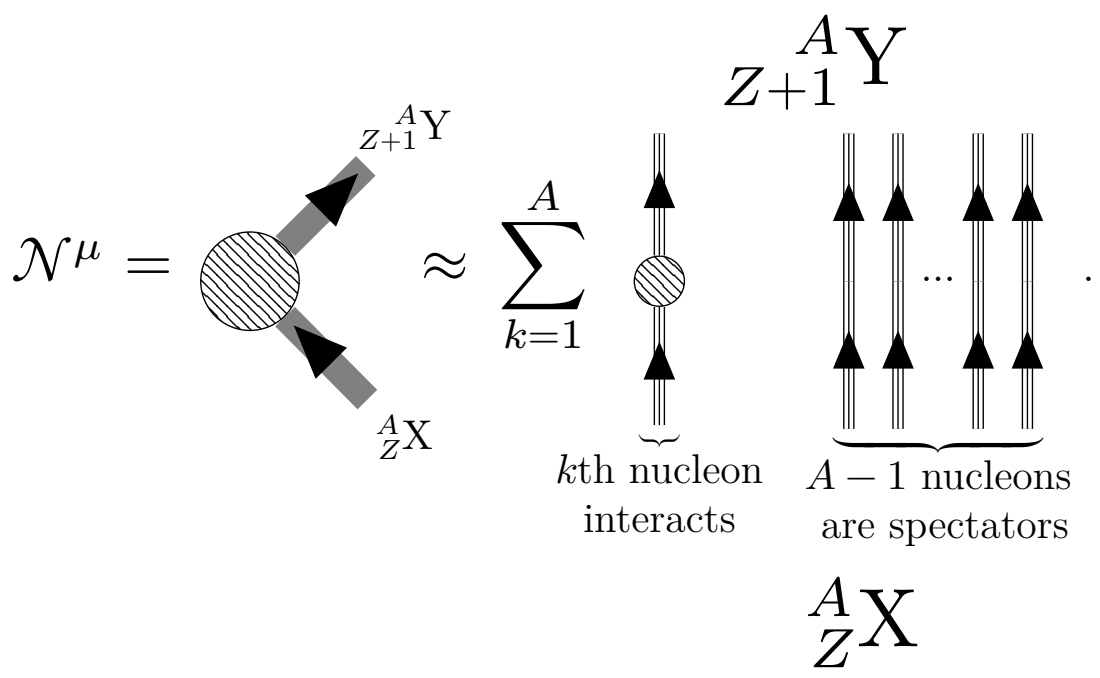

Although a naïve application of the impulse approximation would treat the nucleons as fully noninteracting, such an approach would be far from satisfactory, as they are subject to strong interactions within the nuclear environment. A standard way of correcting the impulse approximation to account for the internucleon forces is to use the positionspace free nucleon transition operator $\hat{\mathcal{O}}^{\mu}(k)$ together with bound nucleon wavefunctions calculated using a suitable model for the nuclear potential. In this way, nuclear effects may be taken into account while still neglecting the two-, three-, and many-body terms that are ignored in the impulse approximation's simple sum over individual nucleons [264].

When applying the operator $q^{0}$ to bound nucleon wavefunctions, however, because the nucleons are off their mass shell, ${ }^{18}$ it becomes unclear [231] whether one should use nucleon kinematics (the operator definition in eq. (3.90)) or lepton kinematics, i.e., the non-operator value

$$
q^{0}=E_{\nu_{\ell}}-E_{\ell}
$$

known from the leptonic part of the problem via conservation of energy. For the present calculation, we will choose to use lepton kinematics, noting that differences between ob-

\footnotetext{
${ }^{18} \mathrm{~A}$ nucleon with total energy $E_{N}$ and 3-momentum $\mathbf{p}_{N}$ is considered off-shell if $E_{N} \neq \sqrt{\left|\mathbf{p}_{N}\right|^{2}-m_{N}^{2}}$. The inequality holds for bound nucleons due to the presence of the nuclear potential.
} 
servables calculated using the two approaches are typically small [231]. The initial nucleon momentum will continue to be treated as an operator (see eq. (3.87)).

It should be pointed out that, as long as plane-wave wavefunctions are used for the initial and final leptons, there is no similar nucleon vs. lepton kinematics ambiguity for the 3-momentum transfer $\mathbf{q}$. This is because, when choosing to work with nucleon kinematics, one may use partial integration to move the gradient operators from the nucleon wavefunctions to the lepton wavefunctions, yielding a result for the 3-momentum transfer that is identical to its value under lepton kinematics [231].

As discussed in section 3.3, to determine the differential cross section for charged current neutrino-nucleus scattering, one must evaluate the absolute square of the amplitude $\mathcal{M}$, summing or averaging over unobserved spins (helicities) as appropriate. Because only left-handed neutrinos (and right-handed antineutrinos) participate in weak interactions, and because neutrinos are approximated as massless in the Standard Model, the neutrino helicity is known a priori. An average should not, therefore, be computed for the initial neutrino spin orientations, although one is free to sum over them since the projection operator $1-\gamma_{5}$ that appears in $\ell_{\mu}$ will ensure that only contributions from the appropriate helicity state survive. Averaging over the unknown initial nuclear spin and summing over the final particle spins gives

$$
|\overline{\mathcal{M}}|^{2}=\frac{G_{F}{ }^{2}\left|V_{u d}\right|^{2}}{2 J_{i}+1} \sum_{M_{i}} \sum_{M_{f}} \mathcal{N}^{\mu} \mathcal{N}^{\nu *} \mathrm{~L}_{\mu \nu}=4 E_{i} E_{f} \frac{G_{F}{ }^{2}\left|V_{u d}\right|^{2}}{2 J_{i}+1}\left|\overline{\mathcal{T}_{f i}}\right|^{2} .
$$

Here the lepton tensor $\mathrm{L}_{\mu \nu}$ for the neutrino charged current interaction has been defined by

$$
\mathrm{L}_{\mu \nu} \equiv \frac{1}{2} \sum_{h_{\nu_{\ell}}} \sum_{h_{\ell}} \ell_{\mu} \ell_{\nu}^{*}
$$

where $h_{\nu_{\ell}}$ is the neutrino helicity, $h_{\ell}$ is the same for the charged lepton, and the factor of $1 / 2$ comes from the absolute square of the coupling constant factor $G_{F} V_{u d} / \sqrt{2}$ that appears in eq. (3.95), not an average over the incident neutrino helicities, as discussed above. The quantity $\left|\overline{\mathcal{T}_{f i}}\right|^{2}$ is defined by

$$
\left|\overline{\mathcal{T}}_{f i}\right|^{2} \equiv \sum_{M_{i}} \sum_{M_{f}} O^{\mu} O^{\nu *} \mathrm{~L}_{\mu \nu}
$$


where

$$
O^{\mu} \equiv\left\langle J_{f} M_{f}\left|\hat{\mathcal{N}}^{\mu}\right| J_{i} M_{f}\right\rangle=\left(2 E_{i}\right)^{-1 / 2}\left(2 E_{f}\right)^{-1 / 2} \mathcal{N}^{\mu}
$$

\subsection{Charged current lepton tensor}

The lepton tensor $\mathrm{L}_{\mu \nu}$ defined in eq. (3.101) may expressed in terms of the four-momenta of the neutrino $\left(p_{\nu_{\ell}}^{\mu}\right)$ and charged lepton $\left(p_{\ell}^{\mu}\right)$ as

$$
\mathrm{L}_{\mu \nu}=4\left[p_{\ell, \mu} p_{\nu_{\ell}, \nu}+p_{\ell, \nu} p_{\nu_{\ell}, \mu}-g_{\mu \nu}\left(p_{\ell} \cdot p_{\nu_{\ell}}\right)-i \epsilon_{\mu \nu \rho \sigma} p_{\ell}^{\rho} p_{\nu_{\ell}}^{\sigma}\right]
$$

Equation (3.104) provides an expression for the lepton tensor in terms of Cartesian components of the four-vectors, i.e., $\mu, \nu \in\{0,1,2,3\}$, with

$$
\begin{array}{ll}
p_{\ell, 0}=p_{\ell, t}=E_{\ell} & p_{\nu_{\ell}, 0}=p_{\nu_{\ell}, t}=E_{\nu_{\ell}} \\
p_{\ell, 1}=p_{\ell, x} & p_{\nu_{\ell}, 1}=p_{\nu_{\ell}, x} \\
p_{\ell, 2}=p_{\ell, y} & p_{\nu_{\ell}, 2}=p_{\nu_{\ell}, y} \\
p_{\ell, 3}=p_{\ell, z} & p_{\nu_{\ell}, 3}=p_{\nu_{\ell}, z} .
\end{array}
$$

In this basis the metric tensor ${ }^{19} g_{\mu \nu}$ is given by

$$
g_{\mu \nu}=\delta_{\mu 0} \delta_{\nu 0}-\delta_{\mu 1} \delta_{\nu 1}-\delta_{\mu 2} \delta_{\nu 2}-\delta_{\mu 3} \delta_{\nu 3}=\left(\begin{array}{cccc}
1 & 0 & 0 & 0 \\
0 & -1 & 0 & 0 \\
0 & 0 & -1 & 0 \\
0 & 0 & 0 & -1
\end{array}\right)_{\mu=3}^{\mu=0}{ }_{\mu=1}^{\mu=0}
$$

where the Kronecker delta $\delta_{\mu \nu}$ is defined by

$$
\delta_{\mu \nu}=\left\{\begin{array}{ll}
0 & \mu \neq \nu \\
1 & \mu=\nu
\end{array} .\right.
$$

In section 3.8, it will be convenient to work in spherical coordinates. In anticipation of those future calculations, this section will provide expressions for the spherical components

\footnotetext{
${ }^{19}$ The "West Coast" sign convention (+ - - ) for the Cartesian metric is used throughout this thesis.
} 
of the lepton tensor L. To determine these expressions, first define the spherical unit vectors $^{20}$

$$
\hat{\mathbf{e}}_{\not \phi} \equiv \hat{\mathbf{e}}_{t} \quad \hat{\mathbf{e}}_{0} \equiv \hat{\mathbf{e}}_{z} \quad \hat{\mathbf{e}}_{ \pm 1} \equiv \mp \frac{1}{\sqrt{2}}\left(\hat{\mathbf{e}}_{x} \pm i \hat{\mathbf{e}}_{y}\right)
$$

where $\left\{\hat{\mathbf{e}}_{t}, \hat{\mathbf{e}}_{x}, \hat{\mathbf{e}}_{y}, \hat{\mathbf{e}}_{z}\right\}$ is the standard orthonormal Cartesian basis for four-vectors. Then the set $\left\{\hat{\mathbf{e}}_{\phi}, \hat{\mathbf{e}}_{-1}, \hat{\mathbf{e}}_{0}, \hat{\mathbf{e}}_{1}\right\}$ is an orthonormal basis for four-vectors with metric tensor

$$
g_{\mu \nu}=\delta_{\mu \phi} \delta_{\nu \phi}+\delta_{\mu,-1} \delta_{\nu 1}-\delta_{\mu 0} \delta_{\nu 0}+\delta_{\mu 1} \delta_{\nu,-1}=\left(\begin{array}{cccc}
1 & 0 & 0 & 0 \\
0 & 0 & 0 & 1 \\
0 & 0 & -1 & 0 \\
0 & 1 & 0 & 0
\end{array}\right)_{\mu=1}^{\mu=\varnothing}{ }_{\mu=-1}^{\mu=0}=g^{\mu \nu} .
$$

In this basis, the dot product of two four-vectors $A^{\mu}$ and $B^{\mu}$ may be written as

$$
\begin{aligned}
A \cdot B=A^{\mu} B_{\mu}=g^{\mu \nu} A_{\nu} B_{\mu} & =A_{\phi} B_{\varnothing}+A_{-1} B_{1}-A_{0} B_{0}+A_{1} B_{-1} \\
& =A_{\phi} B_{\varnothing}-\sum_{M}(-1)^{M} A_{M} B_{-M} \\
& =A_{\varnothing} B_{\varnothing}-A^{M} B_{M}=A_{\phi} B_{\varnothing}-\mathbf{A} \cdot \mathbf{B},
\end{aligned}
$$

where the explicit sum is over $M \in\{-1,0,1\}$. The definitions of the spherical basis vectors given in eq. (3.108) imply that the Cartesian components of an arbitrary fourvector $A_{\mu}$ may be written in terms of the spherical components via the relations

$$
A_{t}=A_{\varnothing} \quad A_{x}=\frac{1}{\sqrt{2}}\left(A_{-1}-A_{1}\right) \quad A_{y}=\frac{i}{\sqrt{2}}\left(A_{-1}+A_{1}\right) \quad A_{z}=A_{0} .
$$

Likewise, the spherical components may be written in terms of the Cartesian components via

$$
A_{\varnothing}=A_{t} \quad A_{0}=A_{z} \quad A_{ \pm 1}=\mp \frac{1}{\sqrt{2}}\left(A_{x} \pm i A_{y}\right) .
$$

Recall the definition of $L$ in eq. (3.101), and consider as an example the spherical component

$$
\mathrm{L}_{\phi 1}=\frac{1}{2} \sum_{m_{\nu_{\ell}}} \sum_{m_{\ell}} \ell_{\phi} \ell_{1}^{*}
$$

\footnotetext{
${ }^{20}$ The time index in the spherical basis is denoted by $\emptyset$. This notation is due to Krmpotić et al. [242].
} 
The substitutions shown in eq. (3.112) allow one to rewrite the right-hand side of eq. (3.113) in terms of the Cartesian components of $\ell_{\mu}$, to wit:

$$
\begin{aligned}
\mathrm{L}_{01} & =\frac{1}{2} \sum_{m_{\nu_{\ell}}} \sum_{m_{\ell}} \ell_{z}\left(-\frac{1}{\sqrt{2}}\left[\ell_{x}+i \ell_{y}\right]\right)^{*} \\
& =-\frac{1}{\sqrt{2}}\left[\frac{1}{2} \sum_{m_{\nu_{\ell}}} \sum_{m_{\ell}}\left(\ell_{z} \ell_{x}^{*}-i \ell_{z} \ell_{y}^{*}\right)\right] \\
& =\frac{1}{\sqrt{2}}\left(i \mathrm{~L}_{z y}-\mathrm{L}_{z x}\right) .
\end{aligned}
$$

Substituting the explicit Cartesian expressions from eq. (3.104) into the equation above gives

$$
\mathrm{L}_{01}=2 \sqrt{2}\left[\left(p_{\ell, x}-i p_{\ell, y}\right)\left(p_{\nu_{\ell}, t}-p_{\nu_{\ell}, z}\right)-\left(p_{\ell, t}+p_{\ell, z}\right)\left(p_{\nu_{\ell}, x}+i p_{\nu_{\ell}, y}\right)\right] .
$$

Using eq. (3.111), this expression may in turn be rewritten in terms of the spherical components of $p_{\ell}$ and $p_{\nu_{\ell}}$, yielding

$$
\begin{aligned}
\mathrm{L}_{01} & =4\left(p_{\ell,-1} p_{\nu_{\ell}, \varnothing}-p_{\ell, \varnothing} p_{\nu_{\ell},-1}-p_{\ell, 0} p_{\nu_{\ell},-1}-p_{\ell,-1} p_{\nu_{\ell}, 0}\right) \\
& =4\left(p_{\ell, \varnothing} p_{\nu_{\ell}, 1}^{*}-p_{\ell, 1}^{*} p_{\nu_{\ell}, \varnothing}+p_{\ell, 0} p_{\nu_{\ell}, 1}^{*}+p_{\ell, 1}^{*} p_{\nu_{\ell}, 0}\right) .
\end{aligned}
$$

The identity

$$
A_{ \pm 1}=-A_{\mp 1}^{*} \quad A_{x}, A_{y} \in \mathbb{R}
$$

was used to obtain the second line above. It applies because the Cartesian components of the four-momenta $p_{\ell}$ and $p_{\nu_{\ell}}$ are real.

A similar procedure can be applied to obtain the following expressions [242] for all of the spherical components of $\mathrm{L}$ :

$$
\begin{aligned}
\mathrm{L}_{\phi \emptyset}= & 4\left(E_{\ell} E_{\nu_{\ell}}+p_{\ell}^{M} p_{\nu_{\ell}, M}\right)=4\left(E_{\ell} E_{\nu_{\ell}}+\mathbf{p}_{\ell} \cdot \mathbf{p}_{\nu_{\ell}}\right) \\
\mathrm{L}_{M \emptyset}=4 & \left(E_{\ell} p_{\nu_{\ell}, M}+E_{\nu_{\ell}} p_{\ell, M}+i\left[\mathbf{p}_{\ell} \times \mathbf{p}_{\nu_{\ell}}\right]_{M}\right)=\mathrm{L}_{\phi M}^{*} \\
\mathrm{~L}_{M N}=4 & {\left[\delta_{M N}\left(p_{\ell} \cdot p_{\nu_{\ell}}\right)+p_{\ell, M} p_{\nu_{\ell}, N}^{*}+p_{\ell, N}^{*} p_{\nu_{\ell}, M}\right.} \\
& \left.+K_{M N}\left(E_{\ell} p_{\nu_{\ell}, N-M}^{*}-E_{\nu_{\ell}} p_{\ell, N-M}^{*}\right)\right]
\end{aligned}
$$


The symbol $K_{M N}$ (which appears in eq. (3.120)) is defined by

$$
K_{M N} \equiv \sqrt{6}(-1)^{N+1}\left(\begin{array}{ccc}
1 & 1 & 1 \\
-N & M & N-M
\end{array}\right)=\left(\begin{array}{ccc}
1 & 1 & 0 \\
-1 & 0 & 1 \\
0 & -1 & -1
\end{array}\right)_{M=1}^{M=-1}
$$

where the first definition is given in terms of the Wigner 3 -j symbol and the second uses an ordinary matrix.

\subsection{Charged current nucleon form factors}

The form factors $F_{1}\left(Q^{2}\right), G_{A}\left(Q^{2}\right)$, etc. that appear in eqs. (3.30), (3.77) and (3.78) represent corrections to the free nucleon current that arise because the nucleon has a finite spatial extent rather than being pointlike. Factorizing scattering amplitudes into a value for a pointlike target and a form factor that corrects for a nonzero target size is a common technique in both classical and quantum mechanical scattering problems [265].

In the case of the nucleon form factors of interest for charged current neutrino scattering, it can be shown [59] that, in the limit of perfect isospin invariance, ${ }^{21}$ the form factors associated with second-class currents ${ }^{22}$ must vanish:

$$
F_{3}\left(Q^{2}\right) \approx 0 \quad G_{3}\left(Q^{2}\right) \approx 0
$$

Experimental searches for these second-class currents have confirmed their absence at the level of about $5-10 \%$ of weak magnetism (the $F_{2}\left(Q^{2}\right)$ term) [268]. The remaining vector current form factors $F_{1}\left(Q^{2}\right)$ and $F_{2}\left(Q^{2}\right)$ have been measured using electron scattering experiments (see reference [269] for a detailed review).

Measurements of the axial-vector current form factors $G_{A}\left(Q^{2}\right)$ and $G_{P}\left(Q^{2}\right)$ are more limited $[270,271]$. Current experimental knowledge of the axial-vector form factor $G_{A}\left(Q^{2}\right)$

\footnotetext{
${ }^{21}$ Isospin is an approximate symmetry. Corrections for imperfect isospin invariance are of order $\Delta / m_{N}$, where $\Delta=m_{n}-m_{p}$ is the difference between the neutron and proton masses [266].

${ }^{22}$ This classification is due to Weinberg [267] and refers to the G-parity of the current. The firstclass vector currents have G-parity +1 , and the first-class axial-vector currents have G-parity -1 . The second-class currents have opposite signs for their G-parity eigenvalues.
} 
comes from neutron beta decay, neutrino scattering, pion electroproduction, ${ }^{23}$ and muon capture experiments [272], the last of these also being the primary source for measurements of the pseudoscalar form factor $G_{P}\left(Q^{2}\right)[271,273]$. Our limited understanding of the axial weak form factors of the nucleon is a concern for future precision neutrino experiments, although a high-statistics neutrino measurement using a hydrogen or deuterium target may improve the existing constraints $[178,179]$.

While more sophisticated treatments of the nucleon form factors exist in the literature (e.g., [274]), one commonly-used parameterization assumes that the $Q^{2}$ dependence of the form factors can be described by the dipole shape

$$
G_{D}\left(Q^{2}, M\right)=\left(1+\frac{Q^{2}}{M^{2}}\right)^{-2},
$$

where $M$ is an empirically-determined parameter. Under this assumption, the form factors may be written as $[59,275,276]$

$$
\begin{aligned}
& F_{1}^{n}\left(Q^{2}\right)=\frac{\frac{\mu_{n}}{\mu_{N}} Q^{2}}{4 m_{N}^{2}+Q^{2}} G_{D}\left(Q^{2}, M_{V}\right) \\
& F_{1}^{p}\left(Q^{2}\right)=\frac{4 m_{N}^{2}+\frac{\mu_{p}}{\mu_{N}} Q^{2}}{4 m_{N}^{2}+Q^{2}} G_{D}\left(Q^{2}, M_{V}\right) \\
& F_{1}\left(Q^{2}\right)=F_{1}^{p}\left(Q^{2}\right)-F_{1}^{n}\left(Q^{2}\right)=\frac{4 m_{N}^{2}+\left(\frac{\mu_{p}-\mu_{n}}{\mu_{N}}\right) Q^{2}}{4 m_{N}^{2}+Q^{2}} G_{D}\left(Q^{2}, M_{V}\right) \\
& F_{2}^{n}\left(Q^{2}\right)=\frac{4 m_{N}^{2} \frac{\mu_{n}}{\mu_{N}}}{4 m_{N}^{2}+Q^{2}} G_{D}\left(Q^{2}, M_{V}\right) \\
& F_{2}^{p}\left(Q^{2}\right)=\frac{4 m_{N}^{2}\left(\frac{\mu_{p}}{\mu_{N}}-1\right)}{4 m_{N}^{2}+Q^{2}} G_{D}\left(Q^{2}, M_{V}\right) \\
& F_{2}\left(Q^{2}\right)=F_{2}^{p}\left(Q^{2}\right)-F_{2}^{n}\left(Q^{2}\right)=\frac{4 m_{N}^{2}\left(\frac{\mu_{p}-\mu_{n}}{\mu_{N}}-1\right)}{4 m_{N}^{2}+Q^{2}} G_{D}\left(Q^{2}, M_{V}\right) \\
& G_{A}\left(Q^{2}\right)=g_{A} G_{D}\left(Q^{2}, M_{A}\right)
\end{aligned}
$$

\footnotetext{
${ }^{23}$ For example, the reaction $e^{-}+p \rightarrow e^{-}+n+\pi^{+}$.
} 


$$
G_{P}\left(Q^{2}\right)=\frac{2 m_{N}^{2} g_{A}}{m_{\pi}^{2}+Q^{2}} G_{D}\left(Q^{2}, M_{A}\right)
$$

where $F_{1}^{p}\left(F_{1}^{n}\right)$ is the Dirac electromagnetic form factor of the proton (neutron), $F_{2}^{p}\left(F_{2}^{n}\right)$ is the Pauli electromagnetic form factor of the proton (neutron),

$$
g_{A} \equiv G_{A}(0)=1.2695 \pm 0.0029 .
$$

is the axial coupling constant of the nucleon [59], $\mu_{N}$ is the nuclear magneton, $m_{\pi}$ is the charged pion mass, $\mu_{p}\left(\mu_{n}\right)$ is the magnetic moment of the proton (neutron), and $M_{V}$ and $M_{A}$ are the vector and axial-vector masses, which have the approximate values [59]

$$
\begin{aligned}
& M_{V} \approx 0.84 \mathrm{GeV} \\
& M_{A}=1.026 \pm 0.021 \mathrm{GeV}
\end{aligned}
$$

\subsection{Multipole expansion of the nuclear current}

At this point in the derivation, all of the ingredients needed to compute the differential cross section for charged current neutrino-nucleus scattering at low energies have been assembled. However, the calculation may be made much more feasible if one performs a multipole expansion of the position-space free nucleon transition operator $\hat{\mathcal{O}}^{\mu}=e^{i \mathbf{q} \cdot \mathbf{x}} \hat{\mathrm{j}}^{\mu}$. Doing so allows one to take advantage of the Wigner-Eckart theorem (described below) to eliminate the sums over the initial $\left(M_{i}\right)$ and final $\left(M_{f}\right)$ nucleus helicities in the expression for $\left|\overline{\mathcal{T}_{f i}}\right|^{2}$ (see eq. (3.102)). It also allows one to easily identify the leading-order terms when the Lorentz-invariant amplitude $\mathcal{M}$ is evaluated in the limit of small momentum transfer $(q \rightarrow 0)$, a point that will be revisited in section 3.11 .

\subsubsection{Plane wave expansion}

Consider the quantity $\left|\overline{\mathcal{T}_{f i}}\right|^{2}$ from eq. (3.102). Partially evaluating the sum over $\mu$ and $\nu$ (in spherical coordinates) leads to the expressions

$$
\begin{aligned}
\left|\overline{\mathcal{T}_{f i}}\right|^{2} & =\sum_{M_{i}} \sum_{M_{f}} O^{\mu} O^{\nu *} \mathrm{~L}_{\mu \nu} \\
& =\sum_{M_{i}} \sum_{M_{f}}\left[\left|O^{\phi}\right|^{2} \mathrm{~L}_{\phi \varnothing}+O^{\phi} O^{M *} \mathrm{~L}_{\phi M}+O^{M} O^{\phi *} \mathrm{~L}_{M \varnothing}+O^{M} O^{N *} \mathrm{~L}_{M N}\right]
\end{aligned}
$$




$$
\begin{aligned}
& =\sum_{M_{i}} \sum_{M_{f}}\left[\left|O^{\phi}\right|^{2} \mathrm{~L}_{\phi \phi}+O^{\phi} O^{M *} \mathrm{~L}_{\phi M}+\left(O^{\phi} O^{M *} \mathrm{~L}_{\phi M}\right)^{*}+O^{M} O^{N *} \mathrm{~L}_{M N}\right] \\
& =\sum_{M_{i}} \sum_{M_{f}}\left[\left|O^{\phi}\right|^{2} \mathrm{~L}_{\phi \phi}+2 \Re\left[O^{\phi} O^{M *} \mathrm{~L}_{\phi M}\right]+O^{M} O^{N *} \mathrm{~L}_{M N}\right]
\end{aligned}
$$

To proceed with the multipole expansion, choose a coordinate system in which the momentum transfer $\mathbf{q}$ points along the positive $z$ direction (i.e., the positive 0 direction in the spherical basis). For this choice of coordinates, the plane wave $e^{i \mathbf{q} \cdot \mathbf{x}}$ may be expanded as a series of spherical waves:

$$
e^{i \mathbf{q} \cdot \mathbf{x}}=\sum_{J} i^{J} \sqrt{4 \pi(2 J+1)} j_{J}(\kappa r) Y_{J 0}(\hat{\mathbf{r}})
$$

Here $\kappa \equiv|\mathbf{q}|$ is the magnitude of the 3-momentum transfer, $r=|\mathbf{x}|$ is the radial coordinate, $\hat{\mathbf{r}}=\mathbf{x} / r$ is a unit vector pointing in the direction of $\mathbf{x}, j_{J}(x)$ is the $J$ th spherical Bessel function of the first kind, and $Y_{J 0}(\hat{\mathbf{r}})$ is a spherical harmonic. Using this expansion allows one to write the spherical components of the position-space free nucleon transition operator as

$$
\begin{aligned}
\hat{\mathcal{O}}^{\emptyset}(k) & =\sum_{J} \hat{\mathcal{O}}^{\emptyset}(k, J)=\sum_{J} i^{J} \sqrt{4 \pi(2 J+1)} j_{J}\left(\kappa r_{k}\right) Y_{J 0}\left(\hat{\mathbf{r}}_{k}\right) \hat{j}^{\varnothing}(k) \\
\hat{\mathcal{O}}^{M}(k) & =\sum_{J} \hat{\mathcal{O}}^{M}(k, J)=\sum_{J} i^{J} \sqrt{4 \pi(2 J+1)} j_{J}\left(\kappa r_{k}\right) Y_{J 0}\left(\hat{\mathbf{r}}_{k}\right) \hat{\mathrm{j}}^{M}(k) \\
& =\sum_{J} \sum_{L} i^{L}(L 01 M \mid J M) \sqrt{4 \pi(2 L+1)} j_{L}\left(\kappa r_{k}\right)\left[Y_{L} \otimes \hat{\mathbf{j}}\right](k)_{J M}
\end{aligned}
$$

where the spherical tensor product $\left[Y_{L} \otimes \hat{\mathbf{j}}\right](k)_{J M}$ is given by

$$
\left[Y_{L} \otimes \hat{\mathbf{j}}\right](k)_{J M}=\sum_{r} \sum_{s}(L r 1 s \mid J M) Y_{L r}\left(\hat{\mathbf{r}}_{k}\right) \hat{\mathbf{j}}^{s}(k)
$$

The last expression in eq. (3.138) was obtained by using the result derived in appendix B after noting that the $\hat{\mathrm{j}}^{M}$ are components of a rank 1 spherical tensor (a 3 -vector).

\subsubsection{The Wigner-Eckhart theorem}

Let $\left\langle\zeta^{\prime} j^{\prime} m^{\prime}\left|T_{L M}\right| \zeta j m\right\rangle$ be an arbitrary matrix element where $T_{L M}$ is the $M$ th component of a spherical tensor $T$ of $\operatorname{rank} L$ and the $|\zeta j m\rangle$ are quantum state vectors with definite 
values of total angular momentum $j$ and its projection quantum number $m$. The symbol $\zeta$ denotes any additional quantum numbers needed to fully specify each state vector. The Wigner-Eckart theorem asserts that [260] any such matrix element may be expressed as the product of a geometric factor containing the projection quantum numbers $m^{\prime}, M$, and $m$, and a constant called the reduced matrix element which is independent of the projections:

$$
\left\langle\zeta^{\prime} j^{\prime} m^{\prime}\left|T_{L M}\right| \zeta j m\right\rangle=\frac{1}{\sqrt{2 j^{\prime}+1}}\left(j m L M \mid j^{\prime} m^{\prime}\right)\left\langle\zeta^{\prime} j^{\prime}\left\|\mathbf{T}_{L}\right\| \zeta j\right\rangle .
$$

In the statement of the Wigner-Eckart theorem above, $\left(j m L M \mid j^{\prime} m^{\prime}\right)$ is a ClebschGordan coefficient and $\left\langle\zeta^{\prime} j^{\prime}\left\|\mathbf{T}_{L}\right\| \zeta j\right\rangle$ is the reduced matrix element, denoted by double bars $(\|)$. This thesis uses Racah's convention [277] (which is also used in references [242] and [260]) for the reduced matrix elements. Note that the Wigner-Eckart theorem implies that the reduced matrix element obeys the same selection rule for $j^{\prime}, L$, and $j$ as the Clebsch-Gordan coefficient in eq. (3.140). That is,

$$
\left\langle\zeta^{\prime} j^{\prime}\left\|\mathbf{T}_{L}\right\| \zeta j\right\rangle=0 \text { unless }|j-L| \leq j^{\prime} \leq j+L
$$

\subsubsection{The reduced nuclear matrix element tensor}

Recognizing that $Y_{J M}(\hat{\mathbf{r}})$ and $\left[Y_{L} \otimes \hat{\mathrm{j}}\right]_{J M}$ are both spherical tensors of rank $J$ allows one to use the Wigner-Eckart theorem to write expressions for $O^{\varnothing}$ and $O^{M}$ in terms of reduced nuclear matrix elements:

$$
\begin{aligned}
O^{\phi}=\sum_{J} O^{\phi}(J) & =\sum_{J} \frac{1}{\sqrt{2 J_{f}+1}}\left(J_{i} M_{i} J 0 \mid J_{f} M_{f}\right)\left\langle J_{f}\left\|\hat{\mathcal{N}}^{\phi}(J)\right\| J_{i}\right\rangle \\
& =\sum_{J} \frac{(-1)^{J_{i}-M_{i}}}{\sqrt{2 J+1}}\left(J_{f} M_{f} J_{i}-M_{i} \mid J 0\right)\left\langle J_{f}\left\|\hat{\mathcal{N}}^{\phi}(J)\right\| J_{i}\right\rangle \\
O^{M}=\sum_{J} O^{M}(J) & =\sum_{J} \frac{1}{\sqrt{2 J_{f}+1}}\left(J_{i} M_{i} J M \mid J_{f} M_{f}\right)\left\langle J_{f}\left\|\hat{\mathcal{N}}^{M}(J)\right\| J_{i}\right\rangle \\
& =\sum_{J} \frac{(-1)^{J_{i}-M_{i}}}{\sqrt{2 J+1}}\left(J_{f} M_{f} J_{i}-M_{i} \mid J M\right)\left\langle J_{f}\left\|\hat{\mathcal{N}}^{M}(J)\right\| J_{i}\right\rangle
\end{aligned}
$$


where, under the impulse approximation,

$$
\hat{\mathcal{N}}^{\mu}(J) \equiv \sum_{k=1}^{A} \hat{\mathcal{O}}^{\mu}(k, J)
$$

The identity

$$
\left(j_{1} m_{1} j_{2} m_{2} \mid j m\right)=(-1)^{j_{1}-m_{1}} \sqrt{\frac{2 j+1}{2 j_{2}+1}}\left(j m j_{1}-m_{1} \mid j_{2} m_{2}\right) .
$$

has been used in eqs. (3.142) and (3.143).

The Clebsch-Gordan coefficients obey the orthogonality relation

$$
\sum_{m_{1}} \sum_{m_{2}}\left(j m \mid j_{1} m_{1} j_{2} m_{2}\right)\left(j_{1} m_{1} j_{2} m_{2} \mid j^{\prime} m^{\prime}\right)=\delta_{j j^{\prime}} \delta_{m m^{\prime}}
$$

Inserting the expressions for $O^{\phi}$ and $O^{M}$ given in eqs. (3.142) and (3.143) into eq. (3.135) and using the orthogonality relation from eq. (3.146) gives the result

$$
\left|\overline{\mathcal{T}_{f i}}\right|^{2}=2 \mathfrak{R}\left(\mathrm{O}^{\phi 0} \mathrm{~L}_{\phi 0}\right)+\sum_{\mu} \mathrm{O}^{\mu \mu} \mathrm{L}_{\mu \mu}
$$

where the sum runs over $\mu \in\{\varnothing,-1,0,1\}$, and the reduced nuclear matrix element tensor is defined as

$$
\mathrm{O}^{\mu \nu} \equiv \sum_{J} \frac{1}{2 J+1}\left\langle J_{f}\left\|\hat{\mathcal{N}}^{\mu}(J)\right\| J_{i}\right\rangle\left\langle J_{f}\left\|\hat{\mathcal{N}}^{\nu}(J)\right\| J_{i}\right\rangle^{*}
$$

The spin-summed squared amplitude $|\overline{\mathcal{M}}|^{2}$ may therefore be written as

$$
|\overline{\mathcal{M}}|^{2}=4 E_{i} E_{f} \frac{G_{F}^{2}\left|V_{u d}\right|^{2}}{2 J_{i}+1}\left[2 \Re\left(\mathrm{O}^{\phi 0} \mathrm{~L}_{\phi 0}\right)+\sum_{\mu} \mathrm{O}^{\mu \mu} \mathrm{L}_{\mu \mu}\right] .
$$

\subsubsection{Simplified form of the lepton tensor elements}

For the choice of coordinates used in the multipole expansion above, i.e.,

$$
\mathbf{q} \equiv \mathbf{p}_{N_{f}}-\mathbf{p}_{N_{i}}=\mathbf{p}_{\nu_{\ell}}-\mathbf{p}_{\ell}=\kappa \hat{\mathbf{e}}_{0}
$$

where $\kappa=|\mathbf{q}|$ is the magnitude of the 3-momentum transfer, one may write

$$
\hat{\mathbf{e}}_{0}=\hat{\mathbf{z}}=\frac{\mathbf{q}}{\kappa}=\frac{\mathbf{p}_{\nu_{\ell}}-\mathbf{p}_{\ell}}{\kappa} .
$$


With this expression for $\hat{\mathbf{e}}_{0}$, it is possible to rewrite the lepton tensor elements $L_{\mu \nu}$ needed to evaluate $|\overline{\mathcal{M}}|^{2}$ in the form ${ }^{24}$

$$
\begin{aligned}
\mathrm{L}_{\varnothing \emptyset} & =4\left[E_{\ell} E_{\nu_{\ell}}+\left|\mathbf{p}_{\ell}\right|\left|\mathbf{p}_{\nu_{\ell}}\right| \cos \theta_{\ell}\right] \\
\mathrm{L}_{\phi 0} & =4\left[E_{\ell} p_{\nu_{\ell}, 0}+E_{\nu_{\ell}} p_{\ell, 0}\right] \\
\mathrm{L}_{00} & =4\left[E_{\ell} E_{\nu_{\ell}}-\left|\mathbf{p}_{\ell}\right|\left|\mathbf{p}_{\nu_{\ell}}\right| \cos \theta_{\ell}+2 p_{\ell, 0} p_{\nu_{\ell}, 0}\right] \\
\mathrm{L}_{11} & =4\left[E_{\ell} E_{\nu_{\ell}}-p_{\ell, 0} p_{\nu_{\ell}, 0}-L_{\nu_{\ell}}\left(E_{\ell} p_{\nu_{\ell}, 0}-E_{\nu_{\ell}} p_{\ell, 0}\right)\right] \\
\mathrm{L}_{-1,-1} & =4\left[E_{\ell} E_{\nu_{\ell}}-p_{\ell, 0} p_{\nu_{\ell}, 0}+L_{\nu_{\ell}}\left(E_{\ell} p_{\nu_{\ell}, 0}-E_{\nu_{\ell}} p_{\ell, 0}\right)\right]
\end{aligned}
$$

where

$$
\begin{aligned}
p_{\ell, 0} & =\frac{E_{\nu_{\ell}}\left|\mathbf{p}_{\ell}\right| \cos \theta_{\ell}-\left|\mathbf{p}_{\ell}\right|^{2}}{\kappa}, \\
p_{\nu_{\ell}, 0} & =\frac{E_{\nu_{\ell}}^{2}-E_{\nu_{\ell}}\left|\mathbf{p}_{\ell}\right| \cos \theta_{\ell}}{\kappa},
\end{aligned}
$$

$\theta_{\ell}$ is the angle between $\mathbf{p}_{\ell}$ and $\mathbf{p}_{\nu_{\ell}}, L_{\nu_{\ell}}=+1$ is the lepton number of the neutrino, and $\kappa$ may be written in terms of $\theta_{\ell}$ :

$$
\kappa=\sqrt{E_{\nu_{\ell}}^{2}+\left|\mathbf{p}_{\ell}\right|^{2}-2 E_{\nu_{\ell}}\left|\mathbf{p}_{\ell}\right| \cos \theta_{\ell}} .
$$

\subsection{First-order approximation in $1 / \mathrm{m}_{\mathrm{N}}$}

In principle, one could directly evaluate the charged current neutrino-nucleus differential cross section (see eq. (3.65)) by calculating $|\overline{\mathcal{M}}|^{2}$ using a suitable model for the nuclear wavefunctions and the results shown thus far. However, to avoid dealing with the unwieldy full expressions for the components of the free nucleon transition operator $\mathrm{j}^{\mu}$ given in eqs. (3.77) and (3.78), approximations of this operator to first order in the inverse nucleon mass $1 / m_{N}$ are routinely used in the literature [210, 234, 242].

\footnotetext{
${ }^{24}$ See [241], but note that the lepton tensor presented there is equal to ours divided by $4 E_{\nu_{\ell}} E_{\ell}$. Also, the opposite subtraction order is used for $q$, i.e., $q=p_{\ell}-p_{\nu_{\ell}}$ therein.
} 
When evaluating $\mathrm{j}^{\mu}$ to first order in $1 / m_{N}$, two points are important to consider. First, unlike the other form factors, which are taken to be zeroth order in $1 / m_{N}$ (see eqs. (3.126), (3.129) and (3.130)), $G_{P}\left(Q^{2}\right)$ is intrinsically of order $m_{N}^{2}$ (see eq. (3.131)). Therefore, to maintain consistency, one should keep terms in $\mathrm{j}^{\mu}$ involving $G_{P}\left(Q^{2}\right)$ up to order $1 / m_{N}^{3}$, while terms involving any of the other form factors should be dropped beyond order $1 / m_{N}$. Second, one must treat terms involving factors of $q^{\varnothing}$ carefully. Although for free, nonrelativistic nucleons,

$$
q^{\varnothing}=\frac{\left|\mathbf{p}_{N_{f}}\right|^{2}-\left|\mathbf{p}_{N_{i}}\right|^{2}}{2 m_{N}}=\frac{|\mathbf{q}|^{2}+2 \mathbf{q} \cdot \mathbf{p}_{N_{i}}}{2 m_{N}}
$$

is a first order quantity in $1 / m_{N}$, this is not necessarily true for bound nucleons, which are off their mass shell [231]. Here we will follow the Donnelly-Walecka [210] and KrmpotićSamana [242] formalisms in assuming that $q^{\phi}$ is first order in $1 / m_{N}$ based on its freenucleon value.

Taking these details into account allows one to obtain the following expressions ${ }^{25}$ for the components of the free nucleon transition operator:

$$
\begin{aligned}
\mathrm{j}^{\varnothing}=\left[F_{1}\left(Q^{2}\right)\right. & -\frac{\mathbf{p}_{N_{i}} \cdot \boldsymbol{\sigma}}{m_{N}} G_{A}\left(Q^{2}\right)-\frac{\mathbf{q} \cdot \boldsymbol{\sigma}}{2 m_{N}} G_{A}\left(Q^{2}\right) \\
& \left.-\frac{\mathbf{q} \cdot \boldsymbol{\sigma}}{m_{N}} G_{3}\left(Q^{2}\right)+\frac{q^{\emptyset}}{m_{N}}\left(\frac{\mathbf{q} \cdot \boldsymbol{\sigma}}{2 m_{N}}\right) G_{P}\left(Q^{2}\right)\right] t_{-}+\mathcal{O}\left(1 / m_{N}^{2}\right)
\end{aligned}
$$

and

$$
\begin{aligned}
\mathrm{j}^{M}=\left[-\sigma^{M} G_{A}\left(Q^{2}\right)\right. & +\frac{q^{M}}{m_{N}} F_{3}\left(Q^{2}\right)+\left(F_{1}\left(Q^{2}\right)-F_{2}\left(Q^{2}\right)\right) \frac{i(\boldsymbol{\sigma} \times \mathbf{q})^{M}}{2 m_{N}} \\
& \left.+\left(\frac{2 p_{N_{i}}^{M}+q^{M}}{2 m_{N}}\right) F_{1}\left(Q^{2}\right)+\frac{q^{M}}{m_{N}}\left(\frac{\mathbf{q} \cdot \boldsymbol{\sigma}}{2 m_{N}}\right) G_{P}\left(Q^{2}\right)\right] t_{-}+\mathcal{O}\left(1 / m_{N}^{2}\right) .
\end{aligned}
$$

\footnotetext{
${ }^{25}$ Walecka [210] includes an additional term $+q^{\phi} F_{3}\left(Q^{2}\right) t_{-}$in the first order expression for $\mathrm{j}^{\phi}$ because, in his version of eq. (3.30), the induced scalar form factor $F_{3}\left(Q^{2}\right)$ appears without a prefactor of $1 / m_{N}$, and yet $F_{3}\left(Q^{2}\right)$ is still assumed to be intrinsically zeroth order in $1 / m_{N}$. The corresponding term in the present formalism is $\left(q^{\phi} / m_{N}\right) F_{3}\left(Q^{2}\right) t_{-}$, which is second order in $1 / m_{N}$.
} 
In the spherical basis, the cross product $\mathbf{A} \times \mathbf{B}$ of two 3-vectors is given by [260]

$$
(\mathbf{A} \times \mathbf{B})_{M}=-i \sqrt{2}\left[\mathbf{A}_{1} \otimes \mathbf{B}_{1}\right]_{1 M}=-i \sqrt{2} \sum_{M_{1}, M_{2}}\left(1 M_{1} 1 M_{2} \mid 1 M\right) A_{M_{1}} B_{M_{2}}
$$

where $\left(1 M_{1} 1 M_{2} \mid 1 M\right)$ is a Clebsch-Gordan coefficient. Recall that, during the multipole expansion in section 3.8, a coordinate system was chosen in which the 3-momentum transfer $\mathbf{q}$ was directed along the positive $z$ axis (the positive 0 axis in the spherical basis). With this choice of coordinates, it follows that

$$
q^{M}=\delta_{M 0} \kappa
$$

and

$$
\mathbf{q} \cdot \boldsymbol{\sigma}=\kappa \sigma_{0}
$$

where, like before, $\kappa \equiv|\mathbf{q}|$. Also, invoking eq. (3.163) leads to the result

$$
i(\boldsymbol{\sigma} \times \mathbf{q})^{M}=\kappa M \sigma^{M} .
$$

Combining these results with eqs. (B.19), (3.137), (3.138), (3.161) and (3.162) yields the expressions

$$
\begin{aligned}
& \hat{\mathcal{O}}^{\phi}(k, J)=i^{J} \sqrt{4 \pi(2 J+1)} j_{J}\left(\kappa r_{k}\right) Y_{J 0}\left(\hat{\mathbf{r}}_{k}\right)\left[F_{1}\left(Q^{2}\right)-\frac{\mathbf{p}_{N_{i}} \cdot \boldsymbol{\sigma}}{m_{N}} G_{A}\left(Q^{2}\right)\right] t_{-}(k) \\
& +\sum_{L} i^{L} \sqrt{2 L+1}(L 010 \mid J 0) j_{L}\left(\kappa r_{k}\right)\left[Y_{L} \otimes \boldsymbol{\sigma}\right](k)_{J M} \mathcal{G}^{\phi}\left(Q^{2}\right) t_{-}(k)
\end{aligned}
$$

and

$$
\begin{aligned}
\hat{\mathcal{O}}^{M}(k, J) & =i^{J} \sqrt{4 \pi(2 J+1)} j_{J}\left(\kappa r_{k}\right) Y_{J 0}\left(\hat{\mathbf{r}}_{k}\right)\left[\frac{1}{2} F_{1}\left(Q^{2}\right)+F_{3}\left(Q^{2}\right)\right] \frac{\delta_{M 0} \kappa}{m_{N}} t_{-}(k) \\
& +\sum_{L} i^{L} \sqrt{4 \pi(2 L+1)}(L 01 M \mid J M) j_{L}\left(\kappa r_{k}\right)\left[\mathcal{G}^{M}\left(Q^{2}\right)\left[Y_{L} \otimes \boldsymbol{\sigma}\right](k)_{J M}\right. \\
& \left.+\frac{F_{1}\left(Q^{2}\right)}{m_{N}}\left[Y_{L} \otimes \mathbf{p}_{N_{i}}\right](k)_{J M}\right] t_{-}(k),
\end{aligned}
$$


where

$$
\mathcal{G}_{\phi}\left(Q^{2}\right) \equiv \frac{\kappa}{m_{N}}\left[\frac{q^{\emptyset}}{2 m_{N}} G_{P}\left(Q^{2}\right)-\frac{1}{2} G_{A}\left(Q^{2}\right)-G_{3}\left(Q^{2}\right)\right]
$$

and

$$
\mathcal{G}_{M}\left(Q^{2}\right) \equiv \frac{\kappa}{m_{N}}\left[M \frac{F_{1}\left(Q^{2}\right)-F_{2}\left(Q^{2}\right)}{2 m_{N}}-G_{A}\left(Q^{2}\right)+\frac{\kappa^{2}}{2 m_{N}^{2}} \delta_{M 0} G_{P}\left(Q^{2}\right)\right] .
$$

Equations (3.144), (3.167) and (3.168) imply that

$$
\left\langle J_{f}\left\|\hat{\mathcal{N}}^{\varnothing}(J)\right\| J_{i}\right\rangle=F_{1}\left(Q^{2}\right) \mathcal{N}_{J}^{V}\left(t_{-}\right)-\frac{G_{A}\left(Q^{2}\right)}{m_{N}} \mathcal{N}_{J}^{A}\left(t_{-}\right)+\mathcal{G}_{\phi}\left(Q^{2}\right) \mathcal{N}_{J 0}^{A}\left(t_{-}\right)
$$

and

$$
\begin{aligned}
\left\langle J_{f}\left\|\hat{\mathcal{N}}^{M}(J)\right\| J_{i}\right\rangle & =\frac{\delta_{M 0} \kappa}{m_{N}}\left[\frac{1}{2} F_{1}\left(Q^{2}\right)+F_{3}\left(Q^{2}\right)\right] \mathcal{N}_{J}^{V}\left(t_{-}\right) \\
& +\frac{F_{1}\left(Q^{2}\right)}{m_{N}} \mathcal{N}_{J M}^{V}\left(t_{-}\right)+\mathcal{G}_{M}\left(Q^{2}\right) \mathcal{N}_{J M}^{A}\left(t_{-}\right) .
\end{aligned}
$$

Here the four basic reduced matrix elements are defined by

$$
\begin{gathered}
\mathcal{N}_{J}^{V}(\Theta) \equiv i^{J} \sqrt{4 \pi(2 J+1)}\left\langle J_{f}\left\|\sum_{k=1}^{A} j_{J}\left(\kappa r_{k}\right) Y_{J}\left(\hat{\mathbf{r}}_{k}\right) \Theta(k)\right\| J_{i}\right\rangle \\
\mathcal{N}_{J}^{A}(\Theta) \equiv i^{J} \sqrt{4 \pi(2 J+1)}\left\langle J_{f}\left\|\sum_{k=1}^{A} j_{J}\left(\kappa r_{k}\right) Y_{J}\left(\hat{\mathbf{r}}_{k}\right)\left(\mathbf{p}_{N_{i}} \cdot \boldsymbol{\sigma}\right) \Theta(k)\right\| J_{i}\right\rangle \\
\mathcal{N}_{J M}^{V}(\Theta) \equiv \sum_{L} i^{L} \sqrt{4 \pi(2 L+1)}(L 01 M \mid J M)\left\langle J_{f}\left\|\sum_{k=1}^{A} j_{J}\left(\kappa r_{k}\right)\left[Y_{L} \otimes \mathbf{p}_{N_{i}}\right](k) \Theta(k)\right\| J_{i}\right\rangle \\
\mathcal{N}_{J M}^{A}(\Theta) \equiv \sum_{L} i^{L} \sqrt{4 \pi(2 L+1)}(L 01 M \mid J M)\left\langle J_{f}\left\|\sum_{k=1}^{A} j_{J}\left(\kappa r_{k}\right)\left[Y_{L} \otimes \boldsymbol{\sigma}\right](k)_{J} \Theta(k)\right\| J_{i}\right\rangle .
\end{gathered}
$$

where $\Theta$ is an operator in isospace. As shown above, for charged current neutrino scattering, $\Theta=t_{-}$. 


\subsection{Correction for the final-state Coulomb interaction}

Up to this point in the derivation, the final-state Coulomb interaction between the outgoing charged lepton and the residual nucleus has been ignored. Although a detailed consideration of final-state Coulomb effects is available in the beta-decay literature, ${ }^{26}$ calculations of neutrino-nucleus scattering at low energies often use one of two approximations.

\subsubsection{The Fermi function}

The first of these approximations is a rescaling of the outgoing lepton wavefunction originally proposed by Fermi in his theory of beta decay [21, 22]. Since the amplitudes for beta decay and charged current neutrino-nucleus scattering are related via crossing symmetry, Fermi's approach may also be applied to low-energy neutrino scattering.

To apply Fermi's correction, first note that the momentum space wavefunction for the final-state lepton has been approximated in the previous calculations as the Dirac spinor for a free particle, i.e.,

$$
u_{\ell}\left(p_{\ell}\right)=\sqrt{2 E_{\ell}} \sqrt{\frac{E_{\ell}+m_{\ell}}{2 E_{\ell}}}\left(\begin{array}{c}
1 \\
\frac{\left(\mathbf{p}_{\ell} \cdot \boldsymbol{\sigma}\right)}{E_{\ell}+m_{\ell}}
\end{array}\right) \xi_{\ell}
$$

where $\xi_{\ell}$ is a two-component Pauli spinor and the $\sqrt{2 E_{\ell}}$ prefactor arises because of our relativistic normalization convention. ${ }^{27}$ The corresponding relativistic wavefunction in position space $\psi_{\ell}\left(x_{\ell}\right)$ is the plane wave given by

$$
\psi_{\ell}\left(x_{\ell}\right)=V^{-1 / 2} T^{-1 / 2} u_{\ell}\left(p_{\ell}\right) e^{-i p_{\ell} \cdot x_{\ell}}
$$

where the factors $V$ and $T$ have been introduced so that the wavefunction is correctly

\footnotetext{
${ }^{26}$ See, e.g., reference [278].

${ }^{27}$ See eq. (3.72) and the discussion immediately following it.
} 
normalized (see eq. (3.73)) within the box of section 3.3, i.e.,

$$
\begin{aligned}
\left\langle\psi_{\ell} \mid \psi_{\ell}\right\rangle & =\int \psi_{\ell}^{\dagger}(x) \psi_{\ell}(x) d^{4} x=V^{-1} T^{-1} \int e^{i p_{\ell} \cdot x_{\ell}} u_{\ell}^{\dagger}\left(p_{\ell}\right) u_{\ell}\left(p_{\ell}\right) e^{-i p_{\ell} \cdot x_{\ell}} d^{4} x \\
& =2 E_{\ell} \xi_{\ell}^{\dagger} \xi_{\ell} V^{-1} T^{-1} \int e^{i p_{\ell} \cdot x_{\ell}} e^{-i p_{\ell} \cdot x_{\ell}} d^{4} x=2 E_{\ell} \xi_{\ell}^{\dagger} \xi_{\ell} V^{-1} T^{-1} \int d^{4} x \\
& =2 E_{\ell} \xi_{\ell}^{\dagger} \xi_{\ell} .
\end{aligned}
$$

Choose a coordinate system in which the final-state nucleus is at rest at the origin, called the "final nucleus rest frame" (FNR frame). Then, if the residual nucleus is modeled as a point with electric charge $Z_{f} e$, where $e$ is the elementary charge and $Z_{f}$ is the proton number of the final-state nucleus, it can be shown [279] that the exact charged lepton wavefunction approaches the following limiting value ${ }^{28}$ near the origin:

$$
\lim _{x_{\ell} \rightarrow 0} \psi_{\ell}\left(x_{\ell}\right)=\frac{N}{\sqrt{V T}} \sqrt{2 E_{\ell}} \sqrt{\frac{E_{\ell}+m_{\ell}}{2 E_{\ell}}}\left(1+\frac{i Z_{f} \alpha}{1+S} \frac{\mathbf{a} \cdot \mathbf{x}_{\ell}}{r_{\ell}}\right)\left(\begin{array}{c}
1 \\
M \frac{\left(\mathbf{p}_{\ell} \cdot \boldsymbol{\sigma}\right)}{E_{\ell}+m_{\ell}}
\end{array}\right) \xi_{\ell} .
$$

(FNR frame, 3.180)

Here, $N$ is a function that will be discussed momentarily, $\alpha$ is the fine structure constant, $r_{\ell} \equiv\left|\mathbf{x}_{\ell}\right|$ is the charged lepton radial coordinate, $S \equiv \sqrt{1-\alpha^{2} Z_{f}^{2}}, \mathbf{a} \equiv\left(\begin{array}{cc}0 & \boldsymbol{\sigma} \\ \boldsymbol{\sigma} & 0\end{array}\right)$, and

$$
M \equiv \frac{E_{\ell}+m_{\ell}}{E_{\ell}+S m_{\ell}}\left(1+i \frac{Z_{f} \alpha m_{\ell}}{\left|\mathbf{p}_{\ell}\right|}\right) .
$$

Since the term proportional to $\mathbf{a} \cdot \mathbf{x}_{\ell}$ in eq. (3.180) can only contribute to transitions in which the nuclear parity changes, [279] it may be ignored under the allowed approximation, which, as will be discussed in section 3.11, includes only parity-conserving nuclear transitions. This term is also sometimes neglected in calculations that include the possibility of parity-changing nuclear transitions. ${ }^{29}$ A possible justification for this is that the Fermi function (obtained below) "exhibits the main dependence on the energy and the nuclear charge number," for the charged lepton radial wavefunction $\psi_{\ell}(x)$, independent

\footnotetext{
${ }^{28}$ Note that reference [279] uses Gaussian natural units, so the value of the elementary charge used therein is $e=\sqrt{\alpha}$. To avoid confusion, the relevant factors of $e$ have been rewritten here in terms of the fine structure constant, which has the same value in all systems of units.

${ }^{29}$ Examples include [242, 280-282], which employ the Fermi function derived here and therefore implicitly neglect the $\mathbf{a} \cdot \mathbf{x}_{\ell}$ term.
} 
of the model of the nuclear charge distribution used in the calculation [278]. Since the fine structure constant is small ( $\alpha \approx 1 / 137)$, the approximation $M \approx 1$ is typically good if the charged lepton is relativistic. Neglecting the $\mathbf{a} \cdot \mathbf{x}_{\ell}$ term and making the approximation $M \approx 1$ in eq. (3.180) yields the expression

$$
\lim _{x_{\ell} \rightarrow 0} \psi_{\ell}\left(x_{\ell}\right) \approx \frac{N}{\sqrt{V T}} \sqrt{2 E_{\ell}} \sqrt{\frac{E_{\ell}+m_{\ell}}{2 E_{\ell}}}\left(\begin{array}{c}
1 \\
\frac{\left(\mathbf{p}_{\ell} \cdot \boldsymbol{\sigma}\right)}{E_{\ell}+m_{\ell}}
\end{array}\right) \xi_{\ell}=N V^{-1 / 2} T^{-1 / 2} u_{\ell}\left(p_{\ell}\right) .
$$

(FNR frame, 3.182)

Comparing eqs. (3.178) and (3.182), one finds that the effect of the Coulomb interaction on the charged lepton wavefunction in the vicinity of the nucleus may be approximated by scaling the original plane wave expression by a factor $N$. Since the absolute square of the charged lepton momentum space wavefunction appears in the lepton tensor $\mathrm{L}_{\mu \nu}$, the cross section described above should be multiplied by the correction factor $|N|^{2}$, which turns out to be [279]

$$
|N|^{2}=\frac{E_{\ell}+S m_{\ell}}{E_{\ell}+m_{\ell}} F\left(Z_{f}, E_{\ell}\right) \approx F\left(Z_{f}, E_{\ell}\right)
$$

where the approximation in the second step is made for consistency with the earlier choice of $M \approx 1$, and the Fermi function [21,22] may be written as

$$
F\left(Z_{f}, E_{\ell}\right)=\frac{2(1+S)}{[\Gamma(1+2 S)]^{2}}\left(2\left|\mathbf{p}_{\ell}\right| R\right)^{2 S-2} e^{-\pi \eta}|\Gamma(S+i \eta)|^{2} .
$$

Here,

$$
R \approx 1.2 A^{1 / 3} \frac{\mathrm{fm}}{\hbar c}
$$

is the nuclear radius (in natural units), and the Sommerfeld parameter $\eta$ is given by

$$
\eta=\frac{\alpha Z_{f} Z_{\ell} E_{\ell}}{\left|\mathbf{p}_{\ell}\right|}
$$

where $Z_{\ell}$ is the charge of the outgoing lepton in units of the elementary charge, e.g., $Z_{\ell}=-1$ for electrons and $Z_{\ell}=+1$ for positrons.

Although the cross section derivation in this chapter has been done in the centerof-momentum frame, the Fermi function presented here was obtained in the rest frame of the final-state nucleus. Because the Coulomb potential seen by the outgoing lepton 
depends only on its motion relative to the nucleus, however, the Fermi function should be regarded as a Lorentz invariant correction factor. That is, $F\left(Z_{f}, E_{\ell}\right)$ should be evaluated in the FNR frame when computing the cross section in any frame of reference. This may be made more explicit by rewriting the expression for the Fermi function given above in terms of the Lorentz-invariant relative speed $\beta_{f, \text { rel }}$ of the final nucleus and lepton, i.e.,

$$
F\left(Z_{f}, \beta_{f, \text { rel }}\right)=\frac{2(1+S)}{[\Gamma(1+2 S)]^{2}}\left(2 \gamma_{f, \text { rel }} \beta_{f, \text { rel }} m_{\ell} R\right)^{2 S-2} e^{-\pi \eta}|\Gamma(S+i \eta)|^{2}
$$

where $\gamma_{f, \text { rel }} \equiv\left(1-\beta_{f, \text { rel }}^{2}\right)^{-1 / 2}$, the Sommerfeld parameter is now written as

$$
\eta=\frac{\alpha Z_{f} Z_{\ell}}{\beta_{f, \mathrm{rel}}}
$$

and $\beta_{f \text {,rel }}$ is given by eq. (3.45) with the substitutions $i, 1 \rightarrow \ell$ for the final lepton 4-momentum and $i, 2 \rightarrow f$ for the final nucleus 4-momentum. In the FNR frame, of course, $\beta_{f, \text { rel }}$ is simply the speed of the final-state lepton.

Fermi's correction for final-state Coulomb effects in the neutrino charged current cross section may therefore be expressed in any frame of reference as the substitution

$$
|\overline{\mathcal{M}}|^{2} \rightarrow|\overline{\mathcal{M}}|^{2} F\left(Z_{f}, \beta_{f, \text { rel }}\right)
$$

where $F\left(Z_{f}, \beta_{f, \text { rel }}\right)$ is defined as in eq. (3.187).

\subsubsection{Effective momentum approximation}

The procedure used above to derive the Fermi function implicitly assumes that the final lepton wavefunction may be adequately described using only its s-wave (partial wave with zero orbital angular momentum) component. This approximation arises because only the s-wave part of the wavefunction survives [279] when the $x_{\ell} \rightarrow 0$ limit is taken in eq. (3.180). Although this approach works well for low-energy electrons, a more careful theoretical treatment of the Coulomb distortion in charged current neutrino scattering shows that the Fermi function overestimates the Coulomb effects for high-energy electrons and performs particularly poorly for muons [283].

For situations where the Fermi function is inadequate, an alternative to performing 
a very involved calculation of the Coulomb effects ${ }^{30}$ is to apply an effective momentum approximation (EMA) first developed to describe electron-nucleus scattering (see, e.g., [286] and references therein) and later applied to the charged current neutrino-nucleus case by Engel [283].

Under this approximation, the lab-frame outgoing lepton energy and momentum are replaced by the effective values

$$
E_{\ell}^{\mathrm{eff}}=E_{\ell}-V_{C}(0) \quad\left|\mathbf{p}_{\ell}^{\mathrm{eff}}\right|=\sqrt{\left(E_{\ell}^{\mathrm{eff}}\right)^{2}-m_{\ell}^{2}} \quad \text { (lab frame, 3.190) }
$$

when the Lorentz-invariant amplitude $\mathcal{M}$ is calculated, resulting in a larger effective value of the momentum transfer $q$. The Coulomb potential evaluated at the nuclear center of mass, $V_{C}(0)$, is usually taken to be that at the center of a uniformly-charged sphere, i.e.,

$$
V_{C}(0) \approx \frac{3 Z_{f} Z_{\ell} \alpha}{2 R}
$$

where $R, Z_{f}$ and $Z_{\ell}$ are defined as in eqs. (3.185)-(3.186). The amplitude of the outgoing lepton wavefunction is also rescaled by a factor

$$
f_{\mathrm{EMA}}=\frac{\left|\mathbf{p}_{\ell}^{\mathrm{eff}}\right|}{\left|\mathbf{p}_{\ell}\right|}
$$

which results in the cross section being multiplied by the factor $f_{\mathrm{EMA}}^{2}$.

Using these adjustments in place of the Fermi function has been found to be a good approximation for final-state electrons in most cases where the Fermi function is inappropriate [283]. For final-state muons, a modified effective momentum approximation (MEMA) is recommended [283] to be used at all energies. Under the MEMA, the rescaling factor $f_{\mathrm{EMA}}$ from the ordinary EMA is replaced by

$$
f_{\mathrm{MEMA}}=\sqrt{\frac{\left|\mathbf{p}_{\ell}^{\mathrm{eff}}\right| E_{\ell}^{\mathrm{eff}}}{\left|\mathbf{p}_{\ell}\right| E_{\ell}}}
$$

while the rest of the procedure is unchanged.

\footnotetext{
${ }^{30}$ For example, by using the Distorted Wave Born Approximation (DWBA), which is discussed in, e.g., references $[284,285]$.
} 
To combine the EMA and MEMA recommendations in the present formalism, define the Coulomb correction factor $f_{C}$ by

$$
f_{C} \equiv \begin{cases}f_{\mathrm{EMA}} & \ell=e \\ f_{\mathrm{MEMA}} & \ell=\mu .\end{cases}
$$

Thus, $f_{C}=f_{\mathrm{EMA}}$ when the final-state lepton is an (anti)electron, and $f_{C}=f_{\mathrm{MEMA}}$ when it is a muon or antimuon. Because tau leptons have a mass of about $1.777 \mathrm{GeV}$, charged current reactions that produce them are far above the energy scale at which the MARLEY models are expected to be appropriate.

\subsubsection{Coulomb-corrected differential cross section}

The final-state Coulomb interaction increases the charged current cross section for matter neutrinos and decreases it for antineutrinos. Because the EMA overestimates the size of this effect for electrons at low energies while the Fermi function does the same at high energies, a reasonable way of combining the two approximate Coulomb corrections described above is, for a given neutrino energy, to choose the approach which gives the smaller cross section for neutrinos and the larger cross section for antineutrinos. ${ }^{31}$

To incorporate this treatment of Coulomb effects into the present cross section calculation, define the Coulomb-corrected spin-summed squared amplitude

$$
|\overline{\mathcal{M}}|_{C}^{2} \equiv \min \left\{|\overline{\mathcal{M}}|^{2} F\left(Z_{f}, \beta_{f, \text { rel }}\right),\left|\overline{\mathcal{M}_{\text {eff }}}\right|^{2} f_{C}^{2}\right\}
$$

where $F\left(Z_{f}, \beta_{f, \text { rel }}\right)$ is defined in eq. (3.187), and

$$
\mathcal{M}_{\text {eff }}=\left.\mathcal{M}\right|_{E_{\ell}=E_{\ell}^{\text {eff }},\left|\mathbf{p}_{\ell}\right|=\left|\mathbf{p}_{\ell}^{\text {eff }}\right|}
$$

is the Lorentz-invariant amplitude evaluated using the effective values of the final-state lepton energy and 3-momentum.

To obtain the value of $\mathcal{M}_{\text {eff }}$ in the center-of-momentum (CM) frame, one may perform a Lorentz transformation of the effective lepton energy $E_{\ell}^{\text {eff }}$ from the lab to the CM frame.

\footnotetext{
${ }^{31}$ Publications which have previously used this method for combining the approximate Coulomb corrections include $[230,237]$.
} 
To do so, note that the velocity $\boldsymbol{\beta}_{\mathrm{CM}}$ of the CM frame as viewed in the lab frame is given by

$$
\beta_{\mathrm{CM}}=\frac{\mathbf{p}_{\nu_{\ell}}}{E_{\nu_{\ell}}+m_{i}}
$$

where $m_{i}$ is the mass of the initial nucleus. For

$$
\gamma_{\mathrm{CM}} \equiv\left(1-\left|\boldsymbol{\beta}_{\mathrm{CM}}\right|^{2}\right)^{-1 / 2}
$$

the effective charged lepton energy in the CM frame $E_{\ell}^{\text {eff,CM }}$ is given in terms of its labframe value $E_{\ell}^{\text {eff }}$ and the effective lab-frame 3 -momentum $\mathbf{p}_{\ell}^{\text {eff }}$ by

$$
E_{\ell}^{\mathrm{eff}, \mathrm{CM}}=\gamma_{\mathrm{CM}}\left[E_{\ell}^{\mathrm{eff}}-\left|\boldsymbol{\beta}_{\mathrm{CM}}\right|\left|\mathbf{p}_{\ell}^{\mathrm{eff}}\right| \cos \theta_{\ell}^{\mathrm{lab}}\right],
$$

where $\theta_{\ell}^{\text {lab }}$ is the angle between the 3-momenta of the charged lepton and the incident neutrino as measured in the lab frame. From this result, one may also obtain the CM frame effective 3-momentum:

$$
\left|\mathbf{p}_{\ell}^{\mathrm{eff}, \mathrm{CM}}\right|=\sqrt{\left(E_{\ell}^{\mathrm{eff}, \mathrm{CM}}\right)^{2}-m_{\ell}^{2}} .
$$

To first order in $1 / m_{N}$, the charged current neutrino-nucleus differential cross section may now be written in the form

$$
\frac{d \sigma}{d \cos \theta_{\ell}}=\frac{|\overline{\mathcal{M}}|_{C}^{2}}{32 \pi s} \frac{\left|\mathbf{p}_{\ell}\right|}{E_{\nu_{\ell}}} .
$$

where $|\overline{\mathcal{M}}|_{C}^{2}$ is defined in eq. (3.195), $|\overline{\mathcal{M}}|^{2}$ is given in eq. (3.149), the elements of the reduced nuclear matrix element tensor $\mathrm{O}^{\mu \nu}$ may be computed using eqs. (3.171)-(3.176), and the elements of the lepton tensor $\mathrm{L}_{\mu \nu}$ may be evaluated in terms of $\cos \theta_{\ell}$ using eqs. (3.152)-(3.159).

\subsection{Allowed approximation cross section}

The first-order expression given in (3.201) may be used to calculate cross sections using a suitable model for the four basic nuclear matrix elements defined in eqs. (3.173)-(3.176). The details of such a calculation for a particular nucleus could easily form the basis for 
a complete $\mathrm{PhD}$ thesis ${ }^{32}$ on their own, and, unfortunately, most publications discussing low-energy neutrino-nucleus cross sections do not include information about the individual nuclear matrix elements that would be needed to create a fully-detailed event generator.

To work around these difficulties, we will apply the allowed approximation to the cross section calculation outlined in earlier sections. Doing so will allow us to use published values for the leading-order nuclear matrix elements to calculate an approximate differential cross section for charged current $\nu_{e}$ scattering on ${ }^{40}$ Ar. This approximate cross section will then be applied in chapters 6 and 7 to the simulation of supernova neutrino events in liquid argon, demonstrating via practical calculations the feasibility of creating a realistic Monte Carlo event generator for neutrinos in this energy range.

To begin using the allowed approximation in the cross section calculation described previously, first note that the $n$th spherical Bessel function of the first kind $j_{n}(x)$ may be expressed as the Maclaurin series

$$
j_{n}(x)=x^{n} \sum_{k=0}^{\infty} \frac{(-1)^{k} x^{2 k}}{(2 k) ! !(2 n+2 k+1) ! !} .
$$

Therefore, as $x \rightarrow 0$, the function $j_{n}(x)$ approaches the limiting form

$$
j_{n}(x) \rightarrow \frac{x^{n}}{(2 n+1) ! !} \quad(\text { for small } x) .
$$

Now consider the multipole expansion of the position-space free nucleon transition operator given in eqs. (3.137) and (3.138). Each term in the expansion contains a spherical Bessel function $j_{n}\left(\kappa r_{k}\right)$ for $n \in\{J, L\}$, where $\kappa \equiv|\mathbf{q}|$ is the magnitude of the 3-momentum transfer and $r_{k}$ is the radial position operator for the $k$ th nucleon. Equation (3.203) implies that

$$
\lim _{\kappa \rightarrow 0} j_{n}\left(\kappa r_{k}\right)=\left\{\begin{array}{ll}
1 & \text { if } n=0 \\
0 & \text { otherwise }
\end{array} .\right.
$$

To obtain a rough estimate of the range of validity of the allowed approximation $\kappa \approx 0$, note that one may obtain a conservative upper limit on the magnitude of the 3 -momentum transfer by using $\kappa \lesssim 2 E_{\nu}$, i.e., the lab-frame value of $\kappa$ for elastic scattering

\footnotetext{
${ }^{32}$ See, e.g., $[227,287]$ for recent examples of such theses.
} 
of the neutrino straight backward from an infinitely-massive wall. For ${ }^{40} \mathrm{Ar}$, one may approximate the nuclear radius using eq. (3.185) to obtain $r_{k} \lesssim 0.021 \mathrm{MeV}$. For a $10 \mathrm{MeV}$ neutrino, setting these quantities equal to their upper limits yields $j_{0}\left(\kappa r_{k}\right) \approx 0.97$ and $j_{1}\left(\kappa r_{k}\right) \approx 0.14$, with smaller values $(<0.02)$ for $n>1$. Thus, the allowed approximation (which includes only the $n=0$ terms) should work reasonably well at this energy. For a $50 \mathrm{MeV}$ neutrino, however, applying the same procedure gives $j_{0}\left(\kappa r_{k}\right) \approx 0.41$ and $j_{1}\left(\kappa r_{k}\right) \approx 0.44$, demonstrating that the allowed approximation is no longer adequate.

While the estimates above provide a convenient means of checking whether the allowed approximation might be valid for a given neutrino energy, they are somewhat pessimistic. On average, $\kappa$ and $r_{k}$ will be signficantly smaller than their upper bounds. Nevertheless, more sophisticated calculations (e.g., $[225,288])$ of the cross section for charged current $\nu_{e}$ scattering on ${ }^{40} \mathrm{Ar}\left(\nu_{e}{ }^{40} \mathrm{ArCC}\right)$ suggest that, although the allowed $(n=0)$ terms remain dominant, forbidden $(n \neq 0)$ transitions become important at neutrino energies around $50 \mathrm{MeV}$ to $60 \mathrm{MeV}$.

Under the allowed approximation, the operator $\mathrm{j}^{\mu}$ is evaluated in the low momentum transfer limit $q \rightarrow 0$, and only terms that are zeroth order in $1 / m_{N}$ are kept. Applying these changes to eqs. (3.137) and (3.138) yields the following expressions for the positionspace free nucleon transition operator:

$$
\begin{aligned}
\hat{\mathcal{O}}^{\phi}(k) & =\sum_{J} \hat{\mathcal{O}}^{\phi}(k, J) \longrightarrow \hat{\mathcal{O}}^{\phi}(k, 0)=g_{V} t_{-}(k) \\
\hat{\mathcal{O}}^{M}(k) & =\sum_{J} \hat{\mathcal{O}}^{M}(k, J) \longrightarrow \hat{\mathcal{O}}^{M}(k, 1)=-g_{A} \sigma^{M}(k) t_{-}(k)
\end{aligned}
$$

Here $g_{V} \equiv F_{1}(0) \approx 1$ is the vector coupling constant of the nucleon. The components of the reduced nuclear matrix element tensor $\mathrm{O}^{\mu \nu}$ needed to compute $\left|\overline{\mathcal{T}}_{f i}\right|^{2}$ are given by the following expressions in this limit:

$$
\begin{aligned}
& \mathrm{O}^{\phi \phi} \longrightarrow \mathcal{M}_{F} \equiv g_{V}^{2}\left|\left\langle J_{f}\left\|\sum_{k=1}^{A} t_{-}(k)\right\| J_{i}\right\rangle\right|^{2} \\
& \mathrm{O}^{\phi 0} \longrightarrow 0
\end{aligned}
$$




$$
\mathrm{O}^{M M} \longrightarrow \mathcal{M}_{G T} \equiv \frac{g_{A}^{2}}{3}\left|\left\langle J_{f}\left\|\sum_{k=1}^{A} \boldsymbol{\sigma}(k) t_{-}(k)\right\| J_{i}\right\rangle\right|^{2}
$$

The quantity $\left|\overline{\mathcal{T}_{f i}}\right|^{2}$ may be written in this limit as

$$
\left|\overline{\mathcal{T}_{f i}}\right|^{2}=4 \mathcal{M}_{F}\left(E_{\ell} E_{\nu_{\ell}}+\mathbf{p}_{\ell} \cdot \mathbf{p}_{\nu_{\ell}}\right)+4 \mathcal{M}_{G T}\left(E_{\ell} E_{\nu_{\ell}}-\frac{1}{3} \mathbf{p}_{\ell} \cdot \mathbf{p}_{\nu_{\ell}}\right) .
$$

Therefore, the spin-summed squared amplitude $|\overline{\mathcal{M}}|^{2}$ becomes

$$
\begin{aligned}
|\overline{\mathcal{M}}|^{2} \approx 16 & G_{F}^{2}\left|V_{u d}\right|^{2} E_{\nu_{\ell}} E_{i} E_{\ell} E_{f} \\
& \times\left(B\left(\mathrm{~F}_{-}\right)\left[1+\boldsymbol{\beta}_{\ell} \cdot \boldsymbol{\beta}_{\nu_{\ell}}\right]+B\left(\mathrm{GT}_{-}\right)\left[1-\frac{1}{3} \boldsymbol{\beta}_{\ell} \cdot \boldsymbol{\beta}_{\nu_{\ell}}\right]\right),
\end{aligned}
$$

where the velocity $\boldsymbol{\beta}_{x}$ of particle $x$ is given by

$$
\beta_{x}=\frac{\mathbf{p}_{x}}{E_{x}} .
$$

The reduced Fermi and Gamow-Teller matrix elements $B\left(\mathrm{~F}_{-}\right)$and $B\left(\mathrm{GT}_{-}\right)$are given in the position-space representation by

$$
B\left(\mathrm{~F}_{-}\right) \equiv \frac{g_{V}^{2}\left|\left\langle J_{f}\left\|\sum_{k=1}^{A} t_{-}(k)\right\| J_{i}\right\rangle\right|^{2}}{2 J_{i}+1}
$$

and

$$
B\left(\mathrm{GT}_{-}\right) \equiv \frac{g_{A}^{2}\left|\left\langle J_{f}\left\|\sum_{k=1}^{A} \boldsymbol{\sigma}(k) t_{-}(k)\right\| J_{i}\right\rangle\right|^{2}}{2 J_{i}+1} .
$$

These matrix elements obey the selection rules

$$
B\left(\mathrm{~F}_{-}\right)=0 \text { unless } J_{f}=J_{i} \text { and } \pi_{f}=\pi_{i}
$$

and

$$
B\left(\mathrm{GT}_{-}\right)=0 \text { unless }\left|J_{i}-1\right| \leq J_{f} \leq J_{i}+1 \text { and } \pi_{f}=\pi_{i}
$$

where $\pi_{i}\left(\pi_{f}\right)$ is the intrinsic parity of the initial (final) nucleus. The angular momentum rules shown here follow from the Wigner-Eckart theorem (see section 3.8.2). The nuclear 
parity is conserved in both cases because the Fermi and Gamow-Teller operators are even under parity transformations.

Combining the results from eqs. (3.65) and (3.211) allows one to write

$$
\begin{aligned}
\frac{d \sigma}{d \cos \theta_{\ell}}= & \frac{G_{F}^{2}\left|V_{u d}\right|^{2}}{2 \pi}\left[\frac{E_{i} E_{f}}{s}\right] E_{\ell}\left|\mathbf{p}_{\ell}\right| \\
& \times\left(\left[1+\beta_{\ell} \cos \theta_{\ell}\right] B\left(\mathrm{~F}_{-}\right)+\left[1-\frac{1}{3} \beta_{\ell} \cos \theta_{\ell}\right] B\left(\mathrm{GT}_{-}\right)\right)
\end{aligned}
$$

where $\theta_{\ell}$ is the angle between the 3 -momenta of the incident neutrino and the outgoing lepton.

When applying the Coulomb corrections described in section 3.10 .3 to the allowed approximation cross section shown above, one should recall that the Lorentz-invariant amplitude $\mathcal{M}$ was evaluated in the $\kappa \rightarrow 0$ limit. To preserve this limit within the effective momentum approximation (for which a modified value of the final lepton 4-momentum $p_{\ell}$ is used), the calculation of $\mathcal{M}$ is left unchanged and only the rescaling factor $f_{C}^{2}$ is applied to the cross section. Furthermore, at the high neutrino energies needed for charged current $\nu_{\mu}$ scattering to occur, the allowed approximation is expected to be inadequate. Therefore, only charged current $\nu_{e}$ scattering for which $f_{C}=f_{\mathrm{EMA}}$ will be considered here.

We define the allowed approximation Coulomb correction factor $F_{C}$ by

$$
F_{C} \equiv \begin{cases}F\left(Z_{f}, \beta_{f, \mathrm{rel}}\right) & f_{\mathrm{EMA}}^{2}>F\left(Z_{f}, \beta_{f, \mathrm{rel}}\right) \\ f_{\mathrm{EMA}}^{2} & \text { otherwise }\end{cases}
$$

where $F\left(Z_{f}, \beta_{f, \text { rel }}\right)$ is defined in eq. (3.187) and $f_{\mathrm{EMA}}$ is evaluated in the laboratory frame as described in eq. (3.192). The allowed approximation differential cross section for charged current neutrino-nucleus scattering in the CM frame may now be written as

$$
\begin{aligned}
\frac{d \sigma}{d \cos \theta_{\ell}}= & \frac{G_{F}^{2}\left|V_{u d}\right|^{2}}{2 \pi}\left[\frac{\left(\sqrt{s}-E_{\ell}\right) E_{i}}{s}\right] F_{C} E_{\ell}\left|\mathbf{p}_{\ell}\right| \\
& \times\left(\left[1+\beta_{\ell} \cos \theta_{\ell}\right] B\left(\mathrm{~F}_{-}\right)+\left[1-\frac{1}{3} \beta_{\ell} \cos \theta_{\ell}\right] B\left(\mathrm{GT}_{-}\right)\right) .
\end{aligned}
$$

where it has been noted that $E_{f}=\sqrt{s}-E_{\ell}$ in the CM frame. The total cross section $\sigma$ 
is obtained by integrating eq. (3.219) over $\cos \theta_{\ell} \in[-1,1]$, yielding

$$
\sigma=\frac{G_{F}^{2}\left|V_{u d}\right|^{2}}{\pi}\left[\frac{\left(\sqrt{s}-E_{\ell}\right) E_{i}}{s}\right] F_{C} E_{\ell}\left|\mathbf{p}_{\ell}\right|\left[B\left(\mathrm{~F}_{-}\right)+B\left(\mathrm{GT}_{-}\right)\right] . \quad(\mathrm{CM} \text { frame, 3.220) }
$$

\subsection{Extension to charged current antineutrino scattering}

The differential cross section for charged current antineutrino-nucleus scattering may also be calculated using the formalism developed in the previous sections. To do so, first consider the Lorentz-invariant amplitude $\mathcal{M}$ for charged current scattering of an antineutrino on a free up quark. At tree level, the amplitude is given by

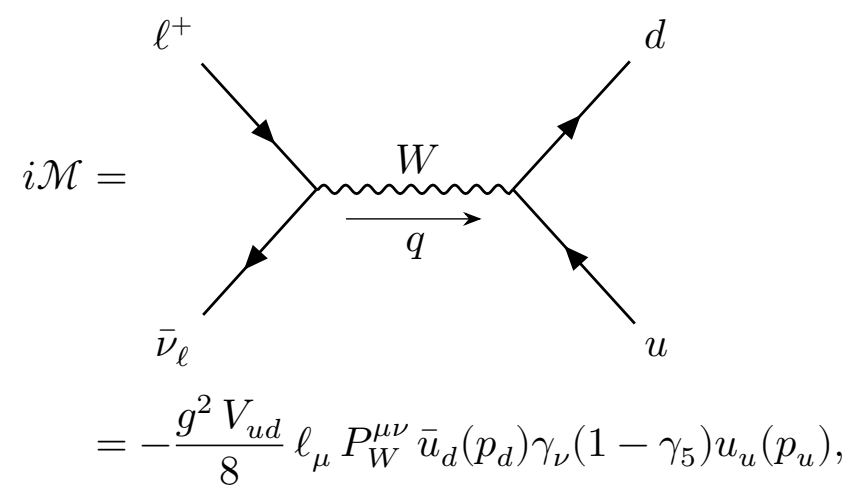

where

$$
\ell_{\mu}=\bar{v}_{\bar{\nu}_{\ell}}\left(p_{\bar{\nu}_{\ell}}\right) \gamma_{\mu}\left(1-\gamma_{5}\right) v_{\ell}\left(p_{\ell}\right)
$$

is the leptonic matrix element for antineutrino charged current scattering, and the other factors are defined as in eq. (3.3). Taking the low-energy limit of the $W$ boson propagator as was done in eq. (3.5) leads to the diagram

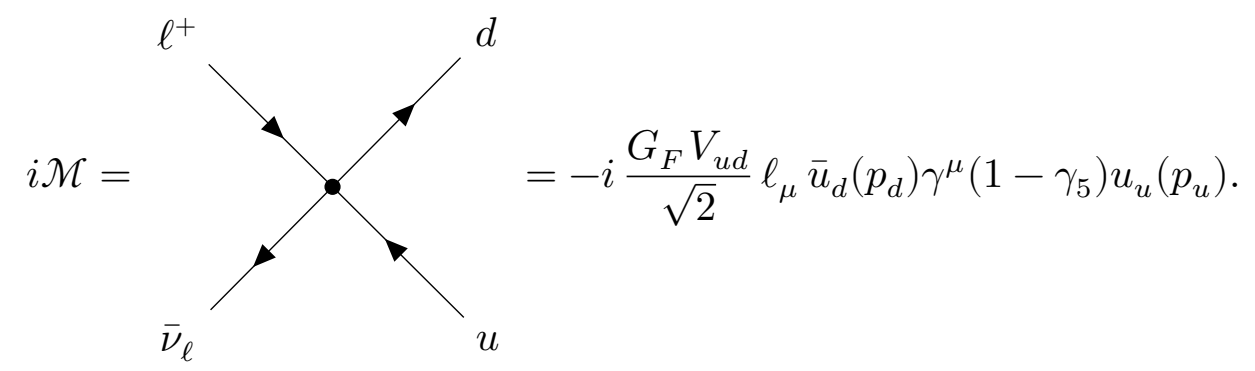

Comparing eqs. (3.6) and (3.223), one finds that the quark matrix element for the antineutrino case is the complex conjugate of that for the neutrino case, i.e.,

$$
\bar{u}_{d}\left(p_{d}\right) \gamma^{\mu}\left(1-\gamma_{5}\right) u_{u}\left(p_{u}\right)=\left[\bar{u}_{u}\left(p_{u}\right) \gamma^{\mu}\left(1-\gamma_{5}\right) u_{d}\left(p_{d}\right)\right]^{*}
$$


For any general bilinear of Dirac spinors $\bar{w}_{2} M w_{1}$, where $w \in\{u, v\}$ and $M$ is an arbitrary $4 \times 4$ matrix, the complex conjugate of the bilinear is given by [49]

$$
\left(\bar{w}_{2} M w_{1}\right)^{*}=\bar{w}_{1} \bar{M} w_{2}
$$

where $\bar{M}=\gamma^{0} M^{\dagger} \gamma^{0}$ is the Dirac adjoint of $M$.

Therefore, when considering the diagram describing antineutrino-nucleon scattering,

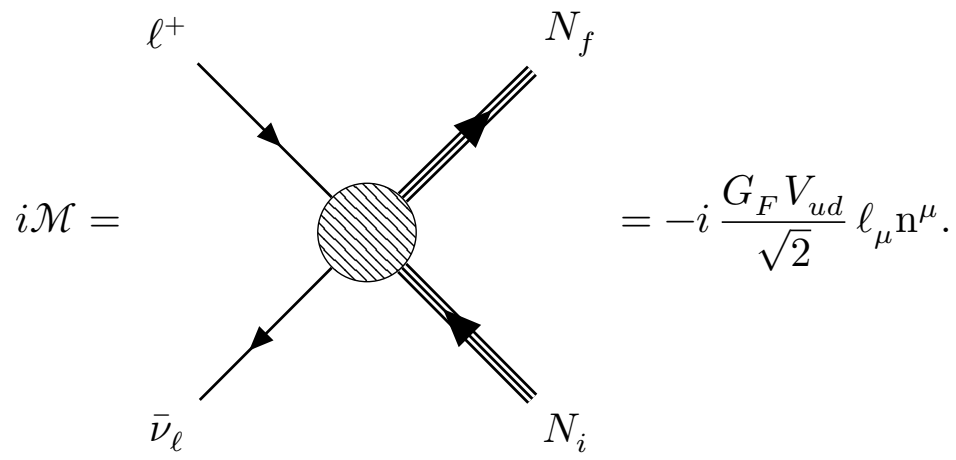

the free nucleon matrix element $\mathrm{n}^{\mu}$ will have the same form as in eq. (3.30) except for two needed changes. First, one must take the Dirac adjoint of the quantity in square brackets in order to account for the complex conjugation of the quark matrix element relative to the matter neutrino case. Making this change is equivalent to the substitutions $F_{2}\left(Q^{2}\right) \rightarrow-F_{2}\left(Q^{2}\right)$ and $G_{P}\left(Q^{2}\right) \rightarrow-G_{P}\left(Q^{2}\right)$. Second, one must make the substitution $t_{-} \rightarrow t_{+}$in order to switch from a $n \rightarrow p$ transition to a $p \rightarrow n$ transition in the isospin representation. Explicitly, for the antineutrino charged current case, the free nucleon matrix element $\mathrm{n}^{\mu}$ is given by

$$
\begin{aligned}
\mathrm{n}^{\mu}= & \chi_{N_{f}}^{\dagger} \bar{u}_{N_{f}}\left(p_{N_{f}}\right)\left[\gamma^{\mu} F_{1}\left(Q^{2}\right)-\frac{i}{2 m_{N}} \sigma^{\mu \nu} q_{\nu} F_{2}\left(Q^{2}\right)+\frac{q^{\mu}}{m_{N}} F_{3}\left(Q^{2}\right)\right. \\
& \left.-\gamma^{\mu} \gamma^{5} G_{A}\left(Q^{2}\right)+\frac{q^{\mu}}{m_{N}} \gamma^{5} G_{P}\left(Q^{2}\right)+\frac{i}{m_{N}} \sigma^{\mu \nu} q_{\nu} \gamma^{5} G_{3}\left(Q^{2}\right)\right] t_{+} u_{N_{i}}\left(p_{N_{i}}\right) \chi_{N_{i}} .
\end{aligned}
$$

To calculate the elements of the reduced nuclear matrix element tensor $\mathrm{O}^{\mu \nu}$ (see eq. (3.148)) for charged current antineutrino scattering, one need only make the same substitutions, i.e., $F_{2}\left(Q^{2}\right) \rightarrow-F_{2}\left(Q^{2}\right), G_{P}\left(Q^{2}\right) \rightarrow-G_{P}\left(Q^{2}\right)$, and $t_{-} \rightarrow t_{+}$wherever these quantities appear in the equations describing the neutrino-nucleus case.

For antineutrinos, the lepton tensor $\mathrm{L}_{\mu \nu}$ defined in eq. (3.101) may be expressed in Cartesian coordinates using the same expression as the neutrino case (see eq. (3.104)) 
except for a change in the sign of the last term:

$$
\mathrm{L}_{\mu \nu}=4\left[p_{\ell}^{\mu} p_{\bar{\nu}_{\ell}}^{\nu}+p_{\ell}^{\nu} p_{\bar{\nu}_{\ell}}^{\mu}-g^{\mu \nu}\left(p_{\ell} \cdot p_{\bar{\nu}_{\ell}}\right)+i \epsilon^{\mu \nu \rho \sigma} p_{\ell, \rho} p_{\bar{\nu}_{\ell}, \sigma}\right]
$$

The expressions for the spherical components of the lepton tensor in eqs. (3.118)-(3.120) may be used for the antineutrino case if the substitution $i \rightarrow-i$ is made for $\mathrm{L}_{M \varnothing}$, the substitution $K_{M N} \rightarrow-K_{M N}$ is made for $\mathrm{L}_{M N}$, and the subscript $\nu_{\ell}$ is replaced by $\bar{\nu}_{\ell}$ everywhere. The simplified expressions for the lepton tensor in eqs. (3.152)-(3.159) may be used for antineutrinos if the subscript substitution $\nu_{\ell} \rightarrow \bar{\nu}_{\ell}$ is made, which changes the lepton number factor to $L_{\bar{\nu}_{\ell}}=-1$.

With all of the adjustments mentioned above, the derivation of the first-order and allowed approximation cross sections for charged current antineutrino-nucleus scattering proceeds in the same manner as in the neutrino case. The allowed approximation cross section turns out to be exactly the same as in eqs. (3.219)-(3.220) except that (1) the reduced nuclear matrix elements $B\left(\mathrm{~F}_{+}\right)$and $B\left(\mathrm{GT}_{+}\right)$both contain the isospin raising operator $t_{+}$instead of $t_{-}$, and (2) the Coulomb correction factor $F_{C}$ from eq. (3.218) should now be redefined as

$$
F_{C} \equiv \begin{cases}F\left(Z_{f}, \beta_{f, \text { rel }}\right) & f_{\mathrm{EMA}}^{2}<F\left(Z_{f}, \beta_{f, \text { rel }}\right) \\ f_{\mathrm{EMA}}^{2} & \text { otherwise }\end{cases}
$$

\subsection{Extension to neutral current scattering}

The Standard Model describes the neutral current interaction using the Lagrangian density

$$
\mathcal{L}_{Z}=\frac{g}{\cos \theta_{W}}\left[J_{\mu}^{3}-\sin ^{2}\left(\theta_{W}\right) J_{\mu}^{\mathrm{EM}}\right] Z^{\mu}
$$

where $Z^{\mu}$ is the $Z$ boson field and $g$ is the $\mathrm{SU}(2)$ gauge coupling constant. The weak mixing angle $\theta_{W}$ is related to the masses of the $W$ and $Z$ bosons via

$$
\cos \theta_{W}=\frac{m_{W}}{m_{Z}}
$$

The weak neutral current $J_{\mu}^{3}$ and the electromagnetic current $J_{\mu}^{\mathrm{EM}}$ are given by

$$
J_{\mu}^{3}=\frac{1}{2} \sum_{F} I_{F}^{3} \bar{F} \gamma_{\mu}\left(1-\gamma^{5}\right) F
$$


and

$$
J_{\mu}^{\mathrm{EM}}=\sum_{F} q_{F} \bar{F} \gamma_{\mu} F
$$

where the sums are over all fundamental fermion fields ${ }^{33} F$ included in the Standard Model, and $q_{F}\left(I_{F}^{3}\right)$ is the electric charge (third component of weak isospin ${ }^{34}$ ) of the particle associated with the $F$ field (see table 3.1).

The Lorentz-invariant amplitude $\mathcal{M}$ for neutral current scattering of a neutrino on a free quark $\mathcal{Q}$ (of arbitrary flavor) may be computed at tree-level using the diagram

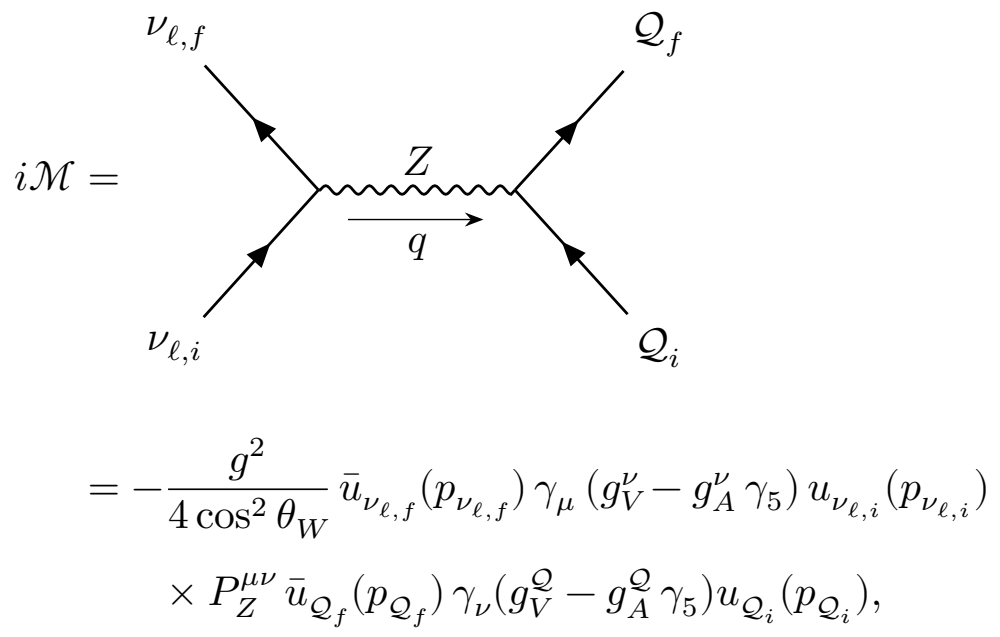

where

$$
P_{Z}^{\mu \nu}=i \frac{-g^{\mu \nu}+\frac{q^{\mu} q^{\nu}}{m_{Z}^{2}}}{q^{2}-m_{Z}^{2}+i \epsilon}
$$

is the $Z$ boson propagator. The vector $g_{V}^{F}$ and axial-vector $g_{A}^{F}$ coupling constants for neutral current interactions between fermion fields are given by

$$
g_{V}^{F}=I_{F}^{3}-2 q_{F} \sin ^{2} \theta_{W}
$$

and

$$
g_{A}^{F}=I_{F}^{3}
$$

Note that the flavor of the struck quark is conserved in this interaction, i.e., $\mathcal{Q}_{i}$ and $\mathcal{Q}_{f}$ have the same flavor.

\footnotetext{
${ }^{33}$ These may be expressed using either weak-eigenstate or mass-eigenstate fields since the unitary transformation that connects the two bases cancels out in the combination $\bar{F} F[59]$.

${ }^{34}$ Not to be confused with (strong) isospin
} 


\begin{tabular}{ccc}
\hline Fermions $F$ & Electric charge $q_{F}$ & Weak isospin $I_{F}^{3}$ \\
\hline$\nu_{e}, \nu_{\mu}, \nu_{\tau}$ & 0 & $1 / 2$ \\
$e^{-}, \mu^{-}, \tau^{-}$ & -1 & $-1 / 2$ \\
$u, c, t$ & $2 / 3$ & $1 / 2$ \\
$d, s, b$ & $-1 / 3$ & $-1 / 2$ \\
\hline
\end{tabular}

Table 3.1: Quantities needed to compute the vector $g_{V}^{F}$ and $g_{A}^{F}$ axial-vector coupling constants for neutral current scattering amplitudes.

As was the case for charged current neutrino scattering, it is adequate to approximate the propagator for the intermediate vector boson by its low-energy limit:

$$
P_{Z}^{\mu \nu} \approx \frac{i g^{\mu \nu}}{m_{Z}^{2}} .
$$

In this limit, the diagram shown in eq. (3.234) becomes

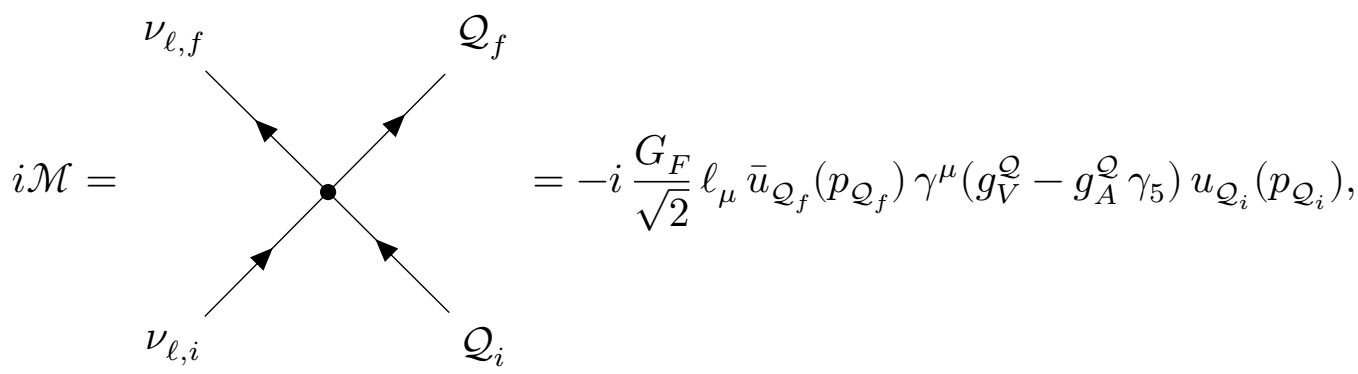

where the Fermi constant $G_{F}$ is given by

$$
\frac{G_{F}}{\sqrt{2}}=\frac{g^{2}}{8 m_{W}^{2}}=\frac{g^{2}}{8 m_{Z}^{2} \cos ^{2} \theta_{W}},
$$

and the neutrino neutral current leptonic matrix element is given by

$$
\ell_{\mu}=\bar{u}_{\nu_{\ell, f}}\left(p_{\nu_{\ell, f}}\right) \gamma_{\mu}\left(1-\gamma_{5}\right) u_{\nu_{\ell, i}}\left(p_{\nu_{\ell, i}}\right)
$$

To transition to neutrino-nucleon scattering, one must once again replace the quark part of the amplitude $\mathcal{M}$ with a hadronic matrix element $\mathrm{n}^{\mu}$ :

$$
\bar{u}_{\mathcal{Q}_{f}}\left(p_{\mathcal{Q}_{f}}\right) \gamma^{\mu}\left(g_{V}^{\mathcal{Q}}-g_{A}^{\mathcal{Q}} \gamma_{5}\right) u_{\mathcal{Q}_{i}}\left(p_{\mathcal{Q}_{i}}\right) \rightarrow \mathrm{n}^{\mu}
$$


After making this substitution, the diagram in eq. (3.239) becomes

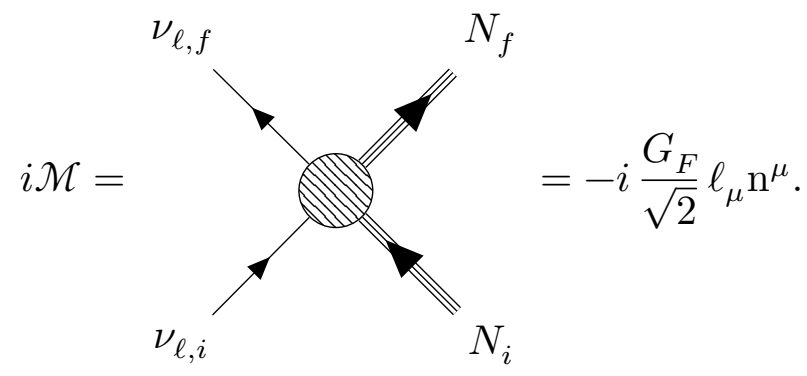

Since the leptonic matrix element $\ell_{\mu}$ for neutrino neutral current scattering has the same form as that for neutrino charged current scattering (compare eqs. (3.8) and (3.241)), it follows that eqs. (3.118)-(3.120) may be used to calculate the spherical components of the neutrino neutral current lepton tensor $\mathrm{L}_{\mu \nu}$ as long as one makes the substitutions $\nu_{\ell} \rightarrow \nu_{\ell, i}$ and $\ell \rightarrow \nu_{\ell, f}$. The simplified forms given in terms of the final lepton scattering angle from eqs. (3.152)-(3.159) may also be used with the same substitutions.

Symmetry arguments similar to those mentioned in section 3.2 lead to the following form [59] for the neutral current free nucleon matrix element $\mathrm{n}^{\mu}$ :

$$
\begin{aligned}
\mathrm{n}^{\mu}= & \chi_{N_{f}}^{\dagger} \bar{u}_{N_{f}}\left(p_{N_{f}}\right)\left[\gamma^{\mu} F_{1}^{Z}\left(Q^{2}\right)+\frac{i}{2 m_{N}} \sigma^{\mu \nu} q_{\nu} F_{2}^{Z}\left(Q^{2}\right)+\frac{q^{\mu}}{m_{N}} F_{3}^{Z}\left(Q^{2}\right)\right. \\
& \left.-\gamma^{\mu} \gamma^{5} G_{A}^{Z}\left(Q^{2}\right)-\frac{q^{\mu}}{m_{N}} \gamma^{5} G_{P}^{Z}\left(Q^{2}\right)+\frac{i}{m_{N}} \sigma^{\mu \nu} q_{\nu} \gamma^{5} G_{3}^{Z}\left(Q^{2}\right)\right] u_{N_{i}}\left(p_{N_{i}}\right) \chi_{N_{i}} .
\end{aligned}
$$

Note that this expression is identical to the form of $\mathrm{n}^{\mu}$ for neutrino charged current scattering (see eq. (3.30)) except that the isospin-lowering operator $t_{-}$has been removed, ${ }^{35}$ and the neutral current form factors $F_{1}^{Z}\left(Q^{2}\right), G_{A}^{Z}\left(Q^{2}\right)$, etc. have replaced their charged current counterparts. Therefore, by analogy with the procedure shown in section 3.4, we may define the momentum-space free nucleon transition operator $\mathrm{j}^{\mu}$ for neutral current scattering using the same expressions as in the neutrino charged current case but with the substitution $t_{-} \rightarrow 1$ and with the neutral current nucleon form factors substituted for the charged current ones. That is, one must make the substitutions $F_{X}\left(Q^{2}\right) \rightarrow F_{X}^{Z}\left(Q^{2}\right)$ and $G_{Y}\left(Q^{2}\right) \rightarrow G_{Y}^{Z}\left(Q^{2}\right)$ for all $X \in\{1,2,3\}$ and $Y \in\{A, P, 3\}$ in eqs. (3.77) and (3.78).

\footnotetext{
${ }^{35}$ As we shall see, however, the Pauli operator for the third isospin component, $\tau_{3}$, will appear in the isovector pieces of the neutral current form factors.
} 
It can be shown [59] that the neutral current form factors may be written in terms of the charged current ones defined in eqs. (3.124)-(3.131) according to the relations

$$
\begin{aligned}
& F_{i}^{Z N}\left(Q^{2}\right)= \pm \frac{1}{2} F_{i}\left(Q^{2}\right)-2 \sin ^{2}\left(\theta_{W}\right) F_{i}^{N}\left(Q^{2}\right)-\frac{1}{2} F_{i}^{s N}\left(Q^{2}\right) \\
& G_{j}^{Z N}\left(Q^{2}\right)= \pm \frac{1}{2} G_{j}\left(Q^{2}\right)-\frac{1}{2} G_{j}^{s N}\left(Q^{2}\right)
\end{aligned}
$$

where $i \in\{1,2\}, j \in\{A, P\}$, the nucleon type $N \in\{p, n\}$, and $+(-)$ should be chosen for $N=p(N=n)$.

The $F_{i}^{s N}\left(Q^{2}\right)$ and $G_{j}^{s N}\left(Q^{2}\right)$ are the strange form factors. They arise because the $Z$ boson that mediates the neutral weak interaction can couple to both the valence quarks and the sea quarks within the nucleon. It is believed that virtual $s \bar{s}$ pairs in the sea make the dominant contribution [59]. As in the charged current case, the second-class form factors $F_{3}^{Z}\left(Q^{2}\right)$ and $G_{3}^{Z}\left(Q^{2}\right)$ are typically taken to be zero. They will be included in the following discussion for completeness.

To obtain expressions for the neutral current form factors suitable for use in the isospin representation, first define the isospin projection operators:

$$
P_{n} \equiv \frac{1}{2}\left(1+\tau_{3}\right) \quad P_{p} \equiv \frac{1}{2}\left(1-\tau_{3}\right) .
$$

These operators act on neutron and proton states to give

$$
\begin{array}{ll}
P_{n}|n\rangle=|n\rangle & P_{n}|p\rangle=0 \\
P_{p}|n\rangle=0 & P_{p}|p\rangle=|p\rangle .
\end{array}
$$

Since the form factors appear within the free nucleon matrix element (and thus may be taken to act on the initial nucleon state vector), it follows from eqs. (3.245) and (3.246) that they may be rewritten in terms of these operators as

$$
\begin{aligned}
F_{i}^{Z}\left(Q^{2}\right)= & -\frac{1}{2} \tau_{3} F_{i}\left(Q^{2}\right)-2 \sin ^{2}\left(\theta_{W}\right)\left[F_{i}^{n}\left(Q^{2}\right) P_{n}+F_{i}^{p}\left(Q^{2}\right) P_{p}\right] \\
& -\frac{1}{2}\left[F_{i}^{s n}\left(Q^{2}\right) P_{n}+F_{i}^{s p}\left(Q^{2}\right) P_{p}\right]
\end{aligned}
$$




$$
G_{j}^{Z}\left(Q^{2}\right)=-\frac{1}{2} \tau_{3} G_{j}\left(Q^{2}\right)-\frac{1}{2}\left[G_{j}^{s n}\left(Q^{2}\right) P_{n}+G_{j}^{s p}\left(Q^{2}\right) P_{p}\right] .
$$

Plugging in the explicit forms for the isospin projection operators from eq. (3.247) allows one to write

$$
\begin{aligned}
& F_{i}^{Z}\left(Q^{2}\right)=F_{i I}^{Z}\left(Q^{2}\right)+F_{i \tau_{3}}^{Z}\left(Q^{2}\right) \tau_{3} \\
& G_{j}^{Z}\left(Q^{2}\right)=G_{j I}^{Z}\left(Q^{2}\right)+G_{j \tau_{3}}^{Z}\left(Q^{2}\right) \tau_{3}
\end{aligned}
$$

where the isoscalar neutral current form factors are given by

$$
\begin{aligned}
& F_{i I}^{Z}\left(Q^{2}\right)=-\frac{1}{4}\left[F_{i}^{s n}\left(Q^{2}\right)+F_{i}^{s p}\left(Q^{2}\right)\right]-\sin ^{2}\left(\theta_{W}\right)\left[F_{i}^{n}\left(Q^{2}\right)+F_{i}^{p}\left(Q^{2}\right)\right] \\
& G_{j I}^{Z}\left(Q^{2}\right)=-\frac{1}{4}\left[G_{j}^{s n}\left(Q^{2}\right)+G_{j}^{s p}\left(Q^{2}\right)\right]
\end{aligned}
$$

and the isovector neutral current form factors are given by

$$
\begin{aligned}
F_{i \tau_{3}}^{Z}\left(Q^{2}\right) & =\sin ^{2}\left(\theta_{W}\right)\left[F_{i}^{p}\left(Q^{2}\right)-F_{i}^{n}\left(Q^{2}\right)\right]-\frac{1}{2} F_{i}\left(Q^{2}\right)+\frac{1}{4}\left[F_{i}^{s p}\left(Q^{2}\right)-F_{i}^{s n}\left(Q^{2}\right)\right] \\
G_{j \tau_{3}}^{Z}\left(Q^{2}\right) & =\frac{1}{4}\left[G_{j}^{s p}\left(Q^{2}\right)-G_{j}^{s n}\left(Q^{2}\right)\right]-\frac{1}{2} G_{j}\left(Q^{2}\right) .
\end{aligned}
$$

With the replacement $t_{-} \rightarrow 1$ and the neutral current form factors substituted for the corresponding charged current ones, the derivation of the nuclear part of the cross section proceeds in the same way as in the neutrino charged current case until just after eqs. (3.169) and (3.170). In order to write expressions similar to eqs. (3.171) and (3.172) for the neutral current case, first define the isoscalar and isovector pieces of the neutral current $\mathcal{G}$ form factors:

$$
\begin{aligned}
\mathcal{G}_{\mu}^{Z}\left(Q^{2}\right) & =\mathcal{G}_{\mu I}^{Z}\left(Q^{2}\right)+\mathcal{G}_{\mu \tau_{3}}^{Z}\left(Q^{2}\right) \\
\mathcal{G}_{\phi b}^{Z}\left(Q^{2}\right) & \equiv \frac{\kappa}{m_{N}}\left[\frac{q^{\phi}}{2 m_{N}} G_{P b}^{Z}\left(Q^{2}\right)-\frac{1}{2} G_{A b}^{Z}\left(Q^{2}\right)-G_{3 b}^{Z}\left(Q^{2}\right)\right] \\
\mathcal{G}_{M b}^{Z}\left(Q^{2}\right) & \equiv \frac{\kappa}{m_{N}}\left[M \frac{F_{1 b}^{Z}\left(Q^{2}\right)-F_{2 b}^{Z}\left(Q^{2}\right)}{2 m_{N}}-G_{A b}^{Z}\left(Q^{2}\right)+\frac{\kappa^{2}}{2 m_{N}^{2}} \delta_{M 0} G_{P b}^{Z}\left(Q^{2}\right)\right] .
\end{aligned}
$$


where $\mu \in\{\varnothing,-1,0,1\}, M \in\{-1,0,1\}$, and $b \in\left\{I, \tau_{3}\right\}$. Then the elements of the reduced nuclear matrix element tensor $\mathrm{O}^{\mu \nu}$ may be calculated for neutral current scattering via

$$
\begin{gathered}
\left\langle J_{f}\left\|\hat{\mathcal{N}}^{\varnothing}(J)\right\| J_{i}\right\rangle=R^{\varnothing}(I)+R^{\phi}\left(\tau_{3}\right) \\
\left\langle J_{f}\left\|\hat{\mathcal{N}}^{M}(J)\right\| J_{i}\right\rangle=R^{M}(I)+R^{M}\left(\tau_{3}\right)
\end{gathered}
$$

where

$$
\begin{aligned}
R^{\phi}(\Theta) & \equiv F_{1 \Theta}^{Z}\left(Q^{2}\right) \mathcal{N}_{J}^{V}(\Theta)-\frac{G_{A \Theta}^{Z}\left(Q^{2}\right)}{m_{N}} \mathcal{N}_{J}^{A}(\Theta)+\mathcal{G}_{\not \Theta}^{Z}\left(Q^{2}\right) \mathcal{N}_{J 0}^{A}(\Theta) \\
R^{M}(\Theta) & \equiv \frac{\delta_{M 0} \kappa}{m_{N}}\left[\frac{1}{2} F_{1 \Theta}^{Z}\left(Q^{2}\right)+F_{3 \Theta}^{Z}\left(Q^{2}\right)\right] \mathcal{N}_{J}^{V}(\Theta) \\
& +\frac{F_{1 \Theta}^{Z}\left(Q^{2}\right)}{m_{N}} \mathcal{N}_{J M}^{V}(\Theta)+\mathcal{G}_{M \Theta}^{Z}\left(Q^{2}\right) \mathcal{N}_{J M}^{A}(\Theta)
\end{aligned}
$$

$I$ is the identity operator in isospace, $\Theta \in\left\{I, \tau_{3}\right\}$, and the four basic nuclear matrix elements $\mathcal{N}$ are given in eqs. (3.173)-(3.176).

To first order in $1 / m_{N}$ and neglecting the neutrino masses, the neutral current neutrinonucleus differential cross section may be written in the form

$$
\frac{d \sigma}{d \cos \theta_{\ell}}=\frac{|\overline{\mathcal{M}}|^{2}}{32 \pi s} \frac{E_{\nu_{\ell, f}}}{E_{\nu_{\ell, i}}}
$$

where $|\overline{\mathcal{M}}|^{2}$ is given in eq. (3.149) with the substitution $\left|V_{u d}\right|^{2} \rightarrow 1$. The elements of the reduced nuclear matrix element tensor $\mathrm{O}^{\mu \nu}$ defined in eq. (3.148) may be computed using eqs. (3.173)-(3.176) together with eqs. (3.261)-(3.265), and the elements of the lepton tensor $\mathrm{L}_{\mu \nu}$ may be evaluated in terms of $\cos \theta_{\nu_{\ell, f}}$ using eqs. (3.152)-(3.159) with the substitutions $\nu_{\ell} \rightarrow \nu_{\ell, i}$ and $\ell \rightarrow \nu_{\ell, f}$.

Just as in the charged current case, when the allowed approximation is applied to the calculation of the neutral current cross section, the only terms that survive in the free nucleon transition operator $\mathrm{j}^{\mu}$ are those that are proportional to the $Q^{2} \rightarrow 0$ limit of the 
vector $F_{1}^{Z}\left(Q^{2}\right)$ and axial-vector $G_{A}^{Z}\left(Q^{2}\right)$ form factors. In this limit, these two form factors may be written as

$$
\begin{aligned}
& F_{1}^{Z}(0)=g_{V}\left[\left(\tau_{3}-1\right) \sin ^{2}\left(\theta_{W}\right)-\frac{1}{2} \tau_{3}\right] \\
& G_{A}^{Z}(0)=-\frac{1}{2}\left[g_{A} \tau_{3}+G_{A}^{s}(0)\right]
\end{aligned}
$$

where $G_{A}^{s}(0)$ is the strange axial form factor of the nucleon evaluated at zero momentum transfer. In the zero momentum transfer limit $Q^{2} \rightarrow 0$, the strange vector form factor $F_{1}^{s}\left(Q^{2}\right)$ must vanish because the nucleon has no net strangeness. This form factor has therefore been neglected in eq. (3.267).

The strange axial form factor $G_{A}^{s}\left(Q^{2}\right)$ is currently not well understood. The available experimental data favor a small negative value $G_{A}^{s}(0) \approx-0.1$, while theoretical calculations give a value closer to zero [205]. Future measurements by neutrino experiments like MicroBooNE may be capable of improving the existing constraints [289]. Because the contribution of $G_{A}^{s}\left(Q^{2}\right)$ to the neutral current axial form factor $G_{A}^{Z}\left(Q^{2}\right)$ is expected to be small in the limit of zero momentum transfer, we will make the approximation $G_{A}^{s}(0) \approx 0$ in the following calculations.

Under the allowed approximation (and neglecting sea quark contributions), the components of the position-space free nucleon transition operator for neutral current neutrino scattering become

$$
\begin{aligned}
\hat{\mathcal{O}}^{\phi}(k) & =\sum_{J} \hat{\mathcal{O}}^{\varnothing}(k, J) \longrightarrow \hat{\mathcal{O}}^{\phi}(k, 0)=g_{V}\left[\left(\tau_{3}(k)-1\right) \sin ^{2}\left(\theta_{W}\right)-\frac{1}{2} \tau_{3}(k)\right] \\
\hat{\mathcal{O}}^{M}(k) & =\sum_{J} \hat{\mathcal{O}}^{M}(k, J) \longrightarrow \hat{\mathcal{O}}^{M}(k, 1)=\frac{1}{2} g_{A} \sigma^{M}(k) \tau_{3}(k) .
\end{aligned}
$$

The components of the reduced nuclear matrix element tensor $\mathrm{O}^{\mu \nu}$ become

$$
\begin{aligned}
\mathrm{O}^{\phi \phi} & \longrightarrow \mathcal{M}_{F}^{Z} \equiv g_{V}^{2}\left|\left\langle J_{f}\left\|\sum_{k=1}^{A}\left[\left(\tau_{3}(k)-1\right) \sin ^{2}\left(\theta_{W}\right)-\frac{1}{2} \tau_{3}(k)\right]\right\| J_{i}\right\rangle\right|^{2} \\
\mathrm{O}^{\phi 0} & \longrightarrow 0 \\
\mathrm{O}^{M M} & \longrightarrow \mathcal{M}_{G T}^{Z} \equiv \frac{g_{A}^{2}}{12}\left|\left\langle J_{f}\left\|\sum_{k=1}^{A} \boldsymbol{\sigma}(k) \tau_{3}(k)\right\| J_{i}\right\rangle\right|^{2}
\end{aligned}
$$


Since $\tau_{3}|n\rangle=+|n\rangle$ and $\tau_{3}|p\rangle=-|p\rangle$, the sum in eq. (3.271) may be evaluated to obtain

$$
\mathcal{M}_{F}^{Z}=\frac{1}{4} g_{V}^{2} Q_{W}^{2}\left|\left\langle J_{f}\|\mathbf{1}\| J_{i}\right\rangle\right|^{2}
$$

where the weak nuclear charge $Q_{W}$ for a nucleus with neutron number $N$ and proton number $Z$ is given by

$$
Q_{W}=N-\left[1-4 \sin ^{2}\left(\theta_{W}\right)\right] Z .
$$

For massless neutrinos, the quantity $\left|\overline{\mathcal{T}_{f i}}\right|^{2}$ in this limit is given by

$$
\left|\overline{\mathcal{T}}_{f i}\right|^{2} \approx 4 E_{\nu_{\ell, i}} E_{\nu_{\ell, f}}\left(\mathcal{M}_{F}^{Z}\left[1+\cos \theta_{\nu_{\ell, f}}\right]+\mathcal{M}_{G T}^{Z}\left[3-\cos \theta_{\nu_{\ell, f}}\right]\right)
$$

The spin-summed squared amplitude $|\overline{\mathcal{M}}|^{2}$ becomes

$$
|\overline{\mathcal{M}}|^{2} \approx 4 G_{F}^{2} E_{i} E_{f} E_{\nu_{\ell, i}} E_{\nu_{\ell, f}}\left(Q_{W}^{2} B\left(\mathrm{~F}_{3}\right)\left[1+\cos \theta_{\nu_{\ell, f}}\right]+B\left(\mathrm{GT}_{3}\right)\left[1-\frac{1}{3} \cos \theta_{\nu_{\ell, f}}\right]\right)
$$

where the neutral current reduced Fermi and Gamow-Teller matrix elements $B\left(\mathrm{~F}_{3}\right)$ and $B\left(\mathrm{GT}_{3}\right)$ are given in the position-space representation by

$$
B\left(\mathrm{~F}_{3}\right) \equiv \frac{g_{V}^{2}\left|\left\langle J_{f}\|\mathbf{1}\| J_{i}\right\rangle\right|^{2}}{2 J_{i}+1}
$$

and

$$
B\left(\mathrm{GT}_{3}\right) \equiv \frac{g_{A}^{2}\left|\left\langle J_{f}\left\|\sum_{k=1}^{A} \boldsymbol{\sigma}(k) \tau_{3}(k)\right\| J_{i}\right\rangle\right|^{2}}{2 J_{i}+1} .
$$

These reduced matrix elements obey the same selection rules as in the charged current case (see eqs. (3.215)-(3.216)). No final-state Coulomb correction is needed for the neutral current case since the final-state lepton is a neutrino, which is electrically neutral. Using eqs. (3.65) and (3.277), one may write the allowed approximation differential cross section for neutral current neutrino nucleus scattering in the form

$$
\begin{aligned}
\frac{d \sigma}{d \cos \theta_{\ell}}= & \frac{G_{F}^{2}}{8 \pi}\left[\frac{\left(\sqrt{s}-E_{\nu_{\ell, f}}\right) E_{i}}{s}\right] E_{\nu_{\ell, f}}^{2} \\
& \times\left(\left[1+\cos \theta_{\nu_{\ell, f}}\right] Q_{W}^{2} B\left(\mathrm{~F}_{3}\right)+\left[1-\frac{1}{3} \cos \theta_{\nu_{\ell, f}}\right] B\left(\mathrm{GT}_{3}\right)\right)
\end{aligned}
$$

(CM frame, 3.280) 
where $\theta_{\nu_{\ell, f}}$ is the angle between the final neutrino 3-momentum and the incident neutrino 3 -momentum. Integrating eq. (3.280) over $\cos \theta_{\nu_{\ell, f}} \in[-1,1]$ gives the total cross section

$$
\sigma=\frac{G_{F}^{2}}{4 \pi}\left[\frac{\left(\sqrt{s}-E_{\nu_{\ell, f}}\right) E_{i}}{s}\right] E_{\nu_{\ell, f}}^{2}\left[Q_{W}^{2} B\left(\mathrm{~F}_{3}\right)+B\left(\mathrm{GT}_{3}\right)\right] .
$$

For neutral current scattering, $\mathrm{n}^{\mu}$ is the same for both neutrinos and antineutrinos. However, since the leptonic matrix element is given by

$$
\ell_{\mu}=\bar{v}_{\bar{\nu}_{\ell, i}}\left(p_{\bar{\nu}_{\ell, i}}\right) \gamma_{\mu}\left(1-\gamma_{5}\right) v_{\bar{\nu}_{\ell, f}}\left(p_{\bar{\nu}_{\ell, f}}\right)
$$

for the case of antineutrino neutral current scattering, it follows that one should use the same lepton tensor as in the antineutrino charged current case (see eq. (3.228) and the discussion immediately following it) but with the substitutions $\bar{\nu}_{\ell} \rightarrow \bar{\nu}_{\ell, i}$ and $\ell \rightarrow$ $\bar{\nu}_{\ell, f}$. Under the allowed approximation, the neutral current differential cross section for antineutrino-nucleus scattering is the same as that for the neutrino-nucleus case.

It is instructive to note that, since $B(\mathrm{~F})$ in the neutral current case contains the reduced matrix element of the identity operator (see eq. (3.278)), it is nonvanishing only when the initial and final nuclear states are identical. The $B(\mathrm{~F})$ terms in eqs. (3.280) and (3.281) therefore represent the allowed approximation cross section for coherent elastic neutrino-nucleus scattering, a reaction whose existence was first predicted theoretically [290] by Freedman in 1974 and confirmed experimentally [291] by the COHERENT collaboration in 2017. 


\section{Chapter 4}

\section{Nuclear de-excitation model}

After an initial neutrino-nucleus scattering reaction, the final-state nucleus may emit one or more particles as it loses any available excess energy. In order to correctly reconstruct the energy of the original neutrino, the experimentalist must account for this excess energy by detecting the particles from the nuclear de-excitations. While it is straightforward (at least in principle) to measure the energies of de-excitation $\gamma$-rays and charged nuclear fragments, it is often much more difficult to account for energy lost to emitted neutrons. An additional complication arises because, for all but the lightest nuclei, the average binding energy per nucleon is roughly $8 \mathrm{MeV}$. Thus, if one fails to determine that a low-energy neutrino scattering event involved the emission of a nucleon or a light ion, the missing binding energy will create a significant systematic error on the neutrino energy measurement. This error will persist even with perfect reconstruction of all final-state particle kinetic energies. A realistic model of the nuclear de-excitations that may occur in response to low-energy neutrino scattering would therefore be helpful to experimentalists attempting to characterize the response of a detector to neutrinos. This chapter discusses the creation of such a model using a combination of nuclear data and semi-empirical formulae describing global nuclear structure properties.

\subsection{Compound nuclear reactions}

Nuclear reactions are often modeled as proceeding via one of two idealized general mechanisms. In a direct reaction, the incident projectile strikes a single nucleon or a few 
nucleons near the nuclear surface. The reaction products leave the nucleus quickly (perhaps within $10^{-22} \mathrm{~s}$ or so), without experiencing additional collisions on their way out. Compound-nucleus reactions, on the other hand, occur when a projectile scatters on the nucleus with a small impact parameter. The struck particles within the nucleus rescatter many times, creating a relatively long-lived (roughly on the order of $10^{-18}$ to $10^{-16} \mathrm{~s}$ ) state of thermal equilibrium that persists until random fluctuations concentrate enough energy on a nucleon or a light nuclear fragment for it to escape [292, 293].

To the extent that nuclear de-excitations induced by low-energy neutrino scattering are considered in the theoretical literature at all, it is usually assumed that it is adequate to model the neutrino-nucleus scattering process at low energies as proceeding via the formation of a compound nucleus [200, 294-301]. One may possibly justify this approach by noting that neutrinos with energies of tens-of-MeV or lower have de Broglie wavelengths that are considerably longer than a typical nuclear diameter. ${ }^{1}$ At these energies, then, the incident neutrino does not "see" individual nucleons but instead may be thought of as interacting with the nucleus as a whole. One might therefore expect the contribution of direct neutrino-nucleus reactions to the total cross section to be relatively unimportant in this energy range.

Although the available neutrino-nucleus scattering data are too limited $^{2}$ to provide a meaningful constraint on possible direct reaction channels in the tens-of-MeV energy range, the results of electron scattering experiments may shed light on the validity of the compound nucleus assumption in the neutrino case. For example, experimental measurements by Flowers et al. in 1978 found that the energy spectra of alpha particles (shown in fig. 4.1) produced in ${ }^{60} \mathrm{Ni}(e, \alpha) e^{\prime} X$ scattering reactions were well-described by a broad peak predicted by a compound nuclear decay model and a small high-energy "tail" attributable to direct reactions [302]. As expected, the tail representing the direct reaction contribution to the alpha spectrum was obvious in the data for $120 \mathrm{MeV}$ electrons,

\footnotetext{
${ }^{1}$ For example, a $50 \mathrm{MeV}$ neutrino has a de Broglie wavelength of about $25 \mathrm{fm}$, while an $A=40$ nucleus has a diameter of roughly $8 \mathrm{fm}$.

${ }^{2}$ In the energy regime of interest for supernova neutrinos, the only cross section measurements with $\sim 10-20 \%$ uncertainties to date are for ${ }^{12} \mathrm{C}$ [156]. The existing measurements do not include cross sections for processes that involve the emission of nuclear fragments, so they cannot be used to determine the relative contributions of direct and compound reaction mechanisms.
} 


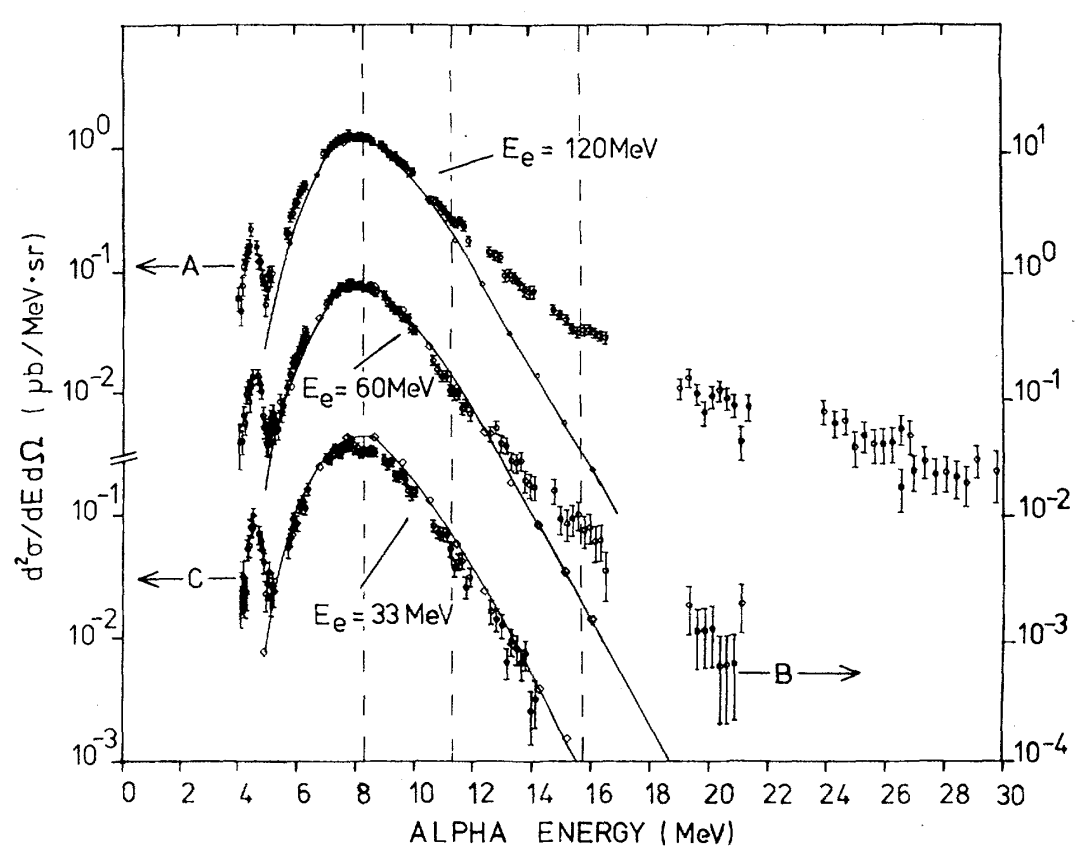

Figure 4.1: Measurement by Flowers et al. [302] of the energy spectra of alpha particles emitted in ${ }^{60} \mathrm{Ni}(e, \alpha) e^{\prime} X$ reactions at $\theta_{\alpha}=90^{\circ}$. Data were obtained for electron energies of $120 \mathrm{MeV}$ (curve A, upper left-hand scale), $60 \mathrm{MeV}$ (curve B, right-hand scale), and

$33 \mathrm{MeV}$ (curve $\mathrm{C}$, lower left-hand scale). The solid lines show predicted spectra obtained using a compound nucleus model. Angular distribution measurements (not shown here) were made at the mean energies marked by the vertical dashed lines.

less prominent for $60 \mathrm{MeV}$ electrons, and essentially absent for $33 \mathrm{MeV}$ electrons. The evidence provided by these results for the formation of compound nuclear states by a tensof-MeV leptonic projectile lends greater plausibility to the naïve de Broglie wavelength argument presented earlier.

At present, published theoretical investigations of the relative importance of direct and compound processes in low energy neutrino scattering appear to be limited to a pair of studies published in 2009 and 2011 by Kim and Cheoun [303, 304]. For tensof- $\mathrm{MeV}$ neutrino scattering on ${ }^{12} \mathrm{C}$, they estimated that the direct contribution to the cross sections is a factor of a few smaller than the compound contribution. From this result, they concluded that, "the direct ... processes might have effects small enough to be neglected" [304]. Given the current dearth of both experimental and theoretical information on this topic, MARLEY assumes, as a first approximation, that low-energy 
neutrino scattering will always result in the formation of a compound nucleus.

The notion of a compound nucleus was first introduced by Niels Bohr in the mid nineteen thirties. Prompted by neutron bombardment experiments performed by Enrico Fermi and others [305], Bohr proposed in a series of three papers [306-308] between 1936 and 1937 that

in order to understand the typical features of nuclear transmutations initiated by impacts of material particles it is necessary to assume that the first stage of any such collision process consists in the formation of an intermediate semistable system composed of the original nucleus and the incident particle. The excess energy must in this state be assumed to be temporarily stored in some complicated motions of all the particles in the compound system. [307]

On account of the strong forces which come into play between any two material particles at the small distances in question, the coupling between the particles of this compound system is in fact so intimate that its eventual disintegration — whether it consists in the release of an "elementary" particle like a proton or a neutron, or of a "complex" nuclear particle like a deuteron or an $\alpha$-ray - must be considered as a separate event, independent of the first stage of the collision process. The final result of the collision may thus be said to depend on a free competition between all the various disintegration and radiation processes of the compound system consistent with the general conservation laws. [308]

This idealized, qualitative description of nuclear scattering has become known in the literature as the Bohr independence hypothesis [309].

For the treatment of nuclear de-excitations presented here, it is important to make two observations about Bohr's compound nucleus model. First, as Bohr himself noted in his early papers, this model assumes that a compound nuclear state may be formed for an arbitrary kinetic energy of the incident projectile. Because the allowed nuclear energy levels, like those of any bound quantum mechanical system, are discrete, it follows that this assumption is only valid at relatively high excitation energies where the nuclear density 
of states is large enough that it is a good approximation to treat the allowed energies as forming a continuum. This condition is satisfied for medium and heavy compound nuclei formed by fast neutrons [307]. It is also satisfied by other projectiles whose kinetic energies are sufficient to create a compound state with an excitation energy that significantly exceeds the neutron emission threshold.

Second, Bohr's treatment assumes that the decay of the compound nucleus may be taken to be an independent event from its formation due to thermal equilibration. Following the initial collision, the projectile's energy is shared so widely among the compound system's constituent nucleons that any "memory" of how the compound state was originally formed (beyond that needed to conserve energy, momentum, etc.) is lost. This "amnesic" quality of the compound nucleus, namely that decays of a compound nuclear state occur in the same way regardless of how the state was originally formed, will prove useful for the quantitative treatment of compound nuclear decays presented in the next section.

\subsection{The Hauser-Feshbach statistical model}

Shortly after Bohr proposed his compound nucleus picture of nuclear reactions, the theory was made more quantitative in a 1937 paper [310] by Victor Weisskopf, who was among the first to point out that one could apply statistical methods to understand the decays of a compound nucleus. For such a nucleus, he argued,

the individual properties of the separate nuclear quantum states are then of no interest, on account of the extremely small distance between the energy levels of highly excited heavy nuclei; it is thus possible to obtain statistical information on the behavior of these nuclei by averaging over many quantum states of approximately the same energy. [310]

Weisskopf then proceeded to derive an expression for the average decay rate of a compound nucleus within an excitation energy interval containing many nuclear levels. Later work by Wolfenstein [311] and Hauser and Feshbach [312] refined Weisskopf's original treatment by considering the angular momentum dependence of compound nuclear decays. 
This improved theory of compound nuclei has come to be known as the Hauser-Feshbach statistical model.

Despite its emergence when nuclear physics was still a relatively young discipline, the Hauser-Feshbach theory (in tandem with other models) is used by modern nuclear reaction codes like TALYS [313, 314], EMPIRE [315], CCONE [316], and $\mathrm{CoH}_{3}$ [317] to calculate cross sections. Hauser-Feshbach calculations performed with these codes have been used in the process of creating evaluated nuclear data libraries, such as ENDF/B-VIII.0 [318] and JENDL-4.0 [319], that are widely used for a variety of applications.

For the present discussion, the observables of interest are the partial decay widths ${ }^{3}$ of a compound nucleus that was formed by a neutrino interaction. In the remainder of this section, we will derive an expression based on the Hauser-Feshbach model that can be used to calculate these decay widths. This derivation largely follows Weisskopf's original chain of reasoning, with two major differences: (1) Hauser and Feshbach's later treatment of the effects of angular momentum and parity conservation is incorporated, and (2) the treatment is fully relativistic.

Consider an arbitrary nucleus $A$ with excitation energy $E_{x, A}$ (measured from the ground state of $A$ ), total energy $E_{A}$, rest mass $m_{A}$, total spin $s_{A}$, and intrinsic parity $\Pi_{A}$. Also consider an arbitrary projectile $a$ (with total energy $E_{a}$, rest mass $m_{a}$, total spin $s_{a}$, and intrinsic parity $\pi_{a}$ ) such that $a$ and $A$ may undergo the capture reaction

$$
a+A \rightarrow C
$$

to form a compound nucleus $C$ with excitation energy $E_{x}$ (measured from the ground state of $C$ ), total energy $E$, total spin $J$, and intrinsic parity $\Pi$. This reaction must satisfy the conservation laws

$$
\begin{aligned}
E_{a}+E_{A} & =E \\
\boldsymbol{\ell}+\boldsymbol{s}_{a}+\boldsymbol{s}_{A} & =\mathbf{J} \\
\pi_{a} \Pi_{A}(-1)^{\ell} & =\Pi
\end{aligned}
$$

\footnotetext{
${ }^{3} \mathrm{Or}$, equivalently in natural units, the partial decay rates
} 
where $\ell$ is the orbital angular momentum of the $a+A$ system. We will also make use of the total projectile angular momentum

$$
\mathbf{j} \equiv \boldsymbol{\ell}+\boldsymbol{s}_{a} .
$$

Suppose that the projectile energy $E_{a}$ is not precisely known, but instead lies within a small interval $\left[E_{a}, E_{a}+d E_{a}\right]$ sufficiently large that there are many accessible states of the compound nucleus $C$ that may be formed but sufficiently small that slowly varying functions of the projectile energy (e.g., the projectile 3-momentum) may be treated as constant. Imagine that $a$ and $A$ are enclosed in a large volume $V$. Then the energy-averaged value (over the range of projectile energies mentioned previously) of the transition rate $\left\langle\Gamma_{a+A \rightarrow C}\right\rangle$ for the capture reaction in eq. (4.1) to occur is given by

$$
\left\langle\Gamma_{a+A \rightarrow C}\right\rangle=\left\langle\sigma_{C}\right\rangle \phi
$$

where the incident particle flux $\phi$ is given by eq. (3.44) with the substitutions $i, 1 \rightarrow a$ and $i, 2 \rightarrow A$. Specializing to the CM frame allows one to write the flux $\phi$ in the form

$$
\phi=\frac{E\left|\mathbf{p}_{\alpha}\right|}{V E_{a} E_{A}} .
$$

The factor $\left\langle\sigma_{C}\right\rangle$ is the energy-averaged ${ }^{4}$ compound nucleus formation cross section. It can be shown (see appendix A) that this cross section may be written in terms of CM frame quantities as

$$
\left\langle\sigma_{C}\right\rangle=\frac{\pi(2 J+1)}{\left|\mathbf{p}_{\alpha}\right|^{2}\left(2 s_{a}+1\right)\left(2 s_{A}+1\right)} \sum_{\ell j} \delta_{\pi} T_{\ell j}(\varepsilon)
$$

where $\left|\mathbf{p}_{\alpha}\right|$ is the magnitude of the 3 -momentum of either $a$ or $A$ in the CM frame, ${ }^{5}$ and $\varepsilon$ is the total kinetic energy in the CM frame:

$$
\varepsilon=E-m_{a}-m_{A} .
$$

The transmission coefficient ${ }^{6} T_{\ell j}(\varepsilon)$ represents the average probability that the incoming projectile's partial wave with orbital angular momentum $\ell$ and total angular

\footnotetext{
${ }^{4}$ In this case, the averaging has been performed over the interval $\left[\varepsilon, \varepsilon+d E_{a}\right]$.

${ }^{5}$ By definition, $\mathbf{p}_{a}=-\mathbf{p}_{A}$ in the CM frame.

${ }^{6}$ Bethe refers to the transmission coefficient as the "sticking probability" [320].
} 
momentum $j$ will be absorbed to create a compound nucleus with total spin $J$. The energy-averaging process allows one to neglect interference between partial waves with different angular momenta. Parity conservation is explicitly enforced in eq. (4.8) by the factor $\delta_{\pi}$, which we define to be one when eq. (4.4) is satisfied and zero when it is not.

The number of states in which the compound nucleus $C$ may be formed is given by

$$
n_{C}=(2 J+1) \rho_{C}\left(E_{x}, J, \Pi\right) d E_{a}
$$

where $\rho_{C}\left(E_{x}, J, \Pi\right)$ is the density of nuclear levels with spin $J$ and parity $\Pi$ for nucleus $C$ near excitation energy $E_{x}$. The factor $2 J+1$ accounts for the number of possible spin orientations for an object with total spin $J$. Now suppose that $a$ is captured by $A$ to form the compound nucleus $C$ and that $a$ will eventually be re-emitted. Let $\left\langle\Gamma_{C \rightarrow a+A}\right\rangle$ denote the energy-averaged transition rate for the decay that results in the re-emission of $a$. The number of states into which the re-emission may occur is given by

$$
n_{a+A}=\left(2 s_{A}+1\right) \rho_{A}\left(E_{x, A}, s_{A}, \Pi_{A}\right) d E_{a}\left[\left(2 s_{a}+1\right) \frac{V}{2 \pi^{2}}\left|\mathbf{p}_{\alpha}\right|^{2} d\left|\mathbf{p}_{\alpha}\right|\right]
$$

where $\rho_{A}\left(E_{x, A}, s_{A}, \Pi_{A}\right)$ is the density of nuclear levels with spin $s_{A}$ and parity $\Pi_{A}$ for nucleus $A$ at excitation energy $E_{x, A}$. The factor in square brackets represents the number of states available ${ }^{7}$ to the projectile $a$ within the finite volume $V$. A similar factor for $A$ is not needed here because the state that it occupies within the volume $V$ is fixed by momentum conservation. The same is true for $C$ in eq. (4.10).

If the Hamiltonian governing the interaction between $a$ and $A$ is invariant under time reversal (which will be the case for particles that interact via the strong force and electromagnetism), then it follows that transitions between individual microstates of $C$ and microstates of the $a+A$ system should occur at the same rate regardless of the direction of time:

$$
\frac{\left\langle\Gamma_{a+A \rightarrow C}\right\rangle}{n_{C}}=\frac{\left\langle\Gamma_{C \rightarrow a+A}\right\rangle}{n_{a+A}} .
$$

\footnotetext{
${ }^{7}$ See eqs. (3.40) and (3.52). Note that we have integrated over all possible directions of $a$ 's 3-momentum and that $d\left|\mathbf{p}_{\alpha}\right|=E_{a}\left|\mathbf{p}_{\alpha}\right|^{-1} d E_{a}$.
} 
Combining the results from eqs. (4.6)-(4.12) allows one to write

$$
\begin{aligned}
\left\langle\Gamma_{C \rightarrow a+A}\right\rangle & =\frac{n_{a+A}}{n_{C}}\left\langle\Gamma_{a+A \rightarrow C}\right\rangle=\frac{n_{a+A}}{n_{C}}\left\langle\sigma_{C}\right\rangle \phi \\
& =\frac{1}{2 \pi} \sum_{\ell j} \frac{E\left|\mathbf{p}_{\alpha}\right| \delta_{\pi} T_{\ell j}(\varepsilon) \rho_{A}\left(E_{x, A}, s_{A}, \Pi_{A}\right)}{E_{a} E_{A} \rho_{C}\left(E_{x}, J, \Pi\right)} d\left|\mathbf{p}_{\alpha}\right| .
\end{aligned}
$$

Since

$$
E=\varepsilon+m_{a}+m_{A}
$$

and

$$
E=E_{a}+E_{A}=\sqrt{\left|\mathbf{p}_{\alpha}\right|^{2}+m_{a}^{2}}+\sqrt{\left|\mathbf{p}_{\alpha}\right|^{2}+m_{A}^{2}}
$$

it follows that

$$
d\left|\mathbf{p}_{\alpha}\right|=\frac{E_{a} E_{A}}{E\left|\mathbf{p}_{\alpha}\right|} d E=\frac{E_{a} E_{A}}{E\left|\mathbf{p}_{\alpha}\right|} d \varepsilon
$$

Equation (4.13) may therefore be simplified to read

$$
\left\langle\Gamma_{C \rightarrow a+A}\right\rangle=\frac{1}{2 \pi \rho_{C}\left(E_{x}, J, \Pi\right)} \sum_{\ell j} \delta_{\pi} T_{\ell j}(\varepsilon) \rho_{A}\left(E_{x, A}, s_{A}, \Pi_{A}\right) d \varepsilon .
$$

The total width for a decay of $C$ into $a+A$ is obtained by integrating eq. (4.17) over all values of $\varepsilon$ consistent with the statement of energy conservation given in eq. (4.2). It should be noted when performing this integral that the excitation energy $E_{x, A}$ of the residual nucleus $A$ is a function of the CM frame total kinetic energy $\varepsilon$. Specifically, in the CM frame (where the kinetic energy of the compound nucleus $C$ is zero), the functional form of $E_{x, A}$ may be written as

$$
E_{x, A}=E_{x, A}(\varepsilon)=E_{x}-Q_{\text {g.s. }}-\varepsilon
$$

The ground-state $\mathrm{Q}$-value $Q_{\text {g.s. }}$ is given by

$$
Q_{\text {g.s. }} \equiv m_{a}+m_{A, \text { g.s. }}-m_{C, \text { g.s. }}
$$

where $m_{A \text {, g.s. }}$ is the ground-state mass of $A$ and $m_{C \text {, g.s. }}$ is the same for $C$. The maximum value of $\varepsilon$ allowed by energy conservation $\varepsilon_{\max }$ occurs when the residual nucleus $A$ is produced in its ground state, i.e., $E_{x, A}=0$. Then eq. (4.18) implies that

$$
\varepsilon_{\max }=E_{x}-Q_{\text {g.s. }} .
$$


The lowest value of $\varepsilon$ consistent with energy conservation is zero, which occurs at the excitation energy threshold for emitting $a$ from the compound nucleus $C$. Integrating eq. (4.17) over the entire allowed energy range yields the expression

$$
\Gamma_{\alpha}=\frac{1}{2 \pi \rho_{C}\left(E_{x}, J, \Pi\right)} \sum_{\ell j} \delta_{\pi} \int_{0}^{\varepsilon_{\max }} T_{\ell j}(\varepsilon) \rho_{A}\left(E_{x, A}(\varepsilon), s_{A}, \Pi_{A}\right) d \varepsilon, \quad \text { (CM frame, 4.21) }
$$

where

$$
\Gamma_{\alpha} \equiv \int_{0}^{\varepsilon_{\max }}\left\langle\Gamma_{C \rightarrow a+A}\right\rangle d \varepsilon
$$

$E_{x, A}(\varepsilon)$ and $\varepsilon_{\max }$ are defined as in eqs. (4.18) and (4.20), and the abbreviation $\alpha \equiv C \rightarrow$ $a+A$ has been introduced to represent the set of decays of $C$ that lead to two-body final states involving $a$ and $A$. The explicit form of the angular momentum sum is

$$
\sum_{\ell j}=\sum_{\ell=0}^{\infty} \sum_{j=\left|\ell-s_{a}\right|}^{\ell+s_{a}}
$$

Because of the centrifugal barrier that appears in the scattering Hamiltonian, the transmission coefficients approach zero as $\ell$ increases. Therefore, in practical calculations, the sum over $\ell$ is terminated when the contribution of the transmission coefficients for higher $\ell$ values may be safely neglected.

Because a compound nucleus retains only enough "memory" of its formation to satisfy basic conservation laws, an identical compound nucleus $C$ formed by a different reaction, say, $b+B \rightarrow C$, will have the same average partial decay width $\Gamma_{\alpha}$ to the final state $a+A$ as in the case just considered. Since we have not made any detailed assumptions about the properties of the particles $a$ and $A$, eq. (4.21) provides a general expression under the Hauser-Feshbach model for the average partial width for a decay of a compound nucleus $C$ into an arbitrary two-body final state $a+A$.

To emphasize the generic nature of this result, define new expressions to represent the initial $\rho_{i}$ and final $\rho_{f}$ nuclear level densities:

$$
\begin{aligned}
\rho_{i}\left(E_{x}, J, \Pi\right) & \equiv \rho_{C}\left(E_{x}, J, \Pi\right) \\
\rho_{f}\left(E_{x}^{\prime}(\varepsilon), J^{\prime}, \Pi^{\prime}\right) & \equiv \rho_{A}\left(E_{x, A}(\varepsilon), s_{A}, \Pi_{A}\right) .
\end{aligned}
$$


Then, using these definitions, eq. (4.21) may be written in the form

$$
\Gamma_{\alpha}=\frac{1}{2 \pi \rho_{i}\left(E_{x}, J, \Pi\right)} \sum_{\ell j} \delta_{\pi} \int_{0}^{\varepsilon_{\max }} T_{\ell j}(\varepsilon) \rho_{f}\left(E_{x}^{\prime}(\varepsilon), J^{\prime}, \Pi^{\prime}\right) d \varepsilon . \quad \text { (CM frame, 4.26) }
$$

If decays involving more than two final particles are neglected, then the energy-averaged total width $\Gamma$ of the initial compound nucleus is simply the sum of the average partial widths to all possible two-body final states $\beta \equiv b+B$ :

$$
\Gamma=\sum_{\beta} \Gamma_{\beta}
$$

Therefore, the probability $P(C \rightarrow \alpha)$ that a compound nucleus $C$ will decay into a twobody final state $\alpha$ may be estimated using the average partial decay widths via

$$
P(C \rightarrow \alpha)=\frac{\Gamma_{\alpha}}{\Gamma}=\frac{\Gamma_{\alpha}}{\sum_{\beta} \Gamma_{\beta}}
$$

The energy-averaged cross section $\left\langle\sigma_{\alpha \beta}\right\rangle$ for a reaction that proceeds via the formation of a compound nucleus, i.e., the cross section for the process

$$
a+A \rightarrow C \rightarrow b+B
$$

for $\alpha \equiv a+A$ and $\beta \equiv b+B$, is given by

$$
\left\langle\sigma_{\alpha \beta}\right\rangle=\left\langle\sigma_{C}\right\rangle P(C \rightarrow \beta)=\left\langle\sigma_{C}\right\rangle \frac{\Gamma_{\beta}}{\Gamma}
$$

with $\left\langle\sigma_{C}\right\rangle$ defined for the initial state $\alpha$ as in eq. (4.8). This expression is a quantitative statement of the Bohr independence hypothesis that was discussed in section 4.1.

Equations (4.26) and (4.28) summarize the MARLEY model of nuclear de-excitations for unbound nuclear states. After a compound nucleus is formed by a neutrino scattering event, it decays to a two-body final state according to (1) tabulated $\gamma$-ray branching ratios (for bound nuclear states) or (2) probabilities computed using the Hauser-Feshbach partial decay widths derived in this section (for unbound states and cases where no $\gamma$-ray data are available). Situations in which multiple particles are emitted from the highly excited nucleus are treated as a sequence of individual Hauser-Feshbach binary decays. 
In order to calculate the partial widths at each decay step, one must first determine the level densities ${ }^{8}\left(\rho_{i}\right.$ and $\left.\rho_{f}\right)$ and the transmission coefficients $\left(T_{\ell j}\right)$ that appear in eq. (4.26). The models used by MARLEY to obtain values for these quantities are described in the following sections. These models are predominantly taken from version 3 of the Reference Input Parameter Library $^{9}$ (RIPL-3) [321], and version 1.8 of the TALYS nuclear reaction code [313, 314].

\subsection{Level density model}

At low excitation energies where discrete nuclear level data are available, MARLEY evaluates the Hauser-Feshbach decay width to each final-state nuclear level individually. This is done by using the following form for the level density $\rho_{f}$ of the final-state nucleus:

$$
\rho_{f}\left(E_{x}^{\prime}, J^{\prime}, \Pi^{\prime}\right)=\sum_{k} \delta\left(E_{x}^{\prime}-E_{x, k}\right) \delta\left(J^{\prime}-J_{k}\right) \delta\left(\Pi^{\prime}-\Pi_{k}\right), \quad \text { (discrete levels, 4.31) }
$$

where $E_{x, k}, J_{k}$, and $\Pi_{k}$ are the excitation energy, total spin, and intrinsic parity of the $k$ th level of the final-state nucleus. Plugging this expression into eq. (4.26) yields

$$
\Gamma_{\alpha}=\frac{1}{2 \pi \rho_{i}\left(E_{x}, J, \Pi\right)} \sum_{k} \sum_{\ell j} \delta_{\pi} T_{\ell j}\left(\varepsilon_{k}\right) \quad \text { (CM frame, discrete levels, 4.32) }
$$

where the sum over $k$ includes all kinematically accessible levels, and $\varepsilon_{k}$, the CM frame total kinetic energy after a decay to the $k$ th level of the final-state nucleus, is given by

$$
\left.\varepsilon_{k}=E_{x}-Q_{\text {g.s. }}-E_{k} . \quad \text { (CM frame, discrete levels, } 4.33\right)
$$

In cases where such data are missing or, in the case of high excitation energies, simply impractical to obtain due to the sheer number of closely-spaced nuclear levels, MARLEY calculates nuclear level densities using the RIPL-3 parameterization [321] of the Backshifted Fermi Gas Model (BSFGM), which is based on the work of Koning et al. [322]

The BSFGM has its roots in a proposal by Hans Bethe [323, 324] to model the nucleus as a gas composed of two distinct species (protons and neutrons) of noninteracting

\footnotetext{
${ }^{8}$ Note that, since each of the partial widths contains the same initial state level density factor $1 /\left[2 \pi \rho_{i}\left(E_{x}, J, \Pi\right)\right]$, this factor will cancel out when computing decay probabilities. One therefore need only compute the final-state level density $\rho_{f}\left(E_{x}^{\prime}, J^{\prime}, \Pi^{\prime}\right)$ when selecting a compound nucleus decay channel in a Monte Carlo simulation.

${ }^{9} \mathrm{~A}$ widely-used collection of nuclear data and parameterized nuclear structure models
} 
fermions. A "back shift" correction was introduced into the model after systematic differences were noticed between the level densities for even-even, odd- $A$, and odd-odd ${ }^{10}$ nuclei. These differences can be attributed $[325,326]$ to the tendency of like nucleons to form spin-zero Cooper pairs. ${ }^{11}$ They are accounted for in the BSFGM by replacing the excitation energy $E_{x}$ of the uncorrected Fermi gas level density with an effective excitation energy $U$ defined by

$$
U \equiv E_{x}-\Delta
$$

where the energy shift

$$
\Delta=\chi_{\text {pair }} \frac{12 \mathrm{MeV}}{\sqrt{A}}+\delta
$$

is an empirical parameter. The constant $\delta$ is adjusted to fit the experimental data for a particular nucleus, and the factor $\chi_{\text {pair }}$ is defined by

$$
\chi_{\text {pair }} \equiv \begin{cases}1 & \text { even-even } \\ 0 & \text { odd- } A \\ -1 & \text { odd-odd }\end{cases}
$$

Under the BSFGM, the total density of nuclear levels near excitation energy $E_{x}$ is given by the expression [322]

$$
\begin{aligned}
\rho^{\mathrm{tot}}\left(E_{x}\right) & =\left[\frac{1}{\rho_{F}^{\text {tot }}\left(E_{x}\right)}+\frac{1}{\rho_{0}\left(E_{x}\right)}\right]^{-1} \\
& =\frac{1}{12 \sigma}\left[\sqrt{2} a_{\mathrm{LD}}^{1 / 4} U^{5 / 4} \exp \left(-2 \sqrt{a_{\mathrm{LD}} U}\right)+\frac{1}{a_{\mathrm{LD}}} \exp \left(-a_{\mathrm{LD}} U-1\right)\right]^{-1},
\end{aligned}
$$

where

$$
\rho_{F}^{\mathrm{tot}}\left(E_{x}\right)=\frac{1}{\sqrt{2 \pi} \sigma} \frac{\sqrt{\pi}}{12} \frac{\exp \left(2 \sqrt{a_{\mathrm{LD}} U}\right)}{a_{\mathrm{LD}}^{1 / 4} U^{5 / 4}}
$$

is the total level density computed according to Bethe's original formula (but using the effective excitation energy $U$ mentioned above), $a_{\mathrm{LD}}$ is the level density parameter, $\sigma$ is

\footnotetext{
${ }^{10}$ These terms refer to the neutron number $N$ and the proton number $Z$ of a nucleus, which are both nonnegative integers. For even-even nuclei, both $N$ and $Z$ are even. For odd- $A$ nuclei, one of $N$ and $Z$ is even and the other is odd, and therefore the mass number $A=N+Z$ is also odd. For odd-odd nuclei, both $N$ and $Z$ are odd.

${ }^{11}$ See reference [327] for a recent review of the nucleon pairing literature.
} 
the spin cutoff parameter, and

$$
\rho_{0}\left(E_{x}\right)=\frac{a_{\mathrm{LD}}}{12 \sigma} \exp \left(a_{\mathrm{LD}} U+1\right)
$$

is a correction intended to suppress the unphysical divergence of $\rho_{F}^{\text {tot }}\left(E_{x}\right)$ at low excitation energies. The traditional derivation of $\rho_{F}^{\text {tot }}\left(E_{x}\right)$ involves the use of the saddle-point method $^{12}$ to approximate the inverse Laplace transform of the nucleon gas's grand canonical partition function. This approximation is inappropriate at low energies and is the source of the problematic divergence [328, 329].

The density of nuclear levels $\rho\left(E_{x}, J, \Pi\right)$ near excitation energy $E_{x}$ with total spin $J$ and intrinsic parity $\Pi$ may be written in the form

$$
\rho\left(E_{x}, J, \Pi\right)=\pi\left(E_{x}, J, \Pi\right) R\left(E_{x}, J\right) \rho^{\text {tot }}\left(E_{x}\right)
$$

where $R\left(E_{x}, J\right)$ is the nuclear spin distribution and $\pi\left(E_{x}, J, \Pi\right)$ is the parity distribution. The total level density is the sum of this quantity over all possible spins and parities, i.e.,

$$
\rho^{\mathrm{tot}}\left(E_{x}, J, \Pi\right)=\sum_{J} \sum_{\Pi} \rho\left(E_{x}, J, \Pi\right) .
$$

Under the assumption that the individual nucleon spins are pointing in random directions, it can be shown [324] that the spin distribution $R\left(E_{x}, J\right)$ is given by [322]

$$
R\left(E_{x}, J\right)=\frac{2 J+1}{2 \sigma^{2}} \exp \left[-\frac{\left(J+\frac{1}{2}\right)^{2}}{2 \sigma^{2}}\right] .
$$

Although at least one attempt has been made [330] to create an energy-dependent empirical model for the parity distribution $\pi\left(E_{x}, J, \Pi\right)$, most calculations assume that both parities occur with equal probability, i.e.,

$$
\pi\left(E_{x}, J, \Pi\right)=\frac{1}{2}
$$

MARLEY adopts this assumption in agreement with the RIPL-3 library [321].

Early versions of the BSFGM typically used a constant value for the level density parameter $a_{\mathrm{LD}}$. However, to correct for the damping of shell effects at high excitation

\footnotetext{
${ }^{12}$ Also often referred to as the method of steepest descent or the stationary-phase method
} 
energies, the RIPL-3 evaluation adopts an energy-dependent functional form (introduced by Ignatyuk et al. [331]) for the level density parameter $a_{\mathrm{LD}}$ :

$$
a_{\mathrm{LD}} \equiv a_{\mathrm{LD}}\left(E_{x}, Z, A\right)=\tilde{a}(A)\left\{1+\frac{\delta W(Z, A)}{U}[1-\exp (-\gamma U)]\right\}
$$

Here $\delta W(Z, A)$ is the shell correction energy, $\tilde{a}(A)$ is the asymptotic value of $a_{\mathrm{LD}}$ at high excitation energies, and $\gamma$ is a damping parameter that represents how quickly $a_{\mathrm{LD}}\left(E_{x}, Z, A\right)$ approaches $\tilde{a}(A)$. The values of these three parameters are determined via the relations

$$
\begin{aligned}
\delta W(Z, A) & =\delta M_{\mathrm{exp}}(Z, A)-\delta M_{\mathrm{LDM}}(Z, A) \\
\tilde{a} & =\alpha A+\beta A^{2 / 3} \\
\gamma & =\frac{\gamma_{0}}{A^{1 / 3}}
\end{aligned}
$$

where $\delta M_{\exp }(Z, A)$ is the measured nuclear mass excess ${ }^{13}$ for the nuclide with proton number $Z$ and mass number $A, \delta M_{\mathrm{LDM}}(Z, A)$ is the corresponding prediction for the nuclear mass excess using the liquid drop model ${ }^{14}$ of Myers and Swiatecki [334], and the empirical parameters $\alpha, \beta$, and $\gamma_{0}$ are determined using fits to nuclear level data. MARLEY adopts the global "BFM effective" values from Koning et al. [322] for $\alpha, \beta$, $\gamma_{0}$, and the constant energy shift $\delta$ (see eq. (4.35)). These values were determined via a systematic study of nuclear level data for 289 nuclides and are given by

$$
\begin{gathered}
\alpha=0.0722396 \mathrm{MeV}^{-1} \\
\beta=0.195267 \mathrm{MeV}^{-1} \\
\gamma_{0}=0.410289 \mathrm{MeV}^{-1} \\
\delta=0.173015 \mathrm{MeV}^{\alpha}
\end{gathered}
$$

\footnotetext{
${ }^{13}$ Experimental nuclear mass values are calculated using the 2012 Atomic Mass Evaluation (AME2012) $[332,333]$. The mass excess $\delta M$ is defined in terms of the mass $M$ via $\delta M \equiv M-A \mathrm{u}$, where u is the unified atomic mass unit.

${ }^{14}$ The RIPL-3 prescription (see reference [321, p. 3164]), which is taken from the work of Mengoni and Nakajima [326], is used for the liquid drop model.
} 
The spin cutoff parameter $\sigma$ determines the width of the angular momentum distribution of the level density. At excitation energies above the neutron separation threshold $S_{n}$ for the nucleus of interest, the RIPL-3 estimate of the spin cutoff parameter may be written as [321]

$$
\sigma^{2}=\sigma_{F}^{2}\left(E_{x}\right) \equiv\left(0.01389 \mathrm{MeV}^{-1}\right) \frac{A^{5 / 3}}{\tilde{a}} \sqrt{a_{\mathrm{LD}} U}=\mathfrak{I}_{\mathrm{eff}} T_{\mathrm{nuc}}
$$

where $T_{\text {nuc }}=\sqrt{U / a}$ is the BSFGM nuclear temperature and

$$
\mathfrak{I}_{\text {eff }}=\left[0.01389 \mathrm{MeV}^{-1}\right] \frac{a_{\mathrm{LD}}}{\tilde{a}} A^{5 / 3}
$$

is the effective nuclear moment of inertia.

At low excitation energies where $E_{x}<\Delta$ (and therefore $U<0$ ), the expression in eq. (4.53) becomes imaginary and therefore unphysical. To get around this difficulty, MARLEY uses linear interpolation to calculate the spin cutoff parameter for excitation energies below $S_{n}$. This interpolation is performed between a low-energy value of the spin cutoff parameter (based on global systematics)

$$
\sigma_{d, \text { global }} \equiv 0.83 A^{0.26}
$$

and the continuum estimate $\sigma_{F}^{2}\left(E_{x}\right)$ evaluated at the neutron separation threshold. This yields the following expression for the spin cutoff parameter $\sigma^{2}$ which is used by MARLEY at all excitation energies $E_{x}$ :

$$
\sigma^{2}=\sigma^{2}\left(E_{x}\right)= \begin{cases}\sigma_{d, \text { global }}^{2}+\frac{E_{x}}{S_{n}}\left[\sigma_{F}^{2}\left(S_{n}\right)-\sigma_{d, \text { global }}^{2}\right] & \text { for } E_{x}<S_{n} \\ \sigma_{F}^{2}\left(E_{x}\right) & \text { for } E_{x} \geq S_{n} .\end{cases}
$$

This is identical to the approach recommended by RIPL-3 [321] when no discrete level data are available. ${ }^{15}$

\footnotetext{
${ }^{15}$ It should be pointed out that the RIPL-3 linear interpolation technique for computing the spin cutoff parameter was first proposed in reference [322]. However, in equation (25) of that paper and in equation (4.257) of the user manual for version 1.8 of the TALYS nuclear code [335] (which is maintained by the same authors), there appears to be a misprint in the expression for $\sigma^{2}$ for the $E_{d} \leq E_{x} \leq S_{n}$ case. Rather than the $\sigma_{F}^{2}\left(S_{n}\right)$ term that appears in the RIPL-3 description (see the equation just below equation (60) in reference [321]) of the BSFGM, Koning et al. write $\sigma_{F}^{2}\left(E_{x}\right)$. Version 1.8 of the TALYS source code, however, appears to agree with the RIPL-3 recommendation.
} 


\subsection{Transmission coefficient model}

In order to determine decay probabilities under the Hauser-Feshbach formalism, one must not only compute the level density for the final-state nucleus, but also the transmission coefficients $T_{\ell j}$ for each particle that may possibly be emitted. For many years, the standard tool used to obtain the transmission coefficients for nucleon and light ion emission has been the nuclear optical model. ${ }^{16}$ Under this model, the nucleus is replaced with a potential well that includes both a real part and an imaginary part, the latter being analogous to the imaginary term that appears in the index of refraction for an optically absorptive material. This simple treatment of the nucleus is inadequate to reproduce the rapid fluctuations of nuclear cross sections in the vicinity of closely-spaced nuclear levels, but it can be used quite successfully to obtain the energy-averaged behavior needed for statistical model calculations [337].

With the exception of elastic scattering, all direct reactions are neglected in the optical model, and therefore particle absorption by the nucleus may be equated with the formation of a compound system. As will be discussed below, because of time-reversal invariance, the transmission coefficient for emitting a particular particle from such a system is equal to the probability of the same particle being absorbed to form the compound nucleus in the time-reversed process. Solving the time-reversed two-body problem for the probability that the emitted particle will be absorbed by the optical potential thus provides a straightforward way of obtaining the transmission coefficients governing the decay of the compound nucleus.

\subsubsection{Optical model parameterization for nucleons ${ }^{17}$}

When calculating partial widths to decay channels that involve the emission of a nuclear fragment (nucleons and light ions up to ${ }^{4} \mathrm{He}$ are considered), MARLEY determines the transmission coefficients needed to evaluate the expression in eq. (4.26) using a global parameterization of the nuclear optical model developed by Koning and Delaroche [338].

\footnotetext{
${ }^{16}$ See reference [336] for an overview of the early history of the nuclear optical model.

${ }^{17}$ Conventional (i.e., non-natural) units where $\hbar \neq c \neq 1$ will be used starting here until the end of the chapter. This choice of units is intended to ease comparison between the results shown here and the many sources which are cited.
} 
Under this parameterization, the optical potential $\mathcal{U}$ used to represent the nucleus may be written as ${ }^{18}$

$$
\mathcal{U}=-\mathcal{V}_{V}-i \mathcal{W}_{V}-i \mathcal{W}_{D}+\left[\mathcal{V}_{S O}+i \mathcal{W}_{S O}\right]\left[\frac{2}{\hbar^{2}} \boldsymbol{\ell} \cdot \boldsymbol{s}\right]+\mathcal{V}_{C}(r)
$$

Here, $\mathcal{V}_{C}(r)$ is the Coulomb potential, and the action of the spin-orbit operator $\frac{2}{\hbar^{2}} \boldsymbol{\ell} \cdot \boldsymbol{s}$ on a state with definite total angular momentum $j$, orbital angular momentum $\ell$, and spin $s$ is given by

$$
\frac{2}{\hbar^{2}} \ell \cdot s|j \ell s\rangle=d_{\ell s}|j \ell s\rangle
$$

where the eigenvalue of the spin-orbit operator may be written as

$$
d_{\ell s}=j(j+1)-\ell(\ell+1)-s(s+1) .
$$

The volume $(V)$, surface $(D)$, and spin-orbit $(S O)$ terms of the optical potential are functions that may be expressed as the product of an energy-dependent well depth and an energy-independent radial part:

$$
\begin{gathered}
\mathcal{V}_{V}=V_{V}\left(\varepsilon_{\text {lab }}\right) f\left(r, R_{V}, a_{V}\right) \\
\mathcal{W}_{V}=W_{V}\left(\varepsilon_{\text {lab }}\right) f\left(r, R_{V}, a_{V}\right) \\
\mathcal{W}_{D}=-4 a_{D} W_{D}\left(\varepsilon_{\text {lab }}\right) \frac{d}{d r} f\left(r, R_{D}, a_{D}\right) \\
\mathcal{V}_{S O}=V_{S O}\left(\varepsilon_{\text {lab }}\right) \frac{\lambda_{\pi}^{2}}{r} \frac{d}{d r} f\left(r, R_{S O}, a_{S O}\right) \\
\mathcal{W}_{S O}=W_{S O}\left(\varepsilon_{\text {lab }}\right) \frac{\lambda_{\pi}^{2}}{r} \frac{d}{d r} f\left(r, R_{S O}, a_{S O}\right)
\end{gathered}
$$

Here, the well depths $V_{V}, W_{V}$, etc. are real-valued functions of the laboratory ${ }^{19}$ kinetic energy $\varepsilon_{\text {lab }}$ of the projectile, $\lambda_{\pi}=\hbar / m_{\pi} c$ is the reduced Compton wavelength of a charged

\footnotetext{
${ }^{18}$ In reference [338], the authors write the spin-orbit terms using the operator $\boldsymbol{\ell} \cdot \boldsymbol{\sigma}$, where $\boldsymbol{\sigma}$ is the Pauli vector and (unlike the expression given here) the eigenvalues of $\ell^{2}$ are taken to be dimensionless (no factor of $\hbar^{2}$ ). While this is neater to write than and, for spin- $\frac{1}{2}$ projectiles, equivalent to the present choice of $\frac{2}{\hbar^{2}} \boldsymbol{\ell} \cdot \boldsymbol{s}$ (since for nucleons, tritons, etc., the spin operator $\mathbf{s}=\frac{\hbar}{2} \boldsymbol{\sigma}$ ), it unnecessarily obscures the transition to composite projectiles with other spins (e.g., deuterons, which have spin 1). The notation shown here for this operator may be used unmodified with a projectile of arbitrary spin.

${ }^{19}$ The laboratory frame is the rest frame of the target nucleus.
} 
pion, and $f\left(r, R, a_{\mathrm{WS}}\right)$ is a Woods-Saxon shape [339]

$$
f\left(r, R, a_{\mathrm{WS}}\right)=\left(1+\exp \left[(r-R) / a_{\mathrm{WS}}\right]\right)^{-1}
$$

with effective radius $R$ and diffuseness parameter $a_{W S}$.

For the sake of brevity, the explicit forms of the well depth functions and the values of the many parameters used to calculate the full optical model potential are omitted from this discussion. The interested reader is encouraged to refer to reference [338] for a complete description of the model. MARLEY uses the global neutron and proton optical potentials defined therein to compute transmission coefficients for nuclear decays involving nucleon emission.

\subsubsection{Optical model parameterization for composite nuclear fragments}

Although most of the effort in the optical model community has focused on creating parameterizations of the optical potential for incident nucleons, there has been some attention paid to complex (i.e., multi-nucleon) projectiles like deuterons and alpha particles. $^{20}$ As an alternative to creating a separate parameterization for deuterons, Watanabe proposed that the single-nucleon optical potentials could instead be averaged over the internal motion of the deuteron using its internal wavefunction [340]. This "folding" approach was soon extended to tritons and helions [341], $\alpha$ particles [342, 343], and ${ }^{6} \mathrm{Li}$ ions [344]. Despite the simplicity of Watanabe's technique, it was nevertheless fairly successful in reproducing the experimental data. ${ }^{21}$

Watanabe's proposed expression for the deuteron optical potential may be written in the form $[346,347]$

$$
\mathcal{U}_{d}\left(\varepsilon_{\mathrm{lab}}, \mathbf{R}\right)=\int d^{3} \mathbf{r}_{d} \psi_{d}^{\dagger}\left(\mathbf{r}_{d}\right)\left[\mathcal{U}_{p}\left(\frac{\varepsilon_{\mathrm{lab}}}{2}, \mathbf{R}+\frac{\mathbf{r}_{d}}{2}\right)+\mathcal{U}_{n}\left(\frac{\varepsilon_{\mathrm{lab}}}{2}, \mathbf{R}-\frac{\mathbf{r}_{d}}{2}\right)\right] \psi_{d}\left(\mathbf{r}_{d}\right)
$$

where $\mathcal{U}_{d}(\mathbf{R})$ is the optical potential for a deuteron projectile, evaluated at the position $\mathbf{R}$ of the deuteron's center of mass. The internal wavefunction $\psi_{d}\left(\mathbf{r}_{d}\right)$ describes the motion of the proton and neutron within the deuteron as a function of their relative position

\footnotetext{
${ }^{20}$ See references $[284,321]$ for lists of some commonly-used parameterizations of the optical potential for complex projectiles.

${ }^{21}$ Reference [345] provides a long list of citations to the early studies.
} 
$\mathbf{r}_{d}$, which is defined to point from the neutron to the proton. The single-nucleon optical potentials $\mathcal{U}_{p}$ and $\mathcal{U}_{n}$ describe the interaction of each individual nucleon with the target nucleus, which is located at the origin. They are evaluated at half the deuteron's kinetic energy (in the laboratory frame) and at the position of each individual nucleon.

For the central terms in the optical model, ${ }^{22}$ there exists a particularly simple approximation to eq. (4.66). Let $\mathcal{U}_{p}^{c}$ and $\mathcal{U}_{n}^{c}$ represent the central terms in the single-nucleon potentials $\mathcal{U}_{p}$ and $\mathcal{U}_{n}$, respectively. If the central potential terms are approximated by their values at the deuteron center of mass, i.e.,

$$
\begin{aligned}
& \mathcal{U}_{p}^{c}\left(\frac{\varepsilon_{\mathrm{lab}}}{2}, \mathbf{R}+\frac{\mathbf{r}_{d}}{2}\right) \approx \mathcal{U}_{p}^{c}\left(\frac{\varepsilon_{\mathrm{lab}}}{2}, \mathbf{R}\right) \\
& \mathcal{U}_{p}^{c}\left(\frac{\varepsilon_{\mathrm{lab}}}{2}, \mathbf{R}-\frac{\mathbf{r}_{d}}{2}\right) \approx \mathcal{U}_{p}^{c}\left(\frac{\varepsilon_{\mathrm{lab}}}{2}, \mathbf{R}\right),
\end{aligned}
$$

then it follows from eq. (4.66) that the central part of the deuteron optical potential $\mathcal{U}_{d}^{c}$ may be written as the sum of the individual nucleon central potentials evaluated at half the deuteron energy:

$$
\mathcal{U}_{d}^{c}(\varepsilon, \mathbf{R}) \approx \mathcal{U}_{p}^{c}\left(\frac{\varepsilon_{\mathrm{lab}}}{2}, \mathbf{R}\right)+\mathcal{U}_{n}^{c}\left(\frac{\varepsilon_{\mathrm{lab}}}{2}, \mathbf{R}\right)
$$

In deriving this result, we have noted that

$$
\int d^{3} \mathbf{r}_{d} \psi_{d}^{\dagger}\left(\mathbf{r}_{d}\right) \psi_{d}\left(\mathbf{r}_{d}\right)=1
$$

If the spin-orbit terms are assumed to be the same for protons and neutrons, then the approach used above may also be applied to them, although the derivation is more involved. The result turns out to be [346]

$$
\mathcal{U}_{d}^{s o} \approx \frac{1}{2} \mathcal{U}_{N}^{s o}\left(\frac{\varepsilon_{\mathrm{lab}}}{2}, \mathbf{R}\right)
$$

where $\mathcal{U}_{N}^{s o}$ represents the spin-orbit terms for a single nucleon. In 1970, Keaton et al. derived similar results for the triton case [346]. In the notation used here, these may be

\footnotetext{
${ }^{22}$ In the parameterization described above, these are $\mathcal{V}_{V}, \mathcal{W}_{V}$, and $\mathcal{W}_{D}$.
} 
written as

$$
\begin{aligned}
\mathcal{U}_{t}^{c} & \approx \mathcal{U}_{p}^{c}\left(\frac{\varepsilon_{\mathrm{lab}}}{3}, \mathbf{R}\right)+2 \mathcal{U}_{n}^{c}\left(\frac{\varepsilon_{\mathrm{lab}}}{3}, \mathbf{R}\right) . \\
\mathcal{U}_{t}^{s o} & \approx \frac{1}{3} \mathcal{U}_{N}^{s o}\left(\frac{\varepsilon_{\mathrm{lab}}}{3}, \mathbf{R}\right) .
\end{aligned}
$$

The expressions seen here for deuterons and tritons are suggestive of a general pattern in which a central term in the optical potential for an arbitrary composite projectile may be approximated as the sum of the corresponding terms for each of its constituent nucleons. If the spin-orbit terms are the same for neutrons and protons, then a spin-orbit term for a composite particle with mass number $A$ may also be approximated by $1 / A$ times its single-nucleon value.

Based on a recommendation by Madland [348], version 1.8 of the TALYS nuclear code [335] implements a superposition model for composite projectile optical potentials much like the approximations shown here. This superposition model is used by default in TALYS and has been adopted for MARLEY calculations of transmission coefficients for composite nuclear fragments. Under this model, the optical model parameters from eqs. (4.60)-(4.64) for nuclear fragments with mass number $A>1$ are given by the following expressions:

$$
\begin{aligned}
& V_{V}\left(\varepsilon_{\text {lab }}\right)=N V_{V}^{n}\left(\varepsilon_{\text {lab }} / A\right)+Z V_{V}^{p}\left(\varepsilon_{\text {lab }} / A\right) \quad W_{V}\left(\varepsilon_{\text {lab }}\right), W_{D}\left(\varepsilon_{\text {lab }}\right) \text { likewise } \\
& V_{S O}\left(\varepsilon_{\text {lab }}\right)=\frac{V_{S O}^{n}\left(\varepsilon_{\text {lab }}\right)+V_{S O}^{p}\left(\varepsilon_{\text {lab }}\right)}{2 A} \quad W_{S O}\left(\varepsilon_{\text {lab }}\right) \text { likewise } \\
& R_{V}=\frac{N R_{V}^{n}+Z R_{V}^{p}}{A} \quad R_{D}, R_{S O} \text { likewise } \\
& a_{v}=\frac{N a_{V}^{n}+Z a_{V}^{p}}{A} \quad a_{D}, a_{S O} \text { likewise. }
\end{aligned}
$$

Here $Z$ and $N$ are the proton and neutron numbers of the fragment, respectively. The superscript ${ }^{n}\left({ }^{p}\right)$ denotes the value of the corresponding parameter for an individual neutron (proton), e.g., $V_{S O}^{n}(E)$ is the spin-orbit well depth for neutrons. Note that the values of the well depths given here match the previous approximations to the Watanabe 
model, except that the spin-orbit depths $V_{S O}$ and $W_{S O}$ are evaluated using the full kinetic energy of the fragment, and the average value $\left(V_{S O}^{n}+V_{S O}^{p}\right) / 2$ is used as the single-nucleon well depth for $V_{S O}$ (with a similar expression for $W_{S O}$ ).

\subsubsection{Optical model calculations}

The optical model potential described above neglects the spin of the target nucleus, treating it as effectively spin zero. Since scattering off of a spin zero target will not alter a projectile's angular momentum quantum numbers, it follows that the incident fragment's radial wavefunction may be expanded as a series of independent spherical partial waves $u_{\ell j}(r)$, each with a definite orbital angular momentum $\ell$ and total angular momentum $j$.

Each of the $u_{\ell j}(r)$ must independently satisfy the radial Schrödinger equation

$$
\left[\frac{d^{2}}{d r^{2}}+k^{2}-\frac{\ell(\ell+1)}{r^{2}}-\frac{k^{2}}{\varepsilon} \mathcal{U}\right] u_{\ell j}(r)=0
$$

where $\mathcal{U}$ is the optical model potential mentioned above, $k=\left|\mathbf{p}_{\alpha}\right| / \hbar$ is the fragment's wavenumber in the $\mathrm{CM}$ frame (with $\mathbf{p}_{\alpha}$ being its $\mathrm{CM}$ frame 3 -momentum), and $\varepsilon$ is the CM frame total kinetic energy. Although the Schrödinger equation is inherently nonrelativistic, MARLEY follows reference [284] in using relativistic kinematics (i.e., relativistic values of $\varepsilon, \varepsilon_{\text {lab }}, k$, and the Sommerfeld parameter $\eta$ defined below) together with the nonrelativistic dynamics of the optical model. MARLEY stops short, however, of applying a relativistic correction to the optical potential itself, although multiple prescriptions exist in the literature for doing so [349, 350].

Far from the nucleus, the optical potential approaches the Coulomb potential, and the fragment's radial wavefunction approaches the asymptotic form [284, 351]

$$
\lim _{r \rightarrow \infty} u_{\ell j}(r)=\frac{i}{2}\left[H_{\ell}^{-}(\eta, k r)-\left\langle S_{\ell j \alpha, \ell j \alpha}^{j}\right\rangle H_{\ell}^{+}(\eta, k r)\right]
$$

where $\left\langle S_{\ell j \alpha, \ell j \alpha}^{j}\right\rangle$ is the energy-averaged S-matrix element, ${ }^{23} H_{\ell}^{ \pm}$are the Coulomb wavefunctions [352], and the Sommerfeld parameter $\eta$ is defined by

$$
\eta \equiv \frac{Z_{\mathrm{nuc}} Z \alpha}{\beta_{\mathrm{rel}}}
$$

\footnotetext{
${ }^{23}$ See appendix A. Also note that, since the nucleus is modeled here as effectively spin zero, the total angular momentum $J$ for the projectile-nucleus system is simply $j$, the projectile's total angular momentum.
} 
where $Z_{\text {nuc }}$ is the proton number of the target nucleus and $\alpha$ is the fine structure constant. The (dimensionless) relative speed of the projectile and target $\beta_{\text {rel }}$ is given by eq. (3.45) if we take the subscripts $i, 1$ to refer to the incident fragment and $i, 2$ to refer to the target nucleus. In the laboratory frame, $\beta_{\text {rel }}$ is simply the speed of the fragment. In terms of the lab frame kinetic energy of the fragment $\varepsilon_{\text {lab }}$, the relative speed may be written as

$$
\beta_{\text {rel }}=\frac{\sqrt{\left(\varepsilon_{\text {lab }}+2 m_{a} c^{2}\right) \varepsilon_{\text {lab }}}}{\varepsilon_{\text {lab }}+m_{a} c^{2}}
$$

where $m_{a}$ is the mass of the fragment.

MARLEY uses the Numerov method [354] to solve for $u_{\ell j}$ numerically at two radii $r_{1}$ and $r_{2}$ that are both sufficiently far from the nucleus that the full optical model potential $\mathcal{U}$ is negligibly different from the Coulomb potential $\mathcal{V}_{C}(r)$. The two solutions, $u_{1} \equiv u_{\ell j}\left(r_{1}\right)$ and $u_{2} \equiv u_{\ell j}\left(r_{2}\right)$, are compared with the asymptotic form from eq. (4.79) and used to compute $^{24}$ the S-matrix element via

$$
\left\langle S_{\ell j \alpha, \ell j \alpha}^{j}\right\rangle \approx \frac{u_{1} H^{-}\left(\eta, k r_{2}\right)-u_{2} H^{-}\left(\eta, k r_{1}\right)}{u_{1} H^{+}\left(\eta, k r_{2}\right)-u_{2} H^{+}\left(\eta, k r_{1}\right)} .
$$

The form of the asymptotic radial wavefunction given in eq. (4.79) lends itself to a straightforward physical interpretation. Since $H_{\ell}^{-}$and $H_{\ell}^{+}$represent ingoing and outgoing spherical waves, respectively, it follows that the absolute square $\left|\left\langle S_{\ell j \alpha, \ell j \alpha}^{j}\right\rangle\right|^{2}$ of the average S-matrix element represents the fraction of the incident flux that emerges after scattering on the nucleus. The transmission coefficient

$$
T_{\ell j} \equiv 1-\left|\left\langle S_{\ell j \alpha, \ell j \alpha}^{j}\right\rangle\right|^{2}
$$

is therefore the probability that the incident fragment is absorbed by the nucleus. Because the scattering Hamiltonian for the optical model is time-reversal invariant, it follows from the reciprocity theorem [357] that the S-matrix elements (and therefore the transmission coefficients) for the forward- and backward-in-time processes (particle absorption by the nucleus and particle emission from the nucleus) must be equal.

\footnotetext{
${ }^{24}$ MARLEY uses the GNU scientific library $[355,356]$ to calculate the Coulomb wavefunctions.
} 


\subsubsection{Gamma-ray transitions in nuclei ${ }^{25}$}

In addition to emitting fragments of the nucleus, compound nuclear states may also deexcite via $\gamma$-ray emission. ${ }^{26}$ The standard theoretical approach used to explain $\gamma$-ray transitions in nuclei considers the interaction of the nuclear current with a perturbing external electromagnetic field. The nuclear current includes both an orbital part (due to the motion of the proton charges within the nucleus) and a spin part (due to the spin magnetic moments of the nucleons). Performing a multipole expansion of the external field allows one to express electromagnetic transition amplitudes between nuclear states in terms of the matrix elements of electric multipole $\left(Q_{\lambda \mu}\right)$ and magnetic multipole $\left(M_{\lambda \mu}\right)$ tensor operators, which may be written in the form

$$
\begin{aligned}
Q_{\lambda \mu} & =\zeta^{(E \lambda)} \sum_{k=1}^{A} e(k) r_{k}^{\lambda} Y_{\lambda \mu}\left(\hat{\mathbf{r}}_{k}\right) \\
M_{\lambda \mu} & =\frac{\mu_{\mathrm{N}}}{\hbar c} \zeta^{(M \lambda)} \sum_{k=1}^{A}\left[\frac{2}{\lambda+1} g_{\ell}^{(k)} \boldsymbol{\ell}(k)+g_{s}^{(k)} \boldsymbol{s}(k)\right] \cdot \nabla_{k}\left[r_{k}^{\lambda} Y_{\lambda \mu}\left(\hat{\mathbf{r}}_{k}\right)\right] .
\end{aligned}
$$

Here, $\zeta^{(E \lambda)}$ and $\zeta^{(M \lambda)}$ are complex phase factors with a magnitude of unity, ${ }^{27}$

$$
\mu_{\mathrm{N}}=\frac{e \hbar}{2 m_{p}}
$$

is the nuclear magneton expressed in terms of the elementary charge $e$ and the proton mass $m_{p}$, the $Y_{\lambda \mu}\left(\hat{\mathbf{r}}_{k}\right)$ are spherical harmonics, and $e(k), \mathbf{r}_{k}, \boldsymbol{\ell}(k)$, and $\boldsymbol{s}(k)$ are respectively the electric charge, position vector (with magnitude $r_{k}$ ), orbital angular momentum, and spin angular momentum of the $k$ th nucleon. The label $\lambda$ is called the multipolarity and refers to the multipole order $2^{\lambda}$ represented by the operator. For example, $Q_{10}$ is component zero of the electric dipole operator, while $M_{2,-1}$ is component -1 of the magnetic quadrupole operator. Each multipole tensor operator has $2 \lambda+1$ components, i.e., $\mu \in\{-\lambda, \lambda+1, \ldots, \lambda\}$. The orbital $\left(g_{\ell}^{(k)}\right)$ and spin $\left(g_{s}^{(k)}\right)$ gyromagnetic ratios for the $k$ th

\footnotetext{
${ }^{25}$ This subsection closely follows Suhonen's presentation given in reference [260].

${ }^{26}$ Electromagnetic nuclear transitions may also result in the ejection of an atomic electron via a process known as internal conversion. However, this decay mode is typically rare for highly-excited nuclear levels. It is neglected in the models adopted for MARLEY.

${ }^{27}$ See reference [260, pp. 119-120] for their explicit definitions according to two commonly used phase conventions.
} 
nucleon are given by [260]

$$
g_{\ell}^{p}=1 \quad g_{s}^{p}=5.586
$$

for protons and

$$
g_{\ell}^{n}=0
$$

for neutrons.

Assuming that the initial and final nuclear spin orientations are not observed, the decay width $\Gamma_{X \lambda}$ for an electromagnetic multipole transition of type $X \in\{E, M\}$ and multipolarity $\lambda$ is given by

$$
\Gamma_{X \lambda}=\frac{2}{\epsilon_{0}} \frac{\lambda+1}{\lambda[(2 \lambda+1) ! !]^{2}}\left(\frac{E_{\gamma}}{\hbar c}\right)^{2 \lambda+1} B\left(X \lambda ; \xi_{i} J_{i} \longrightarrow \xi_{f} J_{f}\right)
$$

where $\epsilon_{0}$ is the vacuum permittivity, $E_{\gamma}$ is the energy of the transition (i.e., the energy of the emitted $\gamma$-ray), $J_{i}\left(J_{f}\right)$ is the initial (final) nuclear spin, and

$$
B\left(X \lambda ; \xi_{i} J_{i} \longrightarrow \xi_{f} J_{f}\right) \equiv \frac{1}{2 J_{i}+1}\left|\left\langle\xi_{f} J_{f}\left\|\mathcal{M}_{X \lambda}\right\| \xi_{i} J_{i}\right\rangle\right|^{2}
$$

is the reduced transition probability. The labels $\xi_{i}$ and $\xi_{f}$ repesent all quantum numbers other than the nuclear spin that are needed to define the initial and final nuclear states, respectively. The electromagnetic multipole operator $\mathcal{M}_{X \lambda}$ is given by

$$
\mathcal{M}_{E \lambda} \equiv \mathbf{Q}_{\lambda} \quad \mathcal{M}_{M \lambda} \equiv \mathbf{M}_{\lambda}
$$

and the reduced matrix element of this operator may be obtained using the Wigner-Eckart theorem, ${ }^{28}$ i.e.,

$$
\left\langle\xi_{f} J_{f}\left\|\mathcal{M}_{\lambda \mu}\right\| \xi_{i} J_{i}\right\rangle=\frac{\sqrt{2 J_{f}+1}\left\langle\xi_{f} J_{f} M_{f}\left|\mathcal{M}_{X \lambda \mu}\right| \xi_{i} J_{i} M_{i}\right\rangle}{\left(J_{i} M_{i} \lambda \mu \mid J_{f} M_{f}\right)}
$$

whenever the Clebsch-Gordan coefficient in the denominator does not vanish. The transition probability is nonzero only when the selection rules

$$
\left|J_{i}-\lambda\right| \leq J_{f} \leq J_{i}+\lambda
$$

\footnotetext{
${ }^{28}$ See section 3.8 .
} 
Table 4.1: Lowest-order multipoles for $\gamma$-ray transitions between an initial nuclear level with spin (parity) $J_{i}\left(\Pi_{i}\right)$ and a final nuclear level with spin (parity) $J_{f}\left(\Pi_{f}\right)$. This table was taken from reference [260].

\begin{tabular}{lcccccc}
\hline$\Delta J \equiv\left|J_{f}-J_{i}\right|$ & $0^{\mathrm{a}}$ & 1 & 2 & 3 & 4 & 5 \\
\hline$\Pi_{i} \Pi_{f}=-1$ & E1 & E1 & M2 & E3 & M4 & E5 \\
$\Pi_{i} \Pi_{f}=+1$ & M1 & M1 & E2 & M3 & E4 & M5 \\
\hline
\end{tabular}

a Gamma-ray transitions for $J_{i}=J_{f}=0$ are not allowed.

and

$$
\Pi_{i} \Pi_{f}= \begin{cases}(-1)^{\lambda} & \text { for } \mathrm{E} \lambda \\ (-1)^{\lambda-1} & \text { for } \mathrm{M} \lambda\end{cases}
$$

are satisfied. In eq. (4.94), $\Pi_{i}\left(\Pi_{f}\right)$ is the intrinsic parity of the initial (final) nuclear state. Because the transition probability decreases rapidly with increasing multipolarity, it is typically sufficient ${ }^{29}$ to consider the lowest-order multipole that satisfies eqs. (4.93)-(4.94) when predicting the electromagnetic decay width between two nuclear levels with definite spins and parities. See table 4.1 for a list of the lowest-order multipoles for typical nuclear transitions.

\subsubsection{Gamma-ray strength functions}

In the context of compound nucleus decays, direct use of eq. (4.89) is impractical due to the large number of accessible levels and the complicated form of the nuclear wavefunctions at high excitation energies. Hauser-Feshbach calculations therefore use a statistical approach in which the electromagnetic decay width for a given transition is approximated by its energy-average value $\left\langle\Gamma_{X \lambda}\right\rangle$. The average behavior of the electromagnetic decay width is then parameterized using a strength function $f_{X \lambda}\left(E_{\gamma}\right)$ such that

$$
\left\langle\Gamma_{X \lambda}\right\rangle=\frac{f_{X \lambda}\left(E_{\gamma}\right) E_{\gamma}^{2 \lambda+1}}{\rho\left(E_{x}, J_{i}, \Pi_{i}\right)}
$$

\footnotetext{
${ }^{29}$ An important exception is competition between the M1 and E2 multipoles. There are known cases where the E2 strength exceeds the M1 strength [260].
} 
where $E_{x}$ is the excitation energy of the initial nuclear level and $\rho\left(E_{x}, J_{i}, \Pi_{i}\right)$ is the level density in its vicinity.

Several phenomenological models of the $\gamma$-ray strength functions are in widespread use $[321,358]$. MARLEY adopts the Standard Lorentzian model ${ }^{30}$ to compute partial decay widths involving $\gamma$-ray emission. This model is based on the work of Brink [359] and Axel [360], who independently [361] proposed that the average E1 decay width could be related to the giant electric dipole resonance, a broad peak that was observed in the cross sections for photonuclear reactions like $(n, \gamma)$ and photofission. ${ }^{31}$ The presence of this resonance may be attributed to a collective vibration of the protons against the neutrons in a dipole pattern. Later experimental searches revealed the presence of additional giant multipole resonances, each of which can be understood as corresponding to its own collective vibration of the nucleons [362].

In the Standard Lorentzian model, $\gamma$-ray emissions of type $X \lambda$ are assumed to take place via de-excitation of the corresponding giant multipole resonance. The strength function $f_{X \lambda}\left(E_{\gamma}\right)$ is therefore parameterized using the Lorentzian shape

$$
f_{X \lambda}\left(E_{\gamma}\right)=\frac{\sigma_{X \lambda}}{(2 \lambda+1) \pi^{2}(\hbar c)^{2}}\left[\frac{\Gamma_{X \lambda}^{2} E_{\gamma}^{3-2 \lambda}}{\left(E_{\gamma}^{2}-E_{X \lambda}^{2}\right)^{2}+E_{\gamma}^{2} \Gamma_{X \lambda}^{2}}\right]
$$

where $E_{X \lambda}, \Gamma_{X \lambda}$, and $\sigma_{X \lambda}$ are respectively the energy, width, and peak cross section of the $X \lambda$ giant resonance. The giant resonance parameters used by MARLEY are identical to the defaults used (in the absence of tabulated data) by version 1.8 of the TALYS nuclear code [314]. They are summarized in table 4.2.

Transmission coefficients for $\gamma$-ray emission may be defined that are analogous to those used for nuclear fragments. Assuming that parity conservation is satisfied, eq. (4.32) implies that, for a transition to a discrete nuclear level in which a nuclear fragment is emitted with orbital angular momentum $\ell$ and total angular momentum $j$, the transmission coefficient $T_{\ell j}$ and the average decay width $\left\langle\Gamma_{\alpha}\right\rangle$ are related via

$$
T_{\ell j}=2 \pi \rho\left(E_{x}, J_{i}, \Pi_{i}\right)\left\langle\Gamma_{\alpha}\right\rangle
$$

\footnotetext{
${ }^{30}$ This name for the model comes from RIPL-3 [321]. It is also often referred to as the Brink-Axel model.

${ }^{31}$ For a historical overview of the early measurements, see reference [362].
} 
Comparing this expression with eq. (4.95), we find that we may write down a $\gamma$-ray transmission coefficient $T_{X \lambda}$ as

$$
T_{X \lambda}\left(E_{\gamma}\right)=2 \pi f_{X \lambda}\left(E_{\gamma}\right) E_{\gamma}^{2 \lambda+1}
$$

The $\gamma$-ray transmission coefficient defined here may be used to compute decay widths according to the Hauser-Feshbach prescription of eq. (4.26), provided that $T_{X \lambda}\left(E_{\gamma}\right)$ is used in place of $T_{\ell j}(\varepsilon)$ and the sum over $\ell j$ is replaced by a sum over multipolarities $X \lambda$. In practice, MARLEY evaluates only the lowest-multipolarity transmission coefficient that is allowed for a particular transition, since the contributions of higher multipoles are expected to be small.

This concludes our discussion of the nuclear de-excitation models adopted for use within MARLEY. The next chapter will present an evaluation of all currently available data that can be used to determine the allowed approximation nuclear matrix elements for the charged current reaction $\nu_{e}+{ }^{40} \mathrm{Ar} \rightarrow e^{-}+{ }^{40} \mathrm{~K}^{*}$. 
Table 4.2: Giant resonance parameters used by MARLEY for $\gamma$-ray strength function calculations. Energies $E_{X \ell}$ and widths $\Gamma_{X \ell}$ are given in $\mathrm{MeV}$, and peak cross sections $\sigma_{X \ell}$ are given in mb.

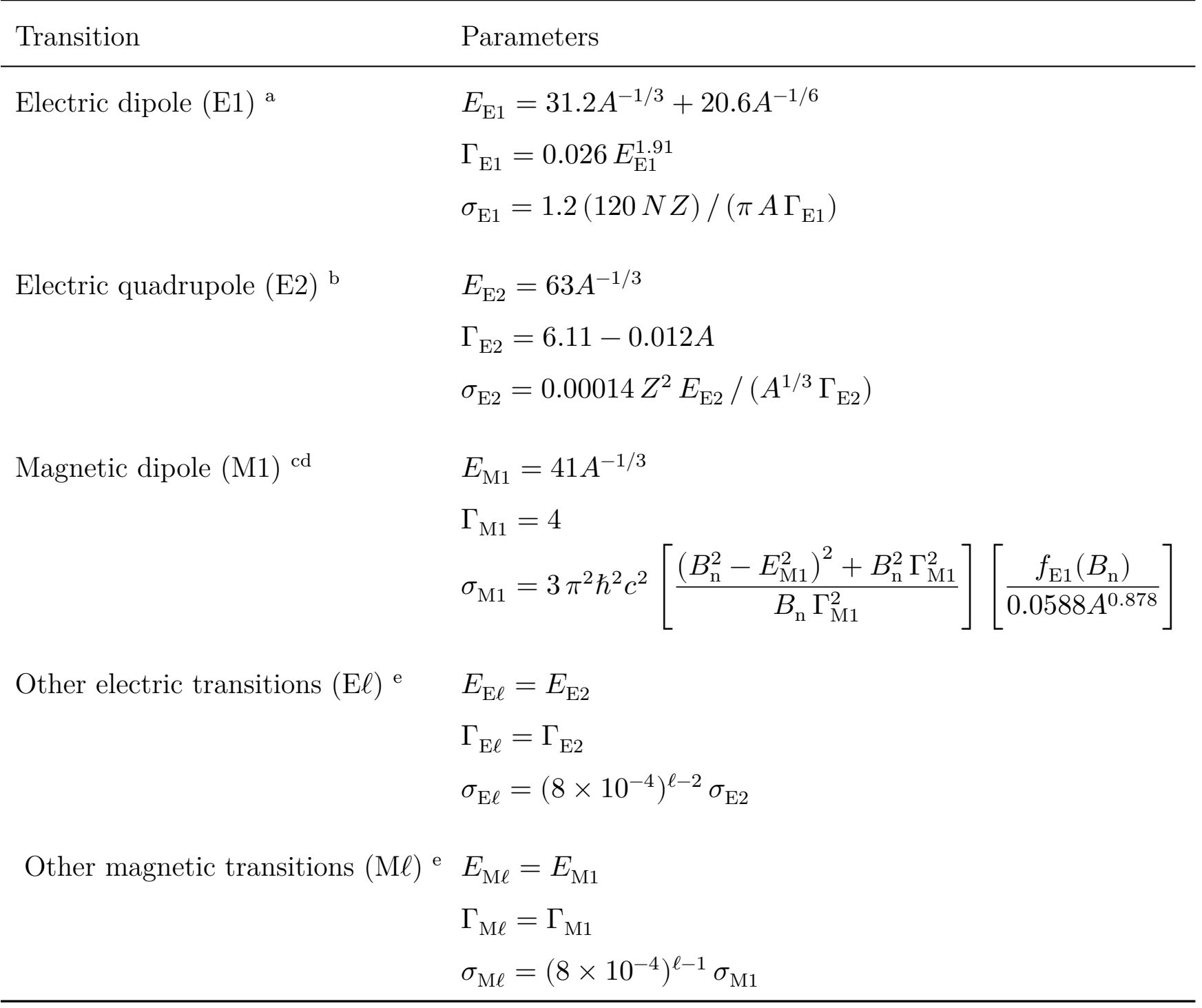

${ }^{\text {a }}$ See [363, p. 129]

${ }^{\text {b }}$ See $[364$, p. 103]

c See $[363$, p. 132]

d $B_{\mathrm{n}}=7 \mathrm{MeV}$ and $f_{\mathrm{E} 1}$ is calculated using the E1 parameters above.

e Default approximation used by TALYS-1.8 


\section{Chapter 5}

\section{Allowed nuclear matrix elements for ${ }^{40} \operatorname{Ar}\left(\nu_{\mathrm{e}}, \mathbf{e}^{-}\right)^{40} \mathbf{K}^{*}$}

Having established a model for neutrino-nucleus scattering cross sections in chapter 3 and for the post-scattering nuclear de-excitations in chapter 4, we need only determine the values of the allowed nuclear matrix elements $B\left(\mathrm{~F}_{-}\right)$and $B\left(\mathrm{GT}_{-}\right)$before we can fully implement a Monte Carlo event generator for charged current $\nu_{e}$ scattering on ${ }^{40} \mathrm{Ar}$. While there exist a number of theoretical calculations of the relevant cross sections (which will be reviewed and compared with the MARLEY model in chapter 7), experimental measurements of the allowed matrix elements have also been made for transitions to lowlying levels of the final-state ${ }^{40} \mathrm{~K}$ nucleus. This chapter reviews the available data and describes how they may be combined with a published theoretical calculation [288] of the Gamow-Teller strength at high excitation energies to create a library of evaluated nuclear matrix elements for MARLEY.

\subsection{Model-independent predictions}

To motivate the experimental techniques used to measure the allowed matrix elements, it will be helpful to examine what can be said theoretically about $B(\mathrm{~F})$ and $B(\mathrm{GT})$ independent of any particular nuclear structure model. 


\subsubsection{The Fermi matrix element}

Consider the reduced Fermi matrix elements for charged current neutrino and antineutrino scattering, which we denote by $B\left(\mathrm{~F}_{-}\right)$and $B\left(\mathrm{~F}_{+}\right)$, respectively, with the sign matching the relevant isospin ladder operator. These reduced matrix elements may be written as

$$
B\left(\mathrm{~F}_{ \pm}\right) \equiv \frac{g_{V}^{2}\left|\left\langle J_{f}\left\|\sum_{k=1}^{A} t_{ \pm}(k)\right\| J_{i}\right\rangle\right|^{2}}{2 J_{i}+1}=\frac{g_{V}^{2}\left|\left\langle J_{f}\left\|T_{ \pm}\right\| J_{i}\right\rangle\right|^{2}}{2 J_{i}+1}
$$

where the $T_{ \pm}$are the total isospin ladder operators defined in eq. (3.26). Note that, since $T_{ \pm}$contains no spin operators, it transforms as a scalar (rank 0 spherical tensor) in spin space, and the Wigner-Eckart theorem eq. (3.140) implies that ${ }^{1}$

$$
B\left(\mathrm{~F}_{ \pm}\right) \delta_{J_{i} J_{f}} \delta_{M_{i} M_{f}}=g_{V}^{2}\left|\left\langle J_{f} M_{f} \mathcal{T}_{f} \mathcal{T}_{3 f}\left|T_{ \pm}\right| J_{i} M_{i} \mathcal{T}_{i} \mathcal{T}_{3 i}\right\rangle\right|^{2}
$$

where $M_{i}\left(M_{f}\right)$ is the spin projection quantum number of the initial (final) nuclear state. The total isospin quantum number $\mathcal{T}_{i}\left(\mathcal{T}_{f}\right)$ and the isospin projection quantum number $\mathcal{T}_{3 i}\left(\mathcal{T}_{3 f}\right)$ are also written explicitly for the initial (final) state. Applying $T_{ \pm}$to the initial state using eq. (3.28) yields

$$
B\left(\mathrm{~F}_{ \pm}\right) \delta_{J_{i} J_{f}} \delta_{M_{i} M_{f}}=g_{V}^{2}\left(\mathcal{T}_{i} \mp \mathcal{T}_{3 i}\right)\left(\mathcal{T}_{i} \pm \mathcal{T}_{3 i}+1\right)\left|\left\langle J_{f} M_{f} \mathcal{T}_{f} \mathcal{T}_{3 f} \mid J_{i} M_{i} \mathcal{T}_{i} \mathcal{T}_{3 i} \pm 1\right\rangle\right|^{2}
$$

The spin eigenstates $|J M\rangle$ are orthonormal, as are the isospin eigenstates $\left|\mathcal{T} \mathcal{T}_{3}\right\rangle$, so this may be rewritten as

$$
B\left(\mathrm{~F}_{ \pm}\right) \delta_{J_{i} J_{f}} \delta_{M_{i} M_{f}}=g_{V}^{2} \delta_{J_{i} J_{f}} \delta_{M_{i} M_{f}} \delta_{\mathcal{T}_{i} \mathcal{T}_{f}} \delta_{\mathcal{T}_{3 i} \pm 1, \mathcal{T}_{3 f}}\left(\mathcal{T}_{i} \mp \mathcal{T}_{3 i}\right)\left(\mathcal{T}_{i} \pm \mathcal{T}_{3 i}+1\right)
$$

Assuming that $J_{f}=J_{i}, M_{f}=M_{i}, \mathcal{T}_{f}=\mathcal{T}_{i}$, and $\mathcal{T}_{3 f}=\mathcal{T}_{3 i} \pm 1$ allows one set all of the Kronecker deltas to unity to obtain

$$
B\left(\mathrm{~F}_{ \pm}\right)=g_{V}^{2}\left(\mathcal{T}_{i} \mp \mathcal{T}_{3 i}\right)\left(\mathcal{T}_{i} \pm \mathcal{T}_{3 i}+1\right)
$$

For a nuclear Hamiltonian that is isospin invariant (so that $\mathcal{T}$ and $\mathcal{T}_{3}$ are good quantum numbers), the results above demonstrate that the reduced Fermi matrix element $B\left(\mathrm{~F}_{ \pm}\right)$

\footnotetext{
${ }^{1}$ The Clebsch-Gordan coefficient identity $\left(J_{i} M_{i} 00 \mid J_{f} M_{f}\right)=\delta_{J_{i} J_{f}} \delta_{M_{i} M_{f}}$ was also used to obtain the result in eq. (5.2).
} 
represents a transition between an initial state $\left|J_{i} M_{i} \mathcal{T}_{i} \mathcal{T}_{3 i}\right\rangle$ and a unique corresponding state $\left|\mathrm{IAS}_{ \pm}\right\rangle=\left|J_{i} M_{i} \mathcal{T}_{i} \mathcal{T}_{3 i} \pm 1\right\rangle$ in the neighboring isobar ${ }^{2}$ with proton number $Z_{f}=$ $Z_{i} \mp 1$. All quantum numbers remain unchanged after the transition except for the isospin projection (and the neutron and proton numbers). The ket for the isobaric analog state may be written in the form

$$
\left|\mathrm{IAS}_{ \pm}\right\rangle=\frac{1}{\sqrt{\left(\mathcal{T}_{i} \mp \mathcal{T}_{3 i}\right)\left(\mathcal{T}_{i} \pm \mathcal{T}_{3 i}+1\right)}} T_{ \pm}\left|J_{i} M_{i} \mathcal{T}_{i} \mathcal{T}_{3 i}\right\rangle
$$

whenever the denominator is nonvanishing. Additional analog states (one for each of the $2 T_{i}+1$ isospin projections) may be found in other isobars by repeated application of the Fermi operators $T_{ \pm}$.

Although the strong interaction is thought to be invariant under rotations in isospace, ${ }^{3}$ this symmetry is broken in the nuclear Hamiltonian because of the proton-neutron mass difference and the Coulomb interaction between the protons. ${ }^{4}$ Thus, the isobaric analog state will not be a true energy eigenstate, and the total Fermi strength $B\left(\mathrm{~F}_{ \pm}\right)$calculated in eq. (5.5) will be somewhat fragmented across multiple nuclear energy levels. Because the symmetry-breaking effects are relatively small, however, isospin invariance remains a good approximation, and experiments (like those that will be discussed momentarily for ${ }^{40} \mathrm{Ar}$ ) typically find that the Fermi strength is concentrated in transitions to a single final energy level or a narrow band of excitation energies.

Based on empirical observations, most nuclear ground states $^{5}$ can be assigned the total isospin quantum number $\mathcal{T}_{i}=\left|\mathcal{T}_{3 i}\right|=\frac{1}{2}\left|N_{i}-Z_{i}\right|$, where $N_{i}$ and $Z_{i}$ are the neutron and proton numbers, respectively. Equation (5.5) implies that the reduced Fermi matrix element $B\left(\mathrm{~F}_{ \pm}\right)$for a transition from such a ground state to its corresponding isobaric

\footnotetext{
${ }^{2}$ Isobars are nuclei that share the same mass number $A$.

${ }^{3} \mathrm{~A}$ small isospin-symmetry-breaking term could possibly exist in the strong Lagrangian, but no experimental evidence for such a term has yet been seen [365].

${ }^{4}$ Both of these effects give rise to an isovector term in the nuclear Hamiltonian, while the Coulomb interaction also leads to an isotensor term [260].

${ }^{5}$ Exceptions exist for some $N=Z$ odd-odd nuclei [366].
} 
analog state is given by

$$
B\left(\mathrm{~F}_{ \pm}\right)= \begin{cases}g_{V}^{2}\left|Z_{i}-N_{i}\right| & \pm\left(Z_{i}-N_{i}\right)>0 \\ 0 & \text { otherwise }\end{cases}
$$

Therefore, in the limit of perfect isospin symmetry, and for $g_{V}=1$, one may estimate model-independently that $B\left(\mathrm{~F}_{-}\right) \approx 4$ for transitions from the ground state of ${ }^{40} \mathrm{Ar}$ (which has $Z_{i}=18$ and $N_{i}=22$ ) to a single isobaric analog state in ${ }^{40} \mathrm{~K}$, and that it will vanish for all other transitions.

\subsubsection{The Gamow-Teller matrix element}

While the Fermi operator $T_{ \pm}$commutes with all but a few small isospin-symmetrybreaking terms in the nuclear Hamiltonian, the Gamow-Teller operator $\sum_{k=1}^{A} \boldsymbol{\sigma}(k) t_{-}(k)$ additionally fails to commute with spin-dependent terms in the effective strong interaction between nucleons. As a result, instead of the modest fragmentation seen for the Fermi strength, one would expect the reduced Gamow-Teller matrix element $B\left(\mathrm{GT}_{ \pm}\right)$ to be nonvanishing for many final nuclear levels (subject to the selection rules given in eq. (3.216)) across a broad range of excitation energies.

This expectation was first experimentally confirmed by Doering et al. [367] in 1975 with the observation of a broad peak attributable to Gamow-Teller transitions in the differential cross section for the ${ }^{90} \operatorname{Zr}(p, n)^{90} \mathrm{Nb}$ reaction (see fig. 5.1). Their discovery of this Gamow-Teller giant resonance (GTGR) was soon replicated in 1980 by Bainum and collaborators [368] at higher proton bombarding energy. Observations of similar resonances in other nuclei (e.g., for a ${ }^{48} \mathrm{Ca}$ target [369]) were made around the same time, confirming that the GTGR is a general feature of nuclear structure. Systematic studies of the GTGR in many nuclei show that the position of its excitation energy centroid $E_{G T}$ relative to the excitation energy of the isobaric analog state ${ }^{6} E_{F}$ may be estimated using the formula $[370]$

$$
E_{G T}-E_{F}=\left[26 A^{-1 / 3}-18.5\left(N_{i}-Z_{i}\right) / A\right] \mathrm{MeV}
$$

\footnotetext{
${ }^{6} \mathrm{Or}$, in cases where the Fermi strength is noticeably fragmented, the centroid of the Fermi resonance
} 

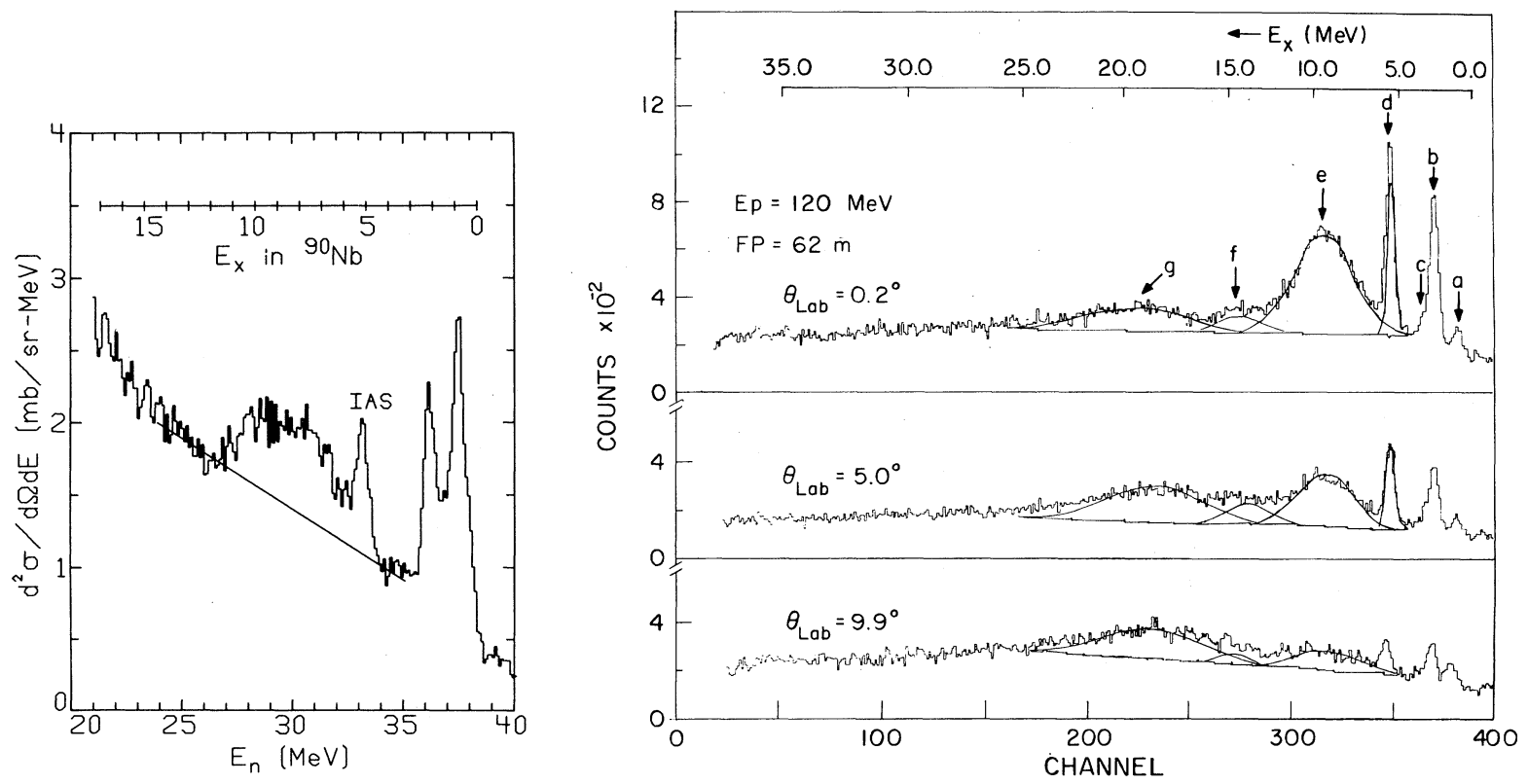

Figure 5.1: Early observations of the Gamow-Teller giant resonance (GTGR) in ${ }^{90} \mathrm{Zr}(p, n)^{90} \mathrm{Nb}$ scattering experiments. LEFT: Measurement of the differential cross section at $0^{\circ}$ performed by Doering et al. [367]. An incident proton kinetic energy of $45 \mathrm{MeV}$ was used. The broad peak to the left of that corresponding to the isobaric analog state (labeled IAS in the figure) is identified as the GTGR. RIGHT: Neutron time-of-flight spectra obtained by Bainum et al. [368] for a proton kinetic energy of $120 \mathrm{MeV}$ and laboratory scattering angles of $0.2^{\circ}, 5.0^{\circ}$, and $9.9^{\circ}$. The peaks labeled $d$ and $e$ correspond to the isobaric analog state and the GTGR, respectively.

Based solely on the commutation properties of the spin and isospin operators, it can be shown [260] that the total Gamow-Teller strength $S_{G T}^{ \pm}$, defined by

$$
S_{G T}^{ \pm} \equiv \sum_{f} B\left(\mathrm{GT}_{ \pm}\right)=\sum_{f} \frac{g_{A}^{2}\left|\left\langle J_{f}\left\|\sum_{k=1}^{A} \boldsymbol{\sigma}(k) t_{ \pm}(k)\right\| J_{i}\right\rangle\right|^{2}}{2 J_{i}+1}
$$

where the sum is over all possible nuclear final states, obeys the Ikeda sum rule ${ }^{7}[371]$

$$
S_{G T}^{-}-S_{G T}^{+}=3 g_{A}^{2}\left(N_{i}-Z_{i}\right)
$$

where $N_{i}\left(Z_{i}\right)$ is the neutron (proton) number of the initial state. This sum rule relies on the assumption that only nucleon states (rather than, e.g., $\Delta$ resonance states) are needed

\footnotetext{
${ }^{7}$ This sum rule appears more commonly in the literature without the factor of $g_{A}^{2}$. In such cases, the definition for the Gamow-Teller reduced matrix element that has been chosen, $B\left(\mathrm{GT}_{-}\right) \equiv$ $\left|\left\langle J_{f}\left\|\sum_{k=1}^{A} \boldsymbol{\sigma}(k) t_{-}(k)\right\| J_{i}\right\rangle\right|^{2} /\left(2 J_{i}+1\right)$, does not include the weak axial-vector coupling constant.
} 
to fully describe the nuclear response to the Gamow-Teller operator, but it is otherwise model-independent [100].

Despite the theoretical robustness of the Ikeda sum rule, experimental measurements have consistently found that the integrated strength of the GTGR is "quenched" with respect to its sum rule value. That is, the ratio

$$
R_{G T} \equiv \frac{S_{G T}^{-}-S_{G T}^{+}}{3 g_{A}^{2}\left(N_{i}-Z_{i}\right)}
$$

is typically found to have the value $R_{G T} \approx 0.5$ when the summed Gamow-Teller strengths $S_{G T}^{ \pm}$include contributions from all levels up to just above the GTGR [372, 373]. Theoretical predictions of the total Gamow-Teller strength and the strengths for transitions to individual nuclear levels are commonly brought into good agreement with beta decay and nuclear scattering measurements by rescaling the axial-vector coupling constant $g_{A}$, that is, by using the effective value

$$
g_{A}^{\mathrm{eff}}=Q_{G T} g_{A}
$$

where the quenching factor $Q_{G T}$ is some fraction, say $Q_{G T}=0.77$ [374] or $Q_{G T}=0.68$ [375]. While satisfactory calculations may be achieved using this method, many years of theoretical work ${ }^{8}$ attempting to account for the "missing" Gamow-Teller strength have not yet resulted in consensus about the correct explanation for the observed quenching [100].

Although a model-independent prediction for the strengths of individual Gamow-Teller transitions is not available, experimental measurements and theoretical calculations of $B(\mathrm{GT})$ have both been performed for ${ }^{40} \mathrm{Ar}$. In the next section, we examine the first of two experimental techniques that have been used to determine the allowed matrix elements: extraction of $B(\mathrm{~F})$ and $B(\mathrm{GT})$ for ${ }^{40} \mathrm{Ar}$ using the closely related $\beta^{+}$decay of ${ }^{40} \mathrm{Ti}$.

\subsection{Measurement via mirror beta decay}

Under the allowed approximation, it can be shown (see appendix C) that the transition rate $\Gamma_{f}$ for $\beta^{ \pm}$decay to a specific final nuclear level $f$ is given in the rest frame of the

\footnotetext{
${ }^{8}$ See, e.g., $[100,376]$ for reviews of the relevant literature.
} 
initial nucleus by

$$
\Gamma_{f}=\frac{G_{F}\left|V_{u d}\right|^{2}}{2 \pi^{3}}\left[B_{f}\left(F_{ \pm}\right)+B_{f}\left(G T_{ \pm}\right)\right] \int_{m_{\ell}}^{Q_{0}} E_{\ell}\left|\mathbf{p}_{\ell}\right|^{2}\left(Q_{0}-E_{\ell}\right)^{2} d E_{\ell}
$$

where the Q-value $Q_{0}=m_{i}-m_{f}$ is the difference between the initial and final nuclear masses, $G_{F}$ is the Fermi coupling constant, $V_{u d}$ is the CKM matrix element connecting the up and down quarks, and $m_{\ell}, E_{\ell}$, and $\mathbf{p}_{\ell}$ are respectively the mass, total energy, and 3-momentum of the final-state (anti)lepton, which is either an electron (for $\beta^{-}$decay) or a positron (for $\beta^{+}$decay). Although beta decay from the ground state of ${ }^{40} \mathrm{Ar}$ is energetically forbidden, ${ }^{9}$ the ground state of ${ }^{40} \mathrm{Ti}$, its mirror nucleus, ${ }^{10}$ is unstable to $\beta^{+}$ decay. Under the assumption of isospin invariance, the ${ }^{40} \mathrm{Ar}$ ground state (with $\mathcal{T}=2$, $\mathcal{T}_{3}=+2$ ) and the ${ }^{40} \mathrm{Ti}$ ground state (with $\mathcal{T}=2, \mathcal{T}_{3}=-2$ ) will be isobaric analogs of each other, and the matrix elements governing allowed $\beta^{+}$decay from ${ }^{40} \mathrm{Ti}$ to final levels in ${ }^{40} \mathrm{Sc}$ will be equal to their counterparts for the charged current reaction ${ }^{40} \operatorname{Ar}\left(\nu_{e}, e^{-}\right)^{40} \mathrm{~K}$, i.e.,

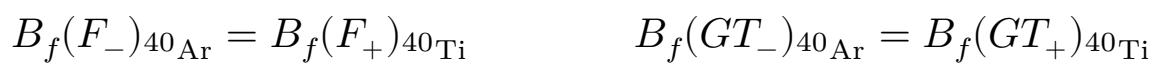

for corresponding transitions.

\subsubsection{Equality of the allowed matrix elements for mirror nuclei}

To see why these matrix elements must be equal if isospin is a good symmetry, note that, since spin and isospin are isomorphic, the Wigner-Eckart theorem may be applied in isospace just as easily as in spin space. For the $M$ th component of a rank $L$ spherical tensor operator $T_{L M}$ in isospace, eq. (3.140) allows one to write

$$
\left\langle\zeta^{\prime} j^{\prime} \mathcal{T}^{\prime} \mathcal{T}_{3}^{\prime}\left\|T_{L M}\right\| \zeta j \mathcal{T} \mathcal{T}_{3}\right\rangle=\frac{1}{\sqrt{2 \mathcal{T}^{\prime}+1}}\left(\mathcal{T} \mathcal{T}_{3} L M \mid \mathcal{T}^{\prime} \mathcal{T}_{3}^{\prime}\right)\left\langle\zeta^{\prime} j^{\prime} \mathcal{T}^{\prime}\left\|\mathbf{T}_{L}\right\| \zeta j \mathcal{T}\right\rangle
$$

where double bars $\|$ are used when writing a spin-reduced matrix element, $\zeta$ and $\zeta^{\prime}$ denote all quantum numbers needed to specify the states that are not written explicitly, and we

\footnotetext{
${ }^{9}$ The neighboring isobars ${ }^{40} \mathrm{~K}$ and ${ }^{4} 0 \mathrm{Cl}$ have ground state masses that are, respectively, about 1.5 MeV and $7.5 \mathrm{MeV}$ above the ${ }^{40} \mathrm{Ar}$ ground state.

10 i.e., the nucleus obtained when all of the protons are replaced with neutrons and vice-versa
} 
have defined the doubly-reduced (i.e., reduced in both spin space and isospace) matrix element $\left\langle\zeta^{\prime} j^{\prime} \mathcal{T}^{\prime}\left\|\mathbf{T}_{L}\right\| \zeta j \mathcal{T}\right\rangle$, which is written with triple bars $\|$. Note that eqs. (3.17) and (3.112) imply that the spherical components of the isospin operator $\mathbf{t}$ are given by

$$
t_{ \pm 1}=\mp \frac{1}{\sqrt{2}} t_{ \pm} \quad t_{0}=t_{3}=t_{z}
$$

with similar expressions for the components of the total isospin operator $\mathbf{T}$ from eq. (3.24).

One may apply eqs. (5.15) and (5.16) to the spin-reduced Fermi matrix elements $B\left(\mathrm{~F}_{ \pm}\right)$ to obtain

$$
\begin{aligned}
B\left(\mathrm{~F}_{ \pm}\right) & =\frac{g_{V}^{2}\left|\left\langle J_{f} \mathcal{T}_{f} \mathcal{T}_{3 f}\left\|T_{ \pm}\right\| J_{i} \mathcal{T}_{i} \mathcal{T}_{3 i}\right\rangle\right|^{2}}{2 J_{i}+1}=\frac{g_{V}^{2}\left|\left\langle J_{f} \mathcal{T}_{f} \mathcal{T}_{3 f}\left\|\mp \sqrt{2} T_{ \pm 1}\right\| J_{i} \mathcal{T}_{i} \mathcal{T}_{3 i}\right\rangle\right|^{2}}{2 J_{i}+1} \\
& =\frac{2 g_{V}^{2}\left|\left(\mathcal{T}_{i} \mathcal{T}_{3 i} 1 \pm 1 \mid \mathcal{T}_{f} \mathcal{T}_{3 f}\right)\left\langle J_{f} \mathcal{T}_{f}\|\mathbf{T}\| J_{i} \mathcal{T}_{i}\right\rangle\right|^{2}}{\left(2 J_{i}+1\right)\left(2 \mathcal{T}_{f}+1\right)}
\end{aligned}
$$

If the matrix element is nonvanishing, then $J_{f}=J_{i}, \mathcal{T}_{f}=\mathcal{T}_{i}$, and $\mathcal{T}_{3 f}=\mathcal{T}_{3 i} \pm 1$. If one assumes that this is the case, then the expression for $B\left(\mathrm{~F}_{ \pm}\right)$becomes

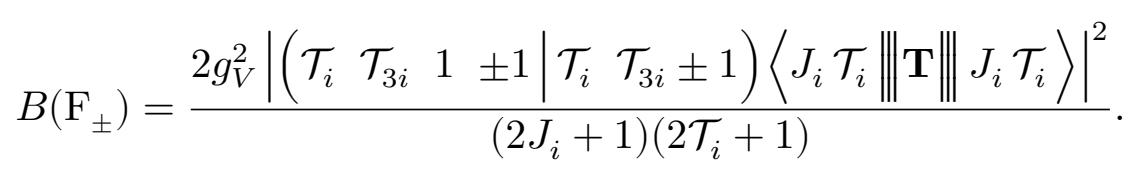

For isospin-lowering Fermi transitions from an initial nuclear state with $\mathcal{T}_{3 i}=\mathcal{T}_{i}$ (e.g., the ground state of ${ }^{40} \mathrm{Ar}$ ), this becomes

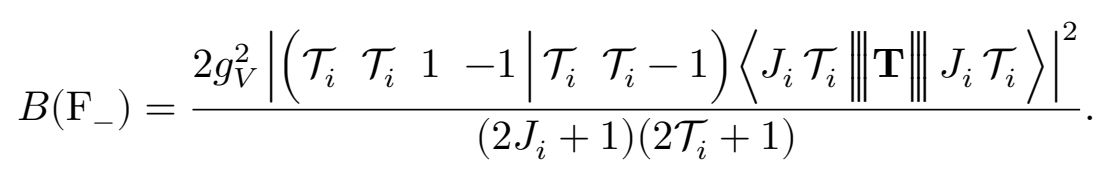

On the other hand, isospin-raising Fermi transitions from the mirror nuclear state with $\mathcal{T}_{3 i}=-\mathcal{T}_{i}$ (e.g., the ground state of ${ }^{40} \mathrm{Ti}$ ) have

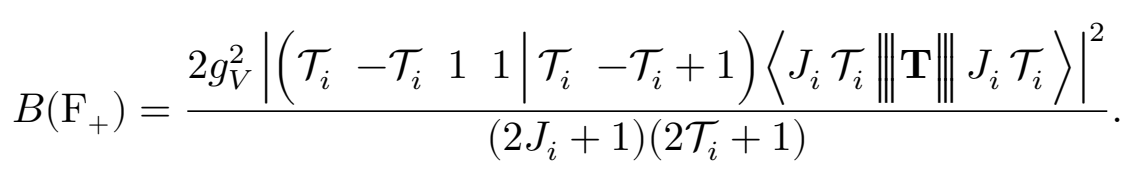

The Clebsch-Gordan coefficients obey the symmetry identity

$$
\left(j_{1} m_{1} j_{2} m_{2} \mid j_{3} m_{3}\right)=(-1)^{j_{1}+j_{2}-j_{3}}\left(j_{1}\left(-m_{1}\right) j_{2}\left(-m_{2}\right) \mid j_{3}\left(-m_{3}\right)\right)
$$


so eq. (5.20) may be rewritten as

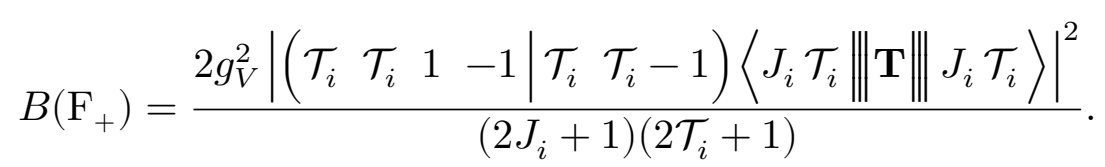

where the factor of $(-1)^{\mathcal{T}_{i}+1-\mathcal{T}_{i}}=-1$ may be dropped because it occurs within the absolute value bars. Comparing eq. (5.19) with eq. (5.22) confirms the equality of the matrix elements for the two types of transitions.

A nearly identical argument shows that matching Gamow-Teller transitions in mirror nuclei will also have equal reduced matrix elements. Thus, to the extent that isospin symmetry is a good approximation, the allowed matrix elements of interest for charged current $\nu_{e}$ scattering on ${ }^{40} \mathrm{Ar}$ may be determined by measuring the strengths of the corresponding transitions from $\beta^{+}$decay in ${ }^{40} \mathrm{Ti}$.

\subsection{2 ${ }^{40} \mathrm{Ti} \beta^{+}$decay measurement}

Motivated by theoretical work [377] pointing out the opportunity to make an indirect measurement of the cross section for charged current $\nu_{e}$ scattering on ${ }^{40} \mathrm{Ar}$ using mirror beta decay, two groups, Liu et al., working at GSI $^{11}[378,379]$, and Bhattacharaya et al., working at GANIL ${ }^{12}$ [380-382], independently made the first detailed measurements ${ }^{13}$ of the allowed matrix elements for ${ }^{40} \mathrm{Ti} \beta^{+}$decay in 1997. Obtaining these data required considerable experimental sophistication, not only because of the short half-life of and difficulty in producing ${ }^{40} \mathrm{Ti}$, but also because $\beta^{+}$decays from this nuclide populate unbound states in ${ }^{40} \mathrm{Sc}$, which immediately decay via proton emission to multiple possible levels in ${ }^{39} \mathrm{Ca}$ [384]. Because the techniques used by the two groups were similar, only the approach used by Bhattacharya et al. [382] will be summarized here.

\subsubsection{Experimental procedure}

To produce the ${ }^{40} \mathrm{Ti}$ ions needed for their measurement, Bhattacharya and collaborators bombarded a nickel target with a $82.6 \mathrm{MeV} /$ nucleon ${ }^{50} \mathrm{Cr}$ beam. Reaction products in the mass range of interest were selected by the LISE 3 spectrometer [385] using a combination

\footnotetext{
${ }^{11}$ Gesellschaft für Schwerionenforschung (Society for Heavy Ion Research), Darmstadt, Germany

${ }^{12}$ Grand Accélérateur National d'Ions Lourds (Large Heavy Ion National Accelerator), Caen, France

${ }^{13}$ Détraz et al. [383] were the first to study ${ }^{40} \mathrm{Ti} \beta^{+}$decay experimentally, but only 190 events were observed.
} 
of dipole magnets and a Wien filter. The selected ions were then sent to a stack of siliconbased charged-particle detectors labeled $D_{1}$ through $D_{5}$, with $D_{1}$ the first to be struck by the beam. A combination of time-of-flight and deposited energy was used to identify ${ }^{40} \mathrm{Ti}$ ions in the beam and reject contaminants. The thicknesses of the silicon detectors were chosen so that the ${ }^{40} \mathrm{Ti}$ ions were implanted within $D_{4}$. Positrons from $\beta^{+}$decay events within $D_{4}$ were detected using $D_{3}$ and $D_{5}$. Beta decay events were selected by requiring (1) a ${ }^{40} \mathrm{Ti}$ candidate to be implanted in $D_{4}$ at least five half-lives after the preceding proton emitter, (2) a proton candidate to deposit at least $800 \mathrm{keV}$ in $D_{4}$ no sooner than $4 \mathrm{~ms}$ after ion implantation, ${ }^{14}$ and (3) a positron candidate to deposit at least $800 \mathrm{keV}$ in exactly one of $D_{3}$ and $D_{5}$ in coincidence with the $D_{4}$ signal.

\subsubsection{Data analysis}

Two results were needed from the data analysis in order to obtain the allowed matrix elements. The first of these was a measurement of the half-life of ${ }^{40} \mathrm{Ti}$, which was determined using a fit to the distribution of time intervals between the implantation of a ${ }^{40} \mathrm{Ti}$ candidate in $D_{4}$ and the detection of a subsequent proton candidate. The fit was performed using two exponentials in order to remove a component from the relatively long-lived contaminant ${ }^{38} \mathrm{Ca}$, which has a half-life of $440 \mathrm{~ms}$ [386].

The second item needed to determine the allowed matrix elements was a set of branching ratios to each of the energy levels in the daughter ${ }^{40} \mathrm{Sc}$ nucleus accessed via $\beta^{+}$decay. Although the relative sizes of the peaks in the $D_{4}$ proton energy distribution provided a simple means of obtaining these branching ratios, associating each proton energy group with a ${ }^{40} \mathrm{Sc}$ level was somewhat challenging. In the energy range of interest for the experiment, single proton emission was expected to be the dominant de-excitation mechanism for the ${ }^{40}$ Sc nucleus, but this emission could occur to either the ${ }^{39}$ Ca ground state (a " $p_{0}$ " decay) or to its first excited state (a " $p_{1}$ " decay). Figure 5.2 is a decay scheme depicting these two beta-delayed proton transitions.

After fitting the peaks observed in the $D_{4}$ proton energy spectrum, Bhattacharya et al. assigned a label of $p_{0}$ or $p_{1}$ to the proton transition associated with each peak.

\footnotetext{
${ }^{14} \mathrm{~A}$ correction was made for the dead time associated with making this timing cut.
} 


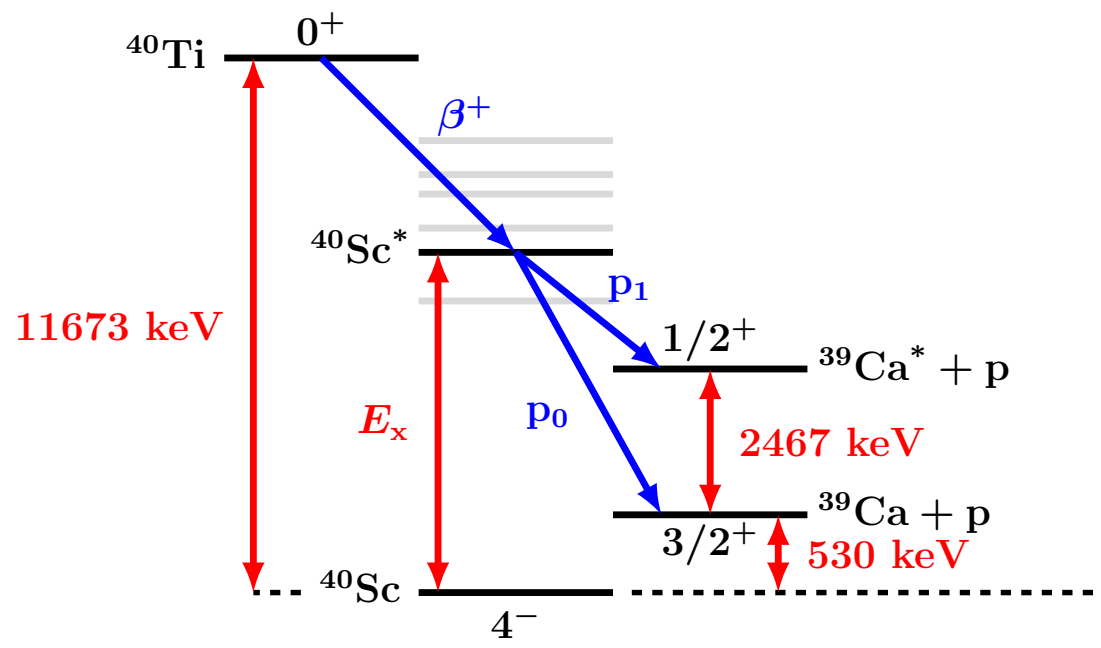

Figure 5.2: Decay scheme for ${ }^{40} \mathrm{Ti} \beta^{+}$decay. Bhattacharya et al. [382] observed beta-delayed proton transitions to both the ground state (" $p_{0}$ ") and the first excited state (" $p_{1}$ ") of ${ }^{39} \mathrm{Ca}$. Without a determination of whether a particular proton line corresponds to a $p_{0}$ or a $p_{1}$ decay, the energy level of the excited ${ }^{40} \mathrm{Sc}^{*}$ nucleus originally accessed by the $\beta^{+}$decay is ambiguous.

The matching ${ }^{40} \mathrm{Sc}$ level was then determined by summing the measured proton energy, the mass difference between the ${ }^{40} \mathrm{Sc}$ and ${ }^{39} \mathrm{Ca}$ ground states, and (in the case of $p_{1}$ transitions) the energy of the $\gamma$-ray emitted during de-excitations from the first excited state to the ground state of ${ }^{39} \mathrm{Ca}$. Although detection of the coincident $\gamma$-rays from $p_{1}$ decays was attempted using a set of five high-purity germanium detectors, poor statistics forced all but one of the $p_{0}$ versus $p_{1}$ assignments to be made based on which of the two possible ${ }^{40} \mathrm{Sc}$ level energies had a more plausible corresponding level in ${ }^{40} \mathrm{~K}$, the mirror nucleus for ${ }^{40} \mathrm{Sc}$.

Having measured the half-life of ${ }^{40} \mathrm{Ti}$ and branching ratios to a number of final nuclear levels in ${ }^{40} \mathrm{Sc}$, Bhattacharya et al. determined values for the allowed matrix elements $B\left(\mathrm{~F}_{+}\right)$and $B\left(\mathrm{GT}_{+}\right)$using eq. (C.27). Based on the nuclear structure data available at the time, they also assigned final levels for the mirror ${ }^{40} \mathrm{Ar} \rightarrow{ }^{40} \mathrm{~K}$ transitions when a plausible match (based on the excitation energy, spin, and parity of the corresponding ${ }^{40} \mathrm{Sc}$ and ${ }^{40} \mathrm{~K}$ levels) could be found.

Before discussing the results obtained from ${ }^{40} \mathrm{Ti} \beta^{+}$decay by the Liu and Bhattacharya 
groups, we will review a complementary experimental probe of ${ }^{40} \mathrm{Ar}$ Gamow-Teller transitions in the next section.

\subsection{Measurement via $(\mathbf{p}, \mathbf{n})$ scattering}

A second method for measuring the allowed matrix elements was first developed around 1980 by Goodman et al. They noted that the $0^{\circ} \operatorname{cross}_{\operatorname{section}}{ }^{15}$ for $(p, n)$ reactions with a proton energy of about $100 \mathrm{MeV}$ could be written in terms of CM frame quantities in the form ${ }^{16}[387]$

$$
\frac{d \sigma\left(0^{\circ}\right)}{d \Omega}=\left(\frac{\mu}{\pi \hbar^{2}}\right)^{2} \frac{\left|\mathbf{k}_{f}\right|}{\left|\mathbf{k}_{i}\right|}\left[N_{\tau}^{D}\left|J_{\tau}\right|^{2} g_{V}^{-2} B\left(\mathrm{~F}_{-}\right)+N_{\sigma \tau}^{D}\left|J_{\sigma \tau}\right|^{2} g_{A}^{-2} B\left(\mathrm{GT}_{-}\right)\right]
$$

where $\mu$ is the reduced energy, $\mathbf{k}_{i}\left(\mathbf{k}_{f}\right)$ is the momentum of the initial (final) nucleon, $N_{\tau}^{D}$ and $N_{\sigma \tau}^{D}$ are distortion factors, and $J_{\tau}$ and $J_{\sigma \tau}$ are interaction strengths. The proportionality between the $0^{\circ}$ cross section and the allowed matrix elements suggested that the latter might be determined by measuring the former, but direct use of this expression by experimentalists was complicated by the presence of the distortion factors and interaction strengths, which would need to be measured themselves. ${ }^{17}$ This situation was soon improved by Taddeucci et al. [389, 390], who, by combining $0^{\circ}(p, n)$ cross section measurements with known Gamow-Teller strengths $B\left(\mathrm{GT}_{-}\right)$observed via $\beta$ decay, found that the available data were well-described by the empirical relationship

$$
\frac{\sigma\left(G T_{-}\right) g_{A}^{2} / B\left(\mathrm{GT}_{-}\right)}{\sigma\left(F_{-}\right) g_{V}^{2} / B\left(\mathrm{~F}_{-}\right)}=\left(\frac{E_{p}}{E_{0}}\right)^{2}
$$

Here $\sigma\left(G T_{-}\right)$and $\sigma\left(F_{-}\right)$are $(p, n)$ cross sections at $0^{\circ}$ for a Gamow-Teller and a Fermi transition, respectively, $E_{p}$ is the incident proton kinetic energy in the lab frame (which, to ensure validity of the formula, should be in the low hundreds of $\mathrm{MeV}$ ), and the empirical

\footnotetext{
${ }^{15}$ i.e., the cross section for $(p, n)$ reactions where the angle between the incident proton and the outgoing neutron is $0^{\circ}$

${ }^{16}$ The notation from reference [387] has been altered here to account for our use of natural units and the inclusion of the weak coupling constants $g_{V}$ and $g_{A}$ in the definitions of the allowed matrix elements (see eqs. (3.213)-(3.214)). The cross section given here does not depend on these constants since the $(p, n)$ reaction is governed by the residual strong force.

${ }^{17}$ Although one could potentially calculate the needed quantities, Goodman et al. claim that "reaction theory does not have the precision required to convert reaction cross-sections to GT matrix elements with the desired accuracy" [388].
} 
parameter $E_{0}$ had the best-fit value

$$
E_{0}=(55.0 \pm 1.7) \mathrm{MeV}
$$

Since the early studies described here, a sizeable literature ${ }^{18}$ has emerged on experimental probes of nuclear spin-isospin excitations, of which Gamow-Teller transitions are an example. Based on the original technique developed for $(p, n)$ scattering, $0^{\circ}$ cross section measurements for a number of other charge-exchange reactions have been used to extract Gamow-Teller matrix elements, including $\left({ }^{3} \mathrm{He}, \mathrm{t}\right)$ for isospin-lowering transitions and $(\mathrm{n}, \mathrm{p}),\left(\mathrm{d},{ }^{2} \mathrm{He}\right)$, and $\left(\mathrm{t},{ }^{3} \mathrm{He}\right)$ for isospin-raising transitions ${ }^{19}$ [397]. Recently, it has also been pointed out [398] that the neutral Gamow-Teller strength

$$
B\left(\mathrm{GT}_{0}\right) \equiv \frac{g_{A}^{2}\left|\left\langle J_{f}\left\|\sum_{k=1}^{A} \boldsymbol{\sigma}(k) t_{3}(k)\right\| J_{i}\right\rangle\right|^{2}}{2 J_{i}+1}
$$

of interest for neutral current reactions may be obtained using measurements of $0^{\circ}$ inelastic proton scattering cross sections.

\subsubsection{Technique for extraction of Gamow-Teller strength}

For the present discussion, it is sufficient to consider a method presented by Goodman and collaborators [388] in 2001 for determining Gamow-Teller strengths $B\left(\mathrm{GT}_{-}\right)$using measured neutron time-of-flight spectra from $(p, n)$ scattering at $0^{\circ}$. The method is appropriate for all target nuclei with a neutron excess, i.e., $N_{i}>Z_{i}$. First, the time-of-flight spectra are converted to outgoing neutron energy spectra and then to excitation energy spectra using the length traveled from the target to the neutron detector and elementary kinematical calculations. Second, the number of observed counts in the Fermi peak (i.e., the peak corresponding to transitions to the isobaric analog state) is determined. Because the Fermi peak may not be fully resolved from a nearby Gamow-Teller peak, ${ }^{20}$ this is done

\footnotetext{
${ }^{18}$ References [362, 372, 391-395] are some of the many reviews on the subject.

${ }^{19}$ The present author's colleagues at UC Davis may be interested to learn that, in their review of the literature on nucleon charge-exchange reactions, Alford and Spicer [393] state that the Crocker cyclotron was the "first system in [the high tens to low hundreds of $\mathrm{MeV}$ ] energy range to produce useful measurements of (n,p) cross sections." Those early measurements are described in reference [396].

${ }^{20}$ Additionally, in the case of an initial nuclear spin $J_{i} \neq 0$, the Fermi peak may contain counts from both Fermi and Gamow-Teller transitions (see the selection rules in eqs. (3.215) and (3.216)) to the isobaric analog state.
} 
by comparing two excitation energy spectra recorded using different proton bombarding energies $E_{p}$ and $E_{p}^{\prime}$. The Fermi peak and a pure Gamow-Teller peak are identified in both spectra. Then, for the $E_{p}\left(E_{p}^{\prime}\right)$ spectrum, if $f\left(f^{\prime}\right)$ is the number of counts in the Fermi peak that arise from true Fermi transitions, $g_{1}\left(g_{1}^{\prime}\right)$ is the number of counts in the Fermi peak that are due to Gamow-Teller transitions, and $g_{2}\left(g_{2}^{\prime}\right)$ is the number of counts in the pure Gamow-Teller peak, one may write [388]

$$
\left(\frac{f}{g_{2}}\right)=\left(\frac{E_{p}^{\prime}}{E_{p}}\right)^{2}\left(\frac{f^{\prime}}{g_{2}^{\prime}}\right) .
$$

This expression may be obtained using eq. (5.24) if one considers that the observed numbers of counts will be proportional to the corresponding cross sections. It implies that

$$
f=\frac{\left(g_{2} / g_{2}^{\prime}\right)\left(f^{\prime}+g_{1}^{\prime}\right)-\left(f+g_{1}\right)}{\left(E_{p} / E_{p}^{\prime}\right)-1}
$$

and

$$
f^{\prime}=\frac{\left(g_{2}^{\prime} / g_{2}\right)\left(f+g_{1}\right)-\left(f^{\prime}+g_{1}^{\prime}\right)}{\left(E_{p}^{\prime} / E_{p}\right)-1} .
$$

Since $\left(f+g_{1}\right)$ and $g_{2}$ are the observed counts in the Fermi peak and the pure GamowTeller peak, respectively, for the spectrum measured using the proton kinetic energy $E_{p}$, and since $\left(f^{\prime}+g_{1}^{\prime}\right)$ and $g_{2}^{\prime}$ are the corresponding quantities for the spectrum measured using $E_{p}^{\prime}$, the number of counts in each Fermi peak attributable to Fermi transitions, $f$ and $f^{\prime}$, may be determined for both spectra. If one applies the substitutions

$$
\frac{\sigma\left(G T_{-}\right)}{\sigma\left(F_{-}\right)} \rightarrow \frac{g_{2}}{f}
$$

and (invoking eq. (5.7))

$$
B\left(\mathrm{~F}_{-}\right)=g_{V}^{2}\left(N_{i}-Z_{i}\right)
$$

to eq. (5.24), the Gamow-Teller strength $B_{2}\left(G T_{-}\right)$to the nuclear level represented by the peak containing $g_{2}$ counts may be calculated via

$$
B_{2}\left(G T_{-}\right)=\left(\frac{E_{0}}{E_{p}}\right)^{2} \frac{g_{A}^{2} g_{2}\left(N_{i}-Z_{i}\right)}{f} .
$$


In the expression above, $g_{A}=1.26$ should be used, since this value was assumed during the determination of $E_{0}$ (see eq. (5.25)) in reference [390]. Measurements of the GamowTeller strength for the $g_{1}$ peak and for the Gamow-Teller peaks in the spectrum for $E_{p}^{\prime}$ may be obtained using a similar procedure. If systematic errors can be neglected, then the $B\left(\mathrm{GT}_{-}\right)$values obtained using either spectrum will match within statistics.

\subsubsection{Experimental procedure}

In a 2009 paper [399], Bhattacharya, Goodman, and García presented a measurement of $B\left(\mathrm{GT}_{-}\right)$strengths in ${ }^{40} \mathrm{Ar}$ obtained using $0^{\circ}(p, n)$ scattering. The neutron time-of-flight data needed for their analysis were obtained at $\mathrm{IUCF}^{21}$ using proton kinetic energies of 120 and $160 \mathrm{MeV}$ and an $81 \mathrm{~m}$ flight path for the neutrons. Twelve plastic scintillator bars were used to detect neutrons with scattering angles of $(0.0 \pm 0.2)^{\circ}$ via proton recoils. A plastic scintillator was also placed near the target to detect elastically scattered protons. A gas cell filled with high-purity argon at $300 \mathrm{kPa}$ and cooled using liquid nitrogen was used as the target. Data were taken with the cell filled and with it evacuated to isolate the contribution of the argon to the observed time-of-flight spectra. Reference spectra for the ${ }^{13} \mathrm{C}(p, n){ }^{13} \mathrm{~N}$ reaction, whose level structure is well understood, were used to define an empirical peak shape. This shape was then used to fit spectra recorded using the argon target. Gamow-Teller strengths $B\left(\mathrm{GT}_{-}\right)$were determined by comparing the peak integrals from the two time-of-flight spectra for the ${ }^{40} \mathrm{Ar}$ target, as described in section 5.3.1.

\subsection{Evaluated nuclear matrix elements for MARLEY}

In the publications describing all three available measurements [379, 382, 399] of the allowed matrix elements governing the reaction ${ }^{40} \operatorname{Ar}\left(\nu_{e}, e^{-}\right)^{40} \mathrm{~K}$, attempts were made to assign each observed transition to a known energy level in the final-state ${ }^{40} \mathrm{~K}$ nucleus. While many of the previous level assignments remain plausible at present, new nuclear structure data for the $A=40$ nuclides have become available in recent years. To keep MARLEY as up-to-date as possible, a full re-evaluation of the level assignments was

\footnotetext{
${ }^{21}$ The Indiana University Cyclotron Facility
} 
performed based on the 2017 revision [400] of the ${ }^{40} \mathrm{~K}$ level scheme adopted for use in the ENSDF database. ${ }^{22}$

Table 5.1 presents the experimental matrix element data together with the ${ }^{40} \mathrm{~K}$ energy level assignments for MARLEY, which were made on the basis of excitation energy and (if known) spin-parity. Although Gamow-Teller transitions from the initial ${ }^{40}$ Ar nucleus can only access $1^{+}$levels ${ }^{23}$ in ${ }^{40} \mathrm{~K}$, ENSDF levels with different spin-parity assignments were matched to measured $B(\mathrm{GT})$ values in cases where (1) the ENSDF evaluator regarded the evidence for the spin-parity assignment of the candidate level as "weak," and (2) another suitable level could not be found in the appropriate excitation energy range.

\subsubsection{Disagreements between the ${ }^{40} \mathrm{Ti} \beta^{+}$decay and ${ }^{40} \mathrm{Ar}(p, n)$ experiments}

Figures 5.3 and 5.4 compare the Gamow-Teller strengths $B(\mathrm{GT})$ from the three competing measurements discussed in this chapter. Although the two ${ }^{40} \mathrm{Ti} \beta^{+}$decay experiments show reasonable agreement, obvious differences can be seen between either of those measurements and the $(p, n)$ data of Bhattacharya et al. [399]. Beyond the generally less fragmented $B(\mathrm{GT})$ distribution seen in the $(p, n)$ data, which may be attributed to limited resolution, there is also a disagreement between the beta decay and $(p, n)$ datasets over the relative strength of the two strongest Gamow-Teller transitions. All of the measurements agree that the transition to the ${ }^{40} \mathrm{~K}$ level at $2.73 \mathrm{MeV}$ has $B(\mathrm{GT}) \approx 1.5$. However, the $(p, n)$ data suggest that the strength to the level at $2.29 \mathrm{MeV}$ is larger, $(B(\mathrm{GT})=1.64(16))$ while the two beta decay datasets both report smaller values consistent with $B(\mathrm{GT}) \approx 0.90$.

The large discrepancy in the ratio

$$
R=\frac{B(\mathrm{GT})_{2.73 \mathrm{MeV}}}{B(\mathrm{GT})_{2.29 \mathrm{MeV}}}
$$

obtained from the beta decay data $(R \approx 1.7$ for either dataset) and the $(p, n)$ data ( $R=0.91)$ was recently investigated by Karakoç et al. [401]. On the basis of theoretical

\footnotetext{
${ }^{22}$ The Evaluated Nuclear Structure Data File database (see http://www.nndc.bnl.gov/ensdf/) is maintained by the National Nuclear Data Center of Brookhaven National Laboratory.

${ }^{23}$ This follows immediately from the selection rules in eq. (3.216) if one recalls that the ground state of ${ }^{40}$ Ar has spin-parity $0^{+}$.
} 
Figure 5.3: Gamow-Teller strengths $B(\mathrm{GT})$ from two independent measurements of ${ }^{40} \mathrm{Ti}$ $\beta^{+}$decay. The second dataset is shown with an inverted vertical axis to facilitate comparisons.

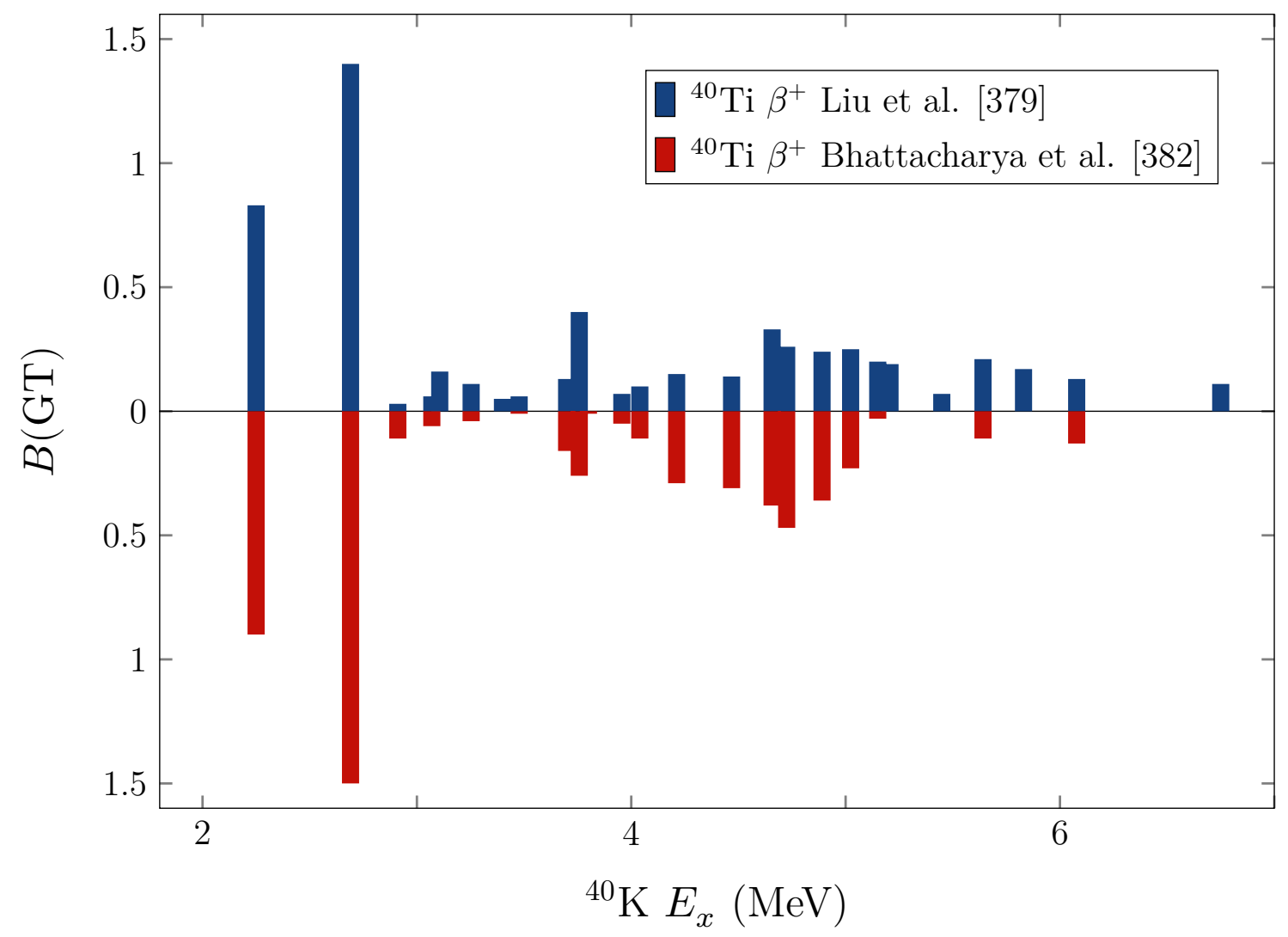

calculations and data from a ${ }^{40} \operatorname{Ar}\left({ }^{3} \mathrm{He}, \mathrm{t}\right){ }^{40} \mathrm{~K}$ scattering experiment, ${ }^{24}$ they argue that one should "use Gamow-Teller strengths extracted from the ${ }^{40} \operatorname{Ar}(p, n)$ data, not the ${ }^{40} \mathrm{Ti}$ $\beta$-decay data, for the calculation of neutrino capture rates." While it may be prudent for MARLEY users to heed their recommendation, matrix element data files based on the results of all three of the published measurements discussed above are nevertheless distributed with the current version of the code. By comparing the results of MARLEY calculations performed using the different experimental datasets, one may estimate the degree to which the remaining uncertainties in the measurements of the Gamow-Teller strength will impact future low-energy neutrino analyses.

\footnotetext{
${ }^{24}$ As of this writing, a detailed report of this experiment remains unpublished.
} 
Table 5.1: Level assignments and measured $B(\mathrm{~F})$ and $B(\mathrm{GT})$ values for ${ }^{40} \operatorname{Ar}\left(\nu_{e}, e^{-}\right)^{40} \mathrm{~K}$

\begin{tabular}{|c|c|c|c|c|c|c|c|}
\hline \multirow{2}{*}{$\begin{array}{c}\text { Assigned } \\
{ }^{40} \mathrm{~K} E_{x} \\
(\mathrm{keV})\end{array}$} & \multirow{2}{*}{$\begin{array}{l}\text { ENSDF [400] } \\
\text { spin-parity } \\
\text { assignment }^{a}\end{array}$} & \multicolumn{2}{|c|}{$\begin{array}{l}\text { Liu et al. }[379] \\
{ }^{40} \mathrm{Ti} \beta^{+} \text {decay }\end{array}$} & \multicolumn{2}{|c|}{$\begin{array}{l}\text { Bhattacharya et al. }[382] \\
{ }^{40} \mathrm{Ti} \beta^{+} \text {decay }\end{array}$} & \multicolumn{2}{|c|}{$\begin{array}{l}\text { Bhattacharya et al. [399] } \\
{ }^{40} \operatorname{Ar}(p, n){ }^{40} \mathrm{~K}\end{array}$} \\
\hline & & $\begin{array}{c}{ }^{40} \mathrm{Sc} E_{x} \\
(\mathrm{keV})\end{array}$ & $\begin{array}{l}B(\mathrm{~F})+ \\
B(\mathrm{GT})\end{array}$ & $\begin{array}{c}{ }^{40} \mathrm{Sc} E_{x} \\
(\mathrm{keV})\end{array}$ & $\begin{array}{l}B(\mathrm{~F})+ \\
B(\mathrm{GT})\end{array}$ & $\begin{array}{c}{ }^{40} \mathrm{~K} E_{x} \\
(\mathrm{keV})\end{array}$ & $\begin{array}{l}\text { Weak }^{b} \\
B(\mathrm{GT})\end{array}$ \\
\hline $2289.868(11)$ & $1^{+}$ & $2287(10)$ & $0.83(8)$ & $2281(8)$ & $0.90(4)$ & $2333(30)$ & $1.64(16)$ \\
\hline $2730.357(19)$ & 1 & $2761(10)$ & $1.40(10)$ & $2752(8)$ & $1.50(6)$ & $2775(30)$ & $1.49(14)$ \\
\hline $2950.9(6)$ & & $2966(40)$ & $0.03(1)$ & $2937(13)$ & $0.11(2)$ & & \\
\hline $3109.56(4)$ & $1^{+}, 2^{+}$ & $3121(46)$ & $0.06(3)$ & $3143(20)$ & $0.06(1)$ & & \\
\hline $3146.50(5)$ & $1^{(-)}$ & $3235(50)$ & $0.16(4)$ & & & $3204(32)$ & $0.06(2)$ \\
\hline $3293(10)$ & unnatural $^{c}$ & $3342(40)$ & $0.11(8)$ & $3334(19)$ & $0.04(1)$ & & \\
\hline $3439.18(3)$ & $\left(2^{+}\right)$ & $3418(60)$ & $0.05(2)$ & & & & \\
\hline $3517(15)$ & & $3521(40)$ & $0.06(2)$ & $3569(56)$ & $0.01(1)$ & $3503(30)$ & $0.16(2)$ \\
\hline $3738.49(3)^{d}$ & $1^{+}$ & $3662(40)$ & $0.13(7)$ & $3652(10)$ & $0.16(2)$ & & \\
\hline $3797.48(3)$ & $1^{+}$ & $3782(40)$ & $0.40(16)$ & $3786(10)$ & $0.26(3)$ & & \\
\hline $3840.27(3)$ & $\left(1,2^{+}\right)$ & & & $3861(49)$ & $0.01(1)$ & $3870(30)$ & $0.44(5)$ \\
\hline $3996(10)$ & unnatural $^{c}$ & $4033(88)$ & $0.07(4)$ & $4067(24)$ & $0.05(2)$ & & \\
\hline $4080(5)$ & & $4194(60)$ & $0.10(6)$ & $4111(30)$ & $0.11(3)$ & & \\
\hline $4251.70(15)$ & $\left(1,2^{-}\right)$ & $4264(46)$ & $0.15(4)$ & $4267(10)$ & $0.29(3)$ & & \\
\hline $4383.7(7)^{e}$ & $0^{+}$ & $4365(10)$ & $4.01(31)$ & $4364(8)$ & $3.84(17)$ & & \\
\hline $4508(15)$ & & $4540(86)$ & $0.14(5)$ & $4522(16)$ & $0.31(5)$ & $4421(30)$ & $0.86(14)$ \\
\hline $4697(10)$ & unnatural $^{c}$ & $4628(40)$ & $0.33(9)$ & $4655(12)$ & $0.38(6)$ & & \\
\hline $4765(5)$ & $(1)^{+}$ & $4782(60)$ & $0.26(11)$ & $4825(21)$ & $0.47(8)$ & $4763(30)$ & $0.48(5)$ \\
\hline 4930(10) & unnatural $^{c}$ & $4997(72)$ & $0.24(10)$ & $5017(27)$ & $0.36(9)$ & & \\
\hline $5063.37(7)$ & $\left(2^{-}, 3^{+}\right)$ & $5051(40)$ & $0.25(11)$ & $5080(35)$ & $0.23(7)$ & & \\
\hline $5189.89(5)$ & $\left(2^{-}\right)$ & $5135(86)$ & $0.20(6)$ & $5223(32)$ & $0.03(3)$ & $5162(30)$ & $0.59(6)$ \\
\hline $5247.1(6)$ & & $5362(60)$ & $0.19(7)$ & & & & \\
\hline $5488.65(17)$ & $\left(2^{-}, 3,4^{-}\right)$ & $5574(40)$ & $0.07(4)$ & & & & \\
\hline $5681(32)$ & & $5777(60)$ & $0.21(15)$ & $5696(23)$ & $0.11(4)$ & $5681(32)$ & $0.21(3)$ \\
\hline $5870(20)$ & & $5886(80)$ & $0.17(7)$ & & & & \\
\hline $6118(30)$ & & $6126(60)$ & $0.13(7)$ & $6006(21)$ & $0.13(5)$ & $6118(30)$ & $0.48(5)$ \\
\hline $6790(30)$ & & $6426(60)$ & $0.11(6)$ & & & $6790(30)$ & $0.71(8)$ \\
\hline $7468(37)$ & & & & & & $7468(37)$ & $0.06(2)$ \\
\hline $7795(33)$ & & & & & & $7795(33)$ & $0.14(2)$ \\
\hline $7952(32)$ & & & & & & $7952(32)$ & $0.97(10)$ \\
\hline Cotal $^{f} B(\mathrm{GT})$ & & & $5.84(39)$ & & $5.52(20)$ & & $8.29(31)$ \\
\hline
\end{tabular}

${ }^{a}$ Parenthesized values are based upon weak arguments [402].

${ }^{b}$ Reference [399] uses a definition of $B(\mathrm{GT})$ different from our "weak $B(\mathrm{GT})$ " convention. See section 5.3.

${ }^{c}$ A nuclear level with parity $\Pi$ and spin $J$ has natural parity if $\Pi=(-1)^{J}$. Otherwise it has unnatural parity.

${ }^{d}$ Another candidate ${ }^{40} \mathrm{~K}$ level for this transition has $E_{x}=3663.88 \mathrm{keV}$ and $J^{\pi}=\left(1^{-}, 2,3,4^{+}\right)$.

${ }^{e}$ This level is the isobaric analog of the ${ }^{40} \mathrm{Ar}$ ground state.

${ }^{f}$ Gamow-Teller transitions are assumed for all levels other than the isobaric analog state. 
Figure 5.4: Comparison of the Gamow-Teller strengths $B(\mathrm{GT})$ measured using ${ }^{40} \mathrm{Ti} \beta^{+}$ decay (see fig. 5.3) with those obtained using a $0^{\circ}(p, n)$ scattering experiment. The sensitivity of the $\beta$ decay experiments above $6.5 \mathrm{MeV}$ (marked by the dashed line) was very limited. Liu et al. note that, for a ${ }^{40} \mathrm{Sc}$ level at this excitation energy, a $B(\mathrm{GT})$ value of 0.1 corresponds to a branching fraction of only $0.1 \%$ due to the phase space factor for allowed $\beta$ decay. Detector effects further complicate extraction of $B(\mathrm{GT})$ values from $\beta$ decays to higher excitation energies [379].
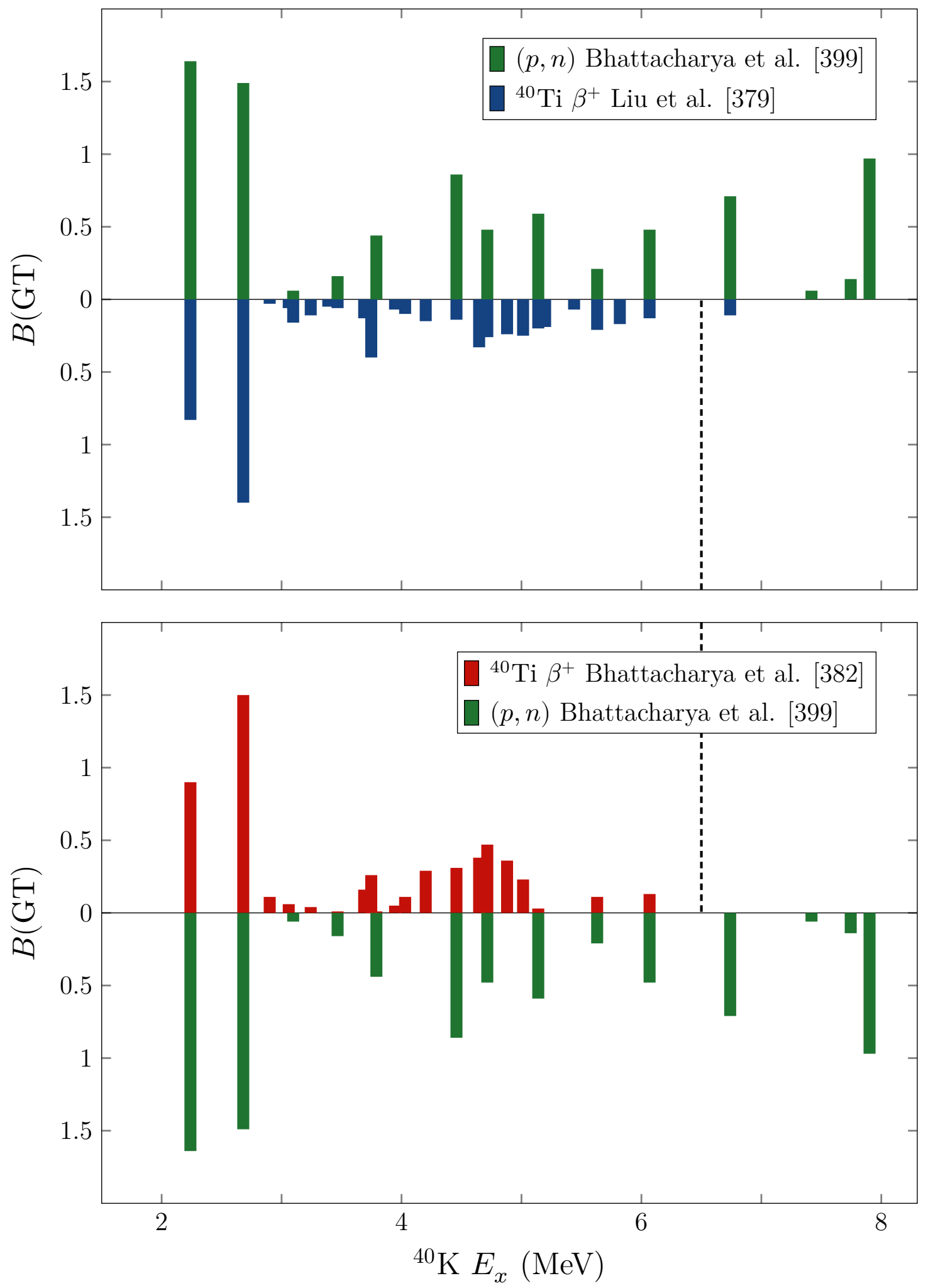


\subsubsection{High-lying Gamow-Teller strength}

Notwithstanding the need to carefully examine the differences between the existing measurements of the ${ }^{40} \mathrm{Ar}$ allowed matrix elements, a more challenging consideration for supernova neutrino detection is the currently unmeasured portion of the Gamow-Teller strength predicted by the Ikeda sum rule. Equation (5.10) implies that the integral of $B\left(\mathrm{GT}_{-}\right)$ over all final ${ }^{40} \mathrm{~K}$ levels must have as its minimum possible value $S_{G T}^{-}=12 g_{A}^{2} \approx 19$. Comparing this lower limit to the $B\left(\mathrm{GT}_{-}\right)$sums given in the last row of table 5.1 reveals that the currently available measurements can account for less than $50 \%$ of the total expected strength. The remaining Gamow-Teller transitions will access highly-excited levels in the final-state ${ }^{40} \mathrm{~K}$ nucleus beyond the 6 to $8 \mathrm{MeV}$ maximum excitation energy probed to date by experiments.

Since, according to eq. (5.8), the centroid of the GTGR for ${ }^{40}$ Ar should be located at an excitation energy of about $10 \mathrm{MeV}$ in the daughter ${ }^{40} \mathrm{~K}$ nucleus, one can expect that many of the unmeasured Gamow-Teller transitions will be kinematically accessible for supernova $\nu_{e}$ with tens-of-MeV energies. These transitions will populate nucleon-unbound ${ }^{25}$ states for which neutron emission will be a dominant de-excitation mode. Because such deexcitations will be challenging to accurately reconstruct in a LArTPC, simply neglecting the high-lying GT strength in a model of the response of ${ }^{40} \mathrm{Ar}$ to supernova neutrinos would lead to overly optimistic sensitivity estimates.

While a number of theoretical predictions of the ${ }^{40} \operatorname{Ar}\left(\nu_{e}, e^{-}\right){ }^{40} \mathrm{~K}$ cross section exist in the literature (see chapter 7 for a brief review), most publications on the subject do not include an explicit calculation of the reduced matrix elements $B\left(\mathrm{GT}_{-}\right)$needed to evaluate the allowed approximation cross section given in eq. (3.220). An exception is a 2012 paper by Cheoun, Ha, and Kajino [288] in which both $B\left(\mathrm{GT}_{-}\right)$and $B\left(\mathrm{GT}_{+}\right)$ strengths are obtained for ${ }^{40} \mathrm{Ar}$ over the entire energy range of interest for supernova neutrinos. Their results, which were calculated using the Quasiparticle Random Phase Approximation (QRPA), are shown in fig. 5.5 and compared with the experimental data

\footnotetext{
${ }^{25}$ The proton and neutron separation energies for ${ }^{40} \mathrm{~K}$ are $7.582(5) \mathrm{MeV}$ and $7.79962(6) \mathrm{MeV}$, respectively [400].
} 
Figure 5.5: Theoretical Gamow-Teller strengths $B\left(\mathrm{GT}_{-}\right)$for ${ }^{40} \operatorname{Ar}\left(\nu_{e}, e^{-}\right){ }^{40} \mathrm{~K}$ calculated using the Quasiparticle Random Phase Approximation (QRPA)

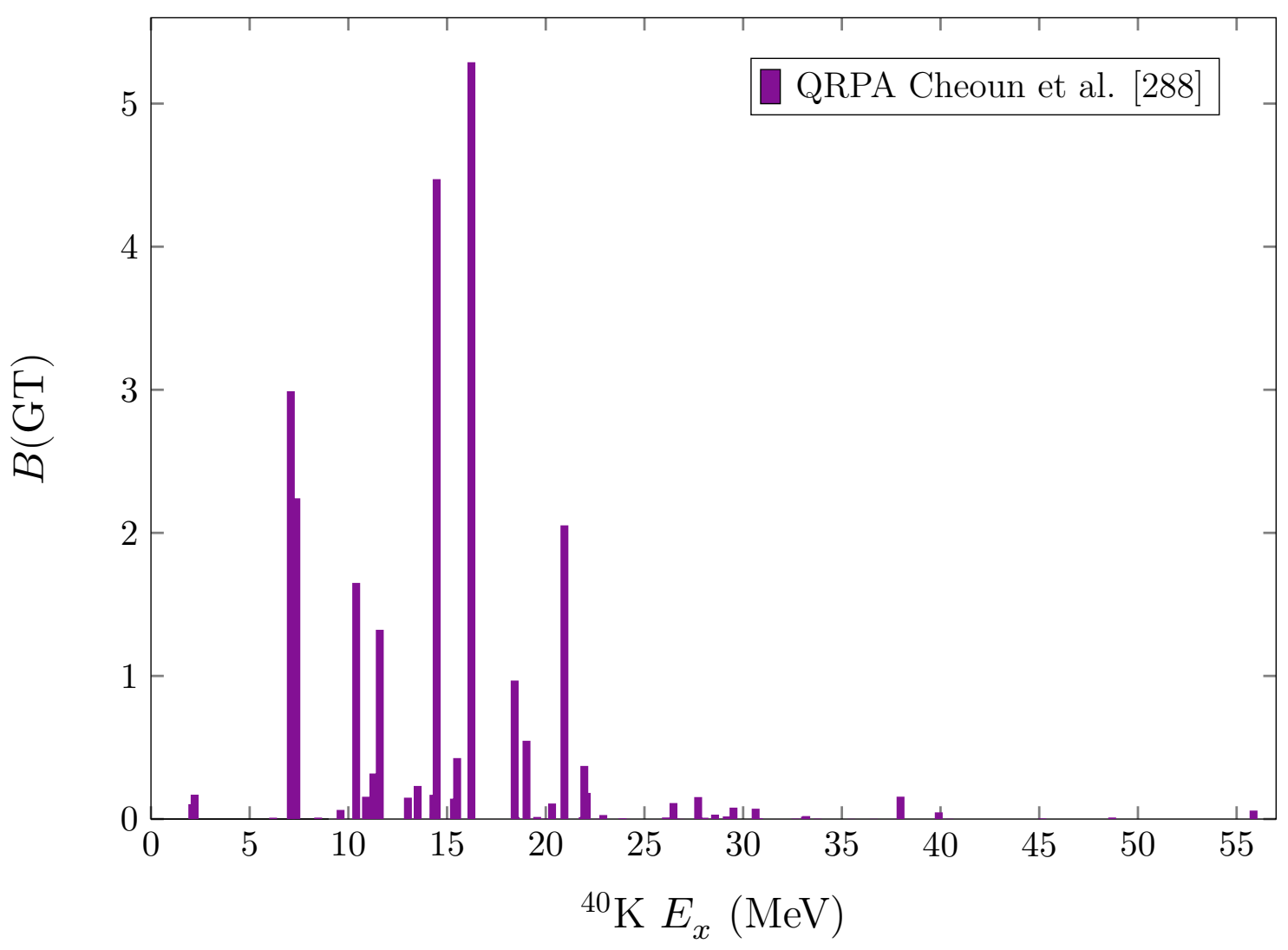

in fig. 5.6.

An examination of the latter figure reveals reasonably close agreement at $7.5 \mathrm{MeV}$ between the integrated $B\left(\mathrm{GT}_{-}\right)$strengths obtained from the QRPA calculation and from the $\beta$ decay data. However, the theoretical prediction concentrates nearly all of this strength in two transitions to ${ }^{40} \mathrm{~K}$ levels around $7 \mathrm{MeV}$, while the experiments demonstrate that the strength is actually highly fragmented. Although results obtained using the nuclear shell model show better agreement $[282,377]$ with the data ${ }^{26}$ nuclear effective potentials used in the shell model are typically designed with only low energies in mind, and calculations at several tens of $\mathrm{MeV}$ are computationally infeasible due to the large number of states that must be considered [225]. The QRPA solves the second of these two

\footnotetext{
${ }^{26}$ This is achieved by applying a quenching factor of $Q_{G T}=0.775$ to the weak axial-vector coupling constant (see section 5.1.2). The validity of this procedure is unclear for neutrinos at supernova energies [225]. Cheoun et al. [288] did not use a quenching factor in their QRPA calculation.
} 
Figure 5.6: Running sums of $B(\mathrm{GT})$ from three experimental measurements and a QRPA calculation as a function of ${ }^{40} \mathrm{~K}$ excitation energy. Although the theoretical prediction fails to reproduce the observed fragmentation of the Gamow-Teller strength at low energies, the predicted integrated strength at $7.5 \mathrm{MeV}$ nearly matches that of the ${ }^{40} \mathrm{Ti} \beta^{+}$decay measurements at around the same excitation energy.

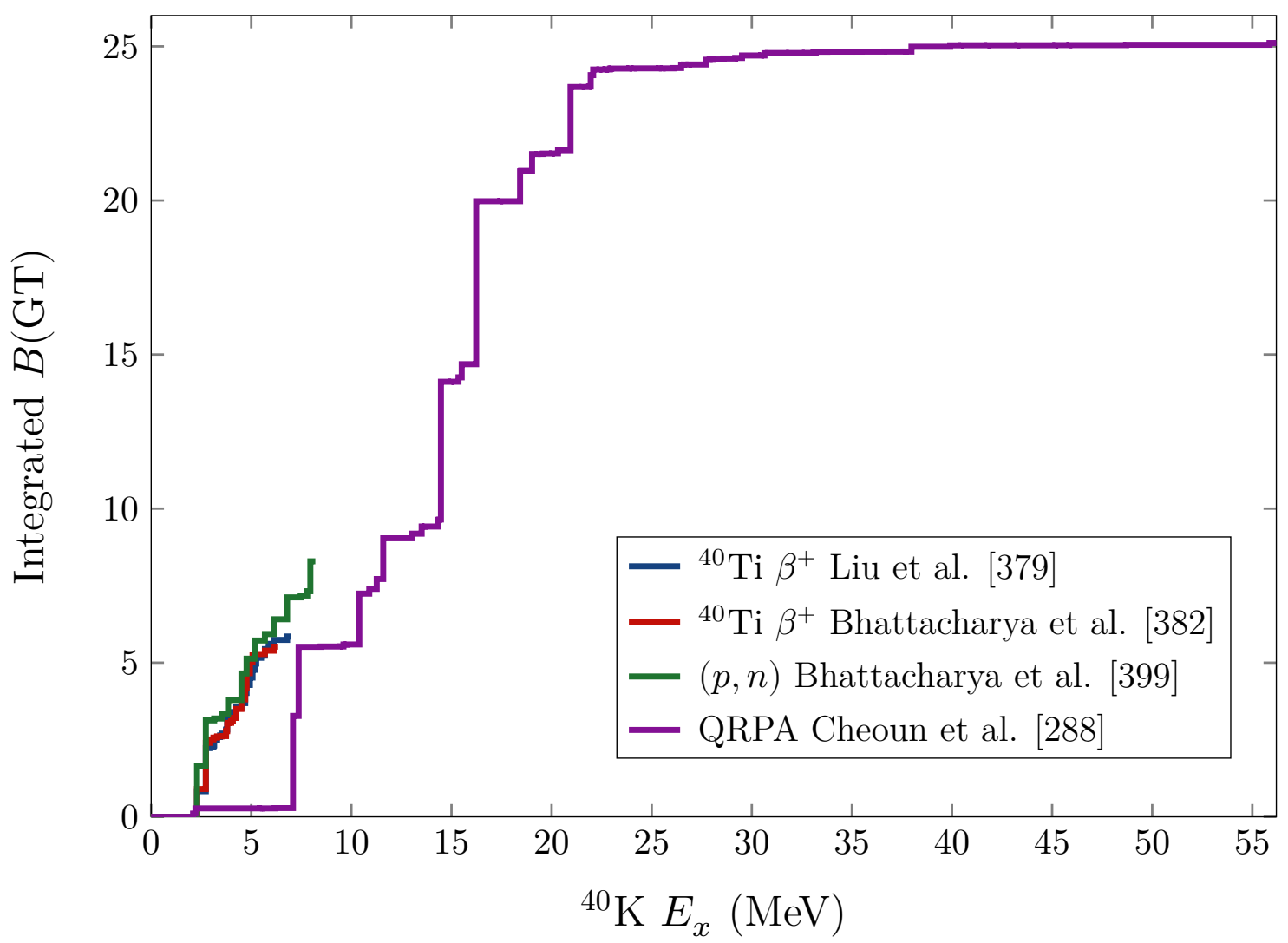

problems by considering only a small subset of the possible nuclear configurations. This choice extends the excitation energy reach of the model but artificially restricts the degree of fragmentation that can be produced [200]. The disagreement between the data and the QRPA prediction over how the $\mathrm{GT}_{-}$strength is distributed below $7 \mathrm{MeV}$ is symptomatic of this defect of the model.

If one is willing to tolerate the limitations of the QRPA, the calculation of Cheoun et al. may be used together with the experimental data to create a complete set of GT matrix elements. Because the Ikeda sum rule is nearly satisfied (to about $7 \%$ ) by the theoretical calculation, ${ }^{27}$ such a set of matrix elements will include essentially all of the

\footnotetext{
${ }^{27}$ Cheoun et al. suggest that this discrepancy might arise because of the lack of exact nucleon number conservation in the QRPA [288].
} 
expected total strength. While combining experimental and theoretical matrix elements in this way may be difficult to do in general, the good agreement between the QRPA and the $\beta$ decay data seen in the integrated $\mathrm{GT}_{-}$strength at $7.5 \mathrm{MeV}$ suggests that the two may be straightforwardly merged by (1) computing the total $\mathrm{GT}_{-}$strength measured by a particular experiment, (2) finding the ${ }^{40} \mathrm{~K}$ excitation energy $E_{x, \mathrm{QRPA}}$ for which the theoretical integrated $\mathrm{GT}_{\text {- }}$ strength first exceeds the experimental total, (3) including only measured $B\left(\mathrm{GT}_{-}\right)$values (from the experiment of interest) for transitions to ${ }^{40} \mathrm{~K}$ levels below the "theory threshold" $E_{x, \mathrm{QRPA}}$, and (4) using QRPA B(GT_) values for transitions to ${ }^{40} \mathrm{~K}$ levels above $E_{x, \mathrm{QRPA}}$.

Using the procedure outlined above, three complete matrix element datasets, one based on each of the experiments discussed in this chapter, were prepared for the current version of MARLEY. Figure 5.7 shows the matrix elements included in each dataset, with different colors used to distinguish experimental from theoretical values of $B\left(\mathrm{GT}_{-}\right)$. Since both ${ }^{40} \mathrm{Ti} \beta^{+}$decay experiments measured values of $B\left(\mathrm{~F}_{-}\right)$consistent with the sum rule prediction (see section 5.1.1), a single Fermi transition to the isobaric analog state $^{28}$ with $B\left(\mathrm{~F}_{-}\right)=4$ was included in each dataset. Figure 5.8 compares the running sums of $B\left(\mathrm{GT}_{-}\right)$from each dataset to that of the QRPA calculation by Cheoun et al. [288]. As expected, "stitching together" the theoretical matrix elements and either of the two $\beta$ decay measurements (as was done to create datasets $\mathrm{B}$ and $\mathrm{C}$ ) yields an integrated $B\left(\mathrm{GT}_{-}\right)$curve that is nearly the same as the original QRPA calculation above $E_{x, \mathrm{QRPA}} \approx$ 7.5 MeV. Less satisfactory results are obtained for MARLEY dataset A, which is based on the $(p, n)$ data. However, given the inability of the QRPA to reproduce the individual $B(\mathrm{GT})$ strengths below $7 \mathrm{MeV}$, one should regard the theoretical calculation as only a rough estimate of the true GT strength distribution. As more detailed calculations (or, better still, measurements) of the high-lying GT strength distribution become available for ${ }^{40} \mathrm{Ar}$, MARLEY will be able to move beyond its use of these QRPA strengths as a first approximation.

\footnotetext{
${ }^{28} 4.3837 \mathrm{MeV}$ above the ${ }^{40} \mathrm{~K}$ ground state
} 
Figure 5.7: The three allowed matrix element datasets for charged current $\nu_{e}$ scattering on ${ }^{40} \mathrm{Ar}$ distributed with the current version of MARLEY
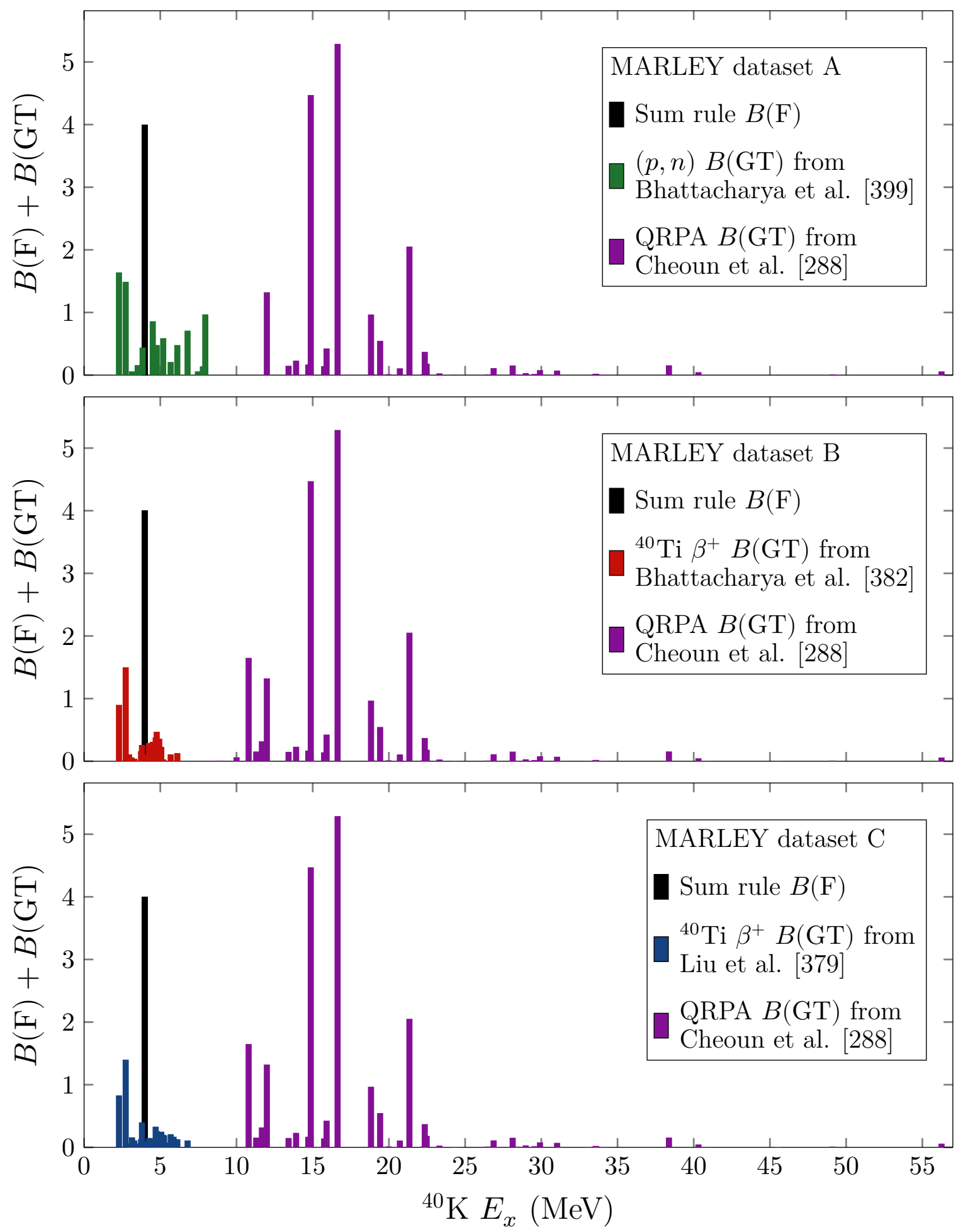
Figure 5.8: Comparison of the integrated $B(\mathrm{GT})$ curves for the three allowed matrix element datasets for ${ }^{40} \operatorname{Ar}\left(\nu_{e}, e^{-}\right)^{40} \mathrm{~K}$ distributed with the current version of MARLEY.

A corresponding curve for the QRPA calculation that provided $B(\mathrm{GT})$ values for

high-lying Gamow-Teller transitions in all three datasets is also shown.

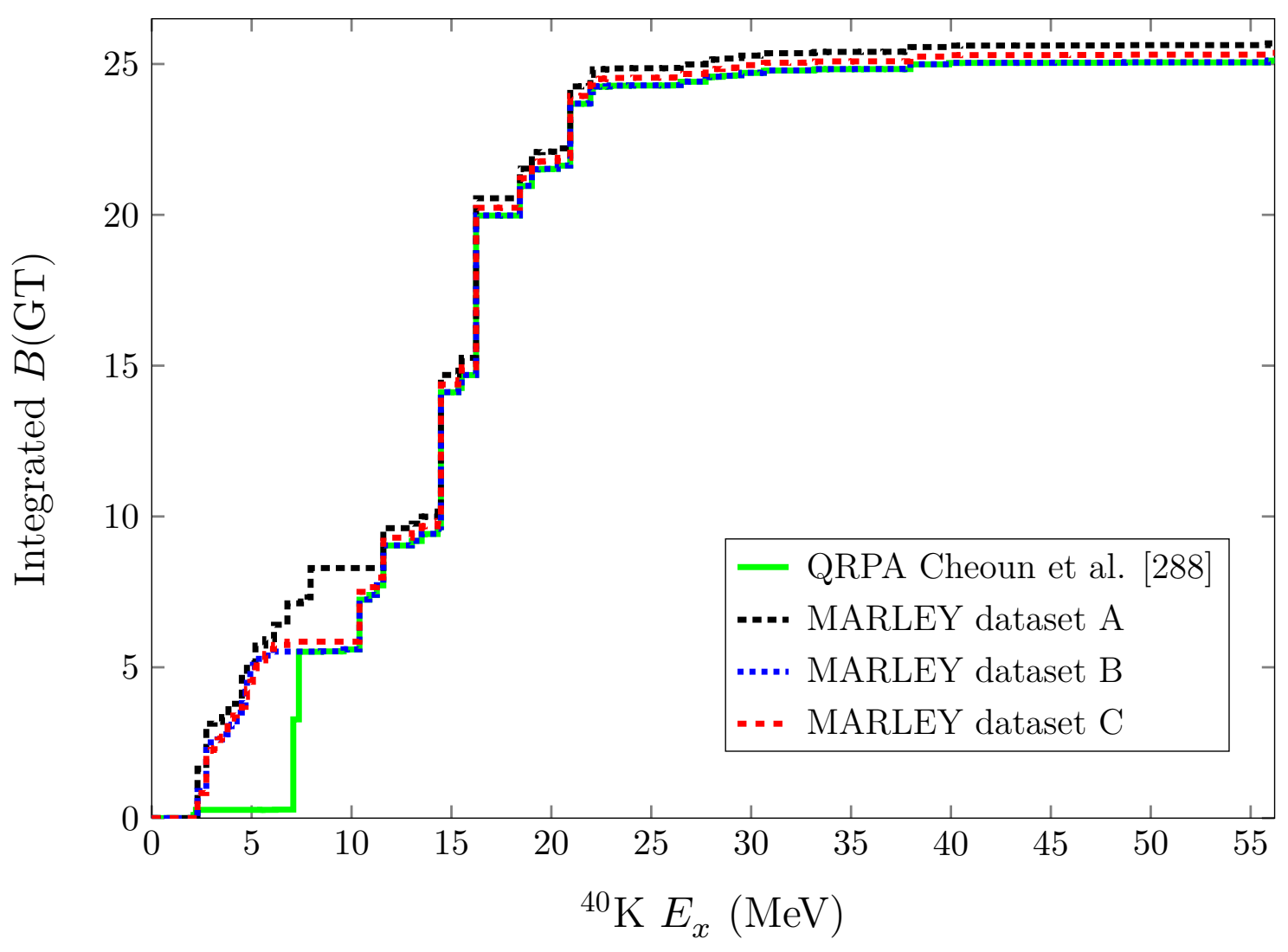




\section{Chapter 6}

\section{Monte Carlo implementation}

To enable practical calculations to be performed using the models and nuclear data discussed in the previous three chapters, a Monte Carlo event generator called MARLEY (Model of Argon Reaction Low Energy Yields) was created. While the ultimate goal of the code is to become a general-purpose generator for neutrino-nucleus scattering at tens-of-MeV and below, the current version focuses solely on simulating the charged cur-

rent reaction ${ }^{40} \operatorname{Ar}\left(\nu_{e}, e^{-}\right)^{40} \mathrm{~K}$. In the following sections, the procedure followed by the MARLEY code for generating neutrino scattering events is briefly reviewed. A summary of the event generation workflow is given in the flowchart in fig. 6.1.

\subsection{Generator initialization}

The MARLEY command-line program takes as input the name of a configuration file which contains, at a minimum, two pieces of information. The first of these is the name of a reaction data file that specifies the set of allowed matrix elements to be used when calculating cross sections. Although multiple reactions may be defined in the configuration file, only reaction data files for ${ }^{40} \operatorname{Ar}\left(\nu_{e}, e^{-}\right)^{40} \mathrm{~K}$ are currently distributed with the code. Each reaction data file lists the initial and final particle ID numbers (using the conventions defined by the Particle Data Group [78]), a descriptive string, and a table of the allowed nuclear matrix elements.

The second required piece of input data is a specification of the incident (i.e., not weighted by the reaction cross section) neutrino energy spectrum. This may be done 


\section{MARLEY event generation flowchart}

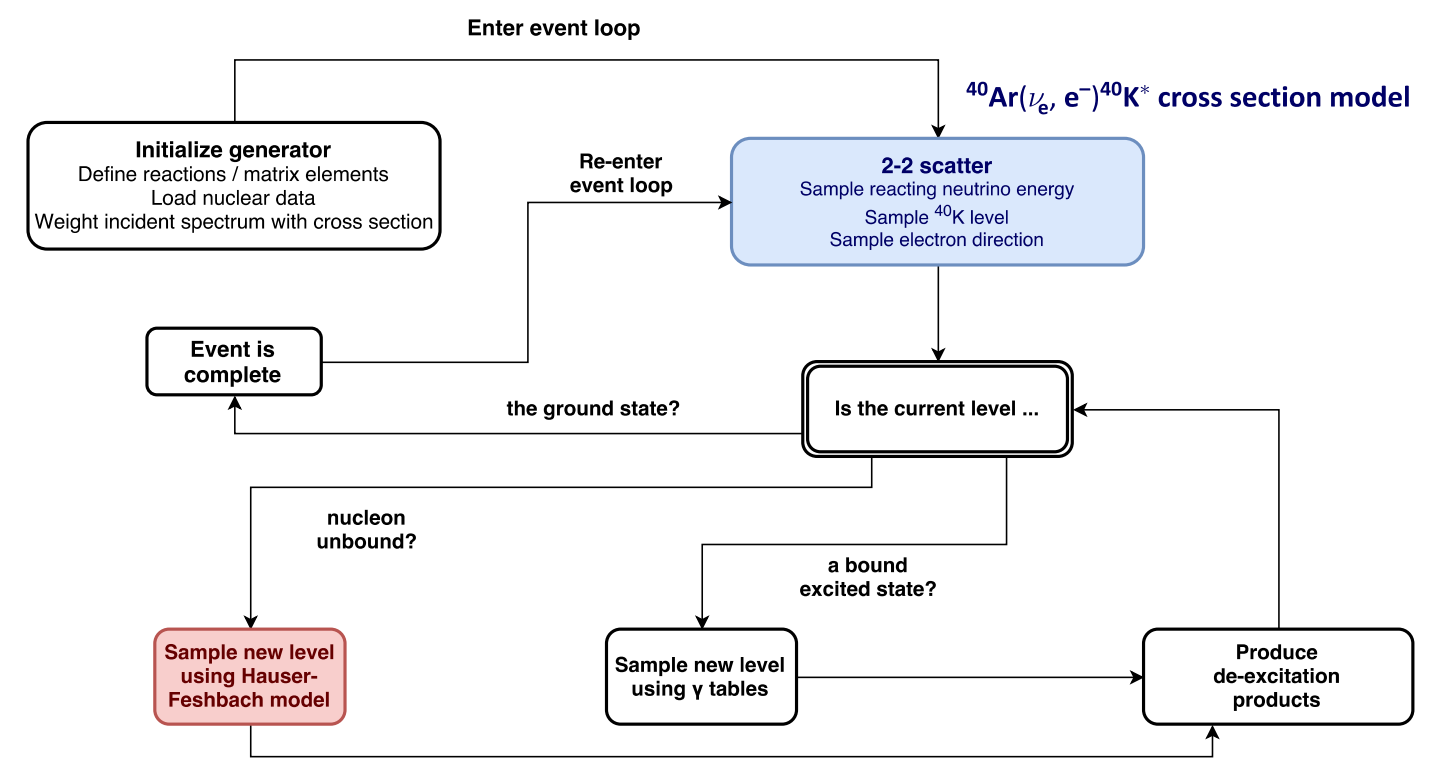

Nuclear de-excitation model

Figure 6.1: Flowchart showing the procedure followed by MARLEY for creating simulated neutrino-nucleus scattering events.

using one of several built-in spectra (e.g., a muon decay-at-rest spectrum, a Fermi-Dirac distribution), a user-defined histogram, or a user-defined function evaluated by interpolating between a list of (energy, flux) ordered pairs.

Beyond the two required inputs, the user may specify the paths to one or more nuclear structure data files (which contain energies, spin-parities, and $\gamma$-ray branching ratios for the nuclear energy levels of one or more nuclides), a desired number of events, one of several output formats, and a variety of other configuration options. Documentation describing the configuration file format is available from the official MARLEY website (https://www.marleygen.org) and in the forthcoming MARLEY user manual.

Before entering the event loop, the reaction data and incident neutrino spectrum specified by the user are used to calculate an energy spectrum for reacting neutrinos. This spectrum is computed by multiplying the incident neutrino spectrum by the sum of the total cross sections for each of the defined reactions. The resulting spectrum is 
normalized to unity to obtain a probability density function $P\left(E_{\nu}\right)$ for sampling reacting neutrino energies:

$$
P\left(E_{\nu}\right)=\frac{\psi_{\text {reacting }}\left(E_{\nu}\right)}{\int_{E_{\min }}^{E_{\max }} \psi_{\text {reacting }}(E) d E}=\frac{\psi_{\text {incident }}\left(E_{\nu}\right) \sum_{r} \sigma_{r}\left(E_{\nu}\right)}{\int_{E_{\min }}^{E_{\max }}\left[\psi_{\text {incident }}(E) \sum_{r} \sigma_{r}(E)\right] d E} .
$$

Here the sums $\sum_{r}$ include all defined reactions, and the integration in the denominator is over the full energy interval over which the incident spectrum is defined. The total cross sections are computed by using the allowed approximation (see eq. (3.220)) and summing the contributions of all kinematically-accessible final nuclear levels.

\section{$6.22 \rightarrow 2$ scattering reaction}

After preparing the reacting neutrino energy spectrum, the MARLEY code enters the start of the event loop, which repeats until the desired number of events specified by the user have been created. To begin a new event, a reacting neutrino energy $E_{\nu}$ is sampled from the probability density $P\left(E_{\nu}\right)$ given in eq. (6.1). This sampling, like all other sampling from continuous probability distributions in the MARLEY code, is performed using a simple rejection technique. First, the maximum of the probability density $P_{\max }$ is found numerically using Brent's method ${ }^{1}$ [403]. After the maximum is found, ordered pairs $\left(E_{\nu}, y\right)$ are sampled from uniform distributions on $E_{\nu} \in\left[E_{\min }, E_{\max }\right]$ and $y \in\left[0, P_{\max }\right]$. If a sampled point has $y \leq P\left(E_{\nu}\right)$, the sampled neutrino energy $E_{\nu}$ is accepted, and the rest of the simulation proceeds. If $y>P\left(E_{\nu}\right)$, the sampled energy is rejected, and a new energy is sampled repeatedly until one is accepted. Although the incident spectrum (and therefore reacting neutrino energy $E_{\nu}$ ) is specified in the lab frame by the user, all calculations for simulating the $2 \rightarrow 2$ scattering reaction are evaluated internally in the CM frame.

Once a reacting neutrino energy has been found, if multiple reactions are defined, then a specific reaction $r$ is chosen with probability

$$
P(r)=\frac{\sigma_{r}\left(E_{\nu}\right)}{\sum_{s} \sigma_{s}\left(E_{\nu}\right)}
$$

\footnotetext{
${ }^{1} \mathrm{~A}$ robust minimization algorithm for one-dimensional functions. The method does not involve the use of numerical differentiation.
} 
where the $\sigma_{r}\left(E_{\nu}\right)$ are total reaction cross sections (including contributions from all accessible final-state nuclear levels) and the sum in the denominator is over all defined reactions. To sample from the discrete distribution defined by the reaction probability mass function shown in eq. (6.2), MARLEY computes the cumulative distribution function for the $r$ th reaction via

$$
C(r)=\sum_{k=1}^{r} P(k)
$$

for $k \in\{1,2,3, \cdots, r\}$ and $C(0) \equiv 0$. After sampling a uniform random number $\xi$ on the interval $(0,1]$, the reaction $r$ for which $C(r-1)<\xi \leq C(r)$ is selected. A similar technique is used to sample from all other discrete probability distributions of interest for MARLEY.

Once a unique reaction has been determined for the current event, MARLEY samples a specific energy level $L$ of the final-state nucleus with probability

$$
P(L)=\frac{\sigma_{r}\left(E_{\nu}, L\right)}{\sigma_{r}\left(E_{\nu}\right)}
$$

where $\sigma_{r}\left(E_{\nu}, L\right)$ is the partial cross section to the $L$ th final level, and the total cross section $\sigma_{r}\left(E_{\nu}\right)$ may be expressed in terms of the partial cross sections via

$$
\sigma_{r}\left(E_{\nu}\right)=\sum_{L} \sigma_{r}\left(E_{\nu}, L\right)
$$

Following selection of the final-state nuclear level $L$, an azimuthal angle for the outgoing charged lepton (currently always an electron) is sampled uniformly on the half-open interval $[0,2 \pi)$. None of the differential cross sections of interest for MARLEY have any dependence on the electron's azimuthal angle, as discussed in chapter chapter 3. A polar angle cosine $\cos \theta_{e}$ for the outgoing electron is also sampled on the interval $[-1,1]$ from the probability density function

$$
P\left(\cos \theta_{e}\right)=\frac{1}{\sigma_{r}\left(E_{\nu}, L\right)} \frac{d \sigma_{r}\left(E_{\nu}, L\right)}{d \cos \theta_{e}}
$$

where the differential cross section for the $L$ th final level $d \sigma_{r}\left(E_{\nu}, L\right) / d \cos \theta_{e}$ is computed according to eq. (3.219). As the notation for the differential cross section suggests, the integral of the differential cross section over all possible polar angle cosines is the partial 
reaction cross section to level $L$ :

$$
\sigma_{r}\left(E_{\nu}, L\right)=\int_{-1}^{1} \frac{d \sigma_{r}\left(E_{\nu}, L\right)}{d \cos \theta_{e}} d \cos \theta_{e}
$$

After obtaining a direction in the CM frame for the outgoing electron, MARLEY uses conservation of 4-momentum to determine the full 4-momenta of the electron and final-state nucleus in the CM frame. A Lorentz boost is then applied to re-express the 4-momenta in the laboratory frame.

\subsection{Nuclear de-excitations}

Once simulation of the initial $2 \rightarrow 2$ scattering reaction is complete, MARLEY enters a nuclear de-excitation loop. At each stage of the loop, information about the current energy level of the recoiling nucleus is obtained from the nuclear structure data loaded into memory during the initialization step. If the current level corresponds to the nuclear ground state, the de-excitation loop terminates, and the event is complete.

If the current level is an unbound excited state, i.e., it has an excitation energy above at least one of the emission thresholds for nuclear fragments with mass number $A \leq 4,{ }^{2}$ then MARLEY computes Hauser-Feshbach decay widths $\Gamma_{\alpha}$ for each possible de-excitation channel $\alpha$ using the expressions given in chapter 4 . These are computed individually for accessible discrete energy levels included in the input nuclear structure data. Deexcitations to the continuum (which is considered to begin for each nuclide just above its tabulated nuclear level with the highest excitation energy) are also considered by integrating over the accessible continuum as shown in eq. (4.26). A particular de-excitation channel $\alpha$ is sampled using the calculated decay widths with probabilty

$$
P(\alpha)=\frac{\Gamma_{\alpha}}{\sum_{\beta} \Gamma_{\beta}}
$$

If a de-excitation to the continuum bin is selected, then a unique value of the total kinetic energy in the CM frame $\varepsilon$ is sampled from the probability density function

$$
P(\varepsilon)=\frac{\sum_{\ell j} \delta_{\pi} T_{\ell j}(\varepsilon) \rho_{f}\left(E_{x}^{\prime}(\varepsilon), J^{\prime}, \Pi^{\prime}\right)}{\sum_{\ell j} \delta_{\pi} \int_{0}^{\varepsilon_{\max }} T_{\ell j}(\varepsilon) \rho_{f}\left(E_{x}^{\prime}(\varepsilon), J^{\prime}, \Pi^{\prime}\right) d \varepsilon .}
$$

\footnotetext{
${ }^{2}$ Explicitly, these are neutrons, protons, deuterons, tritons, helions, and alpha particles.
} 
where all symbols are defined as in eq. (4.26) except that the maximum total CM frame kinetic energy $\varepsilon_{\max }$ is the maximum possible within the range of excitation energies defined by the boundaries of the continuum. Once a value of $\varepsilon$ has been determined, elementary kinematical calculations are used to assign 4-momenta to the outgoing fragment and the daughter nucleus.

If the current level is a bound excited state, i.e., it has a nonzero excitation energy but is below all of the nuclear fragment emission thresholds, then MARLEY consults its internal nuclear structure database to determine whether a table of $\gamma$-ray intensities is available for the level. If such a table was included in the input data provided in the initialization step, then a $\gamma$-ray transition $\gamma_{j}$ is sampled with probability

$$
P\left(\gamma_{j}\right)=\frac{I_{j}}{\sum_{k} I_{k}}
$$

where $I_{j}$ is the tabulated intensity of the $j$ th $\gamma$-ray for the current level and the sum in the denominator runs over all $\gamma$-ray transitions from that level that are included in the tabulated data. If a table of $\gamma$-ray intensities cannot be found for the current level, then MARLEY follows the same procedure as for the unbound nuclear levels.

At the end of each de-excitation step, all particle 4-momenta are Lorentz transformed into the laboratory frame. The de-excitation loop continues until the ground state is reached, or, in cases where no discrete level data are available for a particular final-state nucleus, until the excitation energy of the current level falls below $1 \mathrm{keV}$.

\subsection{Completing the event}

For convenience during its internal calculations, MARLEY assumes that the incident neutrino direction lies along the positive $\mathrm{z}$ axis. This is also assumed in the output unless the user specifies another direction in the configuration file. After the de-excitation loop has terminated, all particle momentum vectors are appropriately rotated as needed to account for the neutrino direction selected by the user.

At the end of every iteration of the event loop, a marley: :Event $\mathrm{C}++$ object is created in memory. This object stores all of the particle 4-momenta for the initial and final states, and it also includes the initial excitation energy of the recoil nucleus created 
during the $2 \rightarrow 2$ scattering step. When MARLEY is used via its command-line executable, the marley: :Event object is written to disk in one of several possible formats. When MARLEY is called as an external library within a particle transport framework such as Geant4, the marley: : Event object may be used to supply initial 4-momenta for particles produced during a low-energy neutrino scattering event.

After the generated event is saved to disk or used as an input for a particle transport simulation, the event generation loop repeats until the desired number of events specified by the user has been reached. In the following chapter, example simulation results obtained using MARLEY on its own and in combination with LArSoft [165], a Geant4-based LArTPC simulation code (see section 2.5), will be presented. 


\section{Chapter 7}

\section{Example simulation results}

To conclude the presentation of MARLEY in this thesis, this chapter briefly reviews the existing literature on low-energy neutrino-argon scattering, presents some example simulation results obtained using the MARLEY generator, and discusses future possiblities for its improvement.

\subsection{Previous studies of low-energy neutrino- ${ }^{40}$ Ar cross sections}

Interest in liquid argon as a detection medium for low-energy neutrinos, including supernova neutrinos, began shortly after the LArTPC was originally proposed [129] by Carlo Rubbia in 1977. The first neutrino cross section calculation in this energy range for a ${ }^{40} \mathrm{Ar}$ target emerged in a pioneering 1979 AT\&T Bell Laboratories technical report [404] by Ramaswamy Raghavan. In the report, Raghavan pointed out the relatively large cross section for ${ }^{40} \operatorname{Ar}\left(\nu_{e}, e^{-}\right)^{40} \mathrm{~K}$ scattering proceeding via Fermi transitions to the isobaric analog state (see section 5.1.1) and argued that events of this kind could be readily identified in a LArTPC using the final-state electron and the accompanying de-excitation $\gamma$-ray cascade. Building on this early work, Bahcall et al. published a study of solar neutrino event rates in the ICARUS detector [405] in 1986. During that same year, in addition to publishing an updated version [406] of his previous report, Raghavan and collaborators produced a second article [407] that considered inelastic neutral current neutrino scattering ${ }^{1}$ on ${ }^{40} \mathrm{Ar}$ using a shell model calculation of the Gamow-Teller transition matrix elements. While

\footnotetext{
${ }^{1}$ This process is referred to as "NUEX" in the publication.
} 
the predicted rate of inelastic NC events generated by solar neutrinos in a LArTPC was not entirely negligible, this early calculation was deemed "not encouraging," partially because much of the neutral Gamow-Teller strength was predicted to lie above the neutron emission threshold of ${ }^{40} \mathrm{Ar}$ and partially because the solar neutrino $\mathrm{NC}$ event rate was found to be nearly two orders of magnitude smaller than the rate for CC transitions to the ${ }^{40} \mathrm{~K}$ isobaric analog state.

The first theoretical calculation of a cross section for charged current $\nu_{e}$ scattering on ${ }^{40} \mathrm{Ar}$ that included contributions from Gamow-Teller transitions was that of Ormand et al. [377], who in 1995 used the nuclear shell model to predict solar neutrino reaction rates in ICARUS under the allowed approximation. By 1998, two independent experiments $[379,382]$ had made indirect measurements of the low-lying Gamow-Teller strengths using ${ }^{40} \mathrm{Ti}$ beta decay (see section 5.2.2). Further publications on low-energy neutrinoargon cross sections would not emerge until 2003, when Gil-Botella and Rubbia, building on work reported in an earlier preprint [408], published the first study [409] of supernova neutrino event rates in a LArTPC to consider all reaction channels, including $\nu_{e}$ and $\bar{\nu}_{e}$ charged current absorption, neutral current neutrino-argon scattering, and neutrinoelectron elastic scattering. ${ }^{2}$ Soon thereafter, Athar and Singh produced an $\mathrm{LDA}^{3}$ calculation of charged current cross sections for supernova electron (anti)-neutrinos on ${ }^{40} \mathrm{Ar}$ [280]. This approach was also applied to muon decay at rest neutrinos in a 2006 paper that they published together with Ahmad [410]. Before the end of the decade, a second experimental probe, $(p, n)$ scattering, had been used [399] to study the allowed matrix elements for the ${ }^{40} \operatorname{Ar}\left(\nu_{e}, e^{-}\right)^{40} \mathrm{~K}$ reaction (see section 5.3).

Theoretical work on supernova neutrino cross sections for ${ }^{40} \mathrm{Ar}$ has grown in recent years, likely due in part to the decision by the LBNE Collaboration (the forerunner of the present DUNE Collaboration) in January 2012 to use LArTPCs as the far detectors for the experiment [411]. Since then, new cross section calculations have been performed using a

\footnotetext{
${ }^{2}$ Note that the cross sections used for the studies in references $[408,409]$ were provided to the authors by Martinez-Pinedo et al. via private communication.

${ }^{3}$ Local Density Approximation
} 
variety of nuclear models, including the $\mathrm{QRPA}^{4}[288,412,413]$, the $\operatorname{RQRPA}^{5}[281,414]$, the PQRPA ${ }^{6}$ [415], the GTDB ${ }^{7}$ [415], the $\mathrm{LDA}^{3}$ [416], the nuclear shell model [225], and a "hybrid" approach [282, 417]. The last of these used a shell model calculation to evaluate the contributions of Fermi and Gamow-Teller transitions to the cross section, while the $\mathrm{RPA}^{8}$ was used for other transitions. A similar "hybrid" technique was employed much earlier [418] by Kolbe, Langanke, and Martinez-Pinedo to compute the ${ }^{56} \mathrm{Fe}\left(\nu_{e}, e^{-}\right){ }^{56} \mathrm{Co}$ total cross section.

\subsection{Total cross section comparison}

Given the current absence of any direct experimental measurements of neutrino-argon cross sections in the energy range of interest for MARLEY, it is impossible to definitively evaluate the quality of the ${ }^{40} \operatorname{Ar}\left(\nu_{e}, e^{-}\right)^{40} \mathrm{~K}$ cross section model implemented in the generator. However, one may make a tentative judgment by comparing the MARLEY model to other more detailed theoretical calculations. Figure 7.1 shows the MARLEY total cross section for CC absorption of $\nu_{e}$ on ${ }^{40} \mathrm{Ar}$ compared to several other predictions over a wide energy range. Except for the $\mathrm{GTBD}^{7}$ cross section, which is obtained using a very simple macroscopic nuclear model, all other non-MARLEY cross sections shown in the figure are based on sophisticated microscopic calculations that go beyond MARLEY's impulse approximation treatment. Despite MARLEY's relative simplicity, it reproduces the general trend seen in the other calculations remarkably well, yielding a cross section that is consistently near the middle of the various predictions. This is true even above the 50-60 MeV energy range in which the impulse approximation begins to become inadequate (see section 3.11).

The agreement seen between MARLEY and the other cross section models at energies above the former's nominal range of validity can be attributed to two compensating deficiencies. Although the impulse approximation used in the current version of MARLEY

\footnotetext{
${ }^{4}$ Quasiparticle Random Phase Approximation

${ }^{5}$ Relativistic Quasiparticle Random Phase Approximation

${ }^{6}$ Projected Quasiparticle Random Phase Approximation

${ }^{7}$ Gross Theory of Beta Decay

${ }^{8}$ Random Phase Approximation
} 
treats the nuclear matrix elements as constant, a calculation accurate to first order in the inverse nucleon mass $\left(1 / m_{N}\right)$ yields nuclear matrix elements (see eqs. (3.173)-(3.176)) that depend on the spherical Bessel function $j_{J}\left(\kappa r_{k}\right)$, where $\kappa$ is the magnitude of the 3 -momentum transfer, $r_{k}$ is the radial coordinate of the $k$ th nucleon, and $J$ is the multipole order. As the neutrino energy increases, $\kappa$ will also increase, and the matrix elements for allowed $(J=0)$ transitions (corresponding to Fermi and Gamow-Teller transitions under the impulse approximation) will be suppressed, while those for forbidden transitions $(J>$ 0) will be enhanced. At high energies, then, MARLEY's overestimate of the contributions of the allowed transitions will be offset by its complete neglect of the forbidden ones. Although there is no a priori reason to believe that these two effects will cancel out, they counteract each other sufficiently well to yield a total cross section value for MARLEY in good agreement with competing calculations.

\subsection{Exclusive cross sections}

In addition to the inclusive CC cross section, MARLEY's Monte Carlo implementation of the Hauser-Feshbach statistical model allows one to perform straightforward calculations of partial cross sections for a number of exclusive channels. For a given neutrino energy $E_{\nu}$ and exit channel $\alpha$, the partial cross section for that channel $\sigma\left(E_{\nu}, \alpha\right)$ is given by

$$
\sigma\left(E_{\nu}, \alpha\right)=\sigma\left(E_{\nu}\right) \sum_{L} P(L) P(\alpha \mid L)
$$

where $\sigma\left(E_{\nu}\right)$ is the CC total cross section for $\nu_{e}, P(L)$ is the probability that the CC reaction will proceed via a transition to the $L$ th excited level of ${ }^{40} \mathrm{~K}$ (see eq. (6.4)), and $P(\alpha \mid L)$ is the probability that the $L$ th excited level will de-excite into a final state corresponding to channel $\alpha$. The quantities $\sigma\left(E_{\nu}\right)$ and $P(L)$ may be easily calculated using the cross section formulae derived in this thesis. The channel probability $P(\alpha \mid L)$, on the other hand, may be estimated using a large sample of Monte Carlo events in which a decay of level $L$ is simulated.

Figure 7.2 shows the MARLEY $\nu_{e}$ CC total cross section (computed using dataset B) together with the corresponding partial cross sections for several exclusive channels obtained using the expression in eq. (7.1). All of these channels are defined to include $X$ 
${ }^{40} \operatorname{Ar}\left(\nu_{e}, e^{-}\right){ }^{40} \mathrm{~K}$ total cross section

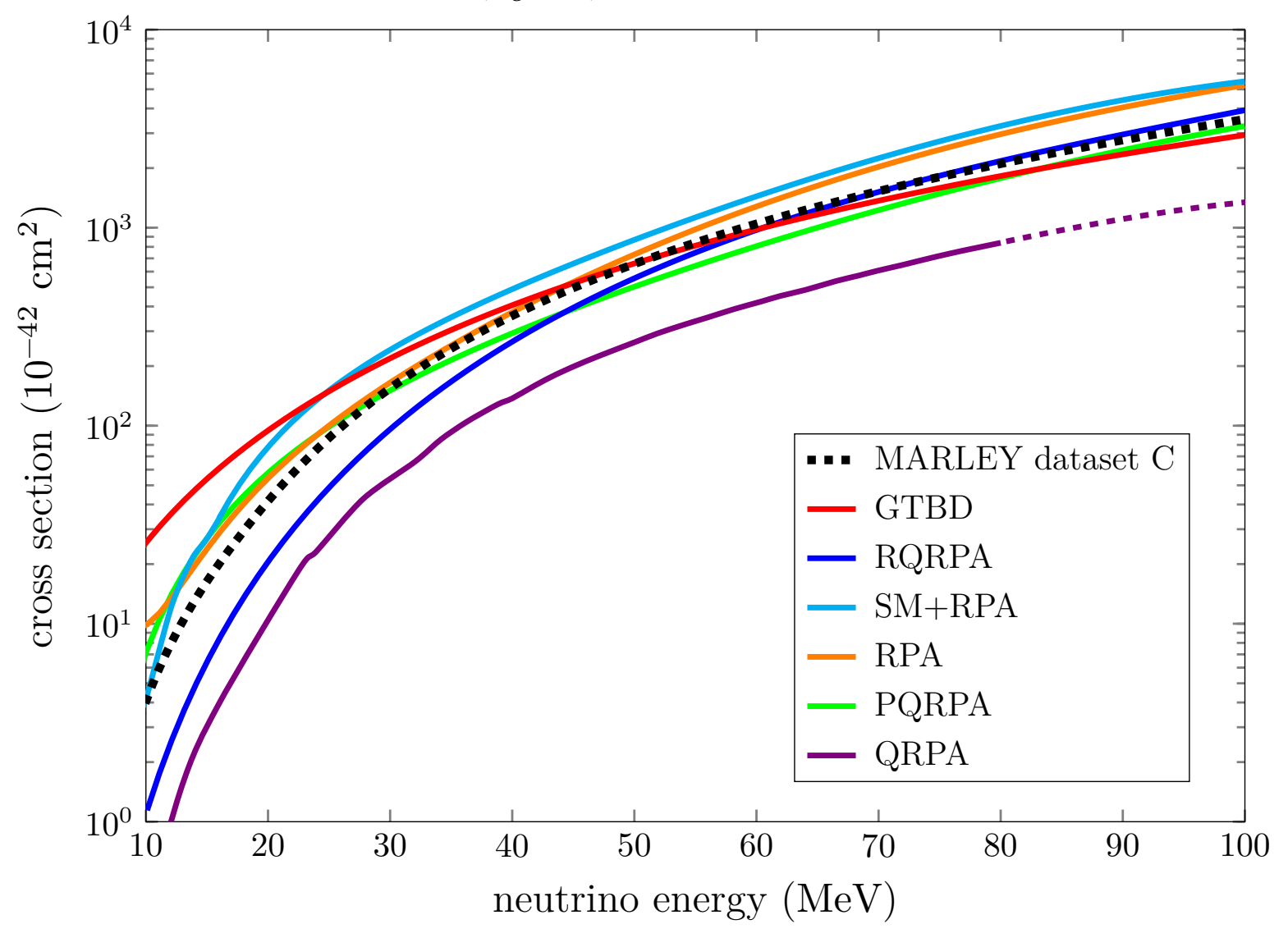

Figure 7.1: MARLEY ${ }^{40} \operatorname{Ar}\left(\nu_{e}, e^{-}\right)^{40} \mathrm{~K}$ total cross section compared to other calculations. Datasets A and B give similar results for the total cross section. The compilation of non-MARLEY cross sections shown here was originally presented in reference [415] and obtained via private communication with the author. GTBD: Unpublished calculation by A. Samana et al. that used a macroscopic neutrino cross section model based on the Gross Theory of Beta Decay. Reference [255] describes the model and presents some energy-averaged cross sections. RQRPA: Relativistic

Quasiparticle Random Phase Approximation calculation by N. Paar et al. Energy-averaged cross sections obtained using this approach were published in reference [281]. SM+RPA: A "hybrid model" calculation by T. Suzuki and M. Honma [282]. Partial cross sections for $0^{+}$and $1^{+}$multipole transitions (which correspond to Fermi and Gamow-Teller transitions, respectively, in the low momentum transfer limit) were obtained using the nuclear shell model, while those for other multipoles were computed using the Random Phase Approximation. RPA: Random Phase Approximation calculation by Martinez-Pinedo et al. [409]. PQRPA: Unpublished Projected Quasiparticle Random Phase Approximation calculation performed by A. Samana et al. using the QRAP computer code [245]. QRPA: Quasiparticle Random Phase Approximation calculation by M. Cheoun et al. [412]. The dashed portion of the curve shows an extrapolation (based on a sixth degree polynomial fit) of this cross section beyond the maximum energy of $80 \mathrm{MeV}$ tabulated by A. Samana. 
$\gamma$-rays in the final state, where $X \geq 0$. Although similar calculations have been performed for other nuclides (e.g., ${ }^{16} \mathrm{O}$ [294]), to the present author's knowledge, the results shown here represent the first calculation of these exclusive cross sections for charged current $\nu_{e}$ scattering on argon in this energy range.

Despite the agreement seen up to $100 \mathrm{MeV}$ between MARLEY and other calculations of the ${ }^{40} \operatorname{Ar}\left(\nu_{e}, e^{-}\right)^{40} \mathrm{~K}$ total cross section, invoking the allowed approximation to calculate the exclusive cross sections at high energies is problematic. As discussed above in section 7.2, as the neutrino energy increases, forbidden transitions governed by $J>0$ terms in the multipole expansion of the nuclear current (see section 3.8) become increasingly important. Just as the majority of the Gamow-Teller strength in ${ }^{40} \mathrm{~K}$ is concentrated in an excitation energy region near $12 \mathrm{MeV}$ (the Gamow-Teller giant resonance, see section 5.1.2), there are corresponding giant resonances for the forbidden multipole operators. As the multipole order $J$ grows, the centroid energy of the associated giant resonance also

grows, roughly like $(J+1) \hbar \omega$, where $\hbar \omega \approx 41 A^{-1 / 3} \mathrm{MeV}$ is a typical nuclear energy shell splitting [198]. Thus, for neutrino scattering in the energy region where forbidden transitions start to become important (above $50 \mathrm{MeV}$ or so), considering only Fermi and Gamow-Teller transitions will cause one to underestimate the average energy transfer to the nucleus. This in turn will lead to an underestimate of the average numbers of emitted nucleons and heavy fragments. Based on this reasoning, the cross sections shown in fig. 7.2 are only plotted for neutrino energies up to $50 \mathrm{MeV}$.

\subsection{Neutrino energy reconstruction}

Beyond its usefulness as a tool to calculate $\nu_{e}$ CC cross sections for ${ }^{40} \mathrm{Ar}$, MARLEY's status as a full-fledged event generator enables a variety of detailed simulation studies to be performed. Because the success of DUNE as a supernova neutrino detector will largely depend on its ability to reconstruct the incident neutrino energies, simulations that assess the degree to which the physics of low-energy neutrino-argon scattering imposes intrinsic limitations on the energy reconstruction will provide a useful input for future analyses and help the collaboration to evaluate the adequacy of the detector design for supernova 


$$
\begin{aligned}
& - \text { total } \\
& -{ }^{40} \mathrm{Ar}\left(\nu_{e}, e^{-} X \gamma\right){ }^{40} \mathrm{~K} \\
& -{ }^{40} \mathrm{Ar}\left(\nu_{e}, e^{-} n X \gamma\right){ }^{39} \mathrm{~K} \\
& -{ }^{40} \mathrm{Ar}\left(\nu_{e}, e^{-} p X \gamma\right){ }^{39} \mathrm{Ar} \\
& -{ }^{40} \mathrm{Ar}\left(\nu_{e}, e^{-} \alpha X \gamma\right){ }^{36} \mathrm{Cl} \\
& -{ }^{40} \operatorname{Ar}\left(\nu_{e}, e^{-} 2 n X \gamma\right)^{38} \mathrm{~K} \\
& -{ }^{40} \operatorname{Ar}\left(\nu_{e}, e^{-} n p X \gamma\right)^{38} \mathrm{Ar} \\
& -{ }^{40} \operatorname{Ar}\left(\nu_{e}, e^{-} 2 p X \gamma\right){ }^{38} \mathrm{Cl}
\end{aligned}
$$

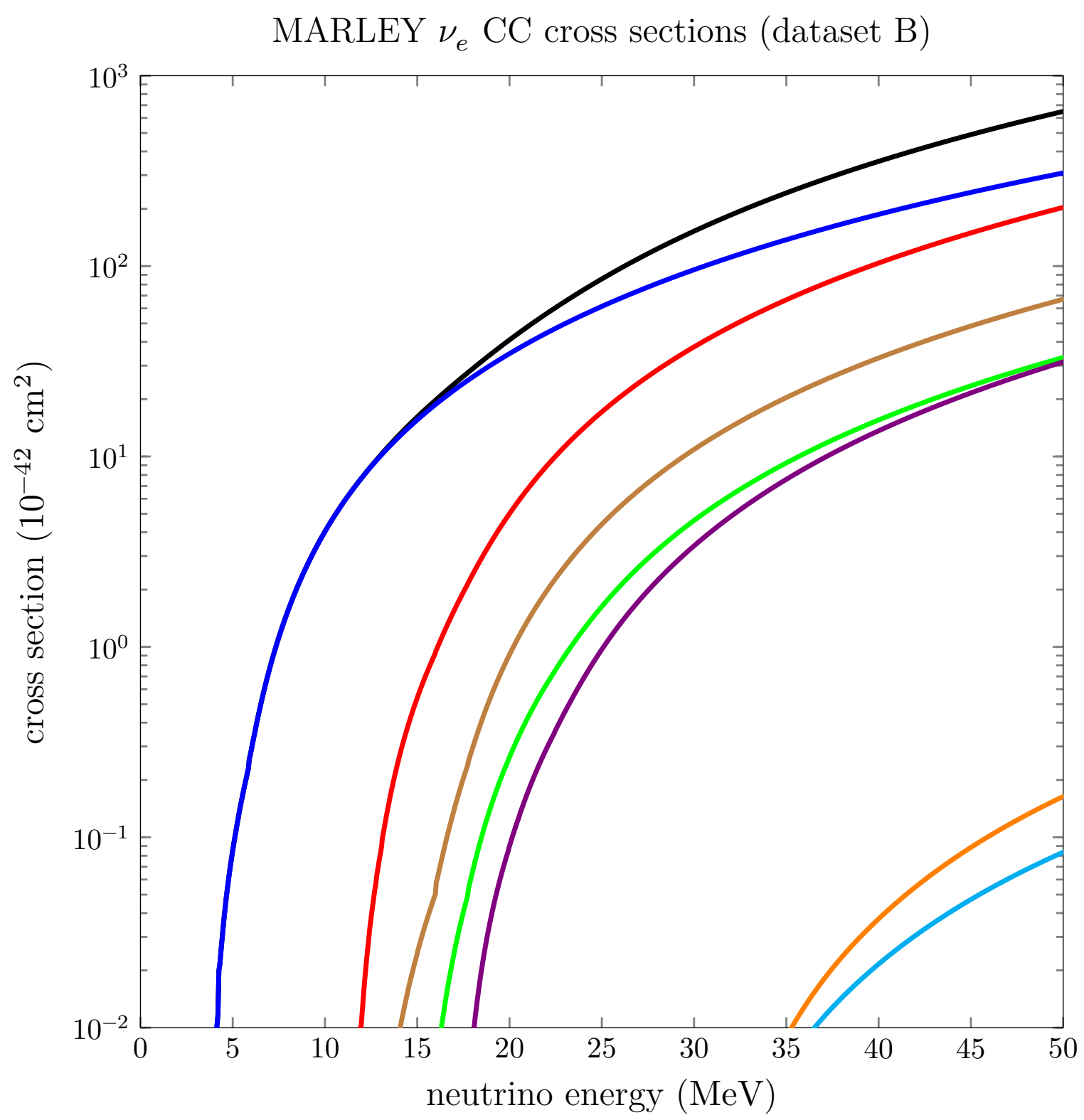

Figure 7.2: MARLEY prediction for the total cross section for $\nu_{e} \mathrm{CC}$ absorption on ${ }^{40} \mathrm{Ar}$, together with several partial cross sections to exclusive final states. For all of the exclusive channels considered here, the final state contains $X \gamma$-rays, where $X \geq 0$. 
physics.

Figure 7.3 shows the results from two examples of such simulations. In the top panel, the black histogram shows the true neutrino energy spectrum obtained from a sample of $10^{6}$ MARLEY events generated using the time-integrated $\nu_{e}$ flux predicted by the Livermore supernova model [419]. The red histogram shows the corresponding reconstructed energy spectrum obtained using only the electron kinetic energy $T_{e}$ and the difference $m_{\text {g.s. } \rightarrow \text { g.s. }}$ (see eq. (2.14)) between the ground-state atomic masses of ${ }^{40} \mathrm{Ar}$ and ${ }^{40} \mathrm{~K}$ :

$$
E_{\nu, \text { reco }}=T_{e}+m_{\text {g.s. } \rightarrow \text { g.s. }}
$$

As was discussed in section 2.3, this expression for the reconstructed neutrino energy, which ignores the excitation energy of the final-state ${ }^{40} \mathrm{~K}^{*}$ nucleus, is akin to that used to reconstruct inverse beta decay events in water Cherenkov and hydrocarbon scintillator neutrino detectors. While perfectly adequate when used in that context, the application of this expression to CC $\nu_{e}$ absorption in a LArTPC introduces a clear bias in the reconstructed energy distribution, shifting the peak about $8 \mathrm{MeV}$ downward relative to the true value and somewhat distorting the shape.

The blue histogram in the top panel of fig. 7.3 gives the reconstructed energy distribution under the very optimistic assumption that the kinetic energies of the $\gamma$-rays and all charged particles produced in each event are perfectly reconstructed:

$$
E_{\nu, \text { reco }}=m_{\text {g.s. } \rightarrow \text { g.s. }}+\sum_{p} T_{p}
$$

Here $T_{p}$ is the kinetic energy of the $p$ th neutrino reaction product, and the sum runs over all particle types except for neutrons. While the agreement with the true distribution is much better in this case, the reconstructed spectrum remains mildly distorted due to the missing kinetic energy of the neutrons and the missing binding energy required to emit each nuclear fragment. While each of these kinds of missing energy could in principle be at least partially recovered (neutron kinetic energies by measuring the energies of $\gamma$-rays produced in inelastic scattering, binding energies by tagging and identifying the emitted fragments), doing so in practice is likely to be very difficult. Since some of the kinetic energy imparted to charged particles and $\gamma$-rays is likely to be poorly reconstructed in 
a LArTPC (e.g., Compton-scattered electrons below the detection threshold), the blue histogram may be interpreted as an estimated upper limit for the accuracy of the energy reconstruction that might be achieved for these events. Assuming that the energy of the primary electron may be precisely measured (which may not be true for the lowest-energy electrons), the red histogram likewise serves as an estimate of the lower limit.

In the bottom panel of fig. 7.3, histograms with identical definitions are shown, but the simulation was performed using the spectrum for $\nu_{e}$ produced by muon decays at rest, which is much harder than the spectrum for the Livermore supernova model and may be described by the normalized distribution [420]

$$
\phi\left(E_{\nu_{e}}\right)=96 E_{\nu_{e}}^{2} m_{\mu}^{-4}\left(m_{\mu}-2 E_{\nu_{e}}\right)
$$

As the mean energy of the incident neutrinos increases, MARLEY predicts that a larger fraction of events will involve the emission of nucleons and heavier nuclear fragments (see table 7.1). The larger amount of missing binding energy (and neutron kinetic energy) in the muon decay at rest events leads to greater distortions in the reconstructed energy spectra relative to the spectra calculated for the Livermore supernova model. Future measurements of low-energy $\nu_{e}$ CC cross sections using a LArTPC at a stopped pion source, ${ }^{9}$ such as that proposed by the CAPTAIN experiment [422], will, according to MARLEY, have to contend with the energy reconstruction difficulties afforded by nuclear fragment emission in nearly $40 \%$ of their signal events.

\subsection{Truth-level studies in LArSoft}

When incorporated into a full detector simulation chain, MARLEY is able to provide additional insights about the experimental techniques needed to reconstruct supernova neutrino events in a LArTPC. In an effort to make the generator readily usable by the experimental community, an interface has been created between MARLEY and the LArSoft $^{10}$ simulation and data analysis framework for LArTPCs. Starting with LArSoft

\footnotetext{
${ }^{9}$ The decay $\pi^{+} \rightarrow \mu^{+}+\nu_{\mu}$ produces a monoenergetic $4.12 \mathrm{MeV} \mu^{+}$if the initial $\pi^{+}$is at rest. The range of the $\mu^{+}$in typical target materials is very small (e.g., about $1 \mathrm{~mm}$ in scintillator), so it will also stop and decay at rest. The dominant decay mode $\mu^{+} \rightarrow e^{+}+\nu_{e}+\bar{\nu}_{\mu}$ yields an electron neutrino in the tens-of-MeV energy range of interest for supernovae [421].

${ }^{10}$ See section 2.5 for a description of LArSoft.
} 

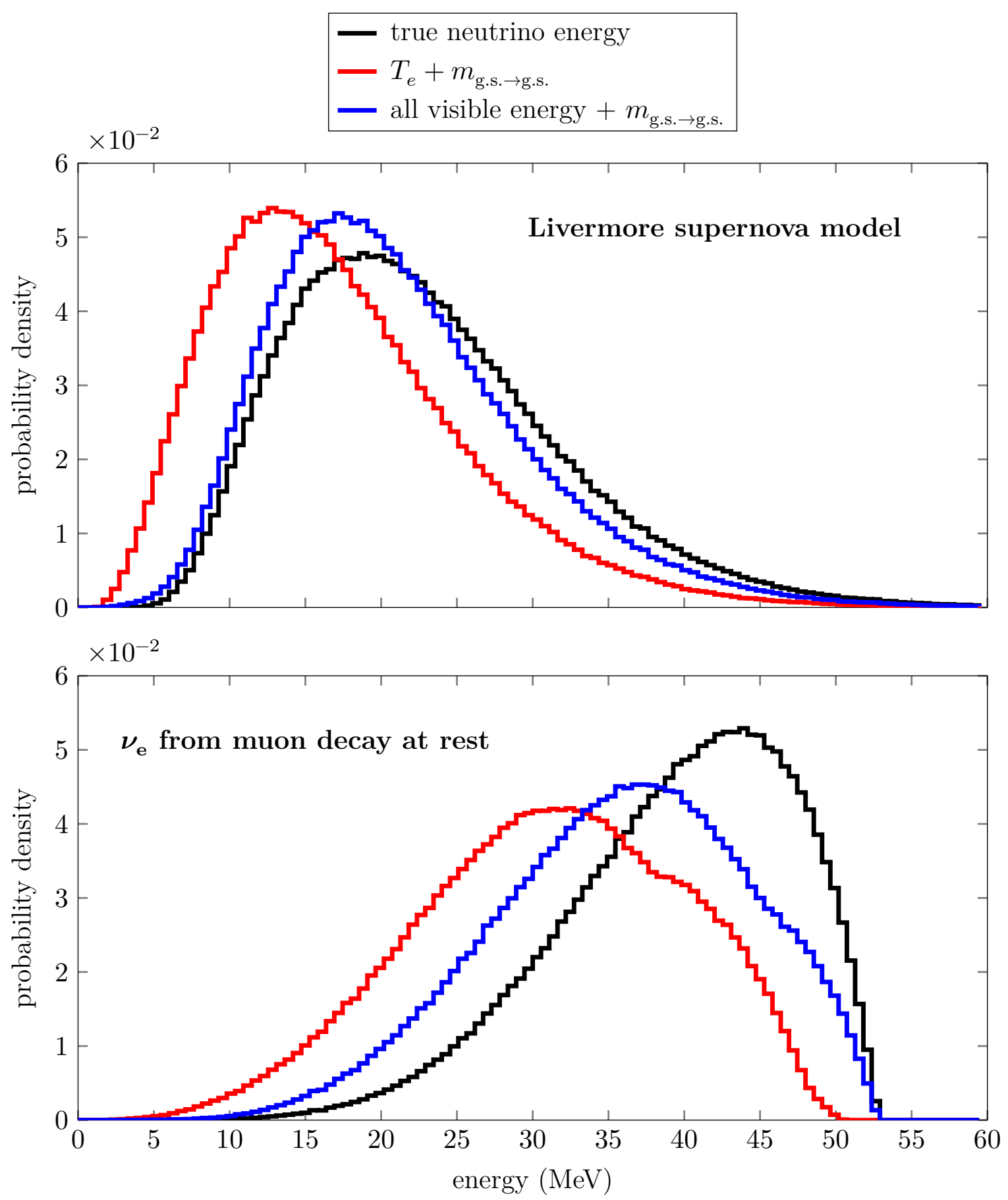

Figure 7.3: TOP: Truth-level energy reconstruction study performed using MARLEY for simulated events generated according to the time-integrated $\nu_{e}$ spectrum predicted by the Livermore supernova model [419]. The definitions of the three histograms shown in the plot are described at length in the text. BOTTOM: A similar MARLEY study performed using the $\nu_{e}$ spectrum for muon decays at rest. 


\begin{tabular}{|c|c|c|}
\hline De-excitation products & $\begin{array}{l}\text { Branching ratio } \% \text { for } \\
\text { Livermore supernova model }\end{array}$ & $\begin{array}{c}\text { Branching ratio } \% \text { for } \\
\mu \text { decay at rest }\end{array}$ \\
\hline gammas only & 82.3 & 60.7 \\
\hline single neutron emission & 12.7 & 25.6 \\
\hline single proton emission & 3.3 & 8.3 \\
\hline other & 1.7 & 5.3 \\
\hline
\end{tabular}

Table 7.1: Branching ratios to final states from the MARLEY simulations shown in fig. 7.3. The "single neutron emission" and "single proton emission" channels include cases where a single nucleon emission is followed by a gamma cascade. The channel labeled "other" includes both multi-step nucleon emissions (e.g., the emission of a neutron followed by the emission of a proton) and the emission of deuterons, alphas, etc.

version 6.03.00, MARLEY has been distributed with every new release of LArSoft, and it has seen widespread use within the DUNE collaboration for supernova neutrino sensitivity studies.

While LArSoft is capable of performing full detector response simulations and realistic event reconstruction, in this section we will briefly consider only a few truth-level results from a sample of $8 \times 10^{4}$ MARLEY events. These were simulated within the geometry provided by LArSoft for one of the $10 \mathrm{kt}$ single-phase LArTPC modules planned for the DUNE far detector. Figure 7.4 shows illustrations of the true trajectories for the finalstate particles produced in representative MARLEY events for the three dominant exit channels: an event involving only a de-excitation $\gamma$-ray cascade in addition to the recoiling nucleus and primary electron (top left), an event involving single neutron emission (top right), and an event involving single proton emission (bottom).

In the event shown in the top left of fig. 7.4, five de-excitation $\gamma$-rays scatter many times, creating small electron tracks in the LArTPC. While some of these will fall below the energy threshold for detection, others will lead to one or a few wire hits that can be used to identify the $\gamma$-ray cascade and potentially measure its total energy. One may characterize the size of the supernova neutrino events (and thus study their containment) by defining a "visible energy sphere" centered on the neutrino vertex. The radius of this 
sphere is defined to be the minimum needed to fully contain all of the energy deposited in the event. For the MARLEY event shown in the top left of fig. 7.4, the visible energy sphere radius is $48.4 \mathrm{~cm}$. In fig. 7.5, the blue histogram shows the distribution of visible energy sphere radii observed for all MARLEY events that did not include a primary neutron. For such events, the mean radius of the visible energy sphere is about $60 \mathrm{~cm}$.

The example single neutron emission event, shown in the upper right plot of fig. 7.4, includes only three primary particles: an electron, a neutron, and the recoiling ${ }^{39} \mathrm{~K}$ nucleus. Although MARLEY predicts that neutrons produced by charged current $\nu_{e}$ scattering on ${ }^{40} \mathrm{Ar}$ will often be accompanied by one or more de-excitation $\gamma$-rays, in this case, all of the $\gamma$-rays produced in the event originated either via inelastic scattering of the primary neutron or via its capture on an ${ }^{40} \mathrm{Ar}$ nucleus far from the neutrino vertex. Although the large size of the DUNE LArTPC modules will aid neutron containment (nearly all of the simulated primary neutrons were eventually captured within the detector in this study), the distance of nearly $1.5 \mathrm{~m}$ seen in this event between the neutrino and neutron capture vertices is typical (see fig. 7.6). Even if the neutrons originating from supernova neutrino interactions can be successfully tagged in liquid argon, associating them with the correct neutrino vertex may be challenging.

Unlike neutrons, protons emitted in supernova neutrino events will generate ionization tracks in a LArTPC and will therefore directly contribute to the visible energy observed in each event. However, because $\sim 10 \mathrm{MeV}$ protons have a short range in liquid argon, these tracks will be no larger than a few $\mathrm{mm}$ and potentially hard to distinguish from the start of the primary electron track (see the illustration at the bottom of fig. 7.4). Although MARLEY predicts that supernova $\nu_{e}$ will generate final-state protons less frequently than neutrons (due to the Coulomb barrier experienced by the former), since each emitted proton is accompanied by the invisible loss of roughly $8 \mathrm{MeV}$ of binding energy, it may still be useful for future experimental efforts to develop a means of tagging them in a LArTPC. 


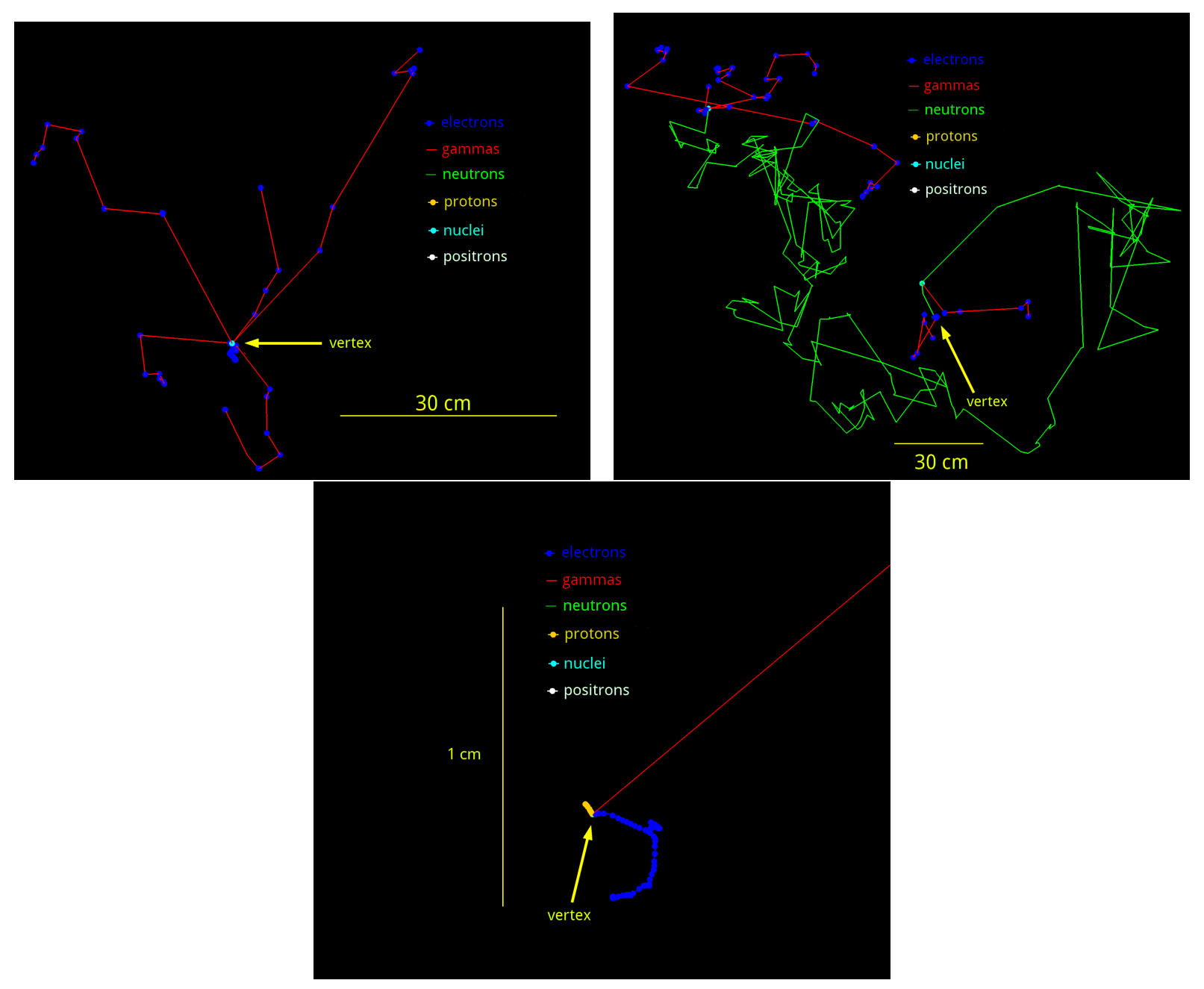

Figure 7.4: Displays of the true trajectories of the outgoing particles for representative MARLEY events simulated using LArSoft. The particles were tracked through the simulated geometry of one of the proposed $10 \mathrm{kt}$ single-phase LArTPC modules for the DUNE far detector. Event vertices were sampled uniformly throughout the active TPC volumes within the simulated detector. TOP LEFT: A ${ }^{40} \operatorname{Ar}\left(\nu_{e}, e^{-} 5 \gamma\right)^{40} \mathrm{~K}$ event induced by a $16.1 \mathrm{MeV}$ neutrino. TOP RIGHT: A ${ }^{40} \operatorname{Ar}\left(\nu_{e}, e^{-} n\right)^{39} \mathrm{~K}$ event induced by a 16.3 $\mathrm{MeV}$ neutrino. The secondary $\gamma$-rays emitted close to the vertex were produced via inelastic scatters of the primary neutron. Those emitted far away were produced when the primary neutron was captured on an ${ }^{40} \mathrm{Ar}$ nucleus. BOTTOM: A ${ }^{40} \operatorname{Ar}\left(\nu_{e}, e^{-} p \gamma\right){ }^{39} \mathrm{Ar}$ event induced by a $17.8 \mathrm{MeV}$ neutrino. 


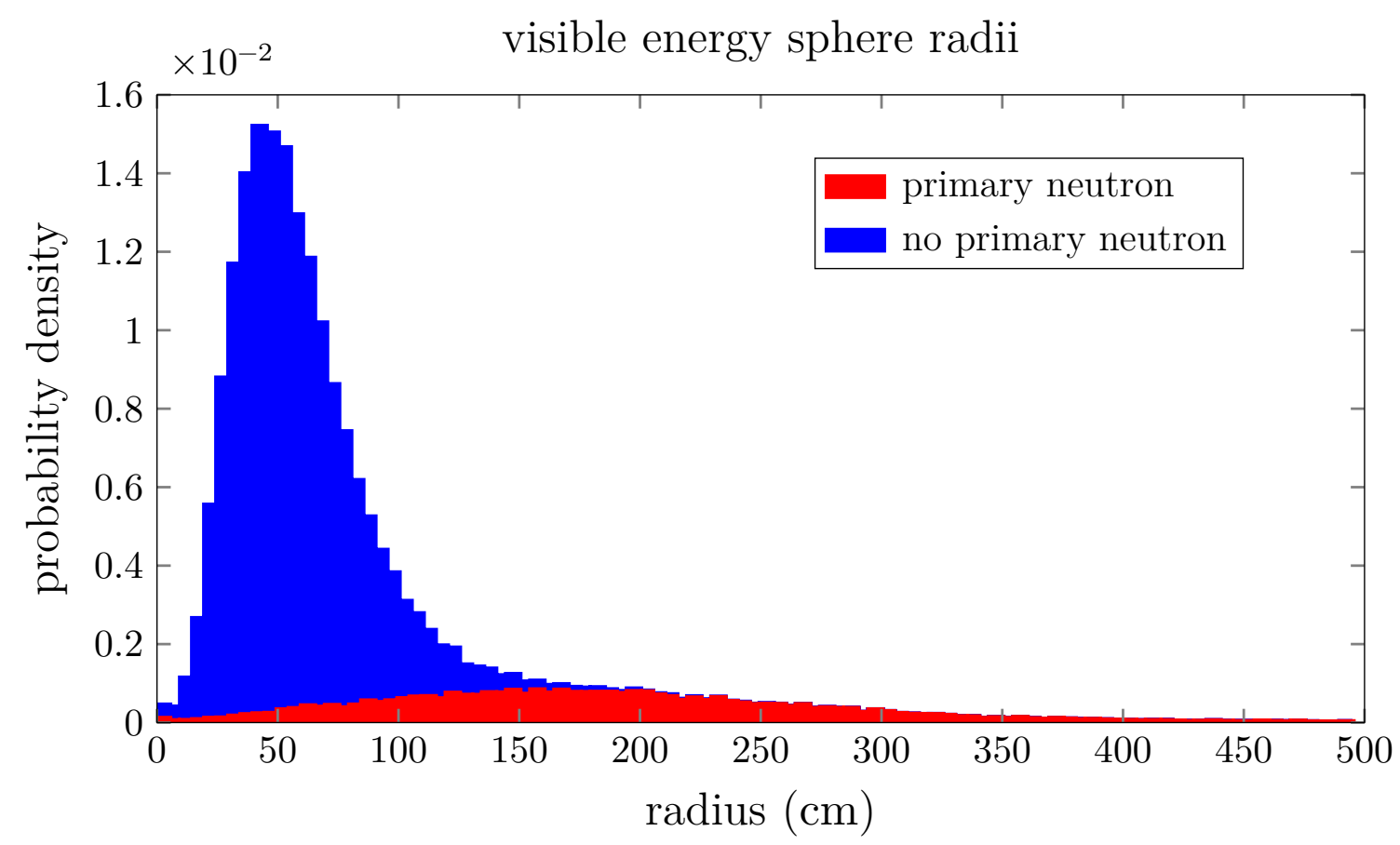

Figure 7.5: Stacked plot showing the separate contributions of MARLEY events involving at least one primary neutron (red) and zero primary neutrons (blue) to the total distribution of "visible energy sphere" radii observed in the LArSoft simulations discussed in this section.

\subsection{Future prospects for MARLEY}

Although the MARLEY physics models and event generator implementation described in this thesis represent a reasonable starting point for simulation-based investigations into the sensitivity of future LArTPCs to low-energy neutrinos, including supernova neutrinos, there remain a number of clear avenues through which the present treatment may be expanded and improved. First, incorporating a cross section calculation into the MARLEY generator which takes forbidden transitions into account will allow more theoretically rigorous predictions to be made for the event signatures produced by high-energy $(\sim 50 \mathrm{MeV}$ and above) supernova neutrinos. While a full event-by-event calculation of the nuclear matrix elements may be computationally infeasible in the generator itself, an alternative approach would require precomputed tables of matrix elements evaluated as a function of the final-state nuclear excitation energy and of the magnitude of the 3-momentum trans- 


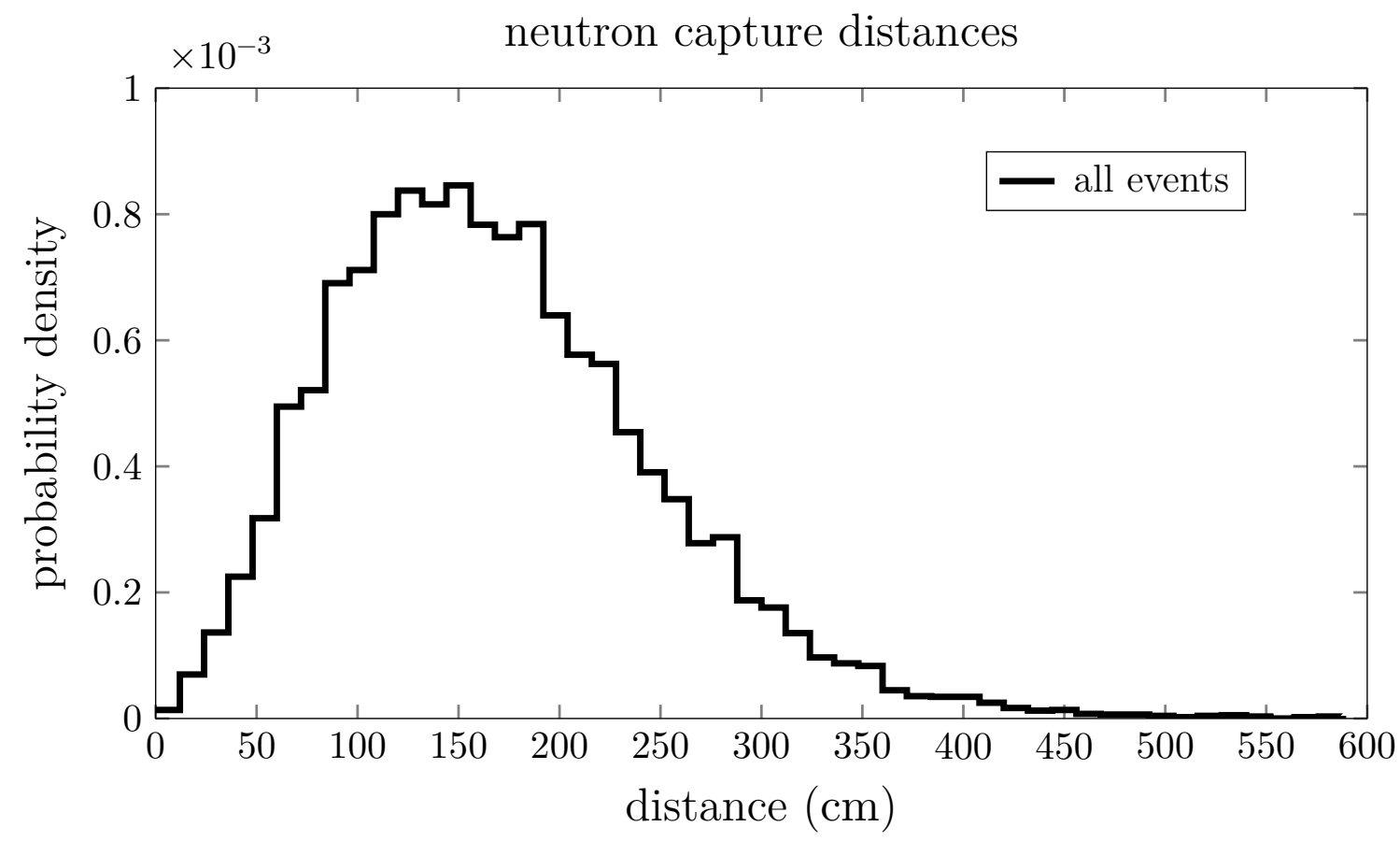

Figure 7.6: Distribution of neutron capture distances (defined as the distance between the neutrino vertex and the neutron capture vertex) observed in the LArSoft simulations described in this section.

fer. Numerical interpolation methods could then be used to obtain an approximate value for the matrix elements when computing cross sections.

Second, adding the capability to simulate channels other than the dominant CC $\nu_{e}$ reaction will enable MARLEY to provide a more complete picture of low-energy neutrino scattering. While the current structure of the generator code could easily allow for such an enhancement, adding channels other than neutrino-electron scattering (for which the cross section is well known) will not be trivial. Unlike the $\mathrm{CC} \nu_{e}$ reaction, there are currently no available experimental data which could be used to determine the GamowTeller matrix elements for $\mathrm{CC} \bar{\nu}_{e}$ reactions on ${ }^{40} \mathrm{Ar}$, and only a single measured transition [423] which could be used for the NC reactions. ${ }^{11}$ In the absence of such data, which could potentially be obtained (see section 5.3) via a charge exchange (CC $\left.\bar{\nu}_{e}\right)$ or an

\footnotetext{
${ }^{11} \mathrm{~A}$ second measurement of interest for determining the neutral Gamow-Teller matrix elements for ${ }^{40} \mathrm{Ar}$ was recently discussed in a talk at the APS Division of Nuclear Physics Fall Meeting [424], but the results have not yet been published.
} 
inelastic proton (NC) scattering experiment at a suitable intermediate-energy nuclear beam facility, ${ }^{12}$ one could attempt to provide a set of purely theoretical Gamow-Teller matrix elements for MARLEY. This could be done by combining shell model calculations at low excitation energies (which should reproduce the expected fragmentation of the GT strength better than competing models) with an RPA-like model (e.g., the QRPA) calculation at high excitation energies (where using the shell model becomes impractical).

Third, although the creation of the MARLEY generator was motivated by the desire to understand supernova neutrino events in liquid argon, the code could be applied to the simulation of low-energy neutrino scattering on other nuclear targets with little needed modification. Because the nuclear de-excitation simulation used in MARLEY relies on tabulated structure data and nuclear model parameterizations that are intended to work for essentially all nuclei, simply providing new tabulated level energies and $\gamma$-ray branching ratios should be sufficient to simulate de-excitations of any target nucleus likely to be of interest. ${ }^{13}$ The main obstacle to extending the MARLEY generator to handle other nuclear targets is the need to prepare an input table of reaction matrix elements for each nuclide individually. Since the cross sections for low-energy neutrino scattering are sensitive to the details of nuclear structure, this requirement is unavoidable but not insurmountable if there is sufficient interest in a particular nucleus.

Finally, future experimental measurements using low-energy neutrinos will provide invaluable constraints on the MARLEY physics models. Barring the actual observation of supernova neutrinos in a currently operating LArTPC, the most convenient means of making a direct test of the generator's predictions will be to measure neutrino-argon cross sections by exposing a $\mathrm{LArTPC}^{14}$ to $\nu_{e}$ produced at a stopped pion source. Such an experiment could be carried out at an existing neutron spallation or neutrino beam facility, with possible sites in the United States including the Spallation Neutron Source at

\footnotetext{
${ }^{12}$ Examples include iThemba LABS in South Africa and the Research Center for Nuclear Physics (RCNP) in Japan.

${ }^{13}$ The one major exception is that, for very heavy nuclei, the possibility of neutrino-induced fission [425] may become important. To properly study low-energy neutrino scattering on a ${ }^{208} \mathrm{~Pb}$ target, for instance, this channel would probably need to be added to MARLEY's compound nucleus model.

${ }^{14} \mathrm{Or}$, perhaps, another kind of liquid-argon-based detector. An alternative design optimized for lowenergy neutrino-argon cross section measurements is proposed in reference [426].
} 
Oak Ridge National Laboratory and an off-axis location at the NuMI or Booster Neutrino beamlines at Fermilab [422, 427]. Current efforts by the COHERENT collaboration to obtain an improved measurement of the $\mathrm{CC} \nu_{e}$ cross section on ${ }^{127} \mathrm{I}$ and to measure the cross sections for neutrino-induced neutron production on lead, iron, and copper [428] will also provide much-needed data in MARLEY's energy range of interest. However, in order to properly interpret these data in a way that can lead to improvements in future versions of the generator, the creation of new MARLEY reaction matrix element datasets, similar to those presented for ${ }^{40} \mathrm{Ar}$ in chapter 5, will likely be required. 


\section{Part II}

ANNIE 


\section{Chapter 8}

\section{Neutron background characterization in ANNIE Phase I}

To help ensure the success of its proposed neutron yield measurement, the ANNIE collaboration must carefully consider potential sources of background events, including true neutron captures that do not originate from a neutrino interaction vertex inside the water volume. While one may greatly reduce backgrounds due to natural radioactivity (e.g., neutrons produced by cosmic rays, neutrons from uranium fissions in concrete) by requiring neutron capture candidate events to occur in a small time window shortly after the arrival of each beam spill, timing cuts of this kind will not allow one to eliminate background neutrons that are correlated in time with the beam itself. Because these beam-correlated backgrounds are difficult to accurately predict and potentially disastrous for the experiment if they are too large, a direct characterization of them is needed to maintain the integrity of the planned Phase II measurements. This chapter describes the neutrino beam used for the ANNIE experiment (see fig. 8.6 for a pictorial overview of beam production) and examines possible sources of beam-correlated neutron backgrounds in the ANNIE detector. It then describes the goals and design of ANNIE Phase I, an experiment to measure those backgrounds that is the subject of the remainder of this thesis. 


\subsection{The Fermilab Booster Neutrino Beam}

The Booster accelerator is a $74.5 \mathrm{~m}$ radius proton synchrotron located at Fermi National Accelerator Laboratory in Batavia, Illinois. Protons are injected into the Booster at $400 \mathrm{MeV}$ kinetic energy from an upstream linear accelerator system, and they are extracted at $8 \mathrm{GeV}$ kinetic energy into the MI- $8^{1}$ transfer line, where they may be sent to a beam dump, the Main Injector synchrotron, the Recycler ring, or the beamline for the Booster Neutrino Beam (BNB) [429, 430]. Figure 8.1 shows a schematic of the proton accelerators in operation at Fermilab at the time of this writing.

To produce neutrinos for the BNB, protons extracted from the Booster accelerator are directed at a $71 \mathrm{~cm}$ thick, $1 \mathrm{~cm}$ diameter cylindrical target (see fig. 8.2) consisting of seven slugs of beryllium encased in a beryllium sleeve. Beryllium was chosen as the material for the primary target elements because of its high pion production yield, its ability to withstand a large number of beam spills before failing, the relative ease with which it may be cooled, and the desire to minimize the radiological hazards associated with exposing the target to $10^{21}$ protons or more over its lifetime [431]. Protons are sent to the target from the Booster in a $1.6 \mu$ s spill that consists of a series of 81 bunches, each lasting about $2 \mathrm{~ns}$ and arriving in $19 \mathrm{~ns}$ intervals. A single beam spill delivers a total of roughly $5 \times 10^{12}$ protons to the target. The spills repeat at an average rate of $5 \mathrm{~Hz}$ under normal operating conditions [84].

Protons interacting in the target produce a secondary beam of short-lived mesons, particularly pions. Charged particles emerging from the target are focused by by a device called a horn. First invented by Simon van der Meer in 1961 [432], a horn uses a large pulsed current (174 kA [431] for the BNB horn) to generate a toroidal magnetic field that focuses charged particles of one sign while defocusing those of the opposite sign. The sign to be focused can be changed by reversing the direction of the horn current. When the BNB is running in neutrino mode, positive particles are focused, while negative particles are focused when running in antineutrino mode. A photograph of a horn used in the BNB is shown in fig. 8.3.

\footnotetext{
${ }^{1}$ Main Injector $8-\mathrm{GeV}$
} 
After being focused by the horn, charged particles from the beam are allowed to drift down a $50 \mathrm{~m}$ decay pipe. The majority of the pions (almost entirely of the sign selected by the horn) in the pipe will undergo the decays

$$
\pi^{+} \rightarrow \mu^{+}+\nu_{\mu} \quad \pi^{-} \rightarrow \mu^{-}+\bar{\nu}_{\mu}
$$

to produce muon-flavor neutrinos or antineutrinos. While these neutrinos are the primary component of the final beam, some muons will also decay in the pipe via

$$
\mu^{+} \rightarrow e^{+}+\bar{\nu}_{\mu}+\nu_{e} \quad \mu^{-} \rightarrow e^{-}+\nu_{\mu}+\bar{\nu}_{e},
$$

creating some contamination of the beam from electron flavor neutrinos and muon flavor neutrinos of the wrong species (for example, $\bar{\nu}_{\mu}$ in a beam composed of mainly $\nu_{\mu}$ ). Hadrons other than pions may also contribute to neutrino production in the beam, e.g., via the charged kaon decays

$$
K^{+} \rightarrow \mu^{+}+\nu_{\mu} \quad K^{-} \rightarrow \mu^{-}+\bar{\nu}_{\mu} .
$$

At the far end of the decay pipe, a thick barrier constructed using steel and concrete is used to absorb essentially all of the remaining hadrons. A second retractable absorber is placed halfway down the decay pipe (at $25 \mathrm{~m}$ ), allowing users to reduce the available decay time for hadrons in the beam [84]. While useful for studying systematic effects in the neutrino beam flux, the retractable absorber is typically left outside of the decay pipe during normal operation. Because the BNB target is located about $7 \mathrm{~m}$ underground, [433] muons that penetrate the final barrier eventually stop in the unexcavated rock downstream from the target hall while the beam neutrinos pass through it unimpeded. Readers interested in more detailed information about the production of the Booster Neutrino Beam are encouraged to consult references [429-431, 434-436].

The Booster Neutrino Beam is the primary source of neutrinos for three currently operating experiments: ANNIE, MicroBooNE, and MiniBooNE. In the near future, two additional detectors, ICARUS-T600 and SBND, will begin taking data in the BNB beamline as part of the proposed Short-Baseline Neutrino Program [84]. Figure 8.5 depicts the 


\section{Fermilab Accelerator Complex}

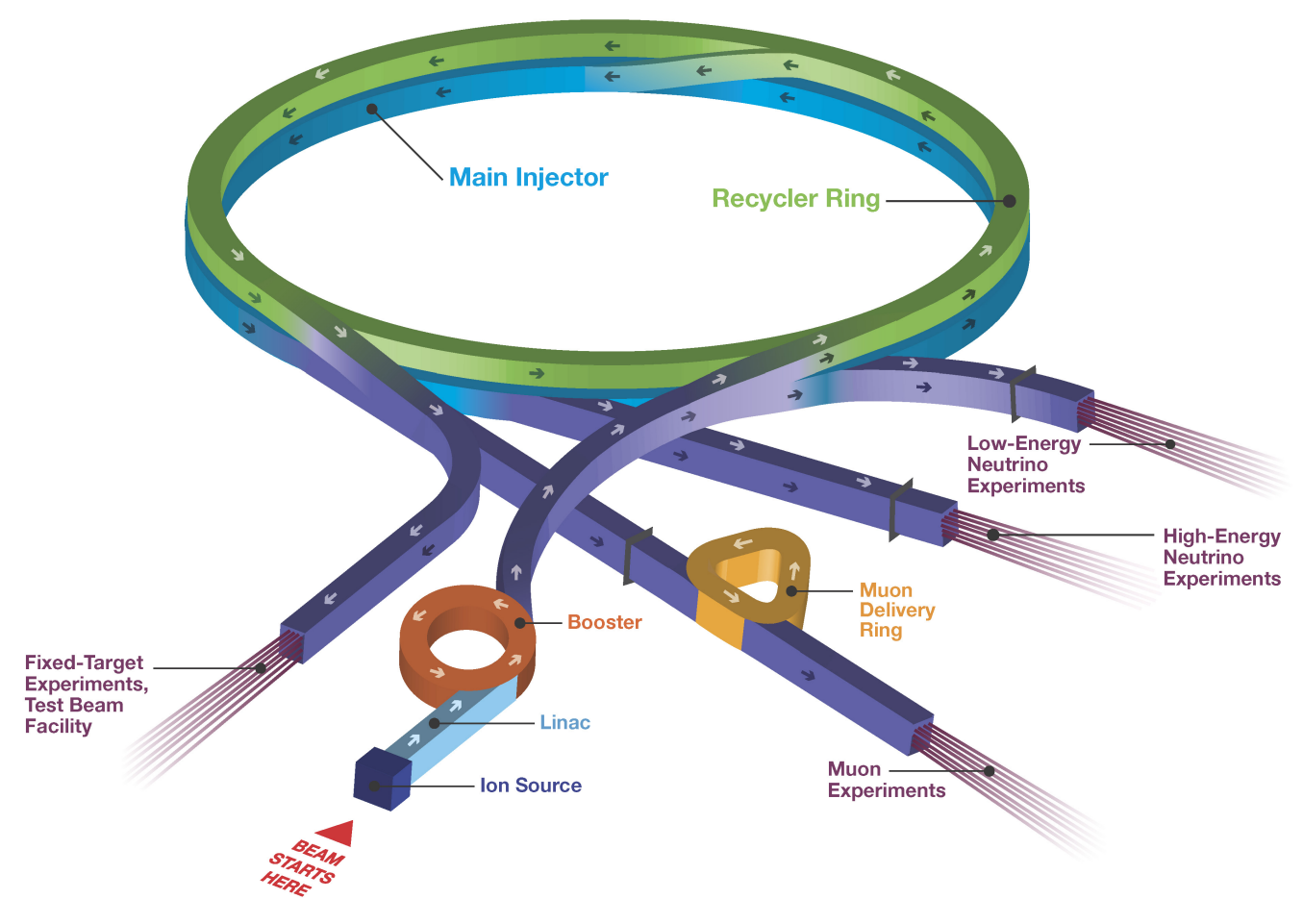

Figure 8.1: Diagram of the currently-operating proton accelerator beamlines at Fermilab. The line labeled "Low-Energy Neutrino Experiments" is used to produce neutrinos for the Booster Neutrino Beam. Figure taken from http://www.fnal.gov/ pub/science/particle-accelerators/accelerator-complex.html. 


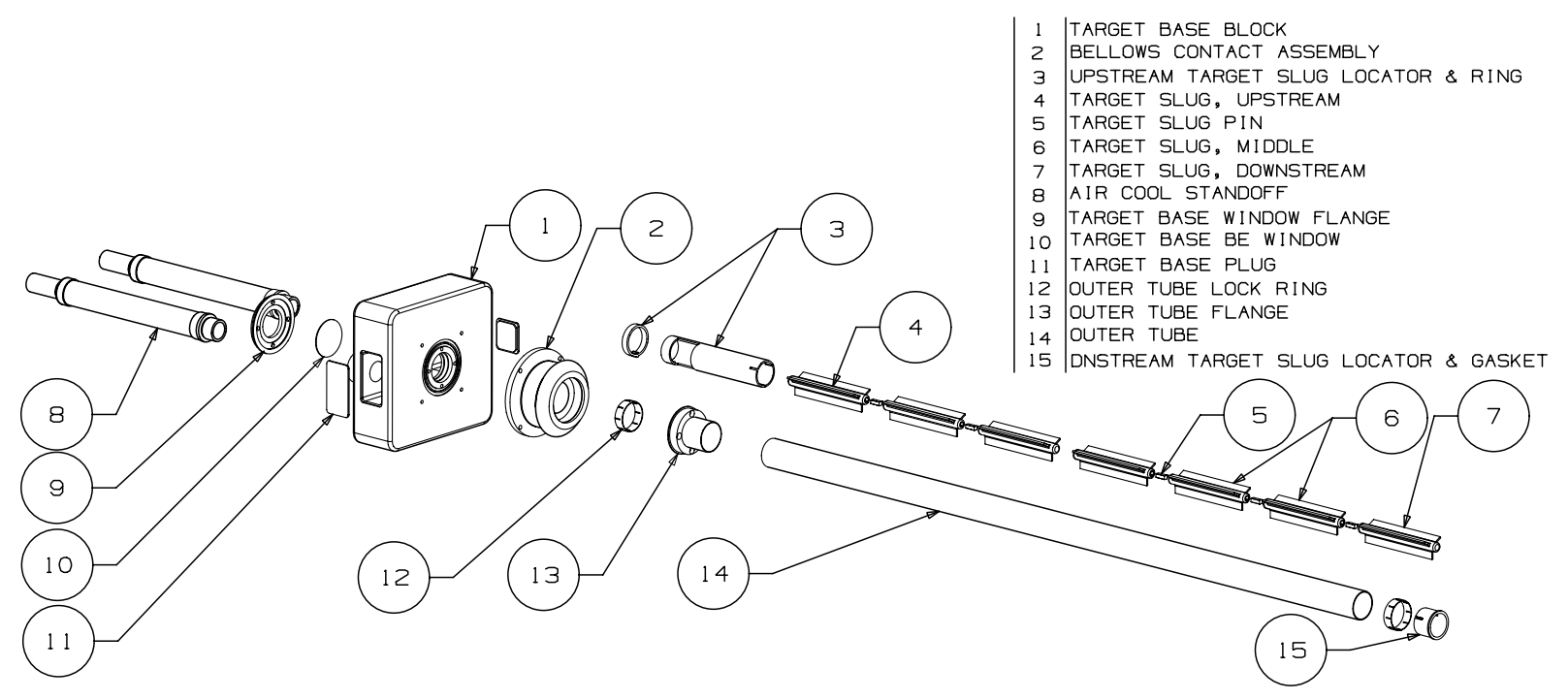

Figure 8.2: Exploded view of the Booster Neutrino Beam target components. Figure taken from reference [436].

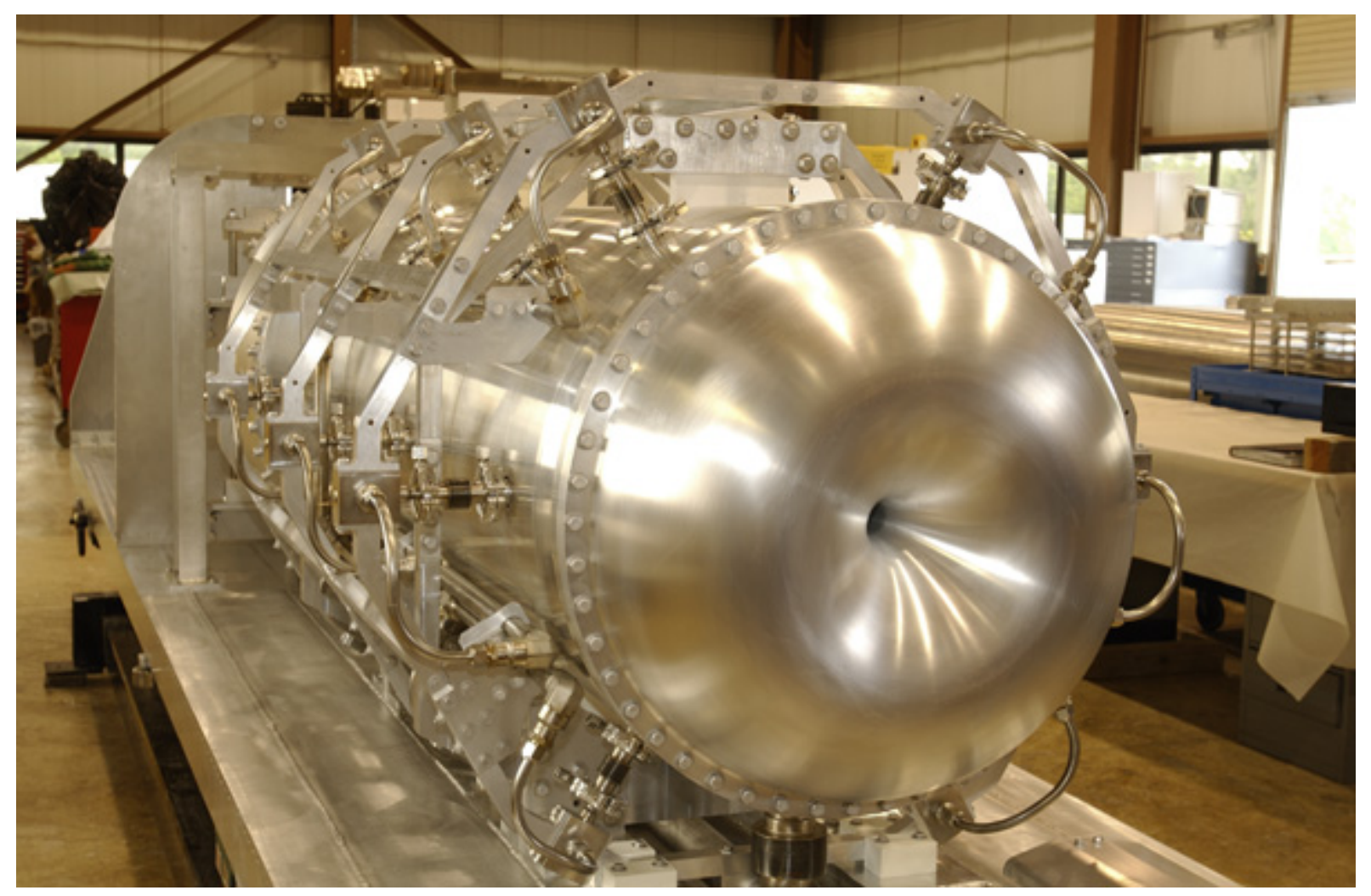

Figure 8.3: A magnetic horn used to produce the Booster Neutrino Beam. Figure taken from https://web.fnal.gov/project/TargetSystems/BNB_target_hall/SitePages/ BNB $\% 20$ Horn . aspx. 


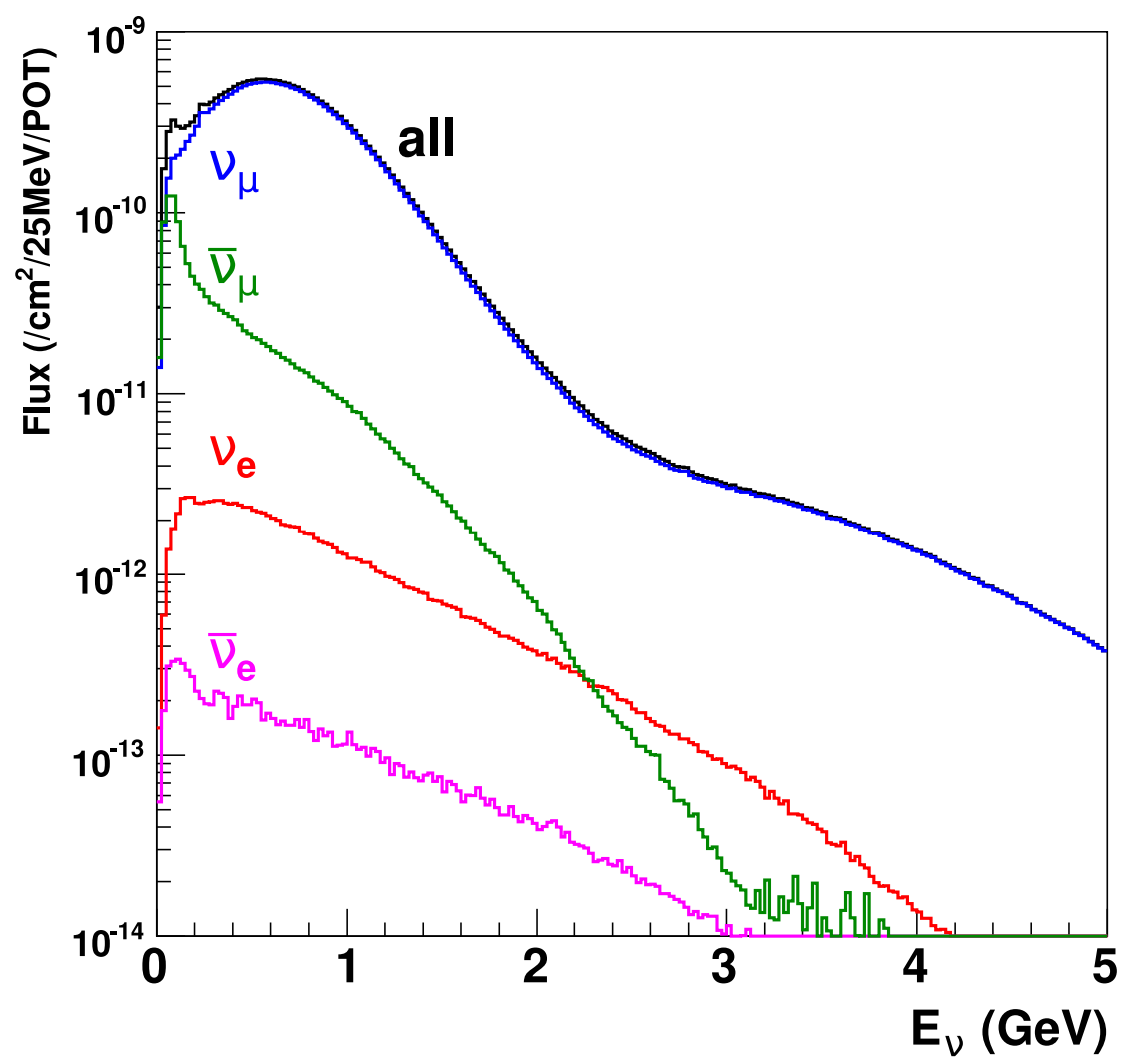

Figure 8.4: Predicted neutrino flux (total and for individual neutrino species) from the Booster Neutrino Beam (running in neutrino mode) at SciBooNE hall, the location of the ANNIE detector. The spectra shown here represent averages within a $2.12 \mathrm{~m}$ radius of the beam center. They are normalized per unit area, per unit energy, and per proton on target (POT). Figure taken from reference [437].

expected site layout for the experiments that will be using the BNB over the next few years.

The ANNIE detector is the first BNB experiment to receive neutrinos from each beam spill, being located in SciBooNE hall only $100 \mathrm{~m}$ away from the BNB target. A calculation of the expected neutrino flux in SciBooNE hall when the Booster Neutrino Beam is running normally in neutrino mode (positive horn polarity) is shown in fig. 8.4. The total estimated neutrino flux of $2.2 \times 10^{-8} \mathrm{~cm}^{-2}$ per proton on target is dominated by muon neutrinos $(93 \%)$, with a modest number of muon antineutrinos present $(6.4 \%)$ and a small amount of contamination from electron neutrinos and antineutrinos ( $0.6 \%$ total) [437]. 


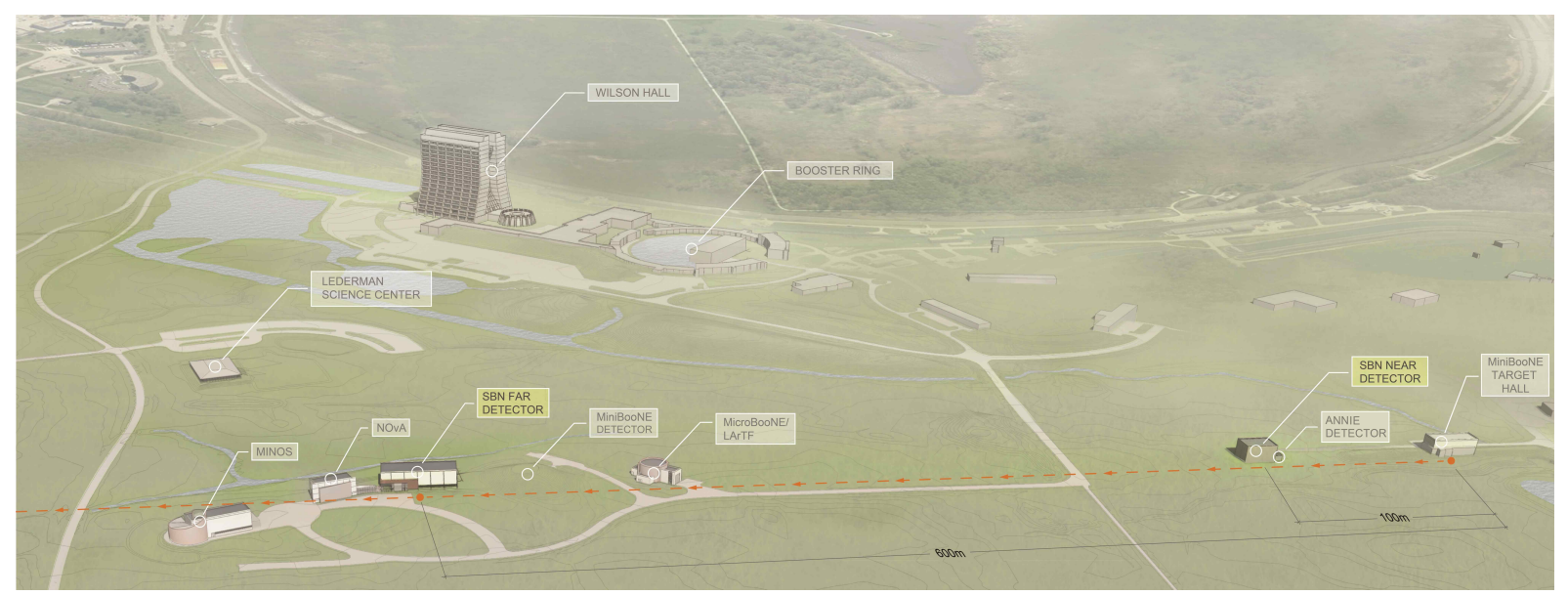

Figure 8.5: Map showing the detectors along the path of the Booster Neutrino Beam, including the planned sites of the far detector (ICARUS-T600) and near detector (SBND) for the Short-Baseline Neutrino Program at Fermilab. Although the near detectors for the MINOS and NOvA experiments are also relatively close to the production site of the BNB, their primary source of neutrinos is the Fermilab NuMI (Neutrinos at the Main Injector) beam. Figure taken from reference [84] and updated to include the ANNIE detector (shown near the bottom right of the drawing), which occupies an experimental hall previously used by the SciBooNE experiment [196].

\subsection{Beam monitoring}

Many diagnostic instruments are used at all stages of producing the BNB to achieve and maintain the highest possible intensity, provide useful information for beam simulations, alert operators to problems, and ensure safety. For the ANNIE experiment, two beam monitoring systems are particularly important: the resistive wall current monitor (RWM) and the beam current toroids.

\subsubsection{Resistive wall current monitor}

A resistive wall current monitor upstream of the BNB target is used to record the arrival of each beam spill. The device consists of a set of resistors connected across a gap in the wall of the beam pipe (see figs. 8.7 and 8.8). The gap is sealed with a ceramic insulator to prevent foreign objects from entering the beam pipe and to force induced currents to flow across the resistors. As protons from the beam pass through the beam pipe, they induce a current of negative image charges on the pipe's conducting inner surface (see fig. 8.9). 


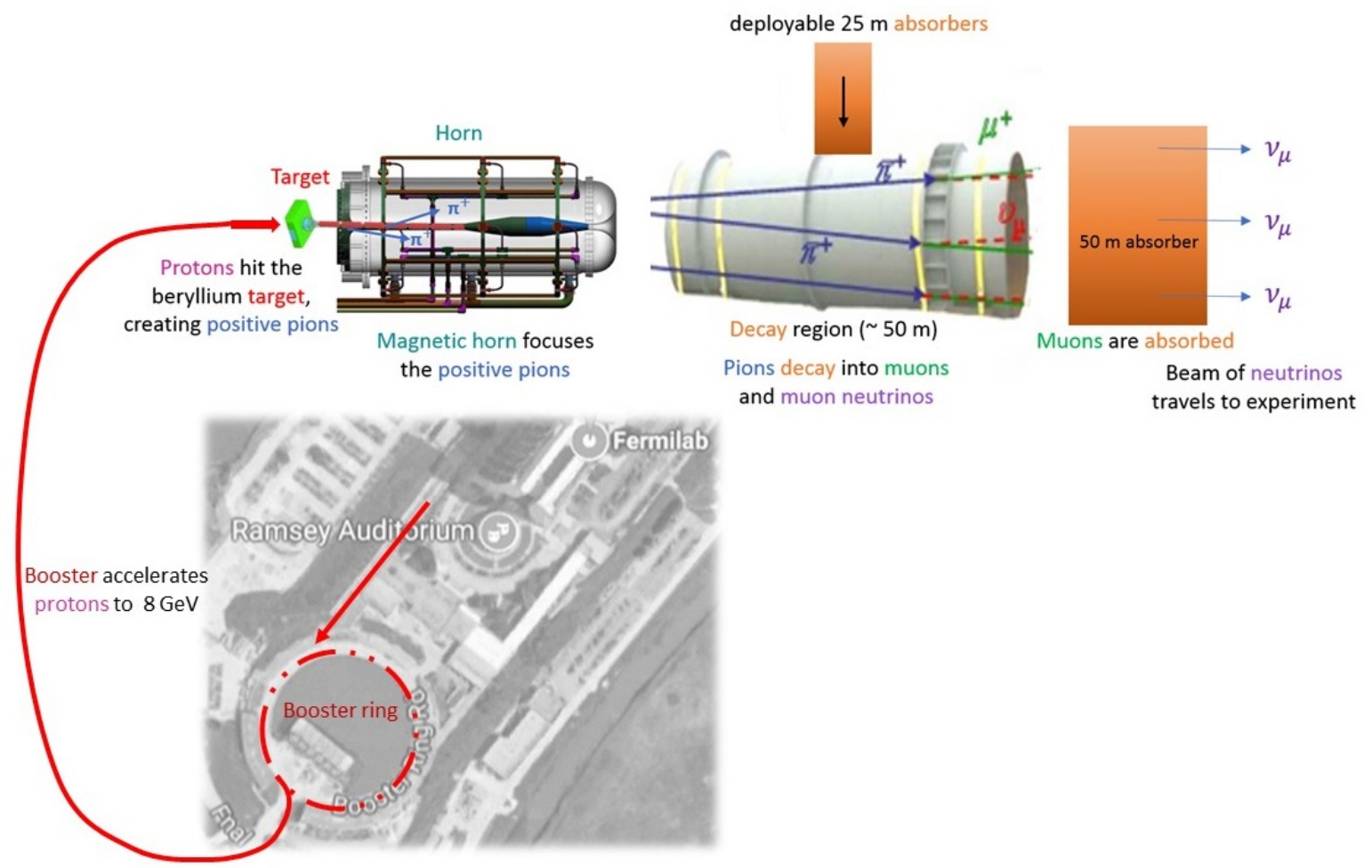

Figure 8.6: Summary of the procedure used to produce neutrinos for the Fermilab Booster Neutrino Beam. Figure taken from http://targets.fnal.gov/BNB_neutrino_beam.html.

This image current is proportional to the beam intensity. One may therefore obtain a measurement of the longitudinal profile of the beam by measuring the image current flowing through the resistors that bridge the gap in the beam pipe wall [430]. Signals from the RWM measuring the proton beam arriving at the BNB target are recorded by the ANNIE data acquisition system when taking beam data.

\subsubsection{Beam current toroids}

The beam current toroids provide a way to measure the number of protons arriving at the target in each beam spill. Each toroid consists of a ferrite ring positioned so that the beam travels perpendicularly through its center (see fig. 8.10). The moving protons in the beam create a circular magnetic field concentric with the beam that enters the ferrite ring and is amplified by it. Because the protons from the beam are grouped into discrete bunches, the amplitude of the magnetic field changes as each bunch approaches 


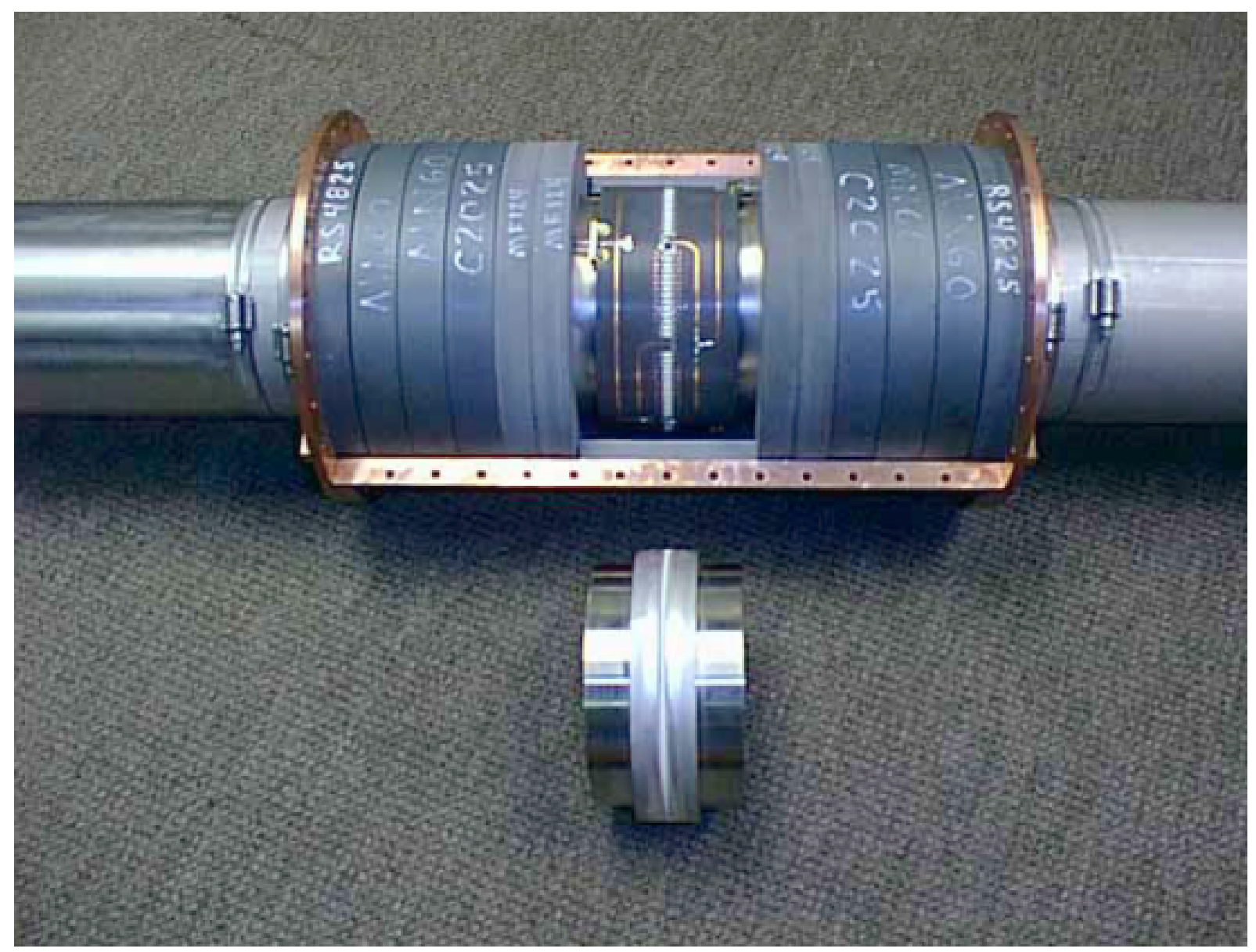

Figure 8.7: Photograph of the resistive wall current monitor used to measure the proton beam arriving at the BNB target. The outer cover has been removed to expose the gap and the RWM electronics. Figure taken from reference [438].

and leaves the toroid. This changing magnetic field induces an electrical current in a pickup loop wrapped around part of the ferrite ring. By measuring the voltage across a resistor connected to the pickup loop, one may use Ohm's law to obtain the current in the pickup loop. Faraday's law of induction may then be used to obtain a measurement of the beam current [430].

Two beam current toroids are used to monitor the BNB. Toroid 875 is located $5 \mathrm{~m}$ upstream of the target, while Toroid 860 is placed $150 \mathrm{~m}$ further upstream [439]. Both toroids are continuously calibrated at $5 \mathrm{~Hz}$ and typically agree with each other to within $2 \%$. Semiannual verifications of their absolute calibrations have shown them to be stable (deviations are less than 0.5\%) [436]. 


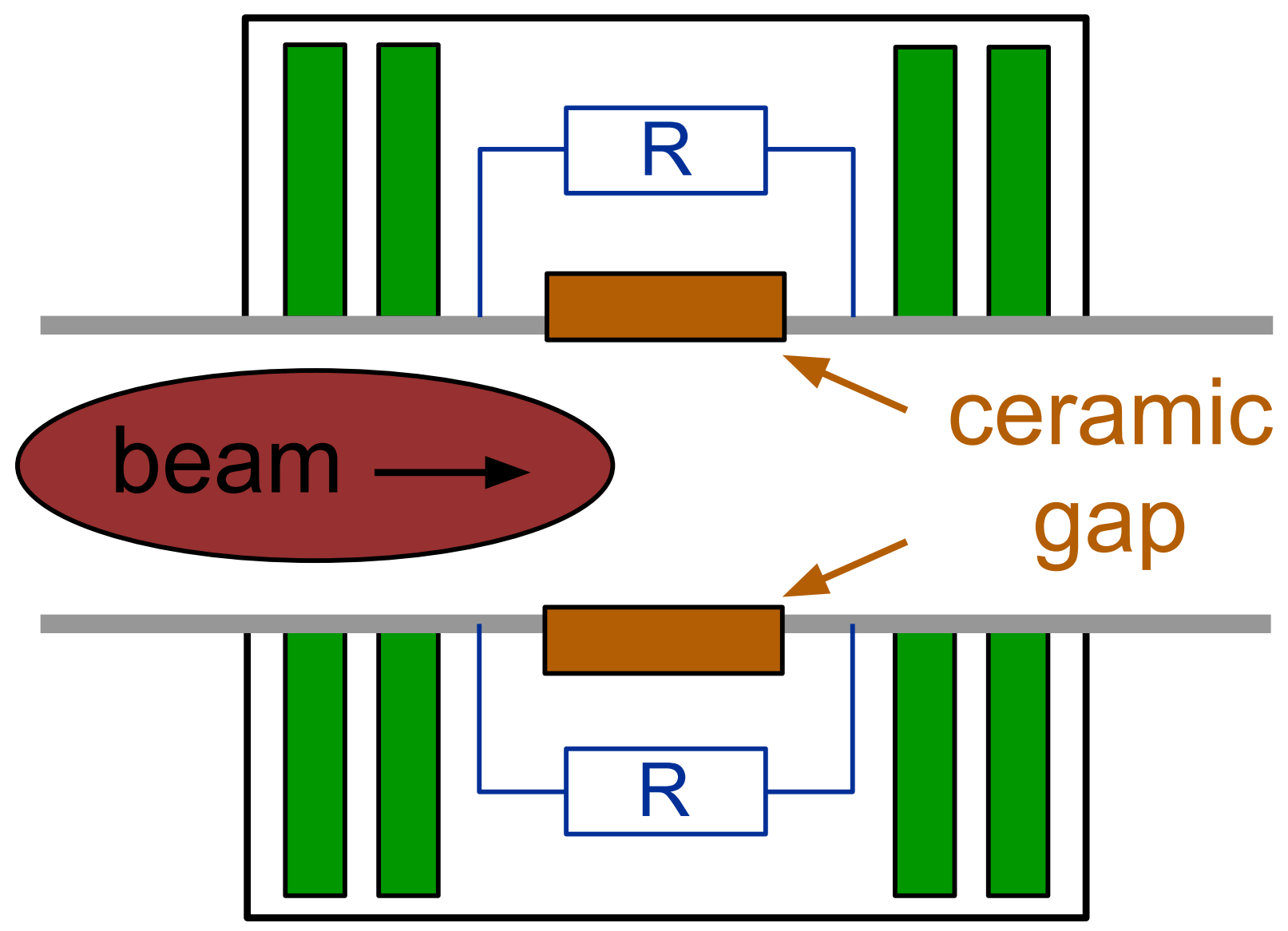

Figure 8.8: Basic design of a resistive wall current monitor (RWM). Resistors are connected across a ceramic gap in the beam pipe wall, and the current flowing through them is measured. Figure taken from reference [430].

\subsection{Beam-associated neutron backgrounds}

Several kinds of neutron backgrounds are expected to exist in the ANNIE detector. Neutrons that are the result of natural radioactivity will form a constant-in-time background that can be measured using an off-beam trigger and greatly reduced by vetoing events outside of a small time window after the arrival of the beam. Two more worrisome kinds of neutron backgrounds, illustrated in fig. 8.11, are not mitigated by such timing cuts because they are correlated in time with the beam. 


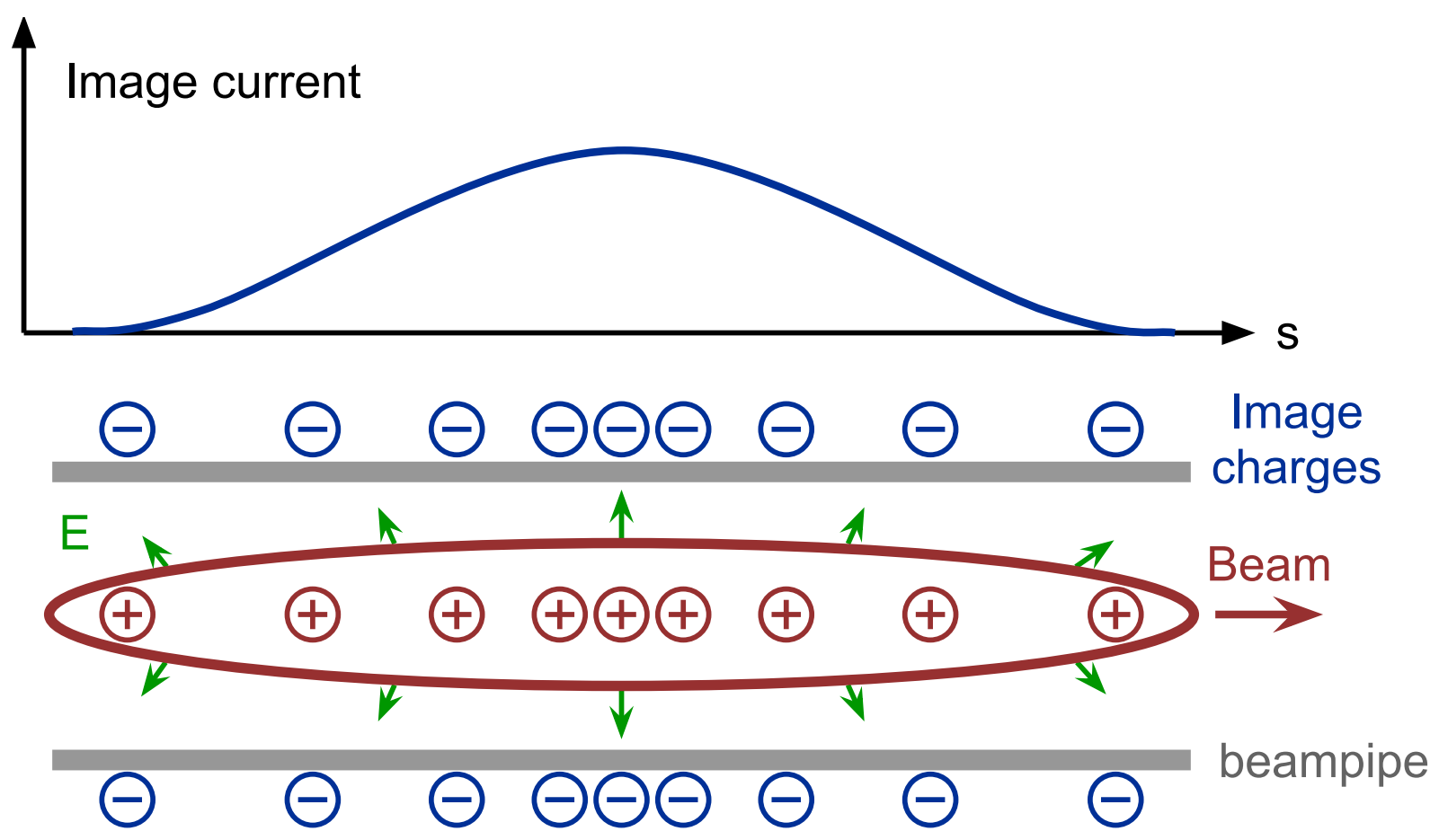

Figure 8.9: As protons from the beam pass through the beam pipe, they induce a negatively-charged image current on the wall of the pipe. Figure taken from reference [430].

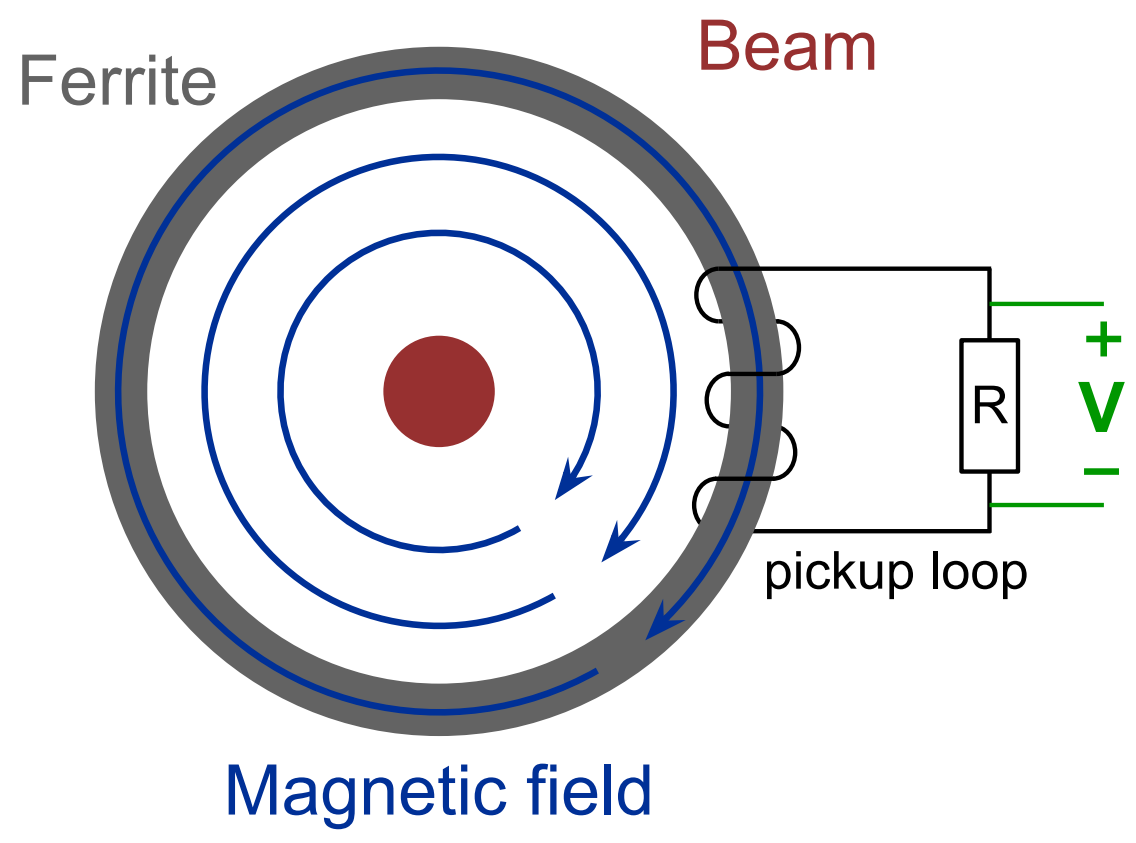

Figure 8.10: Conceptual design of a beam current toroid. The proton beam is directed perpendicularly into the page. Figure taken from [430]. 


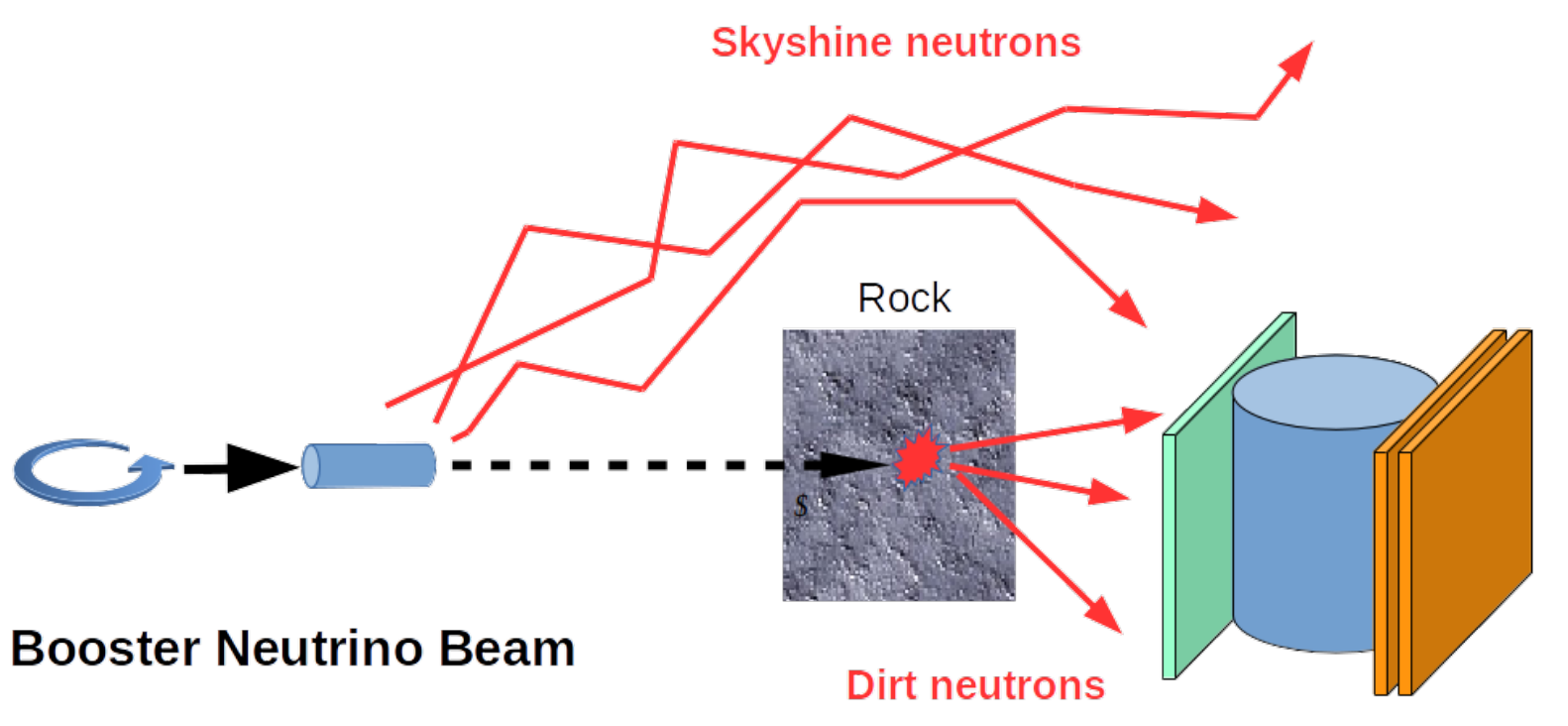

ANNIE

Figure 8.11: Beam-induced neutron backgrounds in the ANNIE detector. "Skyshine neutrons" originate from hadron interactions in the beam target and absorber, while "dirt neutrons" originate from neutrino interactions in the dirt and rock upstream of the ANNIE detector. Figure by Vincent Fischer.

\subsubsection{Dirt neutrons}

The first of these beam-associated backgrounds consists of dirt neutrons, that is, neutrons produced by neutrino interactions with the dirt and rock upstream of the ANNIE detector, which is positioned several meters underground in line with the beam axis. Because the signal neutrons produced by neutrino interactions in the tank will typically take tens of $\mu$ s to be detected via capture on Gd nuclei, even dirt neutrons that arrive fairly late with respect to the beam may nevertheless pose a problem for an accurate neutron yield measurement.

To predict the size of the background produced by dirt neutrons, ANNIE collaborators Erika Cataño Mur, Robert Hatcher, and Mayly Sanchez produced simulations of neutrinos from the BNB passing through SciBooNE hall, the ANNIE detector, and the surrounding area using the GENIE neutrino event generator [174] and the Geant4 particle transport code [440]. A sample of simulated events corresponding to about $3.05 \times 10^{20}$ protons on 
target $^{2}$ (POT) was included in the study. Although the simulations used a preliminary geometry for the ANNIE water tank that had a smaller diameter than the final design (9 $\mathrm{ft}$ in the simulation vs. $10 \mathrm{ft}$ as built), they nevertheless provide a useful rough estimate for the expected rates of dirt and signal neutrons entering the ANNIE detector.

The simulated neutrino interaction vertices and points where neutrons entered the water tank are shown in fig. 8.12. Though about $28 \%$ of the simulated dirt neutrons enter the tank within the first 24 ns after the arrival of the beam (with $78.5 \%$ entering within the first $70 \mu \mathrm{s}$ ), the right panel of fig. 8.13 shows that some dirt neutrons will continue to trickle in for up to tens of ms. As expected, cuts requiring signal events to occur shortly after the arrival of the beam will be ineffective at eliminating the dirt neutron background. Figure 8.14 shows the predicted energies for both signal and dirt neutrons, which have similar distributions.

For a nominal beam spill intensity of $5 \times 10^{12}$ POT, the simulations showed that about $1 \%$ of spills will create a dirt neutron that enters the ANNIE tank, while about $0.8 \%$ of spills will create a signal neutron. To a good approximation, these two processes may be treated as independent, since the interaction of one or even a few beam neutrinos upstream of the ANNIE detector will not appreciably change the probability that a neutrino interaction will occur in the tank during the same spill. Therefore, one may estimate based on these simulations that roughly $1 \%$ of all neutrino events will be contaminated by a dirt neutron entering the water volume.

This result suggests that dirt neutrons will be only a minor nuisance in ANNIE Phase II. However, because significant uncertainties exist in the models used to create the dirt neutron simulations (including the nuclear models governing neutrino-induced neutron emission, which ANNIE Phase II intends to measure), obtaining an experimental constraint for this background would still be prudent.

\subsubsection{Skyshine neutrons}

The second type of beam-induced neutron background that may be observed in the ANNIE detector is due to skyshine neutrons. These neutrons, produced via hadronic interactions

\footnotetext{
${ }^{2}$ For a year of operating the BNB, the nominal number of protons on target is $2 \times 10^{20}$.
} 


\section{$v$ vertex}

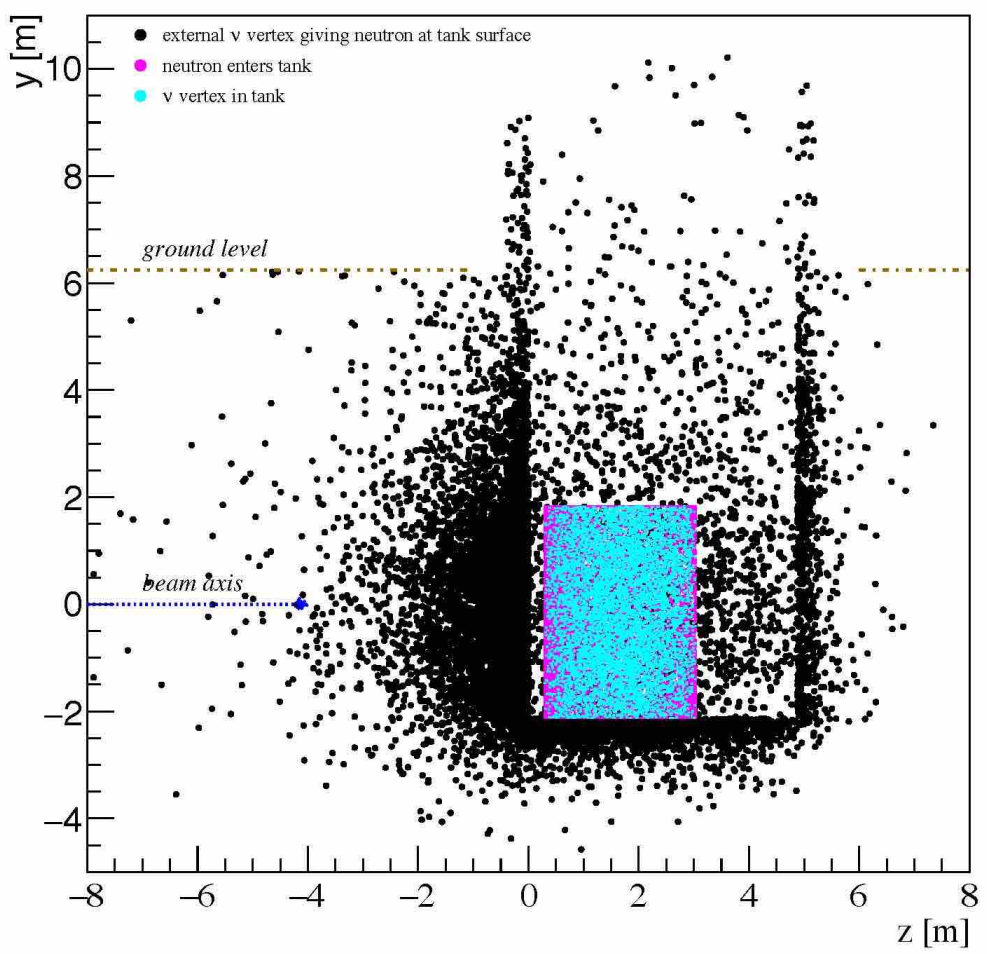

Figure 8.12: Simulated spatial distributions of neutrino vertices outside the tank that produce neutrons that reach the tank (black points), neutrino vertices inside the tank

(cyan points), and points where neutrons enter the tank (magenta). Figure from reference [126].

in the target or beam aborber, initially leak out of the target hall into the air and then scatter downward toward the detector (see fig. 8.11).

Skyshine neutrons from high-energy accelerators have been recognized ${ }^{3}$ as a potential radiological hazard since the 1950s [443, 444] and as a troublesome background for neutrino experiments for at least forty years [445]. Figure 8.15 illustrates an observation of skyshine in the SciBar plastic scintillator detector during its use in the K2K long-baseline neutrino oscillation experiment. The plot shows the time distribution of hits on a single scintillator strip at the top of the detector. Individual beam bunches can be observed as the narrow peaks on the left side of the plot. The peaks lie on top of an increasing baseline attributed to skyshine neutrons.

\footnotetext{
${ }^{3}$ For reviews of the early literature on skyshine, see references [441, 442].
} 

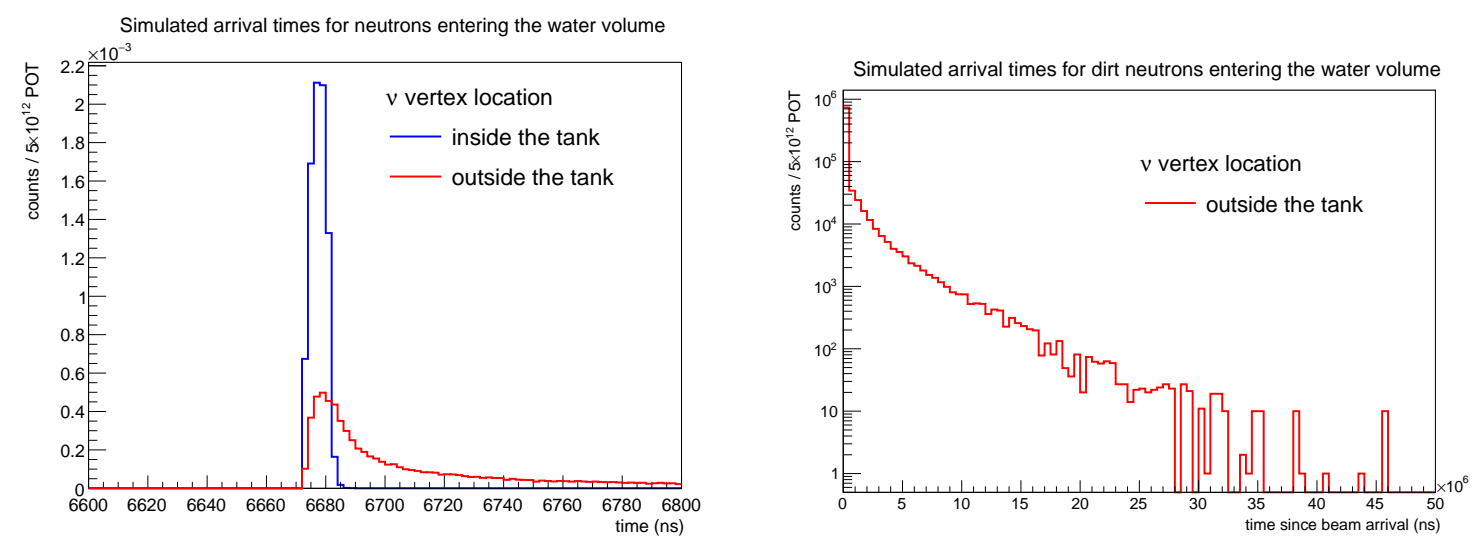

Figure 8.13: Simulated arrival times for neutrino-induced neutrons entering the water volume. LEFT: All of the signal neutrons produced by neutrino interactions in the tank are emitted within a few ns. Most of the background dirt neutrons enter the water around the same time as the signal neutrons. RIGHT: The dirt neutron arrival time distribution includes a long tail that continues until tens of ms after the beam arrives in the detector.

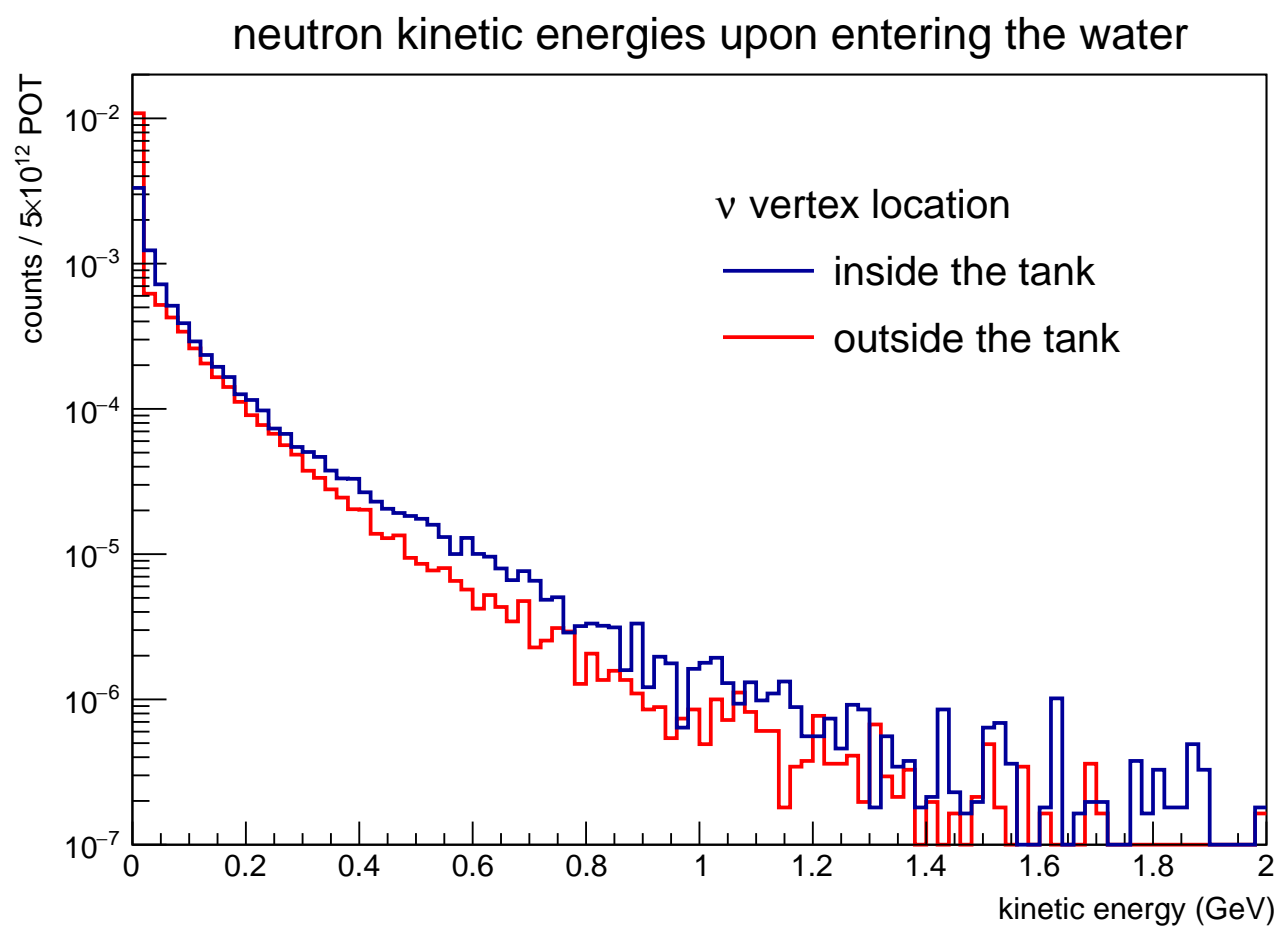

Figure 8.14: Simulated distribution of the kinetic energies of neutrino-induced neutrons at the moment that they enter the ANNIE water volume. The signal neutrons (blue) produced by neutrino interactions in the tank have a similar energy distribution to the background dirt neutrons (red) produced outside of the tank. 


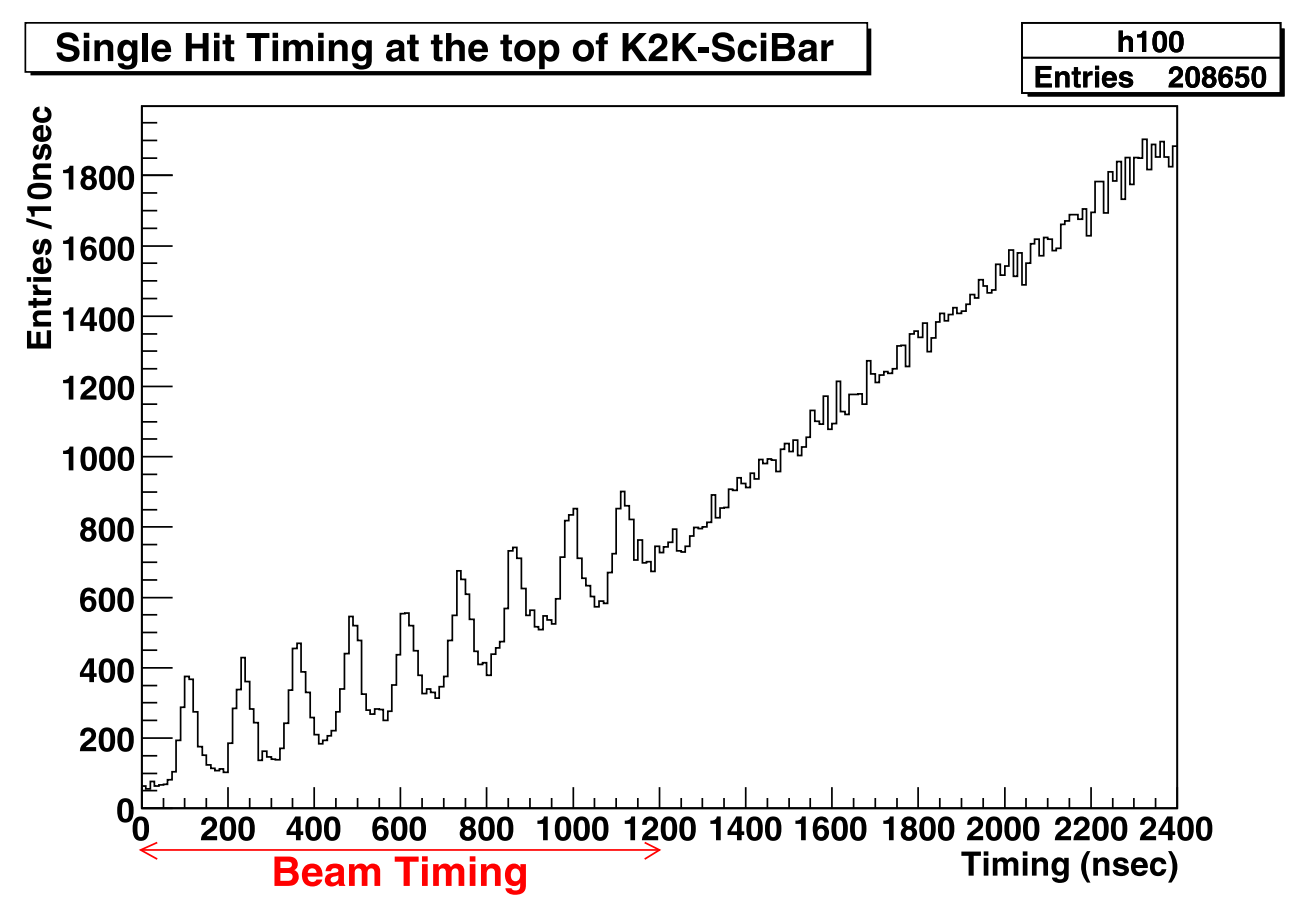

Figure 8.15: Single-strip hit time distribution at the top of the SciBar plastic scintillator detector for a measurement at KEK. The beam bunch peaks sit on top of an increasing pedestal attributable to skyshine neutrons. Figure from reference [196].

Preliminary studies performed by the SciBooNE collaboration at the BNB also revealed noticeable skyshine event rates (see fig. 8.16) $[196,446]$. Because it will be difficult to predict the expected skyshine neutron flux as a function of position within the ANNIE detector, an experimental measurement of this background is needed.

\subsection{Phase I detector description}

The goal of Phase I of the ANNIE experiment is to determine whether the neutron backgrounds at the BNB will be sufficiently low for the Phase II neutron yield measurement to be feasible. The Phase I configuration of the ANNIE detector was designed to enable this background characterization while requiring relatively minor upgrades for the subsequent physics measurement. In fig. 8.17, concept drawings of the detector configuration for the two phases of the experiment are compared. In the remainder of this section, each of the major components of the Phase I detector are described. 

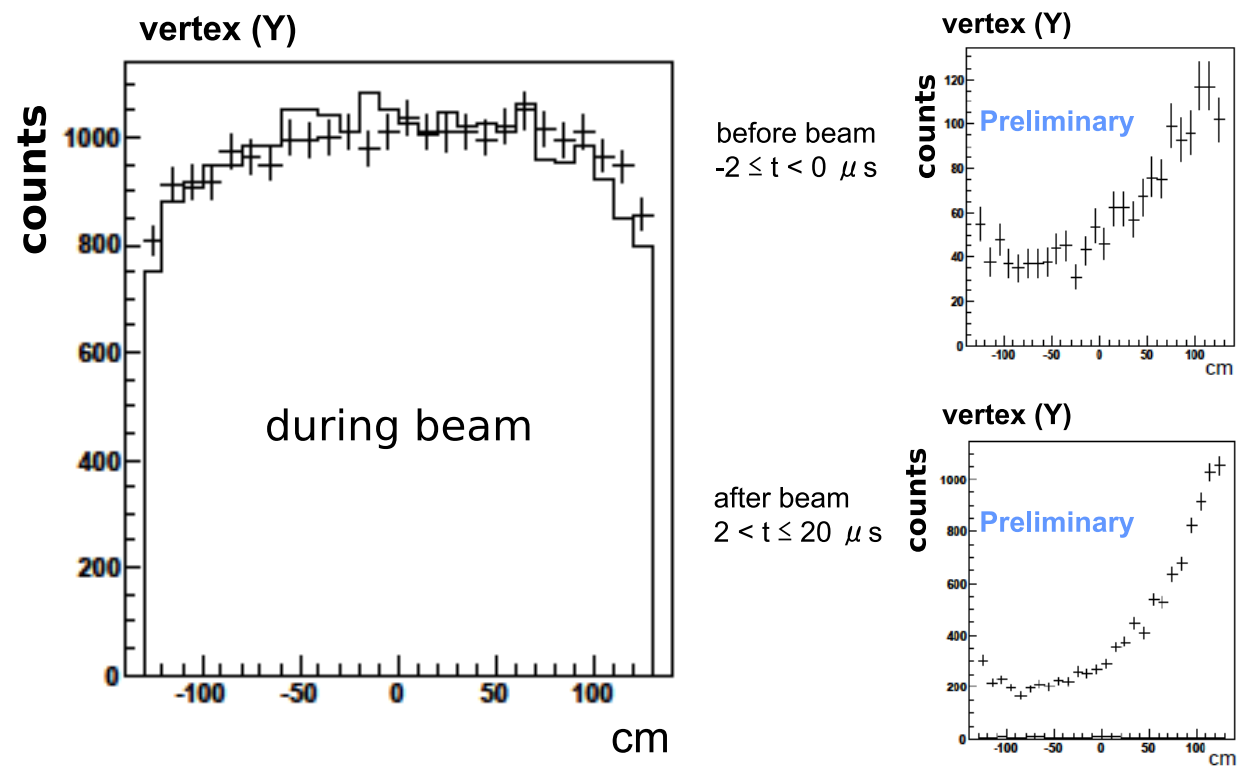

Figure 8.16: Reconstructed $y$ coordinates of event vertices in the SciBooNE detector while running at the BNB. Events that occur in time with the beam have a nearly uniform distribution of $y$ coordinates, while those that occur before and after have distributions that are biased toward the top of the detector. This bias is attributable to cosmic rays in the pre-beam timing region and to a combination of cosmic rays and skyshine in the post-beam timing region. Figure adapted from reference [447], which obtained the plots from reference [446].

\subsubsection{Water tank}

The target for the Phase II neutrino interaction measurements will consist of 26 tons of water loaded with gadolinium sulfate $\left(\mathrm{Gd}_{2} \mathrm{O}_{12} \mathrm{~S}_{3}\right)$ at a concentration of $0.2 \%$ by weight, which corresponds to a concentration of $0.1 \%$ of pure Gd by weight. In Phase I, 26 tons of ultra-pure water were used without any gadolinium sulfate present. In the detector design for both phases, the water volume is stored in an upright cylindrical steel tank that is $13 \mathrm{ft}$ tall and has a diameter of $10 \mathrm{ft}$. The inner surface of the tank is covered with a reflective white $\mathrm{PVC}^{4}$ liner to maximize light collection and to prevent the leaching of transparencyreducing materials into the water from the tank walls. In Phase I, the inner bottom surface of the tank is instrumented with sixty Hamamatsu 8-inch R5912 photomultiplier tubes. These are mounted on a stainless steel inner structure (see fig. 8.18) that was assembled

\footnotetext{
${ }^{4}$ Polyvinyl chloride
} 

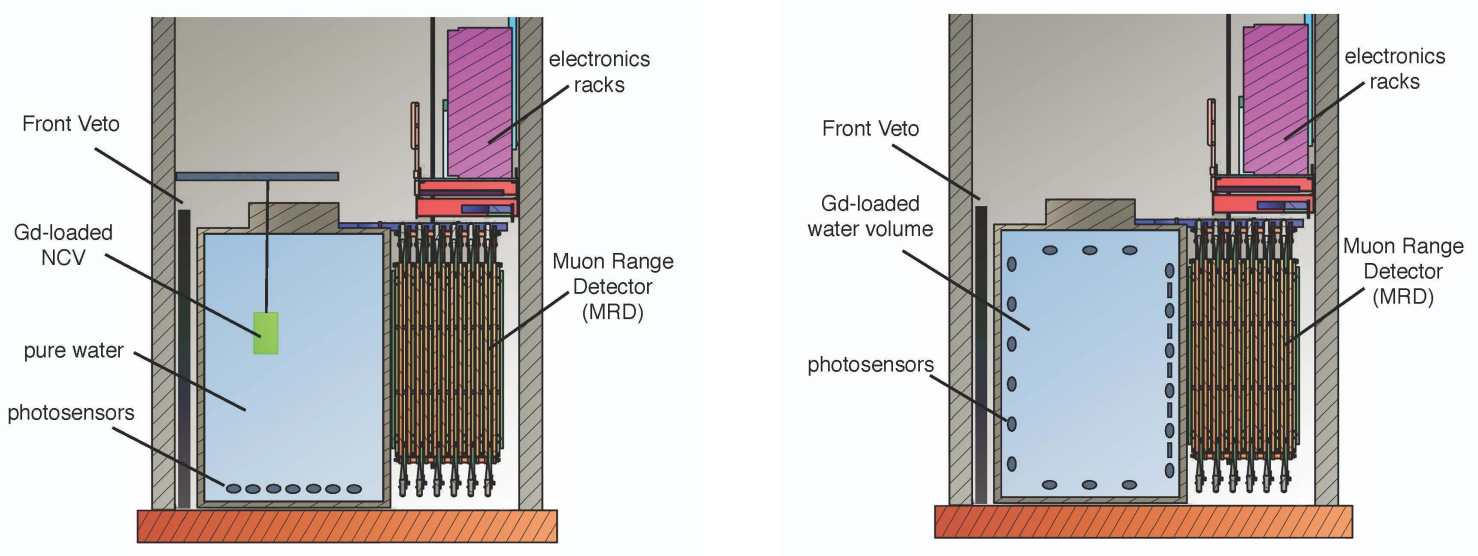

Figure 8.17: Concept drawings of the detector configuration used for each phase of the ANNIE experiment. In both drawings, the beam travels from left to right. LEFT: The

Phase I configuration of the ANNIE detector. For Phase I, the tank contains pure water, and the bottom of the tank is instrumented with sixty PMTs. The Neutron Capture Volume (NCV), a movable neutron-sensitive subvolume of the detector, is used to measure position-dependent neutron backgrounds within the tank. RIGHT: The Phase II configuration of the ANNIE detector. For Phase II, the tank is filled with gadolinium-loaded water, all inner surfaces of the tank are instrumented with PMTs, and LAPPDs (represented by thin gray rectangles in the drawing) are installed on the far side of the tank from the beam. Figure from reference [126].

separately and then lowered by crane into the tank.

\subsubsection{Neutron Capture Volume (NCV)}

The main detector component that is unique to ANNIE Phase I is the Neutron Capture Volume (NCV), a cast acrylic upright cylindrical vessel reinforced with a frame of stainless steel (see fig. 8.19). The vessel consists of a 24 in long acrylic tube with an outer diameter of 20 in and a $3 / 8$ in thick wall. Both ends of the tube are sealed using 20 in acrylic squares that are 1 in thick. The vessel is filled with EJ-335 liquid scintillator, a gadolinium-loaded (0.25\% Gd by weight) scintillator manufactured by Eljen Technology [448]. The top of the vessel is instrumented with two photomultiplier tubes placed in waterproof housings. The NCV PMTs are labeled as "\#1" and "\#2" in the chapters describing the neutron background analysis, with \#1 referring to the PMT that was installed first. A white wrapper placed around the acrylic vessel maximizes the light collected by the two PMTs. As shown in the right panel of fig. 8.20, the NCV and its two PMTs were enclosed in 


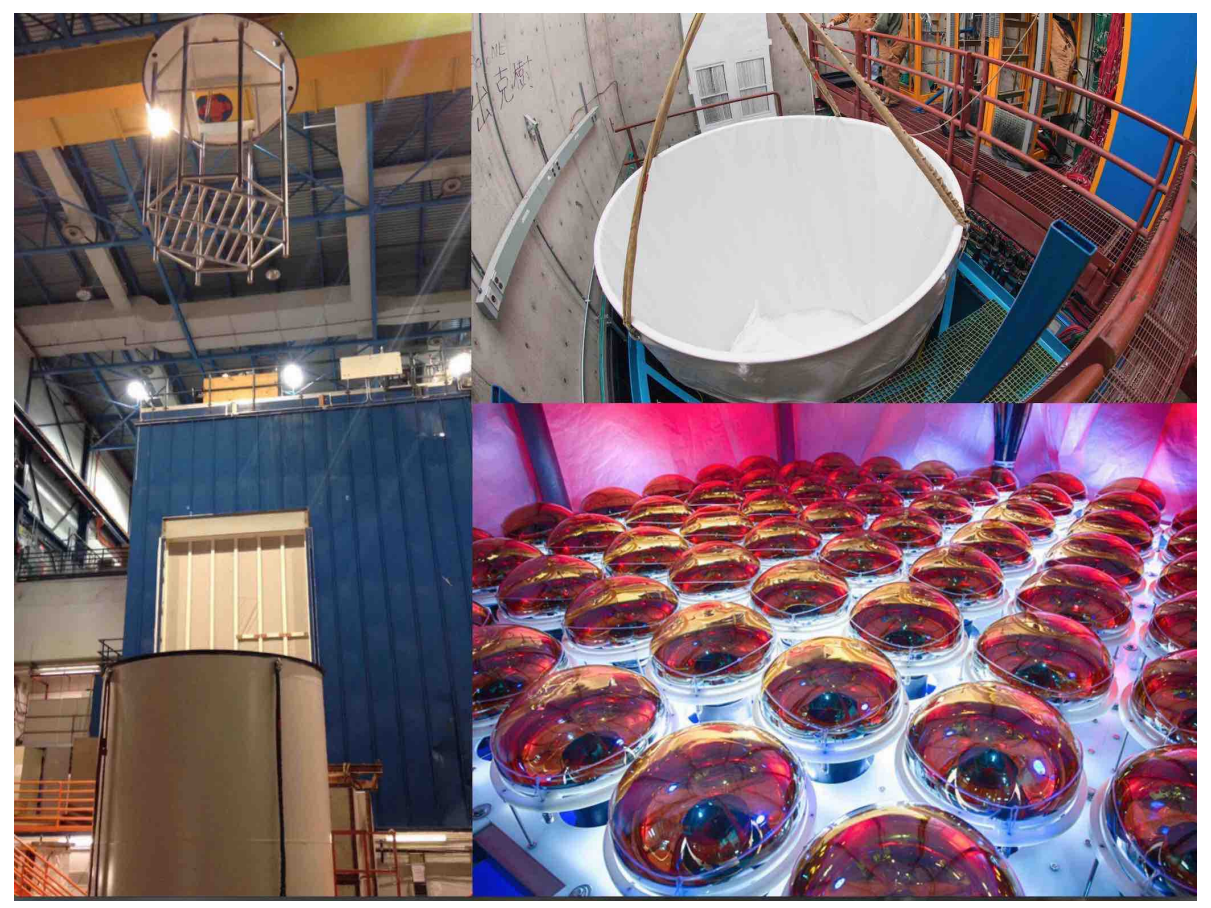

Figure 8.18: LEFT: The Phase I stainless steel inner structure being lowered into the ANNIE water tank. UPPER RIGHT: The ANNIE water tank being installed in the experimental hall. LOWER RIGHT: The 8-inch PMTs used to instrument the bottom of the tank for Phase I. Figure from reference [126].

a black plastic bag to optically isolate them from the rest of the ANNIE tank. The steel NCV frame was connected to a winch system installed in the ANNIE detector hall. The winch allowed the NCV assembly to be moved to various positions within the water volume and stably held in place during data taking.

\subsubsection{Front veto}

To veto muons produced by neutrino interactions in the dirt and rock upstream of the ANNIE detector, 26 plastic scintillator paddles were mounted on the beam-side wall of the ANNIE experimental hall just outside of the water tank. The paddles, each of which has an active area of $10 \mathrm{ft} 6$ in by $1 \mathrm{ft} 1 / 8$ in and a thickness of $7 / 8$ in, were mounted in a single layer 13 paddles high and 2 paddles thick to form a barrier that completely covers the water tank. Each paddle is instrumented with a light guide and a 2 inch PMT on one side. The muon detection efficiency of the paddles is about $90 \%$ near the light guide to about $50 \%$ near the far end. Figure 8.21 shows a concept drawing of the front 


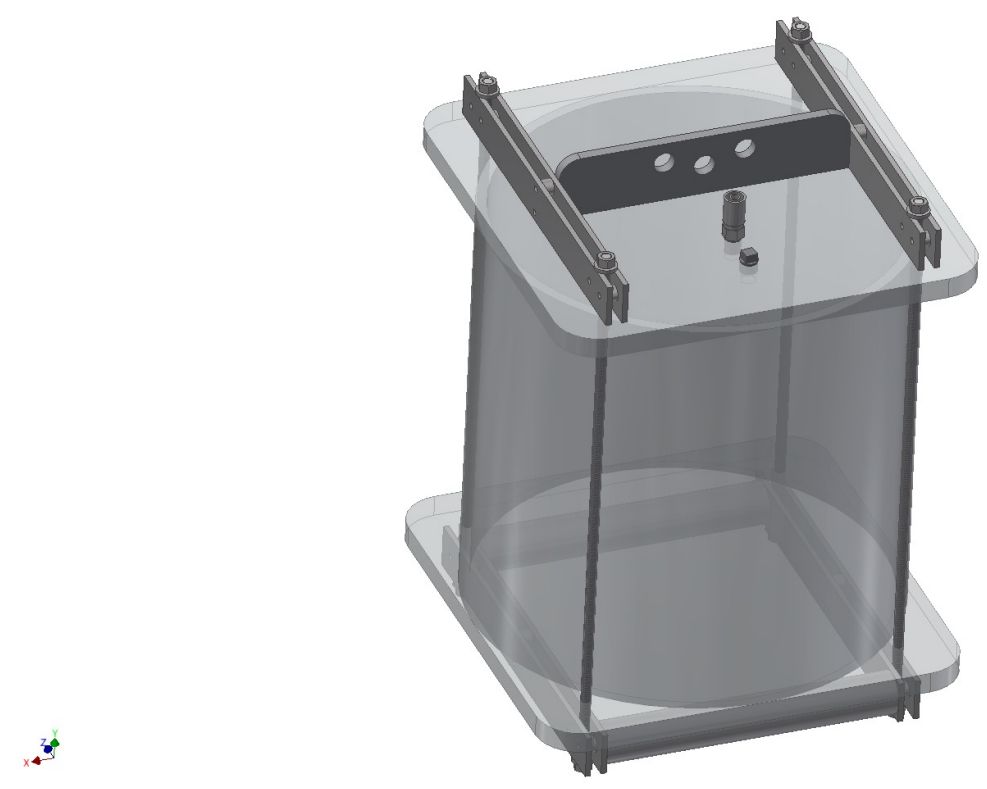

Figure 8.19: CAD (Computer Assisted Design) drawing of the Neutron Capture Volume from ANNIE Phase I. Figure by David Hemer.

veto and a photograph of it after installation.

While the front veto was fully operational during ANNIE Phase I, an alternative means of vetoing cosmic muons using the tank PMTs was found to be sufficiently effective for the neutron background measurements. The front veto will become much more important in Phase II, since the signals of interest for the physics measurements will be detected by PMTs and LAPPDs observing the water volume, and therefore an external muon veto will be needed.

\subsubsection{Muon Range Detector}

During ANNIE Phase II, the direction and energy of muons produced by charged current neutrino interactions inside the water volume will be measured using a device called the Muon Range Detector (MRD). Originally built for the SciBooNE experiment (which previously occupied the same experimental hall used by ANNIE), the MRD consists of a "sandwich" of twelve $274 \times 305 \mathrm{~cm}^{2}$ iron plates placed between thirteen layers of plastic scintillator paddles, the latter being read out by a total of 362 two-inch PMTs. The pattern of hit paddles combined with the depth at which a muon ranges out in the 

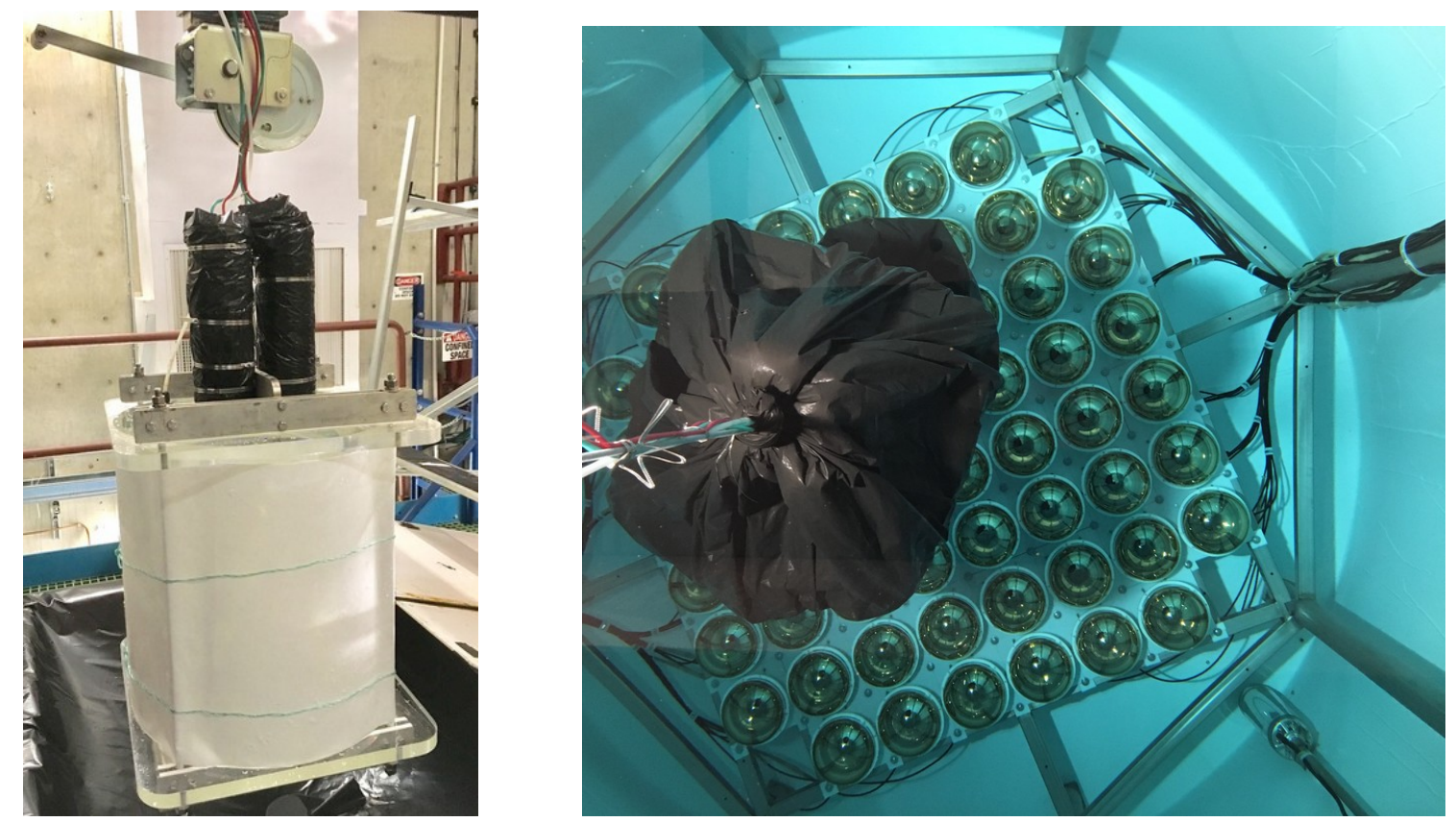

Figure 8.20: LEFT: The Neutron Capture Volume (NCV) from ANNIE Phase I before its installation in the water tank. Two PMTs, encased in waterproof housings and wrapped with black plastic, observe scintillation light induced by neutron captures in the acrylic vessel, which is filled with Gd-loaded liquid-scintillator. The NCV frame is attached to a winch system (partially shown near the top of the photograph), allowing the NCV to be moved to different locations inside the tank. RIGHT: The NCV after installation in the water tank. To optically isolate the NCV from the rest of the tank, the acrylic vessel and two PMTs were wrapped with a black plastic bag.

MRD will be used for kinematical event reconstruction in ANNIE Phase II. Although not needed for the background neutron event rate analysis, two layers of the MRD were fully instrumented and operated for testing purposes during Phase I. The MRD has been fully refurbished in preparation for Phase II data taking.

\subsubsection{Cosmic ray trigger}

To enable triggering of the ANNIE detector in response to cosmic muons traveling in a known direction, six plastic scintillator paddles, each with an attached light guide and PMT, were mounted in a metal frame as shown in fig. 8.23. The six PMT outputs were connected to a $\mathrm{NIM}^{5}$ logic module that issued trigger signals to the ANNIE data acquisition system in response to three distinct sets of coincident pulses from the PMTs.

\footnotetext{
${ }^{5}$ Nuclear Instrumentation Module
} 

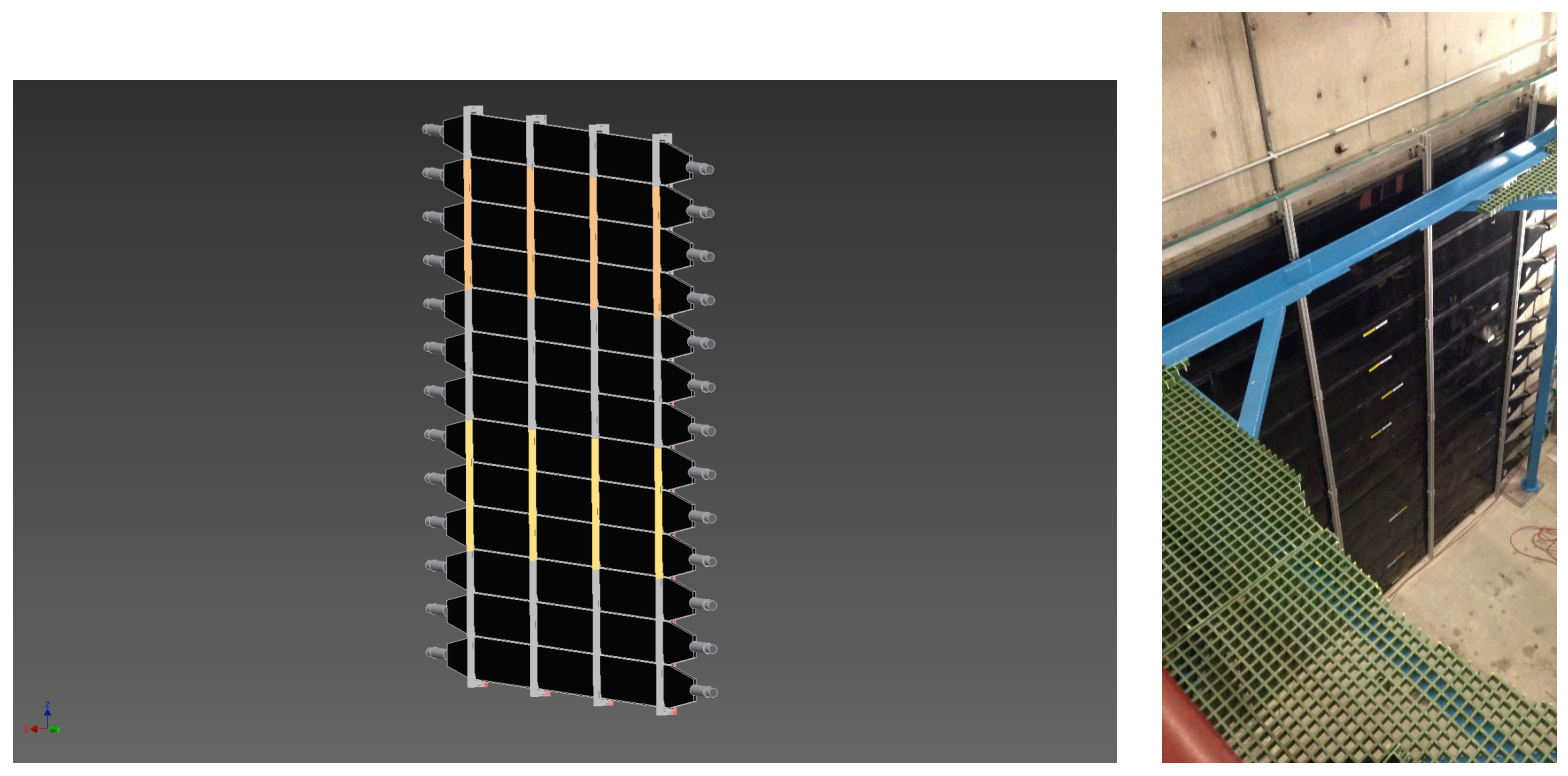

Figure 8.21: LEFT: Concept drawing of the ANNIE front veto. RIGHT: A photograph of the front veto after its installation in the experimental hall for ANNIE Phase I.
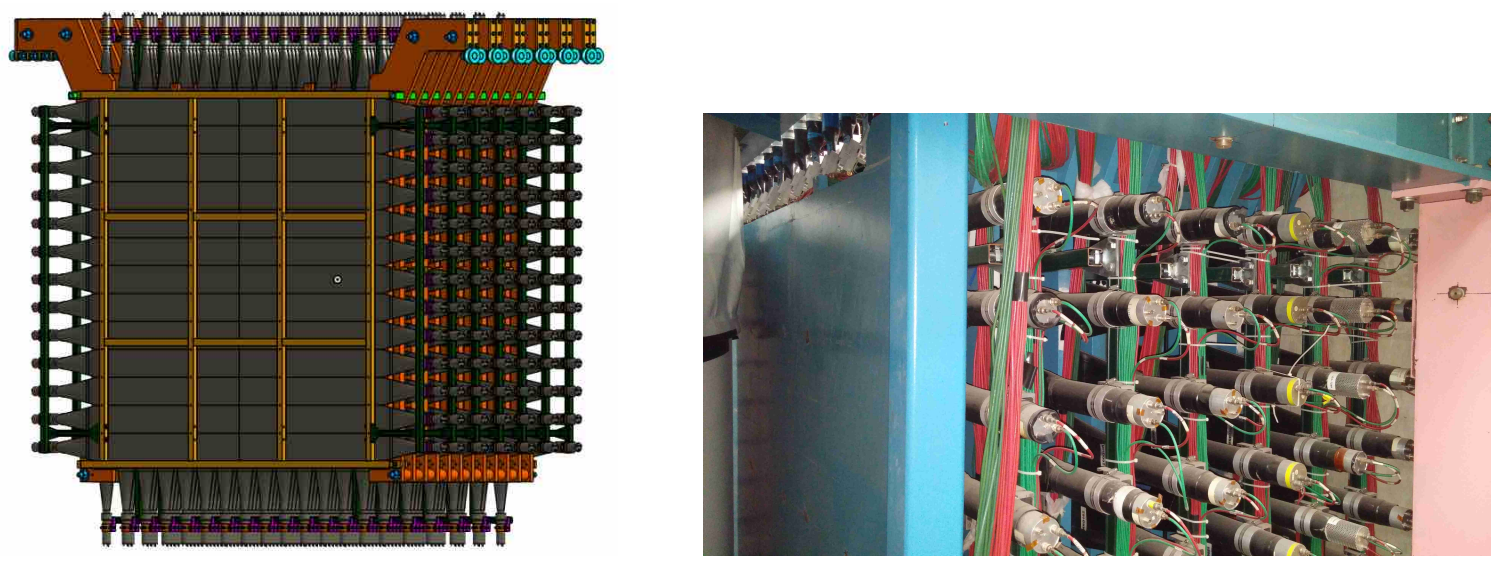

Figure 8.22: LEFT: Concept drawing of the SciBooNE Muon Range Detector, now repurposed for ANNIE. RIGHT: A side view of the MRD as it appeared during Phase I data taking. 
Table 8.1: The three distinct combinations of coincident paddle hits that gave rise to cosmic muon triggers in ANNIE Phase I. The three combinations were distinguished during data taking by the width of the NIM logic pulse issued by the cosmic ray trigger.

Each combination is indicative of a different physical situation, as described in the "Interpretation" column of the table.

\begin{tabular}{cccc}
\hline $\begin{array}{c}\text { Combination } \\
\text { number }\end{array}$ & $\begin{array}{c}\text { Hit } \\
\text { paddles }^{a}\end{array}$ & $\begin{array}{c}\text { Trigger logic } \\
\text { pulse width (ns) }\end{array}$ & Interpretation \\
\hline 1 & $1,3,4,6$ & 10 & Downward muon toward NCV \\
2 & $2,3,5,6$ & 20 & Downward muon outside NCV \\
3 & $1,3,5,6$ & 40 & $45^{\circ}$ muon toward MRD \\
\hline
\end{tabular}

${ }^{a}$ See the left panel of fig. 8.23 for the paddle numbering scheme.

Table 8.1 lists the combinations of paddle hits that gave rise to a NIM logic pulse in ANNIE Phase I. The width of the logic pulse was used to indicate which of the three combinations of interest was observed. Of particular importance to the Phase I analysis was combination 1, which was consistent with downward muons passing through the scintillator paddles toward the NCV. Data taken in response to cosmic triggers of this kind were used to estimate the neutron detection efficiency of the NCV, as described in section 10.2.

\subsection{6 $\quad{ }^{252} \mathrm{Cf}$ calibration trigger}

In addition to the NCV calibration based on data taken with the cosmic muon trigger, efficiency calibrations were also performed using a ${ }^{252} \mathrm{Cf}$ fission neutron source. Because a description of the triggering apparatus used in those measurements is most directly relevant to the discussion of the NCV efficiency analysis, we will postpone giving a description of this portion of the detector until section 10.1.1.

\subsection{Phase I Data Acquisition}

The data acquisition system (DAQ) for ANNIE Phase I uses two sets of readout electronics in parallel. The first of these, a $500 \mathrm{MHz}$ analog-to-digital converter (ADC) system 

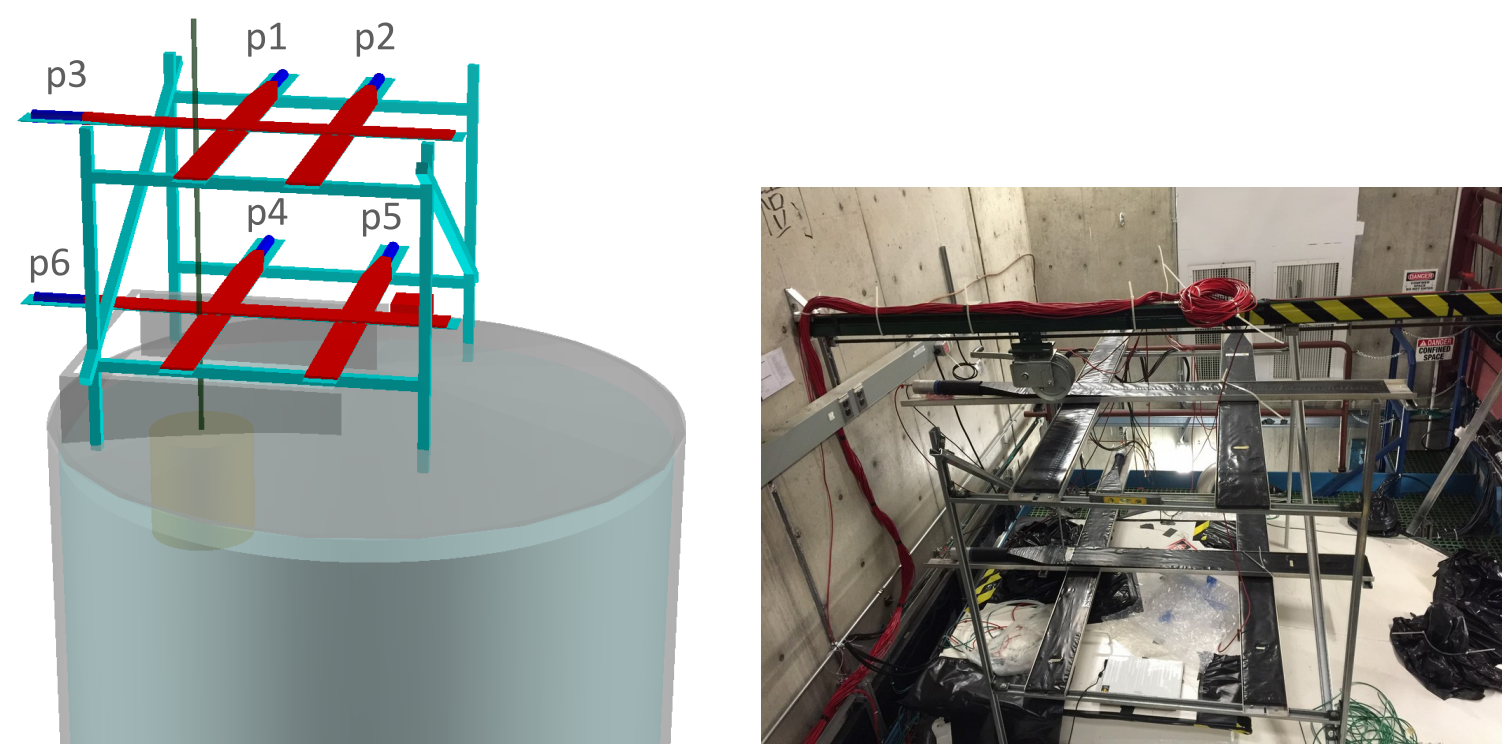

Figure 8.23: ANNIE Phase I cosmic ray trigger. LEFT: Concept drawing showing the paddle numbering scheme. As described in the text and in table 8.1, coincidences of multiple paddles are used to select cosmic muons traveling in three different directions.

The yellow cylinder in the drawing represents the NCV. Drawing by Jingbo Wang.

RIGHT: Photograph of the installed cosmic ray trigger during Phase I data taking.

implemented using $\mathrm{VME}^{6}$ electronics, is used to digitize the PMT signals. The second, a time-to-digital converter (TDC) system originally developed for the SciBooNE experiment using $\mathrm{CAMAC}^{7}$ electronics, records signals from the front veto and MRD. Both sets of readout electronics share a centralized triggering and timing system implemented using a CAEN V1495 FPGA ${ }^{8}$ VME card running custom firmware. Triggers used by the DAQ include those indicating the arrival of the beam (highest priority), coincidences of the cosmic ray paddles (see section 8.4.5), and pulses on the PMT monitoring the ${ }^{252} \mathrm{Cf}$ calibration source (see section 10.1), among others. Timestamps recorded by the trigger card and the ADC and CAMAC systems allow the data streams to be synchronized and combined in the offline analysis. The DAQ computers use an ANNIE-specific version of the ToolDAQ framework ${ }^{9}$ to configure, monitor, and receive data from the readout electronics. Raw data files from the DAQ are saved to disk in ROOT format [449] and

\footnotetext{
${ }^{6}$ Versa Module Europa

${ }^{7}$ Computer-Aided Measurement and Control

${ }^{8}$ Field-programmable gate array

${ }^{9}$ http://github.com/ToolDAQ/ToolDAQFramework
} 
analyzed offline using procedures described in chapter 9. More details about the Phase I readout electronics and DAQ software are available in references [126, 450].

During early Phase I data taking, the ADC system was configured to respond to trigger events by recording data from the PMTs continuously during an $80 \mu$ s time window. This normal mode readout scheme operated in essentially the same way for all of the enabled triggers, except that the portion of the window in which pre-trigger data were digitized differed between trigger types. Part of the way through Phase I, however, the DAQ software and firmware were upgraded to enable data taking using a zero suppression mode, nicknamed "Hefty mode" in reference [126], which was used during all of the remaining beam runs.

Zero suppression mode introduces a new multiplicity trigger for the ADC system, which is issued in response to pulses above a user-defined ADC threshold on one or more PMTs. The ADC multiplicity trigger is temporarily enabled during a configurable time window following the receipt of a trigger of a different type, e.g., a beam trigger. During ANNIE Phase I, the ADC multiplicity trigger was used as a self-trigger for the NCV: a multiplicity trigger signal was issued whenever either of the two NCV PMTs recorded a signal above $357 \mathrm{ADC}$ counts ${ }^{10}$ within $100 \mu$ s of a preceding beam, cosmic ray, or ${ }^{252} \mathrm{Cf}$ calibration source trigger.

While running in zero suppression mode, the ADC system digitizes the PMT signals during a $2 \mu$ s minibuffer following any enabled trigger, including NCV self-triggers. The time when each minibuffer began is also recorded with ns precision. When 40 minibuffers have been read out (so that the complete $80 \mu$ s buffer used to store a single event in normal mode has been filled), the data must be transferred to the DAQ computers before further minibuffers may be recorded. If additional triggers that would have resulted in the creation of a new minibuffer are received during the dead time associated with the data transfer, a boolean flag is set in the DAQ output that allows incomplete events to be identified and excluded during the offline analysis.

To reconstruct a beam event taken in zero suppression mode, a minibuffer recorded

\footnotetext{
${ }^{10}$ This corresponds to a threshold of about $6 \mathrm{mV}$ for NCV PMT \#1 and about $8.6 \mathrm{mV}$ for NCV PMT $\# 2$.
} 
in response to a beam trigger is considered together with zero or more NCV self-trigger minibuffers that followed. The start times of each of the self-trigger minibuffers relative to the beam trigger are determined in a pre-processing step using timing information recorded by the ADC system and trigger card. The start times for PMT pulses, which are assigned by the ANNIE reconstruction software relative to the start of the minibuffer in which they are found, are then corrected using the appropriate minibuffer offsets to obtain event times relative to the beam.

\subsection{Measurement strategy}

During the planned Phase II physics measurements, photomultiplier tubes will be used to count the number of neutron captures observed in tens of $\mu$ s following charged current neutrino interactions in the water tank. The goal of ANNIE Phase I is to determine whether the rate of background neutron captures (in particular, those correlated in time with the beam) during the same time period of interest will be sufficiently low for the Phase II measurements to proceed. For definiteness, a time region of interest (ROI) from $10 \mu \mathrm{s}$ to $70 \mu \mathrm{s}$ after the beam begins to cross the ANNIE detector was chosen for Phase I.

To study how the background neutron event rate varies with position inside the ANNIE water tank, Phase I beam data were taken with the NCV placed at seven different positions, as shown in fig. 8.24, where the positions are numbered in chronological order. In an attempt to separate the contributions of skyshine and dirt neutrons to the measured background, the NCV was used to perform vertical (positions 1, 2, 4, 5, and 6) and horizontal (position 2, 3, and 7) scans of the detector. Cosmic trigger data were recorded at each position in addition to beam data. Table 8.2 lists the number of recorded cosmic triggers and the total POT delivered by the beam with the NCV at each position.

Assuming that captures of neutrons above thermal energies $(\sim 0.025 \mathrm{eV})$ and thermal neutron captures outside of the NCV liquid may be neglected, ${ }^{11}$ the expected number of

\footnotetext{
${ }^{11}$ The estimated NCV energy threshold of $4.76 \mathrm{MeV}$ determined in section 10.2.2, which is comfortably above the energy of the $2.2 \mathrm{MeV} \gamma$-ray emitted following thermal neutron capture on ${ }^{1} \mathrm{H}$, lends support to the latter assumption.
} 


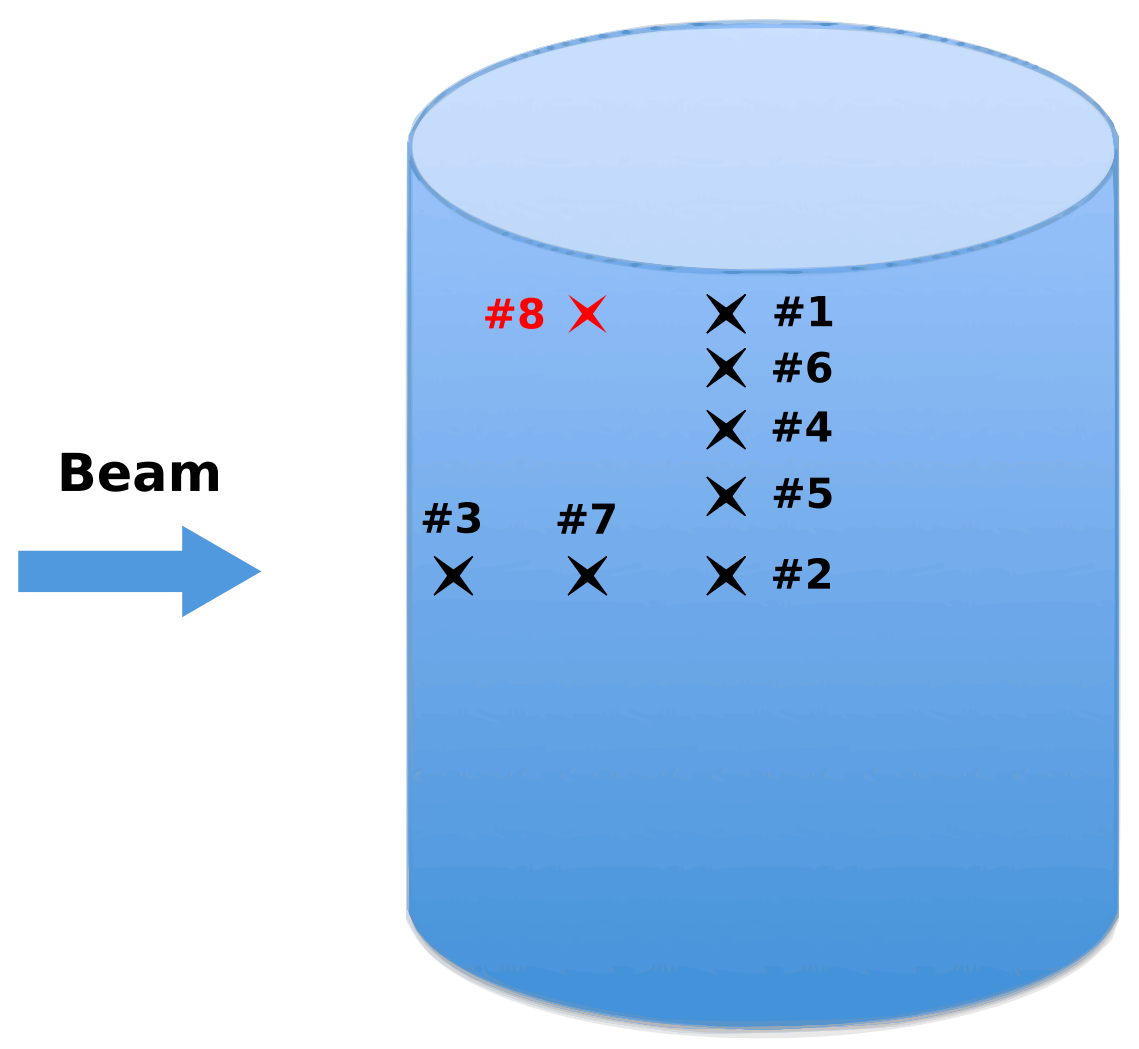

Figure 8.24: NCV position scan performed during beam data taking in ANNIE Phase I. Each of the NCV positions, which are not drawn to scale, is numbered in chronological order. The beam had been shut down for maintenance before any data were taken at position \#8. Despite the lack of beam, additional test runs were taken with the NCV at position \#8 before the Phase I detector was decommissioned. Position \#8 is shown in red in the figure to indicate that data taken there are not included in the analysis described in this thesis.

neutron capture events observed in the $\mathrm{NCV} N_{n}$ at a given position may be written as

$$
N_{n}=\phi_{n} \mathcal{P} V_{\mathrm{NCV}} \epsilon_{\mathrm{NCV}} \Sigma_{\mathrm{NCV}}
$$

where the exposure $\mathcal{P}$ represents the total number of protons delivered to the Booster Neutrino Beam target while data were being taken with the NCV at the position of interest, $V_{\mathrm{NCV}}$ is the volume of the NCV liquid, and $\epsilon_{\mathrm{NCV}}$ is the detection efficiency of the NCV for neutron captures. $\Sigma_{\mathrm{NCV}}$ is the macroscopic neutron capture cross section of the NCV liquid, defined by

$$
\Sigma_{\mathrm{NCV}}=\sum_{j} g_{j} \sigma_{j} \rho_{j}=37.2(1) \mathrm{m}^{-1}
$$


Table 8.2: Summary of beam and cosmic data taken during Phase I. See fig. 8.24 for a diagram of the NCV positions. An uncertainty of $2 \mathrm{~cm}$ was estimated for all NCV position measurements.

\begin{tabular}{ccccccc}
\hline $\begin{array}{c}\text { NCV } \\
\text { position }\end{array}$ & $\begin{array}{c}\text { Water } \\
\text { overburden }^{a} \\
(\mathrm{~cm})\end{array}$ & $\begin{array}{c}\text { Water }_{\text {shielding }}{ }^{b} \\
(\mathrm{~cm})\end{array}$ & $\begin{array}{c}\text { Beam } \\
\text { spills }\end{array}$ & $\begin{array}{c}\text { Total exposure } \\
\left(10^{18} \text { POT }\right)\end{array}$ & $\begin{array}{c}\text { Average POT } \\
\text { per spill } \\
\left(10^{12} \text { POT }\right)\end{array}$ & $\begin{array}{c}\text { Cosmic } \\
\text { triggers }\end{array}$ \\
\hline 1 & $6(2)$ & $104(2)$ & 1779098 & 7.88 & 4.43 & 17217 \\
2 & $138(2)$ & $104(2)$ & 4568327 & 17.66 & 3.87 & 33437 \\
3 & $138(2)$ & $10(2)$ & 4383135 & 13.24 & 3.02 & 40451 \\
4 & $36(2)$ & $104(2)$ & 3476203 & 10.98 & 3.16 & 59387 \\
5 & $67(2)$ & $104(2)$ & 2023082 & 6.79 & 3.36 & 26610 \\
6 & $21(2)$ & $104(2)$ & 973057 & 3.63 & 3.73 & 11502 \\
7 & $138(2)$ & $58(2)$ & 650378 & 2.20 & 3.39 & 6575 \\
\hline
\end{tabular}

${ }^{a}$ The thickness of water above the NCV

${ }^{b}$ The thickness of water between the beam-side wall of the tank and the NCV

where $\sigma_{j}$ is the microsopic cross section ${ }^{12}$ for capture of $2.2 \mathrm{~km} / \mathrm{s}$ neutrons on nuclide $j$, $g_{j}$ is the Westcott factor ${ }^{13}$ for nuclide $j, \rho_{j}$ is the atom density of nuclide $j$, and the sum runs over all nuclides present in the NCV liquid scintillator. The numerical value given in eq. (8.5) was calculated using the atom densities given in reference [448] and the cross section data given in reference [451]. The symbol $\phi_{n}$ denotes the average fluence ${ }^{14}$ of thermal neutrons per proton on target within the NCV liquid. That is, if $\Phi_{n}(\mathbf{r}, t)$ denotes the average instantaneous flux of thermal neutrons in the NCV liquid ${ }^{15}$ per proton on target at position $\mathbf{r}$ and time $t$, then the average value of $\Phi_{n}(\mathbf{r}, t)$ over the NCV liquid

\footnotetext{
${ }^{12}$ i.e., the cross section for a single target nucleus

${ }^{13}$ The ratio of the average cross section for room-temperature thermal neutrons obeying a MaxwellBoltzmann distribution to the cross section for $2.2 \mathrm{~km} / \mathrm{s}$ neutrons.

${ }^{14}$ i.e., the time-integrated flux

${ }^{15}$ The total length traveled by all thermal neutrons in the NCV liquid per unit time and volume. This definition is the standard one in nuclear reactor physics. It is equivalent to the number of neutrons penetrating a sphere with unit cross sectional area per unit time from all sides [452].
} 
volume $\psi_{n}(t)$ is given by

$$
\psi_{n}(t)=\frac{1}{V_{\mathrm{NCV}}} \int_{\text {NCV liquid }} \Phi_{n}(\mathbf{r}, t) d^{3} \mathbf{r}
$$

and the average thermal neutron fluence per proton on target $\phi_{n}$ is given by

$$
\phi_{n}=\int_{\mathrm{ROI}} \psi_{n}(t) d t
$$

where the integration is over the time region of interest for Phase I (10 $\mu$ s to $70 \mu \mathrm{s}$ postbeam). The quantity $\phi_{n}$ may be expressed in units of thermal neutrons per $\mathrm{cm}^{2}$ per POT.

The average neutron capture rate in the NCV liquid $\mathcal{R}_{n}^{\mathrm{NCV}}$, which may expressed in units of neutron captures per $\mathrm{m}^{3}$ per POT, is given by

$$
\mathcal{R}_{n}^{\mathrm{NCV}}=\phi_{n} \Sigma_{\mathrm{NCV}}=\frac{N_{n}}{\mathcal{P} \epsilon_{\mathrm{NCV}} V_{\mathrm{NCV}}} .
$$

To convert $\mathcal{R}_{n}^{\mathrm{NCV}}$ into the corresponding predicted neutron capture rate within the Phase II water volume $\mathcal{R}_{n}^{\text {II }}$, two changes must be made. First, since the gadolinium-loaded water that will be used in Phase II has a macroscopic cross section ${ }^{16} \Sigma_{\mathrm{II}}=18.70(5) \mathrm{m}^{-1}$ that is different from that of the NCV liquid, the measured NCV capture rate must be scaled by the ratio of the macroscopic cross sections. Second, because of the difference in the thermal neutron capture cross sections for the two materials, the average thermal neutron fluence per proton on target $\phi_{n}$ will also have a different value $\phi_{n}^{\text {II }}$ for the Phase II water.

Although the exact value of $\phi_{n}^{\text {II }}$ may be difficult to predict, we may estimate the ratio $f=\phi_{n}^{\mathrm{II}} / \phi_{n}$ by assuming that the different thermal capture cross sections for the two materials do not appreciably affect the spatial averaging performed in eq. (8.6). We further assume that, for both materials, the average thermal neutron flux per proton on target $\psi_{n}(t)$ may be written as the convolution of some unknown function $T(t)$, representing the time distribution of thermal neutrons arriving in the NCV liquid (or the corresponding volume of the Phase II gadolinium-loaded water) and a decaying exponential that

\footnotetext{
${ }^{16}$ Cross section data from reference [451] were used to obtain the numerical value of $\Sigma_{\text {II }}$ given here.
} 
represents the distribution of capture times for thermal neutrons within the material of interest:

$$
\begin{aligned}
& \psi_{n}(t)=\int_{0}^{t} T(s) e^{-(t-s) / \tau_{\mathrm{NCV}}} d s \\
& \psi_{n}^{\mathrm{II}}(t)=\int_{0}^{t} T(s) e^{-(t-s) / \tau_{\mathrm{II}}} d s
\end{aligned}
$$

Here the time constants $\tau_{\mathrm{NCV}}$ and $\tau_{\mathrm{II}}$ are defined by

$$
\begin{gathered}
\tau_{\mathrm{NCV}}=\Sigma_{\mathrm{NCV}} v_{\text {thermal }}=12.2 \mu \mathrm{s} \\
\tau_{\mathrm{II}}=\Sigma_{\mathrm{II}} v_{\text {thermal }}=24.3 \mu \mathrm{s}
\end{gathered}
$$

where $v_{\text {thermal }}=2.2 \mathrm{~km} / \mathrm{s}$ is the most probable speed for thermal neutrons at room temperature. Using the definitions above, one may write

$$
f=\frac{\phi_{n}^{\mathrm{II}}}{\phi_{n}}=\frac{\int_{\mathrm{ROI}} \psi_{n}^{\mathrm{II}}(t) d t}{\int_{\mathrm{ROI}} \psi_{n}(t) d t}=\frac{\int_{0}^{60 \mu \mathrm{s}} \int_{0}^{t} T(s) e^{(s-t) / \tau_{\mathrm{II}}} d s d t}{\int_{0}^{60 \mu \mathrm{s}} \int_{0}^{t} T(s) e^{(s-t) / \tau_{\mathrm{NCV}}} d s d t} .
$$

The arrival time distribution for the thermal neutrons $T(t)$ is unknown, but it may be reasonably assumed to lie between two extreme cases: (1) a delta function at time $t=0$, which corresponds to all of the thermal neutrons arriving at the beginning of the Phase I time region of interest, and (2) a delta function at the end of the ROI, time $t=60 \mu \mathrm{s}$. Evaluating eq. (8.13) for the first case gives

$$
f=\frac{\int_{0}^{60 \mu \mathrm{s}} \int_{0}^{t} A \delta(s) e^{(s-t) / \tau_{\mathrm{II}}} d s d t}{\int_{0}^{60 \mu \mathrm{s}} \int_{0}^{t} A \delta(s) e^{(s-t) / \tau_{\mathrm{NCV}}} d s d t}=\frac{\int_{0}^{60 \mu \mathrm{s}} e^{-t / \tau_{\mathrm{II}}} d t}{\int_{0}^{60 \mu \mathrm{s}} e^{-t / \tau_{\mathrm{NCV}}} d t}=1.83 .
$$

The integrals in the second case are trivial, and one obtains the exact result $f=1$ regardless of the numerical values of $\tau_{t} \operatorname{ext} I I$ and $\tau_{\mathrm{NCV}}$. For comparison, assuming $T(t)=$ $A$ for some constant $A$ (which implies a uniform distribution of thermal neutron arrivial times) yields the result

$$
f=\frac{\int_{0}^{60 \mu \mathrm{s}} \int_{0}^{t} A e^{(s-t) / \tau_{\mathrm{II}}} d s d t}{\int_{0}^{60 \mu \mathrm{s}} \int_{0}^{t} A e^{(s-t) / \tau_{\mathrm{NCV}}} d s d t}=\frac{\int_{0}^{60 \mu \mathrm{s}} \tau_{\mathrm{II}}\left(1-e^{-t / \tau_{\mathrm{II}}}\right) d t}{\int_{0}^{60 \mu \mathrm{s}} \tau_{\mathrm{NCV}}\left(1-e^{-t / \tau_{\mathrm{II}}}\right) d t}=1.59 .
$$


As noted above, one may obtain the neutron capture rate for Phase II by multiplying $\mathcal{R}_{n}^{\mathrm{NCV}}$ by suitable correction factors:

$$
\mathcal{R}_{n}^{\mathrm{II}}=\mathcal{R}_{n}^{\mathrm{NCV}} \frac{\Sigma_{\mathrm{II}}}{\Sigma_{\mathrm{NCV}}} f=\phi_{n} \Sigma_{\mathrm{NCV}} \frac{\Sigma_{\mathrm{II}} \phi_{n}^{\mathrm{II}}}{\Sigma_{\mathrm{NCV}} \phi_{n}}=\phi_{n}^{\mathrm{II}} \Sigma_{\mathrm{II}} .
$$

Since $\Sigma_{\mathrm{II}} / \Sigma_{\mathrm{NCV}}=0.50$ and, based on the extreme cases above, $f \in[1,1.83]$, it follows from eq. (8.16) that $\mathcal{R}_{n}^{\mathrm{II}}<\mathcal{R}_{n}^{\mathrm{NCV}}$ regardless of the functional form of the arrival time distribution $T(t)$ of thermal neutrons reaching the NCV liquid.

The measured neutron capture rate in the $\mathrm{NCV} \mathcal{R}_{n}^{\mathrm{NCV}}$ therefore represents a robust upper limit on the expected neutron capture rate at the corresponding location in the Phase II water tank. Chapter 9 will present an analysis of the Phase I beam data leading to values for $N_{n}$, the observed number of candidate neutron capture events, at each of the seven NCV positions studied in ANNIE Phase I. In chapter 10, the NCV detection efficiency $\epsilon_{\mathrm{NCV}}$ will be studied using two different methods. Finally, in chapter 11, these results will be combined using eq. (8.8) to obtain $\mathcal{R}_{n}^{\mathrm{NCV}}$, an upper limit for the expected background neutron event rate in ANNIE Phase II, at each of the NCV positions where beam data were recorded. 


\section{Chapter 9}

\section{Neutron candidate event selection}

As discussed in the previous chapter, one of the key ingredients for a determination of the position-dependent neutron backgrounds in the Phase I ANNIE detector is the rate of observed neutron captures in the NCV. In this chapter, a procedure is described for identifying candidate neutron capture events while removing backgrounds due to cosmic rays, beam-induced muons, etc. Measurements of the neutron capture rates in the NCV at different positions in the Phase I tank are then presented. In the following chapter, a determination of the NCV efficiency will be made that will allow the raw rates discussed here to be converted into expected background neutron event rates in the Phase II detector.

\subsection{PMT waveform processing}

The primary raw data of interest for the Phase I measurement consist of digitized wave-

forms from the two photomultiplier tubes (PMTs) fixed to the top of the NCV and the 60 PMTs placed at the bottom of the water tank. During early Phase I operations, four of the water tank PMTs were found to be damaged and were disconnected from the DAQ, leaving 56 active water tank PMTs. As described in section 8.5, the DAQ readout scheme was upgraded during Phase I data taking. Early beam runs used a normal mode in which the PMT signals were continuously digitized during an $80 \mu$ s time window. Later runs used a zero suppression mode in which $2 \mu$ s minibuffers of interest were digitized and read out from the detector in a buffer consisting of 40 minibuffers. For both readout schemes, 
all of the PMT waveforms were sampled by an analog-to-digital converter (ADC) at a rate of $500 \mathrm{MHz}$. The digitized signals were saved to disk and analyzed offline using the ROOT data analysis framework [449] and an ANNIE-specific software package called ToolAnalysis. ${ }^{1}$

\subsubsection{Waveform calibration}

The first step needed to reconstruct the flashes of light detected by the photomultiplier tubes is to convert the ADC counts recorded in the raw data files into voltages. This may be done for the ANNIE ADC cards using the relation

$$
V_{\mathrm{PMT}}=\frac{2.415 \mathrm{~V}}{2^{12} \mathrm{ADC} \text { counts }}\left(x_{\mathrm{ADC}}-x_{0}\right),
$$

where $x_{\mathrm{ADC}}$ is the recorded $\mathrm{ADC}$ value and $V_{\mathrm{PMT}}$ is the corresponding voltage. The parameter $x_{0}$, which represents the baseline $\mathrm{ADC}$ value (i.e., the $\mathrm{ADC}$ reading when no charge is being deposited on the PMT anode), must be estimated using the raw data. Because the value of $x_{0}$ may fluctuate over time due to electronic noise, pickup of external interference, etc., this must be done for each PMT in each DAQ readout buffer individually.

To estimate the ADC baseline $x_{0}$ of a PMT trace, ToolAnalysis uses an algorithm created for ZE3RA (ZEPLIN 3 Reduction Analysis) [453], a data analysis computer code developed for the ZEPLIN-III dark matter experiment. When analyzing normal mode data, the ANNIE implementaton of the algorithm splits the pre-trigger portion of the PMT trace into $M$ regions, each containing $N$ samples. The sample mean $\mu_{j}$ and variance $\sigma_{j}^{2}$ for the $\mathrm{ADC}$ values recorded in the $j$ th region are given by the formulas

$$
\begin{aligned}
\mu_{j} & \equiv \frac{1}{N} \sum_{k=1}^{N} x_{j, k} \\
\sigma_{j}^{2} & \equiv \frac{\sum_{k=1}^{N}\left(x_{j, k}-\mu_{j}\right)^{2}}{N-1}
\end{aligned}
$$

where $x_{j, k}$ is the $k$ th ADC value in the $j$ th region of the PMT trace. To enable efficient calculation of these statistics in a single pass over the raw data, a technique due to Welford

\footnotetext{
${ }^{1}$ ToolAnalysis is based on the ToolDAQ framework (http://github.com/ToolDAQ/ ToolDAQFramework) developed by Benjamin Richards.
} 
[454] is used, in which the quantities

$$
\begin{array}{lr}
\mu_{j, k}=\mu_{j, k-1}+\frac{x_{j, k}-\mu_{j, k-1}}{k} & \text { with } \mu_{j, 0}=0 \\
A_{j, k}=A_{j, k-1}+\left(x_{j, k}-\mu_{j, k-1}\right)\left(x_{j, k}-\mu_{j, k}\right) & \text { with } A_{j, 0}=0
\end{array}
$$

are computed iteratively for the data points in the $j$ th region, yielding the desired values

$$
\begin{gathered}
\mu_{j}=\mu_{j, N} \\
\sigma_{j}^{2}=\frac{A_{j, N}}{N-1}
\end{gathered}
$$

when $k=N$.

After the sample statistics for all of the $M$ regions are calculated, the sample variances of neighboring regions are compared with each other for statistical consistency. This check is intended to exclude electrical transients and true PMT pulses (which would bias the baseline estimation) that occur during the pre-trigger time window. Under the assumption that the $\mathrm{ADC}$ values from the $j$ th and $(j+1)$ th regions are independent simple random samples drawn from normal populations with the same variance, the ratio $R$ of their sample variances

$$
R=\frac{\sigma_{j}^{2}}{\sigma_{j+1}^{2}}
$$

follows an $\mathrm{F}$ distribution on the domain $[0, \infty)$ with $n=N-1$ degrees of freedom for the numerator in eq. (9.8) and another $m=N-1$ degrees of freedom for the denominator [455]. This distribution has the cumulative distribution function [456]

$$
F_{n, m}(R)=I\left(\frac{n R}{m+n R} ; \frac{n}{2}, \frac{m}{2}\right)
$$

where $I(z ; a, b)$ is the regularized beta function

$$
I(z ; a, b)=\frac{\Gamma(a+b)}{\Gamma(a) \Gamma(b)} \int_{0}^{z} t^{a-1}(1-t)^{b-1} d t
$$

and the gamma function $\Gamma(n)$ for a complex number $z$ with a positive real part is given by

$$
\Gamma(z)=\int_{0}^{\infty} x^{z-1} e^{-x} d x
$$


A useful property of the regularized beta function is that [457]

$$
I(z ; a, b)=1-I(1-z ; b, a)
$$

For the $M-1$ pairs of neighboring regions, therefore, the probability $Q_{j}$ that the test statistic

$$
R_{j} \equiv \begin{cases}\sigma_{j}^{2} / \sigma_{j+1}^{2} & \sigma_{j}>\sigma_{j+1} \\ \sigma_{j+1}^{2} / \sigma_{j}^{2} & \sigma_{j} \leq \sigma_{j+1}\end{cases}
$$

would be at least as large as actually observed (accepting the null hypothesis that ADC values in the two regions were drawn from normal distributions with equal variances) is given by

$$
\begin{aligned}
Q_{j} & =1-F_{N-1, N-1}\left(R_{j}\right)=1-I\left(\frac{[N-1] R_{j}}{[N-1]+[N-1] R_{j}} ; \frac{N-1}{2}, \frac{N-1}{2}\right) \\
& =1-I\left(\frac{R_{j}}{1+R_{j}} ; \nu, \nu\right)=I\left(\frac{1}{1+R_{j}} ; \nu, \nu\right)=\frac{\Gamma(2 \nu)}{2 \Gamma(\nu)} \int_{0}^{\left(1+R_{j}\right)^{-1}}\left(t-t^{2}\right)^{\nu-1} d t
\end{aligned}
$$

where $\nu \equiv(N-1) / 2$. Equation (9.12) was used in the penultimate step above.

In order to adequately test the equality of variance hypothesis, unusually small values of $R_{j}$ should be rejected as well as unusually large ones. Conveniently, eqs. (9.9) and (9.12) imply that such a two-tailed hypothesis test may be performed by simply doubling $Q_{j}$ [457]. The quantity $P_{j} \equiv 2 Q_{j}$ represents, assuming the truth of the null hypothesis, the probability of observing a deviation from equality of the sample variances at least as extreme as that actually obtained.

Thus, $P_{j}$ is a p-value that may be used to test the hypothesis that the population variances for the $j$ th and the $(j+1)$ th regions are equal. This hypothesis is rejected ${ }^{2}$ for a given critical value $P_{\text {crit }}$ whenever $P_{j} \leq P_{\text {crit }}$. The $L$ regions for which the F-test

\footnotetext{
${ }^{2}$ Confusingly, the sole publication [453] describing in detail the algorithms implemented in the ZE3RA code claims that only regions for which (in the notation used in the present discussion) $Q_{j}<Q_{\text {crit }}$ (where $Q_{\text {crit }} \equiv 10^{-4}$ ) should be used to compute the estimates for $x_{0}$ and $\sigma_{x_{0}}$. This appears to indicate that only regions whose variances differ quite noticeably from their neighbors are accepted by ZE3RA as suitable for use in estimating the baseline. Because this runs counter to the statement in reference [453] that the F-test is used "to check if the variances are statistically consistent," however, it appears that there is a misprint in the original description of the algorithm. Unfortunately, the ZE3RA code has not been made publicly available so that its true behavior may be verified.
} 
described above fails to reject the null hypothesis are then used to estimate the baseline for the PMT trace of interest via the formulas

$$
x_{0}=\frac{1}{L} \sum_{\ell=1}^{L} \mu_{\ell} \quad \sigma_{x_{0}}=\left(\frac{N-1}{N L-1} \sum_{\ell=1}^{L} \sigma_{\ell}^{2}\right)^{1 / 2}
$$

where $\sigma_{x_{0}}$ is the estimated error on $x_{0}$ and the sums are over all regions for which $P_{j}>$ $P_{\text {crit }}$. The expressions given in eq. (9.16) are equivalent to computing the mean $\left(x_{0}\right)$ and standard deviation $\left(\sigma_{x_{0}}\right)$ of the combined sample of all ADC values in the $L$ regions for which $P_{j}>P_{\text {crit }}$.

For the analysis of the ANNIE Phase I data, $M=40$ regions of $N=25$ samples were used, with a significance level of $P_{\text {crit }}=0.01$ required to reject the null hypothesis. When zero-suppressed data were analyzed, instead of using the first $M$ sets of $N$ samples to estimate the baseline, each minibuffer contributed one of the $M=40$ regions, with the first $N=25$ samples of the minibuffer being used to compute the sample statistics. The estimated baseline for zero suppression mode, which was otherwise computed using the same technique as for normal mode, was assumed to remain constant over the full PMT trace.

Using the estimated baseline ADC value $x_{0}$ defined in eq. (9.16), ToolAnalysis produces calibrated versions of the PMT traces, stored in memory alongside the raw traces, by applying the conversion formula eq. (9.1) to each of the raw samples.

\subsubsection{PMT pulse finding}

When a photon strikes the photocathode of a PMT and successfully initiates an electron cascade, the current generated at the anode will appear in the PMT waveform as a pulse whose area is proportional to the total deposited charge. Characterizing the pulses that appear in a PMT trace therefore provides information about the arrival time and intensity of the flashes of light detected by the PMT.

ToolAnalysis finds pulses in PMT traces using a simple procedure. Starting with the first sample in a trace, ADC values are checked one by one until a given threshold is exceeded. The sample for which this first occurs is defined as the start of the pulse. The following samples continue to be checked until one of the following conditions occurs: 
(1) an ADC value is found that falls below the estimated baseline plus its 1-sigma error (i.e., below $x_{0}+\sigma_{x_{0}}$ in the notation of the previous section), (2) the end of the PMT trace is reached, or (3) for zero-suppressed data, the end of the current minibuffer is reached. The first sample which fulfills any of these criteria is considered the end of the pulse.

To ensure that normal and zero-suppressed data (the latter of which used a hardware threshold of 357 ADC counts for both NCV PMT channels) are treated on an equal footing, pulses are found using the raw PMT traces (which continue to use ADC units) rather than the calibrated ones. This ensures that, as long as the software pulse finding threshold is set at or above 357 ADC counts for the two NCV PMTs, the same set of pulses would be reconstructed for an event regardless of which triggering mode was used to record it.

For the ANNIE Phase I analysis shown in this thesis, a constant threshold of 357 ADC counts was used when finding pulses on traces from both NCV PMTs. For all other PMT channels, a threshold of $x_{0}+7$ ADC counts (rounded to the nearest integer) was used, where $x_{0}$ was the estimated baseline for the channel.

After finding the starting and ending samples for a pulse in a given PMT trace, ToolAnalysis characterizes the pulse using the following parameters:

start time The time since the beginning of the trace (or, in zero suppression mode, the beginning of the current minibuffer) at which the pulse began. This is defined as the starting sample index multiplied by the sampling period (2 ns for $500 \mathrm{MHz}$ sampling).

peak time The time at which the maximum ADC value occurred within the pulse. If the maximum $\mathrm{ADC}$ value was reached more than once during the pulse, then the earliest sample for which this occurred is used to calculated the peak time.

end time The time corresponding to the first sample after the start of the pulse at which either the ADC signal fell below $x_{0}+\sigma_{x_{0}}$, the PMT trace ended, or, in the case of zero-suppressed data, the current minibuffer ended.

raw amplitude The maximum ADC value recorded during the pulse 
calibrated amplitude The maximum voltage recorded during the pulse. This is calculated using eq. (9.1) with $x_{\mathrm{ADC}}$ set equal to the raw amplitude of the pulse.

charge The time integral of the calibrated version of the pulse, divided by the ADC input impedance, which is taken to be $50 \Omega$.

\subsection{Determination of beam conditions}

Before performing any further analysis on the PMT pulses, the ANNIE reconstruction software associates information from the instruments monitoring production of the Booster Neutrino Beam (see section 8.1) with the PMT traces. This information is retrieved from the Beam Conditions Database for Intensity Frontier Experiments (IFBeam DB) [458], a web-accessible collection of monitoring data for all of the neutrino beamlines at Fermilab.

The data server for the IFBeam $\mathrm{DB}^{3}$ retrieves beam monitoring data for a single device or for a "bundle" of related devices in response to HTTP requests. Data from the monitoring devices of primary interest for users of the ANNIE detector are included in the BNBBPMTOR ("Booster Neutrino Beam: Beam Position Monitors and Toroids") bundle. Data in the BNBBPMTOR bundle may be retrieved from the IFBeam DB in $\mathrm{CSV}^{4}$ format by accessing the $\mathrm{URL}^{5}$

http://ifb-data.fnal.gov:8100/ifbeam/data/data?e=e\%2C1d\&b=BNBBPMTOR

$\& \mathrm{f}=\mathrm{csv} \& \mathrm{t} z=\&$ action $=$ Show + devi ce\&t $0=\mathrm{T} 0 \& \mathrm{t} 1=\mathrm{T} 1$

where T0 and T1 should be replaced with the initial and final times of interest, respectively, expressed in terms of the number of seconds since the Unix epoch. ${ }^{6}$ Queries with values of $\mathrm{T} 0$ and $\mathrm{T} 1$ that differ by more than one day are likely to be rejected by the server.

\footnotetext{
${ }^{3}$ http://ifb-data.fnal.gov:8100/ifbeam/data/index

${ }^{4}$ Comma-separated values

${ }^{5}$ Uniform Resource Locator

${ }^{6}$ That is, the number of seconds since 00:00:00 Coordinated Universal Time (UTC), Thursday, 1 January 1970. Timestamps of this kind are easily calculated using command-line programs such as GNU date (included by default with most Linux distributions) or web applications like https://www . epochconverter.com/.
} 
To avoid overwhelming the IFBeam DB data server with frequent requests for beam status information during the offline ANNIE data analysis, the entire contents of the database covering the time period of interest for ANNIE Phase I data taking were downloaded and stored in a compressed format. Copies of the compressed file were then used to obtain beam status information during Phase I data processing.

To ensure that the beam was operating normally for all spills included in the background neutron rate analysis, three beam quality checks were applied to each set of PMT traces recorded in response to a beam trigger. The first check required the number of protons on target (POT) measured by Toroid 875 (referred to as E:TOR875 in the IFBeam $\mathrm{DB}$ ) to be between $0.5 \times 10^{12}$ and $6 \times 10^{12} \mathrm{POT}$. Typical intensity values for the Booster Neutrino Beam observed during ANNIE Phase I running were between $4 \times 10^{12}$ POT to $5 \times 10^{12}$ POT. The second check, adopted from earlier studies using the SciBooNE de-

tector [459], required the POT measurements recorded by the two beam current toroids (E:TOR875 and E:TOR860) to agree within 10\%, i.e.,

$$
2 \times \frac{\mid \text { TOR } 875-\text { TOR } 860 \mid}{\text { TOR } 875+\text { TOR } 860}<10 \%
$$

where TOR875 and TOR860 are the POT measurements for the beam spill of interest made using the two toroids. The third check, intended to ensure that the horn was focusing the beam properly, required that the peak horn current for each beam spill was between $172 \mathrm{kA}$ and $176 \mathrm{kA}$, with $174 \mathrm{kA}$ being the nominal value.

Data from beam spills that failed any one of these beam quality checks were flagged and excluded from the rest of the analysis. When the minimum POT requirement was fulfilled, only a very small fraction of spills failed any of the remaining beam quality checks.

\subsection{Event selection}

After searching for pulses on all of the PMT traces contained in a full DAQ readout, the ToolAnalysis code then searches for neutron capture candidate events. Four criteria were adopted for identifying neutron candidate events during the ANNIE Phase I analysis: 
NCV coincidence requirement Both NCV PMTs must fire within 40 ns of each other. The event time is defined as the start time for the earlier of the two coincident pulses.

Afterpulsing cut A new event must occur at least $10 \mu$ s after the preceding event.

Total charge cut The sum of the charges of the two coincident NCV PMT pulses must be less than $150 \mathrm{pC}$.

Outer PMT veto cut Less than 8 tank PMTs must fire within 40 ns of the event time.

The rationales for each of these criteria are discussed below using beam data from NCV position \#2 (the center of the tank) for illustration.

\subsubsection{NCV coincidence requirement}

The black curve in fig. 9.1 shows the time distribution (relative to the start of the beam crossing the ANNIE detector) for all of the reconstructed pulses found on NCV PMT \#1 during beam data taking in zero suppression mode at NCV position \#2 (the center of the tank). Two features of the distribution are immediately apparent. First, there is a dominant flat component composed primarily of dark pulses. Second, in addition to the beam crossing peak beginning at time $t=0 \mu \mathrm{s}$, a second peak attributable to afterpulsing appears roughly $5 \mu$ s later.

To minimize the number of spurious events caused by dark noise and afterpulsing, neutron capture candidate events were selected by looking for coincident pulses on both NCV PMTs. An NCV coincidence of this kind was defined as a pair of pulses, one from the trace of NCV PMT \#1 and the second from the trace of NCV PMT \#2, whose start times (calculated as described in section 9.1.2) differed by less than 40 ns. The start time

for the earlier of the two coincident pulses is considered the time of the NCV coincidence event. The blue curve in fig. 9.1 shows the time distribution of NCV coincidences for the same dataset as the black curve. While the beam crossing peak at $t=0 \mu \mathrm{s}$ is still present, the second peak at $5 \mu$ s has disappeared, suggesting that it was largely composed of afterpulses on NCV PMT \#1. 


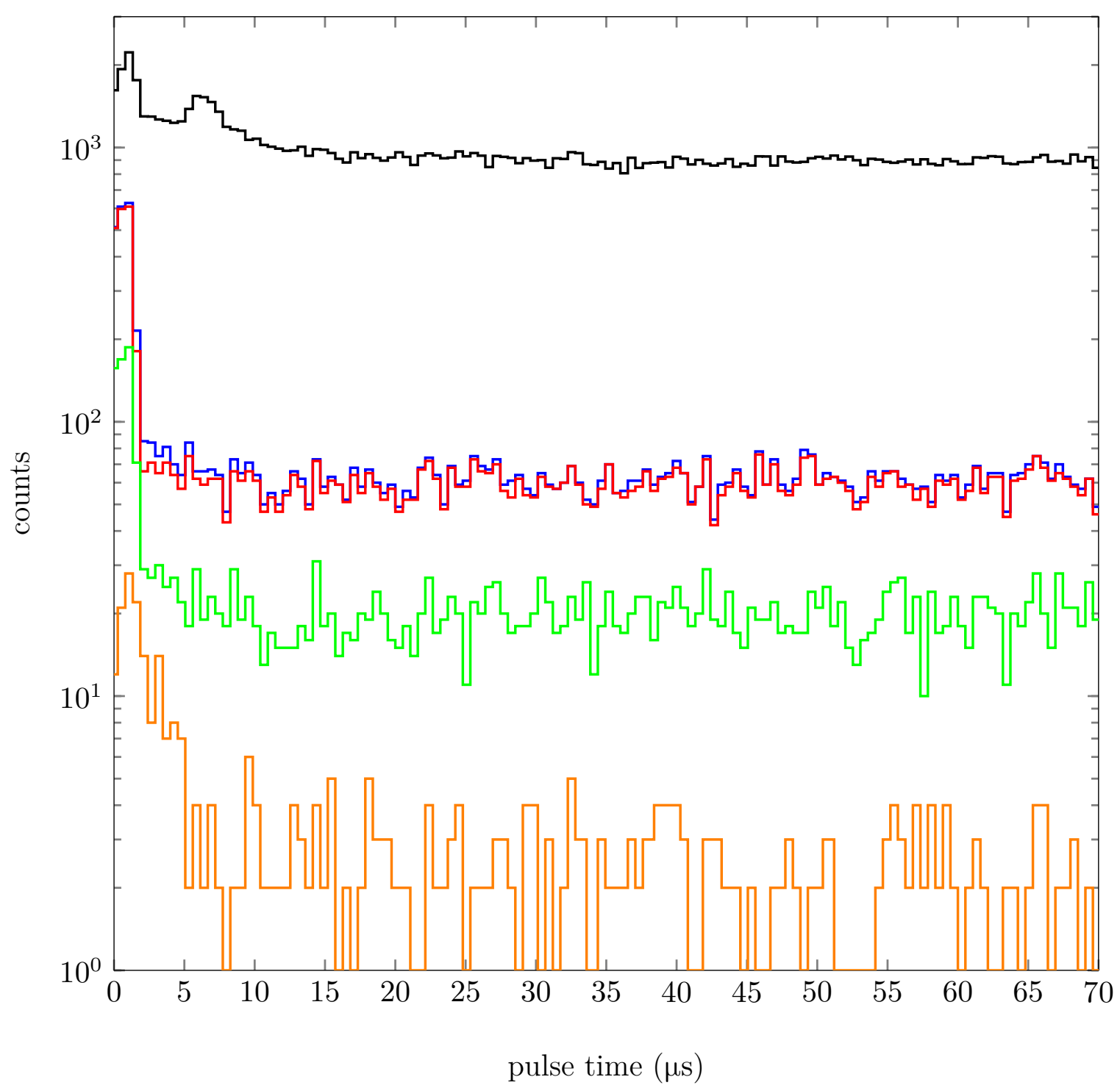

Figure 9.1: Successive applications of each of the neutron candidate event criteria for zero-suppressed beam data taken at position \#2 (center of the tank). BLACK: Time distribution of all pulses recorded in zero suppression mode on NCV PMT \#1 at position \#2. No analysis cuts have been applied to these data. Starting from the left hand side of the plot, the first peak coincides with the neutrino beam crossing the

ANNIE detector, while the second is attributable to afterpulsing. BLUE: Time distribution of all NCV coincidences from the same dataset. RED: Events from the blue histogram that passed the afterpulsing cut. GREEN: Events from the red histogram that passed the total charge cut. ORANGE: Events from the green histogram that passed the outer PMT veto cut. These are considered neutron candidates in the background event rate analysis. 


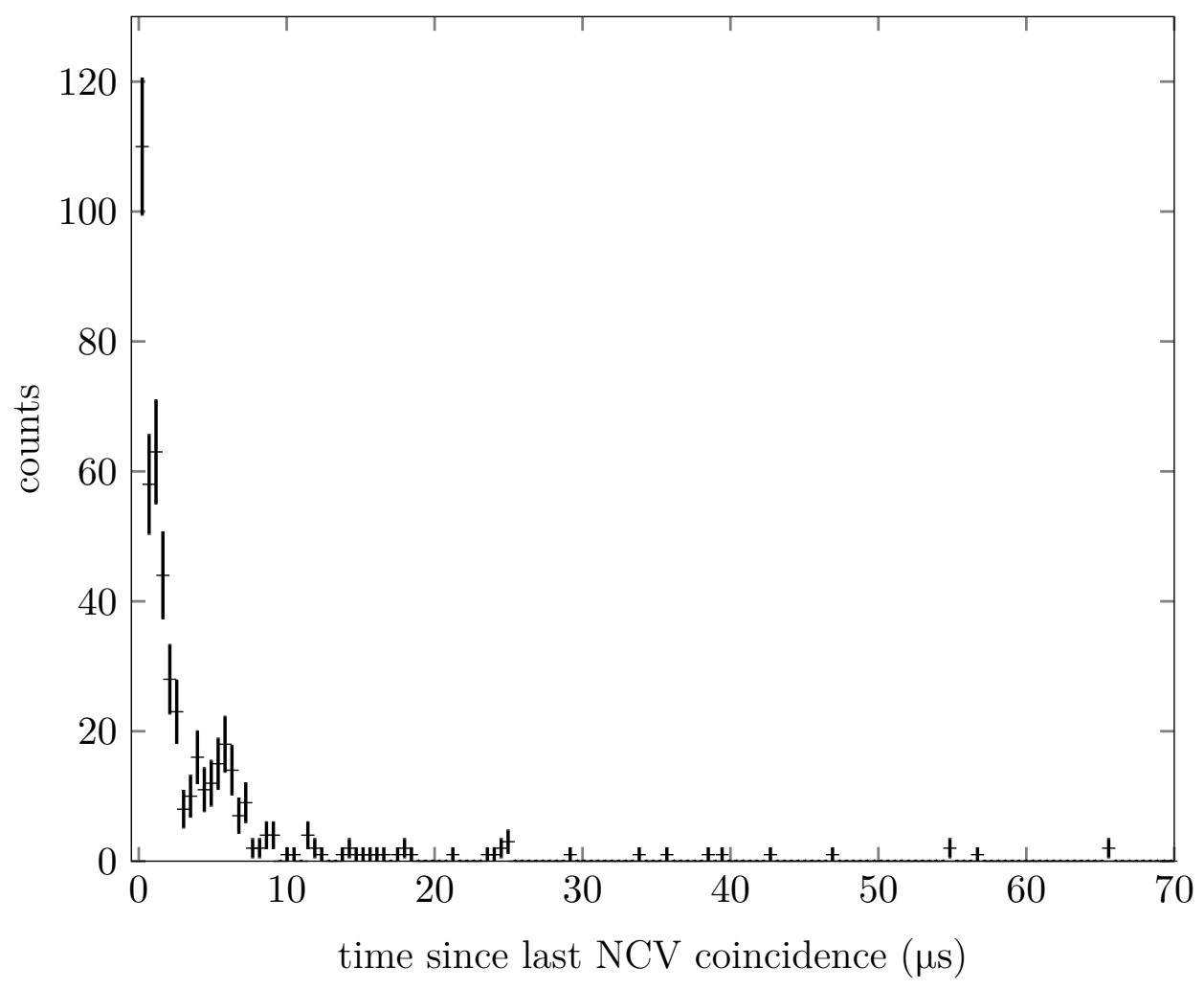

Figure 9.2: Distribution of the elapsed time since the previous event for NCV coincidences from the blue histogram in fig. 9.1. The plot includes only the small minority (about 4\%) of the NCV coincidence events that occurred within the first $100 \mu \mathrm{s}$. The remaining events, not shown in the plot, occurred far later (at least $10 \mathrm{~ms}$ since the previous event).

\subsubsection{Afterpulsing cut}

Although requiring the two NCV PMTs to fire in coincidence during a neutron candidate event was sufficient to suppress the obvious afterpulsing peak seen in the black histogram from fig. 9.1, this requirement will not fully eliminate false events due to afterpulses. Evidence for their continuing contribution to the event rate can be seen in fig. 9.2, which shows the distribution of elapsed times since the preceding event for the small fraction of NCV coincidences in the blue histogram from fig. 9.1 that occurred within the first $100 \mu \mathrm{s}$. The distribution is dominated by the $92 \%$ of these NCV coincidences that happened less than $10 \mu \mathrm{s}$ after the previous event, as one would expect if afterpulsing remains a significant problem. 
To ensure that spurious events due to afterpulsing were eliminated as much as possible from the measured NCV event rates, only NCV coincidences that occurred at least $10 \mu \mathrm{s}$ after the previous event were accepted as neutron candidates. The red histogram in fig. 9.1 shows the small effect of applying this cut to the position \#2 beam data.

\subsubsection{Total charge cut}

While the event criteria discussed so far are useful for removing NCV coincidences attributable to dark counts and afterpulsing, they do little to suppress backgrounds caused by cosmic and beam-induced muons crossing the NCV. Such muons will deposit relatively large amounts of energy in the scintillator, leading to intense light production and often to large pulses on both NCV PMTs. To veto events with energy deposits in the scintillator far higher than the maximum of nearly $9 \mathrm{MeV}$ that would be expected from a fully-contained neutron capture $\gamma$-ray cascade (see fig. 10.11), a cut on the total charge collected by the two NCV PMTs was imposed. Based on the NCV charge-to-energy calibration described in section 10.2.1, a conservative maximum total charge $Q_{\max }=150 \mathrm{pC}$ was adopted, which corresponds to (see eq. (10.24) and table 10.2) a deposited energy of about $34 \mathrm{MeV}$. The green histogram in fig. 9.1 shows the events that remain after applying the cut $Q_{1}+Q_{2}<Q_{\max }$ (where $Q_{1}$ and $Q_{2}$ are the charges collected by NCV PMTs \#1 and \#2, respectively) to the events in the red histogram.

\subsubsection{Outer PMT veto cut}

Beyond the easily recognized muon candidate events that deposit large amounts of energy in the scintillator, there will also be significant numbers of "corner clippers," i.e., muons that exit the NCV after traveling only a short distance through the scintillator. Because these muons may deposit an energy low enough to be mistaken for a neutron capture, the NCV total charge cut will not be an effective means of excluding them. However, although some muons will stop in the NCV, and some will enter the tank with energies below the Cherenkov threshold (about $55 \mathrm{MeV}$ kinetic energy), many will produce enough light within the water volume to be detected by the PMTs at the bottom of the tank. Because neutron captures occurring in the optically isolated NCV are expected to produce 


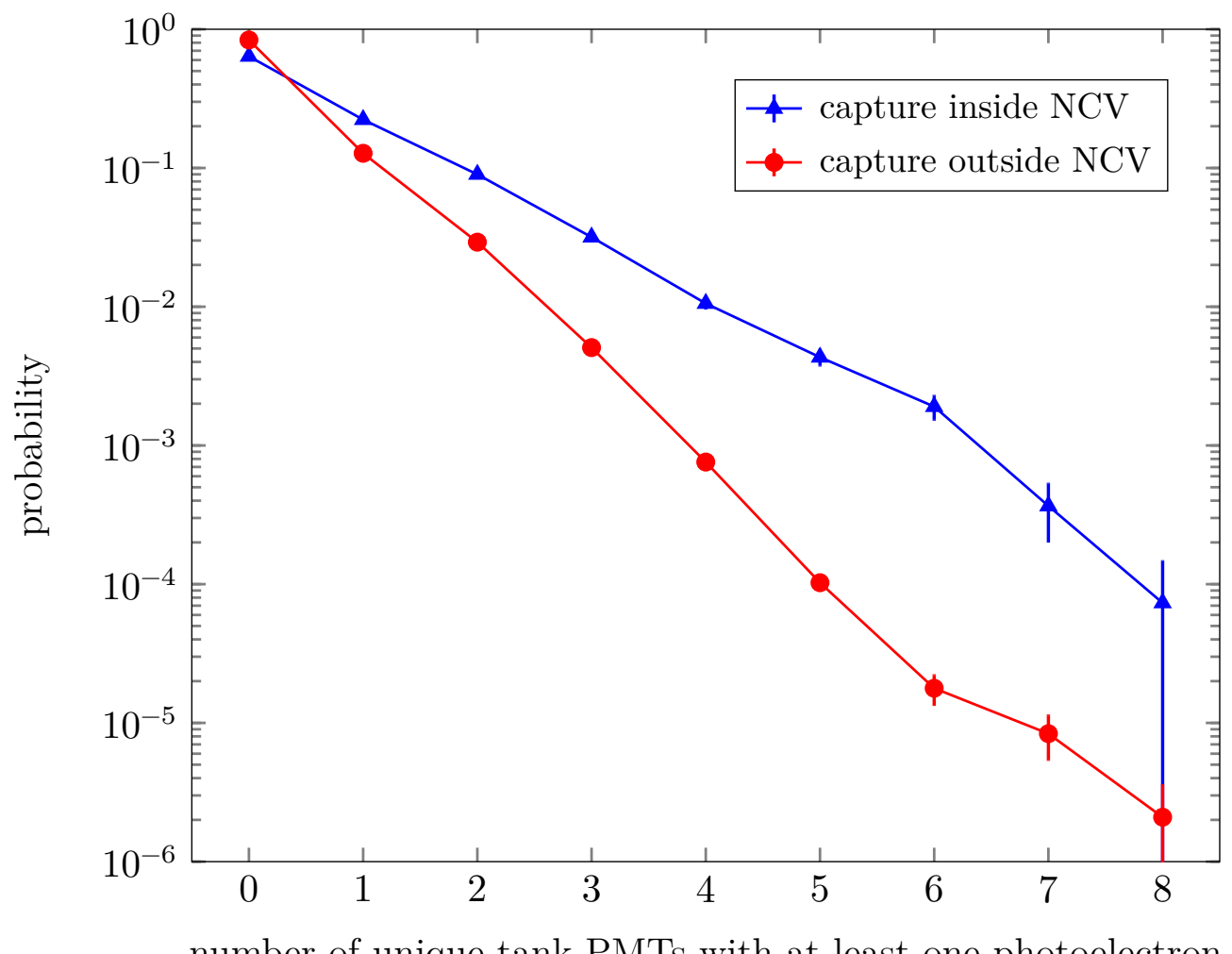

Figure 9.3: Results of an NCV containment simulation study performed using RAT-PAC. Thermal neutron tracks were started one at a time from locations sampled uniformly within a $50 \mathrm{~cm}$ radius sphere centered on the NCV, which was placed at position \#2 (center of the tank). BLUE: Monte Carlo estimate of the probability distribution for the number of unique tank PMTs that will collect at least one photoelectron as a result of a neutron capture occurring within the NCV liquid. RED: The same distribution for neutrons that capture outside the NCV liquid. Only photoelectrons whose origin can be traced back to the neutron capture are considered in the simulation.

pulses on few, if any, of the water tank PMTs (see fig. 9.3), the tank PMTs may therefore serve as a muon veto for the NCV.

The blue data points in fig. 9.4 show a histogram of the number of water tank PMTs that recorded a pulse within $40 \mathrm{~ns}$ of an NCV coincidence event. The bimodal shape of the histogram, with clear peaks at zero and fifty-five coincident tank PMTs, agrees well with the expected topology of two kinds of NCV events: neutron captures within the NCV, which will typically result in only a modest amount of activity on the tank PMTs; and muons crossing the NCV, which are likely to generate pulses on many of the tank 


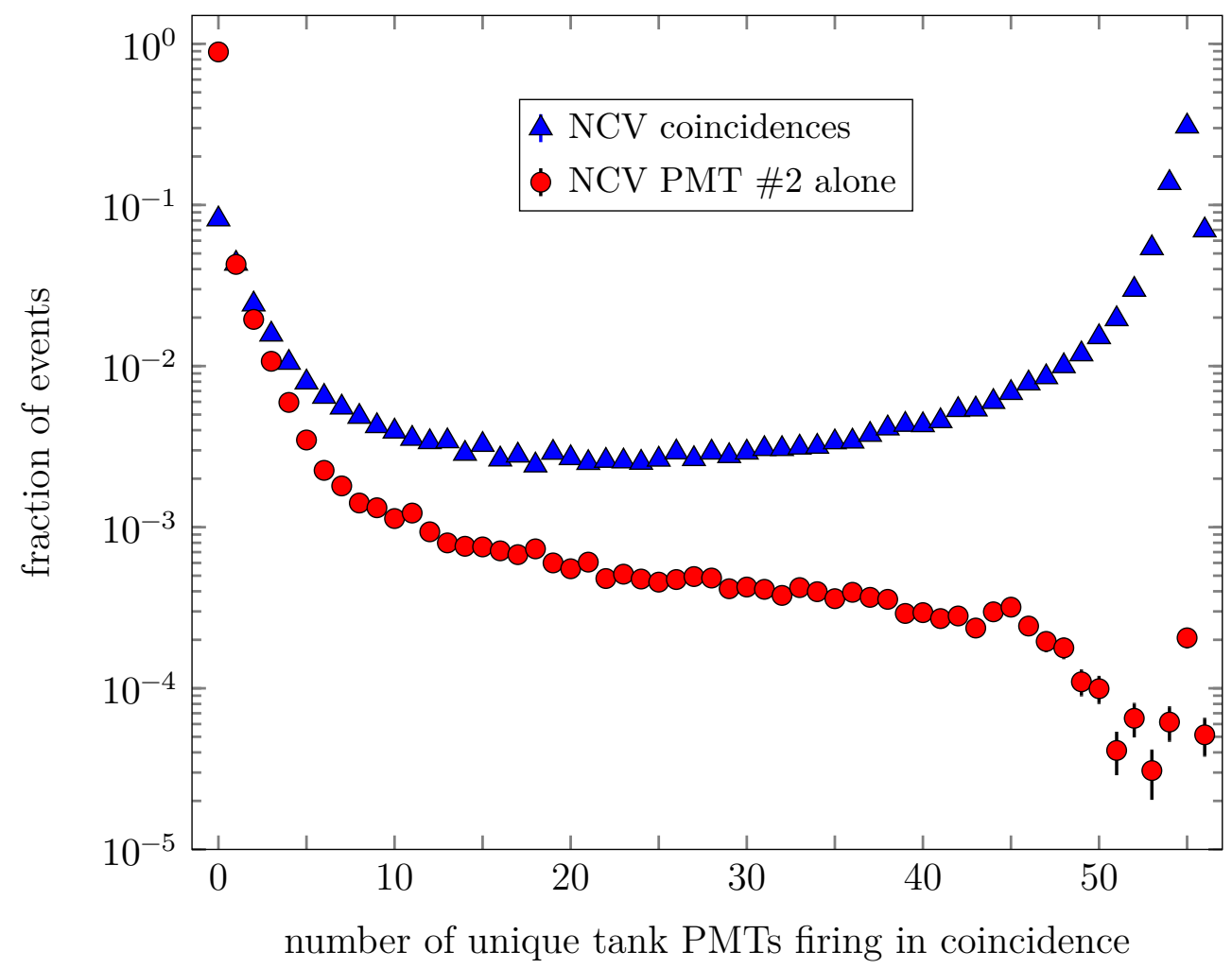

Figure 9.4: Distributions of the number of unique water tank PMTs that recorded at least one pulse within 40 ns of activity in the NCV. For the blue dataset, "activity" is defined as an NCV coincidence, while for the red dataset it is defined as a pulse on NCV

PMT \#2 without a coincident pulse on NCV PMT \#1. Both datasets were created using all runs analyzed for this thesis. No analysis cuts have been applied.

PMTs in addition to the two NCV PMTs.

To veto the contribution of "corner clipper" cosmic muons to the observed NCV event rate, only NCV coincidences for which less than eight tank PMTs fired within 40 ns of the start of the event were accepted as neutron candidates. The orange histogram in fig. 9.1 shows the result of applying this cut to the events in the green histogram.

Confirmation that the total charge cut and the outer PMT veto cut are indeed effective for removing muons can be obtained from data taken using the cosmic ray trigger described in section 8.4.5. In fig. 9.5, the distribution of the total charge collected on both NCV PMTs is shown for NCV coincidence events recorded within the $2 \mu$ s following a trigger consistent with a cosmic muon traveling downward through the NCV. While many events pass the afterpulsing cut (as shown by the blue dataset), applying the total charge and 


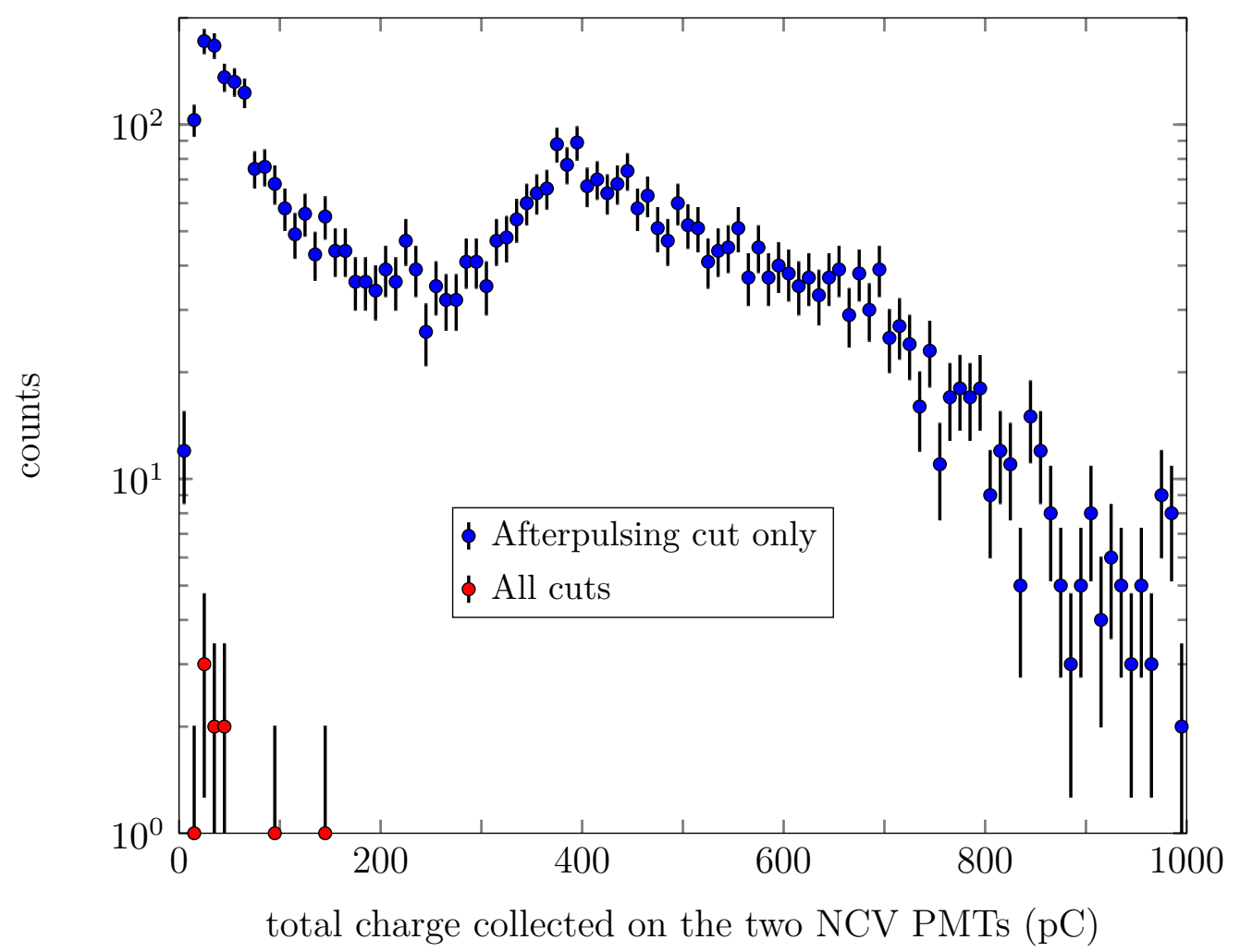

Figure 9.5: BLUE: Distribution of the total charge collected on both NCV PMTs for NCV coincidence events that passed the afterpulsing cut. All events shown here were recorded within $2 \mu$ s of a cosmic trigger consistent with a downward muon passing through the NCV. RED: The corresponding charge distribution when all neutron candidate event cuts have been applied.

outer PMT veto cuts yields the red dataset, in which only a handful of events remain.

\subsection{Cut corrections}

Before presenting the measured neutron capture rates obtained using the event criteria discussed above, we must consider and correct for possible systematic errors that could be introduced by each of the analysis cuts. Because the goal of the ANNIE Phase I measurement is to establish whether or not the background neutron event rates are sufficiently low for Phase II to proceed, we will be primarily interested in systematic effects that could result in an underestimate of the true rates. In the sections below, the possible introduction of such a negative bias by each of the three analysis cuts is considered. 


\subsubsection{Afterpulsing cut}

The afterpulsing cut introduces $10 \mu$ s of artificial dead time after each NCV coincidence event. Under the assumption that the events occur independently of one another, the probability $P(k)$ that $k$ events will occur in the region of interest during a single beam spill is given by the Poisson distribution

$$
P(k)=\frac{\lambda^{k} e^{-\lambda}}{k !}
$$

where $\lambda$ is the average number of events in the region of interest during a single beam spill. The probability $P(k \geq 1)$ of observing at least one event is given by

$$
P(k \geq 1)=P(k>0)=1-P(0)=1-e^{-\lambda} .
$$

If it is known that at least one event was observed, then the conditional probability of observing at least two events is given by

$$
P(k \geq 2 \mid k \geq 1)=\frac{P(k \geq 2)}{P(k \geq 1)}=\frac{1-P(0)-P(1)}{1-P(0)}=\frac{1-e^{-\lambda}-\lambda e^{-\lambda}}{1-e^{-\lambda}} .
$$

For small $\lambda$, the probability of observing more than two events may be neglected, and one may write

$$
P(2 \mid k \geq 1) \approx P(k \geq 2 \mid k \geq 1) .
$$

If it is known that two events were observed, then the conditional probability $P(\Delta t<$ $10 \mu \mathrm{s} \mid 2$ ) that the second event occurs within $10 \mu \mathrm{s}$ of the first may be roughly estimated assuming a uniform distribution of event times within the region of interest:

$$
P(\Delta t<10 \mu \mathrm{s} \mid 2) \approx \frac{10 \mu \mathrm{s}}{\Delta T_{\mathrm{ROI}}} .
$$

Here $\Delta T_{\mathrm{ROI}}=60 \mu \mathrm{s}$ is the duration of the region of interest for thermal neutron captures. Under the assumptions described above, the probability $P_{\text {fail afterpulse }}$ that a true event will be vetoed by the afterpulsing cut is approximately given by

$$
P_{\text {fail afterpulse }} \approx P(\Delta t<10 \mu \mathrm{s} \mid 2) \cdot P(2 \mid k \geq 1)=\frac{1}{6}\left(\frac{1-e^{-\lambda}-\lambda e^{-\lambda}}{1-e^{-\lambda}}\right)
$$


where, for a given NCV position, the average number of events $\lambda$ may be estimated using the expression

$$
\lambda=\frac{\mathcal{N}_{\text {no afterpulsing cut }}}{N_{\text {spills }}} .
$$

Here, $\mathcal{N}_{\text {no afterpulsing cut }}$ is the number of NCV coincidence events in the region of interest after all cuts except the afterpulsing cut have been applied, and $N_{\text {spills }}$ is the number of beam spills for which data were recorded at the given NCV position.

One could correct for the dead time introduced by the afterpulsing cut by adding to the number of observed neutron candidates the product of $P_{\text {fail afterpulse }}$ and the number of events observed at a particular NCV position that passed all cuts except for the afterpulsing cut. For the measurements taken in ANNIE Phase I, however, this correction was found to be negligible at all NCV positions.

At the beginning of this section, the assumption was made that all of the NCV coincidence events are independent. While spurious events due to afterpulsing badly violate this assumption, the cut under discussion is specifically designed to remove them, so this is of no concern. A more problematic violation of the independence assumption, however, can be seen if one examines the orange histogram in fig. 9.1, which represents the time distribution (relative to the beam crossing at $t=0 \mu \mathrm{s}$ ) of neutron candidate events recorded at $\mathrm{NCV}$ position \#2 (center of the tank).

Although a significant number of neutron candidate events occur approximately in time with the beam (consider the relatively large peak that is present during the first $5 \mu \mathrm{s}$ ), the cause of these events is unlikely to be neutron captures within the NCV. For high energy neutrons that may arrive in the detector nearly in time with the beam crossing, the probability of immediate capture will be low. On the other hand, thermal neutrons will have a relatively high capture probability, but they will arrive in the detector much later than the beam.

Rather than being caused by neutron captures, the neutron candidate events that occur during the first several $\mu$ s after the beam crossing are more readily explained as being the result of proton recoils induced by high-energy neutrons within the NCV liquid. Because a neutron that caused such a proton recoil event could subsequently capture 
within the NCV liquid, a second NCV event that appears within $10 \mu$ s of the proton recoil cannot always be attributed to afterpulsing.

As a conservative means of ensuring that captures following the proton recoils seen in time with the beam are never misidentified as afterpulses, all events that occurred within the first $10 \mu$ s of the region of interest and failed the afterpulsing cut but passed all other cuts were added to the total number of observed neutron candidates. Because this approach is overly cautious, a $100 \%$ systematic error on the correction is also adopted, i.e., if $N_{10 \mu \mathrm{s}}$ is the number of events within the first $10 \mu \mathrm{s}$ of the region of interest that

failed only the afterpulsing cut, then the quantity $N_{10 \mu \mathrm{s}} \pm N_{10 \mu \mathrm{s}}$ (syst) is added to the number of events in the region of interest that pass all cuts.

\subsubsection{Outer PMT veto cut}

The outer PMT veto cut introduces at least two ways in which true neutron capture events within the NCV may be misidentified as originating from cosmic muons: (1) some neutron capture $\gamma$-rays will escape the $\mathrm{NCV}$, scatter within the water volume, and produce light detected by the tank PMTs, and (2) some true neutron captures within the NCV will occur in coincidence with unrelated tank activity, e.g., a cosmic muon entering the tank.

To obtain a conservative upper bound on the probability that a poorly-contained neutron capture within the NCV would generate pulses on at least 8 tank PMTs (and therefore fail the cut), simulations of thermal neutron captures occurring in and around the NCV were performed using RAT-PAC (Reactor Analysis Tool, Plus Additional Codes) [460], a water Cherenkov detector simulation framework based on Geant4 [440]. The simulated NCV was placed at position \#2 (the center of the tank) to maximize the number of tank PMTs that would collect photons as a result of each neutron capture. The simulation results, shown in fig. 9.3 , indicate that less than $2 \%$ of neutron captures within the NCV liquid will lead to more than three tank PMTs detecting at least one photoelectron each, while even fewer (less than $0.1 \%$ ) of neutron captures outside the liquid scintillator will do the same.

While the RAT-PAC results suggest that the leakage of neutron capture $\gamma$-rays from the NCV will only very infrequently lead to an event failing the outer PMT veto cut, 
unrelated tank PMT activity in coincidence with a true neutron capture could have a more important effect. To estimate the probability that eight or more tank PMTs would fire in accidental coincidence with an unrelated NCV event, the full population of pulses (from the Phase I data considered in this thesis) recorded by NCV PMT \#2 without a coincident (within $40 \mathrm{~ns}$ ) pulse on NCV PMT \#1 was analyzed. The red dataset from fig. 9.4 shows a histogram of the number of unique tank PMTs that fired within 40 ns of each NCV PMT \#2 pulse. Since a large fraction of the pulses on one of the NCV PMTs that do not have a corresponding pulse on the second will be due to dark counts, the histogram approximately represents a probability distribution for the number of tank PMTs that will fire in accidental coincidence with the NCV PMTs. Integrating the histogram from 8, the minimum number of tank PMTs needed to fail the outer PMT veto cut, to 56, the number of active tank PMTs used in Phase I, yields the probability of accidental failure

$$
\sum_{n=8}^{56} P(n)=2.28(3) \%
$$

where $P(n)$ is the fraction of events from the red histogram in fig. 9.4 with $n$ unique tank PMTs firing in coincidence. The statistical error given in eq. (9.25) was determined assuming that the number of accidental failures of the outer PMT veto cut in a given number of trials is a binomial random variable.

To be as conservative as possible, the small contribution to the failure probability from poorly-contained neutron captures within the NCV was incorporated via the expression

$$
P_{\text {tank fail }}=\sum_{k=0}^{8} P(k)\left[\sum_{n=8-k}^{56} P(n)\right]=2.42 \pm 0.03 \text { (stat) } \pm 0.14 \text { (syst) } \%
$$

where $P(k)$ is the RAT-PAC estimate from fig. 9.3 of the probability that a neutron capture within the NCV will lead to $k$ unique tank PMTs detecting at least one photoelectron. Since the RAT-PAC event containment study overestimates the true contribution of uncontained neutron captures to the outer PMT veto cut failure rate (because, e.g., more than one photoelectron may be needed to generate reconstructable pulses on the tank PMTs), the systematic error assigned above was chosen to be equal to the difference in the values of $P_{\text {tank fail }}$ obtained with (see eq. (9.26)) and without (see eq. (9.25)) the 
small RAT-PAC correction. To account for the accidental failure rate, the number of observed neutron candidate events should be increased by $P_{\text {tank fail }}$ times the number of NCV coincidence events that passed all cuts except for the outer PMT veto cut.

\subsubsection{Total charge cut}

Although the total charge cut was designed to remove only the most obvious muon candidate events (those that deposit about $34 \mathrm{MeV}$ or more in the scintillator), it could conceivably reduce the number of true neutron capture events if there is a very large unrecognized error in the NCV charge-to-energy calibration described in section 10.2.1, e.g., the total charge collected on the NCV PMTs deviates substantially from being, on average, a linear function of the total energy deposited in the liquid scintillator. However, because the NCV efficiency estimate from section 10.2.3 based on the charge-to-energy

calibration agrees reasonably well with the value obtained using a ${ }^{252} \mathrm{Cf}$ calibration source, an error of the magnitude needed for even the highest-energy signal events (which deposit nearly $9 \mathrm{MeV}$ ) to fail the total charge cut appears unlikely.

Another possibile means by which the total charge cut could reduce the number of signal events is if a cosmic muon enters the NCV within $40 \mathrm{~ns}$ of the start of a true neutron capture event. For realistic values of the rate at which cosmic muons enter the $\mathrm{NCV}$ (say, a few tens of $\mathrm{Hz}$ ), however, this pileup effect is negligible. Unlike the other two analysis cuts, therefore, no systematic corrections associated with the total charge cut will be applied to the observed neutron candidate event rate.

\subsection{Results}

Based on the discussion in the previous section, the accepted value $N_{n}$ for the number of neutron candidate events observed at each NCV position may be written as

$$
N_{n}=N_{\text {pass }}+N_{10 \mu \mathrm{s}}+P_{\text {tank fail }} N_{\text {tank }}
$$

where, in the region of interest (10 $\mu$ s to $70 \mu$ s after the start of the beam crossing), $N_{\text {pass }}$ is the number of observed NCV coincidences that pass all cuts, $N_{10 \mu \mathrm{s}}$ is the number of events that fail only the afterpulsing cut and occur within the first $10 \mu \mathrm{s}, N_{\text {tank }}$ is the 


\begin{tabular}{cccccc}
\hline $\begin{array}{c}\text { NCV } \\
\text { position }\end{array}$ & $\begin{array}{c}\text { Exposure } \\
\left(10^{18} \mathrm{POT}\right)\end{array}$ & $N_{\text {pass }}$ & $N_{10 \mu \mathrm{s}}$ & $N_{\text {tank }}$ & $N_{n}$ \\
\hline 1 & 7.88 & 3768 & 57 & 1602 & $3864 \pm 61$ (stat) \pm 57 (syst) \\
2 & 17.66 & 296 & 43 & 2549 & $401 \pm 17$ (stat) \pm 43 (syst) \\
3 & 13.24 & 723 & 20 & 2234 & $797 \pm 27$ (stat) \pm 20 (syst) \\
4 & 10.98 & 825 & 41 & 2428 & $925 \pm 29$ (stat) \pm 41 (syst) \\
5 & 6.79 & 241 & 13 & 1276 & $285 \pm 16$ (stat) \pm 13 (syst) \\
6 & 3.63 & 356 & 12 & 664 & $384 \pm 19$ (stat) \pm 12 (syst) \\
7 & 2.20 & 55 & 5 & 348 & $68 \pm 7$ (stat) \pm 5 (syst) \\
\hline
\end{tabular}

Table 9.1: Neutron candidate events observed at each NCV position. Starting with the third column, the quantities $N_{\text {pass }}, N_{10 \mu \mathrm{s}}, N_{\text {tank }}$, and $N_{n}$ listed in the table represent event counts observed in the region of interest. They are, respectively, the number of events that pass all cuts, the number of events that fail only the afterpulsing cut and occur within the first $10 \mu \mathrm{s}$, the number of events that fail only the outer PMT veto cut, and the accepted number of neutron candidate events.

number of events that fail only the outer PMT veto cut, and $P_{\text {tank fail }}$ is given in eq. (9.26). The uncertainty on the number of observed neutron candidate events, $\sigma_{N_{n}}$, is computed using

$$
\sigma_{N_{n}}^{2}=N_{\text {pass }}(\text { stat })+N_{10 \mu \mathrm{s}}^{2} \text { (syst) }+P_{\text {tank fail }}^{2} N_{\text {tank }}(\text { stat })+N_{\text {tank }}^{2} \sigma_{P_{\text {tank fail }}}^{2}
$$

where $\sigma_{P_{\text {tank fail }}}$ is the error given in eq. (9.26), and the last term includes both a statistical and a systematic contribution to the uncertainty.

Table 9.1 lists the measured neutron candidate event counts at each NCV position together with the corresponding number of protons on target (the exposure) delivered by the beam. After a determination of the NCV neutron detection efficiency is made in the following chapter, these two quantities will be used to compute an upper limit on the expected background neutron event rates for ANNIE Phase II. 


\section{Chapter 10}

\section{Efficiency calibration of the Neutron Capture Volume}

In addition to the measured event rates for neutron capture candidates in the NCV, a determination of the detection efficiency of the NCV is needed before the background neutron event rates at each position may be calculated. This determination was made using two independent calibration techniques: exposing the $\mathrm{NCV}$ to neutrons from a ${ }^{252} \mathrm{Cf}$ fission source and using cosmic ray muon data to determine an energy threshold. This chapter reviews both of these calibration techniques and describes the data analysis that led to an accepted value of the NCV efficiency for ANNIE Phase I.

\section{1 ${ }^{252}$ Cf source calibration method}

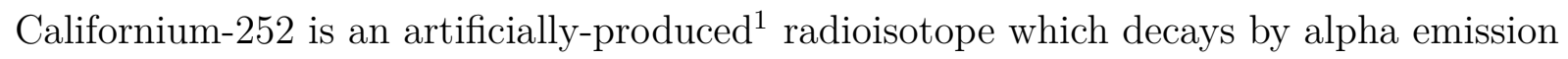
(96.908(8)\% branching fraction) and spontaneous fission (3.092(8)\% branching fraction) with a total half-life of $2.645(8)$ years [462]. Spontaneous fissions of ${ }^{252} \mathrm{Cf}$ produce an average of 3.7675(40) neutrons each [463]. The energy spectrum of these neutrons follows the Watt distribution $N(E)$, which may be written in the form

$$
N(E)=e^{-E / a} \sinh (\sqrt{b E})
$$

\footnotetext{
${ }^{1}$ Only two institutions worldwide currently produce californium: Oak Ridge National Laboratory in the United States and the Research Institute of Atomic Reactors in Russia [461].
} 
where $E$ is the neutron kinetic energy in $\mathrm{MeV}$, and, for ${ }^{252} \mathrm{Cf}$, the parameters $a$ and $b$ may be taken to have the values [461] $a=1.18 \mathrm{MeV}$ and $b=1.03419 \mathrm{MeV}^{-1}$. Gamma-rays are also produced by each fission of ${ }^{252} \mathrm{Cf}$ with an average multiplicity of $7.98(20)$ [464].

A straightforward method for calibrating a neutron detector is to expose it to a neutron source with a precisely known activity, count the number of detected neutrons, and compare the result to the expected number of neutron events in the detector. While such an approach could in principle have been used to calibrate the NCV, a major obstacle was the lack of precision with which the source activity was known. Because the ${ }^{252} \mathrm{Cf}$ source selected for the NCV calibration measurements was found on January 28, 1988 to have a neutron emission rate $^{2}$ of $2.31 \times 10^{7}$ neutrons/s, one might attempt to determine its current activity via a naïve application of the radioactive decay law

$$
A(t)=A_{0} \exp \left[-\ln (2) t / t_{1 / 2}\right]
$$

where $A(t)$ is the activity of the source at time $t, A_{0}$ is the initial activity, and $t_{1 / 2}$ is the half-life of the source. However, because californium sources are manufactured by irradiating curium oxide targets with neutrons and then chemically separating Cf from the other transmutation products, the sources are never isotopically pure. In young sources, ${ }^{252} \mathrm{Cf}$ will completely dominate the neutron emission rate (see fig. 10.1) due to its comparatively short half-life, but in sources that are several decades old, the contributions of ${ }^{250} \mathrm{Cf}$ and ${ }^{248} \mathrm{Cm}$ become appreciable, eventually overwhelming the remaining neutron emissions from ${ }^{252} \mathrm{Cf}$ [461]. Since the initial isotopic composition of the californium source used for ANNIE Phase I is unknown, only an estimate of its activity may be obtained using eq. (10.2).

To avoid the need to know the source activity precisely in the NCV efficiency analysis, a different calibration technique was used. Because a ${ }^{252} \mathrm{Cf}$ fission event involves the nearsimultaneous emission of both neutrons and $\gamma$-rays from the daughter nuclei, the detection of a fission $\gamma$-ray may be used to trigger a neutron detector. Neutron captures observed in the detector shortly after the $\gamma$-ray trigger will often be due to neutrons from the same fission event that produced the $\gamma$-ray. If the source activity and the unrelated background

\footnotetext{
${ }^{2}$ Unfortunately, no uncertainty is given for this calibration measurement in the original documents.
} 


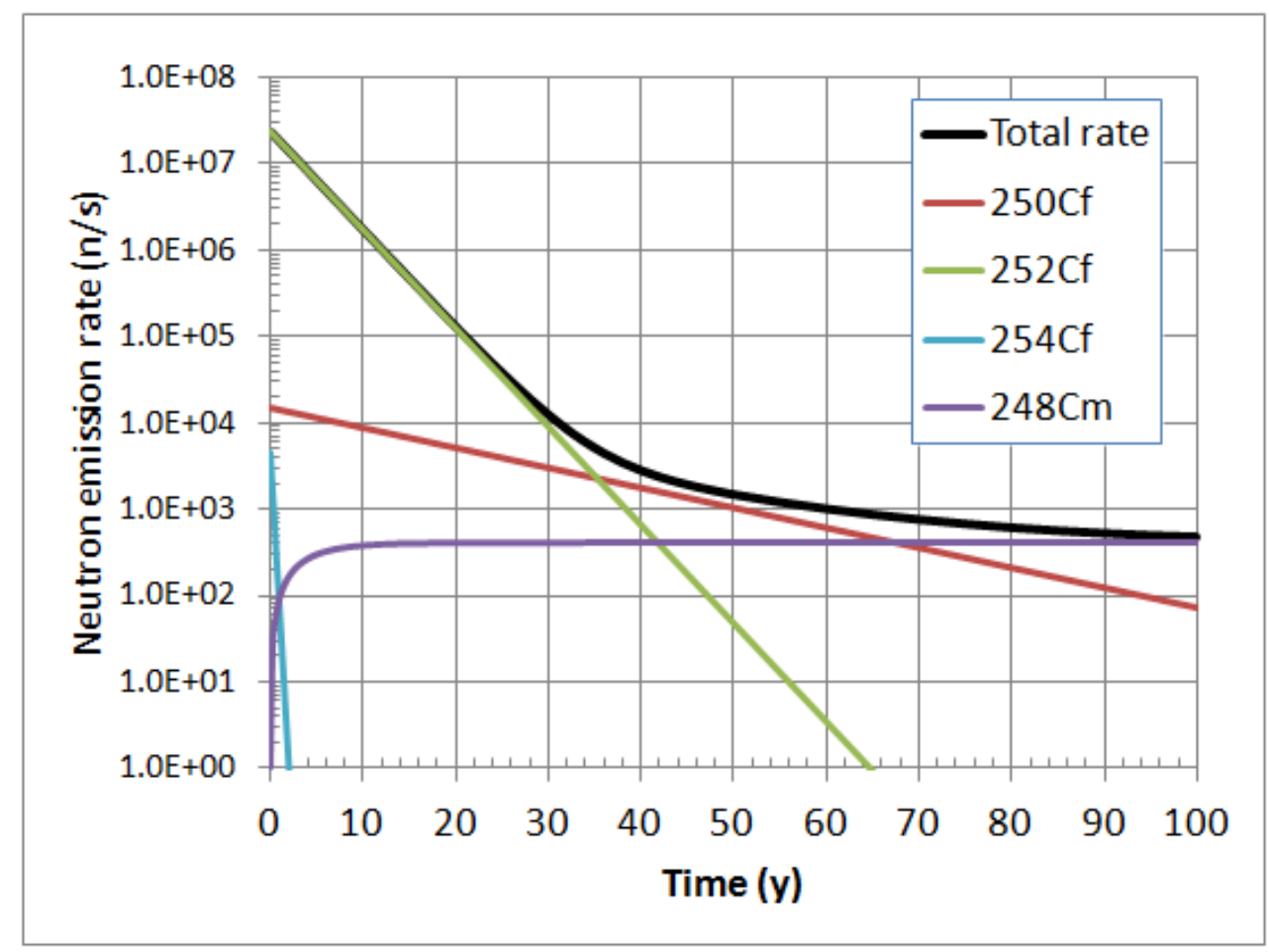

Figure 10.1: Calculated neutron emission rate of a californium source with an initial isotopic composition of $4.32 \%{ }^{249} \mathrm{Cf}, 10.82 \%{ }^{250} \mathrm{Cf}, 3.31 \%{ }^{251} \mathrm{Cf}, 81.50 \%{ }^{252} \mathrm{Cf}, 0.04 \%$ ${ }^{253} \mathrm{Cf}$, and $0.01 \%{ }^{254} \mathrm{Cf}$. Figure taken from [461].

event rate are both sufficiently small, the distribution of neutron event times relative to the $\gamma$-ray trigger will include a flat component and an excess with a characteristic shape that is due to the prompt neutrons. The size of this excess may be compared with simulations to extract the neutron detector efficiency. This technique was used to calibrate the NCV by (1) acquiring data using a ${ }^{252} \mathrm{Cf}$ source and a suitable triggering device, and (2) comparing the measured event time distribution to simulations produced using the FREYA event generator [465] and the RAT-PAC detector simulation package [460].

\subsubsection{Triggering apparatus}

During the NCV calibration measurements, an $\mathrm{LYSO}^{3}$ scintillation crystal coupled to a small photomultiplier tube was used to detect the fission $\gamma$-rays. A discriminator connected to the PMT output was used to trigger the full ANNIE detector. The crystal and

\footnotetext{
${ }^{3}$ Lutetium-yttrium oxyorthosilicate $\left(\mathrm{Lu}_{1.8} \mathrm{Y}_{0.2} \mathrm{SiO}_{5}(\mathrm{Ce})\right)$
} 
PMT were placed in a light-tight cardboard box which was positioned directly on top of the ANNIE water tank hatch. The ${ }^{252} \mathrm{Cf}$ source was then placed on top of the box just above the LYSO crystal. All of the efficiency measurements considered here were performed with the NCV at position \#1 (just below the surface of the water at the center of the tank).

Background signals in the LYSO crystal are produced not only by cosmic ray muons (which provide an irreducible background at a relatively low rate) but also by $\beta^{-}$decays of ${ }^{178} \mathrm{Lu}$ [466], which has a natural abundance of $0.02599(13) \%$ [160]. To mitigate the

intrinsic ${ }^{178} \mathrm{Lu}$ beta decay background, measurements of the calibration trigger rate were performed before each run with and without the source present. The discriminator threshold and source placement relative to the LYSO crystal were both adjusted to minimize the contamination $\mathcal{C}$, defined as

$$
\mathcal{C} \equiv \frac{R_{\text {no source }}}{R_{\text {source }}},
$$

where $R_{\text {source }}$ is the LYSO crystal trigger rate when the ${ }^{252} \mathrm{Cf}$ source was present and $R_{\text {no source }}$ is the trigger rate when the source was removed. The contamination was measured again at the end of each calibration run to check its consistency. Because the average of the pre- and post-run measurements of the contamination never exceeded $0.52 \%$, the false LYSO trigger rate due to cosmic muons and ${ }^{178} \mathrm{Lu}$ beta decays will be neglected in the source data analysis.

\subsubsection{Timing data}

A total of 206732 LYSO triggers were recorded using the apparatus described in section 10.1.1. In response to each trigger, an $80 \mu$ s readout window was digitized by the ANNIE DAQ, including traces from both of the NCV PMTs and all of the water tank PMTs. The time distribution within this window of all events passing the neutron candidate criteria described in chapter 9 is shown in fig. 10.2. Although the distribution appears to be flat near the end of the $80 \mu$ s window, two features are obvious at early times: a large excess in the third bin and a smaller, broad bump that extends from roughly $4 \mu \mathrm{s}$ to $30 \mu \mathrm{s}$. Because the first two bins in the distribution represent times before the LYSO trigger was issued, the large value in the third bin may be attributed to prompt 


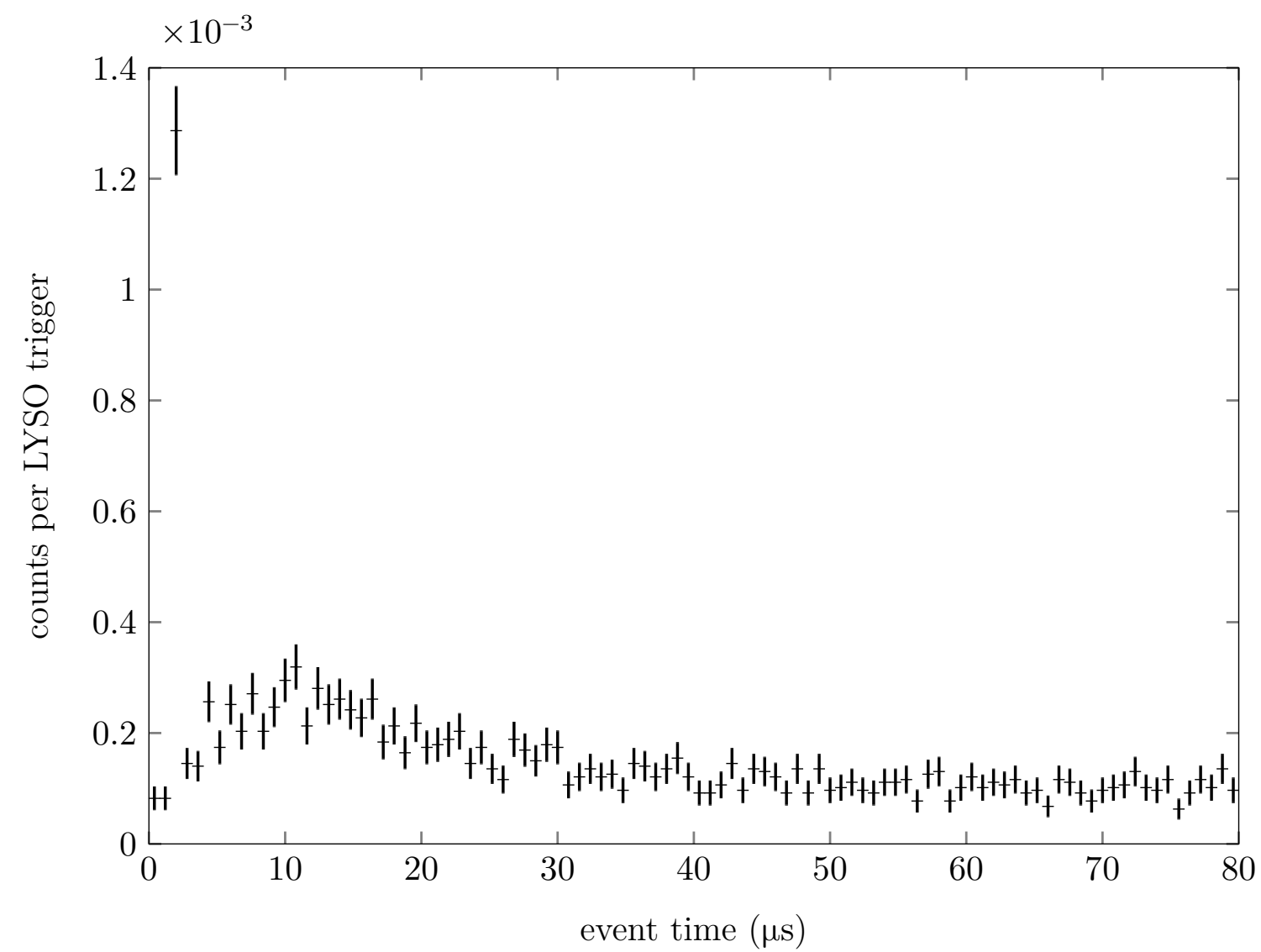

Figure 10.2: Distribution of neutron candidate event times from the ${ }^{252} \mathrm{Cf}$ source calibration runs taken with the NCV at position \#1. The large event rate in the third bin may be attributed to prompt fission $\gamma$-rays entering the NCV.

fission $\gamma$-rays (which will arrive in coincidence with the LYSO trigger) scattering within the NCV.

Evidence for the origin of the second feature, the broad bump at early times, may be obtained by fitting an exponential plus a flat background to the right side (the region later than about $15 \mu \mathrm{s}$ ) of the bump. The result of such a fit, shown in fig. 10.3, yields a value for the exponential time constant of $(12.8 \pm 2.8) \mu \mathrm{s}$, which is consistent with the nominal value of $12.2 \mu$ s for thermal neutron captures in the NCV liquid. This observation suggests that the broad bump seen at early times corresponds to ${ }^{252} \mathrm{Cf}$ neutrons thermalizing and then capturing within the NCV. Stronger evidence for this conclusion was obtained using Monte Carlo simulations, as described below. 


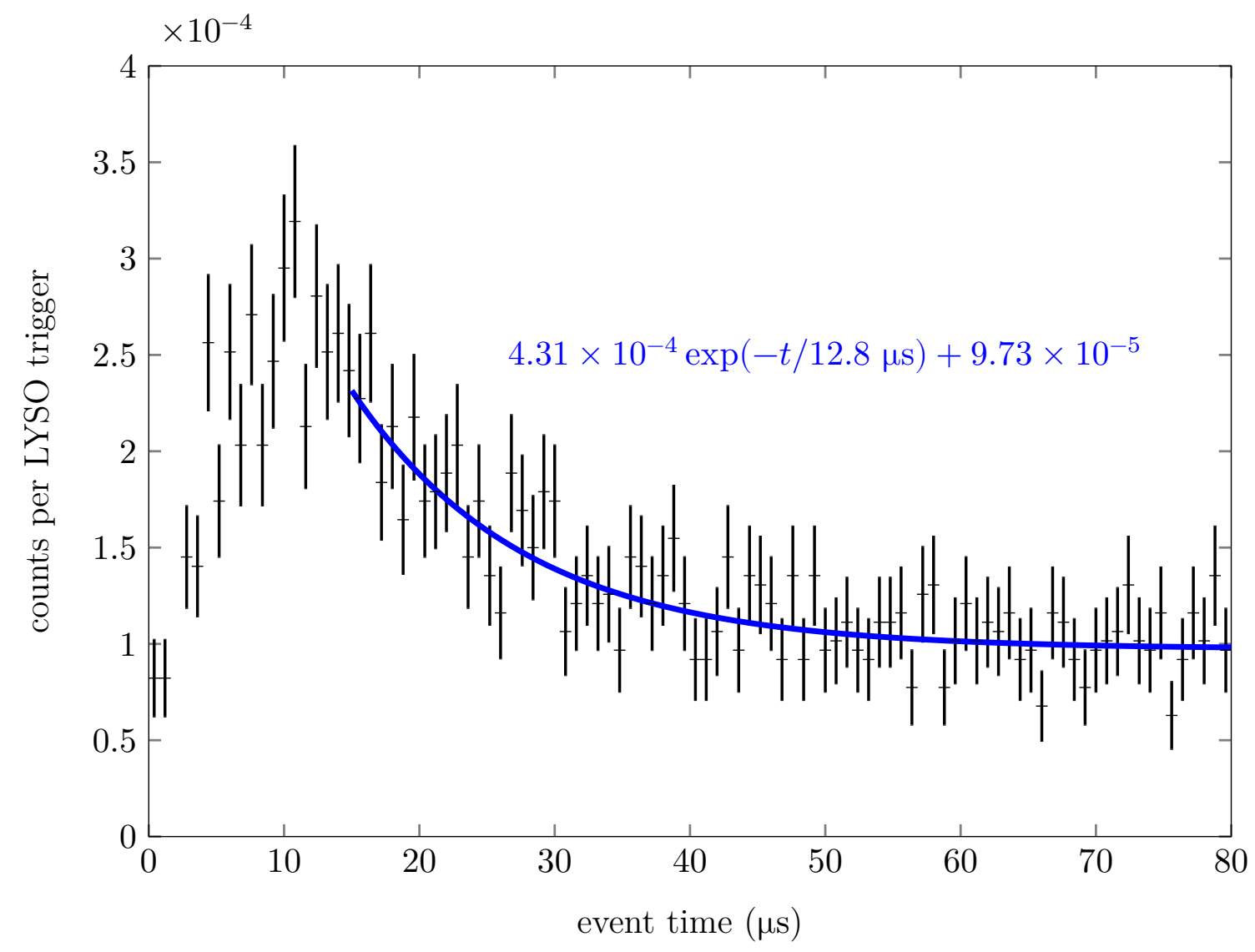

Figure 10.3: Exponential fit of the time distribution from fig. 10.2 after $15 \mu \mathrm{s}$.

\subsubsection{Simulations}

To measure the NCV efficiency, data from the ${ }^{252} \mathrm{Cf}$ source calibration runs were compared with simulations produced using version 2.0.3 of the FREYA (Fission Reaction Event Yield Algorithm) event generator and an ANNIE-specific version of RAT-PAC.

\subsubsection{FREYA model for ${ }^{252} \mathrm{Cf}$ spontaneous fission}

The FREYA event generator, which was introduced by Randrup and Vogt [467] in 2009 and has undergone continuing development $[465,468]$ and testing against experimental data [469-474] up to the present, implements realistic models of both spontaneous fission and neutron-induced fission. ${ }^{4}$ Version 2.0.3 of FREYA is capable of generating spontaneous fission events for 6 nuclides $\left({ }^{238} \mathrm{U},{ }^{238} \mathrm{Pu},{ }^{240} \mathrm{Pu},{ }^{242} \mathrm{Pu},{ }^{244} \mathrm{Cm}\right.$, and $\left.{ }^{252} \mathrm{Cf}\right)$ and

\footnotetext{
${ }^{4}$ Fissions induced by neutrons with incident kinetic energies up to $E_{n}=20 \mathrm{MeV}$ may be simulated.
} 
neutron-induced fission events for 5 nuclides $\left({ }^{233} \mathrm{U},{ }^{235} \mathrm{U},{ }^{238} \mathrm{U},{ }^{239} \mathrm{Pu}\right.$, and $\left.{ }^{241} \mathrm{Pu}\right)$. A brief summary of the procedures used by FREYA to model spontaneous fission of ${ }^{252} \mathrm{Cf}$ is given below. For a complete description of the code, the interested reader is encouraged to consult the publications cited above and the FREYA 2.0.3 user manual [475].

To begin a simulated ${ }^{252} \mathrm{Cf}$ fission event, FREYA samples a mass number $A_{f}$ for one of the fragments using the empirical distribution shown in fig. 10.4. The proton number $Z_{f}$ of the selected fragment is then sampled on the restricted interval $\left|Z_{f}-\bar{Z}_{f}\left(A_{f}\right)\right| \leq 5 \sigma_{Z}$ from the Gaussian distribution

$$
P\left(Z_{f} \mid A_{f}\right) \propto \exp \left(-\frac{\left[Z_{f}-\bar{Z}_{f}\left(A_{f}\right)\right]^{2}}{2 \sigma_{Z}^{2}}\right)
$$

recommended by reference [476]. In the expressions above, the mean fragment proton number $\bar{Z}_{f}\left(A_{f}\right)$ is chosen so that, on average, the fragment will have the same charge-tomass ratio as the parent nucleus, i.e.,

$$
\bar{Z}_{f}\left(A_{f}\right)=A_{f}\left(\frac{Z_{0}}{A_{0}}\right),
$$

where $Z_{0}=98$ is the initial proton number and $A_{0}=252$ is the initial mass number of the fissioning ${ }^{252} \mathrm{Cf}$ nucleus. The parameter $\sigma_{Z}$ has the value 0.47 for ${ }^{252} \mathrm{Cf}$.

After $A_{f}$ and $Z_{f}$ values have been chosen for the first fragment, conservation of electric charge $\left(Z_{f}^{\prime}=Z_{0}-Z_{f}\right)$ and baryon number $\left(A_{f}^{\prime}=A_{0}-A_{f}\right)$ allow an immediate determination of the proton number $Z_{f}^{\prime}$ and the mass number $A_{f}^{\prime}$ for the second fragment. Once both are identified, the two fragments may be referred to as the "light" and "heavy" fragment, where the heavy fragment has mass number $A_{H}=\max \left(A_{f}, A_{f}^{\prime}\right)$.

The energy released by the fission reaction is given by the Q-value

$$
Q=m_{0}-m_{H, \text { g.s. }}-m_{L, \text { g.s. }}
$$

where $m_{0}$ is the mass of the initial ${ }^{252} \mathrm{Cf}$ nucleus and $m_{H, \text { g.s. }}\left(m_{L, \text { g.s. }}\right)$ is the groundstate mass of the heavy (light) fission fragment. The available energy, which is exactly conserved in the model, is partitioned into kinetic energy (using the appropriate value of the mean total kinetic energy measured in reference [477]), rotational energy (assuming 
statistical population of the dinuclear modes available during scission), and excitation energy of the fragments, allowing for thermal fluctuations in the last of these.

Once the released energy has been divided between the fission fragments, both of them are made to de-excite via repeated single neutron emission. A neutron emission step is simulated by sampling a kinetic energy $E_{n}$ from the black-body spectrum

$$
\frac{d N_{n}}{d E_{n}} \propto E_{n} \exp \left(-E_{n} / T_{\max }\right)
$$

The maximum possible temperature $T_{\max }$ of the daughter nucleus is given by

$$
T_{\max }=\sqrt{Q_{n} / a_{d}}
$$

where $a_{d}$ is the level density parameter of the daughter nucleus (see section 4.3) and $Q_{n}$ is the maximum possible excitation energy of the daughter nucleus (corresponding to the emission of a neutron at rest). A direction for the emitted neutron is also chosen under the assumption that the emission process is isotropic in the rest frame of the parent fragment. Momentum four-vectors are stored for the final-state neutron and daughter nucleus. If the excitation energy of the daughter nucleus exceeds $S_{n}+Q_{\min }$, where $S_{n}$ is the neutron separation energy and $Q_{\min }=0.01 \mathrm{MeV}$, then another neutron emission step is simulated using the daughter nucleus as the new parent. This process continues for both fragments for as many steps as are energetically possible.

After insufficient excitation energy remains for neutron evaporation to continue, FREYA simulates the emission of $\gamma$-rays from the fission fragments. For high excitation energies (near the neutron emission threshold $S_{n}$ ) this is done by sampling $\gamma$-ray energies from the spectrum $^{5}$

$$
\frac{d N_{\gamma}}{d E_{\gamma}} \propto \frac{\Gamma_{\mathrm{GDR}}^{2} E_{\gamma}^{2}}{\left(E_{\gamma}^{2}-E_{\mathrm{GDR}}^{2}\right)^{2}-\Gamma_{\mathrm{GDR}}^{2} E_{\gamma}^{2}} E_{\gamma}^{2} e^{-E_{\gamma} / T}
$$

where the energy $E_{\mathrm{GDR}}$ and width $\Gamma_{\mathrm{GDR}}$ of the giant dipole resonance are taken to be

$$
E_{\mathrm{GDR}}=\left(31.2 A^{-1 / 3}+20.6 A^{-1 / 6}\right) \mathrm{MeV}
$$

and

$$
\Gamma_{\mathrm{GDR}}=5 \mathrm{MeV}
$$

\footnotetext{
${ }^{5}$ This is similar to the MARLEY treatment of E1 $\gamma$-ray emission in the Hauser-Feshbach statistical model. See section 4.4.5.
} 


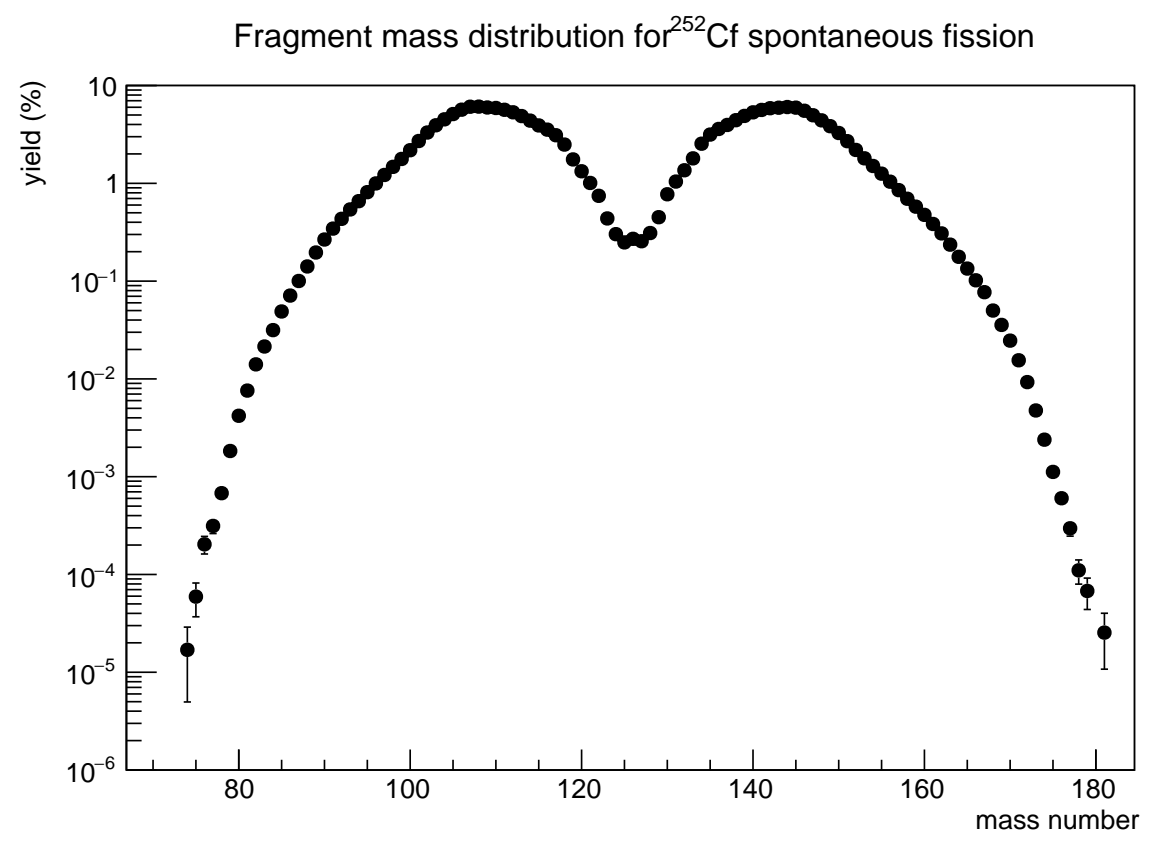

Figure 10.4: Experimental distribution measured by Hambsch and Oberstedt [477] of the mass number $A$ of fission fragments produced by spontaneous fission of ${ }^{252} \mathrm{Cf}$.

for a fragment with mass number $A$. When the remaining excitation energy falls below one of the discrete nuclear levels given in the RIPL-3 database [321], FREYA uses the tabulated branching ratios therein to complete the $\gamma$-ray cascade to the ground state.

\subsubsection{Particle tracking with RAT-PAC}

To enable FREYA to be used with the existing ANNIE simulation tools, the recommended interface between FREYA and Geant4 provided in version 2.0.3 of the LLNL ${ }^{6}$ Fission Library [478] was incorporated into a fork of RAT-PAC. While models of the ANNIE tank, water volume, PMTs, and NCV were all included in the simulated detector geometry, the dark box containing the LYSO crystal and trigger PMT was not simulated. Fission events were created one at a time in RAT-PAC using neutron and $\gamma$-ray tracks with initial 4-momenta taken from a single fission simulated by FREYA. These tracks were started at a point above the tank hatch corresponding to the location of the ${ }^{252} \mathrm{Cf}$ source used for the calibration measurements. Neutron reactions were simulated using version 4.5 of

\footnotetext{
${ }^{6}$ Lawrence Livermore National Laboratory
} 


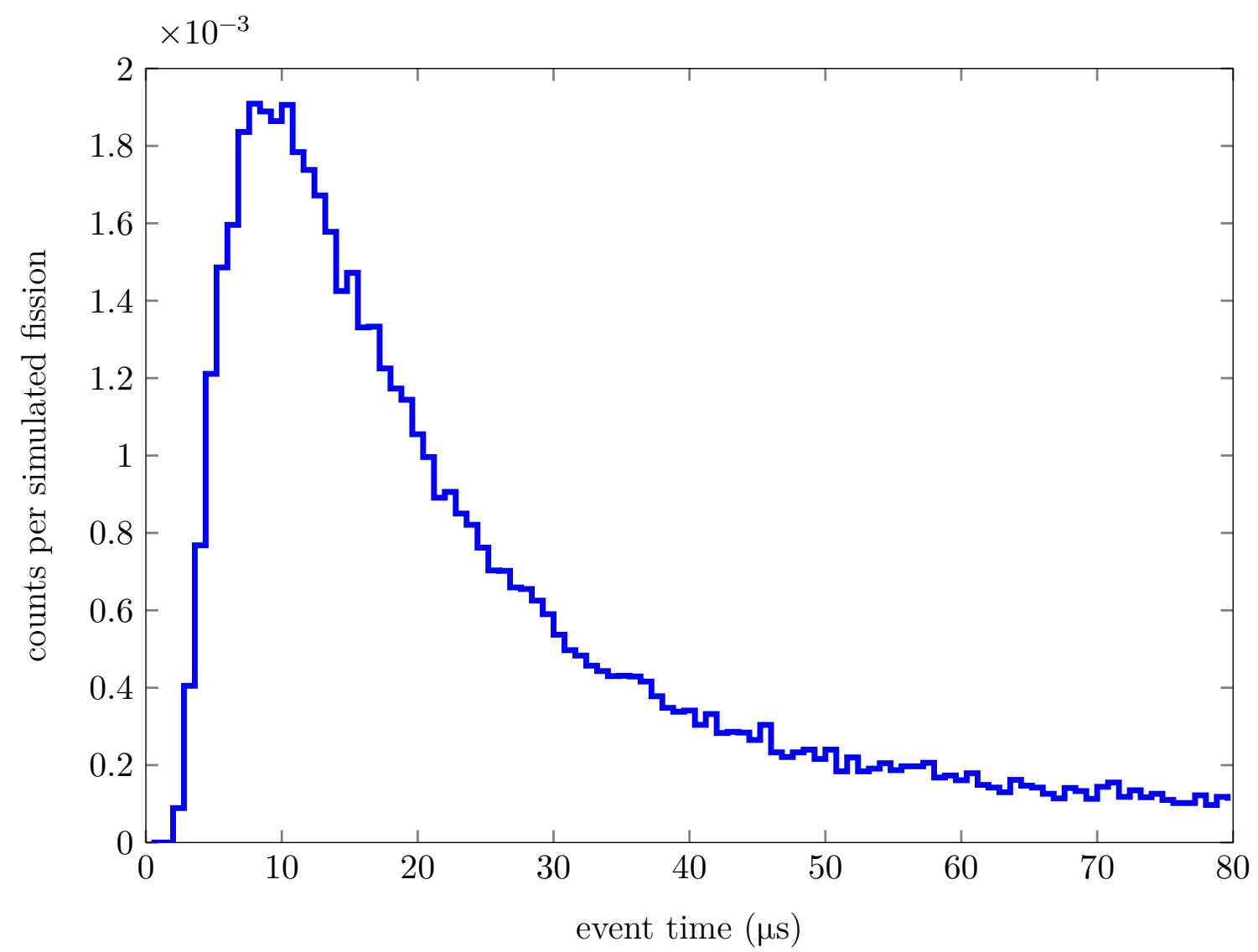

Figure 10.5: Predicted time distribution for neutron capture events in the NCV liquid. Time $t=0$ corresponds to a fission event simulated using the FREYA event generator.

G4NDL ${ }^{7}$ a collection of continuous-energy neutron cross sections and final-state particle production data primarily based on the ENDF/B-VII.1 evaluated nuclear data library $[479]$.

\subsubsection{Simulation results}

Using the approach described above, a sample of $10^{6}$ simulated ${ }^{252} \mathrm{Cf}$ fission events was produced using FREYA and RAT-PAC. Within this sample, a total of 66608 neutron captures were observed inside of the NCV liquid volume. The time distribution for these neutron captures during the first $80 \mu$ s after fission, ${ }^{8}$ a time period which includes $84 \%$ of all of the NCV captures, is shown in fig. 10.5. After adding an offset of 2014 ns to the

\footnotetext{
${ }^{7}$ Geant4 Neutron Data Library

${ }^{8}$ All fission neutrons and $\gamma$-rays were treated in the simulations as being emitted simultaneously at time $t=0$.
} 
simulated capture times to account for the pre-trigger part of the readout window, the data shown in fig. 10.2 were fit with a model based on the simulation results. According to this model, the expected number of counts observed after $N_{\text {trigs }}$ LYSO triggers in the $j$ th time bin $C_{j}$ is given by

$$
C_{j}\left(\epsilon_{\mathrm{NCV}}, R, P_{\gamma}\right)=N_{\text {trigs }}\left(\epsilon_{\mathrm{NCV}} P_{n, j}+\delta_{j, \gamma \text { bin }} P_{\gamma}+\Delta t_{j} R\right)
$$

where $\delta_{j, \gamma \text { bin }}$ is one for the bin containing the gamma flash and zero otherwise, $\Delta t_{j}$ is the duration of the $j$ th time bin, and $\epsilon_{\mathrm{NCV}}, R$, and $P_{\gamma}$ are fitting parameters representing, respectively, the NCV efficiency (the fraction of true neutron captures within the NCV liquid that are detected), the background event rate, ${ }^{9}$ and the fraction of fissions that result in a $\gamma$-ray event being detected by the NCV. The probability $P_{n, j}$ that a ${ }^{252} \mathrm{Cf}$ fission leads to a true neutron capture inside the NCV during the $j$ th time bin was estimated from the simulation results via the formula

$$
P_{n, j}=\frac{N_{j}}{N_{\text {simulated }}},
$$

where $N_{j}$ is the number of simulated captures that occurred in the $j$ th time bin (with the pre-trigger correction to the simulated times applied as described above), and $N_{\text {simulated }}=$ $10^{6}$ is the number of simulated fissions.

To obtain the best-fit values for the parameters $\epsilon_{\mathrm{NCV}}, R$, and $P_{\gamma}$, a maximum likelihood fit was performed using the ROOT [449] implementation of the MINUIT [480] function minimization and error analysis software package. The negative log-likelihood function

$$
-\ln (L)=\sum_{j} B_{j} \ln \left(C_{j}\right)-C_{j}-\ln \left(B_{j} !\right)
$$

minimized during the fit corresponds to the product of Poisson probabilities

$$
L \equiv L\left(\epsilon_{\mathrm{NCV}}, R, P_{\gamma}\right)=\prod_{j} \frac{C_{j}^{B_{j}} e^{-C_{j}}}{B_{j} !},
$$

where $B_{j}$ is the observed number of counts in the $j$ th bin, the expected number of counts $C_{j}$ is given by the model in eq. (10.12), and the product in eq. (10.15) (the sum in

\footnotetext{
${ }^{9}$ This rate includes a contribution from "pileup fissions" that did not trigger the detector but nevertheless led to a neutron capture or a $\gamma$-ray event in the NCV that was recorded.
} 
Table 10.1: Results of the maximum likelihood fit (described in section 10.1.3.3) to the ${ }^{252} \mathrm{Cf}$ source calibration data. Only statistical errors are given here.

\begin{tabular}{ccc}
\hline Parameter & Variable & Best-fit value \\
\hline NCV efficiency & $\epsilon_{\mathrm{NCV}}$ & $9.60(57) \times 10^{-2}$ \\
Background event rate $(\mathrm{Hz})$ & $R$ & $1.12(4) \times 10^{2}$ \\
$\gamma$-ray event probability & $P_{\gamma}$ & $1.19(8) \times 10^{-3}$ \\
Reduced chi-squared statistic & $\chi^{2} / \nu$ & 0.753 \\
\hline
\end{tabular}

eq. (10.14)) includes all 100 time bins ${ }^{10}$ used to create the data histogram shown in fig. 10.2. The best-fit parameter estimates, including statistical errors estimated by MINUIT using numerical differentiation at the minimum of $-\ln (L)$, are shown in table 10.1. The best-fit model is plotted together with the source data in fig. 10.6.

To validate the fitting procedure, fictitious ${ }^{252} \mathrm{Cf}$ source datasets were generated by sampling bin counts from a Poisson distribution for which the expected number of counts $D_{j}$ for the $j$ th bin was given by

$$
\begin{aligned}
D_{j} & =N_{\text {trigs }}\left(\epsilon_{\mathrm{NCV}} P_{n, j}+\delta_{j, \gamma \text { bin }} P_{\gamma}\right) \\
& +N_{\text {trigs }} \Delta t_{j}\left(R_{\text {fission }}\left[\epsilon_{\mathrm{NCV}} P_{n}+P_{\gamma}\right]+R_{\text {other }}\right)
\end{aligned}
$$

where $P_{n}$ is the probability that a ${ }^{252} \mathrm{Cf}$ fission event will lead to a neutron capture at any time in the NCV liquid, $R_{\text {fission }}$ is the fission rate of the source, $R_{\text {other }}$ is the rate of background events unrelated to ${ }^{252} \mathrm{Cf}$ fissions, and all other symbols in the equation have the same meanings as in eq. (10.12). Note that the model presented in eq. (10.16) is the same as in eq. (10.12) except that the flat background term has now been separated into contributions from pileup fission ${ }^{11}$ neutrons, pileup fission $\gamma$-rays, and other causes. To generate each fictitious dataset, a new set of model parameters $\epsilon_{\mathrm{NCV}}, R_{\mathrm{fission}}, R_{\mathrm{other}}, P_{\gamma}$,

\footnotetext{
${ }^{10}$ The fit procedure described here was repeated using a subset of the time bins representing the first $25 \mu$ s after an LYSO trigger. Only slight differences in the best-fit parameters were observed.

${ }^{11}$ That is, fissions other than those that activated the trigger apparatus during the source calibration measurements
} 


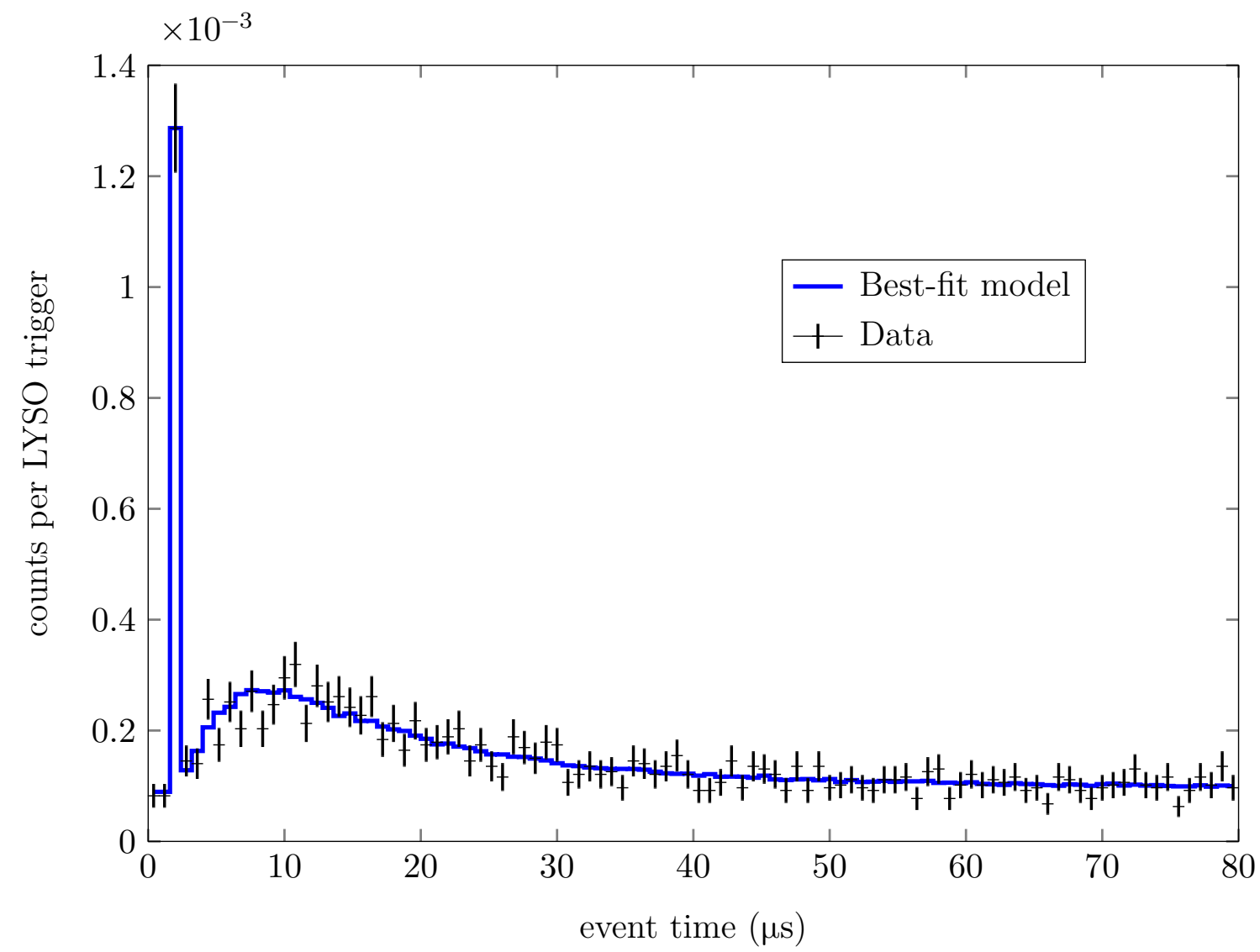

Figure 10.6: Comparison of the ${ }^{252} \mathrm{Cf}$ calibration source data with the result of a maximum likelihood fit of the model defined in eq. (10.12).

and $N_{\text {trigs }}$ were sampled from the distributions

$$
\begin{aligned}
\epsilon_{\mathrm{NCV}} & \sim \mathcal{U}(0.03,0.25) \\
R_{\mathrm{fission}} & \sim \mathcal{N}(3000 \mathrm{~Hz}, 700 \mathrm{~Hz}) \\
R_{\mathrm{other}} & \sim \mathcal{U}(10 \mathrm{~Hz}, 150 \mathrm{~Hz}) \\
P_{\gamma} & \sim \mathcal{U}\left(5 \times 10^{-4}, 5 \times 10^{-3}\right) \\
N_{\text {trigs }} & \sim \mathcal{U}\left(1 \times 10^{5}, 3 \times 10^{5}\right)
\end{aligned}
$$

where $\mathcal{U}(a, b)$ is a uniform distribution defined on the interval $[a, b]$ and $\mathcal{N}(\mu, \sigma)$ is a normal distribution with mean $\mu$ and standard deviation $\sigma$. The mean of the normal 
distribution for $R_{\text {fission }}$ was chosen based on the approximate fission rate that the ${ }^{252} \mathrm{Cf}$ calibration source would have had at the time of data taking if it were truly isotopically pure.

After each fictitious dataset was generated, a maximum likelihood fit was performed using MINUIT in the same manner as was done for the real data. If an obvious error occurred during fitting (as signaled by a nonzero return code from one of the MINUIT subroutines), the problematic dataset was discarded, and a new one was sampled before continuing. After each successful fit, the pull $g_{\mathrm{NCV}}$ was computed, which is defined by

$$
g_{\mathrm{NCV}} \equiv \frac{\epsilon_{\mathrm{NCV}, \mathrm{fit}}-\epsilon_{\mathrm{NCV}, \mathrm{true}}}{\sigma_{\mathrm{NCV}, \mathrm{fit}}},
$$

where $\epsilon_{\mathrm{NCV} \text {,fit }}$ is the fitted value of the NCV efficiency, $\epsilon_{\mathrm{NCV} \text {,true }}$ is the true value (sampled from the uniform distribution given in eq. (10.17)) of the NCV efficiency, and $\sigma_{\mathrm{NCV} \text {,fit }}$ is the MINUIT estimate of the 1-sigma error on $\epsilon_{\mathrm{NCV} \text {,fit }}$. In the absence of bias and assuming that the estimated error on $\epsilon_{\mathrm{NCV}}$ is accurate, the pull $g_{\mathrm{NCV}}$ will follow a standard normal distribution $\mathcal{N}(0,1)$.

A histogram showing the empirical pull distribution for 10000 fictitious datasets is shown in the top panel of fig. 10.7, together with a Gaussian fit to the results. While the fitted mean of the distribution is consistent with zero, the fitted standard deviation of 0.164 is well below the expected value of 1 , suggesting that the estimated statistical error returned by MINUIT is overly pessimistic. If one rescales the MINUIT error estimate by a factor of 0.165 for each of the 10000 trials, one obtains the corrected pull histogram shown in the bottom panel of fig. 10.7, which is consistent with a standard normal distribution.

These results suggest that, for a reasonably large range of possible true NCV efficiencies, background event rates, etc. (see eqs. (10.17)-(10.21)), and assuming that the simulation produced using FREYA and RAT-PAC is an accurate representation of the underlying physics, the fitting procedure described above will yield an unbiased estimator of the true NCV efficiency. Although, based on the empirical pull distribution, one could justify rescaling the MINUIT estimate of the statistical error for the NCV efficiency by the factor 0.165 , we will opt to be more conservative and use the unaltered value. According 

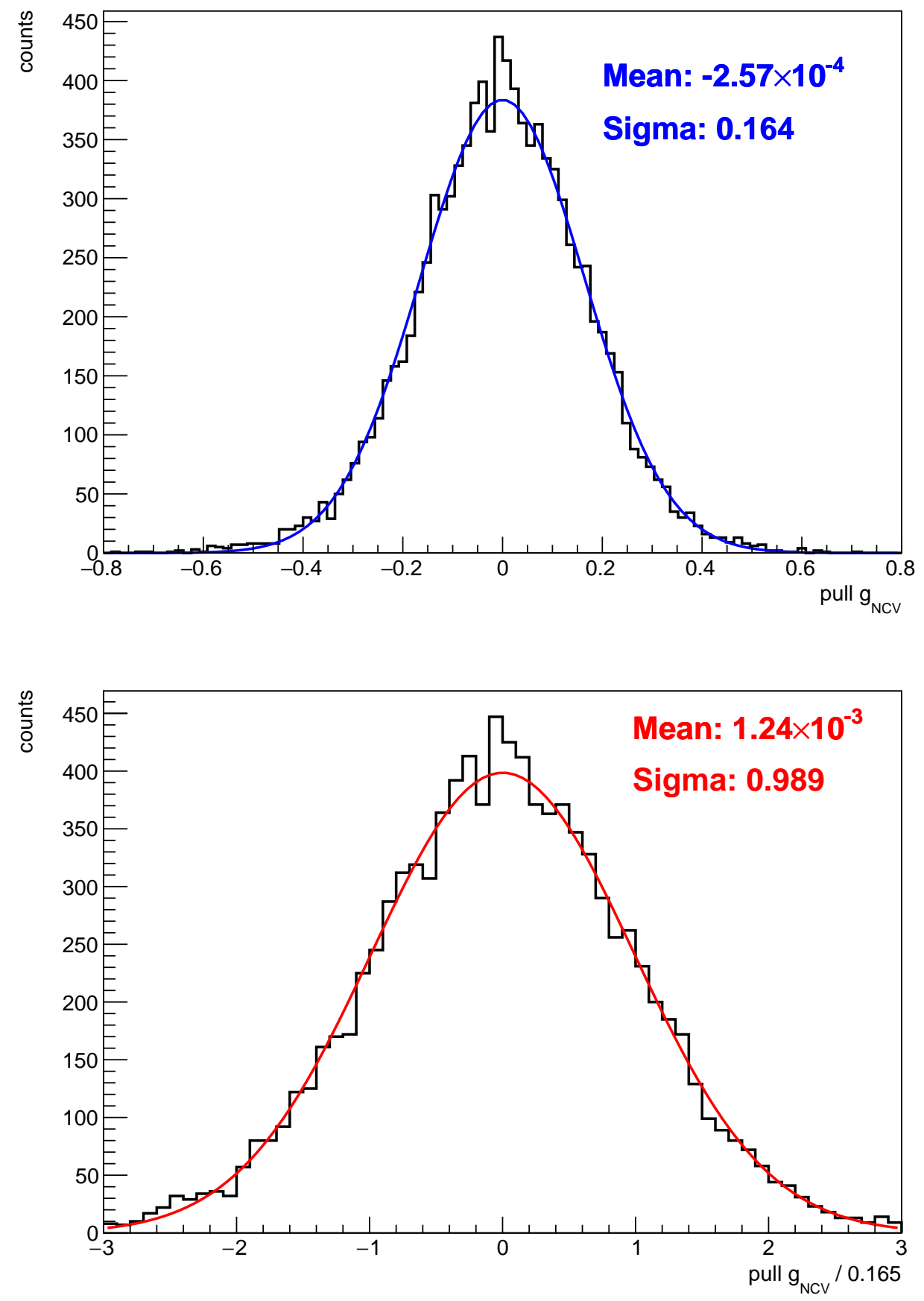

Figure 10.7: TOP: The distribution of the pull $g_{\mathrm{NCV}}$ (see eq. (10.22)) for 10000 fictitious ${ }^{252} \mathrm{Cf}$ calibration source datasets generated using a toy Monte Carlo simulation. A Gaussian fit to the simulation results is shown in blue. BOTTOM: The pull distribution with the estimated 1-sigma error rescaled by a factor of 0.165 . Correcting the error in this way improves the agreement with the expected standard normal distribution. 
to table 10.1, then, the fit to the calibration source data yields the value

$$
\epsilon_{\mathrm{NCV}}=9.60 \pm 0.57(\text { stat }) \%
$$

for the NCV efficiency. To obtain an estimate of the systematic error, we will compare the value obtained using the ${ }^{252} \mathrm{Cf}$ source data with another efficiency calibration method described in the following section.

\subsection{Cosmic muon calibration method}

Alongside the measurements performed using the ${ }^{252} \mathrm{Cf}$ source apparatus described above, a second technique, based on data taken using the cosmic muon trigger, was also used to estimate the NCV neutron detection efficiency. This was done by (1) comparing simulations of muons passing through the NCV with cosmic trigger data to obtain a conversion factor between the summed charge on the two NCV PMTs and the energy deposited in the liquid scintillator, (2) estimating the charge threshold for NCV events and converting it to an energy threshold, and (3) performing simulations to predict the fraction of true neutron captures that will result in an energy deposition above threshold in the NCV liquid.

\subsubsection{Estimate of the charge-to-energy conversion factor}

As was discussed in section 8.4.5, the cosmic ray trigger for ANNIE Phase I allows one to select muon tracks passing nearly directly downward through the NCV. To estimate the charge-to-energy conversion factor for the NCV, simulations of muons passing through the NCV were compared with cosmic trigger data. In the simulations, a cosmic muon event generator originally written for the G4beamline code [481] was adapted for use with RATPAC. Starting locations for each muon track were sampled from a disk high above the ANNIE detector. The initial muon momenta were determined using a $\cos ^{2} \theta$ distribution ${ }^{12}$ for the polar angle $\theta \in\left[0^{\circ}, 70^{\circ}\right.$ ) (with $0^{\circ}$ being straight downward), a uniform distribution for the azimuthal angle $\phi \in[0,2 \pi)$, and an empirical distribution for the momentum magnitude measured using the CAPRICE94 spectrometer as described in reference [482].

\footnotetext{
${ }^{12}$ See section 29.3 .1 of the "Reviews, Tables, and Plots" in reference [78].
} 
Table 10.2: Results of the fits used to estimate an energy threshold for the NCV

\begin{tabular}{cccccc}
\hline Parameter & Variable & Best-fit value $^{a}$ & Fit $^{2}$ & DOF $^{b}$ & p-value \\
\hline $\begin{array}{c}\text { Simulated downward muon } \\
\text { energy deposition peak }\end{array}$ & $E_{\mu, \text { peak }}$ & $91.1(2) \mathrm{MeV}$ & 2.3 & 6 & 0.89 \\
$\begin{array}{c}\text { Downward muon summed } \\
\text { NCV PMT charge peak }\end{array}$ & $Q_{\mu, \text { peak }}$ & $400(8) \mathrm{pC}$ & 7.7 & 7 & 0.36 \\
$\begin{array}{c}\text { Threshold summed } \\
\text { NCV PMT charge peak }\end{array}$ & $Q_{\text {thresh }}$ & $20.9(3) \mathrm{pC}$ & 5.1 & 14 & 0.98 \\
\hline
\end{tabular}

${ }^{a}$ Parameter errors are statistical only

${ }^{b}$ Degrees of freedom

Figure 10.8 shows a histogram of the energy deposited in the NCV by simulated muons that passed through the cosmic trigger paddles in a way that would result in an event being recorded by the ANNIE DAQ. The histogram of deposited energies revealed a clear peak around $91 \mathrm{MeV}$ which was used as a reference point when comparing the simulation results to the cosmic trigger data. To precisely estimate the peak location $E_{\mu \text {,peak }}$ and obtain an associated statistical error, a Gaussian fit (shown in red in the figure) was performed on the simulation results in the vicinity of the peak. The results of the fit are shown in the first row of table 10.2 .

To obtain the charge $Q_{\mu \text {,peak }}$ associated with the corresponding peak in the cosmic trigger data, a sample of $4841 \mathrm{NCV}$ coincidence events ${ }^{13}$ recorded at position \#2 (center of the tank) was analyzed. Each of the selected events occurred within $2 \mu$ s of a downward muon candidate being observed by the cosmic ray trigger. Figure 10.9 shows a histogram of the summed charge observed on the two NCV PMTs for events within that sample. Using the same procedure as for the RAT-PAC simulations, a Gaussian was fit to the peak seen in the data, yielding the results shown in the second row of table 10.2.

Assuming that the total charge observed on the NCV PMTs is approximately a linear function of the energy deposited in the liquid scintillator, the NCV energy threshold

\footnotetext{
${ }^{13}$ All cuts were applied to these events except for the outer PMT veto cut (see chapter 9).
} 


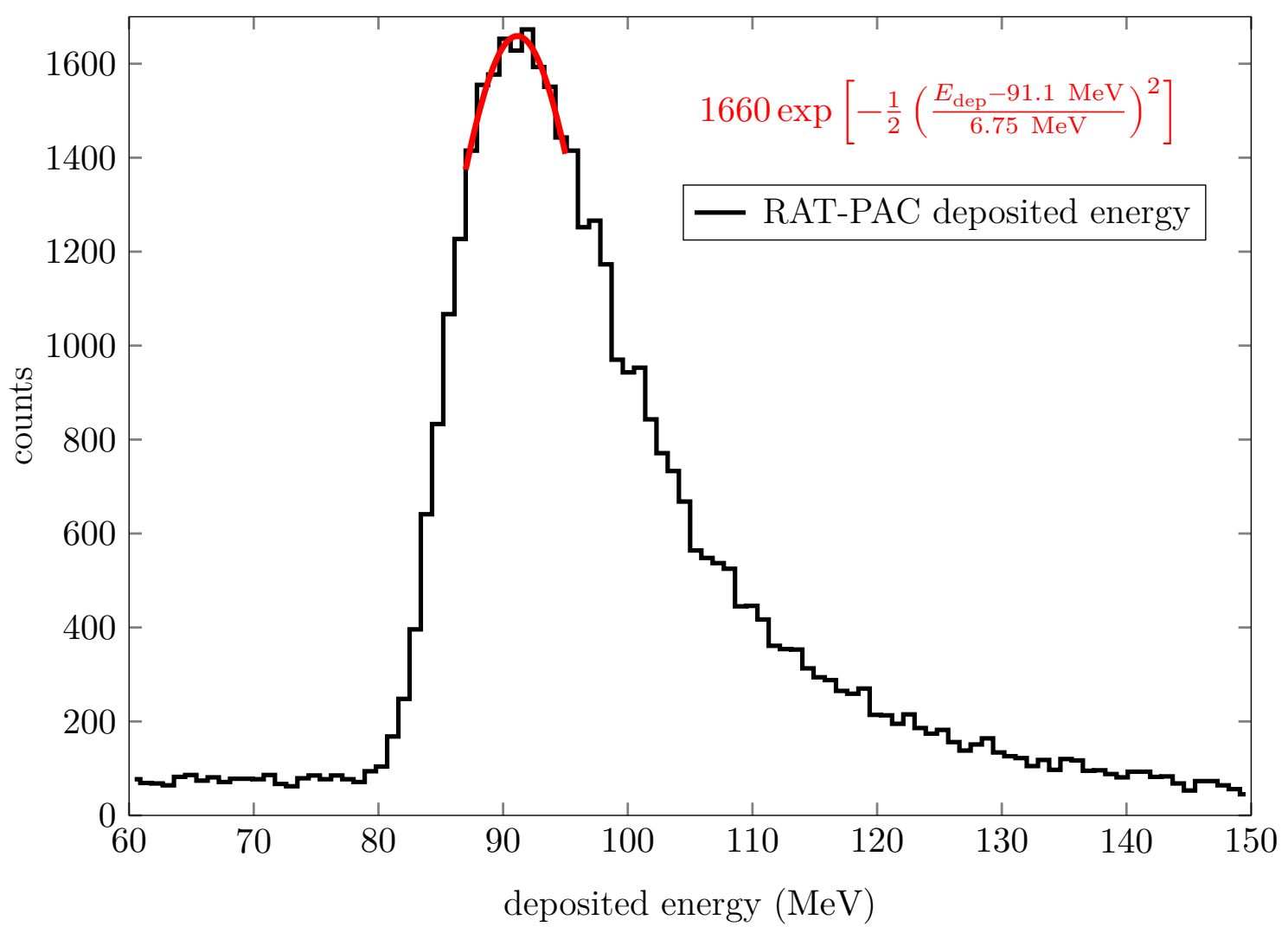

Figure 10.8: Distribution of deposited energies for simulated cosmic muons passing through the cosmic trigger paddles and through the NCV at position \#2 (center of the tank).

$E_{\text {thresh }}$ may be written in the form

$$
E_{\text {thresh }}=Q_{\text {thresh }} \frac{E_{\mu, \text { peak }}}{Q_{\mu, \text { peak }}}
$$

where $Q_{\text {thresh }}$ is the summed charge on the NCV PMTs at threshold, and the ratio $E_{\mu, \text { peak }} / Q_{\mu, \text { peak }}$ is used as a charge-to-energy conversion factor.

\subsubsection{Charge and energy thresholds}

Because the algorithms used to reconstruct PMT pulses in ANNIE Phase I rely on a threshold based on pulse amplitude rather than charge, the total charge collected by the two NCV PMTs for events at threshold will be distributed about some mean value. Figure 10.10 shows a histogram of the total charge $Q_{\text {sum }}$ collected on the two NCV PMTs for a large sample of NCV coincidence events (from all positions) with $Q_{\text {sum }}<100 \mathrm{pC}$. 


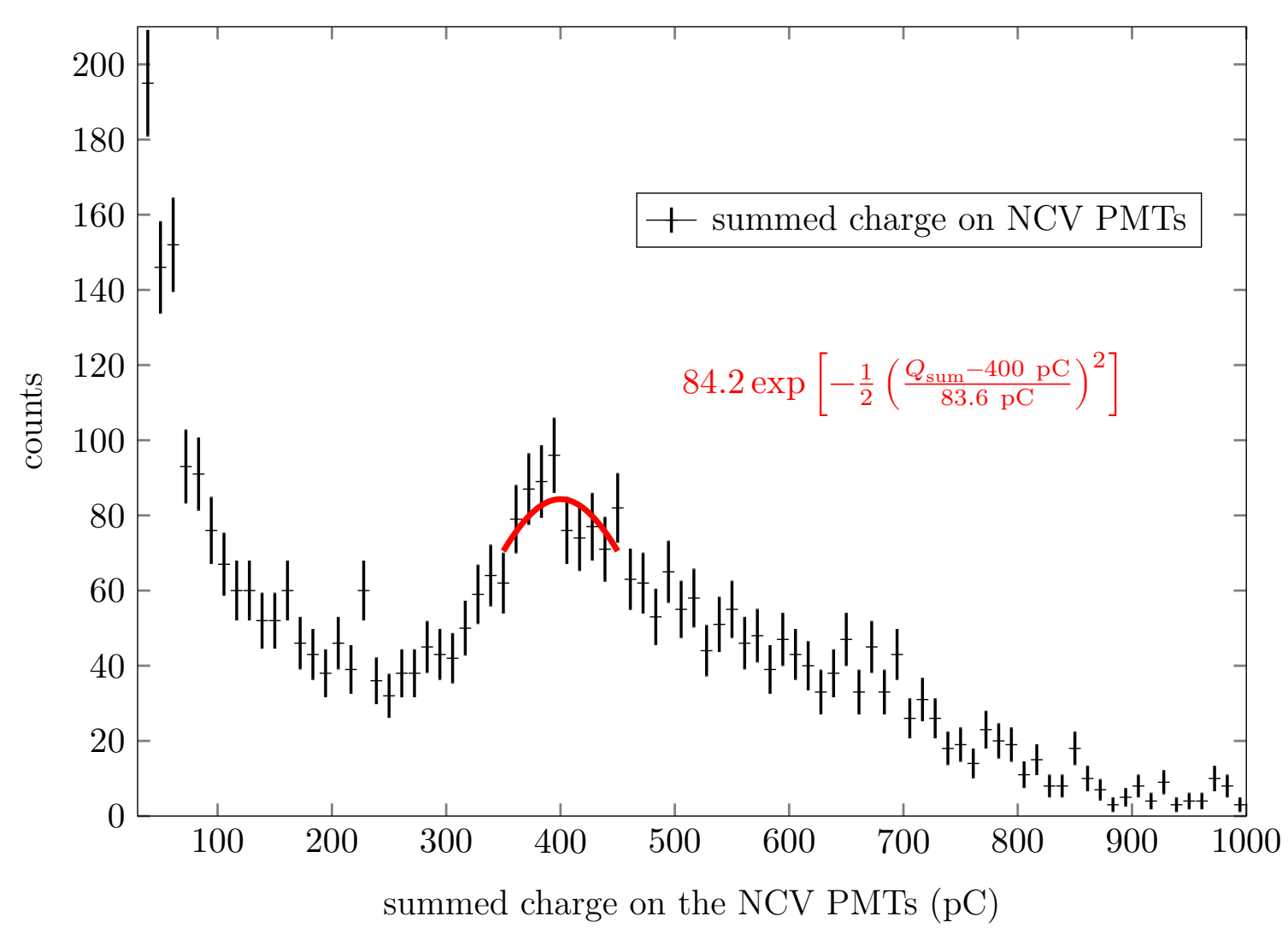

Figure 10.9: Distribution of the total charge collected on the two NCV PMTs at position \#2 (center of the tank) for NCV coincidence events recorded in response to cosmic muon triggers. Only triggers consistent with a muon traveling nearly directly downward through the NCV are included in the plot.

Because the presence of afterpulses (which will generally have small charges) would bias the charge distribution toward an erroneously low mean value, only NCV events that passed the afterpulsing cut described in chapter 9 are included in the histogram. To estimate the mean $Q_{\text {thresh }}$ of the threshold charge distribution, a Gaussian fit (shown in red) was performed in the vicinity of the peak seen at about $20 \mathrm{pC}$ in fig. 10.10. The results of the fit are given in the final row of table 10.2.

Plugging the best-fit parameter values from table 10.2 into eq. (10.24) yields the NCV energy threshold

$$
E_{\text {thresh }}=4.76 \pm 0.12 \text { (stat) } \mathrm{MeV} \text {. }
$$

Here the statistical error $s_{E_{\text {thresh }}}$ was estimated using the relation (valid for independent 


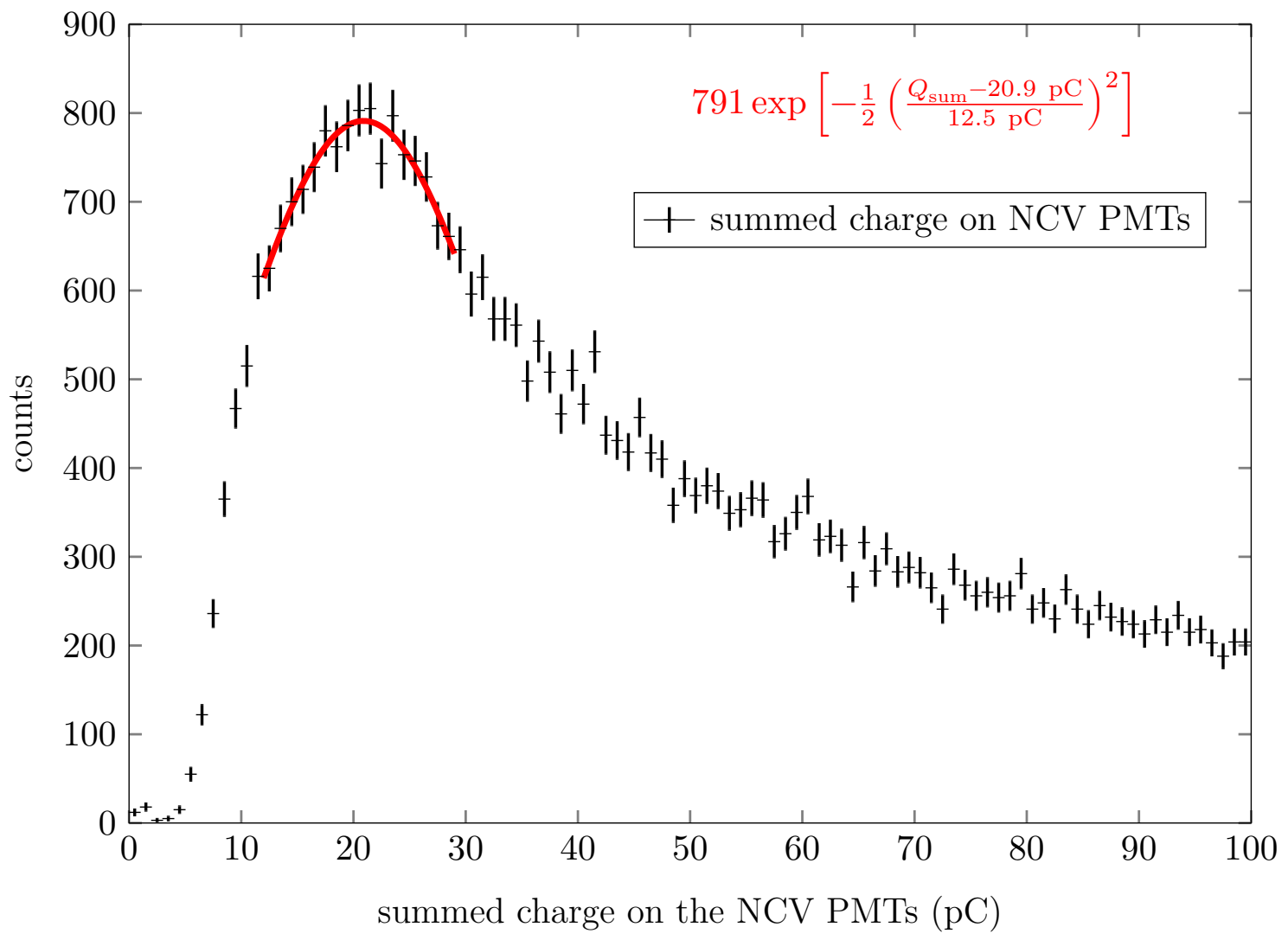

Figure 10.10: Distribution of the total charge collected on the two NCV PMTs for NCV coincidence events recorded at all positions. Only events with low charges (less than $100 \mathrm{pC}$ ) are shown. The afterpulsing cut has been applied to these data to avoid biasing the distribution toward an erroneously low mean charge.

fitted parameters)

$$
\begin{aligned}
s_{E_{\text {thresh }}} & =\sqrt{\left(\frac{\partial E_{\text {thresh }}}{\partial Q_{\text {thresh }}}\right)^{2} s_{Q_{\text {thresh }}}^{2}+\left(\frac{\partial E_{\text {thresh }}}{\partial Q_{\mu, \text { peak }}}\right)^{2} s_{Q_{\mu, \text { peak }}}^{2}+\left(\frac{\partial E_{\text {thresh }}}{\partial E_{\mu, \text { peak }}}\right)^{2} s_{E_{\mu, \text { peak }}}^{2}} \\
& =\sqrt{\left(\frac{E_{\text {peak }}}{Q_{\text {peak }}}\right)^{2} s_{Q_{\text {thresh }}}^{2}+\left(\frac{E_{\text {peak }} Q_{\text {thresh }}}{Q_{\text {peak }}^{2}}\right)^{2} s_{Q_{\mu, \text { peak }}}^{2}+\left(\frac{Q_{\text {thresh }}}{Q_{\text {peak }}}\right)^{2} s_{E_{\mu, \text { peak }}}^{2}}
\end{aligned}
$$

\subsubsection{Efficiency determination}

To determine the NCV neutron detection efficiency $\epsilon_{\mathrm{NCV}}$ that corresponds to the energy threshold given in eq. (10.25), simulations of thermal neutron captures occurring within and around the NCV were generated using RAT-PAC. In each of the $5 \times 10^{6}$ simulated 


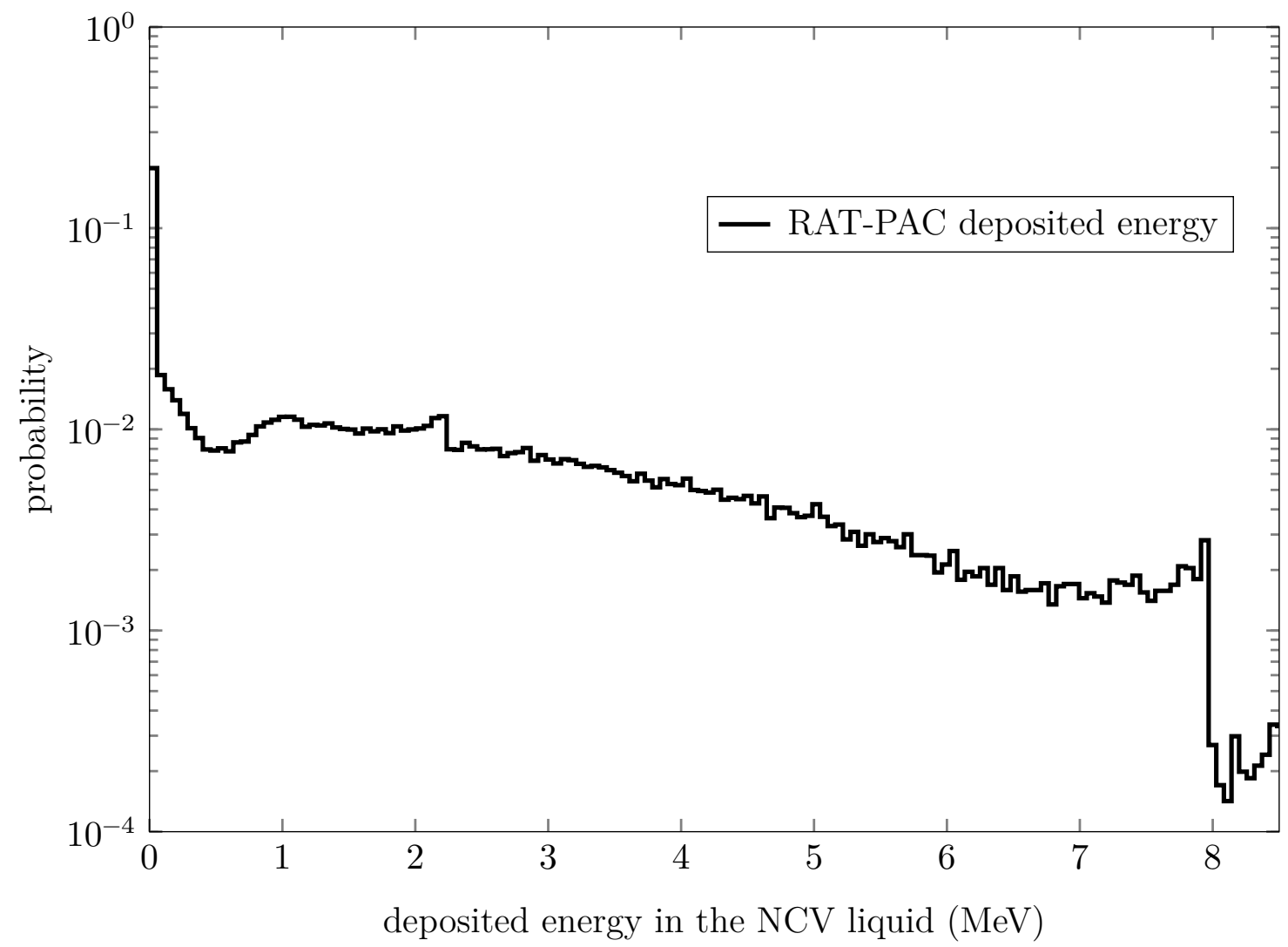

Figure 10.11: Histogram of the total energy deposited in the scintillator for simulated neutron captures that occurred within the NCV liquid volume. Two events above $8.6 \mathrm{MeV}$, attributable to rare neutron captures on ${ }^{14} \mathrm{~N}$, are not shown.

events, a single thermal neutron (with $0.025 \mathrm{eV}$ kinetic energy) track was started with an initial direction sampled isotropically. The starting location of each thermal neutron was sampled uniformly from a $1.5 \mathrm{~m}$ radius sphere centered on the NCV. A total of $N_{\text {liquid }}=70470$ of the simulated events ended with a neutron capture inside the NCV liquid volume. A negligibly small number of external neutron captures led to energy deposits within the NCV liquid, so these were ignored in the efficiency analysis.

Figure 10.11 shows the distribution of the total energy deposited in the liquid scintillator for each of the $N_{\text {liquid }}$ simulated neutron captures that occurred within the NCV liquid volume. The sharp drops seen in the histogram near $2.2 \mathrm{MeV}$ and $7.9 \mathrm{MeV}$ correspond to full absorption of the $\gamma$-rays produced by thermal neutron capture on ${ }^{1} \mathrm{H}$ and ${ }^{157} \mathrm{Gd}$, respectively, the latter nuclide being the Gd isotope with the largest capture cross section. 
For a given NCV energy threshold $E_{\text {thresh }}$, the NCV efficiency $\epsilon_{\mathrm{NCV}}$ may be obtained from the RAT-PAC deposited energy spectrum via

$$
\epsilon_{\mathrm{NCV}}=\frac{\int_{E_{\text {thresh }}}^{\infty} \mathcal{N}_{\text {liquid }}(E) d E}{\int_{0}^{\infty} \mathcal{N}_{\text {liquid }}(E) d E}=\frac{\int_{E_{\text {thresh }}}^{\infty} \mathcal{N}_{\text {liquid }}(E) d E}{N_{\text {liquid }}}
$$

where $\mathcal{N}_{\text {liquid }}(E)$ is the number of simulated neutron captures in the NCV liquid that deposited an energy between $E$ and $E+d E$. Using eq. (10.25) and eq. (10.27) with the simulation results shown in fig. 10.11 yields the result

$$
\epsilon_{\mathrm{NCV}}=12.8 \pm 0.9 \text { (stat) } \% \text {. }
$$

The statistical error shown in eq. (10.28) was found by computing $\epsilon_{\mathrm{NCV}}$ according to eq. (10.27) with the value of $E_{\text {thresh }}$ adjusted by plus or minus its one-sigma error.

\subsection{Adopted value of the NCV efficiency}

While reasonable efforts have been made to estimate statistical errors for both of the NCV efficiency measurements discussed in this chapter, the importance of systematic errors becomes immediately apparent when one compares eq. (10.23) with eq. (10.28). An accepted value of the NCV efficiency with an associated systematic error was determined by treating the values of $\epsilon_{\mathrm{NCV}}$ obtained using the ${ }^{252} \mathrm{Cf}$ source data and using the cosmic muon data as two independent measurements. If one weights each of the two measurements $x_{i}$ by the reciprocal of its squared statistical error, i.e., if one associates the weight

$$
w_{i}=\frac{1}{\sigma_{i}^{2}}
$$

with the $i$ th measurement (where $\sigma_{i}$ is the quoted statistical error), then the weighted sample mean $\bar{x}$ is given by

$$
\bar{x}=\frac{\sum_{i} w_{i} x_{i}}{\sum_{i} w_{i}}
$$

and has the standard error

$$
\sigma_{\bar{x}}=\sqrt{\frac{1}{\sum_{i} w_{i}}} .
$$


In terms of the weights $w_{i}$ and the weighted sample mean $\bar{x}$, the sample standard deviation $s$ (which we will take as an estimate of the systematic error on $\bar{x}$ ) is given by

$$
s=\sqrt{\frac{\sum_{i} w_{i}\left(x_{i}-\bar{x}\right)^{2}}{\left(\sum_{i} w_{i}\right)-\left(\frac{\sum_{i} w_{i}^{2}}{\sum_{i} w_{i}}\right)}} .
$$

Applying eqs. (10.29)-(10.32) to the two measurements of the NCV efficiency gives

$$
\epsilon_{\mathrm{NCV}}=\bar{x} \pm \sigma_{\bar{x}}(\text { stat }) \pm s(\text { syst })=10.5 \pm 0.5(\text { stat }) \pm 2.3(\text { syst }) \% .
$$

In the following chapter, this efficiency value will be used with the results from chapter 9 to compute position-dependent background neutron event rates. 


\section{Chapter 11}

\section{Background neutron event rates}

Having determined in previous chapters values of $\epsilon_{\mathrm{NCV}}$, the NCV detection efficiency, and $N_{n}$, the number of observed neutron candidate events at each NCV position, we are now in a position to convert these quantities into an expected event rate in the NCV. In eq. (8.8), the neutron event rate (in neutron captures per unit volume per POT) in the NCV was written in the form

$$
\mathcal{R}_{n}^{\mathrm{NCV}}=\frac{N_{n}}{\mathcal{P} \epsilon_{\mathrm{NCV}} V_{\mathrm{NCV}}},
$$

where the exposure $\mathcal{P}$ is the total number of protons on target and $V_{\mathrm{NCV}}$ is the volume of the NCV liquid. Assuming that the errors on these quantities are uncorrelated allows one to analytically compute the estimated uncertainty $\sigma_{\mathcal{R}_{n}^{\mathrm{NCV}}}$ on $\mathcal{R}^{\mathrm{NCV}}$ via the relation

$$
\begin{aligned}
\sigma_{\mathcal{R}_{n}^{\mathrm{NCV}}} & =\sqrt{\left(\frac{\partial \mathcal{R}_{n}^{\mathrm{NCV}}}{\partial N_{n}}\right)^{2} \sigma_{N_{n}}^{2}+\left(\frac{\partial \mathcal{R}_{n}^{\mathrm{NCV}}}{\partial \epsilon_{\mathrm{NCV}}}\right)^{2} \sigma_{\epsilon_{\mathrm{NCV}}}^{2}+\left(\frac{\partial \mathcal{R}_{n}^{\mathrm{NCV}}}{\partial \mathcal{P}}\right)^{2} \sigma_{\mathcal{P}}^{2}+\left(\frac{\partial \mathcal{R}_{n}^{\mathrm{NCV}}}{\partial V_{\mathrm{NCV}}}\right)^{2} \sigma_{V_{\mathrm{NCV}}}^{2}} \\
& =R_{n}^{\mathrm{NCV}} \sqrt{\left(\frac{\sigma_{N_{n}}}{N_{n}}\right)^{2}+\left(\frac{\sigma_{\epsilon_{\mathrm{NCV}}}}{\epsilon_{\mathrm{NCV}}}\right)^{2}+\left(\frac{\sigma_{\mathcal{P}}}{\mathcal{P}}\right)^{2}+\left(\frac{\sigma_{V_{\mathrm{NCV}}}}{V_{\mathrm{NCV}}}\right)^{2}} .
\end{aligned}
$$

The uncertainties on $N_{n}$ and $\epsilon_{\mathrm{NCV}}$ have been discussed at length in previous chapters. For the exposure $\mathcal{P}$, we adopt the $2 \%$ systematic uncertainty found during routine calibrations of the beam current toroids (see section 8.2.2). For $V_{\mathrm{NCV}}$, we note that, when the NCV is full, the volume of liquid scintillator contained therein is given by

$$
V_{\mathrm{NCV}}=\pi\left(\frac{D}{2}-W\right)^{2} L
$$


Table 11.1: Measured rates of neutron candidate events from ANNIE Phase I. A nominal value of $5 \times 10^{12}$ POT per spill is assumed in the second column.

\begin{tabular}{cc}
\hline NCV position & $\begin{array}{c}\text { Neutron candidate event rate } \mathcal{R}_{n}^{\mathrm{NCV}} \\
\left(\% \mathrm{~m}^{-3} \mathrm{spill}^{-1}\right)\end{array}$ \\
\hline 1 & $20.5 \pm 1.0($ stat $) \pm 4.7$ (syst) \\
2 & $0.95 \pm 0.06($ stat $) \pm 0.23$ (syst) \\
3 & $2.5 \pm 0.1($ stat $) \pm 0.6$ (syst) \\
4 & $3.5 \pm 0.2($ stat $) \pm 0.8$ (syst) \\
5 & $1.8 \pm 0.1($ stat $) \pm 0.4$ (syst) \\
6 & $4.4 \pm 0.3($ stat $) \pm 1.0$ (syst) \\
7 & $1.3 \pm 0.2($ stat $) \pm 0.3$ (syst) \\
\hline
\end{tabular}

where $D$ is the outer diameter of the acrylic tube, $W$ is the tube wall thickness, and $L$ is the length of the tube. Using the numerical values given in section 8.4.2 and estimating uncorrelated uncertainties of 0.5 in for $D$ and $L$ and $1 / 16$ in for $W$, we find that the relative systematic uncertainty on the NCV liquid volume $V_{\mathrm{NCV}}$ is given by

$$
\begin{aligned}
\frac{\sigma_{V_{\mathrm{NCV}}}}{V_{\mathrm{NCV}}} & =\frac{1}{V_{\mathrm{NCV}}} \sqrt{\left(\frac{\partial V_{\mathrm{NCV}}}{\partial D}\right)^{2} \sigma_{D}^{2}+\left(\frac{\partial V_{\mathrm{NCV}}}{\partial W}\right)^{2} \sigma_{W}^{2}+\left(\frac{\partial V_{\mathrm{NCV}}}{\partial L}\right)^{2} \sigma_{L}^{2}} \\
& =\frac{\sqrt{4(D-2 W)^{2} L^{2}\left(\sigma_{D}^{2}+4 \sigma_{W}^{2}\right)+(D-2 W)^{4} \sigma_{L}^{2}}}{(D-2 W)^{2} L}=5.7 \% .
\end{aligned}
$$

By combining the accepted value of $\epsilon_{\mathrm{NCV}}$ from eq. (10.33) and the neutron candidate event counts $N_{n}$ tabulated in table 9.1, one may use eqs. (11.1) and (11.2) to obtain the measured rates $\mathcal{R}_{n}^{\mathrm{NCV}}$ of neutron candidate events at each NCV position. The results of this calculation are shown in table 11.1 and fig. 11.1.

A striking feature in fig. 11.1 is the much larger background event rate observed at position \#1 (the top center of the tank) compared to all other NCV positions. Since the NCV had only $6 \mathrm{~cm}$ of water above it at this position, compared with more than three times this amount at position \#6, the next-shallowest position (see table 8.2), the large event rate suggests that skyshine neutrons, particularly low-energy ones that 


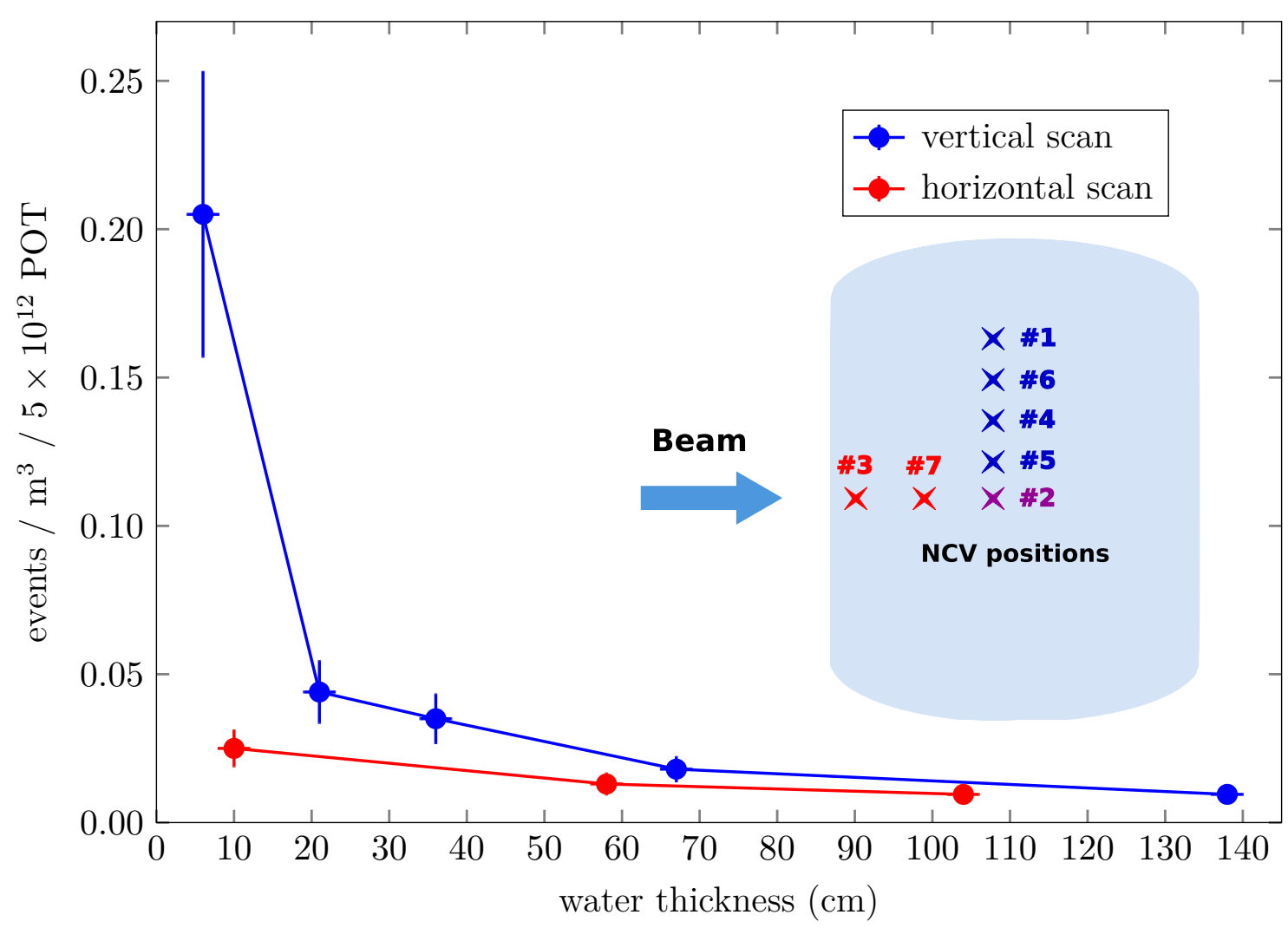

Figure 11.1: Neutron candidate event rates measured during ANNIE Phase I. The inset diagram shows the NCV positions included in the red and blue datasets. Position \#2 (the center of the tank) is shown in purple to indicate that it is included in both the red and blue data. For the blue dataset, the "water thickness" is the depth of the water above the top of the NCV. For the red dataset, it is the smallest distance between the side of the tube forming the NCV vessel and the beam side of the tank. Error bars shown in the plot include both statistical and systematic contributions. 
cannot penetrate deeply into the tank, provide a sizeable background in the absence of appropriate shielding. While difficult to compare quantitatively with previous SciBooNE results indicating the presence of significant skyshine background at the BNB, the large excess of events observed by that experiment near the top of the detector briefly after the beam crossing (see fig. 8.16) is at least qualitatively consistent with our results.

Although the separate horizontal and vertical scans of the tank performed during Phase I data taking will not fully separate the contributions of dirt and skyshine neutrons, the dramatic difference in the rates measured at position \#1 (the top center of the tank) and at position \#3 (vertically centered and close to the beam-side wall of the tank) suggests that skyshine is the more dominant of the two beam-associated neutron backgrounds.

When considering the practical implications of these results for ANNIE Phase II, it should be noted that, because no subtraction of the constant-in-time (as opposed to beam-correlated) background rate was performed for these data, the rates reported in this chapter include an unknown but potentially significant contribution from cosmic-ray induced neutrons, which arrived randomly in the NCV with respect to the beam. This component of the background may be easily characterized using a random off-beam trigger in Phase II. While a measurement of the constant-in-time component of the background was attempted during Phase I, insufficient random trigger data were taken for the zero suppression mode runs. As a result, large statistical errors on the constant-in-time background rates prevented a meaningful subtraction of the constant-in-time component from the measured Phase I event rates.

Despite the limitations of the Phase I data, the measured rates nevertheless allow unambiguous statements about the feasibility of the planned Phase II physics measurements. Examination of table 11.1 and fig. 11.1 demonstrates that, if the entired planned $2.5 \mathrm{~m}^{3}$ fiducial volume is chosen to be at least $60 \mathrm{~cm}$ from all of the tank walls, then no more than about $6 \%$ of spills will involve a background neutron capture within the fiducial volume. If the measurement at position \#2 (the center of the tank) is more reflective of the average background event rate of the chosen fiducial volume, then this 
fraction drops to less than $3 \%$. It should be recognized that these rates are likely to be overly pessimistic, given the unknown constant-in-time component of the background and the fact that, as argued in section 8.6, the measured NCV rates represent only an upper limit on the corresponding neutron capture rates in the Phase II Gd-loaded water. Because the presence of background neutrons in the fiducial volume and the occurrence of a charged current neutrino interaction therein may be treated as uncorrelated events, one may conclude from the Phase I results that only a few percent of signal events in Phase II will be contaminated by a background neutron. 
Appendices 


\section{Appendix A}

\section{Derivation of the compound nucleus formation cross section}

Consider an arbitrary two-body reaction

$$
a+A \rightarrow b+B
$$

in which a projectile $a$ strikes a target $A$ to produce an ejectile $b$ and a residue $B$. Assume that $a$ and $A$ are distinguishable (in the sense that they are not identical particles), that $b$ and $B$ are distinguishable, and that all four particles have nonzero masses. Let the label $\alpha$ refer to the initial channel, i.e., the set of quantum numbers (apart from angular momenta, which we will consider separately) needed to specify the initial configuration of the $a+A$ system, including the masses, charges, etc. of the two particles. Let the label $\beta$ refer to the final channel, which we define in terms of the same set of quantum numbers as $\alpha$. Then it can be shown [262] that the total cross section for the reaction given in eq. (A.1) is given by ${ }^{1}[309]$

$$
\sigma_{\alpha \beta}=\frac{\pi}{\left|\mathbf{p}_{\alpha}\right|^{2}\left(2 s_{a}+1\right)\left(2 s_{A}+1\right)} \sum_{J \ell j \ell^{\prime} j^{\prime}}(2 J+1)\left|\delta_{\ell^{\prime} \ell} \delta_{j^{\prime} j} \delta_{\alpha \beta}-S_{\ell^{\prime} j^{\prime} \beta, \ell j \alpha}^{J}\right|^{2} \text { (CM frame, A.2) }
$$

\footnotetext{
${ }^{1}$ The expression shown here uses the "J basis" of reference [309], in which the third angular momentum quantum number (in addition to the total angular momentum $J$ and the orbital angular momentum $\ell$ ) used to define the entrance channel is the projectile's total angular momentum $\mathbf{j} \equiv \boldsymbol{\ell}+\boldsymbol{s}_{a}$ (with a similar definition for the exit channel). In reference [262], Weinberg uses the "S basis," in which the third angular momentum quantum number defining the entrance channel is instead chosen to be the "channel spin" $\boldsymbol{s}=\boldsymbol{s}_{a}+\boldsymbol{s}_{A}$.
} 
where $\left|\mathbf{p}_{\alpha}\right|$ is the magnitude of the 3-momentum of one of the initial particles in the CM frame, and $S_{\ell^{\prime} j^{\prime} \beta, \ell j \alpha}^{J}$ is the S-matrix element representing the amplitude for the reaction to occur for a particular configuration of angular momenta. The composite angular momenta $j, j^{\prime}$, and $J$ are given by

$$
\begin{array}{ll}
\boldsymbol{j}=\boldsymbol{\ell}+\boldsymbol{s}_{a} & \Longrightarrow\left|\ell-s_{a}\right| \leq j \leq \ell+s_{a} \\
\boldsymbol{j}^{\prime}=\boldsymbol{\ell}^{\prime}+\boldsymbol{s}_{b} & \Longrightarrow\left|\ell^{\prime}-s_{b}\right| \leq j^{\prime} \leq \ell^{\prime}+s_{b} \\
\mathbf{J}=\boldsymbol{\ell}+\boldsymbol{s}_{A}=\boldsymbol{\ell}^{\prime}+\boldsymbol{s}_{B} & \Longrightarrow\left|\ell-s_{A}\right| \leq J \leq \ell+s_{A}
\end{array}
$$

where $\ell$ is the relative orbital angular momentum between $a$ and $A, \ell^{\prime}$ is the same for $b$ and $B$, and $s_{a}, s_{A}, s_{b}$, and $s_{B}$ are the total spins of $a, A, b$, and $B$, respectively. The sum over all possible angular momenta in eq. (A.2) may be written explicitly as

$$
\sum_{J \ell j \ell^{\prime} j^{\prime}} \equiv \sum_{J=\left|\ell-s_{A}\right|}^{\ell+s_{A}} \sum_{\ell=0}^{\infty} \sum_{j=\left|\ell-s_{a}\right|}^{\ell+s_{a}} \sum_{\ell^{\prime}=0}^{\infty} \sum_{j^{\prime}=\left|\ell-s_{b}\right|}^{\ell+s_{b}} .
$$

The factors of $\left(2 s_{a}+1\right),\left(2 s_{A}+1\right)$, and $(2 J+1)$ appear in our expression for $\sigma_{\alpha \beta}$ because we have assumed that the initial and final spin orientations of the particles are unobserved.

Although eq. (A.2) is often derived in a nonrelativistic context by assuming a particular form for a scattering potential, ${ }^{2}$ the result depends only on symmetries (e.g., rotational invariance) and unitarity of the S-matrix, the latter of which is implied by conservation of probability. It remains fully valid for relativistic scattering [262].

Equation (A.2) allows us to write the cross section $\sigma_{\text {el }}$ for elastic scattering (in which the initial and final channels are the same) in the form

$$
\sigma_{\mathrm{el}}=\sigma_{\alpha \alpha}=\frac{\pi}{\left|\mathbf{p}_{\alpha}\right|^{2}\left(2 s_{a}+1\right)\left(2 s_{A}+1\right)} \sum_{J \ell j}(2 J+1)\left|1-S_{\ell j \alpha, \ell j \alpha}^{J}\right|^{2} . \quad(\text { CM frame, A.7) }
$$

Since the S-matrix is unitary, i.e.,

$$
\left(S^{J}\right)^{\dagger} S^{J}=I
$$

\footnotetext{
${ }^{2}$ See, e.g., reference [284].
} 
where $I$ is the identity matrix, it follows that ${ }^{3}$

$$
\sum_{\ell^{\prime} j^{\prime} \beta}\left|S_{\ell^{\prime} j^{\prime} \beta, \ell j \alpha}^{J}\right|^{2}=1
$$

and

$$
\sum_{\beta \neq \alpha} \sum_{\ell^{\prime} j^{\prime}}\left|S_{\ell^{\prime} j^{\prime} \beta, \ell j \alpha}^{J}\right|^{2}=1-\left|S_{\ell j \alpha, \ell j \alpha}^{J}\right|^{2} .
$$

The reaction cross section $\sigma_{R}$, defined as the cross section for all final channels $\beta$ other than the entrance channel $\alpha$, may therefore be written in the form

$$
\sigma_{R}=\sum_{\beta \neq \alpha} \sigma_{\alpha \beta}=\frac{\pi}{\left|\mathbf{p}_{\alpha}\right|^{2}\left(2 s_{a}+1\right)\left(2 s_{A}+1\right)} \sum_{J \ell j}(2 J+1)\left(1-\left|S_{\ell j \alpha, \ell j \alpha}^{J}\right|^{2}\right) .
$$

(CM frame, A.11)

The total cross section $\sigma_{\text {tot }}$ is the sum of the elastic scattering and reaction cross sections:

$$
\sigma_{\text {tot }}=\sigma_{\text {el }}+\sigma_{R}=\frac{2 \pi}{\left|\mathbf{p}_{\alpha}\right|^{2}\left(2 s_{a}+1\right)\left(2 s_{A}+1\right)} \sum_{J \ell j}(2 J+1) \Re\left(1-S_{\ell j \alpha, \ell j \alpha}^{J}\right) .
$$

(CM frame, A.12)

In the regime of high excitation energies where the compound nucleus picture is appropriate, the spacing between the nuclear levels is small, and it becomes useful to consider the average values of these cross sections over an energy window that contains many states of the intermediate compound nucleus with approximately the same excitation energy. Let $\Delta E_{x}$ be the excitation energy interval of interest, chosen large enough that it includes many compound nuclear levels but small enough that slowly varying functions of energy (including $\left|\mathbf{p}_{\alpha}\right|^{2}$ ) may be approximated as constant within it. Then the energy average of the S-matrix element $S_{\ell j \alpha, \ell j \alpha}^{J}$ may be defined by

$$
\left\langle S_{\ell j \alpha, \ell j \alpha}^{J}(\varepsilon)\right\rangle \equiv \frac{1}{\Delta E_{x}} \int_{\varepsilon-\Delta E_{x} / 2}^{\varepsilon+\Delta E_{x} / 2} S_{\ell j \alpha, \ell j \alpha}^{J}\left(\varepsilon^{\prime}\right) d \varepsilon^{\prime}
$$

where the S-matrix element is expressed as a function of the total kinetic energy in the CM frame $\varepsilon$.

\footnotetext{
${ }^{3}$ For notational simplicity and later convenience, we have written the sum in eq. (A.9) to include all possible two-body final channels. If other final channels (e.g., a three-body channel) cannot be neglected, then the sum should be understood to include these possibilities as well. The same is true for the sum in eq. (A.10).
} 
Since

$$
\begin{aligned}
\left\langle\left|1-S_{\ell j \alpha, \ell j \alpha}^{J}\right|^{2}\right\rangle & =\left|1-\left\langle S_{\ell j \alpha, \ell j \alpha}^{J}\right\rangle\right|^{2}+\left\langle\left|S_{\ell j \alpha, \ell j \alpha}^{J}\right|^{2}\right\rangle-\left|\left\langle S_{\ell j \alpha, \ell j \alpha}^{J}\right\rangle\right|^{2} \\
& =\left|1-\left\langle S_{\ell j \alpha, \ell j \alpha}^{J}\right\rangle\right|^{2}+\operatorname{Var}\left(S_{\ell j \alpha, \ell j \alpha}^{J}\right),
\end{aligned}
$$

where

$$
\operatorname{Var}\left(S_{\ell j \alpha, \ell j \alpha}^{J}\right) \equiv\left\langle\left|S_{\ell j \alpha, \ell j \alpha}^{J}\right|^{2}\right\rangle-\left|\left\langle S_{\ell j \alpha, \ell j \alpha}^{J}\right\rangle\right|^{2}
$$

is the variance of $S_{\ell j \alpha, \ell j \alpha}^{J}$, it follows that the energy-averaged elastic scattering cross section $\left\langle\sigma_{\mathrm{el}}\right\rangle$ may be decomposed into two contributions:

$$
\left\langle\sigma_{\mathrm{el}}\right\rangle=\left\langle\sigma_{\mathrm{se}}\right\rangle+\left\langle\sigma_{\mathrm{ce}}\right\rangle .
$$

Here the shape elastic scattering cross section $\sigma_{\mathrm{se}}$ is given by

$$
\left\langle\sigma_{\mathrm{se}}\right\rangle=\frac{\pi}{\left|\mathbf{p}_{\alpha}\right|^{2}\left(2 s_{a}+1\right)\left(2 s_{A}+1\right)} \sum_{J \ell j}(2 J+1)\left|1-\left\langle S_{\ell j \alpha, \ell j \alpha}^{J}\right\rangle\right|^{2},
$$

and the compound elastic cross section $\sigma_{\text {ce }}$ is given by

$$
\left\langle\sigma_{\mathrm{ce}}\right\rangle=\frac{\pi}{\left|\mathbf{p}_{\alpha}\right|^{2}\left(2 s_{a}+1\right)\left(2 s_{A}+1\right)} \sum_{J \ell j}(2 J+1) \operatorname{Var}\left(S_{\ell j \alpha, \ell j \alpha}^{J}\right) .
$$

This decomposition of the elastic scattering cross section separates it into a smooth, non-fluctuating piece $\sigma_{\text {se }}$ representing a direct reaction (without the formation of an intermediate compound nucleus) and a piece that rapidly fluctuates with energy due to the presence of many closely-spaced compound nucleus states that may be temporarily formed before re-emission of the projectile in the entrance channel [337, 483].

If one assumes that direct reaction channels other than shape elastic scattering may be neglected, then the energy-averaged compound nucleus formation cross section $\left\langle\sigma_{C}\right\rangle$ is given by the sum of the average reaction cross section and the compound elastic cross section:

$$
\begin{aligned}
\left\langle\sigma_{C}\right\rangle & =\left\langle\sigma_{R}\right\rangle+\left\langle\sigma_{\text {ce }}\right\rangle \\
& =\frac{\pi}{\left|\mathbf{p}_{\alpha}\right|^{2}\left(2 s_{a}+1\right)\left(2 s_{A}+1\right)} \sum_{J \ell j}(2 J+1)\left(1-\left|\left\langle S_{\ell j \alpha, \ell j \alpha}^{J}\right\rangle\right|^{2}\right) .
\end{aligned}
$$


For reactions involving the formation of a compound nucleus with a specific total spin $J$ and intrinsic parity $\Pi$, one may drop the sum over $J$ and write

$$
\left\langle\sigma_{C}\right\rangle=\frac{\pi(2 J+1)}{\left|\mathbf{p}_{\alpha}\right|^{2}\left(2 s_{a}+1\right)\left(2 s_{A}+1\right)} \sum_{\ell j} \delta_{\pi} T_{\ell j}(\varepsilon)
$$

where the transmission coefficient $T_{\ell j}(\varepsilon)$ is defined by

$$
T_{\ell j}(\varepsilon) \equiv 1-\left|\left\langle S_{\ell j \alpha, \ell j \alpha}^{J}\right\rangle\right|^{2} .
$$

The factor $\delta_{\pi}$ is defined as in eq. (4.8) and has been introduced to explicitly enforce parity conservation.

During the derivation of these results, it has been assumed that interference between different $\ell j$ values and different $J$ values may be neglected. This may be justified by assuming that the elements of the S-matrix have random phases so that the interference terms will average to zero [337]. For a review of the connection between random matrix theory and statistical treatments of compound-nucleus reactions, see reference [484]. 


\section{Appendix B}

\section{Tensor product lemma}

Let $\mathbf{x}$ be an arbitrary 3 -vector with magnitude

$$
r \equiv|\mathbf{x}|
$$

and let $\mathbf{q}$ be a 3-vector directed along the positive $z$ axis, i.e., let

$$
\mathbf{q}=\kappa \hat{\mathbf{z}}
$$

for some $\kappa \geq 0$. Since $\mathbf{q}$ lies along the positive $z$ axis, one may write

$$
e^{i \mathbf{q} \cdot \mathbf{x}}=\sum_{J} i^{J} \sqrt{4 \pi(2 J+1)} j_{J}(\kappa r) Y_{J 0}(\hat{\mathbf{r}})
$$

where $j_{J}(x)$ is the $J$ th spherical Bessel function of the first kind, $Y_{J 0}(\hat{\mathbf{r}})$ is a spherical harmonic, and

$$
\widehat{\mathbf{r}} \equiv \frac{\mathbf{x}}{r}
$$

is a unit vector in the direction of $\mathbf{x}$. Although explicit limits for the sums in this appendix will be omitted for tidiness, every sum shown here should be understood to include all nonnegative integer values of the index/indices of summation, e.g.,

$$
\begin{aligned}
& \sum_{J} \equiv \sum_{J=0}^{\infty} \\
& \sum_{p n} \equiv \sum_{p=0}^{\infty} \sum_{n=0}^{\infty} .
\end{aligned}
$$


Consider the $M$ th component $T_{L M}$ of an arbitrary spherical tensor operator $T$ of rank $R$. From eq. (B.3), it follows that

$$
\begin{aligned}
e^{i \mathbf{q} \cdot \mathbf{x}} T_{R M} & =\sum_{J} i^{J} \sqrt{4 \pi(2 J+1)} j_{J}(\kappa r) Y_{J 0}(\hat{\mathbf{r}}) T_{R M} \\
& =\sum_{J} i^{J} \sqrt{4 \pi(2 J+1)} j_{J}(\kappa r) \sum_{p n} \delta_{p 0} \delta_{M n} Y_{J p}(\hat{\mathbf{r}}) T_{R n}
\end{aligned}
$$

where the Kronecker delta function is defined as in eq. (3.107). Since angular momentum eigenstates are orthonormal to each other, it follows that the inner product of the state vectors $\mid J 0 R M)$ and $\mid J p R n)$ is given by

$$
(J 0 R M \mid J p R n)=\delta_{p 0} \delta_{M n} .
$$

Substituting this result into eq. (B.5) yields the expression

$$
\begin{aligned}
e^{i \mathbf{q} \cdot \mathbf{x}} T_{R M} & =\sum_{J} i^{J} \sqrt{4 \pi(2 J+1)} j_{J}(\kappa r) \sum_{p n}(J 0 R M \mid J p R n) Y_{J p}(\hat{\mathbf{r}}) T_{R n} \\
& =\sum_{J} i^{J} \sqrt{4 \pi(2 J+1)} j_{J}(\kappa r) \Sigma_{p n}
\end{aligned}
$$

where $\Sigma_{p n}$ has been defined as

$$
\Sigma_{p n} \equiv \sum_{p n}(J 0 R M \mid J p R n) Y_{J p}(\hat{\mathbf{r}}) T_{R n} .
$$

Recall that the identity operator may be written as a sum over a complete set of states, i.e.,

$$
\left.\sum_{L k} \mid L k\right)(L k \mid=1 .
$$

One may therefore insert a complete set of states into the sum over $p$ and $n$ to obtain

$$
\begin{aligned}
\Sigma_{p n} & =\sum_{p n}\left(J 0 R M\left|\left[\sum_{L k} \mid L k\right)(L k \mid]\right| J p L n\right) Y_{J p}(\hat{\mathbf{r}}) T_{R n} \\
& =\sum_{L k}(J 0 R M \mid L k) \sum_{p n}(L k \mid J p L n) Y_{J p}(\hat{\mathbf{r}}) T_{R n} .
\end{aligned}
$$

Since the Clebsch-Gordan coefficients may always be chosen to be real, [485] it follows that

$$
(L k \mid J p L n)=(J p L n \mid L k)^{*}=(J p L n \mid L k),
$$


and therefore

$$
\Sigma_{p n}=\sum_{L k}(J 0 R M \mid L k) \sum_{p n}(J p L n \mid L k) Y_{J p}(\hat{\mathbf{r}}) T_{R n} .
$$

Since the outer Clebsch-Gordan coefficient $(J 0 R M \mid L k)$ vanishes unless $k=M$, the sum over $k$ may be removed:

$$
\Sigma_{p n}=\sum_{L}(J 0 R M \mid L M) \sum_{p n}(J p L n \mid L M) Y_{J p}(\widehat{\mathbf{r}}) T_{R n} .
$$

The tensor product $\left[\mathbf{T}_{L_{1}} \otimes \mathbf{T}_{L_{2}}\right]_{L M}$ of two spherical tensors $\mathbf{T}_{L_{1}}$ (of rank $L_{1}$ ) and $\mathbf{T}_{L_{2}}\left(\right.$ of rank $\left.L_{2}\right)$ is defined by [260]

$$
\left[\mathbf{T}_{L_{1}} \otimes \mathbf{T}_{L_{2}}\right]_{L M} \equiv \sum_{M_{1} M_{2}}\left(L_{1} M_{1} L_{2} M_{2} \mid L M\right) T_{L_{1} M_{1}} T_{L_{2} M_{2}}
$$

Since the spherical harmonic $Y_{J p}(\hat{\mathbf{r}})$ is the $p$ th component of a spherical tensor of rank $J$, eq. (B.15) implies that

$$
\sum_{p n}(J p L n \mid L M) Y_{J p}(\widehat{\mathbf{r}}) T_{L n}=\left[Y_{J} \otimes \mathbf{T}_{R}\right]_{L M},
$$

and therefore

$$
\Sigma_{p n}=\sum_{L}(J 0 R M \mid L M)\left[Y_{J} \otimes \mathbf{T}_{R}\right]_{L M} .
$$

Combining the results from eqs. (B.8) and (B.17) allows one to write

$$
e^{i \mathbf{q} \cdot \mathbf{x}} T_{R M}=\sum_{J} \sum_{L} i^{J} \sqrt{4 \pi(2 J+1)} j_{J}(\kappa r)(J 0 R M \mid L M)\left[Y_{J} \otimes \mathbf{T}_{R}\right]_{L M} .
$$

Since $J$ and $L$ are dummy summation indices, it is permissible to relabel them. Choosing the substitutions $J \rightarrow L$ and $L \rightarrow J$, and swapping the order in which the two sums are carried out leads to the expression

$$
\begin{aligned}
e^{i \mathbf{q} \cdot \mathbf{x}} T_{R M} & =\sum_{J} i^{J} \sqrt{4 \pi(2 J+1)} j_{J}(\kappa r) Y_{J 0}(\hat{\mathbf{r}}) T_{R M} \\
& =\sum_{J} \sum_{L} i^{L} \sqrt{4 \pi(2 L+1)}(L 0 R M \mid J M) j_{L}(\kappa r)\left[Y_{L} \otimes \mathbf{T}_{R}\right]_{J M} .
\end{aligned}
$$




\section{Appendix C}

\section{Allowed beta decay}

Although this thesis focuses on neutrino-nucleus interactions, the formalism presented in chapter 3 is sufficiently general that it may be used in calculations of other semileptonic nuclear processes. Because of the relevance of ${ }^{40} \mathrm{Ti} \beta^{+}$decay to a determination of the allowed approximation matrix elements for charged current $\nu_{e}$ scattering on ${ }^{40} \mathrm{Ar}$ (see chapter 5), a brief derivation of the total decay rate for $\beta$ decay is presented in this appendix.

In the notation of chapter 3 , the Lorentz-invariant amplitude $\mathcal{M}$ for $\beta^{-}$decay is given by the diagram

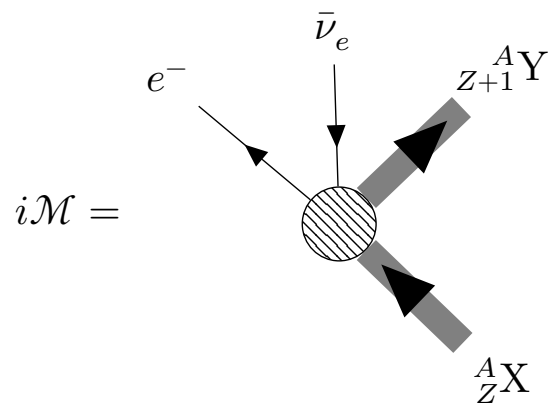

while that for $\beta^{+}$decay is given by

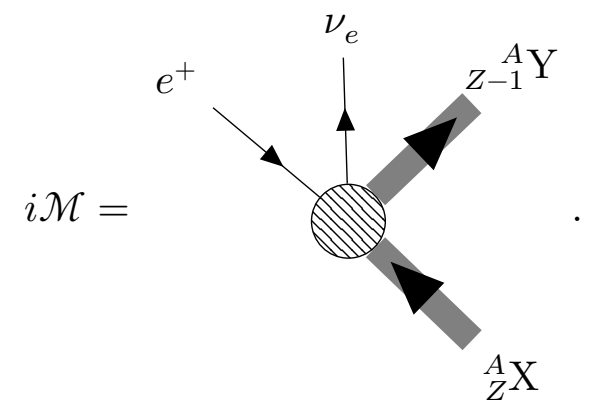


Let $p_{e}$ and $p_{\nu}$ denote the 4-momenta of the final-state (anti)electron and (anti)neutrino, respectively. Then conservation of 4 -momentum ensures that

$$
p_{i}=p_{f}+p_{e}+p_{\nu}
$$

where $p_{i}\left(p_{f}\right)$ is the initial (final) nucleus 4-momentum. The 4-momentum transfer $q$ for $\beta^{ \pm}$decay is defined by

$$
q \equiv p_{f}-p_{i}=-\left(p_{e}+p_{\nu}\right)
$$

For $\beta^{-}$decay, the leptonic part of $\mathcal{M}$ is given by the matrix element

$$
\ell_{\mu}=\bar{u}\left(p_{e}\right) \gamma_{\mu}\left(1-\gamma_{5}\right) v\left(p_{\nu}\right)
$$

while for $\beta^{+}$decay we have

$$
\ell_{\mu}=\bar{u}\left(p_{v}\right) \gamma_{\mu}\left(1-\gamma_{5}\right) v\left(p_{e}\right)
$$

With these changes, the calculation of $|\overline{\mathcal{M}}|^{2}$ proceeds in a similar manner to the charged current neutrino (antineutrino) case for $\beta^{-}\left(\beta^{+}\right)$decay, except that the substitution $\nu_{\ell} \rightarrow \bar{\nu}_{\ell}\left(\bar{\nu}_{\ell} \rightarrow \nu_{\ell}\right)$ should be made for all particle indices in the equations describing the lepton tensor $\mathrm{L}_{\mu \nu}$, and the opposite sign should appear in front of each of the lepton number factors $L_{\nu_{\ell}}$ that appear in eqs. (3.155) and (3.156). Under the allowed approximation, the spin-summed squared amplitude $|\overline{\mathcal{M}}|^{2}$ for $\beta^{ \pm}$decay is given by an expression similar to eq. (3.211). After multiplying by the Coulomb correction factor defined in eq. (3.218) for $\beta^{-}$decay or eq. (3.229) for $\beta^{+}$decay, one may write

$$
\begin{aligned}
|\overline{\mathcal{M}}|^{2} \approx 16 G_{F}^{2}\left|V_{u d}\right|^{2} E_{\nu} E_{i} E_{e} E_{f} F_{C} & \\
& \times\left(B(\mathrm{~F})\left[1+\beta_{e} \cdot \beta_{\nu}\right]+B(\mathrm{GT})\left[1-\frac{1}{3} \beta_{e} \cdot \beta_{\nu}\right]\right)
\end{aligned}
$$

where the Fermi $(B(\mathrm{~F}))$ and Gamow-Teller $(B(\mathrm{GT}))$ reduced matrix elements (see eqs. $(3.213)-(3.214))$ contain the isospin ladder operator $t_{ \pm}$for $\beta^{ \pm}$decay.

The definitions in eqs. (3.39) and (3.42) imply that the differential transition rate (probability per unit time) for $\beta^{ \pm}$decay to a particular nuclear final state $f$ may be 
written as

$$
d \Gamma_{f}=\frac{|\overline{\mathcal{M}}|^{2} \delta^{(4)}\left(p_{i}-p_{f}-p_{e}-p_{\nu}\right)}{16(2 \pi)^{5} E_{i} E_{f} E_{e} E_{\nu}} d^{3} \mathbf{p}_{f} d^{3} \mathbf{p}_{e} d^{3} \mathbf{p}_{\nu}
$$

Integrating over $d^{3} \mathbf{p}_{f}$ is trivial because of the delta function. Doing so gives

$$
d \Gamma_{f}=\frac{|\overline{\mathcal{M}}|^{2} \delta\left(E_{i}-E_{f}-E_{e}-E_{\nu}\right)}{16(2 \pi)^{5} E_{i} E_{f} E_{e} E_{\nu}} d^{3} \mathbf{p}_{e} d^{3} \mathbf{p}_{\nu} .
$$

Plugging in the allowed approximation expression for $|\overline{\mathcal{M}}|^{2}$ from eq. (C.7) yields

$$
\begin{aligned}
d \Gamma_{f} & =\frac{G_{F}^{2}\left|V_{u d}\right|^{2}}{(2 \pi)^{5}} F_{C} \delta\left(E_{i}-E_{f}-E_{e}-E_{\nu}\right) \\
& \times\left(B_{f}(F)\left[1+\beta_{e} \cdot \beta_{\nu}\right]+B_{f}(G T)\left[1-\frac{1}{3} \beta_{e} \cdot \beta_{\nu}\right]\right) d^{3} \mathbf{p}_{e} d^{3} \mathbf{p}_{\nu}
\end{aligned}
$$

where $B_{f}(F)$ and $B_{f}(G T)$ are the Fermi and Gamow-Teller reduced matrix elements connecting the initial nuclear state $i$ with the final nuclear state $f$. Note that

$$
\begin{aligned}
d^{3} \mathbf{p}_{e} d^{3} \mathbf{p}_{\nu} & =\left|\mathbf{p}_{e}\right|^{2}\left|\mathbf{p}_{\nu}\right|^{2} d\left|\mathbf{p}_{e}\right| d\left|\mathbf{p}_{\nu}\right| d \Omega_{e} d \Omega_{\nu} \\
& =E_{\nu}^{2}\left|\mathbf{p}_{e}\right|^{2} d\left|\mathbf{p}_{e}\right| d E_{\nu} d \cos \theta_{\nu e} d \phi_{e} d \Omega_{\nu}
\end{aligned}
$$

where $\theta_{\nu e}$ is the angle between the (anti)neutrino and the (anti)electron. Plugging this result into eq. (C.10) and integrating over $d \phi_{e}$ and $d \Omega_{\nu}$ (which respectively produce factors of $2 \pi$ and $4 \pi$ upon integration) gives

$$
\begin{aligned}
d \Gamma_{f} & =\frac{2 G_{F}^{2}\left|V_{u d}\right|^{2}}{(2 \pi)^{3}} F_{C} E_{\nu}^{2}\left|\mathbf{p}_{e}\right|^{2} \delta\left(E_{i}-E_{f}-E_{e}-E_{\nu}\right) \\
& \times\left(B_{f}(F)\left[1+\beta_{e} \cos \theta_{\nu e}\right]+B_{f}(G T)\left[1-\frac{1}{3} \beta_{e} \cos \theta_{\nu e}\right]\right) d\left|\mathbf{p}_{e}\right| d E_{\nu} d \cos \theta_{\nu e}
\end{aligned}
$$

In the rest frame of the initial nucleus (which, in this case, is both the CM frame and the laboratory frame), $E_{i}=m_{i}$. If one neglects the small recoil kinetic energy of the final nucleus, then $E_{f} \approx m_{f}$, and one may write

$$
\delta\left(E_{i}-E_{f}-E_{e}-E_{\nu}\right)=\delta\left(Q_{f}-E_{e}-E_{\nu}\right)
$$


where the reaction $\mathrm{Q}$-value $Q_{f}$ is defined by

$$
Q_{f} \equiv m_{i}-m_{f}
$$

Since the nuclear recoil has been neglected, $\mathbf{p}_{\nu} \approx-\mathbf{p}_{e}$, and $E_{\nu}$ and $E_{e}$ may be treated as independent of $\cos \theta_{\nu e}$. Integrating over $\cos \theta_{\nu e}$ using this approximation results in the expression

$$
d \Gamma_{f}=\frac{G_{F}^{2}\left|V_{u d}\right|^{2}}{2 \pi^{3}} F_{C} E_{\nu}^{2}\left|\mathbf{p}_{e}\right|^{2} \delta\left(Q_{f}-E_{e}-E_{\nu}\right)\left[B_{f}(F)+B_{f}(G T)\right] d\left|\mathbf{p}_{e}\right| d E_{\nu} .
$$

Integrating over $d E_{\nu}$, which is trivial thanks to the energy-conserving delta function, gives

$$
d \Gamma_{f}=\frac{G_{F}^{2}\left|V_{u d}\right|^{2}}{2 \pi^{3}} F_{C}\left|\mathbf{p}_{e}\right|^{2}\left(Q_{f}-E_{e}\right)^{2}\left[B_{f}(F)+B_{f}(G T)\right] d\left|\mathbf{p}_{e}\right|
$$

Since $\left|\mathbf{p}_{e}\right|=\sqrt{E_{e}^{2}-m_{e}^{2}}$ and $d\left|\mathbf{p}_{e}\right|=\left|\mathbf{p}_{e}\right|^{-1} E_{e} d E_{e}$, this may be rewritten as

$$
d \Gamma_{f}=\frac{G_{F}^{2}\left|V_{u d}\right|^{2}}{2 \pi^{3}} F_{C} E_{e} \sqrt{E_{e}^{2}-m_{e}^{2}}\left(Q_{f}-E_{e}\right)^{2}\left[B_{f}(F)+B_{f}(G T)\right] d E_{e} .
$$

The decay rate to the final state $f$ may be obtained by integrating over the kinematically allowed region $E_{e} \in\left[m_{e}, Q_{f}\right]$, which yields the expression

$$
\Gamma_{f}=\frac{G_{F}^{2}\left|V_{u d}\right|^{2}}{2 \pi^{3}}\left[B_{f}(F)+B_{f}(G T)\right] \int_{m_{e}}^{Q_{f}} F_{C} E_{e} \sqrt{E_{e}^{2}-m_{e}^{2}}\left(Q_{f}-E_{e}\right)^{2} d E_{e}
$$

In the beta decay literature, one often encounters the dimensionless phase space integral $\mathcal{F}\left(Q_{f}\right)$, which is defined by

$$
\mathcal{F}\left(Q_{f}\right) \equiv \frac{1}{m_{e}^{5}} \int_{m_{e}}^{Q_{f}} F_{C} E_{e} \sqrt{E_{e}^{2}-m_{e}^{2}}\left(Q_{f}-E_{e}\right)^{2} d E_{e}
$$

The decay rate $\Gamma_{f}$ to the final nuclear level $f$ may be expressed in terms of $\mathcal{F}\left(Q_{f}\right)$ as

$$
\Gamma_{f}=\frac{G_{F}^{2}\left|V_{u d}\right|^{2} m_{e}^{5}}{2 \pi^{3}} \mathcal{F}\left(Q_{f}\right)\left[B_{f}(F)+B_{f}(G T)\right]
$$

The total decay rate $\Gamma$ is the sum over the decay rates to individual nuclear final states:

$$
\Gamma=\sum_{f} \Gamma_{f}
$$


The half-life $t_{1 / 2}$ is related to the total decay rate $\Gamma$ via

$$
t_{1 / 2}=\frac{\ln (2)}{\Gamma}
$$

The branching ratio $\mathcal{R}_{f}$ to the final nuclear level $f$ is given by

$$
\mathcal{R}_{f}=\frac{\Gamma_{f}}{\Gamma}
$$

Using the definitions above, one may write

$$
\frac{\mathcal{R}_{f}}{\mathcal{F}\left(Q_{f}\right) t_{1 / 2}}=\frac{G_{F}^{2}\left|V_{u d}\right|^{2} m_{e}^{5}}{2 \pi^{3} \ln (2)}\left[B_{f}(F)+B_{f}(G T)\right] .
$$

Switching to conventional units, it follows from this that, if one defines the constant ${ }^{1}$

$$
K \equiv \frac{2 \pi^{3} \ln (2) \hbar}{{G_{F}}^{2}\left|V_{u d}\right|^{2} m_{e}^{5} c^{10}} \approx 6127 \pm 9 \mathrm{~s},
$$

then one may extract the allowed matrix elements $B_{f}(F)$ and $B_{f}(G T)$ from an experimental measurement of $t_{1 / 2}$ and $\mathcal{R}_{f}$ via the relation

$$
B_{f}(F)+B_{f}(G T)=\frac{K \mathcal{R}_{f}}{\mathcal{F}\left(Q_{f}\right) t_{1 / 2}} .
$$

\footnotetext{
${ }^{1}$ The approximate value given in eq. (C.26) is from reference [486].
} 


\section{Bibliography}

1“Ueber eine neue Art von Strahlen. (Vorläufige Mittheilung.) (On a new kind of rays. preliminary communication)", in Sitzungs-Berichte der Physikalisch-medicinischen Gesellschaft zu Würzburg (Proceedings of the Physico-Medical Society of Würzburg), in German (Jan. 1895), pp. 132-141 (cit. on pp. 1, 291).

${ }^{2}$ A. Stanton, "On a new kind of rays", Nature 53, English translation of [1], 274-276 (1896) (cit. on p. 1).

${ }^{3}$ P. Radvanyi and J. Villain, "The discovery of radioactivity", Comptes Rendus Physique 18, 544-550 (2017) (cit. on p. 1).

${ }^{4}$ C. Giunta, "Discovery of radioactivity: Becquerel", in Elements and Atoms: Case Studies in the Development of Chemistry, online book (2002) (cit. on p. 1).

${ }^{5}$ H. Becquerel, "Émission de radiations nouvelles par l'uranium métallique", Comptes rendus de l'Académie des Sciences (Proceedings of the French Academy of Sciences) 122, in French, 1086 (1896) (cit. on pp. 2, 291).

${ }^{6} \mathrm{H}$. Becquerel, "Emission of new radiations by metallic uranium", The Chemical News and Journal of Physical Science 73, English translation of [5], 295 (1896) (cit. on p. 2).

${ }^{7}$ E. Rutherford, "Uranium radiation and the electrical conduction produced by it", The London, Edinburgh, and Dublin Philosophical Magazine and Journal of Science 47, 109-163 (1899) (cit. on p. 2).

${ }^{8}$ E. Rutherford, "The magnetic and electric deviation of the easily absorbed rays from radium", The London, Edinburgh, and Dublin Philosophical Magazine and Journal of Science 5, 177-187 (1903) (cit. on p. 2). 
${ }^{9}$ L. Gerward, "Paul Villard and his discovery of gamma rays", Physics in Perspective 1, 367-383 (1999) (cit. on p. 2).

${ }^{10}$ J. Chadwick, "Intensitätsverteilung im magnetischen Spektrum von -Strahlen von Radium $\mathrm{B}+\mathrm{C}$ (The intensity distribution in the magnetic spectrum of $\beta$-rays from radium B+C)', Verhandlungen der Deutschen Physikalischen Gesellschaft 16, in German, 383391 (1914) (cit. on p. 3).

${ }^{11}$ C. Jensen et al., Controversy and Consensus: Nuclear Beta Decay 1911-1934, Science Networks. Historical Studies (Birkhäuser Basel, 1999) (cit. on p. 3).

${ }^{12}$ A. Franklin, "The spectrum of $\beta$ decay: continuous or discrete? a variety of errors in experimental investigation", in Going Amiss In Experimental Research (Springer, 2009), pp. 211-235 (cit. on p. 3).

${ }^{13}$ L. Brown, "The idea of the neutrino", Physics Today 31, 23 (1978) (cit. on pp. 3, 4).

${ }^{14}$ C. D. Ellis and W. Wooster, "The continuous spectrum of $\beta$-rays", Nature 119, 563 (1927) (cit. on p. 3).

${ }^{15}$ L. Meitner and W. Orthmann, "Über eine absolute Bestimmung der Energie der primären $\beta$-Strahlen von Radium E (on an absolute determination of the energy of the primary $\beta$-rays of radium E)', Zeitschrift für Physik 60, 143-155 (1930) (cit. on p. 3).

${ }^{16}$ N. Bohr, "Faraday lecture. Chemistry and the quantum theory of atomic constitution", Journal of the Chemical Society, 349-384 (1932) (cit. on p. 4).

${ }^{17}$ W. Pauli, Aufsätze und Vorträge über Physik und Erkenntnistheorie, in German (F. Vieweg, Braunschweig, Germany, 1961) (cit. on p. 4).

${ }^{18}$ J. Chadwick, "Possible existence of a neutron", Nature 129, 312 (1932) (cit. on p. 4).

${ }^{19}$ J. Chadwick, "The existence of a neutron", Proceedings of the Royal Society of London A: Mathematical, Physical and Engineering Sciences 136, 692-708 (1932) (cit. on p. 4).

${ }^{20}$ E. Amaldi, "From the discovery of the neutron to the discovery of nuclear fission", Physics Reports 111, 1-331 (1984) (cit. on pp. 4, 5). 
${ }^{21}$ E. Fermi, "Versuch einer Theorie der $\beta$-Strahlen. I. (An attempt of a theory of beta radiation. I.)", Zeitschrift für Physik 88, in German, 161-177 (1934) (cit. on pp. 5, 95, 97, 293).

${ }^{22}$ F. L. Wilson, "Fermi's theory of beta decay", American Journal of Physics 36, English translation of [21], 1150 (1968) (cit. on pp. 5, 95, 97).

${ }^{23}$ G. Wick, "Sugli elementi radioattivi di F. Joliot e I. Curie (On the radioactive elements of F. Joliot and I. Curie)", Atti della Reale Accademia Nazionale dei Lincei, Rendiconti 19, in Italian, 319-324 (1934) (cit. on p. 5).

${ }^{24}$ H. Yukawa and S. Sakata, "On the theory of the $\beta$-disintegration and the allied phenomenon", Proceedings of the Physico-Mathematical Society of Japan. 3rd Series 17, 467-479 (1935) (cit. on p. 5).

${ }^{25}$ J. S. Allen, "Experimental evidence for the existence of a neutrino", Physical Review 61, 692-697 (1942) (cit. on p. 5).

${ }^{26}$ R. Davis, "Nuclear recoil following neutrino emission from beryllium 7', Physical Review 86, 976-985 (1952) (cit. on p. 5).

${ }^{27}$ H. R. Crane, "An attempt to observe the absorption of neutrinos", Physical Review 55, 501-502 (1939) (cit. on p. 5).

${ }^{28}$ F. Reines and C. L. Cowan, "Detection of the free neutrino", Physical Review 92, 830-831 (1953) (cit. on p. 5).

${ }^{29}$ C. L. Cowan et al., "Detection of the free neutrino: a confirmation", Science 124, 103104 (1956) (cit. on p. 5).

${ }^{30}$ F. Reines et al., "Detection of the free antineutrino", Physical Review 117, 159-173 (1960) (cit. on pp. 5, 18).

${ }^{31}$ S. H. Neddermeyer and C. D. Anderson, "Note on the nature of cosmic-ray particles", Physical Review 51, 884-886 (1937) (cit. on p. 5).

${ }^{32}$ J. Steinberger, "On the range of the electrons in meson decay", Physical Review 74, 500-501 (1948) (cit. on p. 6). 
${ }^{33}$ E. P. Hincks and B. Pontecorvo, "Search for gamma-radiation in the 2.2-microsecond meson decay process", Phys. Rev. 73, 257-258 (1948) (cit. on p. 6).

${ }^{34}$ B. Pontecorvo, "The infancy and youth of neutrino physics: some recollections", Le Journal de Physique Colloques 43, C8-221 (1982) (cit. on p. 6).

${ }^{35}$ G. Danby et al., "Observation of high-energy neutrino reactions and the existence of two kinds of neutrinos", Physical Review Letters 9, 36-44 (1962) (cit. on p. 6).

${ }^{36}$ B. Pontecorvo, "Electron and muon neutrinos", Journal of Experimental and Theoretical Physics (USSR) 10, 1236-1240 (1960) (cit. on p. 6).

${ }^{37}$ M. Schwartz, "Feasibility of using high-energy neutrinos to study the weak interactions", Physical Review Letters 4, 306-307 (1960) (cit. on p. 6).

${ }^{38}$ M. L. Perl, G. S. Abrams, A. M. Boyarski, et al., "Evidence for anomalous lepton production in $e^{+}-e^{-}$annihilation', Physical Review Letters 35, 1489-1492 (1975) (cit. on p. 6).

${ }^{39}$ K. Kodama, N. Ushida, C. Andreopoulos, et al., "Observation of tau neutrino interactions", Physics Letters B 504, 218-224 (2001) (cit. on p. 6).

${ }^{40}$ D. Decamp, B. Deschizeaux, et al., ALEPH Collaboration, "A precise determination of the number of families with light neutrinos and of the Z boson partial widths", Physics Letters B 235, 399-411 (1990) (cit. on p. 6).

${ }^{41}$ P. Aarnio, P. Abreu, et al., DELPHI Collaboration, "Measurement of the mass and width of the $Z^{0}$-particle from multihadronic final states produced in $e^{+} e^{-}$annihilations", Physics Letters B 231, 539-547 (1989) (cit. on p. 6).

${ }^{42}$ B. Adeva, O. Adriani, et al., L3 Collaboration, "A determination of the properties of the neutral intermediate vector boson $Z^{0}$ ", Physics Letters B 231, 509-518 (1989) (cit. on p. 6).

${ }^{43}$ M. Akrawy, G. Alexander, et al., OPAL Collaboration, "Measurement of the $Z^{0}$ mass and width with the OPAL detector at LEP', Physics Letters B 231, 530-538 (1989) (cit. on p. 6). 
${ }^{44}$ S. L. Glashow, "The renormalizability of vector meson interactions", Nuclear Physics 10, 107-117 (1959) (cit. on p. 7).

${ }^{45}$ S. L. Glashow, "Partial-symmetries of weak interactions", Nuclear Physics 22, 579-588 (1961) (cit. on p. 7).

${ }^{46}$ A. Salam and J. C. Ward, "Weak and electromagnetic interactions", Il Nuovo Cimento 11, 568-577 (1959) (cit. on p. 7).

${ }^{47}$ A. Salam and J. Ward, "Electromagnetic and weak interactions", Physics Letters 13, 168-171 (1964) (cit. on p. 7).

${ }^{48}$ S. Weinberg, "A model of leptons", Physical Review Letters 19, 1264-1266 (1967) (cit. on p. 7).

${ }^{49}$ P. Langacker, The Standard Model and Beyond, Series in High Energy Physics, Cosmology and Gravitation (CRC Press, 2009) (cit. on pp. 7, 62, 67, 107).

${ }^{50}$ F. Hasert, S. Kabe, W. Krenz, et al., "Observation of neutrino-like interactions without muon or electron in the Gargamelle neutrino experiment', Nuclear Physics B 73, 1-22 (1974) (cit. on p. 8).

${ }^{51}$ J. Blietschau, H. Deden, H. Faissner, et al., "Evidence for the leptonic neutral current reaction $\nu_{\mu}+e^{-} \rightarrow \nu_{\mu}+e^{-}$, Nuclear Physics B 114, 189-198 (1976) (cit. on p. 8).

${ }^{52}$ G. Arnison, A. Astbury, B. Aubert, et al., UA1 Collaboration, "Experimental observation of isolated large transverse energy electrons with associated missing energy at $s=540 \mathrm{GeV}$ ', Physics Letters B 122, 103-116 (1983) (cit. on p. 8).

${ }^{53}$ M. Banner, R. Battiston, P. Bloch, et al., UA2 Collaboration, "Observation of single isolated electrons of high transverse momentum in events with missing transverse energy at the CERN pp collider", Physics Letters B 122, 476-485 (1983) (cit. on p. 8).

${ }^{54}$ G. Arnison, A. Astbury, B. Aubert, et al., UA1 Collaboration, "Experimental observation of lepton pairs of invariant mass around $95 \mathrm{GeV} / c^{2}$ at the CERN SPS collider", Physics Letters B 126, 398-410 (1983) (cit. on p. 8). 
${ }^{55}$ P. Bagnaia, M. Banner, R. Battiston, et al., UA2 Collaboration, "Evidence for $Z^{0} \rightarrow$ $e^{+} e^{-}$at the CERN pp collider", Physics Letters B 129, 130-140 (1983) (cit. on p. 8).

${ }^{56}$ G. Aad, T. Abajyan, B. Abbott, et al., "Observation of a new particle in the search for the Standard Model Higgs boson with the ATLAS detector at the LHC", Physics Letters B 716, 1-29 (2012) (cit. on p. 8).

${ }^{57}$ S. Chatrchyan, V. Khachatryan, A. Sirunyan, et al., CMS Collaboration, "Observation of a new boson at a mass of $125 \mathrm{GeV}$ with the CMS experiment at the LHC", Physics Letters B 716, 30-61 (2012) (cit. on p. 8).

${ }^{58}$ J. Berryhill and A. Oh, "Electroweak physics at the LHC", Journal of Physics G: Nuclear and Particle Physics 44, 023001 (2017) (cit. on p. 8).

${ }^{59}$ C. Giunti and C. Kim, Fundamentals of Neutrino Physics and Astrophysics (Oxford University Press, 2007) (cit. on pp. 9, 15, 16, 18, 30, 67, 85-87, 109, 111, 112).

${ }^{60}$ B. Pontecorvo, "Mesonium and antimesonium", Journal of Experimental and Theoretical Physics (USSR) 33, 549-551 (1957) (cit. on p. 10).

${ }^{61}$ B. Pontecorvo, "Neutrino experiments and the problem of conservation of leptonic charge", Journal of Experimental and Theoretical Physics (USSR) 26, 165 (1968) (cit. on p. 10).

${ }^{62}$ Z. Maki, M. Nakagawa, and S. Sakata, "Remarks on the unified model of elementary particles", Progress of Theoretical Physics 28, 870-880 (1962) (cit. on p. 10).

${ }^{63}$ V. Gribov and B. Pontecorvo, "Neutrino astronomy and lepton charge", Physics Letters B 28, 493-496 (1969) (cit. on p. 10).

${ }^{64}$ R. Davis, "Solar neutrinos. II. Experimental", Physical Review Letters 12, 303-305 (1964) (cit. on p. 10).

${ }^{65}$ R. Davis, D. S. Harmer, and K. C. Hoffman, "Search for neutrinos from the Sun", Physical Review Letters 20, 1205-1209 (1968) (cit. on p. 10).

${ }^{66}$ J. N. Bahcall, "Solar neutrinos. I. Theoretical", Physical Review Letters 12, 300-302 (1964) (cit. on p. 11). 
${ }^{67}$ K. S. Hirata, K. Inoue, T. Kajita, et al., Kamiokande-II Collaboration, "Results from one thousand days of real-time, directional solar-neutrino data", Physical Review Letters 65, 1297-1300 (1990) (cit. on p. 11).

${ }^{68}$ A. I. Abazov, O. L. Anosov, E. L. Faizov, et al., "Search for neutrinos from the sun using the reaction ${ }^{71} \mathrm{Ga}\left(\nu_{e}, e^{-}\right){ }^{71} \mathrm{Ge}$ ", Physical Review Letters 67, 3332-3335 (1991) (cit. on p. 11).

${ }^{69}$ P. Anselmann, W. Hampel, G. Heusser, et al., GALLEX Collaboration, "Solar neutrinos observed by GALLEX at Gran Sasso", Physics Letters B 285, 376-389 (1992) (cit. on p. 11).

${ }^{70}$ P. Anselmann, W. Hampel, G. Heusser, et al., GALLEX Collaboration, "Implications of the GALLEX determination of the solar neutrino flux", Physics Letters B 285, 390397 (1992) (cit. on p. 11).

${ }^{71}$ T. J. Haines, R. M. Bionta, G. Blewitt, et al., Irvine-Michigan-Brookhaven Collaboration, "Calculation of atmospheric neutrino-induced backgrounds in a nucleon-decay search", Physical Review Letters 57, 1986-1989 (1986) (cit. on p. 11).

${ }^{72}$ K. Hirata, T. Kajita, M. Koshiba, et al., Kamiokande-II Collaboration, "Experimental study of the atmospheric neutrino flux", Physics Letters B 205, 416-420 (1988) (cit. on p. 11).

${ }^{73}$ K. Hirata, K. Inoue, T. Ishida, et al., Kamiokande-II Collaboration, "Observation of a small atmospheric $\nu_{\mu} / \nu_{e}$ ratio in Kamiokande", Physics Letters B 280, 146-152 (1992) (cit. on p. 11).

${ }^{74}$ J. G. Learned, "The atmospheric neutrino anomaly: Muon neutrino disappearance", in Current Aspects of Neutrino Physics, edited by D. O. Caldwell (Springer, Berlin, Heidelberg, 2001), pp. 89-130 (cit. on p. 11).

${ }^{75}$ J. M. LoSecco, "The history of "anomalous" atmospheric neutrino events: A first person account", Physics in Perspective 18, 209-241 (2016) (cit. on p. 11). 
${ }^{76}$ Y. Fukuda, T. Hayakawa, E. Ichihara, et al., Super-Kamiokande Collaboration, "Evidence for oscillation of atmospheric neutrinos", Physical Review Letters 81, 1562-1567 (1998) (cit. on p. 11).

${ }^{77}$ Q. R. Ahmad, R. C. Allen, T. C. Andersen, et al., SNO Collaboration, "Direct evidence for neutrino flavor transformation from neutral-current interactions in the Sudbury Neutrino Observatory", Physical Review Letters 89, 011301 (2002) (cit. on p. 11).

${ }^{78}$ M. Tanabashi, K. Hagiwara, K. Hikasa, et al., Particle Data Group, "Review of particle physics", Physical Review D 98, 030001 (2018) (cit. on pp. 12, 14, 42, 172, 264).

${ }^{79}$ P. de Salas, D. Forero, C. Ternes, et al., "Status of neutrino oscillations 2018: $3 \sigma$ hint for normal mass ordering and improved CP sensitivity", Physics Letters B 782, 633640 (2018) (cit. on p. 13).

${ }^{80}$ K. N. Abazajian, M. Acero, S. Agarwalla, et al., "Light sterile neutrinos: a white paper", arXiv preprint arXiv:1204.5379 (2012) (cit. on p. 13).

${ }^{81}$ A. Aguilar-Arevalo, B. Brown, L. Bugel, et al., MiniBooNE Collaboration, "Observation of a significant excess of electron-like events in the MiniBooNE short-baseline neutrino experiment", arXiv preprint arXiv:1805.12028 (2018) (cit. on p. 13).

${ }^{82}$ G. Mention et al., "Reactor antineutrino anomaly", Physical Review D 83, 073006 (2011) (cit. on p. 13).

${ }^{83}$ F. P. An, A. B. Balantekin, H. R. Band, et al., Daya Bay Collaboration, "Improved measurement of the reactor antineutrino flux and spectrum at Daya Bay", Chinese Physics C 41, 013002 (2017) (cit. on p. 13).

${ }^{84}$ R. Acciari et al., The ICARUS-WA104, LAr1-ND, and MicroBooNE Collaborations, "A proposal for a three detector short-baseline neutrino oscillation program in the Fermilab Booster Neutrino Beam", arXiv preprint arXiv:1503.01520v1 (2015) (cit. on pp. 13, 198, 199, 203).

${ }^{85} \mathrm{~J}$. Ashenfelter et al., "First search for short-baseline neutrino oscillations at HFIR with PROSPECT', arXiv preprint arXiv:1806.02784 (2018) (cit. on p. 13). 
${ }^{86}$ V. N. Aseev, A. I. Belesev, A. I. Berlev, et al., "Upper limit on the electron antineutrino mass from the Troitsk experiment", Physical Review D 84, 112003 (2011) (cit. on p. 14).

${ }^{87}$ L. Bornschein, B. Bornschein, et al., KATRIN Collaboration, "Status of the Karlsruhe Tritium Neutrino Mass Experiment KATRIN", Fusion Science and Technology 71, 485-490 (2017) (cit. on p. 14).

${ }^{88}$ A. A. Esfahani, D. M. Asner, et al., "Determining the neutrino mass with cyclotron radiation emission spectroscopy —Project 8", Journal of Physics G: Nuclear and Particle Physics 44, 054004 (2017) (cit. on p. 14).

${ }^{89}$ Ade, P. A. R., Aghanim, N., et al., Planck Collaboration, "Planck 2015 results - XIII. Cosmological parameters", Astronomy \& Astrophysics 594, A13 (2016) (cit. on p. 14).

${ }^{90}$ J. Schechter and J. W. F. Valle, "Neutrino-oscillation thought experiment", Physical Review D 23, 1666-1668 (1981) (cit. on p. 16).

${ }^{91}$ J. Schechter and J. W. F. Valle, "Neutrinoless double- $\beta$ decay in $\mathrm{SU}(2) \times \mathrm{U}(1)$ theories", Physical Review D 25, 2951-2954 (1982) (cit. on p. 17).

${ }^{92}$ M. Hirsch, S. Kovalenko, and I. Schmidt, "Extended black box theorem for lepton number and flavor violating processes", Physics Letters B 642, 106-110 (2006) (cit. on p. 17).

${ }^{93}$ S. Dell'Oro et al., "Neutrinoless double beta decay: 2015 review", Advances in High Energy Physics 2016, 2162659 (2016) (cit. on p. 17).

${ }^{94}$ M. Agostini, A. M. Bakalyarov, et al., GERDA Collaboration, "Improved limit on neutrinoless double- $\beta$ decay of ${ }^{76}$ Ge from GERDA Phase II', Physical Review Letters 120, 132503 (2018) (cit. on p. 17).

${ }^{95}$ C. E. Aalseth, N. Abgrall, E. Aguayo, et al., MAJoranA Collaboration, "Search for neutrinoless double- $\beta$ decay in ${ }^{76}$ Ge with the Majorana Demonstrator', Physical Review Letters 120, 132502 (2018) (cit. on p. 17).

${ }^{96}$ J. Hartnell et al., SNO+ Collaboration, "Neutrinoless double beta decay with SNO+", Journal of Physics: Conference Series 375, 042015 (2012) (cit. on p. 17). 
${ }^{97}$ A. Gando, Y. Gando, et al., KamLAND-Zen Collaboration, "Search for Majorana neutrinos near the inverted mass hierarchy region with KamLAND-Zen", Physical Review Letters 117, 082503 (2016) (cit. on p. 17).

${ }^{98}$ J. B. Albert, G. Anton, et al., EXO-200 Collaboration, "Search for neutrinoless doublebeta decay with the upgraded EXO-200 detector", Physical Review Letters 120,072701 (2018) (cit. on p. 17).

${ }^{99}$ O. Cremonesi and M. Pavan, "Challenges in double beta decay", Advances in High Energy Physics 2014, 951432 (2014) (cit. on p. 17).

${ }^{100}$ J. Engel and J. Menéndez, "Status and future of nuclear matrix elements for neutrinoless double-beta decay: a review", Reports on Progress in Physics 80, 046301 (2017) (cit. on pp. 17, 152).

${ }^{101}$ S. Fukuda, Y. Fukuda, T. Hayakawa, et al., Super-Kamiokande Collaboration, "The Super-Kamiokande detector", Nuclear Instruments and Methods in Physics Research Section A: Accelerators, Spectrometers, Detectors and Associated Equipment 501, 418462 (2003) (cit. on p. 18).

${ }^{102}$ P. A. Cherenkov, "Visible emission of clean liquids by action of $\gamma$ radiation", Doklady Akademii Nauk SSSR (Proceedings of the USSR Academy of Sciences) 2, 451 (1934) (cit. on p. 18).

${ }^{103}$ I. Tamm and I. Frank, "Coherent radiation of fast electrons in a medium", Doklady Akademii Nauk SSSR (Proceedings of the USSR Academy of Sciences) 14, 107-112 (1937) (cit. on p. 18).

${ }^{104}$ J. Jackson, Classical Electrodynamics (Wiley, 2012) (cit. on p. 19).

${ }^{105}$ C. Grupen and B. Shwartz, Particle Detectors, Vol. 26 (Cambridge University Press, 2008) (cit. on p. 20).

${ }^{106}$ Cherenkov radiation - Wikipedia, the free encyclopedia, 2018 (cit. on p. 20).

${ }^{107}$ File:advanced test reactor.jpg — Wikimedia Commons, the free media repository, 2017 (cit. on p. 21). 
${ }^{108}$ Hamamatsu Photonics K. K., Photomultiplier tubes, basics and applications, 2007 (cit. on pp. 22,23$)$.

${ }^{109}$ T. Wright and A. Wright, The Photomultiplier Handbook (Oxford University Press, 2017) (cit. on pp. 22, 23).

${ }^{110}$ F. Ardellier, I. Barabanov, J. C. Barrière, et al., Double Chooz Collaboration, "Double Chooz, a search for the neutrino mixing angle $\theta_{13}$ ", arXiv preprint hep-ex/0606025 (2006) (cit. on p. 23).

${ }^{111}$ K. Eguchi, S. Enomoto, K. Furuno, et al., KamLAND Collaboration, "First results from KamLAND: Evidence for reactor antineutrino disappearance', Physical Review Letters 90, 021802 (2003) (cit. on p. 23).

${ }^{112}$ T. Adam et al., JUNO Collaboration, "JUNO conceptual design report', arXiv preprint arXiv:1508.07166 (2015) (cit. on p. 23).

${ }^{113}$ G. Knoll, Radiation Detection and Measurement (John Wiley \& Sons, 2010) (cit. on p. 23).

${ }^{114}$ M. Fechner, K. Abe, Y. Hayato, et al., Super-Kamiokande Collaboration, "Kinematic reconstruction of atmospheric neutrino events in a large water Cherenkov detector with proton identification", Physical Review D 79, 112010 (2009) (cit. on p. 24).

${ }^{115}$ G. D. O. Gann et al., "Physics potential of an advanced scintillation detector: Introducing THEIA", arXiv preprint arXiv:1504.08284 (2015) (cit. on p. 24).

${ }^{116} \mathrm{M}$. Yeh et al., "A new water-based liquid scintillator and potential applications", Nuclear Instruments and Methods in Physics Research Section A: Accelerators, Spectrometers, Detectors and Associated Equipment 660, 51-56 (2011) (cit. on p. 24).

${ }^{117}$ J. Alonso, N. Barros, M. Bergevin, et al., "Advanced scintillator detector concept (ASDC): a concept paper on the physics potential of water-based liquid scintillator", arXiv preprint arXiv:1409.5864 (2014) (cit. on p. 24).

${ }^{118}$ M. Hyman and J. J. Ryan, "Heavy elements in plastic scintillators", IRE Transactions on Nuclear Science 5, 87-90 (1958) (cit. on p. 24). 
${ }^{119}$ A. Bernstein, T. West, and V. Gupta, "An assessment of antineutrino detection as a tool for monitoring nuclear explosions", Science \& Global Security 9, 235-255 (2001) (cit. on p. 24).

${ }^{120}$ J. F. Beacom and M. R. Vagins, "Antineutrino spectroscopy with large water Čerenkov detectors", Physical Review Letters 93, 171101 (2004) (cit. on pp. 24, 25, 49).

${ }^{121}$ S. Dazeley et al., "Observation of neutrons with a gadolinium doped water Cherenkov detector', Nuclear Instruments and Methods in Physics Research Section A: Accelerators, Spectrometers, Detectors and Associated Equipment 607, 616-619 (2009) (cit. on pp. 24, 25).

${ }^{122}$ S. Mughabghab, Thermal neutron capture cross sections resonance integrals and $g$ factors, tech. rep. (International Atomic Energy Agency, 2003) (cit. on p. 25).

${ }^{123}$ J. Cao and K.-B. Luk, "An overview of the Daya Bay reactor neutrino experiment", Nuclear Physics B 908, 62-73 (2016) (cit. on p. 25).

${ }^{124}$ Magro, Lluís Martí, "EGADS approaching GADZOOKS!", EPJ Web of Conferences 95, 04041 (2015) (cit. on p. 25).

${ }^{125}$ S. Dazeley et al., "A search for cosmogenic production of $\beta$-neutron emitting radionuclides in water", Nuclear Instruments and Methods in Physics Research Section A: Accelerators, Spectrometers, Detectors and Associated Equipment 821, 151-159 (2016) (cit. on p. 25).

${ }^{126}$ A. Back, J. Beacom, M. Bergevin, et al., "Accelerator Neutrino Neutron Interaction Experiment (ANNIE): Preliminary results and physics phase proposal", arXiv preprint arXiv:1707.08222 (2017) (cit. on pp. 25, 46, 50-53, 210, 214, 215, 221).

${ }^{127}$ M. Askins, M. Bergevin, A. Bernstein, et al., WATCHMAN Collaboration, "The physics and nuclear nonproliferation goals of WATCHMAN: A WAter CHerenkov Monitor for ANtineutrinos", arXiv preprint arXiv:1502.01132 (2015) (cit. on p. 25). 
${ }^{128}$ T. Mori, "Status of the Super-Kamiokande gadolinium project", Nuclear Instruments and Methods in Physics Research Section A: Accelerators, Spectrometers, Detectors and Associated Equipment 732, Vienna Conference on Instrumentation 2013, 316-319 (2013) (cit. on p. 25).

${ }^{129} \mathrm{C}$. Rubbia, The liquid-argon time projection chamber: A new concept for neutrino detectors, tech. rep. CERN-EP-INT-77-08 (European Organization for Nuclear Research (CERN), Geneva, Switzerland, 1977) (cit. on pp. 25, 179).

${ }^{130}$ B. Fleming et al., MicroBooNE Collaboration, The MicroBooNE technical design report, tech. rep. FERMILAB-DESIGN-2012-04 (2012) (cit. on pp. 25, 41).

${ }^{131}$ A. Ciocio, M. B. Ceolin, L. Bassi, et al., ICARUS Collaboration, "ICARUS liquid argon imaging chamber: A novel detector technology", in 13th International Conference on Neutrino Physics and Astrophysics (Neutrino 88) Boston, Massachusetts, June 5-11, 1988 (1988), pp. 280-288 (cit. on pp. 25, 42).

${ }^{132}$ B. Abi et al., "The DUNE far detector interim design report volume 1: physics, technology and strategies", arXiv preprint arXiv:1807.10334 (2018) (cit. on pp. 25, 34).

${ }^{133}$ R. Acciarri, C. Adams, R. An, et al., MicroBooNE Collaboration, "Noise characterization and filtering in the MicroBooNE liquid argon TPC', Journal of Instrumentation 12, P08003 (2017) (cit. on p. 26).

${ }^{134}$ R. Acciarri, C. Adams, R. An, et al., "Michel electron reconstruction using cosmic-ray data from the MicroBooNE LArTPC", Journal of Instrumentation 12, P09014 (2017) (cit. on p. 26).

${ }^{135}$ F.-Y. Zhao, R. G. Strom, and S.-Y. Jiang, "The Guest Star of AD185 must have been a Supernova", Chinese Journal of Astronomy and Astrophysics 6, 635 (2006) (cit. on p. 29).

${ }^{136}$ W. Baade and F. Zwicky, "On super-novae", Proceedings of the National Academy of Sciences 20, 254-259 (1934) (cit. on p. 29).

${ }^{137}$ T. Kunugise and K. Iwamoto, "Neutrino emission from type Ia supernovae", Publications of the Astronomical Society of Japan 59, L57-L61 (2007) (cit. on p. 29). 
${ }^{138}$ O. Pols, Pre-supernova evolution of massive stars, Lecture notes for a graduate course taught at Utrecht University, 2011 (cit. on p. 29).

${ }^{139}$ W. P. Wright, J. P. Kneller, et al., "Neutrinos from type Ia supernovae: The gravitationally confined detonation scenario", Physical Review D 95, 043006 (2017) (cit. on p. 29).

${ }^{140}$ H.-T. Janka, K. Langanke, A. Marek, et al., "Theory of core-collapse supernovae", Physics Reports 442, 38-74 (2007) (cit. on p. 31).

${ }^{141}$ A. Burrows, "Supernova explosions in the universe", Nature 403, 727-733 (2000) (cit. on p. 31).

${ }^{142}$ K. Hirata, T. Kajita, M. Koshiba, et al., Kamiokande-II Collaboration, "Observation of a neutrino burst from the supernova SN1987A', Physical Review Letters 58, 14901493 (1987) (cit. on p. 32).

${ }^{143}$ K. S. Hirata, T. Kajita, M. Koshiba, et al., Kamiokande-II Collaboration, "Observation in the Kamiokande-II detector of the neutrino burst from supernova SN1987A", Physical Review D 38, 448-458 (1988) (cit. on p. 32).

${ }^{144}$ R. M. Bionta, G. Blewitt, C. B. Bratton, et al., IMB Collaboration, "Observation of a neutrino burst in coincidence with supernova 1987A in the Large Magellanic Cloud", Physical Review Letters 58, 1494-1496 (1987) (cit. on p. 32).

${ }^{145}$ E. Alexeyev et al., "Detection of the neutrino signal from SN 1987A in the LMC using the INR Baksan underground scintillation telescope", Physics Letters B 205, 209-214 (1988) (cit. on p. 32).

${ }^{146}$ T. J. Loredo and D. Q. Lamb, "Bayesian analysis of neutrinos observed from supernova SN 1987A", Physical Review D 65, 063002 (2002) (cit. on p. 32).

${ }^{147}$ J. M. Lattimer and J. Cooperstein, "Limits on the neutrino magnetic moment from SN1987A", Physical Review Letters 61, 23-26 (1988) (cit. on p. 32). 
${ }^{148}$ A. M. Nobili and A. Anselmi, "Relevance of the weak equivalence principle and experiments to test it: Lessons from the past and improvements expected in space", Physics Letters A 382, 2205-2218 (2018) (cit. on p. 32).

${ }^{149}$ L. M. Krauss and S. Tremaine, "Test of the weak equivalence principle for neutrinos and photons", Physical Review Letters 60, 176-177 (1988) (cit. on p. 32).

${ }^{150} \mathrm{~S}$. Horiuchi and J. P. Kneller, "What can be learned from a future supernova neutrino detection?", Journal of Physics G: Nuclear and Particle Physics 45, 043002 (2018) (cit. on p. 32$)$.

${ }^{151}$ G. G. Raffelt, "Supernova neutrino observations: What can we learn?", Nuclear Physics B - Proceedings Supplements 221, The Proceedings of the 22nd International Conference on Neutrino Physics and Astrophysics, 218-229 (2011) (cit. on p. 32).

${ }^{152}$ J. M. Blondin, A. Mezzacappa, and C. DeMarino, "Stability of standing accretion shocks, with an eye toward core-collapse supernovae", The Astrophysical Journal 584, 971 (2003) (cit. on p. 32).

${ }^{153}$ I. Tamborra et al., "Self-sustained asymmetry of lepton-number emission: A new phenomenon during the supernova shock-accretion phase in three dimensions", The Astrophysical Journal 792, 96 (2014) (cit. on p. 32).

${ }^{154}$ P. Antonioli, R. T. Fienberg, et al., "SNEWS: the SuperNova Early Warning System", New Journal of Physics 6, 114 (2004) (cit. on p. 32).

${ }^{155}$ SNEWS: SuperNova Early Warning System, Official website, 2018 (cit. on p. 32).

${ }^{156}$ K. Scholberg, "Supernova neutrino detection", Annual Review of Nuclear and Particle Science 62, 81-103 (2012) (cit. on pp. 32, 33, 56, 119).

${ }^{157}$ L. Hüdepohl et al., "Neutrino signal of electron-capture supernovae from core collapse to cooling", Physical Review Letters 104, 251101 (2010) (cit. on p. 35).

${ }^{158}$ K. Scholberg, "Supernova signatures of neutrino mass ordering", Journal of Physics G: Nuclear and Particle Physics 45, 014002 (2017) (cit. on p. 35). 
${ }^{159}$ A. Friedland, SN signal modeling: collective effects, Talk presented at the May 2018 DUNE Collaboration Meeting, Batavia, Illinois, May 17, 2018 (cit. on p. 35).

${ }^{160}$ M. Berglund and M. E. Wieser, "Isotopic compositions of the elements 2009 (IUPAC technical report)", Pure and Applied Chemistry 83, 397-410 (2011) (cit. on pp. 36, 252).

${ }^{161}$ P. J. Mohr, D. B. Newell, and B. N. Taylor, "CODATA recommended values of the fundamental physical constants: 2014', Reviews of Modern Physics 88, 035009 (2016) (cit. on p. 36).

${ }^{162}$ M. J. Kuusela, "Statistical issues in unfolding methods for high energy physics", MA thesis (Aalto University, 2012) (cit. on p. 41).

${ }^{163}$ M. J. Kuusela, "Uncertainty quantification in unfolding elementary particle spectra at the Large Hadron Collider', PhD thesis (École Polytechnique Fédérale de Lausanne, 2016) (cit. on p. 41).

${ }^{164}$ K. Mahn, C. Marshall, and C. Wilkinson, "Progress in measurements of $0.1-10 \mathrm{GeV}$ neutrino-nucleus scattering and anticipated results from future experiments", arXiv preprint arXiv:1803.08848, accepted for publication in Annual Review of Nuclear and Particle Science (2018) (cit. on pp. 41, 47).

${ }^{165}$ E. D. Church, "LArSoft: A software package for liquid argon time projection drift chambers", arXiv preprint arXiv:1311.6774 (2013) (cit. on pp. 41, 178).

${ }^{166}$ J. Paley et al., "LArIAT: Liquid Argon In A Testbeam", arXiv preprint arXiv:1406.5560 (2014) (cit. on p. 42).

${ }^{167}$ N. McConkey et al., SBND Collaboration, "SBND: Status of the Fermilab ShortBaseline Near Detector", 888, 012148 (2017) (cit. on p. 42).

${ }^{168}$ R. Acciarri, C. Adams, J. Asaadi, et al., ArgoNeuT Collaboration, "Measurement of $\nu_{\mu}$ and $\bar{\nu}_{\mu}$ neutral current $\pi^{0} \rightarrow \gamma \gamma$ production in the ArgoNeuT detector", Physical Review D 96, 012006 (2017) (cit. on p. 42). 
${ }^{169}$ C. Anderson, M. Antonello, B. Baller, et al., ArgoNeuT Collaboration, "First measurements of inclusive muon neutrino charged current differential cross sections on argon", Physical Review Letters 108, 161802 (2012) (cit. on p. 42).

${ }^{170}$ R. Acciarri, C. Adams, J. Asaadi, et al., ArgoNeuT Collaboration, "Detection of backto-back proton pairs in charged-current neutrino interactions with the ArgoNeuT detector in the NuMI low energy beam line", Physical Review D 90, 012008 (2014) (cit. on p. 42).

${ }^{171}$ J. S. Marshall and M. A. Thomson, "The Pandora software development kit for pattern recognition", The European Physical Journal C 75, 439 (2015) (cit. on p. 42).

${ }^{172}$ M. Diwan et al., "A novel method for event reconstruction in Liquid Argon Time Projection Chamber", 762, 012033 (2016) (cit. on p. 42).

${ }^{173}$ I. Lepetic et al., ArgoNeuT Collaboration, First demonstration of LArTPC MeV-scale physics in ArgoNeuT, Talk presented at the April Meeting of the American Physical Society, 2018 (cit. on p. 42).

${ }^{174}$ C. Andreopoulos et al., GENIE Collaboration, "The GENIE Neutrino Monte Carlo Generator", Nuclear Instruments and Methods A614, 87-104 (2010) (cit. on pp. 42, 208).

${ }^{175}$ T. Golan, J. Sobczyk, and J. Żmuda, "NuWro: the Wrocław Monte Carlo generator of neutrino interactions", Nuclear Physics B - Proceedings Supplements 229-232, 499 (2012) (cit. on p. 42).

${ }^{176}$ O. Buss, T. Gaitanos, K. Gallmeister, et al., "Transport-theoretical description of nuclear reactions", Physics Reports 512, 1-124 (2012) (cit. on p. 42).

${ }^{177}$ Y. Hayato, "A neutrino interaction simulation program library NEUT", Acta Physica Polonica B 40, 2477-2489 (2009) (cit. on p. 42).

${ }^{178}$ L. Alvarez-Ruso et al., NuSTEC: Neutrino Scattering Theory Experiment Collaboration, "NuSTEC white paper: Status and challenges of neutrino-nucleus scattering", Progress in Particle and Nuclear Physics 100, 1-68 (2018) (cit. on pp. 42, 43, 45, 47, 86). 
${ }^{179}$ U. Mosel, "Neutrino interactions with nucleons and nuclei: importance for long-baseline experiments", Annual Review of Nuclear and Particle Science 66, 171-195 (2016) (cit. on pp. 42,86$)$.

${ }^{180}$ A. A. Aguilar-Arevalo, B. C. Brown, L. Bugel, et al., MiniBooNE Collaboration, "First measurement of monoenergetic muon neutrino charged current interactions", Physical Review Letters 120, 141802 (2018) (cit. on p. 44).

${ }^{181} \mathrm{~K}$. Abe et al., T2K Collaboration, "Observation of electron neutrino appearance in a muon neutrino beam", Physical Review Letters 112, 061802 (2014) (cit. on p. 47).

${ }^{182}$ K. Abe, J. Amey, C. Andreopoulos, et al., T2K Collaboration, "Measurement of neutrino and antineutrino oscillations by the $\mathrm{T} 2 \mathrm{~K}$ experiment including a new additional sample of $\nu_{e}$ interactions at the far detector", Physical Review D 96, 092006 (2017) (cit. on p. 47).

${ }^{183}$ K. Babu, E. Kearns, U. Al-Binni, et al., "Baryon number violation", arXiv preprint arXiv:1311.5285, Report of the Community Summer Study (Snowmass 2013), Intensity Frontier - Baryon Number Violation Group (2013) (cit. on p. 48).

${ }^{184}$ K. Abe, Y. Haga, Y. Hayato, et al., Super-Kamiokande Collaboration, "Search for proton decay via $p \rightarrow e^{+} \pi^{0}$ and $p \rightarrow \mu^{+} \pi^{0}$ in 0.31 megaton $\cdot$ years exposure of the Super-Kamiokande water Cherenkov detector", Physical Review D 95, 012004 (2017) (cit. on p. 48).

${ }^{185}$ H. Ejiri, "Nuclear deexcitations of nucleon holes associated with nucleon decays in nuclei", Physical Review C 48, 1442-1444 (1993) (cit. on p. 48).

${ }^{186}$ J. F. Beacom, "The diffuse supernova neutrino background", Annual Review of Nuclear and Particle Science 60, 439-462 (2010) (cit. on pp. 49, 50).

${ }^{187}$ H. Zhang, K. Abe, et al., Super-Kamiokande Collaboration, "Supernova relic neutrino search with neutron tagging at Super-Kamiokande-IV', Astroparticle Physics 60, 4146 (2015) (cit. on p. 49). 
${ }^{188}$ A. Gando, Y. Gando, K. Ichimura, et al., KamLAND Collaboration, "Search for extraterrestrial antineutrino sources with the KamLAND detector', The Astrophysical Journal 745, 193 (2012) (cit. on p. 49).

${ }^{189}$ A. Mirizzi, I. Tamborra, et al., "Supernova neutrinos: production, oscillations and detection", La Rivista del Nuovo Cimento 39, 1-112 (2016) (cit. on p. 49).

${ }^{190}$ C. Lunardini, "Diffuse supernova neutrinos at underground laboratories", Astroparticle Physics 79, 49-77 (2016) (cit. on p. 49).

${ }^{191}$ H. Watanabe, H. Zhang, K. Abe, et al., Super-Kamiokande Collaboration, "First study of neutron tagging with a water Cherenkov detector", Astroparticle Physics 31, 320328 (2009) (cit. on p. 50).

${ }^{192}$ B. Adams et al., "Timing characteristics of Large Area Picosecond Photodetectors", Nuclear Instruments and Methods in Physics Research Section A: Accelerators, Spectrometers, Detectors and Associated Equipment 795, 1-11 (2015) (cit. on pp. 52, 53).

${ }^{193}$ E. Oberla et al., "A $15 \mathrm{GSa} / \mathrm{s}, 1.5 \mathrm{GHz}$ bandwidth waveform digitizing ASIC", Nuclear Instruments and Methods in Physics Research Section A: Accelerators, Spectrometers, Detectors and Associated Equipment 735, 452-461 (2014) (cit. on p. 52).

${ }^{194}$ J. Xie et al., "Rate capability and magnetic field tolerance measurements of fast timing microchannel plate photodetectors', Nuclear Instruments and Methods in Physics Research Section A: Accelerators, Spectrometers, Detectors and Associated Equipment (2017) 10.1016/j.nima.2017.10.059 (cit. on p. 52).

${ }^{195} \mathrm{~J}$. Wang, ANNIE reconstruction using LAPPDs, Talk presented at the 12-14 April 2018 Theia Workshop at the University of California, Davis, 2018 (cit. on p. 54).

${ }^{196}$ A. Aguilar-Arevalo et al., Bringing the SciBar detector to the Booster Neutrino Beam, tech. rep. FERMILAB-PROPOSAL-0954 (Fermi National Accelerator Laboratory, Batavia, Illinois, 2006) (cit. on pp. 53, 203, 212).

${ }^{197}$ P. Vogel, "Neutrino-nucleus cross section at low energies", Nuclear Physics A 777, 340-355 (2006) (cit. on p. 56). 
${ }^{198}$ E. Kolbe et al., "Neutrino-nucleus reactions and nuclear structure", Journal of Physics G: Nuclear and Particle Physics 29, 2569-2596 (2003) (cit. on pp. 56, 184).

${ }^{199}$ K. Kubodera and S. Nozawa, "Neutrino-nucleus reactions", International Journal of Modern Physics E 3, 101-148 (1994) (cit. on p. 56).

${ }^{200}$ K. Balasi, K. Langanke, and G. Martínez-Pinedo, "Neutrino-nucleus reactions and their role for supernova dynamics and nucleosynthesis", Progress in Particle and Nuclear Physics 85, 33-81 (2015) (cit. on pp. 56, 119, 168).

${ }^{201}$ J. A. Formaggio and G. P. Zeller, "From eV to EeV: Neutrino cross sections across energy scales", Reviews of Modern Physics 84, 1307-1341 (2012) (cit. on pp. 56, 57, $59)$.

${ }^{202}$ J. R. Distel et al., "Measurement of the cross section for the reaction ${ }^{127} \mathrm{I}\left(\nu_{e}, e^{-}\right){ }^{127} \mathrm{Xe}_{\text {bound states }}$ with neutrinos from the decay of stopped muons", Physical Review C 68, 054613 (2003) (cit. on p. 56).

${ }^{203}$ R. Acciarri et al., DUNE Collaboration, "Long-Baseline Neutrino Facility (LBNF) and Deep Underground Neutrino Experiment (DUNE) conceptual design report volume 2: The physics program for DUNE at LBNF", arXiv preprint arXiv:1512.06148 (cit. on p. 56).

${ }^{204}$ A. Samana, "QRPA-based calculations for neutrino scattering and electroweak excitations of nuclei", in Proceedings of the XVII International Workshop on Neutrino Factories and Future Neutrino Facilities (NuFact15) (Aug. 2015) (cit. on pp. 57, 59).

${ }^{205}$ S. Nakamura et al., "Towards a unified model of neutrino-nucleus reactions for neutrino oscillation experiments", Reports on Progress in Physics 80, 056301 (2017) (cit. on pp. 57, 115).

${ }^{206}$ K. Kubodera, "Neutrino-nucleus reactions and effective field theory", in Proceedings of the First Yamada Symposium on Neutrinos and Dark Matter in Nuclear Physics (2003) (cit. on p. 57).

${ }^{207}$ J. S. O'Connell, T. W. Donnelly, and J. D. Walecka, "Semileptonic weak interactions with C ${ }^{12}$, , Physical Review C 6, 719-733 (1972) (cit. on p. 57). 
${ }^{208}$ T. W. Donnelly and J. D. Walecka, "Electron scattering and nuclear structure", Annual Review of Nuclear Science 25, 329-405 (1975) (cit. on p. 57).

${ }^{209}$ T. Donnelly et al., "Nuclear excitation by neutral weak currents", Physics Letters B 49, 8-12 (1974) (cit. on pp. 57, 62).

${ }^{210}$ J. D. Walecka, "Semileptonic weak interactions in nuclei", in Muon Physics, Volume II: Weak Interactions, edited by V. W. Hughes and C. S. Wu (1975), p. 114 (cit. on pp. 57, 67, 91, 92).

${ }^{211}$ T. Donnelly and R. Peccei, "Neutral current effects in nuclei", Physics Reports 50, 1-85 (1979) (cit. on pp. 57, 62).

${ }^{212}$ J. Walecka, Theoretical Nuclear and Subnuclear Physics (Imperial College Press, 2004) (cit. on p. 57).

${ }^{213}$ A. Botrugno, "Interazione neutrino-nucleo", in Italian, PhD thesis (Università degli Studi di Lecce, 2004) (cit. on p. 57).

${ }^{214}$ T. de Forest Jr. and J. Walecka, "Electron scattering and nuclear structure", Advances in Physics 15, 1-109 (1966) (cit. on p. 57).

${ }^{215}$ J. Luyten, H. Rood, and H. Tolhoek, "On the theory of muon capture by complex nuclei (I)', Nuclear Physics 41, 236-274 (1963) (cit. on p. 57).

${ }^{216}$ J. Luyten and H. Tolhoek, "On the theory of muon capture by complex nuclei (II)", Nuclear Physics 70, 641-657 (1965) (cit. on p. 57).

${ }^{217}$ H. Rood and H. Tolhoek, "The theory of muon capture by complex nuclei: (III). Radiative muon capture", Nuclear Physics 70, 658-692 (1965) (cit. on p. 57).

${ }^{218}$ T. Donnelly and J. Walecka, "Semi-leptonic weak and electromagnetic interactions in nuclei with application to ${ }^{16} \mathrm{O}$ ", Physics Letters B 41, 275-280 (1972) (cit. on p. 57).

${ }^{219}$ T. Donnelly and J. Walecka, "Electromagnetic and weak interactions with nuclei: Onebody densities in ${ }^{6}$ LI', Physics Letters B 44, 330-334 (1973) (cit. on p. 57).

${ }^{220}$ T. Donnelly, "Low-energy neutrino reactions with ${ }^{12} \mathrm{C}$ and ${ }^{16} \mathrm{O}$ ", Physics Letters B 43, 93-97 (1973) (cit. on p. 57). 
${ }^{221}$ T. Donnelly and J. Walecka, "Elastic magnetic electron scattering and nuclear moments", Nuclear Physics A 201, 81-106 (1973) (cit. on p. 57).

${ }^{222}$ T. Donnelly and J. Walecka, "Semi-leptonic weak and electromagnetic interactions with nuclei: Isoelastic processes", Nuclear Physics A 274, 368-412 (1976) (cit. on p. 57).

${ }^{223}$ T. Donnelly and R. Peccei, "Testing the structure of weak neutral currents by inelastic neutrino scattering from nuclei", Physics Letters B 65, 196-200 (1976) (cit. on p. 57).

${ }^{224}$ T. Donnelly and W. Haxton, "Multipole operators in semileptonic weak and electromagnetic interactions with nuclei: Harmonic oscillator single-particle matrix elements", Atomic Data and Nuclear Data Tables 23, 103-176 (1979) (cit. on pp. 57, 58).

${ }^{225}$ J. Kostensalo, J. Suhonen, and K. Zuber, "Shell-model computed cross sections for charged-current scattering of astrophysical neutrinos off ${ }^{40}$ Ar"', Physical Review C 97, 034309 (2018) (cit. on pp. 58, 103, 167, 181).

${ }^{226}$ P. Pirinen, J. Suhonen, and E. Ydrefors, "Neutral-current neutrino-nucleus scattering off Xe isotopes", arXiv preprint arXiv:1804.08995 (2018) (cit. on p. 58).

${ }^{227} \mathrm{~W}$. Almosly, "Theoretical study of supernova neutrino and antineutrino scattering off the stable cadmium and lead isotopes", PhD thesis (2017) (cit. on pp. 58, 102).

${ }^{228}$ W. Almosly et al., "Theoretical estimates of supernova-neutrino cross sections for the stable even-even lead isotopes: Charged-current reactions", Physical Review C 94, 044614 (2016) (cit. on p. 58).

${ }^{229}$ P. C. Divari, "Supernova neutrino reactions on ${ }^{114} \mathrm{Cd}$ and ${ }^{116} \mathrm{Cd}$ isotopes via charge current interaction", Journal of Physics G: Nuclear and Particle Physics 40, 125201 (2013) (cit. on p. 58).

${ }^{230}$ E. Ydrefors and J. Suhonen, "Charged-current neutrino-nucleus scattering off the even molybdenum isotopes", Advances in High Energy Physics 2012, 373946 (2012) (cit. on pp. 58, 100). 
${ }^{231}$ B. D. Serot, "Semileptonic weak and electromagnetic interactions with nuclei: Nuclear current operators through order $(v / c)_{\text {nucleon }}^{2}$ ", Nuclear Physics A 308, 457-499 (1978) (cit. on pp. 58, 80, 81, 92).

${ }^{232}$ Wolfram Research, Incorporated, Mathematica, Champaign, Illinois, 2018 (cit. on p. 58).

${ }^{233}$ W. Haxton and C. Lunardini, "SevenOperators, a Mathematica script for harmonic oscillator nuclear matrix elements arising in semileptonic electroweak interactions", Computer Physics Communications 179, 345-358 (2008) (cit. on p. 58).

${ }^{234}$ T. Kuramoto et al., "Neutrino-induced reaction cross sections at intermediate energies for chlorine and water detectors", Nuclear Physics A 512, 711-736 (1990) (cit. on pp. 58, 91).

${ }^{235}$ M. Fukugita et al., "Reaction cross section for solar flare neutrinos with ${ }^{37} \mathrm{Cl}$ and ${ }^{16} \mathrm{O}$ targets", The Astrophysical Journal 337, L59-L63 (1989) (cit. on p. 58).

${ }^{236} \mathrm{M}$. Fukugita et al., "Reaction cross sections for $\nu{ }^{13} \mathrm{C} \rightarrow e^{-13} \mathrm{~N}$ and $\nu{ }^{13} \mathrm{C} \rightarrow \nu^{\prime}{ }^{13} \mathrm{C}^{*}$ for low energy neutrinos", Physical Review C 41, 1359-1364 (1990) (cit. on p. 58).

${ }^{237} \mathrm{C}$. Volpe et al., "Charged-current neutrino- ${ }^{208} \mathrm{~Pb}$ reactions", Physical Review C 65, 044603 (2002) (cit. on pp. 58, 100).

${ }^{238} \mathrm{C}$. Volpe et al., "Microscopic theories of neutrino- ${ }^{12} \mathrm{C}$ reactions", Physical Review C 62, 015501 (2000) (cit. on p. 58).

${ }^{239}$ J. Engel et al., "Quasielastic neutrino scattering from oxygen and the atmospheric neutrino problem", Physical Review D 48, 3048-3054 (1993) (cit. on p. 58).

${ }^{240} \mathrm{O}$. Civitarese and T. Tarutina, "Multipole decomposition of the cross section of the neutrino-lead scattering process ${ }^{208} \mathrm{~Pb}$ (g.s.) $\left(\nu, \nu^{\prime}\right)^{208} \mathrm{~Pb}^{*}$, , Physical Review C 94, 054603 (2016) (cit. on p. 58).

${ }^{241}$ A. R. Samana et al., "Neutrino and antineutrino charge-exchange reactions on ${ }^{12} \mathrm{C} "$, Physical Review C 83, 024303 (2011) (cit. on pp. 58, 59, 91). 
${ }^{242}$ F. Krmpotić, A. Samana, and A. Mariano, "Neutrino-nucleus reactions and muon capture in ${ }^{12}$ C", Physical Review C 71, 044319 (2005) (cit. on pp. 58, 59, 61, 67, 83, 84, $89,91,92,96)$.

${ }^{243}$ A. Samana, F. Krmpotić, and A. Mariano, "A formalism for weak interactions in nuclei", Brazilian Journal of Physics 34, 862-864 (2004) (cit. on p. 58).

${ }^{244}$ F. Krmpotić, A. Mariano, and A. Samana, "RPA puzzle in ${ }^{12} \mathrm{C}$ weak decay processes", Physics Letters B 541, 298-306 (2002) (cit. on p. 58).

${ }^{245}$ A. Samana, F. Krmpotić, and C. Bertulani, "QRAP: A numerical code for projected (Q)uasiparticle (RA)ndom (P)hase approximation", Computer Physics Communications 181, 1123-1135 (2010) (cit. on pp. 58, 183).

${ }^{246}$ A. Samana et al., "A reanalysis of the LSND neutrino oscillation experiment", Physics Letters B 642, 100-105 (2006) (cit. on p. 59).

${ }^{247}$ A. R. Samana, D. Sande, and F. Krmpotić, "Systematic muon capture rates in PQRPA", AIP Conference Proceedings 1663, 120003 (2015) (cit. on p. 59).

${ }^{248}$ A. Fujii and Y. Yamaguchi, "Muon capture in $\mathrm{He}^{3}$ ", Progress of Theoretical Physics 31, 107-114 (1964) (cit. on p. 59).

${ }^{249}$ C. W. Kim and H. Primakoff, "Theory of muon capture with initial and final nuclei treated as "elementary" particles", Physical Review 140, B566-B575 (1965) (cit. on p. 59).

${ }^{250}$ C. Kim and H. Primakoff, "Nuclei as elementary particles in weak and electromagnetic processes", in Mesons in Nuclei, Vol. 1, edited by M. Rho and D. H. Wilkinson (North Holland Publishing Company, 1979), pp. 67-105 (cit. on p. 59).

${ }^{251}$ M. Fukugita, Y. Kohyama, and K. Kubodera, "Neutrino reaction cross sections on ${ }^{12} \mathrm{C}$ target", Physics Letters B 212, 139-144 (1988) (cit. on p. 59).

${ }^{252}$ J. Engel et al., "Neutrino induced transitions between the ground states of the $A=12$ triad", Physical Review C 54, 2740-2744 (1996) (cit. on p. 59). 
${ }^{253}$ N. Itoh, Y. Kohyama, and A. Fujii, "A statistical theory of nuclear neutrino capture", Nuclear Physics A 287, 501-505 (1977) (cit. on pp. 59, 60).

${ }^{254}$ N. Itoh and Y. Kohyama, "Statistical theory of nuclear neutrino capture: (II). Inclusion of first-forbidden transitions", Nuclear Physics A 306, 527-535 (1978) (cit. on pp. 59, $60)$.

${ }^{255}$ A. R. Samana et al., "The gross theory model for neutrino-nucleus cross-section", New Journal of Physics 10, 033007 (2008) (cit. on pp. 59, 60, 183).

${ }^{256}$ Y.-Z. Qian et al., "Neutrino-induced neutron spallation and supernova $r$-process nucleosynthesis", Physical Review C 55, 1532-1544 (1997) (cit. on p. 60).

${ }^{257}$ E. J. Konopinski, "Beta-decay", Rev. Mod. Phys. 15, 209-245 (1943) (cit. on p. 60).

${ }^{258}$ W. Heisenberg, "Über den Bau der Atomkerne. I. (On the construction of atomic nuclei. I.)', Zeitschrift für Physik 77, in German, 1-11 (1932) (cit. on p. 64).

${ }^{259}$ E. Wigner, "On the consequences of the symmetry of the nuclear Hamiltonian on the spectroscopy of nuclei", Physical Review 51, 106-119 (1937) (cit. on p. 64).

${ }^{260}$ J. Suhonen, From Nucleons to Nucleus: Concepts of Microscopic Nuclear Theory, Theoretical and Mathematical Physics (Springer, 2007) (cit. on pp. 64, 79, 89, 93, 141-143, $149,151,285)$.

${ }^{261}$ İ. Şahin, "Zero-mass limit of a dirac spinor with general spin orientation", European Journal of Physics 37, 065404 (2016) (cit. on p. 68).

${ }^{262}$ S. Weinberg, The Quantum Theory of Fields, vol. 1 (Cambridge University Press, 1995) (cit. on pp. 68, 278, 279).

${ }^{263}$ M. Cannoni, "Lorentz invariant relative velocity and relativistic binary collisions", International Journal of Modern Physics A 32, 1730002 (2017) (cit. on p. 70).

${ }^{264}$ R. Blin-Stoyle and S. Nair, "The fundamentals of $\beta$-decay theory", Advances in Physics 15, 493-545 (1966) (cit. on p. 80).

${ }^{265}$ B. Povh and M. Rosina, Scattering and Structures: Essentials and Analogies in Quantum Physics, Graduate Texts in Physics (Springer, 2017) (cit. on p. 85). 
${ }^{266}$ V. Cirigliano, S. Gardner, and B. R. Holstein, "Beta decays and non-standard interactions in the LHC era', Progress in Particle and Nuclear Physics 71, 93-118 (2013) (cit. on p. 85).

${ }^{267}$ S. Weinberg, "Charge symmetry of weak interactions", Physical Review 112, 1375 (1958) (cit. on p. 85).

${ }^{268}$ T. Donnelly et al., Foundations of Nuclear and Particle Physics (Cambridge University Press, 2017) (cit. on p. 85).

${ }^{269}$ C. Perdrisat, V. Punjabi, and M. Vanderhaeghen, "Nucleon electromagnetic form factors", Progress in Particle and Nuclear Physics 59, 694-764 (2007) (cit. on p. 85).

${ }^{270}$ V. Bernard, L. Elouadrhiri, and U.-G. Meißner, "Axial structure of the nucleon", Journal of Physics G: Nuclear and Particle Physics 28, R1 (2001) (cit. on p. 85).

${ }^{271}$ T. Gorringe and H. W. Fearing, "Induced pseudoscalar coupling of the proton weak interaction", Reviews of Modern Physics 76, 31 (2003) (cit. on pp. 85, 86).

${ }^{272}$ A. S. Meyer et al., "Deuterium target data for precision neutrino-nucleus cross sections", Physical Review D 93, 113015 (2016) (cit. on p. 86).

${ }^{273}$ V. Andreev et al., MuCap Collaboration, "Measurement of muon capture on the proton to $1 \%$ precision and determination of the pseudoscalar coupling $g_{p}{ }^{\prime \prime}$, Physical Review Letters 110, 012504 (2013) (cit. on p. 86).

${ }^{274}$ H. S. Budd, "Modeling quasielastic form-factors for electron and neutrino scattering", in Proceedings of the 2nd International Workshop on Neutrino-Nucleus Interactions in the Few GeV Region (NuInt02) (University of California, Irvine) (cit. on p. 86).

${ }^{275}$ C. H. Llewellyn Smith, "Neutrino reactions at accelerator energies", Physics Reports 3, 261-379 (1972) (cit. on p. 86).

${ }^{276}$ K. Hagiwara, K. Mawatari, and H. Yokoya, "Tau polarization in tau-neutrino nucleon scattering", Nuclear Physics B 668, 364-384 (2003) (cit. on p. 86).

${ }^{277}$ G. Racah, "Theory of complex spectra. II", Physical Review 62, 438 (1942) (cit. on p. 89). 
${ }^{278}$ H. Behrens and W. Bühring, Electron Radial Wave Functions and Nuclear Beta-decay, International Series of Monographs on Physics (Clarendon Press, 1982) (cit. on pp. 95, 97).

${ }^{279}$ J. Jackson, S. Treiman, and H. Wyld, "Note on relativistic coulomb wave functions", Zeitschrift für Physik 150, 640-647 (1958) (cit. on pp. 96-98).

${ }^{280}$ M. S. Athar and S. Singh, " $\nu_{e}\left(\bar{\nu}_{e}\right)-{ }^{40}$ Ar absorption cross sections for supernova neutrinos", Physics Letters B 591, 69-75 (2004) (cit. on pp. 96, 180).

${ }^{281}$ N. Paar et al., "Large-scale calculations of supernova neutrino-induced reactions in $Z=8-82$ target nuclei", Physical Review C 87, 025801 (2013) (cit. on pp. 96, 181, 183).

${ }^{282}$ T. Suzuki and M. Honma, "Neutrino capture reactions on ${ }^{40}$ Ar", Physical Review C 87, 014607 (2013) (cit. on pp. 96, 167, 181, 183).

${ }^{283}$ J. Engel, "Approximate treatment of lepton distortion in charged-current neutrino scattering from nuclei", Phys. Rev. C 57, 2004-2009 (1998) (cit. on pp. 98, 99).

${ }^{284}$ I. Thompson and F. Nunes, Nuclear Reactions for Astrophysics: Principles, Calculation and Applications of Low-Energy Reactions (Cambridge University Press, 2009) (cit. on pp. 99, 136, 139, 279).

${ }^{285}$ C. Bertulani and P. Danielewicz, Introduction to Nuclear Reactions, Graduate Student Series in Physics (Taylor \& Francis, 2004) (cit. on p. 99).

${ }^{286}$ A. Aste and D. Trautmann, "Focusing of high-energy particles in the electrostatic field of a homogeneously charged sphere and the effective momentum approximation", The European Physical Journal A 33, 11-20 (2007) (cit. on p. 99).

${ }^{287}$ E. Ydrefors, "Supernova-neutrino induced reactions on molybdenum via neutral-currents", PhD thesis (Jyväskylän yliopisto (University of Jyväskylä), 2012) (cit. on p. 102).

${ }^{288}$ M.-K. Cheoun, E. Ha, and T. Kajino, "High-lying excited states in Gamow Teller strength and their roles in neutrino reactions", The European Physical Journal A 48, 137 (2012) (cit. on pp. 103, 147, 166-171, 181). 
${ }^{289}$ S. Pate and D. Trujillo, "Strangeness vector and axial-vector form factors of the nucleon", in EPJ Web of Conferences, Vol. 66 (EDP Sciences, 2014), p. 06018 (cit. on p. 115).

${ }^{290}$ D. Z. Freedman, "Coherent effects of a weak neutral current", Phys. Rev. D 9, 13891392 (1974) (cit. on p. 117).

${ }^{291}$ D. Akimov, J. B. Albert, P. An, et al., COHERENT Collaboration, "Observation of coherent elastic neutrino-nucleus scattering", Science 357, 1123-1126 (2017) (cit. on p. 117).

${ }^{292}$ K. S. Krane, Introductory Nuclear Physics (Wiley, 1987) (cit. on p. 119).

${ }^{293}$ P. E. Hodgson, "Direct and compound processes in nuclear reactions", Revista Brasileira de F'́sica 2 (1972) (cit. on p. 119).

${ }^{294}$ K. Langanke, P. Vogel, and E. Kolbe, "Signal for supernova $\nu_{\mu}$ and $\nu_{\tau}$ neutrinos in water Čerenkov detectors", Physical Review Letters 76, 2629 (1996) (cit. on pp. 119, 184).

${ }^{295}$ E. Kolbe et al., "Inelastic neutrino scattering on ${ }^{12} \mathrm{C}$ and ${ }^{16} \mathrm{O}$ above the particle emission threshold", Nuclear Physics A 540, 599-620 (1992) (cit. on p. 119).

${ }^{296}$ E. Kolbe and T. S. Kosmas, "Recent highlights on neutrino-nucleus interactions", in Symmetries in intermediate and high energy physics (Springer, Berlin, Heidelberg, 2000), pp. 199-225 (cit. on p. 119).

${ }^{297}$ E. Kolbe and K. Langanke, "Role of $\nu$-induced reactions on lead and iron in neutrino detectors", Phys. Rev. C 63, 025802 (2001) (cit. on p. 119).

${ }^{298}$ E. Kolbe, "Semileptonic weak interactions in nuclei within continuum RPA", Nuclear Physics A 719, C135-C143 (2003) (cit. on p. 119).

${ }^{299}$ M.-K. Cheoun et al., "Neutrino induced reactions for $\nu$-process nucleosynthesis of ${ }^{92} \mathrm{Nb}$ and ${ }^{98}$ Tc", Physical Review C 85, 065807 (2012) (cit. on p. 119).

${ }^{300}$ A. Bandyopadhyay et al., "Detecting supernova neutrinos with iron and lead detectors", Phys. Rev. D 95, 065022 (2017) (cit. on p. 119). 
${ }^{301}$ D. Vale, T. Rauscher, and N. Paar, "Hybrid method to resolve the neutrino mass hierarchy by supernova (anti)neutrino induced reactions", Journal of Cosmology and Astroparticle Physics 2016, 007 (2016) (cit. on p. 119).

${ }^{302}$ A. G. Flowers et al., "New evidence for a direct process in the $(e, \alpha)$ reaction", Physical Review Letters 40, 709-712 (1978) (cit. on pp. 119, 120).

${ }^{303}$ K. Kim and M.-K. Cheoun, "Roles of one-step process on neutrino scattering off ${ }^{12}$ C", Physics Letters B 679, 330-333 (2009) (cit. on p. 120).

${ }^{304}$ M.-K. Cheoun and K. S. Kim, "One and two-step processes on the neutrino-nucleus scattering near nucleon threshold region", International Journal of Modern Physics: Conference Series 01, 171-176 (2011) (cit. on p. 120).

${ }^{305}$ A. French and P. Kennedy, Niels Bohr: A Centenary Volume (Harvard University Press, 1987) (cit. on p. 121).

${ }^{306}$ N. Bohr, "Neutron capture and nuclear constitution", Nature 137, 344-348 (1936) (cit. on p. 121).

${ }^{307}$ N. Bohr, "Transmutations of atomic nuclei", Science 86, 161-165 (1937) (cit. on pp. 121, $122)$.

${ }^{308}$ N. Bohr and F. Kalckar, "On the transmutation of atomic nuclei by impact of material particles: I. General theoretical remarks", Det Kongelige Danske Videnskabernes Selskab. Mathematisk-fysiske Meddelelser. (The Royal Danish Academy of Sciences and Letters. Mathematical-Physical Messages.) 14 (1937) (cit. on p. 121).

${ }^{309}$ A. Cole, Statistical Models for Nuclear Decay: From Evaporation to Vaporization, Series in Fundamental and Applied Nuclear Physics (CRC Press, 2000) (cit. on pp. 121, 278).

${ }^{310}$ V. Weisskopf, "Statistics and nuclear reactions", Physical Review 52, 295 (1937) (cit. on p. 122).

${ }^{311}$ L. Wolfenstein, "Conservation of angular momentum in the statistical theory of nuclear reactions", Physical Review 82, 690 (1951) (cit. on p. 122). 
${ }^{312}$ W. Hauser and H. Feshbach, "The inelastic scattering of neutrons", Physical Review 87, 366 (1952) (cit. on p. 122).

${ }^{313}$ A. J. Koning, S. Hilaire, and M. C. Duijvestijn, "TALYS-1.0", in Proceedings of the International Conference on Nuclear Data for Science and Technology 200\%, edited by O. Bersillon et al. (EDP Sciences), pp. 211-214 (cit. on pp. 123, 129).

${ }^{314}$ A. J. Koning and D. Rochman, "Modern nuclear data evaluation with the TALYS code system", Nuclear Data Sheets 113, 2841-2934 (2012) (cit. on pp. 123, 129, 144).

${ }^{315}$ M. Herman et al., "EMPIRE: nuclear reaction model code system for data evaluation", Nuclear Data Sheets 108, 2655-2715 (2007) (cit. on p. 123).

${ }^{316} \mathrm{O}$. Iwamoto et al., "The CCONE code system and its application to nuclear data evaluation for fission and other reactions", Nuclear Data Sheets 131, Special Issue on Nuclear Reaction Data, 259-288 (2016) (cit. on p. 123).

${ }^{317}$ T. Kawano et al., "Monte Carlo simulation for particle and $\gamma$-ray emissions in statistical Hauser-Feshbach model", Journal of Nuclear Science and Technology 47, 462-469 (2010) (cit. on p. 123).

${ }^{318}$ D. Brown et al., "ENDF/B-VIII.0: The 8th major release of the nuclear reaction data library with CIELO-project cross sections, new standards and thermal scattering data", Nuclear Data Sheets 148, 1-142 (2018) (cit. on p. 123).

${ }^{319}$ K. Shibata et al., "JENDL-4.0: A new library for nuclear science and engineering", Journal of Nuclear Science and Technology 48, 1-30 (2011) (cit. on p. 123).

${ }^{320}$ H. A. Bethe, "A continuum theory of the compound nucleus", Physical Review 57, 1125-1144 (1940) (cit. on p. 124).

${ }^{321}$ R. Capote et al., "RIPL - Reference Input Parameter Library for calculation of nuclear reactions and nuclear data evaluations", Nuclear Data Sheets 110, 3107-3214 (2009) (cit. on pp. 129, 131-133, 136, 144, 257).

${ }^{322}$ A. J. Koning, S. Hilaire, and S. Goriely, "Global and local level density models", Nuclear Physics A 810, 13-76 (2008) (cit. on pp. 129-133). 
${ }^{323} \mathrm{H}$. Bethe, "An attempt to calculate the number of energy levels of a heavy nucleus", Physical Review 50, 332 (1936) (cit. on p. 129).

${ }^{324}$ H. A. Bethe, "Nuclear physics B. nuclear dynamics, theoretical", Reviews of Modern Physics 9, 69 (1937) (cit. on pp. 129, 131).

$325 \AA$ A. Bohr, B. Mottelson, and D. Pines, "Possible analogy between the excitation spectra of nuclei and those of the superconducting metallic state", Physical Review 110, 936 (1958) (cit. on p. 130).

${ }^{326}$ A. Mengoni and Y. Nakajima, "Fermi-gas model parametrization of nuclear level density", Journal of Nuclear Science and Technology 31, 151-162 (1994) (cit. on pp. 130, $132)$.

${ }^{327}$ A. Sedrakian and J. W. Clark, "Superfluidity in nuclear systems and neutron stars", arXiv preprint arXiv:1802.00017 (2018) (cit. on p. 130).

${ }^{328}$ M. Grossjean and H. Feldmeier, "Level density of a Fermi gas with pairing interactions", Nuclear Physics A 444, 113-132 (1985) (cit. on p. 131).

${ }^{329}$ P. Demetriou and S. Goriely, "Microscopic nuclear level densities for practical applications", Nuclear Physics A 695, 95-108 (2001) (cit. on p. 131).

${ }^{330}$ S. Al-Quraishi et al., "Level densities for $20 \leq A \leq 110$ ", Physical Review C 67, 015803 (2003) (cit. on p. 131).

${ }^{331}$ A. Ignatyuk, G. Smirenkin, and A. Tishin, "Phenomenological description of energy dependence of the level density parameter", Yadernaya Fizika 21, in Russian, 485-490 (1975) (cit. on p. 132).

${ }^{332}$ G. Audi et al., "The AME2012 atomic mass evaluation: (I). Evaluation of input data, adjustment procedures", Chinese Physics C 36, 1287 (2012) (cit. on p. 132).

${ }^{333}$ M. Wang et al., "The AME2012 atomic mass evaluation: (II). Tables, graphs and references", Chinese Physics C 36, 1603 (2012) (cit. on p. 132).

${ }^{334}$ W. D. Myers and W. J. Swiatecki, "Nuclear masses and deformations", Nuclear Physics 81, 1-60 (1966) (cit. on p. 132). 
${ }^{335}$ A. Koning, S. Hilaire, and S. Goriely, TALYS-1.8: A nuclear reaction program, Nuclear Research and Consultancy Group (NRG) (Dec. 2015) (cit. on pp. 133, 138).

${ }^{336}$ H. Feshbach, "The optical model and its justification", Annual Review of Nuclear Science 8, 49-104 (1958) (cit. on p. 134).

${ }^{337}$ H. Feshbach, C. Porter, and V. Weisskopf, "Model for nuclear reactions with neutrons", Physical Review 96, 448 (1954) (cit. on pp. 134, 281, 282).

${ }^{338} \mathrm{~A}$. Koning and J. Delaroche, "Local and global nucleon optical models from $1 \mathrm{keV}$ to 200 MeV", Nuclear Physics A 713, 231-310 (2003) (cit. on pp. 134-136).

${ }^{339}$ R. D. Woods and D. S. Saxon, "Diffuse surface optical model for nucleon-nuclei scattering", Physical Review 95, 577 (1954) (cit. on p. 136).

${ }^{340}$ S. Watanabe, "High energy scattering of deuterons by complex nuclei", Nuclear Physics 8, 484-492 (1958) (cit. on p. 136).

${ }^{341}$ A. Y. Abul-Magd and M. El-Nadi, "Optical model parameters for composite particles", Progress of Theoretical Physics 35, 798-808 (1966) (cit. on p. 136).

${ }^{342}$ S. Kadmenskiı̌, Yadernaya Fizika 8, in Russian, 486 (1969) (cit. on pp. 136, 322).

${ }^{343}$ S. Kadmenskiı̌, "Polarizability and elastic scattering of $\alpha$ particles", Soviet Journal of Nuclear Physics 8, English translation of [342], 284 (1969) (cit. on p. 136).

${ }^{344} \mathrm{~J}$. W. Watson, "Optical potentials for the elastic scattering of ${ }^{6} \mathrm{Li}$ ions", Nuclear Physics A 198, 129-143 (1972) (cit. on p. 136).

${ }^{345}$ P. Keaton Jr and D. Armstrong, "Deuteron optical potential with a tensor term and breakup", Physical Review C 8, 1692 (1973) (cit. on p. 136).

${ }^{346} \mathrm{P}$. Keaton Jr, E. Aufdembrink, and L. Veeser, A model for the optical potential of composite particles, tech. rep. LA-4379-MS (Los Alamos Scientific Laboratory, 1970) (cit. on pp. 136, 137).

${ }^{347}$ M. Ermer et al., "The deuteron optical potential at low energies", Nuclear Physics A 533, 71-94 (1991) (cit. on p. 136). 
${ }^{348}$ D. Madland, "Recent results in the development of a global medium-energy nucleonnucleus optical-model potential', in Proceedings of a Specialists' Meeting on Preequilibrium Nuclear Reactions, NEA/NEANDC(1988)245/U (Feb. 1988), pp. 103-110 (cit. on p. 138).

${ }^{349}$ D. G. Madland, "Progress in the development of global medium-energy nucleon-nucleus optical model potentials", in Proceedings of the Specialists' Meeting on the Nucleon Nucleus Optical Model up to $200 \mathrm{MeV}$ (Nov. 1996) (cit. on p. 139).

${ }^{350} \mathrm{~V}$. Lukyanov et al., "Calculations of the $\mathrm{K}^{+}$-nucleus microscopic optical potential and of the corresponding differential elastic cross sections", in Proceedings of the 19th International Baldin Seminar on High Energy Physics Problems: Relativistic Nuclear Physics and Quantum Chromodynamics (2009) (cit. on p. 139).

${ }^{351}$ B. V. Carlson, "Optical model calculations with the code ECIS95", in Proceedings of the Workshop on Nuclear Reaction Data and Nuclear Reactors: Physics, Design, and Safety, part 1, edited by N. Paver, M. Herman, and A. Gandini (The Abdus Salam International Centre for Theoretical Physics), pp. 61-104 (cit. on p. 139).

${ }^{352}$ NIST Digital Library of Mathematical Functions, http://dlmf.nist.gov/, Release 1.0.11 of 2016-06-08, Online companion to [353], 2016 (cit. on pp. 139, 323).

${ }^{353}$ F. W. J. Olver et al., eds., NIST Handbook of Mathematical Functions, Print companion to [352] (Cambridge University Press, New York, NY, 2010) (cit. on p. 323).

${ }^{354}$ J. Thijssen, Computational Physics, 2nd ed. (Cambridge University Press, 2007) (cit. on p. 140).

${ }^{355}$ M. Galassi and B. Gough, GNU Scientific Library: Reference Manual (Network Theory, 2009) (cit. on p. 140).

${ }^{356}$ M. Galassi, J. Theiler, et al., GSL - GNU Scientific Library, 2018 (cit. on p. 140).

${ }^{357}$ J. Blatt and V. Weisskopf, Theoretical Nuclear Physics (Springer-Verlag, 1979) (cit. on p. 140). 
${ }^{358}$ B. Baramsai, "Neutron capture reactions on gadolinium isotopes", PhD thesis (North Carolina State University, 2010) (cit. on p. 144).

${ }^{359}$ D. M. Brink, "Some aspects of the interaction of light with matter", PhD thesis (Oxford University, 1955) (cit. on p. 144).

${ }^{360} \mathrm{P}$. Axel, "Electric dipole ground-state transition width strength function and 7-MeV photon interactions", Physical Review 126, 671 (1962) (cit. on p. 144).

${ }^{361}$ D. Brink, "Giant resonances in excited nuclei", in Proceedings of the International Workshop on Chaos and Collectivity in Many-Body Systems (CCM08) (2008) (cit. on p. 144).

${ }^{362}$ M. Harakeh and A. Woude, Giant Resonances: Fundamental High-frequency Modes of Nuclear Excitation, Oxford science publications (Oxford University Press, 2001) (cit. on pp. 144, 159).

${ }^{363}$ T. Belgya et al., Handbook for calculations of nuclear reaction data, RIPL-2, tech. rep. IAEA-TECDOC-1506 (International Atomic Energy Agency, Vienna, Austria, 2006) (cit. on p. 146).

${ }^{364}$ M. B. Chadwick et al., Reference Input Parameter Library: Handbook for calculations of nuclear reaction data, tech. rep. IAEA-TECDOC-1034 (International Atomic Energy Agency, Vienna, Austria, 1998) (cit. on p. 146).

${ }^{365}$ P. Siemens, Elements Of Nuclei: Many-body Physics With The Strong Interaction (CRC Press, 2018) (cit. on p. 149).

${ }^{366}$ Y. Lam, "Isospin symmetry breaking in sd shell nuclei", PhD thesis (Université de Bordeaux (University of Bordeaux), 2011) (cit. on p. 149).

${ }^{367}$ R. R. Doering et al., "Observation of giant Gamow-Teller strength in $(p, n)$ reactions", Physical Review Letters 35, 1691-1693 (1975) (cit. on pp. 150, 151).

${ }^{368}$ D. E. Bainum et al., "Observation of giant particle-hole resonances in ${ }^{90} \mathrm{Zr}(p, n){ }^{90} \mathrm{Nb}$ ", Physical Review Letters 44, 1751-1754 (1980) (cit. on pp. 150, 151). 
${ }^{369}$ B. D. Anderson et al., "Observation of a $T$, Gamow-Teller state in ${ }^{48} \mathrm{Ca}(p, n){ }^{48} \mathrm{Sc}$ at 160 MeV", Physical Review Letters 45, 699-702 (1980) (cit. on p. 150).

${ }^{370}$ K. Nakayama, A. P. Galeão, and F. Krmpotić, "On the energetics of the Gamow-Teller resonances", Physics Letters B 114, 217-221 (1982) (cit. on p. 150).

${ }^{371}$ K. Ikeda, S. Fujii, and J. Fujita, "The $(p, n)$ reactions and beta decays", Physics Letters 3, 271-272 (1963) (cit. on p. 151).

${ }^{372}$ M. Ichimura, H. Sakai, and T. Wakasa, "Spin-isospin responses via $(p, n)$ and $(n, p)$ reactions", Progress in Particle and Nuclear Physics 56, 446-531 (2006) (cit. on pp. 152, 159).

${ }^{373}$ C. Gaarde, "Gamow-Teller and M1 resonances", Nuclear Physics A 396, 127-144 (1983) (cit. on p. 152).

${ }^{374}$ B. A. Brown and B. H. Wildenthal, "Status of the nuclear shell model", Annual Review of Nuclear and Particle Science 38, 29-66 (1988) (cit. on p. 152).

${ }^{375}$ V. Kumar, P. C. Srivastava, and H. Li, "Nuclear $\beta^{-}$-decay half-lives for $f p$ and $f p g$ shell nuclei", Journal of Physics G: Nuclear and Particle Physics 43, 105104 (2016) (cit. on p. 152).

${ }^{376}$ C. D. Goodman, "Three decades of missing Gamow-Teller strength", in Spin and Isospin in Nuclear Interactions, edited by S. W. Wissink, C. D. Goodman, and G. E. Walker (Springer US, Boston, MA, 1991), pp. 167-179 (cit. on p. 152).

${ }^{377} \mathrm{~W}$. Ormand et al., "Neutrino capture cross sections for ${ }^{40} \mathrm{Ar}$ and $\beta$-decay of ${ }^{40} \mathrm{Ti}$ ", Physics Letters B 345, 343-350 (1995) (cit. on pp. 155, 167, 180).

${ }^{378}$ W. Liu, M. Hellström, R. Collatz, et al., " $\beta$-decay of ${ }^{40} \mathrm{Ti}$ ", Zeitschrift für Physik A Hadrons and Nuclei 359, 1-2 (1997) (cit. on p. 155).

${ }^{379} \mathrm{~W}$. Liu et al., " $\beta$ decay of ${ }^{40} \mathrm{Ti}$ and ${ }^{41} \mathrm{Ti}$ and implication for solar-neutrino detection", Physical Review C 58, 2677-2688 (1998) (cit. on pp. 155, 161, 163-165, 168, 170, 180). ${ }^{380} \mathrm{~W}$. Trinder et al., " ${ }^{40} \mathrm{Ti} \beta$ decay and the neutrino capture cross section of ${ }^{40} \mathrm{Ar}$ ", Physics Letters B 415, 211-216 (1997) (cit. on p. 155). 
${ }^{381}$ M. Lewitowicz, W. Trinder, M. Bhattacharya, et al., "Beta-decay of light nuclei close to the proton drip-line: ${ }^{40} \mathrm{Ti}$ and ${ }^{35} \mathrm{Ca}$ ", Il Nuovo Cimento A 111, 835-840 (1998) (cit. on p. 155).

${ }^{382} \mathrm{M}$. Bhattacharya et al., "Neutrino absorption efficiency of an ${ }^{40}$ Ar detector from the $\beta$ decay of ${ }^{40}$ Ti', Physical Review C 58, 3677-3687 (1998) (cit. on pp. 155, 157, 161, $163-165,168,170,180)$.

${ }^{383}$ C. Détraz et al., "Search for direct two-proton radioactivity from Ti isotopes at the proton drip line", Nuclear Physics A 519, 529-547 (1990) (cit. on p. 155).

${ }^{384}$ C. Dossat et al., "The decay of proton-rich nuclei in the mass $A=36-56$ region", Nuclear Physics A 792, 18-86 (2007) (cit. on p. 155).

${ }^{385}$ R. Anne and A. C. Mueller, "LISE 3: a magnetic spectrometer-Wien filter combination for secondary radioactive beam production", Nuclear Instruments and Methods in Physics Research Section B: Beam Interactions with Materials and Atoms 70, 276-285 (1992) (cit. on p. 155).

${ }^{386}$ J. A. Cameron and B. Singh, "Nuclear data sheets for $A=38$ ", Nuclear Data Sheets 109, 1-170 (2008) (cit. on p. 156).

${ }^{387}$ C. D. Goodman et al., "Gamow-Teller matrix elements from $0^{\circ}(p, n)$ cross sections", Physical Review Letters 44, 1755-1759 (1980) (cit. on p. 158).

${ }^{388}$ C. D. Goodman et al., "Data analysis techniques for extracting Gamow-Teller strengths from $0^{\circ}(p, n)$ data", Nuclear Instruments and Methods in Physics Research Section A: Accelerators, Spectrometers, Detectors and Associated Equipment 462, 545-554 (2001) (cit. on pp. 158-160).

${ }^{389}$ T. N. Taddeucci et al., "Energy dependence of the ratio of isovector effective interaction strengths $\left|\frac{J_{\sigma \tau}}{J_{\tau}}\right|$ from $0^{\circ}(p, n)$ cross sections", Physical Review C 25, 1094-1097 (1982) (cit. on p. 158).

${ }^{390} \mathrm{~T}$. Taddeucci et al., "The $(p, n)$ reaction as a probe of beta decay strength", Nuclear Physics A 469, 125-172 (1987) (cit. on pp. 158, 161). 
${ }^{391}$ D. Frekers et al., "Gamow-Teller strength extraction from $\left({ }^{3} \mathrm{He}, t\right)$ reactions", Nuclear Physics A 916, 219-240 (2013) (cit. on p. 159).

${ }^{392}$ Y. Fujita, B. Rubio, and W. Gelletly, "Spin-isospin excitations probed by strong, weak and electro-magnetic interactions", Progress in Particle and Nuclear Physics 66, 549606 (2011) (cit. on p. 159).

${ }^{393}$ W. P. Alford and B. M. Spicer, "Nucleon charge-exchange reactions at intermediate energy", in Advances in Nuclear Physics, edited by J. W. Negele and E. Vogt (Springer US, Boston, MA, 2002), pp. 1-82 (cit. on p. 159).

${ }^{394}$ J. Rapaport and E. Sugarbaker, "Isovector excitations in nuclei", Annual Review of Nuclear and Particle Science 44, 109-153 (1994) (cit. on p. 159).

${ }^{395}$ F. Osterfeld, "Nuclear spin and isospin excitations", Reviews of Modern Physics 64, 491-557 (1992) (cit. on p. 159).

${ }^{396}$ F. P. Brady, " $(n, p)$ studies at the University of California at Davis", Canadian Journal of Physics 65, 578-587 (1987) (cit. on p. 159).

${ }^{397}$ M. E. Howard, "The joy of CEX: Sharpening the $\left(t,{ }^{3} \mathrm{He}\right)$ probe at $345 \mathrm{MeV}$ for the charge-exchange knife drawer", PhD thesis (The Ohio State University, 2008) (cit. on p. 159).

${ }^{398}$ J. Birkhan et al., "Electromagnetic $M 1$ transition strengths from inelastic proton scattering: The cases of ${ }^{48} \mathrm{Ca}$ and ${ }^{208} \mathrm{~Pb}$ ", Physical Review C 93, 041302 (2016) (cit. on p. 159).

${ }^{399}$ M. Bhattacharya, C. D. Goodman, and A. García, "Weak-interaction strength from charge-exchange reactions versus $\beta$ decay in the $A=40$ isoquintet", Physical Review C 80, 055501 (2009) (cit. on pp. 161, 162, 164, 165, 168, 170, 180).

${ }^{400}$ J. Chen, "Nuclear data sheets for $A=40$ ", Nuclear Data Sheets 140, 1-376 (2017) (cit. on pp. 162, 164, 166).

${ }^{401}$ M. Karakoç et al., "Gamow-Teller transitions in the $A=40$ isoquintet of relevance for neutrino captures in ${ }^{40}$ Ar", Physical Review C 89, 064313 (2014) (cit. on p. 162). 
${ }^{402}$ J. K. Tuli, Evaluated Nuclear Structure Data File: A manual for preparation of data sets, tech. rep. BNL-NCS-51655-01/02-Rev (National Nuclear Data Center, Brookhaven National Laboratory, Upton, New York, 2001) (cit. on p. 164).

${ }^{403}$ R. P. Brent, "Chapter 5: An algorithm with guaranteed convergence for finding a minimum of a function of one variable", in Algorithms for Minimization without Derivatives (Prentice-Hall, 1973), pp. 61-80 (cit. on p. 174).

${ }^{404} \mathrm{R}$. Raghavan, Liquid argon detectors: A new approach to observation of neutrinos from meson factories and collapsing stars, tech. rep. 79-1131-31 (AT\&T Bell Laboratories, 1979) (cit. on p. 179).

${ }^{405}$ J. Bahcall et al., "Predictions for a liquid argon solar neutrino detector", Physics Letters B 178, 324-328 (1986) (cit. on p. 179).

${ }^{406} \mathrm{R}$. S. Raghavan, "Inverse $\beta^{-}$decay of ${ }^{40} \mathrm{Ar}$ : A new approach for observing $\mathrm{MeV}$ neutrinos from laboratory and astrophysical sources", Physical Review D 34, 2088-2091 (1986) (cit. on p. 179).

${ }^{407}$ R. S. Raghavan, S. Pakvasa, and B. A. Brown, "New tools for solving the solar-neutrino problem", Physical Review Letters 57, 1801-1804 (1986) (cit. on p. 179).

${ }^{408}$ A. Bueno, I. Gil-Botella, and A. Rubbia, "Supernova neutrino detection in a liquid argon TPC", arXiv preprint hep-ph/0307222 (2003) (cit. on p. 180).

${ }^{409}$ I. Gil-Botella and A. Rubbia, "Oscillation effects on supernova neutrino rates and spectra and detection of the shock breakout in a liquid argon TPC", Journal of Cosmology and Astroparticle Physics 2003, 009 (2003) (cit. on pp. 180, 183).

${ }^{410}$ M. S. Athar, S. Ahmad, and S. Singh, "Neutrino nucleus cross sections for low energy neutrinos at SNS facilities", Nuclear Physics A 764, 551-568 (2006) (cit. on p. 180).

${ }^{411}$ J. Goon et al., LBNE Collaboration, "The Long Baseline Neutrino Experiment (LBNE) Water Cherenkov Detector (WCD) Conceptual Design Report (CDR)", arXiv preprint arXiv:1204.2295 (2012) (cit. on p. 180). 
${ }^{412}$ M.-K. Cheoun, E. Ha, and T. Kajino, "Reactions on ${ }^{40} \mathrm{Ar}$ involving solar neutrinos and neutrinos from core-collapsing supernovae", Physical Review C 83, 028801 (2011) (cit. on pp. 181, 183).

${ }^{413} \mathrm{~V}$. Tsakstara, T. Kosmas, and J. Vergados, "Weak responses of neutral current neutrinonucleus reactions", Romanian Journal of Physics 58, 1258-1269 (2013) (cit. on p. 181).

${ }^{414}$ H. Đapo and N. Paar, "Neutral-current neutrino-nucleus cross sections based on relativistic nuclear energy density functional", Physical Review C 86, 035804 (2012) (cit. on p. 181).

${ }^{415}$ A. R. Samana, Constraining supernova neutrino detection with weak decay available data, Talk presented at the INT Workshop on Flavor Observations with Supernova Neutrinos (INT-16-61W), Seattle, Washington, Aug. 15, 2016 (cit. on pp. 181, 183).

${ }^{416}$ S. Chauhan, M. S. Athar, and S. K. Singh, "Neutrino-nucleus cross-sections at supernova neutrino energies", International Journal of Modern Physics E 26, 1750047 (2017) (cit. on p. 181).

${ }^{417}$ T. Suzuki et al., "New neutrino-nucleus reaction cross sections at solar, reactor and supernova neutrino energies", EPJ Web of Conferences 66, edited by S. Lunardi et al., 07025 (2014) (cit. on p. 181).

${ }^{418}$ E. Kolbe, K. Langanke, and G. Martínez-Pinedo, "Inclusive ${ }^{56} \mathrm{Fe}\left(\nu_{e}, e^{-}\right){ }^{56} \mathrm{Co}$ cross section", Physical Review C 60, 052801 (1999) (cit. on p. 181).

${ }^{419} \mathrm{~T}$. Totani et al., "Future detection of supernova neutrino burst and explosion mechanism", The Astrophysical Journal 496, 216 (1998) (cit. on pp. 186, 188).

${ }^{420}$ T. S. Kosmas et al., "Probing light sterile neutrino signatures at reactor and Spallation Neutron Source neutrino experiments", Physical Review D 96, 063013 (2017) (cit. on p. 187).

${ }^{421}$ C. Brönnimann, "The pion beta decay experiment: calibrations and developments", PhD thesis (Universität Zürich (University of Zurich), 1996) (cit. on p. 187). 
${ }^{422}$ H. Berns et al., "The CAPTAIN detector and physics program", arXiv preprint arXiv:1309.1740 (2013) (cit. on pp. 187, 195).

${ }^{423}$ T. C. Li et al., "First evidence for spin-flip $M 1$ strength in ${ }^{40}$ Ar", Physical Review C 73, 054306 (2006) (cit. on p. 193).

${ }^{424} \mathrm{~W}$. Tornow, S. Finch, et al., "Three-fold increase of M1 strength in ${ }^{40} \mathrm{Ar}$ at $10 \mathrm{MeV}$ excitation energy", Bulletin of the American Physical Society 62 (2017) (cit. on p. 193).

${ }^{425}$ E. Kolbe, K. Langanke, and G. M. Fuller, "Neutrino-induced fission of neutron-rich nuclei", Physical Review Letters 92, 111101 (2004) (cit. on p. 194).

${ }^{426}$ C. Grant, Low-energy neutrino-argon interactions: a window into stellar collapse, Talk presented at NuEclipse 2017: New Extensions of Coherent scattering and other Lepton Interactions for new Physics SEarches, 20-22 August 2017, Knoxville, Tennessee, 2017 (cit. on p. 194).

${ }^{427}$ C. Grant and B. Littlejohn, "Opportunities with decay-at-rest neutrinos from decayin-flight neutrino beams", arXiv preprint arXiv:1510.08431 (2015) (cit. on p. 195).

${ }^{428}$ D. Akimov, J. Albert, P. An, et al., COHERENT Collaboration, "COHERENT 2018 at the Spallation Neutron Source", arXiv preprint arXiv:1803.09183 (2018) (cit. on p. 195).

${ }^{429}$ B. Worthel et al., Booster rookie book, version 4.1, Batavia, Illinois, 2009 (cit. on pp. 198, 199).

${ }^{430}$ D. Barak, B. Harrison, and A. Watts, Concepts rookie book, Batavia, Illinois, 2013 (cit. on pp. 198, 199, 204-207).

${ }^{431}$ I. Stancu et al., BooNE Collaboration, Technical design report for the MiniBooNE neutrino beam, Batavia, Illinois, May 2001 (cit. on pp. 198, 199).

${ }^{432}$ S. van der Meer, A directive device for charged particles and its use in an enhanced neutrino beam, tech. rep. CERN-61-07 (European Organization for Nuclear Research (CERN), Geneva, Switzerland, 1961) (cit. on p. 198). 
${ }^{433}$ S. J. Brice, R. L. Cooper, F. DeJongh, et al., "A method for measuring coherent elastic neutrino-nucleus scattering at a far off-axis high-energy neutrino beam target", Physical Review D 89, 072004 (2014) (cit. on p. 199).

${ }^{434}$ M. M. Gattuso, MiniBooNE Mini-Rookie Book, tech. rep., version 1.0 (Fermi National Accelerator Laboratory, Batavia, Illinois, 2002) (cit. on p. 199).

${ }^{435}$ I. Stancu et al., BooNE Collaboration, Technical design report for the 8 GeV beam, Batavia, Illinois, May 2001 (cit. on p. 199).

${ }^{436}$ A. A. Aguilar-Arevalo et al., MiniBooNE collaboration, "Neutrino flux prediction at MiniBooNE", Physical Review D 79, 072002 (2009) (cit. on pp. 199, 201, 205).

${ }^{437}$ K. Hiraide et al., SciBooNE Collaboration, "Search for charged current coherent pion production on carbon in a few-GeV neutrino beam”, Physical Review D 78, 112004 (2008) (cit. on p. 202).

${ }^{438}$ M. Backfish, MiniBooNE resistive wall current monitor, tech. rep. FERMILAB-TM2556-AD (Fermi National Accelerator Laboratory, Batavia, Illinois, May 2013) (cit. on p. 205).

${ }^{439}$ J. R. Monroe, "A combined $\nu_{\mu}$ and $\nu_{e}$ oscillation search at MiniBooNE", PhD thesis (Columbia University, 2006) (cit. on p. 205).

${ }^{440}$ S. Agostinelli, J. Allison, K. Amako, et al., "Geant4-a simulation toolkit", Nuclear Instruments and Methods in Physics Research Section A: Accelerators, Spectrometers, Detectors and Associated Equipment 506, 250-303 (2003) (cit. on pp. 208, 245).

${ }^{441}$ A. Rindi and R. H. Thomas, "Skyshine-A paper tiger?", Particle Accelerators 7, 23-39 (1975) (cit. on p. 210).

${ }^{442}$ R. H. Thomas et al., "Radiological environmental impact of high-energy accelerators", CRC Critical Reviews in Environmental Control 9, 51-95 (1979) (cit. on p. 210).

${ }^{443}$ S. Lindenbaum, "Brookhaven National Laboratory proton synchrotron", in Proceedings of the Conference on Shielding of High-Energy Accelerators, USAEC TID-7545 (1957), pp. 28-37 (cit. on p. 210). 
${ }^{444}$ S. Lindenbaum, "Skyshine", in Proceedings of the Conference on Shielding of HighEnergy Accelerators, USAEC TID-7545 (1957), pp. 101-111 (cit. on p. 210).

${ }^{445}$ W. Lee, E. Maddry, W. Sippach, et al., "Observation of the reaction $\nu_{\mu}+p \rightarrow \nu_{\mu}+p$ ", Physical Review Letters 37, 186-189 (1976) (cit. on p. 210).

${ }^{446} \mathrm{H}$. Takei, Identification of recoil proton tracks for a neutrino neutral-current elastic scattering cross-section measurement at SciBooNE, Talk presented at the April Meeting of the American Physical Society, 2008 (cit. on pp. 212, 213).

${ }^{447}$ I. Anghel, J. Beacom, M. Bergevin, et al., ANNIE Collaboration, "Letter of intent: The Accelerator Neutrino Neutron Interaction Experiment (ANNIE)", arXiv preprint arXiv:1504.01480 (2015) (cit. on p. 213).

${ }^{448}$ Gadolinium Loaded EJ-331, EJ-335, http : / / eljentechnology . com / products / liquid-scintillators/ej-331-ej-335, 2016 (cit. on pp. 214, 224).

${ }^{449}$ R. Brun and F. Rademakers, "ROOT - An object oriented data analysis framework", Nuclear Instruments and Methods in Physics Research Section A: Accelerators, Spectrometers, Detectors and Associated Equipment 389, 81-86 (1997) (cit. on pp. 220, $229,259)$.

${ }^{450} \mathrm{~T}$. Boschi, "Phase I of the ANNIE experiment: Data acquisition system and preliminary data analysis", MA thesis (Università degli Studi di Padova, 2016) (cit. on p. 221).

${ }^{451}$ IAEA LiveChart of Nuclides, 2018 (cit. on pp. 224, 225).

${ }^{452}$ K. Beckurts, L. Dresner, and K. Wirtz, Neutron Physics (Springer, 2013) (cit. on p. 224).

${ }^{453}$ F. Neves, D. Y. Akimov, H. M. Araújo, et al., "ZE3RA: the ZEPLIN-III reduction and analysis package", Journal of Instrumentation 6, P11004 (2011) (cit. on pp. 229, 231).

${ }^{454} \mathrm{~B}$. P. Welford, "Note on a method for calculating corrected sums of squares and products", Technometrics 4, 419-420 (1962) (cit. on p. 230).

${ }^{455}$ W. Navidi, Statistics for Engineers and Scientists (McGraw-Hill, 2011) (cit. on p. 230). 
${ }^{456}$ E. W. Weisstein, "F-distribution", in MathWorld-A Wolfram Web Resource (Wolfram Research, 2018) (cit. on p. 230).

${ }^{457}$ W. Press et al., Numerical Recipes: The Art of Scientific Computing (Cambridge University Press, 2007) (cit. on p. 231).

${ }^{458}$ I. Mandrichenko et al., Implementation of beam conditions database for intensity frontier experiments, June 2012 (cit. on p. 234).

${ }^{459} \mathrm{~J}$. J. Walding, "A sub-GeV charged-current quasi-elastic $\nu_{\mu}$ cross-section on carbon at SciBooNE", PhD thesis (Imperial College London, 2009) (cit. on p. 235).

${ }^{460} R A T$ (is an analysis tool) user's guide, http://rat.readthedocs.io/en/latest/ index.html, 2014 (cit. on pp. 245, 251).

${ }^{461}$ R. Radev and T. McLean, Neutron sources for standard-based testing, tech. rep. LLNLTR-664160 (Lawrence Livermore National Laboratory, 2014) (cit. on pp. 249-251).

${ }^{462}$ N. Nica, "Nuclear data sheets for A = 252", Nuclear Data Sheets 106, 813-834 (2005) (cit. on p. 249).

${ }^{463}$ M.-M. Bé et al., Table of Radionuclides, Vol. 4, Monographie BIPM-5 (Bureau International des Poids et Mesures, Pavillon de Breteuil, F-92310 Sèvres, France, 2008) (cit. on p. 249).

${ }^{464}$ T. E. Valentine, "Evaluation of prompt fission gamma rays for use in simulating nuclear safeguard measurements", Annals of Nuclear Energy 28, 191-201 (2001) (cit. on p. 250).

${ }^{465}$ J. Verbeke, J. Randrup, and R. Vogt, "Fission Reaction Event Yield Algorithm FREYA 2.0.2", Computer Physics Communications 222, 263-266 (2018) (cit. on pp. 251, 254). ${ }^{466}$ LYSO scintillator material, http://www.crystals. saint-gobain.com/sites/imdf . crystals.com/files/documents/lyso-material-data-sheet.pdf, 2018 (cit. on p. 252).

${ }^{467}$ J. Randrup and R. Vogt, "Calculation of fission observables through event-by-event simulation", Physical Review C 80, 024601 (2009) (cit. on p. 254). 
${ }^{468}$ J. Verbeke, J. Randrup, and R. Vogt, "Fission Reaction Event Yield Algorithm, FREYA — for event-by-event simulation of fission", Computer Physics Communications 191, 178-202 (2015) (cit. on p. 254).

${ }^{469}$ R. Vogt and J. Randrup, "Event-by-event study of neutron observables in spontaneous and thermal fission", Physical Review C 84, 044621 (2011) (cit. on p. 254).

${ }^{470}$ R. Vogt and J. Randrup, "Event-by-event study of photon observables in spontaneous and thermal fission", Physical Review C 87, 044602 (2013) (cit. on p. 254).

${ }^{471}$ R. Vogt and J. Randrup, "Event-by-event modeling of prompt neutrons and photons from neutron-induced and spontaneous fission with FREYA', Nuclear Data Sheets 118, 220-223 (2014) (cit. on p. 254).

${ }^{472} \mathrm{~T}$. Wang et al., "Correlations of neutron multiplicity and $\gamma$-ray multiplicity with fragment mass and total kinetic energy in spontaneous fission of ${ }^{252} \mathrm{Cf}$ ', Physical Review C 93, 014606 (2016) (cit. on p. 254).

${ }^{473} \mathrm{P}$. Talou et al., "Correlated prompt fission data in transport simulations", The European Physical Journal A 54, 9 (2018) (cit. on p. 254).

${ }^{474}$ M. J. Marcath, R. C. Haight, R. Vogt, et al., "Measured and simulated ${ }^{252} \mathrm{Cf}(\mathrm{sf})$ prompt neutron-photon competition", Physical Review C 97, 044622 (2018) (cit. on p. 254).

${ }^{475}$ J. Verbeke, J. Randrup, and R. Vogt, Fission Reaction Yield Algorithm FREYA 2.0.2 user manual, tech. rep. LLNL-SM-705798 (Lawrence Livermore National Laboratory, Livermore, California, 2016) (cit. on p. 255).

${ }^{476} \mathrm{~W}$. Reisdorf et al., "Fission fragment $\mathrm{K}$ x-ray emission and nuclear charge distribution for thermal neutron fission of ${ }^{233} \mathrm{U},{ }^{235} \mathrm{U},{ }^{239} \mathrm{Pu}$ and spontaneous fission of ${ }^{252} \mathrm{Cf} "$, Nuclear Physics A 177, 337-378 (1971) (cit. on p. 255).

${ }^{477}$ F.-J. Hambsch and S. Oberstedt, "Investigation of the far asymmetric region in ${ }^{252} \mathrm{Cf}(\mathrm{sf})$ ", Nuclear Physics A 617, 347-355 (1997) (cit. on pp. 255, 257). 
${ }^{478}$ J. M. Verbeke, C. Hagmann, and D. Wright, Simulation of neutron and gamma ray emission from fission and photofission: LLNL Fission Library 2.0.2, tech. rep. UCRLAR-228518-REV-1 (Lawrence Livermore National Laboratory, 2016) (cit. on p. 257).

${ }^{479}$ M. Chadwick, M. Herman, P. Obložinský, et al., "ENDF/B-VII.1 nuclear data for science and technology: Cross sections, covariances, fission product yields and decay data", Nuclear Data Sheets 112, Special Issue on ENDF/B-VII.1 Library, 2887-2996 (2011) (cit. on p. 258).

${ }^{480} \mathrm{~F}$. James, MINUIT function minimization and error analysis reference manual version 94.1, tech. rep. CERN-D506 (European Organization for Nuclear Research (CERN), 1994) (cit. on p. 259).

${ }^{481}$ T. J. Roberts and D. M. Kaplan, "G4beamline simulation program for matter-dominated beamlines", in Proceedings of the 2007 IEEE Particle Accelerator Conference (PAC) (June 2007), pp. 3468-3470 (cit. on p. 264).

${ }^{482}$ J. Kremer, M. Boezio, M. L. Ambriola, et al., "Measurements of ground-level muons at two geomagnetic locations", Physical Review Letters 83, 4241-4244 (1999) (cit. on p. 264).

${ }^{483}$ W. Pauli, Niels Bohr and the Development of Physics: Essays Dedicated to Niels Bohr on the Occasion of His Seventieth Birthday (McGraw-Hill, 1955) (cit. on p. 281).

${ }^{484}$ G. E. Mitchell, A. Richter, and H. A. Weidenmüller, "Random matrices and chaos in nuclear physics: Nuclear reactions", Rev. Mod. Phys. 82, 2845-2901 (2010) (cit. on p. 282).

${ }^{485}$ J. Sakurai and J. Napolitano, Modern Quantum Mechanics (Cambridge University Press, 2017) (cit. on p. 284).

${ }^{486} \mathrm{D}$. Wilkinson and B. Macefield, "A parametrization of the phase space factor for allowed ß-decay", Nuclear Physics A 232, 58-92 (1974) (cit. on p. 290). 